\title{
Q-Serie
}

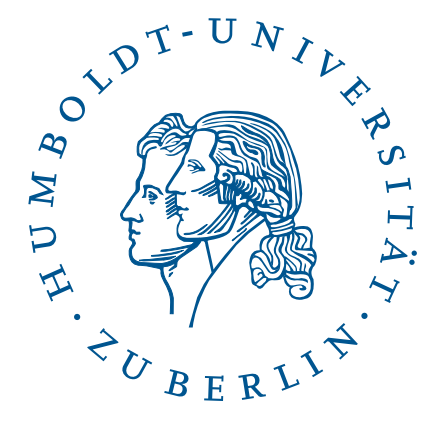

Nr. $15 \mid$ Berlin 2011

\section{Der Einfluss des Aktivierenden Staates auf Familienpolitik in Deutschland und den Niederlanden}

\section{Ansgar Willenborg}


Ansgar Willenborg

Der Einfluss des Aktivierenden Staates auf Familienpolitik in Deutschland und

den Niederlanden 
Ansgar Willenborg

\section{Der Einfluss des Aktivierenden Staates auf Familienpolitik in Deutschland und den Niederlanden}




\section{Bibliografische Information der Deutschen Nationalbibliothek}

Die Deutsche Bibliothek verzeichnet diese Publikation in der

Deutschen Nationalbibliographie; detaillierte bibliografische Daten sind im Internet über http://www.d-nb.de abrufbar.

\section{(c) $(1) \Theta(2) \Theta 211$ Humboldt-Universität zu Berlin}

Diese Publikation ist als elektronische Publikation abrufbar:

http://nbn-resolving.de/urn:nbn:de:kobv:11-100187512 


\section{Zusammenfassung}

Diese Arbeit betrachtet die Umsetzung des politisch-administrativen Leitbildes Aktivierender Staat in der Familienpolitik Deutschlands und der Niederlande. Es wird untersucht, ob es zu Parallelen in den Familienpolitiken kam. Hierdurch kann erstens die Frage nach der Umsetzung des Leitbildes in der Familienpolitik und zweitens die Frage nach familienpolitischen Konvergenzen innerhalb der Europäischen Union beantwortet werden. Um die Fragestellung $\mathrm{zu}$ bearbeiten wird zum Vergleich der beiden sozialstaatlich ähnlich eingerichteten Länder mit unterschiedlichen Kontextbedingungen ein Idealmodell Aktivierender Familienpolitik entwickelt. Es handelt sich hierbei um eine induktive Herangehensweise, bei welcher die Divergenzen und Konvergenzen der beiden Länder herausgearbeitet werden. Zur Entwicklung des Idealmodells werden Bandemers Indikatoren zur Beschreibung des Leitbildes Aktivierender Staat, die klassischen familienpolitischen Zielsetzungen und Instrumente von Kaufmann und die familienpolitischen Staatsinterventionstypologien von Esping-Andersen herangezogen. Die Beschreibung der relevanten familienpolitischen Ziele und Instrumente erfolgt anhand einer Dokumentenanalyse. Durch diese Arbeit wird der Begriff Aktivierende Familienpolitik konkretisiert. Außerdem wird sichtbar, welche Aktivierungsstrategien die beiden untersuchten Länder verfolgten, bzw. wie sie das Leitbild des Aktivierenden Staates umsetzten. Hierdurch kommt es nicht nur zur Bewertung der theoretischen Frage nach der Anwendung aktivierender Politiken in anderen Bereichen als der bereits häufig evaluierten Arbeitsmarktpolitik, sondern auch zu einer Übersicht verschiedener Aktivierungsbeispiele. Anhand einer Analyse der untersuchten Maßnahme und hinzugezogener Literatur werden Schlüsse gezogen im Hinblick auf familienpolitische Konvergenzen und Divergenzen zwischen den beiden Ländern. Die Bewertung der Lücken und Defizite des Leitbildes und die Diskussion über die mögliche Weiterentwicklung des Leitbildes runden die Arbeit ab.

Schlagwörter:

Verwaltungsreformen, Aktivierender Staat, Familienpolitik, Internationaler Vergleich 


\section{Abstract}

This dissertation investigates the influence of the political-administrative ideal of the 'activating state' on German and Dutch family policy. It is studied to what extent parallels can be discerned between the family policies that are pursued by these two countries. It is clarified how the aforementioned ideal is converted into family policy, whilst also offering an answer to the question whether any convergences exist in the field of family policy as pursued within the European Union. In order to be able to answer this question, an ideal model for activating family policy in different contexts has been created to facilitate a comparison between the two countries that are similarly organised from a welfare state perspective. This involves an inductive method in which the divergences and convergences of both countries have been worked out in greater detail. In view of the development of the ideal model, Bandemer's indicators for the ideal of an 'activating state' are studied in combination with Kaufmann's classic family policy goals and instruments, and Esping-Andersen's state intervention typologies for family policy. This is followed by a specification of the relevant family policy goals and instruments based on an analysis of various documents. The goal of this investigation is to concretise the concept of an activating family policy. Additionally, it is shown which 'activating strategies' have been used by both countries and how they have translated the ideal of an activating state. Not only does this offer an answer to the theoretical question about the use of activating policies in areas other than the often-evaluated labour market policies, but it also provides an overview of various examples of activating policy. Using an analysis of the investigated instruments and the corresponding literature, a number of conclusions have been formulated about the convergences and divergences in the family policies that are pursued by these two countries. The dissertation ends with an assessment of the ideal's gaps and shortcomings and a discussion about its possible further development.

Keywords:

Governmental reforms, Activating State, Family Policy, International Comparism 


\section{Abstract (dutch)}

Dit proefschrift onderzoekt de invloed van het politek-administratieve ideaalbeeld van de activerende overheid op het gezinsbeleid van Duitsland en Nederland. Er wordt gekeken of er parallelen zijn waar te nemen tussen het gezinsbeleid van deze landen. Hierdoor kan allereerst worden verduidelijkt hoe het ideaalbeeld in gezinsbeleid is vertaald en kan daarnaast de vraag over het bestaan van convergenties op het terrein van het gezinsbeleid binnen de Europese Unie worden beantwoord. Om de vraagstelling te kunnen beantwoorden wordt ter vergelijking van de twee vanuit welvaartsstaatsperspectief vergelijkbaar ingerichte landen met verschillende contexten een ideaalmodel voor activerend gezinsbeleid ontwikkeld. Het gaat hierbij om een inductieve werkwijze waarbij de divergenties en convergenties van beide landen zijn uitgewerkt. Met het oog op de ontwikkeling van het ideaalmodel wordt gebruik gemaakt van Bandemers indicatoren voor het ideaalbeeld van de activerende overheid, de klassieke gezinsbeleidsdoelen en instrumenten van Kaufmann en de familiepolitieke staatsinterventietypologieën van Esping-Andersen . De beschrijving van de relevante gezinsbeleidsdoelen en instrumenten volgt aan de hand van een documentenanalyse. Door dit onderzoek wordt de term activerend gezinsbeleid geconcretiseerd. Verder wordt zichtbaar gemaakt welke activeringsstrategieën de twee onderzochte landen hebben toegepast en hoe zij het ideaalbeeld van de activerende overheid hebben vertaald. Dit leidt niet alleen tot de beantwoording van de theoretische vraag naar het gebruik van activerend beleid op andere terreinen dan het reeds vaak geëvalueerde arbeidsmarktbeleid maar ook tot een overzicht van verschillende voorbeelden van activering. Door middel van een analyse van de onderzochte instrumenten en de bijbehorende literatuur worden conclusies getrokken over de convergenties en divergenties van het gezinsbeleid in deze twee landen. De beoordeling van de hiaten en tekortkomingen van het ideaalbeeld en de discussie over de mogelijke verdere ontwikkeling van het ideaalbeeld ronden dit onderzoek af. 



\section{Inhaltsverzeichnis}

1 Der aktivierende Wohlfahrtsstaat 7

1.1 Anwendungen politisch-administrativer Leitbilder zur

Steuerung sozialstaatlicher Entwicklungen seit dem Ende des

Zweiten Weltkrieges

1.1.1 Demokratischer Rechtsstaat 8

1.1.2 Aktiver Staat 9

1.1.3 Schlanker Staat 11

1.2 Aktivierender Staat 13

1.2.1 Hintergründe für die Entstehung des neuen Leitbildes 13

1.2.2 Sozialstaatliche Aufgaben und Unterscheidung wohlfahrtsstaatlicher Arrangements $\quad 14$

1.2.3 Ein neues Verhältnis zwischen Staat und Gesellschaft; Einordnung des Leitbildes Aktivierender Staat

1.2.4 Aktivierende politische Praxis 25

1.3 Neue Risiken - neue Verantwortungsteilung 31

1.3.1 Gerechtigkeit und Chancengleichheit 32

1.3.2 Schutz vor neuen Risiken 34

1.3.3 Differenzierung von Verantwortlichkeiten 36

1.3.4 Handlungsakteure im Aktivierenden Staat 38

1.3.5 Das Prinzip der Leistungskette 40

1.4 Verantwortungsstufung und Instrumente zur Aktivierung im Sozialstaat

1.4.1 Neues Staatsverhältnis und entsprechendes politisches Handeln 43

1.4.2 Aufgaben und Instrumente $\quad 46$

2 Entwicklung eines Idealmodells aktivierender Familienpolitik 54 
2.1 Die Entwicklung familienpolitischer Zielsetzungen und Instrumente 54

2.1.1 Allgemeine Hintergründe zur Familienpolitik 54

2.1.2 Allgemeine familienpolitische Zielsetzungen $\quad 56$

2.1.4 Allgemeine familienpolitische Instrumente $\quad 6_{1}$

2.2 Familienpolitik in der vergleichenden Wohlfahrtsforschung Esping-Andersens $\quad 6_{2}$

2.2.1 Einleitung und Beschreibung der Arbeitsschritte $\quad 62$

2.2.2 Analytische Betrachtungen der Werke EspingAndersens $\quad 65$

2.2.3 Familienpolitischer Ansatz Esping-Andersens $\quad 87$

2.3 Zusammenführung der Resultate vergleichender Wohlfahrtsforschung mit den allgemeinen familienpolitischen Erkenntnissen $\quad 91$

2.3.1 Einleitung und Beschreibung der Arbeitsschritte $\quad 91$

2.3.2 Zielsetzungen 94

2.3.3 Instrumente 97

2.3.4 Zusammenführung des Modells 100

2.4 Zusammenführung mit Resultaten Aktivierender Staat 103

2.4.1 Einleitung und Beschreibung der Arbeitsschritte 103

$\begin{array}{ll}2.4 .2 & \text { Zielsetzungen } \\ & 103\end{array}$

$\begin{array}{lll}2.4 .3 & \text { Instrumente } & 108\end{array}$

2.4.4 Zwischenfazit 111

2.6 Das Idealmodell Aktivierender Familienpolitik 114

3 Verwendung familienpolitischer Zielsetzungen und Instrumente in Deutschland 117

3.1 Beschreibung der Arbeitsweise 117

3.2 Familienpolitische Zielsetzungen 119

3.2.1 Bevölkerungspolitische Zielsetzungen 119

3.2.2 Emanzipatorische Zielsetzungen 125

3.2.3 Auf das Kindeswohl gerichtete Zielsetzungen $\quad 130$

3.3 Familienpolitische Instrumente in Deutschland 141 
3.3.1 Instrumente, die die ökonomische Situation der Familienhaushalte betreffen

3.3.2 Instrumente, die die ökologischen Bedingungen des Familienlebens betreffen

3.3.3 Instrumente zur Förderung oder Wiederherstellung von Kompetenzen der Familienangehörigen $\quad 163$

3.3.4 Zeit-Instrumente

4 Verwendung familienpolitischer Zielsetzungen und Instrumente in den Niederlanden

4.1 Beschreibung der Arbeitsweise $\quad 185$

4.2 Familienpolitische Zielsetzungen in den Niederlanden $\quad 188$

4.2.1 Bevölkerungspolitische Zielsetzungen 188

4.2.2 Emanzipatorische Zielsetzungen 190

4.2.3 Auf das Kindeswohl gerichtete Zielsetzungen 196

4.3 Familienpolitische Instrumente in den Niederlanden 207

4.3.1 Instrumente, die die ökonomische Situation der Familienhaushalte betreffen

4.3.2 Instrumente, die die ökologischen Bedingungen des Familienlebens betreffen

4.3.3 Instrumente zur Förderung oder Wiederherstellung von Kompetenzen der Familienangehörigen 223

4.3.4 Zeit-Instrumente 237

5 Analyse 245

5.1 Beschreibung der Arbeitsweise 245

5.2 Analyse der familienpolitischen Zielsetzungen 246

5.2.1 Bevölkerungspolitische Zielsetzungen 246

5.2.2 Emanzipatorische Zielsetzungen 249

5.2.3 Auf das Kindeswohl gerichtete Zielsetzungen 253

5.3 Analyse der familienpolitischen Instrumente 257

5.3.1 Ökonomische Situation der Familienhaushalte 258

5.3.2 Ökologische Situation der Familienhaushalte 266 
5.3.3 Förderung oder Wiederherstellung von Kompetenzen der Familienangehörigen $\quad 275$

5.3.4 Zeit-Instrumente 286

5.4 Zwischenfazit 293

5.4.1 Zwischenfazit Zielsetzungen 294

5.4.2 Zwischenfazit Instrumente 297

5.4.3 Von der Analyse zum Fazit 301

6 Fazit 303

6.1 Aufgreifen der Fragestellungen 303

6.2 Weiterentwicklung des Idealmodells Aktivierender $\begin{array}{ll}\text { Familienpolitik } & 304\end{array}$

6.2.1 Inhaltliche Bewertung der Zielsetzungen 304

6.2.2 Inhaltliche Bewertung der Instrumente 308

6.2.3 Abschließende Bewertung des Idealmodells 315

6.3 Erfahrungen aktivierender Familienpolitik im Zusammenhang mit dem Wohlfahrtsstaatsansatz von EspingAndersen 317

6.3.1 Aufgreifen des Wohlfahrtsstaatsansatzes $\quad 317$

6.3.2 Zur Weiterentwicklung der Themen Pfadabhängigkeit und De-Kommodifizierung $\quad 318$

6.3.3 Zur Bedeutung des Begriffs De-Familialisierung 323

6.3.4 Systemergänzungen auf Mikroniveau 325

6.4 Der Aktivierender Staat: Ein europäisches Sozialmodell in Entwicklung? 332

6.5 Zur Weiterentwicklung des Leitbildes Aktivierender Staat 335

Literaturverzeichnis 353

$\begin{array}{ll}\text { Anlagen } & 381\end{array}$ 


\section{Tabellenverzeichnis}

Tabelle 1: Schematische Darstellung der Arbeitsweise

Tabelle 2: Gemeinwohlkonzepte und Handlungsorientierungen von Staat und Bürgern - Positionierung des Aktivierenden Staates (Lamping/Schridde/Plaß/Blanke 2002: 32)

Tabelle 3: Idealtypen sozialer Risikoverteilung (Schmid 2007: 45)

Tabelle 4: Konzept der arbeitsteiligen Verantwortungsteilung (Quelle in Anlehnung an Reichard 2006) (Schuppert 2005: 39)

Tabelle 5: Verantwortungsstufung und Aktivierung im Sozialstaat - im Spiel von Angebot und Nachfrage (Quelle: in Anlehnung an Blanke: 2001a) (Lamping/Schridde 2004: 55, Bearbeitung Willenborg)

Tabelle 6: Verschobenes Aufgabenspektrum staatlicher Politik und veränderte Vorgehensweisen zur Umsetzung dieser Aufgaben (Walter 2000: 156)

Tabelle 7: Zwei Verhandlungslogiken (Blanke/Schridde/Plaß 2001: 17)

Tabelle 8: Charakteristiken der unterschiedlichen Wohlfahrtsregimes (Lammert 2005: 13), Bearbeitung Willenborg (2008)

Tabelle 9: Kernthemen Esping-Andersens

Tabelle 10: Politische Zielsetzungen und Instrumente (Kaufmann, 2002)

Tabelle 11: Politische Zielsetzungen und Instrumente (Kaufmann) eingefüllt mit Kernpunkten von Esping-Andersen

Tabelle 12: Familienpolitisches Basismodell unter Verwendung der Theorien von Esping-Andersen und Kaufmann

Tabelle 13: Aktivierender Staat - Aktive Gesellschaft (Olk 2000: 115), Bearbeitung Willenborg (2008) 
Tabelle 15: Chronologisches Übersichtstableau der demographischen Zielsetzungen in Deutschland

Tabelle 16: Chronologisches Übersichtstableau der emanzipatorischen Zielsetzungen in Deutschland

Tabelle 17: Chronologisches Übersichtstableau der auf das Kindeswohl gerichteten Zielsetzungen in Deutschland

Tabelle 18: Chronologisches Übersichtstableau der ökonomischen Instrumente in Deutschland

Tabelle 19: Chronologisches Übersichtstableau der ökologischen Instrumente in Deutschland

160

Tabelle 20: Chronologisches Übersichtstableau der Instrumente zur Wiederherstellung oder Förderung der Kompetenzen verschiedener Familienangehörigen in Deutschland

Tabelle 21: Chronologisches Übersichtstableau der Zeit-Instrumente in Deutschland

Tabelle 22: Chronologisches Übersichtstableau der demographischen Zielsetzungen in den Niederlanden

Tabelle 23: Chronologisches Übersichtstableau der emanzipatorischen Zielsetzungen in den Niederlanden

Tabelle 24: Chronologisches Übersichtstableau der auf das Kindeswohl gerichteten Zielsetzungen in den Niederlanden

Tabelle 25: Chronologisches Übersichtstableau der ökonomischen Instrumente in den Niederlanden

Tabelle 26: Chronologisches Übersichtstableau der ökologischen Instrumente in den Niederlanden

Tabelle 27: Chronologisches Übersichtstableau der Instrumente zur Förderung oder Wiederherstellung der Kompetenzen Familienangehöriger in den Niederlanden

Tabelle 28: Chronologisches Übersichtstableau der Zeit-Instrumente in den Niederlanden 
Tabelle 29: Aktivierende ökonomische Instrumente in Deutschland

Tabelle 30: Aktivierende ökonomische Instrumente in den Niederlanden

Tabelle 31: Aktivierende ökologische Instrumente in Deutschland 270

Tabelle 32: Aktivierende ökologische Instrumente in den Niederlanden

Tabelle 33: Aktivierende Instrumente zur Förderung oder Wiederherstellung von Kompetenzen von Familienangehörigen in Deutschland $\quad 278$

Tabelle 34: Aktivierende Instrumente zur Förderung oder Wiederherstellung von Kompetenzen von Familienangehörigen in den Niederlanden $\quad 283$

Tabelle 35: Aktivierende Zeit-Instrumente in Deutschland 290

Tabelle 36: Aktivierende Zeit-Instrumente in den Niederlanden 292

Tabelle 37: Idealmodell Aktivierender Familienpolitik 294

Tabelle 38: Westeuropäische Staaten nach Wohlfahrtsstaats- bzw. Familienregime und Staatsaufbau (Schmidt 2006: 86f, Bearbeitung Willenborg 2009) 



\section{Abkürzungsverzeichnis}

\begin{tabular}{|c|c|}
\hline BaföG & Bundesausbildungsförderungsgesetz \\
\hline Bev.pol.Z. & Bevölkerungspolitische Zielsetzungen \\
\hline BLK & $\begin{array}{l}\text { Bund-Länder Kommission für Bildungspl. und } \\
\text { Forschungsförderung }\end{array}$ \\
\hline BMFSFJ & $\begin{array}{l}\text { Bundesministerium für Familie, Senioren, Frauen und } \\
\text { Jugend }\end{array}$ \\
\hline BZgA & Bundeszentrale für gesundheitliche Aufklärung \\
\hline & Niederländisches Zentrum für Jugend und Familie \\
\hline Emanz.Z. & Emanzipatorische Zielsetzungen \\
\hline$E \& C$ & $\begin{array}{l}\text { Entwicklung und Chancen junger Menschen in sozialen } \\
\text { Brennpunkten }\end{array}$ \\
\hline $\begin{array}{l}\text { Fam.inst.Z. } \\
\text { FLA }\end{array}$ & $\begin{array}{l}\text { Familieninstitutionelle Zielsetzungen } \\
\text { Familienlastenausgleich }\end{array}$ \\
\hline FörMig & $\begin{array}{l}\text { Förderung von Kindern und Jugendlichen mit } \\
\text { Migrationshintergrund }\end{array}$ \\
\hline KAUSA & $\begin{array}{l}\text { Koordinierungsstelle Ausbildung in ausländischen } \\
\text { Unternehmen }\end{array}$ \\
\hline KGSt & $\begin{array}{l}\text { Kommunale Gemeinschaftsstelle für } \\
\text { Verwaltungsmanagement }\end{array}$ \\
\hline KICK & Gesetz zur Weiterentwicklung der Kinder- und Jugendhilfe \\
\hline KW.Z. & Auf das Kindeswohl gerichtete Zielsetzungen \\
\hline I.K. & $\begin{array}{l}\text { Instrumente zur Förderung oder Wiederherstellung von } \\
\text { Kompetenzen der Familienangehörigen }\end{array}$ \\
\hline NPM & New Public Management \\
\hline NSM & Neues Steuerungsmodell \\
\hline Ökol.ı. & $\begin{array}{l}\text { Instrumente, die die ökologischen Bedingungen des } \\
\text { Familienlebens betreffen }\end{array}$ \\
\hline Ökon.I. & $\begin{array}{l}\text { Instrumente, die die ökonomischen Bedingungen des } \\
\text { Familienlebens betreffen }\end{array}$ \\
\hline ÖPP & Öffentlich-Private Partnerschaft \\
\hline PPP & Public Private Partnership \\
\hline PSPP & Public Social Private Partnership \\
\hline R.I. & $\begin{array}{l}\text { Instrumente, die den rechtlichen Status der } \\
\text { Familienmitglieder betreffen }\end{array}$ \\
\hline ROC & $\begin{array}{l}\text { Niederländisches regionales Unterrichtszentrum für } \\
\text { Berufsschulunterricht }\end{array}$ \\
\hline Soz.pol.Z & Sozialpolitische Zielsetzungen \\
\hline TAG & Tagesbetreuungsausbaugesetz \\
\hline TzBfC & Teilzeit- und Befristungsgesetz \\
\hline ZAT & $\begin{array}{l}\text { Niederländische Pflege- und Beratungsteams in der } \\
\text { Jugendhilfe }\end{array}$ \\
\hline & Zeit-Instrumente \\
\hline
\end{tabular}





\section{Einleitung}

Diese Arbeit baut auf den Erkenntnissen des postgraduierten Master of European Governance and Administration (MEGA) und insbesondere auf der Masterarbeit zum Thema ,Dominierende Politisch-Administrative Leitbilder und Ihr Einfluss auf fachpolitische Zielsetzungen; Ein deutsch-niederländischer Vergleich' auf. In dieser Masterarbeit wurde der Einfluss des Leitbildes Aktivierender Staat auf familienpolitische Zielsetzungen Deutschlands und der Niederlande untersucht. Wichtigstes Ergebnis dieser Arbeit war die Feststellung, dass das Leitbild Aktivierender Staat seine Anwendung fand in den Familienpolitiken beider Länder. Da die Frage nach der Art der Umsetzung des Leitbildes in ein politisches Instrumentarium für die beiden ähnlichen Länder mit unterschiedlichen Kontextbedingungen den Rahmen einer Masterarbeit allerdings gesprengt hätte und daher offen blieb, ist sie Thema dieser Dissertation geworden.

Blanke, Schridde und Plaß definieren den Aktivierenden Staat als eine neu gelebte Verantwortungsteilung zwischen Staat und Gesellschaft zur Realisierung gemeinsamer Ziele im Hinblick auf Fortschritt und Solidarität. Sie weisen in diesem Zusammenhang auf die Kooperation und Koproduktion staatlicher, halbstaatlicher und privater Akteure in der Verfolgung des öffentlichen Interesses. Der Aktivierende Staat wolle sein Engagement mit Eigeninitiative und Eigenverantwortung von Bürgerinnen und Bürgern verbinden und eine neue Leistungsaktivierung in allen Gliedern der Wertschöpfungskette öffentlicher Leistungen erreichen (vergl. Blanke/Schridde/Plaß 2001: 8). Forschungsziel dieses Dissertationsvorhabens ist es zu untersuchen, wie das politisch-administrative Leitbild des Aktivierenden Staates in der Familienpolitik Deutschlands und der Niederlande verwendet wurde. Wie wurde die gesellschaftliche Verantwortung zwischen verschiedenen Akteuren geteilt? Die Fragestellung dieser Arbeit lautet dann auch:

"Welche Anwendung fand das Leitbild des Aktivierenden Staates in der Familienpolitik Deutschlands und der Niederlande?" 
Aus den Ergebnissen dieser Fragestellung wird deutlich inwiefern es zu Parallelen und Unterschieden kam und ob die Verwendung desselben Leitbildes eine Angleichung familienpolitischer Instrumente zur Folge hatte.

Bevor das Instrumentarium des Aktivierenden Staates und somit die Frage der Umsetzung von Theorien beantwortet wird, werden in dieser Forschungsarbeit die Hintergründe wohlfahrtstaatlicher Gegebenheiten und das Warum der Reformen erläutert, denn einige sozialstaatliche Eigenschaften und Hintergründe stehen nicht im Zusammenhang mit dem zu untersuchenden Leitbild, haben aber dennoch einen maßgeblichen Einfluss auf sein Wirken bzw. auf die Anwendung seines Instrumentariums.

Um die Fragestellung $\mathrm{zu}$ bearbeiten wird zum Vergleich der beiden sozialstaatlich ähnlich eingerichteten Länder mit unterschiedlichen Kontextbedingungen (Konkordanzmethode) ein Idealmodell Aktivierender Familienpolitik entwickelt. Es handelt sich hierbei um eine induktive Herangehensweise, bei der die Divergenzen und Konvergenzen der beiden Länder herausgearbeitet werden.

Zur Entwicklung des Idealmodells werden Bandemers Indikatoren zur Beschreibung des Leitbildes Aktivierender Staat, die klassischen familienpolitischen Zielsetzungen und Instrumente von Kaufmann und die familienpolitischen Staatsinterventionstypologien herangezogen. Der Schwerpunkt bei der Konstruktion des Idealmodells liegt aber auf den Ausführungen von Esping-Andersen zum Thema aktivierende Familienpolitik. Er betont in seiner Wohlfahrtsstaatstheorie die demographischen, emanzipatorischen und sozialpolitischen Zielsetzungen von Familienpolitik. Entsprechend ihrer in der Vergangenheit geführten Politik unterscheidet er in Europa zwischen sozialdemokratischen, konservativen und liberalen Staaten. Durch ihre Vergangenheit seien die Staaten auch in Zukunft an eine bestimmte Route gebunden (Pfadabhängigkeit). Deutschland und in etwas geringerem Maße auch die Niederlande beschreibt Esping-Andersen als konservative Staaten. Für die Umsetzung aktivierender Maßnahmen verweist er auf Integralität mit den anderen sozialpolitischen Bereichen und betont er vor allem 
die Rolle der Frauen in der Familienpolitik und die Chancengleichheit von Kindern'. Seine Theorien erfahren in dieser Arbeit eine Erweiterung um die allgemeinen Indikatoren des Aktivierenden Staates von Bandemer. Der Fokus dieses Modells liegt auf den Indikatoren, die Bandemer als ,Neue Verantwortungsteilung' und ,Koproduktion' bezeichnet. Im Zusammenhang mit der klassischen Unterscheidung von Zielen und Instrumenten, so wie durch Kaufmann verwendet, ergeben sich allgemein anwendbare Indikatoren für aktivierende Familienpolitik als Teil einer aktivierenden Sozialpolitik.

Für die Beschreibung der relevanten familienpolitischen Zielsetzungen und Instrumente wird eine Dokumentenanalyse verwendet, bei der die Koalitionsvereinbarungen und Regierungserklärungen der rot-grünen Koalitionen, der Großen Koalition, der Schwarz-Gelben Koalition, der beiden violetten Kabinette und drei der vier Kabinette unter Balkenende erörtert werden. Entsprechende Stellungnahmen der Bundesregierung zum Fünften-, Sechsten- und Siebten Familienbericht, zum Elften-, Zwölften- und Dreizehnten Kinder- und Jugendbericht sowie die Familienberichte ,Notitie Gezin', ,Nota Gezin' und 'Nota Gezinsbeleid 2008' erweitern diese Erörterung. An einigen Stellen werden außerdem noch internationale Studien hinzugezogen, um die politischen Instrumente der beiden Länder in ihrem Zusammenhang zu betrachten. Da es sich in dieser Arbeit um eine Dokumentenanalyse politischer Texte handelt, werden Instrumente aus dem Rechtsbereich in dieser Arbeit nicht erörtert. Natürlich sind sie für die Familienpolitik sehr entscheidend, im Hinblick auf den Rahmen dieser Analyse wird sich allerdings auf eine PolicyAnalyse beschränkt.

Im folgenden Schaubild wird die Herangehensweise dieser Arbeit schematisch dargestellt.

1 In seinem Werk ,The Incomplete Revolution - Adapting to Women's New Roles' betont er auch eine grundsätzliche Rentenreform als relevanten Teil der Familienpolitik. Er geht auf diesen neuen Schwerpunkt seiner Arbeiten aber nur so begrenzt ein, dass dieser Bereich in der hier vorliegenden Arbeit nicht berücksichtigt wurde. 
Tabelle 1: Schematische Darstellung der Arbeitsweise

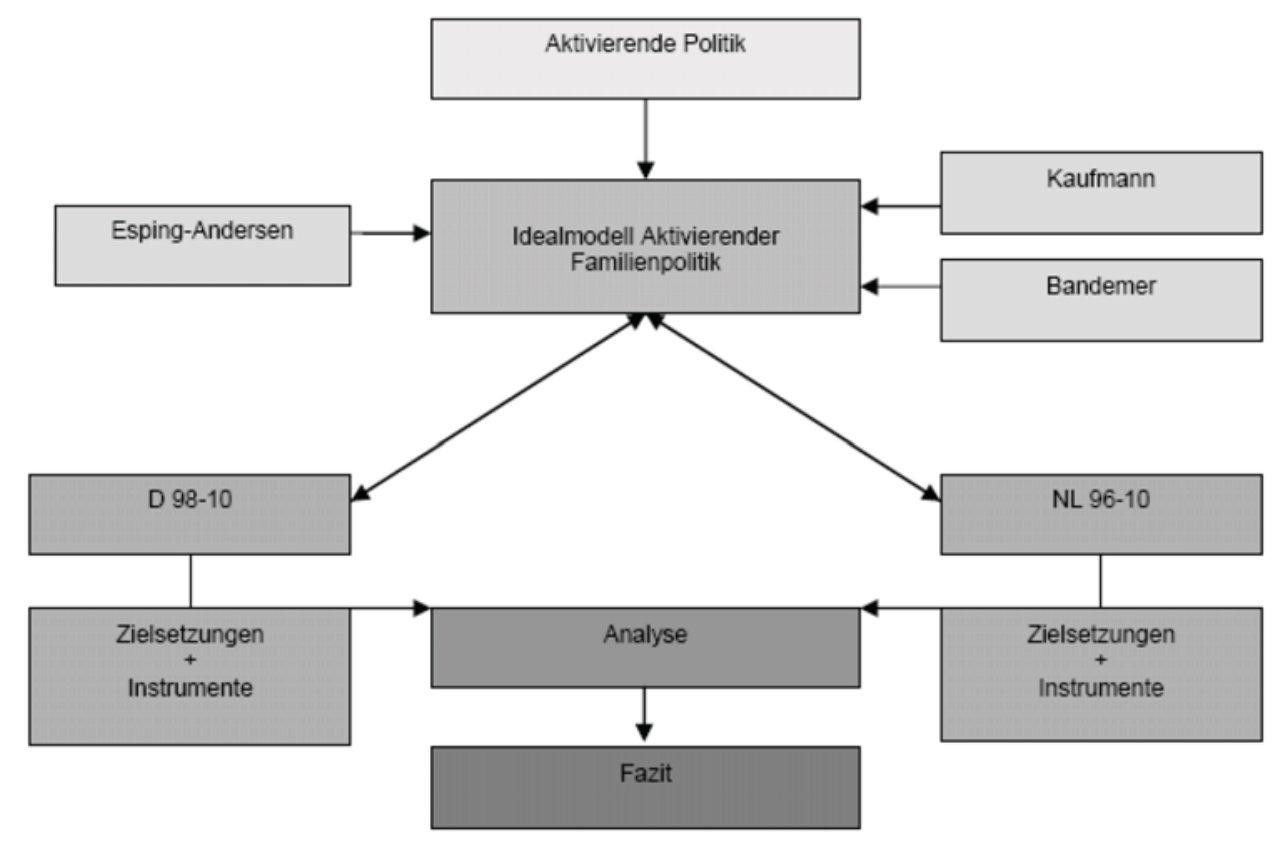

Durch diese Arbeit wird der Begriff Aktivierende Familienpolitik konkretisiert. Welche Ziele und Instrumente kennt dieses Leitbild bzw. dieses Modell? Außerdem wird sichtbar, welche Aktivierungsstrategien die beiden untersuchten Länder verfolgten, bzw. wie sie das Leitbild des Aktivierenden Staates umsetzten. Hierdurch wird nicht nur die theoretische Frage nach der Anwendung aktivierender Politiken in einem anderen Bereich als der bereits häufig evaluierten Arbeitsmarktpolitik beantwortet, sondern auch eine Übersicht verschiedener Herangehensweisen geboten. Reformern in Politik und Verwaltung können hierdurch Unterschiede verdeutlicht und interessante Beispiele genannt werden.

Deutlich werden anhand dieser Arbeit aber auch der unterschiedliche Einfluss und die Verwendung politischer Dokumente. Koalitionsvereinbarungen kennen ein anderes Gewicht als Familienberichte. In Deutschland ist die Familienberichterstattung institutionalisiert während Politiker in den Niederlanden nach eigenem Ermessen Noten zum Thema Familie schreiben. Hinzu kommt die Tatsache, dass es in Deutschland seit einigen Jahrzehnten ein Familienministerium gibt, während das Thema Familie in den Niederlanden auch nach der Einführung eines Programministeriums für Jugend und Familie stark dezentralisiert ist. 
Weiterhin bietet diese Arbeit Hinweise im Bezug auf die politische Umsetzung der Lissabonstrategie auf Mikroniveau. Während Esping-Andersen aufgrund quantitativer Makroanalysen Schlüsse über die Angleichung der europäischen Familienpolitiken zieht, werden in dieser Arbeit die familienpolitischen Routen zweier Länder auf Detailniveau verglichen. Konvergenzen und Divergenzen werden hierdurch verdeutlicht und der familienpolitische Pfad beider Länder dargestellt. 


\section{Der aktivierende Wohlfahrtsstaat}

\subsection{Anwendungen politisch-administrativer Leitbilder zur Steuerung sozialstaatlicher Entwicklungen seit dem Ende des Zweiten Weltkrieges}

Herausforderungen interner und externer Art führten und führen noch heute dazu, dass sich die westlichen Sozialstaaten seit Ende des Zweiten Weltkrieges regelmäßig erneuert haben bzw. erneuern müssen. Die Rolle der Politik und Verwaltung wird und wurde dabei maßgeblich beeinflusst von der Frage nach der Reichweite staatlicher Interventionen. Politik und Verwaltung haben in verschiedenen Reformen regelmäßig nach einer Balance zwischen dem Ideal des Wohlfahrtsstaates einerseits und dem Ideal des neoliberalen Staates andererseits gesucht. Es gab hierbei in der Vergangenheit kein einheitliches europäisches Reformmodell. Allerdings weisen die einzelnen europäischen Länder neben einigen Unterschieden auch deutliche Gemeinsamkeiten auf.

Um das gefundene Gleichgewicht zwischen den beiden genannten Idealen in politisches Handeln zu übersetzen, haben Reformer in Politik und Verwaltung entsprechende Metaphern, bzw. Leitbilder verwendet, die ihre eigene Evolution kennen. "Generell verkörpert ein Leitbild eine Philosophie, einen visionären Endzustand, der durch eine strategische Zielvorgabe als Wegweiser Handlungsgrundsätze für die Zukunft vorgibt“ (Reichard/Schuppan 2000: 84). Werner Jann spricht daher von politischen Steuerungsfaktoren, die er als „Lange Wellen der Leitbildentwicklung" (Jann 2005) beschreibt. Seiner Theorie zufolge kann man seit Ende des Zweiten Weltkrieges in Deutschland die Anwendung folgender dominierender politisch-administrativer Leitbilder unterscheiden:

- Demokratischer Rechtsstaat (1945-1965),

- Aktiver Staat (1965-1979),

- Schlanker Staat (1980-1995) und

- Aktivierender Staat (seit 1996). 
Die Unterscheidung dieser Leitbilder deutet auf den ersten Blick auf eine komplette Erneuerung eines Leitbildes nach 15-20 Jahren. Vielmehr handelt es sich hierbei aber um eine Entwicklung des bestehenden Leitbildes durch Hinzufügung neuer Gedanken. Es ist ein permanenter Lernprozess bei dem durch neue Spielregeln und Strukturen des Zusammenspiels diverser Akteure auf Schwächen, Probleme und Lücken des vorherigen Leitbildes antizipiert bzw. auf verändernde Situationen und Risiken institutionell reagiert wird (vergl. Engelen/Hemerijk/Trommel 2007: 11). Die Kategorisierung der Leitbilder ist relativ und die Einführung eines neuen Leitbildes bedeutet nicht, dass die Reformer das bereits bestehende Leitbild komplett verworfen haben.

Das aktuellste Leitbild, das im Mittelpunkt dieser Arbeit steht, ist das Leitbild des Aktivierenden Staates, welcher sich auszeichnet durch eine neue und prinzipielle Betrachtung der Verantwortlichkeiten in Staat und Gesellschaft. Die Logik dieses Leitbildes hatte einen großen Einfluss auf die Staats- und Verwaltungsmodernisierung seiner Zeit. „Dies gilt sowohl für:

- Die Definition und Abgrenzung dessen, was in den öffentlichen Aufgabenbereich gehört, als auch für

- Die Suche nach den jeweils am besten für die Aufgabenerledigung geeigneten Akteuren sowie für

- Die Erarbeitung von Instrumenten zur Steuerung und Gewährleistung einer anspruchsvollen und sich ständig verbessernden Leistungserbringung“ (Bandemer 2005: 31).

In den folgenden Abschnitten werden die ersten drei von Jann unterschiedenen Leitbilder näher definiert.

\subsubsection{Demokratischer Rechtsstaat}

Durch die traumatisierende Wirkung des Zweiten Weltkrieges bzw. der institutionellen Ursachen, die diesem zu Grunde lagen, war bis in die Mitte der sechziger Jahre die Gewährleistung eines demokratischen Rechtsstaates der zentrale Schwerpunkt des jungen Sozialstaates. Nach Weberschem Beispiel wurde ein bürokratischer Staat aufgebaut, der den Bürgern gerechte Behandlung im Sinne einer Verfahrensgerechtigkeit sicherte. Vor dem 
Weltkrieg vorhandene Ressorts nahmen ihre Arbeit wieder auf und neue Ministerien wurden gegründet (z.B. 1953 das Familienministerium). Aufgrund der funktionierenden Verfahrensgerechtigkeit haben Bevölkerung und Wirtschaft sich in den Folgejahren von den traumatischen politischen Erfahrungen erholen können und sich neuen gesellschaftlichen Entwicklungen bzw. Techniken gewidmet. Obwohl entscheidende Weichen der heutigen Sozialpolitik in beinahe allen europäischen Ländern mit dieser Periode verbunden sind, hat sich kein einheitlich-europäisches Sozialstaatsmodell bzw. einheitlich-europäische Sozialpolitik entwickeln können (vergl. Hemerijk 2007: 88).

\subsubsection{Aktiver Staat}

Starkes wirtschaftliches Wachstum, technischer Fortschritt und politische Stabilität seit Ende des Zweiten Weltkrieges führten $\mathrm{zu}$ neuen Herausforderungen für den Sozialstaat. Die persönlichen Perspektiven der Bevölkerung hatten sich verbessert, und neue gesellschaftliche Probleme taten sich in den sechziger Jahren vor. Die Erwartungen der jungen Bevölkerung an die Problemlösungsfähigkeit des neuen Staates und seiner politischen Administration waren hoch. So entstand auch in der Bundesrepublik das Bedürfnis, die technischen und gesellschaftlichen Entwicklungen sowie das Wachstum der Wirtschaft im Sinne einer keynesianischen Wirtschafts- und Sozialpolitik, in welcher der Staat seinen Einfluss auf die Kräfte der Marktwirtschaft (durch z.B. Kartelle, semiautonomen Staatskonzernen usw.) ausübt, zu steuern. Solche Steuerungsmechanismen wurden in anderen Staaten schon seit längerer Zeit angewandt. Der Keynesianismus bot „eine Reihe wirtschaftspolitischer Instrumente, die die zyklischen Schwankungen kapitalistischer Wirtschaftsentwicklung dämpften und es eine Zeit lang erlaubten, sowohl hohe Wachstumsraten als auch Beschäftigungsquoten zu erzielen. Die entwickelten Industriestaaten konnten so die ,Große Transformation' (im Sinne von Polanyi 1957) vollziehen und die ökonomische Effizienz des dynamischen Kapitalismus nutzen, ohne gleichzeitig dessen Krisen und ungleiche Verteilungseffekte hinnehmen zu müssen“ (Lütz 2004: 11). Vor allem während der Regierungsperiode der ersten Großen Koalition am 
Ende der sechziger Jahre spielte der Keynesianismus in Deutschland eine wichtige Rolle.

Der Aktive Staat griff bis zur wirtschaftlichen Krise der siebziger Jahre durch rationale Planung in sozialwirtschaftliche Abläufe ein „mit dem Ziel, wirtschaftliche Ungleichgewichte und Folgekosten wie Arbeitslosigkeit auszugleichen oder abzufedern. In dieses Paradigma passt nicht nur eine Umverteilung des Bruttosozialprodukts oder Arbeitsbeschaffungsmaßnahmen sondern ebenso Planung und Gestaltung gesellschaftlicher Prozesse" (Schubert/Bandelow 2003: 158). Dem Staat kam hierbei eine zentrale Rolle zu. Er war verantwortlich für das individuelle Wohlergehen seiner Bürger. Das Vertrauen der Bürger in den jungen Staat war höher als in die Kräfte des Marktes bzw. der Selbstorganisation der Gesellschaft. Die Menschen erhofften von dem neuen Staat „die Fähigkeit zur vorausschauenden, aktiven Regelung und Steuerung jener gesellschaftlichen und wirtschaftlichen Prozesse, deren ungesteuerte Dynamik die für das Gesamtsystem relevanten Probleme und Krisen hervorbringt“ (Mayntz/Scharpf 1973: 116). In den Niederlanden wird in diesem Zusammenhang auch gesprochen von der ,gestaltbaren Gesellschaft'. „Die Beziehung von Staat und Gesellschaft im Aktiven Staat wird von einer Kernidee geleitet: der Staat ist ,Garant des Gemeinwohls'. Daran wird staatliches Handeln ausgerichtet, und der Politik fällt in diesem Gefüge eine Führungsrolle zu (...) Profunde Kritik am Aktiven Staat zielt daher darauf, dass effektive Steuerung die Selbstregulierungskräfte der Gesellschaft stärker einbeziehen und somit ,Planung' sich mehr als Bestandteil des jeweiligen gesellschaftlichen Zusammenhangs begreifen muss“ (Lamping/Schridde/Plaß/Blanke 2002: 8f).

Wirtschaft und Gesellschaft galten als gestaltbar. „Die Inanspruchnahme von Sozialleistungen zählte neben den politischen und ökonomischen Freiheitsrechten zum Kanon sozialer Bürgerrechte“ (Lütz 2004: 11f). Diese Inanspruchnahme hat zu einer Flut von Regelungen geführt, die in der gegenwärtigen Politik oft als Blockade erfahren werden. Mit den neuen Aufgaben wuchsen die Ministerien sowohl in ihrer Anzahl als auch in der Vielzahl ihrer Beschäftigten. Eine relativ junge Bevölkerung und eine starke Wirtschaft finanzierten diese Zunahme staatlicher Aufgaben. 


\subsubsection{Schlanker Staat}

In vielen westlichen Sozialstaaten fand der ungezügelte wirtschaftliche Aufschwung in den achtziger Jahren ein abruptes Ende und wurde die öffentliche Haushaltslage der verschiedenen staatlichen Ebenen stets schwieriger. Der Aktive Staat, der Gesellschaft und Wirtschaft durch rationale Planung und Steuerung gestalten wollte, war in eine Krise geraten und entwickelte sich zunehmend „vom Wohlfahrtsstaat zum Wettbewerbsstaat“ (Bode 2004: 74). Die konservativen, politischen Werte von Thatcher und Reagan hatten in diesen Jahren viel Einfluss. Es wurde über das Ende des Wohlfahrtsstaates diskutiert. Er sollte konsequent nach wirtschaftlichem Modell reformiert werden. „Politische Unternehmer wie Thatcher und Reagan bedienten sich eines Triptychons vom ,überlasteten Staat', neoliberaler Ideologie und Globalisierung, um es als machtvolle taktische Waffe im öffentlichen Diskurs einzusetzen und es den Wählern ebenso wie der Öffentlichkeit (...) schmackhaft zu machen. Sie schufen den Wettbewerbsstaat Typ I“ (Evans/Cerny 2004: 210).

Charakteristisch für die Reformdebatte waren Themen wie Entbürokratisierung, Steigerung der Effizienz sowie Einführung von Managementmethoden aus dem privaten Sektor. In den Vereinigten Staaten kam es zur Entwicklung und zum Einsatz des ,New Public Managements' (NPM). Im Gegensatz zu der bisherigen Verwaltungsnorm wird dieses Modell nicht mehr vorrangig durch die Verfahrenssicherheit beherrscht, sondern liegt der Schwerpunkt auf den erzielten Ergebnissen. Outputsteuerung lautet von dem Zeitpunkt an die Maxime. Hiermit geht ein verändertes Rollenverständnis einher, denn nicht mehr die Steuerung über Regeln, sondern über Ziele steht im Mittelpunkt. Beamte und andere Leistungserbringer sollen sich nicht mehr (nur) an der Ordnungsmäßigkeit der Handlungsabläufe sondern (vor allem) an den Wünschen der Bürger bzw. Kunden orientieren. In Deutschland wird im Zusammenhang mit dem veränderten Verwaltungsansatz vom Neuen Steuerungsmodell (NSM) gesprochen (vergl. Jann 2005: 29). Vor allem auf kommunaler Ebene wurde dieses Modell frühzeitig verwendet. Entscheidend mitgewirkt an der Entwicklung dieses Modells hat die Kommunale Gemeinschaftsstelle für Verwaltungsmanagement (KGSt), die auf der Basis der Reformerfahrungen der niederländischen Stadt Tilburg ein deutsches Reformmodell entwickeln konnte. 
Weniger konsequent wurde der Umbau des Sozialstaats im Deutschland der achtziger Jahre auf Bundesebene verfolgt. In seiner Regierungserklärung erklärte Bundeskanzler Kohl 1982 mit einer Rückbesinnung staatlicher Aufgaben auf ursprüngliche Aufgaben beginnen zu wollen. Diese Ankündigung führte allerdings nicht zu einer strukturellen Aufgabenkritik. Erst „nach über einem Jahrzehnt Regierungsverantwortung verkündete Bundeskanzler Kohl im Jahre 1994 die notwendige Rückführung des Staates auf seine originären Aufgaben (...) Im Jahr 1995 wurde der Sachverständigenrat ,Schlanker Staat' einberufen, der die Aktivitäten zum Thema Abbau staatlicher Leistungen und überflüssige Bürokratie fachlich und politisch begleiten sollte“ (Lamping/Schridde/Plaß/Blanke 2002: 12f).

Während der Umbau des Sozialstaates im angelsächsischen Raum dem Prinzip des Neoliberalismus folgt, bei dem das Streben nach individueller Freiheit im Mittelpunkt des politischen Denkens steht und wirtschaftliches Handeln als Maxime staatlicher Koordination gilt, wird das Leitbild des Schlanken Staates in Deutschland vom Prinzip des Ordoliberalismus begleitet, „der sichtbare(n) Hand des Staates, die vom Staat zu konstituierende, von ihm zu gewährleistende und ständig $\mathrm{zu}$ überwachende Ordnung des veranstalteten Wettbewerbs“" (Lamping/Schridde/Plaß/Blanke 2002: 14). Für die politische Praxis bedeutet dies eine Bevorzugung marktkonformer Interventionen durch z.B. subsidiäre Hilfesysteme durch Ordoliberale während Neoliberale prinzipiell die größtmögliche Übernahme öffentlicher Aufgaben durch private Unternehmen befürworteten. Im Gegensatz zum Aktiven Staat wurden die Selbstregulierungsmöglichkeiten der Gesellschaft unterstrichen und Staatsausgaben vermindert bzw. effizienter eingesetzt.

Genau wie das Leitbild des Aktiven Staates weist auch das Leitbild des Schlanken Staates einige kritische Punkte auf. Während das erstgenannte Leitbild vor allem die Handlungsfähigkeit des Staates überbetont, ist das andere Extrem, die gesellschaftlichen Selbstregulierungskräfte, auch nicht allheilend. Weiterhin stellt sich die Frage, wie Aufgaben, die zuvor durch den Staat wahrgenommen wurden verantwortungsvoll an die Privatwirtschaft übergeben werden können nicht immer ganz leicht zu beantworten. 


\subsection{Aktivierender Staat}

Die Entstehung und Entwicklung des Aktivierenden Staates ist das Thema des zweiten Unterkapitels. Hier werden die im vorigen Unterkapitel bereits angedeuteten politisch-administrativen Veränderungen näher erläutert. Vor dem Hintergrund sozialstaatlicher Aufgaben und der Unterscheidung wohlfahrtsstaatlcher Arrangements kommt es $\mathrm{zu}$ einer Definition des Aktivierenden Staates. Da es keine eindeutige und allgemein gültige Definition des Aktivierenden Staates gibt, werden nach einer kurzen Einführung in die Hintergründe die unterschiedlichen Schulen und einflussreichen Theorien zusammengefasst und das Leitbild des Aktivierenden Staates im theoretischen Hintergrund verortet. Konkretisiert und näher verdeutlicht wird die Theorie anhand der politischen Praxis Deutschlands und der Niederlande.

\subsubsection{Hintergründe für die Entstehung des neuen Leitbildes}

Die in den achtziger Jahren noch von allen Seiten befürwortete Reduzierungsund Einsparungsmaxime des Schlanken Staates stieß an ihre Grenzen. "Die Herausforderungen, vor denen die Wohlfahrtsstaaten der EU zu Beginn des 21. Jahrhunderts stehen, sind die Folge eines Zusammentreffens dreier Faktoren. Von außen wird der Wohlfahrtsstaat konfrontiert mit der Zunahme der internationalen Konkurrenz (...) Von innen sind es fließende aber eingreifende Veränderungen in Arbeitsmarktmuster, Familienleben und Lebensstilen sowie dem demographischen Aufbau der Gesellschaft, die neue Anforderungen stellen an Sozialpolitik (...) Aus der Vergangenheit drücken die kollektiven Verpflichtungen, die entworfen wurden auf Basis der nachkriegszeitlichen Konkurrenzverhältnisse, demographischen Muster und Arbeitsmarktmuster, als eine nicht endende Schuld auf dem Haushalt" (Hemerijk 2007: 83ff). Die Gewährleistung der sozialen Gerechtigkeit stand im Mittelpunkt, und das Leitbild des Schlanken Staates konnte dem nicht mehr gerecht werden.

Seit Beginn der neunziger Jahre entwickelte sich daher in den Vereinigten Staaten und seit Mitte der neunziger Jahre dann auch in Europa der Aktivierende Staat. Um diesen Begriff in seiner Vollständigkeit zu erläutern, wird im folgenden Abschnitt eine Umschreibung sozialstaatlicher Aufgaben und eine Unterscheidung wohlfahrtsstaatlicher Arrangements aufgenommen. 
Da der Begriff Aktivierender Staat nicht einheitlich umgesetzt wurde, werden anschließend daran diverse Definitionen aktivierender Politiken und das hiermit einhergehende, verändernde Staatsverständnis umschrieben. Dieser theoretischen Auseinandersetzung mit dem Begriff folgt eine Beschreibung praktischer Politiken in Deutschland und in den Niederlanden am Ende des Unterkapitels.

\subsubsection{Sozialstaatliche Aufgaben und Unterscheidung wohlfahrtsstaatlicher Arrangements}

Die Entstehung des Sozialstaates wird in der Regel in Verbindung gebracht mit den Bismarckschen Sozialgesetzgebungsgrundsätzen, in denen das Schutzprinzip, „also die Absicherung gegen die Standardrisiken der Lohnarbeiterexistenz und das Prinzip der umfassenden Daseinsvorsorge durch ein flächendeckendes System sozialer Dienste und Infrastruktureinrichtungen“ (Olk 2000: 106) verankert war. In diesem Zusammenhang wird oft auch das Beveridge-Modell erwähnt, welches, aufbauend auf den Erkenntnissen von Keynes, die Einrichtung des britischen Wohlfahrtsstaates nach dem Ende des Zweiten Weltkrieges stark beeinflusste und sich durch eine staatlich organisierte Einheitsversicherung "gegen die wichtigsten Lebensrisiken“ (Kaufmann 2003: 83) mit niedrigen Leistungen kennzeichnet. Sozialstaatliche Aufgaben dehnten sich im Laufe der Jahre aus, und somit konnten die Funktionen des Rechtstaates zusammengefasst als: Gewährleistung der sozialen Gerechtigkeit, Förderung von Chancengleichheit, Bereitstellung von Infrastruktur und Handlungsspielräume/strategische Akteure (Arbeitsrecht...) beschrieben werden.

Der Staat nahm die Aufgabenerfüllung wahr. „Im Vordergrund stand eine Vielzahl sozialstaatlicher Leistungen in Form von monetären Transfers (...) Auf diesem Wege wurden die Funktionsfehler der Marktwirtschaft ausgeglichen“ (Grundsatzabteilung, DGB-Bundesvorstand 2000: 201). Esping-Andersen fasst in seinen Studien sieben potentielle Funktionen eines Wohlfahrtsstaates zusammen als: „Armutsbekämpfung, Armutsprävention, Bereitstellung sozialer Sicherheit, Einkommensverteilung, Erhaltung Sozialer Solidarität, Unterstützung von Arbeitsmobilität und Unterstützung von Wirtschafts- und Arbeitsmarktumstrukturierung und -produktivität“ (vergl. Esping-Andersen 
1996: 226). Ziel des Wohlfahrtsstaates ist es also, Armut zu bekämpfen und Einkommensungleichheit $\mathrm{zu}$ nivellieren. „Hinsichtlich der Organisationsstrukturen unterscheidet man zwischen Fürsorge, Versicherung und Versorgung“ (Butterwege 2006: 28).

Die sozialstaatlichen Grundprinzipien wurden im Laufe der Jahre erfolgreich beeinflusst und geprägt durch die verschiedenen Regierungsparteien, bzw. Oppositionen (vergl. Knijn 2004: 4). Vor allem seit dem Zweiten Weltkrieg wirkte der Sozialstaat bei der Schlichtung „sozialer Spannungen, welche durch wirtschaftliche Konjunkturschwankungen, demographische Transitionen und der Entwicklung von der agrarischen zur industriellen und wiederum später dann zur postindustriellen Gesellschaft (Esping-Andersen 1999)“ herrschten (Engelen/Hemerijk 2007: 305). „Wer die Entwicklung der westlichen Wohlfahrtstaaten auf sich einwirken lässt, kann vier Funktionen erkennen, die stets zurückkehren: versorgen, versichern, ermöglichen und verbinden (...) Verbinden und ermöglichen bilden die Achse, die einen positiven Inhalt ans Leben geben. Versorgen und versichern formen die Achse bei der Lösung von Problemen“ (Wetenschappelijke Raad voor het Regeringsbeleid 2006: 35f).

Obgleich die Grundprinzipien der Wohlfahrtsstaaten sich ähneln, erfolgten die sozialpolitische Einrichtung bzw. die sozialstaatlichen Arrangements der westlichen Nationalstaaten in der agrarischen und industriellen Periode unterschiedlich. „Nun wird in komparativen Studien regelmäßig auf die Pfadabhängigkeit sozialstaatlicher Entwicklungen hingewiesen: Demnach gibt es - auch innerhalb Europas - verschiedene Wohlfahrtsregime (EspingAndersen 1990) und auch unterschiedliche Muster der Beziehung zwischen Staat und ,Nonprofit-Sektor' (Salamon/Anheier 1998). Beschrieben wird auch auf die Restsouveränität nationaler Sozialpolitik (Esser 1999: 135ff) sowie die ,Divergenz der Modernisierungsziele' (Loeffler 1998: 327f) bei Verwaltungsreformen. Länderstudien, die den Zusammenhang zwischen ökonomischen Strukturen und deren institutioneller Einbettung beleuchten (vergl. etwa Ebbinghaus/Manow 2001 oder Schmid 2002), legen ebenfalls ein hohes Maß an Divergenz in Bezug auf die Entwicklung von (Wohlfahrts-) Staatlichkeit nahe“ (Bode 2004: 80).

Abhängig von der gewählten Perspektive, kann man diverse wohlfahrtsstaatliche Typologien unterscheiden (zwischen denen es in der Praxis meistens zu 
Mischformen kommt). Wildeboer Schut, Vrooman und de Beer benennen z.B. den liberalen, den korporatistischen und den sozialdemokratischen Wohlfahrtsstaat.

- „Der liberale Wohlfahrtsstaat richtet sich an eine eng umrissene Zielgruppe: die Armen (...),

- Der konservativ-korporatistische Wohlfahrtsstaat ist hierarchisch-selektiv, d.h. an Berufsgruppen orientiert (...),

- Der sozialdemokratische Wohlfahrtsstaat erfasst alle Bürger eines Staates für einen bestimmten Zeitraum und sieht einen relativ hohen Mindestlohn für alle vor (...).

Dreh- und Angelpunkt aller Sozialstaatsmodelle auf Versicherungsbasis sind ein funktionierender Arbeitsmarkt und durchgängige Berufsbiographien mit wenigen Arbeitsplatzwechseln“ (Kevenhörster 2006: 224).

Eine sehr ähnliche Typologie, die in Wissenschaft und Politik viel Aufsehen erregte, und im folgenden Kapitel ausführlich bearbeitet wird, stammt von Esping-Andersen. Er unterscheidet zwischen:

- Dem nordischen Sozialstaatsmodell,

- Dem kontinentaleuropäischen Sozialstaatsmodell und

- Dem liberalen Sozialstaatsmodell (vergl. Esping-Andersen 2002: 13ff).

Am positivsten beurteilte Esping-Andersen die sozialen Leistungen der skandinavischen Länder. „Diese zeichneten sich aus durch einen universalistischen, egalitären und durch großzügige, steuerfinanzierte Transferleistungen charakterisierten Sozialstaat, der eine aktive Familien- und Arbeitsmarktpolitik zur Förderung der Frauenerwerbstätigkeit und Fortbildung betreibt und auf entwickelten korporatistischen Strukturen im System industrieller Beziehungen beruht" (Lütz 2004: 12). Die Gruppe der kontinentaleuropäischen Länder findet sich abhängig vom konkret gewählten sozialen Arrangement im mittleren Bereich des Leistungsspektrums wieder, 
während Esping-Andersen die liberalen Staaten mit ihren an Mindestleistungen orientieren Instrumenten am unteren Ende der Skala einordnet ${ }^{2}$.

Auch bei den unterschiedlichen Reformpfaden dieser drei Modelle „zeigt sich mithin, dass die Transformationstendenzen in vielerlei Hinsicht vergleichbar sind, dabei indes je spezifische Konturen annehmen bzw. unterschiedliche Wirkungen entfalten. Es gibt überall Tendenzen einer Gewährleistungspolitik“ (Bode 2004: 82) (vergl. SER/WRR-symposium 16.01.2007: 2) bzw. in Richtung eines Wettbewerbsstaates Typ II, Gewährleistungsstaates, bzw. des Aktivierenden Staates, welcher im folgenden Abschnitt näher umschrieben ist.

\subsubsection{Ein neues Verhältnis zwischen Staat und Gesellschaft; Einordnung des Leitbildes Aktivierender Staat}

Schon lange vor der Definition des Leitbildes Aktivierender Staat kam es zu ersten relevanten Entwicklungen. Unter Präsident Johnson wurden in den USA in den siebziger Jahren Programme entworfen, um Bürger in ihrem Handeln zu unterstützen. Der Zielgruppe sollte ermöglicht werden, persönliche Lebensbedingungen besser zu gestalten, um somit selbstständig auf Situationen ihres Lebens vorbereitet zu sein. Dieses Prinzip wurde auch unter der Reaganund Bush-Administration beibehalten bzw. weiterentwickelt.

Parallel zu den Entwicklungen in den Vereinigten Staaten bestimmte Thatcher in Großbritannien maßgeblich das Entstehen des Schlanken Staates und des Neoliberalismus. Den Wohlfahrtsstaat wollte sie konsequent zum Wettbewerbsstaat ausbauen. Ziel der englischen Opposition währenddessen war es, „eine politische und auch auf einzelne Politikfelder anwendbare Strategie zu entwickeln, welche zwar die wohlfahrtsstaatlichen Ideale der alten Linken gerade noch erhielt, gleichzeitig jedoch in eine alternative Globalisierungsvision eingeschmolzen und auf diese Weise umgeformt werden konnte. Damit war der Grundstein für die Entwicklung des Wettbewerbsstaates Typ II gelegt“

2 Esping-Andersen verweist darauf, dass sein Modell die Komplexität der politischen Realität etwas vereinfacht wiedergibt. Die Zuteilung der verschiedenen Staaten zu einem der von ihm unterschiedenen Regimes verläuft nicht immer ganz unproblematisch (vergl. Esping-Andersen 1999: 87). 
(Evans/Cerny 2004: 211). Dieses Modell baut fort auf den Prinzipien des Neoliberalismus. Im Unterschied zum Wettbewerbsstaat Typ I hat der Staat im neuen Modell eine entscheidende Rolle. Hierbei orientierte man sich gedanklich an den Vereinigten Staaten, denn das Instrumentarium, welches den Staat bei der Erfüllung seiner neuen Aufgaben unterstützen sollte, wurde in dem Werk ,Reinventing Government' (das Regieren neu erfinden) beschrieben. Vizepräsident Gore hatte diese Studie in Auftrag gegeben. In dem Werk wurden die Gedanken des Neoliberalismus kombiniert mit staatlichem Interventionismus. Die „neuen politischen Führungsfiguren wie Tony Blair und Gordon Brown in Großbritannien oder Bill Clinton in den USA behaupteten beim Aufbau von ,New Labour' oder den ,New Democrats', ausschließlich die neue Linke sei in der Lage Globalisierung so zu gestalten, dass gleichzeitig die Wettbewerbsfähigkeit der heimischen Industrie verbessert und eine sozial verantwortliche Alternative entwickelt werde“ (Evans/Cerny 2004: 211).

In den USA hat die Grundsatzdiskussion um den Begriff des Wohlfahrtsstaates nicht nur während des Wahlkampfes von Bill Clinton eine große Rolle gespielt, sondern fand durch den „Personal responsibility and work opportunity reconcilitation act” 1996 ihre Institutionalisierung (Wohlfahrt 2007a). Die klassische Definition des Wohlfahrtsstaates erfuhr durch den Begriff ,Enabling state' (Gilbert: 1996) nun eine institutionalisierte Erweiterung. Der Staat unterstützt bzw. ermöglicht durch die Verwendung geeigneter Instrumente Eigeninitiativen der Zivilgesellschaft anstelle einer Ignorierung dieser Kräfte. Die Form der Transfers zwischen Staat und Bürger wurde vielfältiger, und die Regierung erhoffte sich hierdurch bessere Resultate. Allerdings wurde bei diesem Modell noch nicht von einer Kooperation von öffentlichen und privaten Partnern bzw. einer Steuerung durch den Staat gesprochen.

In England wurden inhaltlich vergleichbare Diskussionen unter dem Leitbild des ,Dritten Weges' von Tony Blair bzw. in Deutschland mit dem Term ,Neue Mitte' von Gerhard Schröder beschrieben. „Die behauptete Verbindung zwischen den Anforderungen an den Wettbewerbsstaat und der Haltung der Regierung gegenüber der Erwerbstätigkeit, welche ,Arbeit für Wohlfahrt' impliziert, findet sich nirgendwo besser ausgedrückt als in diesem Zitat aus Blairs New Britain - My Vision of a Young Country (1996). Es verdeutlicht die Beweggründe für Blairs Ziel, das Ausbildungsniveau der britischen Bevölkerung zu heben, um sich im internationalen Wettbewerb zu behaupten“ (Evans/Cerny 
2004: 221). Diese Leitbilder mit soziologischen Grundzügen werden verwendet, um eine neue Aufgabendefinition von Staat und Bürger zu verdeutlichen und die sich ändernde Rolle der Institutionen (mehr Zusammenarbeit zwischen verschiedenen Ebenen) zu erklären. Dieses Prinzip (und das damit einhergehende Menschenbild) findet eine Verdeutlichung im so genannten Blair-Schröder-Papier von 1999. Allen Bürgern, auch den chancenarmen, werden gewisse Verantwortlichkeiten zugesprochen. Chancenlosigkeit ist keine Ausweglosigkeit und chancenreiche Bürger müssen nicht unter allen Umständen permanente Arbeitslosigkeit mitfinanzieren (Surferproblem). Entsprechend persönlicher Möglichkeiten gibt es Aktivierungsmöglichkeiten für alle, die temporär nicht am normalen Arbeits- bzw. Gesellschaftsprozess teilhaben können.

Der Aktivierende Staat geht von einem Menschenbild aus, bei dem aufgrund der problematischen wirtschaftlichen bzw. gesellschaftlichen Situation weniger ein kalkulierender Nutzer von sozialen Programmen im Mittelpunkt steht, sondern eher ein Bürger der aufgrund eigener Interessen aktiv im öffentlichen Interesse handelt. Er begibt sich in ein Feld zwischen dem klassischen neoliberalen und dem kooperativen, wohlfahrtstaatlichen Staatsmodell. Weniger die Frage der Verantwortung des Staates für den Bürger, als die des Bürgers für den Staat steht im Mittelpunkt. „Mehr und mehr rückt die Frage ins Zentrum, wie der gesellschaftliche Zusammenhalt der ,postindustriellen' Nationen und Regionen zu gewährleisten ist. Dies wird vor allem in nationalen Diskurskontexten thematisiert, die auf eine profunde Erfahrung mit dem ,neoliberalen Experiment zurückgreifen können. Insoweit mag Anthony Giddens als Zeuge für diesen Ansatzpunkt dienen, wenn er immer wieder nicht die ,Soziale Sicherheit' ins Zentrum setzt, sondern das Gegensatzpaar von ,Inklusion' und ,Ausschluss' und damit den ,sozialen Zusammenhang' (siehe auch OECD 1997b)“. (Blanke/Schridde/Plaß 2001: 4) Für seine neue Rolle kommt dem Bürger mehr Raum zu. Durch den Staat wird er motiviert und unterstützt bei der Wahrnehmung seiner Eigenverantwortung.

Niklas Luhmann spricht im Zusammenhang mit Aktivierung auch von Inklusionsarbeit. „Der Idee des ,workfare' (,statt welfare') beispielsweise liegt ein deutlich liberales Modell zugrunde, das (allein) auf die Inklusion in ökonomische Funktionen abhebt; die Idee der ,aktivierenden Sozialhilfe' wird von Sozialdemokraten bevorzugt, insoweit sie auf eine ,arbeiterpolitische' 
Inklusion zielt; im konservativen Milieu bevorzugt man eine Normalisierungsoption, die der ,Familienpolitik' eine zentrale Rolle zumisst (Hessische Staatskanzlei 2003). Die garantistische Option wiederum setzt auf ,Empowerment' der Individuen, sie möchte - systematisch gesprochen Inklusion radikalisieren“ (Opielka 2004: 91).

Der Aktivierende Staat zeichnet sich durch eine neue und prinzipielle Diskussion der Verantwortlichkeiten von Staat und Gesellschaft aus, um zu identifizieren, wo die Übernahme gesellschaftlicher Verantwortung möglich ist. Der Staat soll nicht alle typischen Aufgaben des Sozialstaates alleine erfüllen sondern gemeinsam mit den Unternehmen, den Verbänden oder vor allem auch den Bürgern. „Bei der Erörterung der aktivierenden Rolle des Staates geht es darum, inwieweit seine Institutionen gesellschaftliches Engagement mobilisieren und stützen, wieweit sie den Einzelnen zur Wahrnehmung von Eigenverantwortung befähigen, und inwieweit sie gesellschaftlich differenzierten Erwartungen und der Entwicklung individueller Lebensentscheidungen gerecht werden - also um die Konsequenzen aus der Entwicklung, die Anthony Giddens als den Weg von einer Politik der Lebenschancen zu einer Politik der Lebensentwürfe beschreibt“ (Holzapfel 2000: 76). Gleichzeitig erfordert die Aufgabe des Staates auch eine entsprechende Reaktion vom Bürger bzw. der Bevölkerung. Daher wird der Begriff Aktivierender Staat auch oft als Aktivierende Politik bezeichnet, da hierbei weniger die Betonung auf dem Staat liegt. Der Begriff Politik deutet dann eher auf ein Wechselspiel zwischen Bürgern, Wirtschaft, Staat und Drittem Sektor.

Ein weiterer Begriff in diesem Zusammenhang lautet Gewährleistungsstaat (ensuring state). Der Rechts- und Verwaltungswissenschaftler Schuppert versteht unter diesem Leitbild eine Weiterentwicklung bzw. Nuancierung des Aktivierenden Staates. Seines Erachtens scheint „das Bild des Gewährleistungsstaates mehr auszudrücken als das Bild des Aktivierenden Staates. Denn es geht nicht mehr allein um Antworten auf den Minimalstaat. Der Staat sieht seine Rolle auch nicht nur in der Mobilisierung endogener Potentiale der Gesellschaft für das Gemeinwohl, sondern zugleich in einer besonderen Verantwortung für die Gemeinwohlsicherung" (Schuppert, 2005: 54). 
Da die hier vorliegende Abhandlung über den Aktivierenden Sozialstaat aufbaut auf der Theorie der Langen Wellen der Leitbildentwicklung und dort der Begriff Aktivierender Staat verwendet wird, soll dieser Term auch im Folgenden beibehalten werden, obgleich eine Aktivierung von Politik nötig ist, um bürgerliche Partizipation zu ermöglichen (vergl. Evers 2004: 4, Evers 2007: 1).

Um das neue Handeln zwischen den verschiedenen Verantwortlichen zu realisieren, ist ein vom Staat geforderter und geförderter gesellschaftspolitischer Dialog nötig. Ziel ist es, unter der Verantwortlichkeit des Staates Koproduktion zu befördern. Öffentliches Handeln wird weniger als zuvor als eine Alternative zwischen staatlicher und privater Aufgabe verstanden sondern als eine gemeinsame Aufgabenteilung. Diese neue Aufgabenteilung zwischen Staat, Wirtschaft, drittem Sektor und Bürgern wird von Bandemer in seinem Text „Vom expandierenden zum aktivierenden Staat” 2005 in vier Indikatoren aktivierender Politiken definiert.

- „Durch die systematische Organisation eines gesellschaftlichen Dialogs ringt der (...) Staat mit den Bürgern und Institutionen gezielt um öffentliche Aufgabenbereiche an Stelle diese autonom festzulegen oder sich diese von der Gesellschaft übertragen zu lassen.

- Durch eine neue Verantwortungsteilung soll die Aufsplittung in Leistungsempfänger und Leistungserbringer dahingehend überwunden werden, dass der Staat zwar Verantwortung für die Leistungserbringung übernimmt, aber von den Leistungsempfängern entsprechende Gegenleistungen erwarten kann.

- Durch neue Formen der Koproduktion soll gewährleistet werden, dass die unterschiedlichen gesellschaftlichen Akteure gemeinsam an der Leistungserstellung arbeiten und Aufgaben nicht lediglich delegiert werden.

- Im Rahmen der Leistungsaktivierung wird gewährleistet, dass entlang der gesamten Wertschöpfungskette öffentlichen Handelns durch den Einsatz entsprechender Steuerungsinstrumente Effizienz und Effektivität gesichert werden" (Bandemer 2005: 31f). 
Vor dem Gesichtspunkt einer theoretischen Einordnung des Aktivierenden Staates, ist es notwendig, um zwei weitere Theorien, den Kommunitarismus und den Wohlfahrtspluralismus, zu erläutern.

\subsubsection{Kommunitarismus und Wohlfahrtspluralismus}

Der Kommunitarismus entstand im Laufe der achtziger Jahre als Gegenbewegung zum Liberalismus. Dieses Sozialorganisationsmodell betont die Bedeutung gesellschaftlicher Solidarität, moralischer Werte und Verantwortung der Bürger füreinander, welche die Kommunitaristen durch die Zunahme des Liberalismus als bedroht sahen. Jeder Bürger ist an erster Stelle für sich selbst verantwortlich. An zweiter Stelle sind seine Nächsten für ihn verantwortlich. Lokale Zugehörigkeit ist in diesem Zusammenhang entscheidend. „Zwar teilen Liberale und Kommunitarier das Konzept des Minimalstaates. Im Gegensatz zum Liberalismus indes, der den einzelnen als unabhängigen Marktteilnehmer und zur Selbsthilfe fähigen Unternehmer seiner Arbeitskraft sieht, denkt die kommunitaristischen Sozial- und Moralphilosophie Individualismus und individuelles Handeln nur und untrennbar in Kategorien von Gemeinschaftlichkeit und Verantwortungsgemeinschaften“ (Lamping/Schridde/Plaß/Blanke 2002: 17).

In diesem Kontext steht auch das Subsidiaritätsprinzip. Das Konzept der Subsidiarität entstand ursprünglich als Antwort der katholischen Soziallehre auf Entwicklungen wie Säkularisierung, Totalisierung und ungebremster Kapitalismus zu Beginn des 20. Jahrhunderts. Dieses Prinzip theoretisiert die Funktion der sozialen Mikrostruktur. Eingriff durch den Staat ist nur dort nötig, wo die untergeordnete Einheit nicht in der Lage ist die Probleme selbst zu lösen. In Deutschland ist anders als im niederländischen Zentralstaat dieses Prinzip seit langem sehr entscheidend für die staatliche Einrichtung und die Wahrnehmung öffentlicher Aufgaben. Lokal- oder Facheinheiten besitzen hier entscheidende Kompetenzen zur Erfüllung öffentlicher Aufgaben. „Im Zuge der wachsenden gesellschaftlichen Ausdifferenzierung und des Ausbaus des Sozialstaates koppelte sich der Subsidiaritätsbegriff jedoch von seinen ursprünglich naturrechtlich-traditionalistischen Gesellschaftsbezügen ab und verengte sich auf ein verbändezentriertes, formales Subsidiaritätsverständnis (Backhaus-Maul/Olk 1994) (...) Eine Politik der neuen Subsidiarität zielt darauf 
ab, solidarische Handlungsfelder jenseits von Markt und Staat zu begründen bzw. zu stärken“ (Lamping/Schridde 2004: 49f).

Die Theorie des Wohlfahrtspluralismus ist etwas unscharf, kann aber „in einer zeitgeschichtlichen Perspektive auch als Fortentwicklung des in den 8oer Jahren diskutierten Konzepts einer Neuen Subsidiaritätspolitik interpretiert werden.“ (Lamping/Schridde/Plaß/Blanke 2002: 22f). In der Neuen Subsidiaritätspolitik sollte die Aufgabe der Stärkung der zivilen Bürgergesellschaft durch den Dritten Sektor wahrgenommen werden. In der Praxis hatte dieser Ansatz nur eine untergeordnete Relevanz.

Durch die angelsächsischen Debatten im Bereich des ,welfare mix' wurde die Eindimensionalität bestehender Lösungsansätze (wie z.B. der Liberalismus bzw. der Kommunitarismus) kritisiert. Nur durch ein Zusammenspiel unterschiedlicher Parteien der Wohlfahrtsproduktion können öffentliche Aufgaben zufriedenstellend erledigt werden. Die Umstrukturierung der staatlichen Verantwortlichkeiten ist hierfür eine Voraussetzung. „Die Herstellung einer solchen Wohlfahrtsmixtur ist in diesem Konzept Aufgabe des Dritten Sektors, innerhalb dessen die oft mit Konflikt behafteten Logiken und Handlungsanforderungen zwischen Staat, Markt und Familie miteinander vermittelt und organisationsintern miteinander verschränkt werden (Evers/Olk 1996; Dettling 1995)“ (Lamping/Schridde 2004: 50).

Kritiker des Wohlfahrtspluralismus bzw. der Neuen Subsidiarität bemängeln die Effizienzprobleme, welche durch Steuerung durch den Dritten Sektor entstehen können, sowie eventueller demokratietheoretischer Probleme im Falle sozialstruktureller Widersprüche.

\subsubsection{Theoretische Einbettung des Aktivierenden Staates}

Entscheidend für die Zuordnung des Aktivierenden Staates in ein theoretisches Gefüge sind die eher verwendeten Begriffe Aktiver Staat, Schlanker Staat, Kommunitarismus und Wohlfahrtspluralismus.

Die Gruppierung der vier genannten Leitbilder bzw. Theorien auf denen der Aktivierende Staat aufbaut, kann vorgenommen werden anhand zweier Achsen. Einerseits wird unterschieden zwischen der Zivil- bzw. Bürgergesellschaft. Wobei die Frage ist, ob das Konzept primär vom Bürger ausgeht und der der 
Staat eher residual betrachtet wird und andererseits, ob das Konzept primär von den Interventionen des Staates ausgeht (vergl. Blanke/Schridde/Plaß 2001: 11f). Assoziatives oder individuelles Handeln sind die Kriterien nach denen auf der anderen Achse sortiert wird.

Tabelle 2: Gemeinwohlkonzepte und Handlungsorientierungen von Staat und Bürgern Positionierung des Aktivierenden Staates (Lamping/Schridde/Plaß/Blanke 2002: 32)

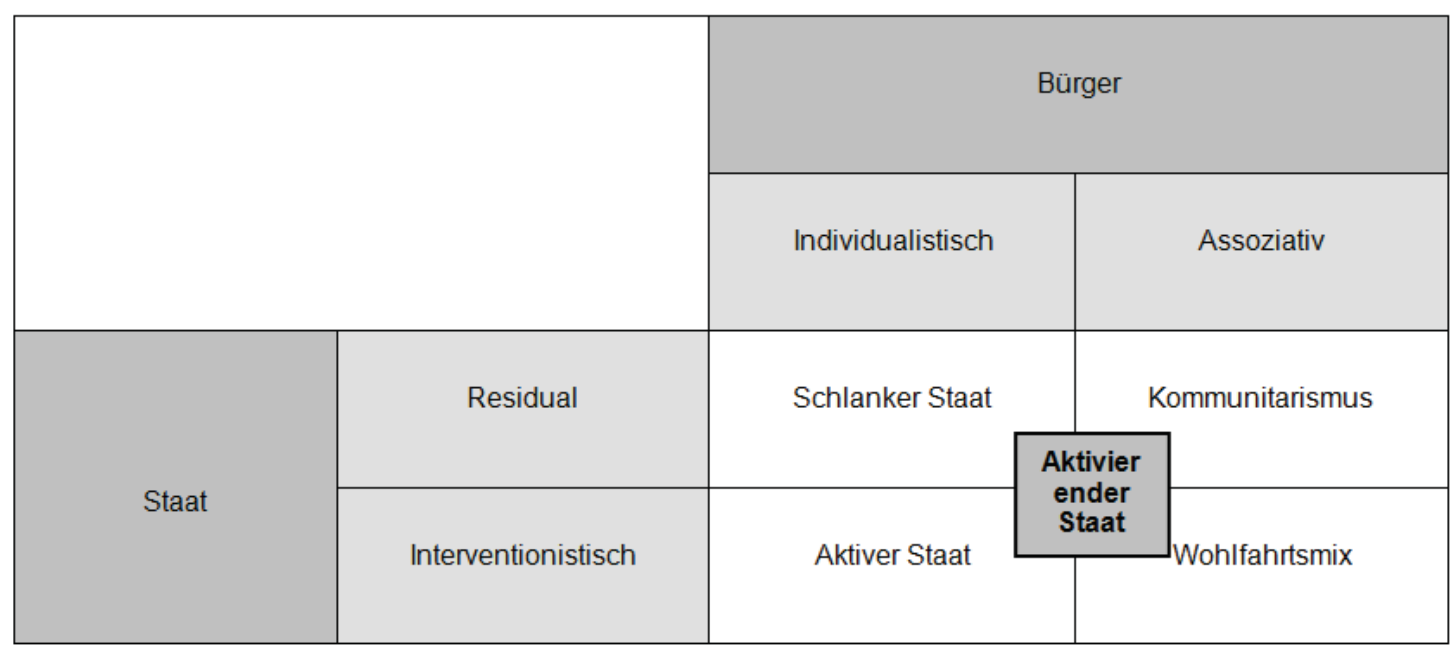

„Stärker als die Positionen eins bis vier thematisiert das Konzept des Aktivierenden Staates ausdrücklich institutionelle Aspekte einer Modernisierungsstrategie. Der Aktivierende Staat fragt danach, wie sich das komplexe System von Institutionen, Organisationen und Akteuren jeweils selbst verändern müsste, um der Idee der Aktivierung gerecht zu werden - ohne dabei die öffentliche (staatliche) Verantwortung für gesellschaftliche Wohlfahrt aufzugeben“ (Lamping/Schridde/Plaß/Blanke 2002: 4). Zur Realisierung dieser Modernisierungsstrategie und seinem Ausmaß ist neben der Selbstaktivierung des Staates eine grundlegende institutionelle Aktivierung über die bisherigen Grenzen praktischer Kompetenzen hinweg (vergl. Wetenschappelijke Raad voor het Regeringsbeleid 2006: 27), sowie ein zweifache Form der gesellschaftlichen Aktivierung nötig. Einerseits handelt es sich bei gesellschaftlicher Aktivierung um die Mobilisierung gesellschaftlicher Ressourcen für den Arbeitsmarkt. Andererseits geht es um eine zivilgesellschaftliche Form der Aktivierung, „die es versteht, Beteiligungsformen $\mathrm{zu}$ entwickeln, entlang derer individuellen Interessen und Gruppenanliegen“ (Evers 2007: 1).

Genau wie bei den vorhergehenden zwei Leitbildern, ist das Ziel des Aktivierenden Staates Staatsmodernisierung, um öffentliche Aufgaben besser 
wahrzunehmen. Im Gegensatz zum Schlanken Staat setzt sich das Leitbild des Aktivierenden Staates allerdings nicht radikal gegen seine Vorgänger ab, sondern versteht sich selbst als hybrides Modell. „Das Konzept des Aktivierenden Staates übernimmt viele Elemente dieser vier unterschiedlichen Denkrichtungen und versucht, diese in ein hybrides Konzept zu integrieren, um einen Ausweg aus dem Dilemma von Allzuständigkeit und Handlungsunfähigkeit des Staates zu weisen“ (Lamping/Schridde 2004: 50f). Aus der Aufstellung des Diagramms und der Positionierung des Aktivierenden Staates wird deutlich, dass gesellschaftlicher Dialog nötig ist, um das Handeln zwischen Staat und Gesellschaft zu ermöglichen.

Im Gegensatz zu den eher residualen Konzepten geht das Leitbild des Aktivierenden Staates aus von staatlicher Verantwortung, die dann allerdings nach wirtschaftlichem Beispiel als Verantwortungsdifferenzierung interpretiert wird. Auf der Grundlage dieser Differenzierung kann es dann zu Koproduktion zwischen den verschiedenen Herstellern öffentlicher Produkte kommen. Der Staat steuert nicht mehr „über umverteilende Transfers, sondern über ergebnisorientierte Anreize; er ist nicht in hierarchischen Bürokratien, sondern in selbststeuernden Netzwerken organisiert; und schließlich wird ein Großteil seiner Leistungen nicht mehr vollfinanziert, sondern nur noch kofinanziert“ (Butterwege 2006: 17). Wie das Leitbild des Aktivierenden Staates in der allgemeinen politischen Praxis Deutschlands und der Niederlande verwendet wurde, wird in den folgenden beiden Paragraphen beschrieben.

\subsubsection{Aktivierende politische Praxis}

\subsubsection{Deutschland}

Nachdem zu Beginn der neunziger Jahre in den Vereinigten Staaten und kurze Zeit später auch in Großbritannien die Staatsmodernisierung dem Leitbild des 'Befähigenden Staates' (enabling state) folgten, stand dieses Thema in Deutschland erst etwas später auf der Agenda. Dafür gibt es verschiedene Ursachen. Scharpf spricht z.B. von ,Politikverflechtung' und er sieht in der „,Politikverflechtungsfalle' eine Schlüsselerklärung für Hindernisse und Versäumnisse bei der Formulierung und Ausführung von Reformprogrammen (vergl. Scharpf 1985)“ (Czada 2004: 134). Eine andere Erklärung könnte die hohe 
finanzielle Reserve Deutschlands vor der Wiedervereinigung sein. „Wie optimistisch die Aussichten waren, wird aus dem Bericht des Sachverständigenrates zur Begutachtung der gesamtwirtschaftlichen Entwicklung erkennbar, der 1989 wenige Wochen vor dem Fall der Berliner Mauer veröffentlicht wurde (SVR 1989). Die ,fünf Wirtschaftsweisen', zumeist neoliberale Gewerkschaftskritiker, legten den Gewerkschaften nahe, von einer Strategie qualitativer Forderungen abzurücken und stattdessen Gehaltsforderungen $\mathrm{zu}$ stellen, $\mathrm{um}$ an den vorangegangenen Steigungen der Unternehmenseinkommen teilzuhaben" (Czada 2004: 135f). Auch die Wiedervereinigung selbst bzw. die Politik der ersten Nachwendejahre haben vielleicht zu einer Euphorie geführt, die keinen Raum bot für Reformstrategien, die früher oder später auch in Deutschland nötig wurden.

Mit der Koalitionsvereinbarung der Rot-Grünen Regierung vom 20.10.1998 hat die neue Bundesregierung dann unter dem Titel: „Moderner Staat - Moderne Verwaltung - Leitbild und Programm der Bundesregierung“ ein Programm beschrieben in dem die Ziele und Aufgaben unter dem Begriff Aktivierender Staat zusammengefasst wurden. Als einen bedeutenden Schritt nach vorne sah die neue Bundesregierung das gewandelte Verständnis von Staat und Verwaltung. Man baute nicht mehr nur auf negative Ansätze (so wie die Reduzierung des Staates) auf. Es kam zu einer inhaltlichen Weiterentwicklung durch das überdachte Aufgabenverständnis von Staat und Gesellschaft.

Zur weiteren Vertiefung seiner Theorien und Gedanken über sein Staats- und Gesellschaftsverständnis hat Gerhard Schröder in dem Text ,Die zivile Bürgergesellschaft. Anregungen zu einer Neubestimmung der Aufgaben von Staat und Gesellschaft' sich dann im Jahr 2000 näher mit der Schnittmenge zwischen Staat und Gesellschaft auseinandergesetzt. Eine rein technische Antwort des Staates auf die gesellschaftlichen Bedürfnisse, wie sie vom Schlanken Staat gegeben wurde, sei nicht mehr ausreichend. Den Bürger beschreibt Bundeskanzler Schröder als ein emanzipiertes Wesen, und der Staat solle den Bürger „(F)fördern aber auch von ihm fordern“ (Schröder 2000: 203).

Auch in der zweiten Regierungserklärung Gerhard Schröders vom 14.03.2003 spielt das Leitbild des Aktivierenden Staates eine weitere Rolle. Die Agenda 2010 bestimmt seine Reformrichtung. Sein Verständnis von Aktivierender Politik wird deutlich bei der Frage nach der Koproduktion im Zusammenhang mit der 
Haushaltskonsolidierung und des Stabilitätspaktes. Für die Lösung dieser Aufgabe sei es nötig, dass auf Wachstumsimpulse gesetzt werde. „Das muss für die Ermunterung privater Investitionen ebenso gelten wie für die öffentlichen Investitionen, insbesondere für die in den Kommunen“ (Bundesregierung 2003: 4). Auf Verantwortungsteilung setze er z.B. im sozialen Bereich. „Gerade weil Eigenverantwortung gestärkt werden muss, sollten wir (...) Instrumente wie differenzierte Praxisgebühren (...) nutzen“ (Bundesregierung 2003: 14).

Nach Neuwahlen und Regierungswechsel kommt es dann im Jahre 2005 zur Bildung der Großen Koalition unter der Leitung von Bundeskanzlerin Merkel. Wie Bundeskanzler Schröder zuvor, verwendet auch sie bei der Beschreibung der Reformbestrebungen der Großen Koalition das Leitbild des Aktivierenden Staates. Aufbauend auf den Prinzipien Verantwortungsteilung und Leistungsaktivierung sagt sie in ihrer Regierungserklärung: „Wir müssen, das wissen wir alle angesichts der kurzen Zeit in der die Zusammenlegung von Arbeitslosen- und Sozialhilfe erst wirkt, den Grundsatz ,Fördern und Fordern“ umfassend umsetzen“ (Bundesregierung 2005b: 7).

\subsubsection{Niederlande}

In den Niederlanden ist der Begriff Aktivierender Staat weniger verbreitet als der Begriff Aktivierende Politik. Diverse Eigenschaften des Leitbildes des Aktivierenden Staates wurden in den Niederlanden auch dem Poldermodell zugeschrieben. Bei diesem Modell handelt es sich nicht um ein Modell an sich, sondern eher um eine Anzahl von Maßnahmen, die sich auf Zielsetzungen in der Wirtschafts- und Arbeitsmarktpolitik richten (vergl. Visser/Hemerijck 1997: 184f). Das so genannte niederländische Leitbild des Poldermodells dem Aktivierenden Staat in Deutschland und dem Leitbild des Dritten Weges im Vereinigten Königreich in gewisser Hinsicht ähnlich. Allerdings kann es nicht ganz mit dem deutschen Leitbild gleichgesetzt werden³.

3 Ähnlich wie beim traditionell-niederländischen Konsensmodell, welches schon zu früheren Zeitpunkten in der niederländischen Geschichte erfolgrleich war, wurde die Zusammenarbeit zwischen Gewerkschaften, Arbeitgebern und unabhängigen von der Regierung ernannten Mitgliedern im Wirtschaftsrat (Sociaal Economische Raad) institutionalisiert und gemeinsame Ziele der Wirtschafts- und Arbeitsmarktpolitik definiert. Die hierbei angewandten Instrumente (z.B. Lohnzurückhaltung und Abbau von 
Als Geburtsstunde des Poldermodells wird das ,Abkommen von Wassenaar' im Jahr 1982 bezeichnet. Da die wirtschaftliche Situation der Niederlande aufgrund großer gesellschaftlicher Differenzen seit den ,langen sechziger Jahren' und den beiden Ölkrisen der siebziger Jahre als sehr schwierig galt, wurde 1982 durch Institutionalisierung der Zusammenarbeit zwischen Gewerkschaften, Arbeitgebern und unabhängigen von der Regierung ernannten Mitgliedern im Wirtschaftsrat (Sociaal Economische Raad) zurückgegriffen auf das traditionellniederländische Konsensmodell. Die hierbei angewandten Instrumente (z.B. Lohnzurückhaltung und Abbau von staatlichen Ausgaben) waren „im Wesentlichen der neoklassischen Theorie entnommen“ (Das Poldermodell, www.niederlandenet.de aufgerufen am 30.07.2007) und galten als ein „entscheidendes Signal der Wirtschaft“ (Visser/Hemerijck 1997: 109).

Der Begriff Poldermodell ist alledings nicht ganz deckungsgleich mit dem hier zu verwendenden Begriff des Aktivierenden Staates, da es sich hierbei nicht um ein Modell an sich handelt, sondern eher um eine Anzahl von Instrumente, die sich auf Zielsetzungen in der Wirtschafts- und Arbeitsmarktpolitik richten (vergl. Visser/Hemerijck 1997: 184f). Bei den niederländischen Wahlen des Jahres 1994 erlitten die beiden großen Volksparteien CDA und PvdA große Stimmenverluste und waren die liberalen Parteien D66 und VVD die großen

\footnotetext{
staatlichen Ausgaben) waren „im Wesentlichen der neoklassischen Theorie entnommen“ (Das Poldermodell, www.niederlandenet.de, aufgerufen am 30.07.2007) und galten als ein „entscheidendes Signal der Wirtschaft“ (Visser/Hemerijck 1997: 109). Die Institutionalisierung der Zusammenarbeit zwischen den verschiedenen Akteuren kann als ,Korporatismus' gedeutet werden. Dieser ,Korporatismus' hat in der niederländischen Gesellschaft tiefe Wurzeln und ist zurückzuführen auf eine Kombination des volkstypischen ,Handelsgeistes‘ und der christlich-sozialen Lehre des Kalvinismus (...) Der Einfluss der christlichsozialen Lehre unterstreicht die gesellschaftliche Verantwortung des einzelnen in der Rolle als Unternehmer und Arbeitnehmer“ (Das Poldermodell, www.niederlandenet.de, aufgerufen am 30.07.2007).

Das Grundmuster der Reformen „ässt sich mit zwei Schlagworten aus der niederländischen Diskussion beschreiben: ,Weniger Staat, mehr Markt' steht für eine Neuverteilung der Verantwortung zwischen Staat und Gesellschaft für die ,Privatisierung sozialer Sicherheit‘. ,Arbeit geht vor Einkommen` steht für die neue sozialpolitische Prioritätensetzung: die Schaffung von Arbeitsplätzen und Instrumenten zur Reintegration von Arbeitslosen und Erwerbsunfähigen in den Arbeitsmarkt haben Vorrang von den Einkommensersatzleistungen“ (Kötter 1997: 14). Kötter schreibt weiterhin in ihrem Buch ,Das niederländische Wohlfahrtsstaatsmodell - kein Vorbild mehr?’, dass bei den Reformen erstens die neue Verantwortungsteilung durch Leistungsbeschränkungen für die Arbeitnehmer entscheidend sei sowie die starke Inanspruchnahme der Arbeitgeber (z.B. für Präventions- und Reintegrationsaufgaben), organisatorische Privatisierung staatlicher Aufgaben und zunehmender Übertragung öffentlicher Aufgaben auf private und gewinnorientierte Träger. Hinzu kommt die Ergänzung der Sicherungsfunktion sozialer Sicherheit durch das Aktivierungsprinzip, bei dem z.B. für die Arbeitgeber finanzielle Anreize geboten werden, um erwerbsunfähige Arbeitnehmer schneller wieder ins Berufsleben zu integrieren (z.B. durch die Einführung von Bonussystemen).
} 
Gewinner der Wahlen. Es kam zur Bildung der ersten Violetten Koalition unter Wim Kok. Unter dieser Regierung wurden die Reformbewegungen unter anderem ausgedehnt auf die sozialen Bereiche und wurde die Aktivierungsstrategie verwendet. Die Prinzipien des sozialen Dialogs und der Kooperation wurden um die Prinzipien der Verantwortlichkeit und der Leistungsaktivierung erweitert (vergl. Visser/Hemerijck 1997: 184f). Daher werden bei dem Vergleich und der Analyse in den kommenden Paragraphen und Kapiteln die politischen Handlungen mit Beginn des ersten Violetten Kabinetts analysiert und nicht seit der vorhergehenden Regierungsperiode von Ruud Lubbers.

Kötter schreibt in ihrem Werk ,Das niederländische Wohlfahrtsstaatsmodell kein Vorbild mehr?', dass bei den Reformen erstens die neue Verantwortungsteilung durch Leistungsbeschränkungen für die Arbeitnehmer entscheidend sei sowie die verstärkte Inanspruchnahme der Arbeitgeber (z.B. für Präventions- und Reintegrationsaufgaben), organisatorische Privatisierung staatlicher Aufgaben und zunehmender Übertragung öffentlicher Aufgaben auf private und gewinnorientierte Träger. Entscheidend sei weiterhin die Ergänzung der sozialen Sicherheit durch die Aktivierungsfunktion, bei der z.B. für die Arbeitgeber finanzielle Anreize geboten werden, um erwerbsunfähige Arbeitnehmer schneller wieder ins Berufsleben zu integrieren.

Auch die Regierungserklärung und Koalitionsvereinbarungen der zweiten Violetten Koalition, im Jahre 1998, unterstreichen deutlich Eigenschaften der Aktivierenden Politik. Das Begriffspaar staatliche Aktivierung und Gewährleistungsverantwortung wird als Schwerpunkt gesehen: „Bürger haben das Recht auf die Sicherheit, dass die Verwaltung/der Staat sie stützt und beschützt wenn es darauf ankommt, jedoch sollen (die Bürger), wenn nötig und möglich, auch selbst Verantwortung übernehmen“ (Ministerie van Algemene Zaken 1998a: 4).

Nach den beiden violetten Koalitionen kam es zu einer turbulenten Zeit in der niederländischen Politik. In den Jahren 2002 und 2003 gab es Neuwahlen, bei denen einmal die LPF und die VVD gemeinsam mit der CDA und einmal die VVD mit D66 den Kabinetten Balkenende I und II angehörten. 2006 kam es nach dem Abtreten der D66-Minister zur Formation des Kabinetts Balkenende III, welches bis zu den Neuwahlen am Ende des Jahres 2006 demissionär 
funktionierte. Dieses Kabinett wurde Anfang 2007 abgelöst vom vierten Kabinett und Leitung des Premierministers Balkenende und kann aufgrund seiner zeitlich begrenzten inhaltlichen Arbeit und Dokumentation in diesem Zusammenhang nicht analysiert werden.

Obwohl Balkenende I sich nach den Wahlen im Jahr 2002 und dem Auftreten der LPF inhaltlich von den violetten Koalitionen absetzte, wollte auch dieses Kabinett den Staat unter Verwendung der Aktivierenden Politik reformieren. Eine deutliche Gewährleistungsverantwortlichkeit ist dieser Regierung sehr wichtig, sie will eine Regierung sein, die „deutlich abgrenzt, wofür sie verantwortlich sein will und kann“ (Ministerie van Algemene Zaken 2002b: 3).

Die Basisprinzipien Kooperation und Leistungsaktivierung werden an verschiedenen Stellen durch diese neue Koalition hervorgehoben. „Das Kabinett stellt nicht die Regierung und die Regulierungen in den Mittelpunkt, sondern die eigene Verantwortung der Menschen und gesellschaftlichen Organisationen. Nicht eine Regierung, die jedermanns Probleme zu lösen versucht, sondern eine Regierung, die Menschen ermöglicht eigene Probleme zu lösen und Verantwortlichkeit zu tragen“ (Ministerie van Algemene Zaken 2002b: 5).

Wie auch ihre Vorgänger im Amt stellt auch das Kabinett Balkenende II, dass Mitte 2003 seine Arbeit aufnahm, seine Reformen unter das Leitbild der Aktivierenden Politik und räumt dem Begriff der Leistungsaktivierung schon in der Regierungserklärung einen wichtigen Platz ein. Es wird schon an dieser Stelle von „mehr aktivierenden sozialen Leistungen“ (Ministerie van Algemene Zaken 2003b: 1) gesprochen. Weiterhin wurde an die Verantwortlichkeit eines jeden appelliert, um „mehr individuelle Verantwortung“ zu übernehmen (Ministerie van Algemene Zaken 2003b: 1). Den Begriff Kooperation braucht man in diesem Zusammenhang wohl nicht zu erläutern. Sowohl in der Überschrift der Regierungsvereinbarung, die den Namen ,Mitmachen' trägt, (Ministerie van Algemene Zaken 2003a) als auch in der kurze Zeit darauf folgenden Regierungserklärung von Balkenende werden alle denkbaren Akteure von ihm zur Kooperation aufgerufen (Ministerie van Algemene Zaken 2003b: $10 f)$.

Zwischen 1994 und 2007 ist es in den Niederlanden insgesamt sechsmal zur Bildung eines neuen Kabinetts gekommen, aber seit der ersten Violetten Regierung unter Kok bis zu Balkenende IV ist das Leitbild der Aktivierenden 
Politik allgegenwärtig. Die letzte hier beschriebene Regierung ist die Koalition Balkenende IV zwischen CDA, PvdA und der ChristenUnie. Direkt auf der ersten Seite der Regierungserklärung wird der Begriff Aktivierung erwähnt (Ministerie van Algemene Zaken 2007b: 1) und verdeutlicht, dass alle Talente im Land erkannt und entwickelt werden sollen.

Zusammenfassend kann man feststellen, dass sowohl in Deutschland seit Beginn der Rot-Grünen Koalition bis zur Großen Koalition unter Merkel als auch in den Niederlanden seit Beginn der ersten Violetten Koalition bis zu Balkenende IV sich alle Kabinette am Leitbild der Aktivierenden Politik orientierten. In Deutschland und den Niederlanden entwickelte sich jeweils ein vergleichbares Grundmuster der angewandten Aktivierungspolitik. Die klassische Versorgung durch den Staat wurde nach und nach ersetzt durch Maßnahmen, die den Bürger bei seiner Aktivierung und Selbsthilfe nicht nur fördern sollten, sondern ihn auch kontrollierten. „Unter Aktivierung wird dabei eine größere Verantwortung der Gesellschaft und die Verbindung von Arbeitsmarkt- und passiver Sozialpolitik verstanden. Das erfordert eine neue Ausrichtung der Leistungen des Wohlfahrtstaats und der Kompetenzen der Akteure“ (Schmid 2002: 187).

\subsection{Neue Risiken - neue Verantwortungsteilung}

Wie im vorherigen Unterkapitel beschrieben, nennt Bandemer vier Indikatoren des Aktivierenden Staates (gesellschaftlicher Dialog, neue Verantwortungsteilung, Koproduktion und Leistungsaktivierung). Blanke, Schridde und Plaß definieren denselben Begriff als „eine neu gelebte Verantwortungsteilung zwischen Staat und Gesellschaft zur Realisierung gemeinsamer Ziele im Hinblick auf Fortschritt und Solidarität. Dabei geht es um Kooperation und Koproduktion staatlicher, halbstaatlicher und privater Akteure in der Verfolgung des öffentlichen Interesses. Der Aktivierende Staat will sein Engagement mit Eigeninitiative und Eigenverantwortung von Bürgerinnen und Bürgern verbinden und eine neue Leistungsaktivierung in allen Stufen der Wertschöpfungskette öffentlicher Leistungen erreichen“ (Blanke/Schridde/Plaß 2001: 8). Entscheidendes Streben des Aktivierenden Staates ist also die Teilung von Verantwortlichkeiten, mit dem Ziel 
Verantwortlichkeiten nicht automatisch dem Staat zuzuordnen, sondern dem geeignetsten Verantwortlichen.

Ausgehend vom sozialstaatlichen Ausgangspunkt der Gerechtigkeit und Chancengleichheit müssen Risiken entsprechend ihrer Ursache und der Kapazität zur bürgerlichen Eigenverantwortung analysiert werden. Sehr aufschlussreich, bei der Frage nach Gerechtigkeit und Chancengleichheit, ist die Differenzierung verschiedener Risiken, die Hintergründe der teilhabenden Akteure und eine Gewichtung bzw. Verteilung von Verantwortlichkeiten. Das betriebswirtschaftliche Prinzip der Leistungskette bildet im letzten Paragrafen des Unterkapitels eine Überleitung zu den aktivierenden Instrumenten und dem neuen Aufgabenverständnis.

\subsubsection{Gerechtigkeit und Chancengleichheit}

Ein wichtiges sozialstaatliches Prinzip ist das der Gerechtigkeit und Chancengleichheit. Durch wirtschaftlich und/oder gesellschaftliche Verhältnisse oder Entwicklungen kann es zu Ungerechtigkeiten bzw. Chancenungleichheiten kommen. Aufgabe des Sozialstaates ist es, diese so gut wie eben möglich zu beheben, um gesellschaftliche Unterschiede auf diesem Gebiet zu nivellieren. „In politisch-philosophischer und in politisch-praktischer Hinsicht können wir uns auf die Gerechtigkeitsprinzipien, insbesondere auf das Differenzprinzip (...) Maximin-Kriterium (...) von John Rawls stützen: Hierbei geht es nicht einfach um Umverteilung, sondern um Gerechtigkeit durch strukturierte und nachvollziehbare Verfahren. Die Grundstruktur einer Gesellschaft ist so zu gestalten, dass keine ungerechtfertigten Ungleichheiten entstehen, die umfangreiche Korrekturen erzwingen. Herkunfts- und klassenbedingte Startvorteile im gesellschaftlichen Wettbewerb sind so weit wie möglich auszugleichen. Einen ,natürlichen Anspruch' gibt es nicht, denn nur unter ,gerechten' Rahmenbedingungen kann jeder Bürger seine Ansprüche bestimmen und festlegen, was er, verdient' (Kevenhörster 2006: 220).

Kevenhörster unterscheidet zwischen drei Formen von Gerechtigkeit: Tauschgerechtigkeit, ausgleichende Gerechtigkeit und Verfahrensgerechtigkeit. Er verbindet mit der ersten Form der Gerechtigkeit das Prinzip der Wechselseitigkeit. Hiermit gemeint ist z.B. das Verhältnis der Generationen 
untereinander. Die zweite Form der Gerechtigkeit kann gesehen werden als eine Form der Entschädigung für die Wahrnehmung gesellschaftlicher Aufgaben wie z.B. Erziehung von Kindern, Pflege in der Familie usw., bzw. Risiken denen der individuelle Bürger nicht gewappnet ist. Im letztgenannten Gerechtigkeitsprinzip steht, wie der Name schon andeutet, die Behandlungsgleichheit politischer und administrativer Verfahren im Mittelpunkt. Wie seit der Anwendung des demokratischen Rechtsstaates verankert, sollen politische und administrative Verfahren für alle Bürger gleich sein (vergl. Kevenhörster 2006: 222).

Aufbauend auf der Gerechtigkeitstheorie von Rawls schließt sich die Theorie von Dworkin an. Er kritisiert Rawls, da dieser nicht unterscheidet zwischen externen Gegebenheiten und persönlichen Entscheidungen, und daher den Gerechtigkeitsgedanken von Rawls mit dem Gedanken der Pflichten erweitert und betont die ethisch vertretbare Balance von Rechten und Pflichten (vergl. Schmid 2007: 44). Vor allem für die Frage des Risikomanagements, das im folgenden Paragraphen eine Rolle spielt, ist diese Hinzufügung relevant.

Beide Theorien sind mit dem eher klassischen Sozialstaat, vor Beginn des Schlanken Staates, verbunden. Sie basieren auf relativ deutlichen gesellschaftlichen Verhältnissen mit einer homogenen Gesellschaft in der allen Bürgern theoretisch vergleichbare Rechte zugesichert werden können. Im Laufe der Jahre hat Rawls seine Theorie gedanklich erweitert und sich mit der Frage befasst, wie sich Gerechtigkeit und Zielmäßigkeit verbinden anstatt ausschließen lassen. Eine weitere Entwicklung dieses Gedankens erfolgte von Sen. Er erweiterte die Gerechtigkeitstheorie um eine gedankliche Ausweitung über die Hilfsmittel, bzw. Instrumente, welchen Bürgern durch den Sozialstaat geboten werden müssten. Er hält universelle Hilfsmittel für wenig sinnvoll und daher nicht geeignet und unterstreicht die individuellen Unterschiede zwischen den Hilfsbedürftigen. Ihnen gelingt es, die in unterschiedlichem Maße angebotenen Instrumente bzw. Mittel zu verwenden und eventuelle Risiken in neue Lebenschancen zu transformieren (vergl. Engelen/Hemerijk 2007: 315ff). 


\subsubsection{Schutz vor neuen Risiken}

Wie im vorherigen Paragraphen beschrieben, hat Dworkin dem Gerechtigkeitsprinzip die Dimension der Eigenverantwortung hinzugefügt. Gewisse Risiken sind selbstverschuldet bzw. (mehr oder weniger bewusst) gewählt, andere Risiken sind externer Art und können nicht vom individuellen Bürger eingeschätzt werden. „Dworkins ethisches Gerechtigkeitsprinzip kann sehr gut als normatives Fundament von sozialem Risikomanagement dienen. Abhängig von der Gegebenheit, ob die Risiken selbstverschuldet sind oder durch externe Umstände und ob die Konsequenzen dieser Risiken selbstständig getragen werden können, oder ob es über den individuellen Rahmen hinausgeht und daher kollektiv verwaltet werden müssen, können vier Typen sozialen Risikomanagements unterschieden werden (...) Natürlich sind die Grenzen dieser Ideale in der Realität unscharf" (Schmid 2007: 44). 
Tabelle 3: Idealtypen sozialer Risikoverteilung (Schmid 2007: 45)

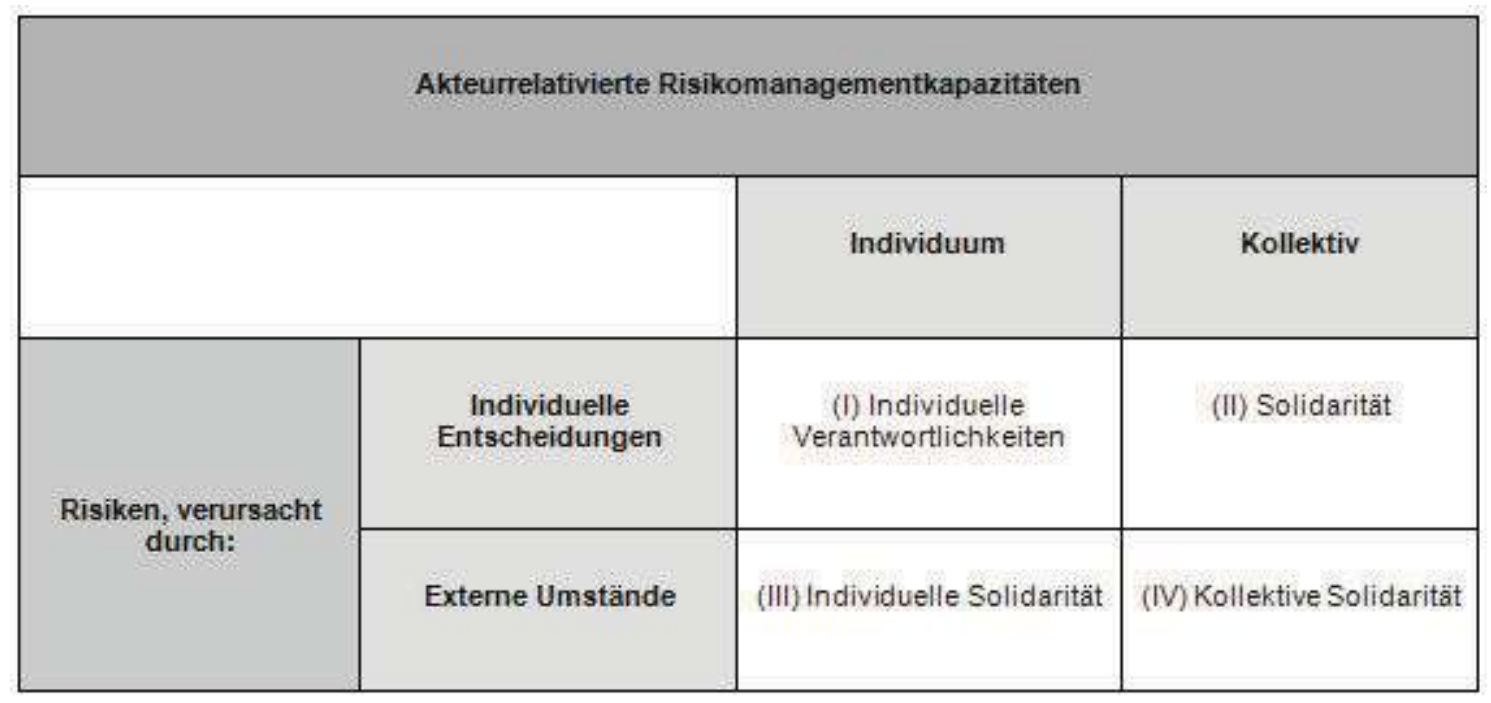

Niklas Luhmann unterscheidet in diesem Zusammenhang zwischen den Begriffen Gefahr und Risiko. Moderne Gesellschaften kennzeichnen sich - laut Luhmann - durch die Umstellung von Gefahren auf Risiken. Von Gefahr spricht man bei einem Schaden der durch die Umwelt verursacht werden kann durch z.B. einem feindlichen Gegenüber, einer Naturkatastrophe usw.. Der Begriff Risiko hingegen kann verwendet werden, wenn der Schaden bzw. der erlittene Verlust Resultat einer individuellen Entscheidung ist (vergl. Schmid 2007: 33).

Wirtschaftliche und gesellschaftliche Verhältnisse nach Ende des Zweiten Weltkrieges erlaubten ein ausgedehntes Maß an sozialen Rechten und den Schutz der Bürger vor individuellen Risiken. „Diese Schutzwirkung teilt sich der Wohlfahrtsstaat mit zwei weiteren gesellschaftlichen Institutionen, der Familie und dem Markt. In der Gegenwart steht der Wohlfahrtsstaat aber vor neuen, nur schwer zu bewältigen Herausforderungen: den Folgen der Globalisierung, der Überalterung der Gesellschaft und der Desintegration der Familien. EspingAndersen spricht in diesem Zusammenhang vom ,Trojanischen Pferd des Wohlfahrtsstaates'. Das „,Trojanische Pferd' birgt (...) Risiken, die die Konstrukteure des Wohlfahrtsstaates nicht vorausgeahnt haben: das Beschäftigungsrisiko gering qualifizierter Arbeitskräfte, die Verlagerung von Haushaltslasten auf künftige Generationen, die wachsende Alterslastquote und die immer schwächer werdende Schutzwirkung der Familie“ (Kevenhörster 2006: 245f). Die vier von Dworkin unterschiedenen Risiken entsprachen eher 
der gesellschaftlichen Situation seiner Zeit mit einer relativ hohen Fertilität bzw. einer jungen Gesellschaft, einer im Vergleich zur heutigen Situation verhältnismäßig niedrigen Lebenserwartung, industrieller Wirtschaft und Arbeit und der Hausfrauenehe (vergl. Engelen/Hemerijk 2007: 310).

Mit dem ,transitionellen Arbeitsmarkt-Ansatz' ist es Schmid dann gelungen soziale Risiken des postindustriellen, bzw. postmodernen Wohlfahrtsstaates nicht nur zu benennen, sondern auch zu beschreiben, welche Rezepte im Falle dieser Risiken helfen könnten (vergl. Trommel 2007: 264). Zu den neuen Risiken zählt Schmid z.B. den späten Übergang von Ausbildung zum Arbeitsmarkt, die Rushhour des Lebens und Ehescheidungen, bzw. die Trennung von seinem Lebensgefährten. Aufgrund der veränderten Bedürfnisse am Arbeitsmarkt kann es außerdem z.B. für ältere Arbeitnehmer bzw. Arbeitnehmern mit niedriger Schulausbildung zu neuen Risiken kommen (vergl. Wetenschappelijke Raad voor het Regeringsbeleid 2006: 158).

Giddens spricht weitergehend auch von einer Wandlung bestehender sozialpolitischer Traditionen. Er führt den Begriff der „Lebenspolitik“ (vergl. Giddens 1997: 272ff) ein, zu der er weitergehende Fähigkeiten als die reine Umverteilung von Mitteln und Ressourcen rechnet. „Sozialpolitische Maßnahmen und Programme können sich daher nicht mehr auf die Versorgung ,Bedürftiger' beschränken, sondern sie müssen Rahmenbedingungen und Gelegenheiten für selbstgesteuerte Problemlösungsund Bewältigungsstrategien bereitstellen, also in gewisser Hinsicht einen Beitrag zu individuellen und kollektiven Strategien des ,Risikomanagements' leisten" (Olk 2000: 111). Um die Risiken gut einzuschätzen und eine Lebenspolitik zu ermöglichen ist es nötig die Verantwortlichkeiten gut zu differenzieren.

\subsubsection{Differenzierung von Verantwortlichkeiten}

Die Erfüllung der sozialstaatlichen Aufgaben und somit das Verhältnis zwischen Staat und Gesellschaft hat sich seit der Entwicklung des Aktivierenden Staates verändert zu einer neuen Form von Verantwortungsteilung. Durch diese neue Form der Verantwortungsteilung und Kooperation entsteht eine neue Steuerungsform, die von allen Akteuren ein angepasstes Handeln erfordert. 
„Ausgehend vom Gedanken der Leistungstiefenpolitik (Naschold 1996) rückt der Aktivierende Staat die Intensitätsgrade staatlicher Verantwortung neu in den Mittelpunkt und gleicht diese fortwährend mit den Beiträgen gesellschaftlicher Akteure ab. Diese neue Stufung von Verantwortung zwischen Staat und Gesellschaft kann man auf der Ebene von Verantwortungskategorien wie folgt beschreiben: Der Aktivierende Staat übernimmt die Gewährleistungsverantwortung für eine öffentliche Aufgabe, wenn im demokratischen Prozess ein gewichtiges öffentliches Interesse dafür festgestellt worden ist. Dies heißt jedoch keinesfalls, dass auch die Finanzierungs- und Vollzugsverantwortung beim Staat liegen muss. Der Staat sollte nur dann die Finanzierungsverantwortung übernehmen, wenn entweder keine marktgerechten Erlöse zu erzielen sind oder aber die staatliche Finanzierung ausdrücklich der politischen Zielsetzung entspricht. Die Vollzugsverantwortung sollte der Staat nur dann übernehmen, wenn (nichtstaatliche) Dritte nicht verfügbar sind oder der Vollzug durch solche Dritte aus Risiko-, Missbrauchsoder Gleichbehandlungsaspekten ausscheidet“ (Lamping/Schridde 2004: 51).

Tabelle 4: Konzept der arbeitsteiligen Verantwortungsteilung (Quelle in Anlehnung an Reichard 2006) (Schuppert 2005: 39)

\begin{tabular}{|c|c|c|c|}
\hline & $\begin{array}{l}\text { Gewährleistungsver- } \\
\text { antwortung }\end{array}$ & $\begin{array}{l}\text { Vollzugsver- } \\
\text { antworgung }\end{array}$ & $\begin{array}{l}\text { Finanzierungsver- } \\
\text { antwortung }\end{array}$ \\
\hline Staatliche Kernaufgabe & \multicolumn{3}{|c|}{ Verantwortung beim Staat } \\
\hline $\begin{array}{c}\text { Staatliche } \\
\text { Gewährleistungsaufgabe }\end{array}$ & $\begin{array}{l}\text { Verantwortung beim } \\
\text { Staat }\end{array}$ & \multicolumn{2}{|c|}{ Verantwortung beim Staat oder bei Privaten } \\
\hline Private Kernaufgabe & \multicolumn{3}{|c|}{ Verantwortung bei Privaten } \\
\hline
\end{tabular}

Wer die möglichen Akteure und somit auch Verantwortungsträger sind, wird im folgenden Abschnitt näher erläutert. 


\subsubsection{Handlungsakteure im Aktivierenden Staat}

Seit dem Zweiten Weltkrieg wurden die sozialpolitischen Aufgaben bereits geteilt zwischen den genannten Akteuren und stellte der Staat neben anderen Akteuren wie Markt, Familie und dem Dritten Sektor nur eine Institution neben anderen dar. Bode schlussfolgert hieraus, dass „der Staat im organisierten Wohlfahrtskapitalismus, anders als häufig unterstellt (vergl. etwa Heinelt 1999), weniger ein Verwaltungs- als (schon längst) ein Verhandlungsstaat“ (Bode 2004: 71) war. Neu allerdings ist die Art der Verträge, welche häufig einen privaten Charakter bekommen und die Art der Aufgabenerteilung und Ausführung. Der Politik, mehr noch als dem Staat, kommt hierbei eine wichtige Rolle zu, da sie laut Priddat zum demokratischen Mittelpunkt von Umverteilungsverträgen wird. „Wir haben es mit drei Basiskollektiven zu tun:

1. Mit dem Kostenkollektiv (KK) der Bürger, die über Steuern, aber auch über Sozialversicherungsbeiträge, private Caritas etc., die Sozialpolitik finanzieren;

2. Mit dem Nutzerkollektiv (NK) derjenigen Bürger, die Anspruch auf social transfer anmelden. $\mathrm{NK}<\mathrm{KK}$;

3. Mit dem Entscheidungskollektiv (EK), das zum einen als ,die Politik' parlamentarisch-repräsentativ ausgegliedert ist, zum anderen aber als Politikprozess wiederum alle Bürger (im Modus KK wie im Modus NK) umspannt.

Das politische Milieu, in dem sich die neue soziale Angebotspolitik: progressive governance etabliert, ist nicht mehr vom Konsens geeint, dass alle ihre Ansprüche realisieren können“ (Priddat 2004: 96f).

„Zur Architektur dessen, was man in Anlehnung an Esping-Andersen (1990) ,Wohlfahrtskapitalismus' nennen könnte, haben während des 20. Jahrhunderts in der Tat zivilgesellschaftliche Kräfte maßgeblich beigetragen. In gewisser Hinsicht sind sie die eigentlichen Urheber von Sozialstaatlichkeit. Für den deutschen Fall beispielsweise ist offenkundig, dass die Infrastruktur der sozialen Daseinsvorsorge in hohem Maße an die Verbandsstrukturen industrieller ,Governance' angekoppelt wurde (...) Die praktische Regulierung der sozialen Frage, konkret: die Verwaltung und Erstellung sozial-reproduktiver Leistungen, gelangte also in die Hände von Instanzen, die zwischen Sozialstaat und 
Zivilgesellschaft angesiedelt waren (...) Die Wohlfahrtsverbände entwickelten sich auf diese Weise zum ,dritten Sozialpartner'“ (Bode 2004: 70).

Auch von den Organisationen des Dritten Sektors wird durch die Entstehung des neuen Leitbildes eine neue Herangehensweise nach wirtschaftlichen Erkenntnissen gefordert. Diese Unternehmenskultur kann eventuell zu Schwierigkeiten führen mit dem originären Charakter dieser Organisationen, die neben des zivilgesellschaftlichen Ursprungs oft auch von bürgerlichem Engagement abhängig ist. Auf den ersten Blick ist dieses Engagement nicht vereinbar mit einer eher wirtschaftlichen Herangehensweise. „Durch die Durchsetzung einer Sozialwirtschaft im Bereich sozialer Dienste verstehen sich die Trägerorganisationen im wachsenden Maße als Unternehmen und diese Selbstdefinition verbietet tendenziell die gleichzeitige Verfolgung davon abweichender Interessen, weil sonst die wirtschaftliche Basis des Unternehmens gefährdet wird“ (Wohlfahrt 2007b: 8). Kritiker des Aktivierenden Staates bemerken, dass die sozialwirtschaftliche Unternehmensstruktur von Wohlfahrtsverbänden im Widerspruch steht zum privaten Engagement. „Diese Neuorientierung von Wohlfahrtsorganisationen anzuregen oder $\mathrm{zu}$ unterstützen, die sowohl von ihrer Geschichte her als auch von ihrem ideellen Auftrag das freiwillige Engagement nicht als Zusatz und Anhängsel professioneller Verbandsstrukturen, sondern als unabdingbare Bedingung des Einsatzes für eine solidarische und an sozialer Inklusion weiterhin interessierte Gesellschaft benötigen, scheint mir eine zentrale Bedingung für die Entwicklung nachhaltiger Förder- und Unterstützungsstrukturen für Engagement im Sozialsektor zu sein“ (Wohlfahrt 2007b: 12).

Dem komplexen Charakter der sozialstaatlichen Dienstleistungen entsprechend muss der Aktivierende Staat sein Handeln einrichten. Während einerseits aus betriebswirtschaftlicher Sicht Innovation, Kundenzufriedenheit und Effizienz entscheidend sind, muss auch politisch-normativen Aufgaben Rechnung getragen werden (vergl. Dijstelbloem/Meurs 2007: 296). Im folgenden Abschnitt werden diese Kriterien miteinander verbunden. 


\subsubsection{Das Prinzip der Leistungskette}

Dem betriebswirtschaftlichen Bereich entnommen ist das Prinzip der Leistungskette, die auch als Wertschöpfungskette umschrieben wird. Die Herstellung sozialpolitischer Produkte bzw. Dienste wird durch die Aufreihung individueller Teilprozesse analysiert bzw. verdeutlicht. Hierdurch werden einzelne Elemente des Prozesses transparanter und können im Hinblick auf Effektivität und Effizienz als prinzipiell notwendig diskutiert werden bzw. wird die Frage der Aufgabenverantwortung und die Frage der erwünschten Wirkung deutlich. „Dabei stellen sich die einzelnen Stufen als Transformationsstufen von öffentlichen Aufträgen und bereitgestellten Finanzmitteln in unterschiedliche sozialpolitische Leistungen (Produkte) dar, die jeweils im Sinne einer inputoutput-impact-Analyse Voraussetzungen für die nächste Stufe sind (...) Eine klare Zuordnung der Verantwortung bildet eine wesentliche Voraussetzung für eine Optimierung der einzelnen Prozessstufen“ (Lamping/Schridde 2004: 54). Übersetzt auf die Verantwortlichkeit der verschiedenen Akteure kommt es bei den einzelnen Stufen dieser Kette zu einem permanenten Wechselspiel. 
Tabelle 5: Verantwortungsstufung und Aktivierung im Sozialstaat - im Spiel von Angebot und Nachfrage (Quelle: in Anlehnung an Blanke: 2001a) (Lamping/Schridde 2004: 55, Bearbeitung Willenborg)

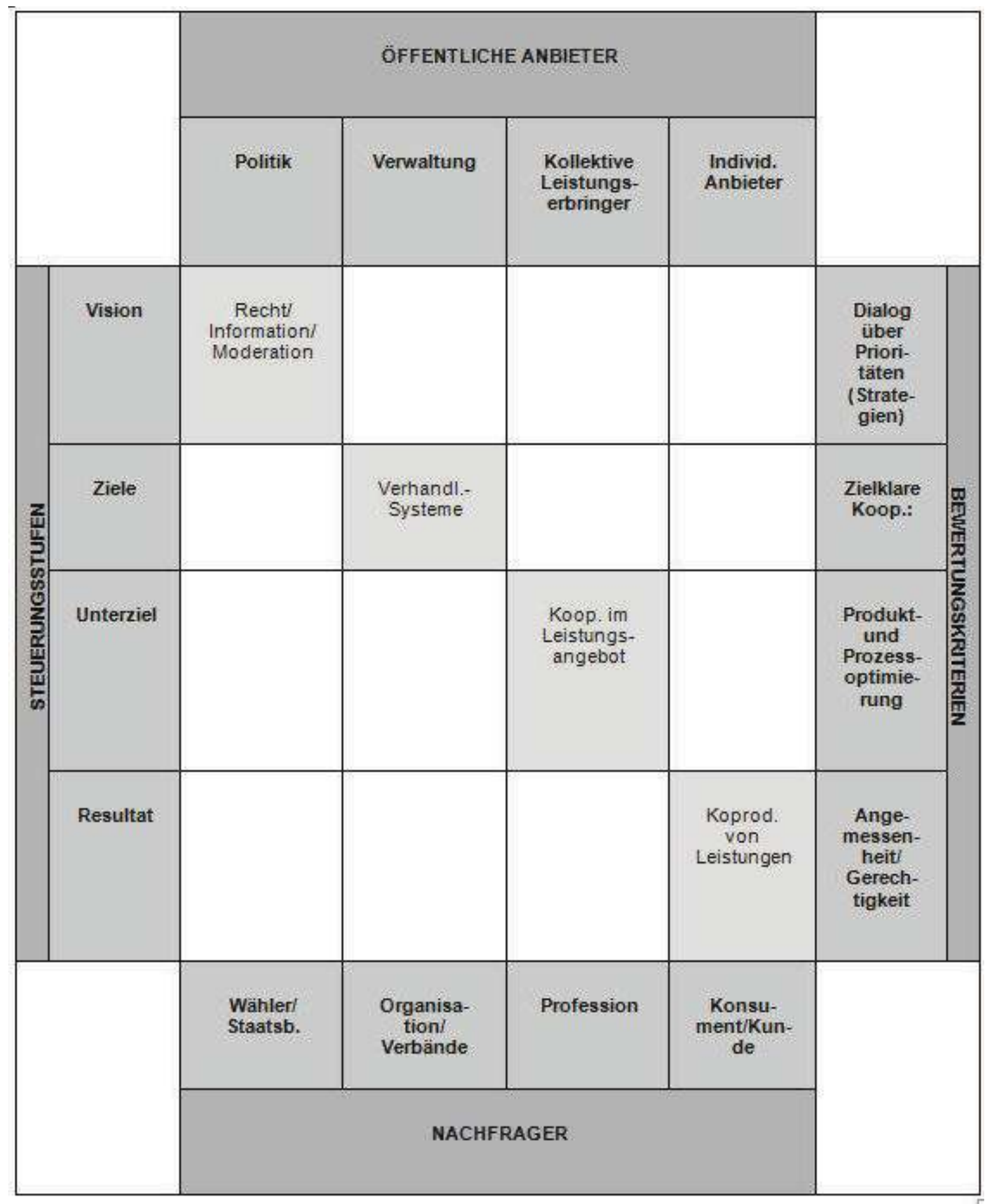


Logischerweise funktioniert die Leistungskette von der Zielsetzung rückwärts, da zu Beginn des Prozesses gemeinsam die erwünschte Wirkung als Vision festgestellt wird. Alle weiteren Stufen der Kette dienen diesem Ziel. „Aufgabe der ,Politik' als strategischer Controllinginstanz wäre es, auf jeder Stufe zu bewirken, dass der Leistungs-(Bewirkungs-)fluss unter optimalen Bedingungen kontinuierlich fließen kann. Diese Formulierung nimmt radikal Abschied von der Regelsteuerung“ (Lamping/Schridde 2004: 54ff). Zu einem guten Funktionieren dieser Kette können Verträge über die Erbringung einzelner Schritte erforderlich sein, denn eine gute Aufgabenteilung und genaue Vereinbarung zwischen den einzelnen Parteien ist nötig. Aus Unverständnis über die Verteilung der Verantwortlichkeiten bzw. Unfähigkeit oder mehr oder weniger bewusste Verweigerung bzw. Störung des Prozesses kann es nämlich $\mathrm{zu}$ erheblichen Steuerungsdefiziten kommen. Um der Komplexität dieser Leistungskette gerecht $\mathrm{zu}$ werden, müssen die Aufgaben weniger zentral, sondern eher dezentral und im permanenten Dialog zwischen den Partnern erfolgen. Ein Instrument hierbei ist das Neue Steuerungsmodell (NSM). „Das in der Bundesrepublik im Umfeld der Verwaltungsreform favorisierte (...) ,NSM' legt hierbei großen Wert auf das Kontraktmanagement, d.h. ein System von Zielvereinbarungen zwischen ,Zentrale' und (teil-)autonomen Funktionsbereichen gepaart mit einem mehrstufigen Controllingprozess, bei welchem zur Bewertung aus dem Feld der erreichten Ergebnisse nur noch diejenigen ausgefiltert werden, die für die allgemeinen Ziele relevant sind“ (Lamping/Schridde/Plaß/Blanke 2002: 33f).

Dem ermöglichenden bzw. befähigenden Charakter des Aktivierenden Staates entsprechend richten sich seine Instrumente darauf, die auch in der Vergangenheit „schon wirksamen Mechanismen und Prozesse der Koproduktion bewusst zu machen und die Strukturen und Abläufe in diesen Einrichtungen derart auszugestalten, dass Koproduktion - wie oft genug - nicht entmutigt und behindert sondern unterstützt und gefördert wird. Während also Partizipationsforderungen in den 7oer-Jahren ,von unten', also von den Bürgerinnen und Bürgern artikuliert und gegen Politik und Verwaltung durchgesetzt werden mussten, besteht das Neue in der gegenwärtigen Situation darin, dass solche Partizipationsförderungen und -angebote im Rahmen aktivierender Konzepte von Politik und Verwaltung an die Bürger herangetragen 
werden“ (Olk 2000: 119f). Die Formgebung dieser Prinzipien in aktivierende Maßnahmen ist im folgenden Unterkapitel beschrieben.

\subsection{Verantwortungsstufung und Instrumente zur Aktivierung im Sozialstaat}

An dieser Stelle wird erläutert, wie sich die beschriebene Verantwortlichkeitsteilung in der praktischen Koproduktion des Aktivierenden Staates manifestiert. Um dieser Verantwortung gerecht $\mathrm{zu}$ werden, bedarf es eines neuen Instrumentariums staatlichen Handelns. Sicherlich sind die Erfahrungen und Erkenntnisse des NSM wichtig und nützlich. Dieses Instrumentarium bedarf allerdings einer Weiterentwicklung, um ihrer politischen Führungsverantwortung gerecht werden zu können in Richtung ,gutes (bzw. kluges) Regieren’. „Der Staat soll daher im Aktivierenden Staat nicht nur Richtungsgeber, Moderator und aktivierende Kraft bleiben, sondern muss zuweilen auch Schiedsrichter sein, der ein ,Spiel' unterbricht, manchmal auch ganz abpfeift und es neu ansetzt“ (Lamping/Schridde/Plaß/Blanke 2002: 29f).

Auch andere Funktionsbereiche staatlichen Handelns, z.B. Rechtsetzung, Rechtsschutzgewährung wurden durch das Leitbild des Aktivierenden Staates beeinflusst. Da diese Arbeit allerdings einen sozialwissenschaftlichen Charakter hat, liegt der Fokus hier primär auf dem Verwaltungshandeln.

Vor der eigentlichen Zusammenfassung der Instrumente für Verwaltungshandeln wird die neue Staatlichkeit verdeutlicht. Eine prinzipielle Umschreibung der neuen Aufgabenwahrnehmung, sowie der Instrumente Privatisierung, Öffentlich-Private Zusammenarbeit und Sozialinvestition, rundet das letzte Unterkapitel ab.

\subsubsection{Neues Staatsverhältnis und entsprechendes politisches Handeln}

Ein wichtiges Element des neuen Staatsverständnisses ist das Verhältnis von Staat und Bürger untereinander. „Das traditionelle hoheitliche Staatsverständnis allein wird der Komplexität einer sich zunehmend differenzierenden, 
dynamisierenden und auch individualisierenden Gesellschaft immer weniger gerecht. Somit verschiebt sich das Aufgabenspektrum staatlicher Politik und es ändern sich Vorgehensweisen zur Umsetzung dieser Aufgaben.

Tabelle 6: Verschobenes Aufgabenspektrum staatlicher Politik und veränderte Vorgehensweisen zur Umsetzung dieser Aufgaben (Walter 2000: 156)

\begin{tabular}{|c|c|}
\hline Versorgungsstaat & Aktivierender Staat \\
\hline Regulative Maßnahme & Kooperativ ausgerichtete Steuerung \\
\hline Dirigistische Entscheidungen & Moderation von Verständigungsprozessen \\
\hline Ordnungspolitische Einzelvorschriften & Grundwerte und -orientierungen \\
\hline Ausdehnung staatlicher Einflussbereiche & Selbstbeschränkung auf Schwerpunkte \\
\hline
\end{tabular}

Kevenhörster betont, dass die Verhandlungen zwischen den Beteiligten das Regieren im modernen Staat ausmacht, da von keinem der betroffenen Akteure eine definitive Erhöhung gegenüber anderen Akteuren erwartet werden kann. Als Voraussetzung für das Funktionieren dieses neuen Staatstyps sieht er: „das wechselseitige Vertrauen der Individuen (...) nicht das Vertrauen auf ,Markt' oder 'Staat', sondern (auf) vielfältige Mischformen aus markt- und staatsähnlichen Institutionen“ (Kevenhörster 2006: 357).

Um ein neues Staatsverhältnis $\mathrm{zu}$ erlangen sind fundamentale sozialwirtschaftliche Veränderungen nötig: von Nachsorge zu Prävention, von Arbeitsplatzgarantie zu Arbeitsgarantie, von einer reaktiven zu einer pro-aktiven und von einer defensiven $\mathrm{zu}$ einer offensiven Politik (vergl. SER/WRRSymposium 16.01.2007: 2). Durch diese Veränderung werden Flexibilität von Staat und Bürger, Eigenverantwortung und Unabhängigkeit der Bürger, Vergrößerung der Wahlmöglichkeiten bzw. der Beteiligungsformen durch den Bürger, Vergrößerung der Effektivität und Effizienz der sozialstaatlichen 
Institutionen und die Verwendung von Ressourcen besser garantiert (vergl. Grundsatzabteilung, DGB-Bundesvorstand 2000: 206).

Lamping, Schridde, Plaß und Blanke formulieren im Zusammenhang mit den Indikatoren des Aktivierenden Staates vier Leitlinien, die das Handeln dieses Leitbildes definieren. Aus ihrer Sichtweise ist der Ersatz des staatlichen Dekrets durch einen Dialog, in dem gemeinsame Prioritäten entwickelt werden, nötig. Eine zielgerichtete Kooperation anstelle gegenseitiger Schuldzuweisung und Kompetenzgerangel ist hierfür erforderlich. Betriebswirtschaftliche Instrumente wie Produkt- und Prozessoptimierung durch Quasi-Märkte und Leistungsvergleiche ebenso wie die Koproduktion, also das Zusammenwirken öffentlicher und privater Leistungserbringer bzw. Bürger halten sie für eine essentielle Voraussetzung (vergl. Lamping/Schridde/Plaß/Blanke 2002: 34).

Da sich durch den Übergang vom Industriellen zum Postindustriellen Zeitalter bzw. der Dienstleistungsgesellschaft neue Anforderungen für den Staat ergeben hatten, wurde eine neue Aufgabenerledigung bzw. ein angepasstes Instrumentarium für die Erfüllung dieser Aufgaben notwendig, denn die „Bestimmung von Solidarität in einer demokratischen Dienstleistungsgesellschaft mit weit gefächerten sozialstaatlichen Leistungen muss zwangsläufig anders aussehen als in einer autoritären Agrargemeinschaft am Beginn der Industrialisierung“ (Badura 2000: 125). Durch die Entstehung und Entwicklung des Leitbildes Aktivierender Staat hat sich das Verhältnis der Akteure staatlichen Handels (Staat, privater Sektor, dritter Sektor und Bürger) gewandelt und ist ein gesellschaftlicher Dialog zwischen diesen Akteuren nötig. „Das Ziel ist es dabei, den Gemeinwohlbezug der Organisationen nicht mehr primär aus ihrem Status und ihrer Funktion, sondern wieder stärker aus ihren Leistungen im Produktionsprozess abzuleiten (...) Weder Staatsüberhöhung noch die Überhöhung der Zivilgesellschaft stehen dem Konzept des Aktivierenden Staates Pate, sondern die Vorstellung durch Aktivierung und intelligente Instrumentalisierung zu erreichen, dass die Beteiligten stärker eine gemeinwohlbezogene Problemlösungsperspektive einnehmen“ (Lamping/Schridde/Plaß/Blanke 2002: 36).

In der bereits genannten Studie ,Reinventing Government, How the Entrepeneurial spirit is transforming the Public Sector' publizierten Osborne und Gaebler im Jahre 1992 wichtige Kriterien für das Funktionieren des 
Aktivierenden Staates. Ihr Buch kann als eine Art Checkliste gelesen werden. Die Autoren betonen in diesem Werk, dass der Staat steuern und nicht rudern soll. Er soll die gesellschaftlichen Kräfte eher stärken, als ihnen zu dienen. Wichtig finden sie auch einen betriebswirtschaftlichen Effekt. Staatliches Handeln sollte ausgehen von Zielen und sich eher an Ergebnissen und Verdiensten als nur an der Einhaltung von Prozessen orientieren, denn es soll dem Bürger und nicht der Bürokratie gedient werden. Man müsse hierfür vorsorglich in den Bürger investieren, und nicht nur im Nachhinein die Schäden reparieren. Marktwirtschaftliches Denken ist für dieses Handeln wichtig, daher ist dezentrales Handeln effektiver als zentrale Steuerung (vergl. Osborne/Gaebler 1992).

\subsubsection{Aufgaben und Instrumente}

Aufbauend auf dem Prinzip des Aktivierenden Staates und den praktischen Möglichkeiten der Leistungskette kann prinzipiell unterschieden werden zwischen zwei Formen von Aufgaben für den Staat. Für die Frage, ob Dienste durch den Staat oder durch andere Akteure ausgeführt werden sollen kann es nützlich sein zu differenzieren zwischen einerseits Kernaufgaben, die „von so großer politischer bzw. strategischer Relevanz sind, dass sie sich der Staat zur eigenständigen Erledigung vorbehält (und) staatliche(n) Gewährleistungsaufgaben, die entweder von staatlichen Einrichtungen oder von nicht-staatlichen Trägern an die Bürger erbracht werden, je nachdem, wer dies in der wirtschaftlichsten Weise leisten kann" (Reichard/Schuppan 2000: 85).

Wohlfahrt schreibt, dass der Aktivierende Staat im Gegensatz zum Schlanken Staat von einer kooperativen Leistungserstellung zwischen Staat und Dritten ausgeht. Daher ist Privatisierung nur in wenigen Fällen die Lösung eines Problems. Der Staat ist verantwortlich, nicht in allen Fällen für die Ausführung aller Aufgaben, wohl aber für die Gewährleistung von Erstellung und Durchführung und sieht sich als Förderer und Unterstützer der Kooperationspartner (vergl. Wohlfahrt 2007b: 3). Gemäß der Prinzipien von Koproduktion und Verantwortungsteilung ist laut Blanke, Schridde und Plaß „die Öffnung des Policy Process auf allen Stufen der Leistungserbringung für eine gemeinwohlbezogene Problemlösungsperspektive im Gegensatz zu einer 
reinen ,bargaining'-Strategie aller Beteiligten“ essentiell (Blanke/Schridde/Plaß 2001: 16).

Tabelle 7: Zwei Verhandlungslogiken (Blanke/Schridde/Plaß 2001: 17)

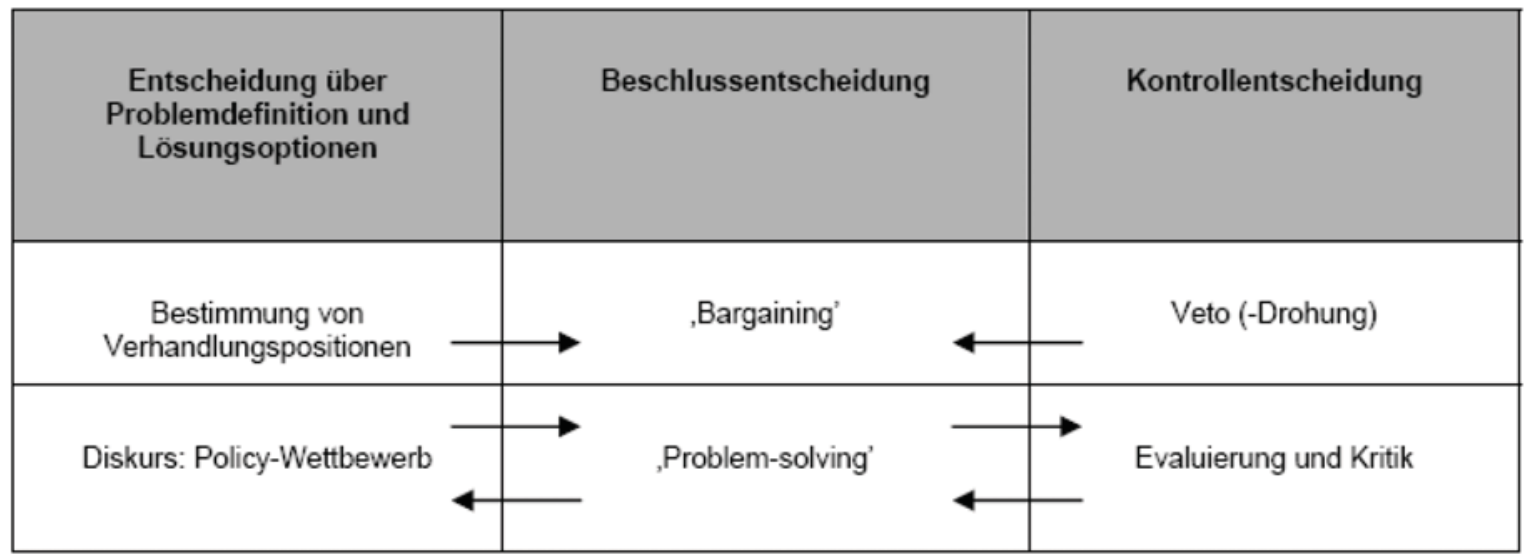

Ziel hierbei ist es den effizientesten und wirksamsten Policy-Mix zu identifizieren und zu verwenden, um bei allen Schritten der Leistungskette die effizientesten und effektivsten Zuständigkeiten $\mathrm{zu}$ erörtern. In diesem Zusammenhang kann auch die Public-Choice-Theorie genannt werden. Diese Theorie ist ein Hilfsmittel zur Analyse kollektiver Entscheidungen. „Hierbei geht es insbesondere um die Ermittlung und Aggregation individueller Präferenzen für öffentliche Güter durch Abstimmungsverfahren, die Festlegung eines optimalen Budgets und die Ermittlung der optimalen Größe von Entscheidungseinheiten“ (Kevenhörster 2006: 245).

Strategisches Vermögen wird vom Staat erwartet, denn er behält die Verantwortung über die verteilten Zuständigkeiten und sollte somit auch den Handlungsspielraum bzw. die persönlichen Ziele (Moral Hazard) anderer Akteure kennen oder zumindest einschätzen können. „Bei unspezifischer Förderung jeder Art von ,Bürgerengagement' kann der Staat nämlich in eine ,Aktivierungsfalle' geraten: Bürgerinnen und Bürger sind heute - zumal, wenn sie über die Ressourcen verfügen - immer mobiler geworden. Die Überspringung von Rollendifferenzierungen und die strategische Nutzung verschiedener ,principal-agent-Beziehungen' (Williamsen 1990) kann dazu führen, dass ,der Staat' in ein Hase-und-Igel-Spiel verstrickt wird, wo die Bürger ,immer schon da sind', zumal dann, wenn die ,Staatsmitarbeiter' entdecken, dass sie ja eigentlich auch Bürger sind und beginnen, sich $\mathrm{zu}$ aktivieren (Blanke/Schridde 2001)“ (Blanke/Schridde/Plaß 2001: 32). Eventuell kann und 
sollte die Entscheidung zwischen dem Stellenwert der Aktivitäten auch dazu führen, dass gewisse Aktivitäten nicht mehr vom Staat unterstützt werden. Vor diesem Hintergrund werden in den folgenden Abschnitten drei Instrumente des Aktivierenden Staates unterschieden: Privatisierung, Öffentlich-Private Partnerschaft (ÖPP)/Public-Private-Partnerships (PPP) und Sozialinvestition.

\subsubsection{Privatisierung}

Privatisierung wird in erster Linie dem Schlanken Staat zugerechnet, ist aber auch Teil des Aktivierenden Staates, da aufgrund der Analyse in der Leistungskette eventuell der Entschluss getroffen werden kann, bestimmte Aufgaben zu privatisieren. Kevenhörster schreibt z.B., dass sich bei „bekannten, einfachen Zielen, die eine Dezentralisierung oder Privatisierung der Leistungserbringung nahe legen, (...) der Aktivierende Staat seiner politischen Verantwortung für seine Leistungserbringung durch Konzepte des Wettbewerbs zwischen den Leistungsanbietern, Qualitätsmanagement und Benchmarking gerecht werden muss. In diesem Rahmen kann er die Leistungserbringung an private oder intermediäre Organisationen übertragen“ (Kevenhörster 2006: $348)$.

Gilbert unterscheidet im Jahr 2002 vier Mechanismen, welche der öffentliche Dienst verwendet, um Privatisierung zu entwickeln bzw. zu stimulieren:

4. Investition öffentlicher Mittel in private Aktivitäten, sowie es teilweise in der Kinderbetreuung üblich ist,

5. Die Regulierung und Mandatierung privat angebotener Dienste und Beaufsichtigung,

6. Die Auslagerung öffentlicher Dienste, z.B. Reintegrationsprojekte von Sozialhilfeempfängern, an private Unternehmen (outsourcing),

7. Das Zuteilen finanzieller Mittel oder Gutscheine zur Verwendung privater Dienstleistungen (vergl. Knijn 2004: 13).

Die verschiedenen Pfade zur Privatisierung können laut Gilbert in zwei Kategorien unterschieden werden. Zu der ersten Kategorie zählt Gilbert direkte Pfade zur Steigerung der privaten Finanzierung (wie z.B. Förderung durch Steuermittel), während die anderen Pfade eher darauf gerichtet sind die Produktion von öffentlichen Produkten und Diensten durch die Privatwirtschaft 
zu fördern (wie z.B. die Anwendung öffentlicher Mittel; durch direkte finanzielle Transfers oder indirekt durch Anwendung von Gutscheinen) (vergl. Gilbert 2005: 7ff). Entsprechend ihres eigenen Charakters verfügen alle Pfade über Vorund Nachteile. Steuerbegünstigungen z.B. gelten für den Bürger als eher unübersichtlich. Das Gutschein-Prinzip hingegen ist einfach zu verstehen, kann aber zu neuer Bürokratie führen.

\subsubsection{2 Öffentliche-Private Partnerschaft(ÖPP)/Public Private Partnership (PPP)}

Auch das zweite Instrument, ÖPP (PPP), ist kein neues Instrument, hat aber seit Entwicklung des Aktivierenden Staates eine weitere Evolution durchlaufen. Budäus unterscheidet zwischen drei Generationen von ÖPP's (Budäus 2006: 12ff). Zu der ersten Generation zählt er die Zusammenarbeit zwischen privaten und öffentlichen Kapitalgebern bei der Wahrnehmung öffentlicher Aufgaben. Im Unterschied zur Privatisierung geht es hierbei nicht um die Vergabe eines Auftrages sondern um die gemeinsame Entwicklung bzw. Abhandlung eines größeren Projektes. Der Hintergrund der zweiten Generation von ÖPP's ergab sich vor allem aus der Haushaltsentwicklung, die auch zum Schlanken Staat führte. Aufgrund der begrenzten Steuermittel wurden auch alternative Finanzierungsquellen verwendet (Wandel von kollektiven $\mathrm{zu}$ individuellen Finanzierungsformen). Die dritte und neue Form einer ÖPP entspricht dem Gedanken der Corporate Social Responsibility. Nach der Verteilung gewisser Aufgaben durch den Staat entstehen neue Märkte, von denen auch private Unternehmen profitieren. Im Falle der Umsetzung spezifisch sozialer Interessen wird eventuell auch von Public Social Private Partnership (PSPP) gesprochen. Dieser Begriff ist dem hier beschriebenen ÖPP inhaltlich sehr ähnlich und wird an dieser Stelle nicht weiter erörtert.

Unterschieden werden muss die ÖPP von der klassischen Auftragsvergabe (Outsourcing), denn ÖPP's zeichnen sich grundsätzlich aus durch Kontinuität und Kooperation. Die oftmals komplexen Aufgaben umfassen in der Regel einen großen Finanzrahmen. Daher können anders als im Rahmen des Outsourcings nicht schon vor Wahrnehmung der Aufgabe die Handlungsschritte detailliert festgelegt werden. Vertrauen in den Partner ist für das Gelingen einer ÖPP essentiell. Dennoch ist es nötig, ÖPP's vertraglich zu regeln. Zwei Formen von Verträgen können hierzu dienen. Das 
vertragsrechtliche Modell, welches auch als Tauschmodell oder Projekt-ÖPP bezeichnet wird, kennzeichnet sich durch den Tausch von Leistung und Gegenleistung. Im Poolmodell, (bzw. Betreibermodell oder Organisaitons-ÖPP), wird ausgegangen von der Teilung der Ressourcen in einem gemeinsamen Pool. Die Kooperation ist in diesen Fällen in der Regel unbefristet.

Der Grundgedanke hierbei ist, dass eine Partnerschaft zwischen privaten und öffentlichen Sektor effizienter ist als die Vergabe von Aufgaben. Verschiedene betriebswirtschaftliche Theorien, wie z.B. die Transaktionskostentheorie von Williamson, die Prinzipal-Agenten-Theorie von Jensen und Meckling sowie die Theorie der Verfügungsrechte von Demsetz bauen auf der Neuen Institutionenökonomie auf und erklären warum eine Zusammenarbeit zwischen öffentlichem und privatem Sektor eventuell gewinnbringender sein kann als die Erfüllung der Aufgaben durch einen der beiden Akteure.

\subsubsection{Sozialinvestition}

Die letzte Gruppe der Instrumente ist gerichtet auf die Zivilgesellschaft. Hier kann unterschieden werden zwischen der Unterstützung einzelner Individuen bei der eigenen Lebensversorgung einerseits und der Unterstützung von Freiwilligkeit und Engagement andererseits. „Die bisherige wohlfahrtsphilosophische Semantik der ,Umverteilung' und ,Solidarität' beginnt sich in Richtung einer ,sozialen Angebotspolitik' zu verschieben: social investment heißt der Begriff der Stunde“" (Priddat 2003: 373).

\subsection{Fördern und Fordern}

Das Schlagwort des Förderns und Forderns geht mit einem konkreten Instrumentarium staatlicher Maßnahmen einher. Ziel ist es dem Bürger nicht nur kurzfristig zu helfen, sondern ihn zur Eigenverantwortlichkeit zu aktivieren. Nicht mehr der Anspruch auf wohlfahrtsstaatliche Leistungen, sondern arbeitsmarktbezogene „Unterstützungsprogrammen (workfare), eine rundumerneuerte Rentenpolitik und monetäre Anreize wie die steuerliche Bezuschussung erwerbstätiger Familien (WVTC) - Dinge, die zusammengenommen den ,Post-Wohlfahrts-Vertragsstaat' (post-welfare contracting state) ausmachen“ (Evans/Cerny 2004: 212), formen die Basis dieses Instrumentes. 
Die klassische Verteilung von Mitteln hat sich erweitert, um den Begriff des Forderns. Soziale Kredite basieren auf dem Gedanken der Gegenseitigkeit, d.h. für die staatliche Investition wird eine Gegenleistung vom Empfänger erwartet. Trommel schreibt in diesem Zusammenhang auch von den produktionistischen Visionen im Sinne des ,social investment states' von Anthony Giddens (1998) der unterscheidet zwischen der Versorgungsverantwortung des Staates einerseits und der zielbewussten staatlichen Investition andererseits. Giddens unterstreicht in diesem Zusammenhang, dass in dieser Form von staatlicher Aufgabenwahrnehmung eine Zusammenführung von sozialen und wirtschaftlichen Investitionen einerseits, sowie eine Gewinnorientierung andererseits im Mittelpunkt stehen sollten. Daher ist eine Politik nötig, die einen produktiven Lebenslauf und soziale Selbstständigkeit unterstützt (vergl. Trommel 2007: 252ff). Priddat unterscheidet bei dieser Form von Sozialpolitik zwischen zwei Typen:

- $\quad$ Sozialpolitik Typ I: durch z.B. Bildungsinvestitionen wird investiert, um die Handlungsfähigkeit wieder herzustellen,

- $\quad$ Sozialpolitik Typ II: Kompensation für ungelungene Sozialinvesition durch z.B. Arbeitslosengeld (vergl. Priddat 2003: 381).

Durch soziale Investitionen können persönliche, sozialwirtschaftliche Rückstände, die z.B. aufgrund Folgen neuer Risiken o.ä. entstanden sein können, behoben werden, „um den Zustand der Bedürftigkeit in einen Zustand des re-entry in selbständige Einkommensgenerierung herzustellen. Aus den returns on investment, das heißt aus dem dann erreichten Einkommen, wird über Steuern und Senkung des sonst anfallenden Sozialtransfers eine Rückzahlung erreicht. Alle Bedürftigen, die keinen eigenen Beitrag zu dieser neuen Kooperation leisten können, werden zwar weiter alimentiert, aber nicht mehr auf dem Wohlfahrtsrentenniveau wie bisher. Hierfür gibt es zwei Gründe: 1. einen Kostengrund, 2. die Stimulierung eines Anreizes. Erst wenn diese Bereitschaft zur Selbsttätigkeit gezeigt wird, investiert der Staat seinen Anteil (...) Der Staat tritt als Kreditgeber auf, als venture-capitalist im Sozialbereich, der dann folgerichtig nur solche Aktivitäten unterstützt, von denen absehbar ist, dass sie sich à la longue rentieren“ (vergl. Priddat 2004: 92).

Auf diese Weise will der Staat Anreize für eine aktive Beteiligung am Arbeitsmarkt schaffen und ändert sich das Sozialmodell. Neben dem üblichen 
Sozialtransfer ohne Retributionen kommt es mehr und mehr zu sozialen Investierungen mit der Erwartung einer längerfristigen Rückzahlung. „An die Stelle von Auszahlungsanspruchsgerechtigkeit (mit den Implikationen: „der hat bekommen, warum bekomme ich nicht?") tritt eine Investitionsgerechtigkeit bzw. eine Investitionsbereitschaftsgerechtigkeit (...) Solidarität wird gegen die tradierte, eher passive Sichtweise, pro-aktiv als Kooperationsprozess rekonstituiert (...) In diesem Sinne macht der Staat ein neues Angebot, dem die Bürger selber mit ihrem eigenen Angebot antworten, um in Kooperation zu treten. Das consuming of the state's services wird in ein prosuming verwandelt (ähnlich wie in der Wirtschaft auch die Kunden in die Zusammenarbeit der Erstellung ihrer Wünsche treten; das IKEA-Modell: man kauft ein Dispositiv, das man selber zuende-produziert" (Priddat 2004: 93f).

\section{Freiwilligkeit und Engagement}

Eine zweite Art sozialer Investierung findet in Form von Förderung bürgerschaftlichen Engagements statt. Hierbei geht es um die Erfüllung von gesellschaftlichen Funktionen durch Ehrenamtliche bzw. Freiwillige in den Institutionen wie z.B. der Verbände, der Jugendhilfe usw., aber auch um das freiwillige Engagement außerhalb dieser Verbände. „Innovative Potentiale der Stadt und Gemeinde, aber v.a. der Nachbarschaften und vor Ort verankerter Initiativen werden immer mehr durch die Perspektive des Sozialkapitals betrachtet. Dies galt zunächst v.a. in den USA, wo eine breite kommunitaristische Tradition längst den Boden für solche Sichtweisen vorbereitet hat" (Wohlfahrt 2007b: 4).

Eine Möglichkeit zur Förderung privater Maßnahmen und Engagements ist es z.B. um ein Startkapital bereitzustellen für Prozesse, die danach selbstständig weiterlaufen. Andererseits ist es auch möglich freiwilliges Engagement (z.B. Großeltern die als Tageseltern auftreten) permanent auf einem niedrigen Niveau zu fördern.

Zur Rolle der Verbände muss in diesem Zusammenhang noch eine kritische Bemerkung hinzugefügt werden. Einerseits wird im Zuge der Verwaltungsreform von ihnen betriebswirtschaftliches und zielbestimmtes Verhalten gefordert. Andererseits sind sie in starkem Maße vom freiwilligen Engagement abhängig, das man nicht immer gleich gut steuern kann. Im Zusammenhang mit den Verbänden stellt sich die Frage, „inwiefern sie als 
intermediäre Organisationen, die sich zwischen dem Sozialstaat auf der einen und der Lebenswelt der Bürger auf der anderen Seite befinden, in der Lage sind, Gemeinsinn und soziale Anteilnahme zu wecken bzw. freiwilliges Engagement anzuregen, $\mathrm{zu}$ organisieren und $\mathrm{zu}$ stabilisieren (vergl. Steinbecher 2000). Kritisch wird dabei darauf hingewiesen, dass die Einbeziehung der Wohlfahrtsverbände in den Sozialstaat inzwischen soweit fortgeschritten sei, dass es diesen immer weniger gelingt direkt freiwilliges Engagement und gesellschaftliche Solidarität in ihren Einrichtungen, Diensten und Vereinsgliederungen anzuregen“ (Wohlfahrt 2007: 6). 


\section{Entwicklung eines Idealmodells aktivierender Familienpolitik}

\subsection{Die Entwicklung familienpolitischer Zielsetzungen und Instrumente}

\subsubsection{Allgemeine Hintergründe zur Familienpolitik}

Die Familienpolitik blickt auf eine lange Geschichte zurück, in der viele Themen für wichtig oder weniger wichtig gehalten wurden. Viele Institutionen und Akteure haben Familienpolitik eingesetzt bzw. sich für sie eingesetzt. Da es sich bei der hier vorliegenden Forschungsarbeit um die Anwendung einer Theorie aus dem allgemeinen politik- bzw. verwaltungswissenschaftlichen Bereich handelt, erfolgt an dieser Stelle keine Beschreibung der allgemeinen Familienpolitik in ihrer Gesamtheit. Der Fokus dieser Arbeit liegt auf der politischen Positionierung dieser Fachpolitik, möglichen Zielsetzungen und theoretisch zu unterscheidenden Instrumenten.

Familienpolitik unterliegt in den europäischen Ländern einer politisch sehr unterschiedlichen Bewertung. Nicht überall werden familienpolitische Instrumente als zusammenhängende Politik verwendet. Kaufmann z.B. unterscheidet im Bezug auf die Familienpolitik drei Herangehensweisen westlicher Staaten:

- „Die wohlfahrtsstaatliche Position: sie postuliert eine grundsätzliche und explizite Verantwortung des Staates für den Schutz und die Förderung familialer Belange und kennt keine Grenzen staatlicher Intervention außer solchen der Zweckmäßigkeit;

- Die Position möglichster Staatsbegrenzung: sie versteht Familien als eine ausschließlich private Angelegenheit, in die sich der Staat ebenso wenig zu mischen habe wie in die Wirtschaft; 
- Die Position einer selektiven Staatsverantwortung: sie fordert ein staatliches Eingreifen lediglich im Hinblick auf sozial schwache oder problembelastete Familien (z.B. Alleinerziehende, Arbeitslose, Erziehungsschwache oder generell durch die Umstände beeinträchtigte Eltern), wobei bald eine Familien fördernde, bald eine eher sozial kontrollierende Absicht damit verbunden ist“ (Kaufmann 1993: 144/Kaufmann 2002: 429f).

Entsprechend dieser Interpretation der Staatsaufgaben wird Familienpolitik explizit, impliziet oder gar nicht eingesetzt. Als Hintergrund für diese unterschiedlichen Bewertungen bzw. Herangehensweisen deutet Bahle „spezifische institutionelle und strukturelle Bedingungen“ (Bahle 1995: 16) der verschiedenen Staaten. Einerseits gibt es Länder, wie z.B. Deutschland, wo es seit 1953 ein eigenes Ministerium für das Fachgebiet Familie gibt. Hierbei handelt es sich um ein Bespiel explizit geführter Familienpolitik. Kaufmann (1993) sieht es als eine Politik, in der sich die „politischen Diskurse ausdrücklich auf Hilfen oder Erwartungen an Familien (und nicht lediglich an Frauen oder Kinder) beziehen und zweitens auf politischer Ebene eine erkennbare Ausdifferenzierung politischer Instanzen mit einer auf die Vertretung spezifisch familialer Belange bezogenen Kompetenz (Ministerium oder wenigstens Abteilung) zu beobachten ist“ (Kaufmann 1993: 145).

In den Niederlanden nimmt das Thema Familienpolitik eine andere Position ein. Genau wie in Deutschland sah man es als eine Querschnittsaufgabe, die allerdings bis zum Kabinett Balkenende IV, durch unterschiedliche Ministerien wahrgenommen und daher implizit geführt wurde (Ministerie van Volksgezondheid, Welzijn en Sport 1996: 5). „Von impliziter Familienpolitik kann gesprochen werden, wo politische Maßnahmen erheblichen Umfangs festzustellen sind, welche sich aus der Perspektive eines wissenschaftlichen Beobachters als auf die Eindämmung oder Beseitigung familialer Problemlagen gerichtet interpretieren lassen, ohne dass sie jedoch von den Beteiligten familienpolitisch legitimiert werden“ (Kaufmann 1993: 145). Im Jahr 2007 hat es hier eine Veränderung ergeben, da erstmals ein 'Programma Ministerie' eingerichtet wurde dem die Aufgabe unterliegt, eine kohärente Familienpolitik zu entwickeln.

Die zweite, von Kaufmann beschriebene Form der Aufgabenwahrnehmung, („möglichster Staatsbegrenzung“) kann z.B. in den Vereinigten Staaten 
observiert werden (vergl. Kaufmann 2002: 433). Diese Interpretation staatlichen Handelns ist für die vorliegende Forschungsarbeit jedoch nur sehr begrenzt relevant und wird an dieser Stelle auch nicht weiter ausgeführt.

\subsubsection{Allgemeine familienpolitische Zielsetzungen}

Pfenning und Bahle unterscheiden Familienpolitiken aufgrund ihrer Zielsetzungen und Interventionsschwerpunke in fünf Gruppen westeuropäischer Familienpolitik:

- „Die skandinavischen Länder mit einer Politik, welche sich stark auf das Kind und auf die Emanzipation von Frauen richtet,

- Großbritannien und Irland mit einer liberalen, nicht interventionistischen Politik, bei der hauptsächlich Risikofamilien im Mittelpunkt der Zielsetzungen stehen,

- Südeuropäische Länder mit einem schwachen Wohlfahrtsstatus und stark ausgebildeten Familienbanden,

- Frankreich und Belgien mit einer starken familienpolitischen Tradition und sowohl traditionellen als auch progressiven Politikelementen,

- Deutschland und Österreich mit einer weniger stark entwickelten, dafür aber konservativen Familienpolitik“ (Pfenning/Bahle 2000: 24).

Wingen analysiert, dass sich die diversen Schemen der Familienpolitik durch die Wertung familialer Leitbilder einerseits und der Position des Individuums, der Familie und der Gesellschaft andererseits unterscheiden. Er identifiziert:

- „ein betont auf den einzelnen und seine Bedürfnisse ausgerichtetes Grundmuster,

- ein vorrangig gesamtgesellschaftlich akzentuiertes Grundmuster,

- $\quad$ ein stark familienzentriertes Grundmuster (und)

- $\quad$ ein am Menschen als einem personalen Wesen orientiertes Grundmuster“ (Wingen 1994: 42ff).

Den hier beschriebenen Kategorisierungen liegt eine Analyse der gestellten Ziele und verwendeten Instrumente zugrunde. Herlth und Kaufmann schreiben 
hierzu: „Differenziert man die pauschale Motivation des jeweiligen Staates Familien bei der Erfüllung dieser Funktionen zu unterstützen, so können die folgenden Teilmotive, bzw. Ziele für Familienpolitik identifiziert werden (Herlth/Kaufmann 1982: 15):

- ein bevölkerungspolitisches Motiv,

- ein emanzipatorisches Motiv,

- $\quad$ ein sozialpolitisches Motiv (und)

- ein familial-institutionelles Motiv“ (Gerlach 2010: 134, Gerlach 2004: 114).

Alle vier Motive haben „eine Veränderung der lebensweltlichen Strukturformen und Leistungsbeziehungen der Familie zum Ziel“ (Kaufmann 1982: 17f). Dementsprechend geht es um die Umsetzung gesellschaftspolitischer Interessen. Zudem gelten diese Motive auch als Zielsetzungen der Familienpolitik. In seinen späteren Werken hat Kaufmann noch weitere Motive der Familienpolitik unterschieden. Inhaltlich ist hiervon im Zusammenhang mit der folgenden Erörterung der Arbeiten Esping-Andersens vor allem auch "the children's welfare motive“ (Kaufmann 2002: 428) als eine weitere familienpolitsche Zielsetzung relevant. Im Folgenden werden dann auch diese fünf hier genannten Zielsetzungen erläutert.

Bevölkerungspolitische Zielsetzungen

Es gibt Politiker, Wissenschaftler und andere Publizisten die Bevölkerungspolitik von Familienpolitik unterscheiden. In der hier vorliegenden Arbeit werden bevölkerungspolitische Motive allerdings als Teil der Familienpolitik angesehen. Man versteht unter diesen Motiven das Einwirken auf den demographischen Aufbau einer Gesellschaft. Bevölkerungspolitische Zielsetzungen gehören zu den ältesten Zielen der Familienpolitik und wurden im Laufe der Geschichte diverse Male missbraucht, um z.B. militärische Stärke $\mathrm{zu}$ sichern. In Deutschland waren bevölkerungspolitische Zielsetzungen für eine lange Zeit aufgrund der negativen Verwendung durch die Nationalsozialisten tabuisiert. Umgekehrt können sich bevölkerungspolitische Zielsetzungen auch auf die Verringerung der Geburtenanzahlen richten. „Den äußeren Rahmen für eine ethisch akzeptable Steuerung des generativen Verhaltens setzt die Erklärung der Menschenrechte, die 1968 in Teheran um einen entsprechenden Passus ergänzt wurde (...) wonach die einzelnen Paare 
das Recht haben, frei, verantwortlich und informiert über die Zahl der Kinder und den zeitlichen Abstand der Geburten zu entscheiden“ (Gerlach 2004: 115f).

Seit dem starken Geburtenrückgang, der in Deutschland (und später auch in den übrigen europäischen Ländern) vor circa vierzig Jahren einsetzte, hat sich eine erneute Diskussion über bevölkerungspolitische Zielsetzungen entwickelt. „Insbesondere die Diskussion der letzten Jahre um die Sicherungsstrukturen des deutschen Sozialstaates hat verdeutlicht, dass das bevölkerungspolitische Segment der Familienpolitik keineswegs Kern konservativer und national orientierter Argumentation ist, sondern wesentliches Element gesellschaftlicher Planung sein sollte" (Gerlach 2004: 115). Verwiesen wird hierbei auf Messinstrumente wie z.B. die Total Fertility Rate. Thomas Malthus schrieb diesbezüglich bereits im 19. Jahrhundert, dass zur demographischen Generationssicherung eine Kinderzahl von ca. 2,1 Kindern nötig sei ${ }^{4}$. In der heutigen Debatte spielt in Deutschland vor allem der Begriff der Nachhaltigkeit im Sinne demographischer Zielsetzungen von Familienpolitik eine wichtige Rolle (vergl. Bertram 2006).

Neben der natürlichen Bevölkerungsentwicklung die sich aus den Geburtenund Sterbeziffern ergibt spielen auch die Wanderbewegungen bzw. Migrationsströme eine wichtige Rolle. Sie werden allerdings nicht dem familienpolitischen Bereich zugeordnet und daher an dieser Stelle auch nicht weiter bearbeitet.

Emanzipatorische Zielsetzungen

Durch die Anbindung familialer Lebensverhältnisse an existierende Machtstrukturen kann sich ein Machtgefälle zwischen den stärkeren und den schwächeren Gliedern einer Familie ergeben (z.B. Frauen gegenüber Männern und Kindern gegenüber Eltern). Zum Ausgleich dieser Strukturen, gehört auch die Neugestaltung von Rollen, die den diversen Akteuren in der Familie zuteil werden. Weiterhin spielt auch das Verhältnis zwischen Elterngeneration, wirtschaftlichen Voraussetzungen und sozialer Sicherung eine wichtige Rolle.

4 Obwohl die Theorien von Maltus heute sehr kritisch betrachtet warden, wird im Bezug auf das Generationenverfangungsniveau im Allgemeinen noch stets die Ziffer 2.1 anstelle von z.B. 2.o genannt, da davon ausgegangen wird, das eine begrenzte Anzahl von Kindern das gebährffähige Alter nicht erreicht. 
Hierbei geht es, aus heutiger Sicht, in erster Linie um die Unterstützung der Vereinbarkeit von Familie und Beruf. Spätestens seit dem Ende des 19. Jahrhunderts hat es verschiedene emanzipatorische Wellenbewegungen gegeben, wodurch verschiedene politische Zielsetzungen zentral standen. Für die hier vorliegende Arbeit sind aus diesem Bereich vor allem zwei Zielsetzungen relevant und das sind erstens die wirtschaftliche Selbstständigkeit von Frauen und zweitens die Vereinbarkeit von Beruf und Familie. Andere Ziele, wie z.B. zum Thema Gewalt gegen Frauen, werden an dieser Stelle nicht bearbeitet.

Auch gibt es außer der Emanzipation von Frauen andere Emanzipationsbewegungen. So hat z.B. in den Niederlanden in der hier untersuchten Periode die Emanzipation von homosexuellen Frauen und Männern eine sehr bedeutende Rolle gespielt, die in den untersuchten Dokumenten vielfältig beschrieben wurden. Aus der für diese Arbeit zentral stehenden wohlfahrtsstaatlichen Perspektive ist diese Form der Emanzipation allerdings weniger relevant und daher an dieser Stelle nicht aufgenommen.

Sozialpolitische Zielsetzungen

Familienpolitik an sich, sowie deren quantitative Erweiterung können zu sozialen Ungleichheiten führen. Nach der Gründung einer Familie nimmt in vielen Fällen das Haushaltseinkommen ab, während die Kosten steigen. Der niederländische Familienrat nannte diese Phase der abnehmenden Kaufkraft ,das Familiental' (Bos/Hooghiemstra 2004: 61).

Während es für sehr viele Familien zu einer Abnahme der Kaufkraft kommt, sind vor allem größere Familien und Alleinerziehende in einigen westlichen Gemeinschaften einem größeren Armutsrisiko ausgesetzt. Die Familienpolitik verfolgt daher unterschiedliche Zielsetzungen, um möglichen Risiken vor zu beugen (z.B. durch die Verwendung des Familienlastenausgleichs).

Viele Instrumente werden in der familienpolitischen Praxis eingesetzt, um sozialwirtschaftliche Differenzen auszugleichen. Man kann z.B. zwischen indirekten Transfers, die sich auf die Höhe der zu bezahlenden Steuern beziehen, und direkten Transfers, (z.B. das Kindergeld) unterscheiden. Weiterhin relevant ist eine dritte Gruppe von Leistungen, die sich nicht auf 
„Einkommensumverteilungspolitik“ (Gerlach 2004: 117) richtet, sondern auf Infrastrukturen und Dienstleistungen.

Familieninstitutionelle Zielsetzungen

Dieses Motiv richtet sich auf die „Binnenstrukturierung der Familien und die Kennzeichnung ihrer Spezifik gegenüber anderen Formen der Lebensorganisation" (Gerlach 2004: 117). Inzwischen hat sich der Begriff Familie erweitert und sich der Realität angepasst. „Während der familienpolitische Diskurs zuvor ein weitgehend normativer und ein wertrationaler gewesen ist, entwickelt er sich zunehmend $\mathrm{zu}$ einem zweckrationalen. Mit der Herausbildung von Elternschaft zu einer Option der Lebensgestaltung u.a. nämlich sind die entsprechenden von den Familien erbrachten und für die Gesellschaft unverzichtbaren Leistungen gegenüber denjenigen Teilen der Bevölkerung in stärkerem Masse als zuvor auszugleichen, die andere Optionen wählen“" (Gerlach 2004: 118).

Kindeswohl

Ein besonderes familiäres Merkmal ist die Abhängigkeit der Angehörigen voneinander. Dies gilt insbesondere für Kinder. In der Regel sind sie noch nicht im Stande, um selbstständig zu leben. Ihnen kommt daher in der Familienpolitik eine besondere Aufmerksamkeit zu. Diese gesteigerte Aufmerksamkeit hat vor allem in den letzten Jahren an politischer Bedeutung gewonnen. In zunehmendem Maße spielt die Perspektive des Kindes bei der Erarbeitung politischer Ziele und Maßnahmen eine Rolle. Unterschieden werden können die Förderung von Kindern bzw. präventive Maßnahmen von denjenigen, die eher dem Bereich der aktiven Jugendhilfe zugeordnet werden müssen. Betont wird heute im Allgemeinen die besondere Bedeutung der Frühförderung von Kindern. Grundsätzlich wird angenommen, dass je älter ein Kind ist, desto weniger effizient bzw. schwerer realisierbar mögliche Entwicklungshilfen sind. Vor diesem Hintergrund wird in zunehmendem Maße auch gewiesen auf die besondere Bedeutung der Qualität der Kinderbetreuung bzw. Früherziehung. Die staatlichen Investitionen in diesem Bereich haben in den vergangenen Jahren dann auch in beinahe allen europäischen Ländern stark zugenommen. 


\subsubsection{Allgemeine familienpolitische Instrumente}

Wie bereits angedeutet, endet dieses Unterkapitel mit einer kurzen Beschreibung möglicher familienpolitischer Interventionsformen. „Aus sozialwissenschaftlicher Sicht lassen sich vier Arten familienpolitischer Interventionen unterscheiden:

- „Maßnahmen, die den rechtlichen Status der Familienmitglieder betreffen",

- „Maßnahmen, die die ökonomische Situation der Familienhaushalte betreffen",

- „Maßnahmen, welche die ökologischen Bedingungen des Familienlebens betreffen",

- „Maßnahmen, welche die Förderung oder Wiederherstellung von

Kompetenzen der Familienangehörigen betreffen“ (Kaufmann 1993:

145/Kaufmann 2002: 435).

$\mathrm{Zu}$ den Instrumenten, die den rechtlichen Status der Familienmitglieder betreffen zählt man Rechtsgebiete wie z.B. die Ausgestaltung des Eherechts, des Erbrechts und des Kindschaftsrechts. Instrumente, die die ökonomische Situation der Familienhaushalte betreffen, gestalten sich in den meisten Fällen wie eine Art Ausgleichszahlung für Menschen mit Kindern, die durch die Erziehung von Kindern eine größere finanzielle Belastung erfahren als Kinderlose. Der Staat unterstützt Eltern finanziell, da die Erziehung von Kindern nicht nur eine private Angelegenheit ist, sondern auch eine große Bedeutung hat für das Zusammenleben in der Gesellschaft. Die Leistungen können als direkte Geldleistungen, oder in der Form von Steuerermäßigungen erstattet werden. Die dritte Kategorie der Instrumente, zielt auf die ökologischen Bedingungen der Familien. Einerseits zählen hierzu die öffentlichen Angebote und Dienstleistungen von Krippen und Kindergärten. Andererseits handelt es sich um Instrumente, die das private Zusammenleben von Familien in der Gesellschaft beeinflussen, wie z.B. die Raum- und Stadtplanung bzw. die Wohnungsbaupolitik. In der letzten Kategorie der Instrumente aufgenommen sind z.B. Bildungsmaßnahmen, die auf die Förderung bzw. Wiederherstellung von Kompetenzen der Familienangehörigen zielen. Durch die Erziehung von Kindern müssen Mütter und Väter eventuell dem Arbeits- oder Bildungsmarkt 
fern bleiben. In einer folgenden Lebensphase können sie durch entsprechende Reintegrationsmaßnahmen unterstützt werden.

\subsection{Familienpolitik in der vergleichenden Wohlfahrtsforschung Esping-Andersens}

\subsubsection{Einleitung und Beschreibung der Arbeitsschritte}

Das Ziel dieser Abhandlung ist der Entwurf eines Idealmodells Aktivierender Familienpolitik. Aufgebaut wird hierbei vor allem auf den Ergebnissen aus der vergleichenden Wohlfahrtsforschung von Esping-Andersen. Dieser beschreibt in seinen Arbeiten Wohlfahrtsstaatsregime und spricht in diesem Zusammenhang nicht von Familienpolitik, sondern von einer sozialpolitischen Vernetzung. Familienpolitik macht hierbei nur Teil des Ganzen aus. „Einen Analyserahmen für den internationalen Vergleich familienpolitischer Inhalte bieten spezifische Typologien. Sie dienen der Systematisierung untersuchter Sachverhalte sowie der Subsumierung empirischer Fälle. Im Bereich der vergleichenden Wohlfahrtsstaatenforschung hat Esping-Andersen grundlegend gearbeitet und drei Typen so genannter Wohlfahrtsstaatsregime identifiziert (...) EspingAndersens Typologie stellt ein Referenzmodell dar, welches die Systematisierung von Wohlfahrtsstaaten ermöglicht (Ministerium für Generationen, Familie, Frauen und Integration des Landes Nordrhein-Westfalen 2006: 20)“" (Schmidt 2006: 86f). Die klassische Familienpolitik hält er für nicht mehr ausreichend, und er beschreibt in Ansätzen, wie aktivierende Familienpolitik aussehen könnte. Ein starkes Gewicht legt er hierbei auf die familienpolitischen Zielsetzungen: Bevölkerungsentwicklung, Emanzipation bzw. Partizipation von Frauen auf dem Arbeitsmarkt und Sozialpolitik. Vor dem Hintergrund der Lissabon-Strategie fragt er sich, ob sich durch den Einfluss des Leitbildes Aktivierender Staat ein europäisches, familienpolitisches Modell entwickeln kann.

Der dänische Wissenschaftler misst der Familienpolitik in seiner vergleichenden Wohlfahrtsforschung eine entscheidende Rolle bei, bietet aber aus politischadministrativer Sicht kein konkretes, aktivierendes familienpolitisches Modell, 
das man zum internationalen Politikvergleich verwenden könnte. In diesem Kapitel folgt daher eine Analyse vier seiner entscheidensten Werke. Dieser Analyse werden die Erörterungen verschiedener Autoren hinzugefügt, die seinen Ansatz aus ihrer Sicht zusammengefasst haben. Knijn unterscheidet z.B. zwischen folgenden politischen Prioritäten:

- Unterstützung der Teilnahme am Arbeitsmarkt und Einkommensschutz für Familien,

- Maximalisierung der Fähigkeiten, um Arbeit und Sorge zu vereinbaren,

- $\quad$ Sozialisierung der Kosten für Kinder und

- $\quad$ Neudefinierung der Verbindung zwischen Arbeit und Freizeit aus der Lebenslaufperspektive.

Ihrer Meinung nach baut Esping-Andersen auf der sozialliberalen Tradition auf, in der vor allem darauf gepocht wird, um in Lebenschancen zu investieren. Diese bilden die Basis für ein neues Verhältnis zwischen Gleichheit und sozialer Grundrechte. Die Verantwortung für eigenes Handeln und deren Stärkung ist ein wichtiger Grundgedanke. Vor diesem Hintergrund sieht sie drei konkrete Themenschwerpunkte:

- Unterhaltskosten von Kindern,

- Übergänge zwischen Teilnahme am Arbeitsmarkt und Pflege der Familie,

- Entwicklung gesellschaftlicher Dienstleistungen (vergl. Knijn 2007: 69ff).

Auch Kevenhörster bietet einen Rahmen zur Analyse des Ansatzes EspingAndersens. „Zur Entscheidung steht die Frage an, wie der Sozialstaat in der postindustriellen Gesellschaft gestaltet werden muss, um eine nachhaltige Sozialpolitik zu ermöglichen. Der dänische Soziologe Esping-Andersen geht von folgenden Entwicklungstendenzen aus: der Überalterung der Gesellschaft, der wachsenden Bedeutung des Dienstleistungssektors und wissensbasierter Ökonomie und der größeren Pluralität von Lebensformen. Da die „Qualität der Kindheit" für die individuellen Lebenschancen ausschlaggebend sei, komme es in einer überalterten Gesellschaft um so mehr darauf an, eine „kinderorientierte soziale Investitionsstrategie“ zu entwerfen und durchzusetzen. Die Verringerung der Kinderarmut werde in der Zukunft eine „individuelle und soziale Dividende abwerfen. Chancengleichheit wird so vorrangig als größere 
Gleichheit der Bildungschancen verstanden, als Förderung der kognitiven und sozialen Fähigkeiten der Menschen. In der Wissensökonomie bleibt das Humankaptial die wichtigste Ressource (...) Dem „Recht auf Arbeit“ steht eine „Pflicht zur Arbeit“ gegenüber (...) Das größte mittelfristige Problem des sozialdemokratischen Wohlfahrtsstaates ist einstweilen die Herstellung des Haushaltsgleichgewichtes, das größte langfristige Problem dagegen der Abgleich der Kostenentwicklung der Sozialpolitik mit den ihr zugrunde liegenden Solidaritätserfordernissen (...) Wie können diese Herausforderungen gemeistert werden? Verschiedene Lösungsstrategien werden vorgeschlagen:

- (...) Größere Flexibilisierung des Arbeitsmarktes und eine größere Spreizung der Löhne,

- (...) Entkoppelung der Finanzierung des Sozialstaates von der Erwerbsarbeit da Sozialbeiträge wie Steuern auf Beschäftigung wirken - ein zentrales Problem des deutschen Wohlfahrtsstaates,

- (...) Eine konsequente Politik der Familienförderung, deren Wirkung darin besteht, die Familien durch schulische Angebote zu entlasten, ihren Zusammenhang zu festigen und den Wunsch nach Kindern erfüllbar zu machen" (Kevenhörster 2006: 250f).

Esping-Andersen hat viel $\mathrm{zu}$ den Themen: Umbau von Sozialstaaten und Familienpolitik geschrieben. Nicht alle Schriften können an dieser Stelle bearbeitet werden. Um in dieser Arbeit zu einem zusammenhängenden und konsequenten Modell Aktivierender Familienpolitik zu gelangen, ist von seinen Werken ,The Three Worlds of Welfare Capitalism' (1990), ,Welfare States in Transition - National Adaptations in Global Economies' (1996), ,Why We Need a New Welfare State' (2002) und ,The Incomplete Revolution - Adapting to Women's New Roles' (2009) eine punktuelle Zusammenfassung der für diese Arbeit relevanten Gedanken erstellt. In der sich anschließenden, analytischen Betrachtung werden die entscheidenden Punkte dieser Zusammenfassungen verarbeitet. Ziel dieser analytischen Betrachtung ist es, anhand einer Evaluierung seiner Gedanken, die Evolution seiner Arbeit im hier zu erstellenden Idealmodell kritisch zu berücksichtigen. Im dritten Unterkapitel werden diese Punkte mit den Ausführungen Kaufmanns zusammengeführt, um ein höheres Abstraktionsniveau aus einem allgemeinen familienwissenschaftlichen Gesichtspunkt zu erreichen. Erweitert wird dieses 
Modell im letzten Unterkapitel um den verwaltungswissenschaftlichen Ansatz des Aktivierenden Staates und seines Instrumentariums.

\subsubsection{Analytische Betrachtungen der Werke Esping-Andersens}

\subsubsection{1 ,The Three Worlds of Welfare Capitalism‘ (1990)}

Dieses erste Werk spielt, aufgrund des systematischen Vergleichs der Investitionen verschiedener Staaten und entsprechender Konsequenzen, eine große Rolle in der Wohlfahrtsforschung. Die beschreibende Analyse erfolgt hier anhand der in diesem Werk zentral stehenden Themen: ,Wohlfahrtsstaatsansatz', ,De-Kommodifizierung', ,Idealregime' und ,die Rolle der Frauen und des Arbeitsmarktregimes'.

\section{Wohlfahrtsstaatsansatz}

Der Autor richtet sich auf einen, wie er es nennt, breiten Wohlfahrtsstaatsansatz. Sein Interesse gilt der integralen Zusammenarbeit von Wirtschaft, Arbeitsmarkt und Wohlfahrtsstaat. „Im breiteren (Wohlfahrtsstaats)Ansatz formen Themen wie Beschäftigung, Gehälter und allgemeine makro-ökonomische Steuerung akzeptierte integrale Komponenten des Wohlfahrtsstaatsgebildes. Zusammenfassend kann diese Interpretation als Keynesianischer Wohlfahrtsstaat oder als Wohlfahrtskapitalismus identifiziert werden“ (vergl. Esping-Andersen 1990: 1f).

Im Zentrum seiner Arbeit steht das Instrumentarium dieses breiten Wohlfahrtskapitalismus, das er verwendet, um achtzehn Wohlfahrtskonglomerate nach soziologischen Gesichtspunkten einem konservativen (z.B. Deutschland), einem liberalen (z.B. Großbritannien) und einem sozialdemokratischen (z.B. Schweden) Regime zuzuordnen (vergl. Esping-Andersen 1990: 3). In seinen Texten unterscheidet er zwischen:

- „Nordic Countries,

- $\quad$,Liberal' Welfare Model,

- Continental European Model“ (Esping-Andersen 2002: 13ff). 
Verschiedene Gründe, wie z.B. die Regierungsformung während des Übergangs von der Agrarier- in eine industrielle Gesellschaft, haben die Formung dieser drei Typen maßgeblich beeinflusst. Eine wichtige Rolle in diesem Zusammenhang spielte auch der Konservativismus der vorkapitalistischen Gesellschaft. Esping-Andersen unterscheidet zwischen Feudalismus, dem Genossenschaftsmodell und dem etatistischen Konservativismus. Das Ziel der herrschenden Klasse im Konservativismus war es, um Armut zu verbannen "nicht um die Arbeiter von ihrer Marktabhängigkeit zu befreien“ (EspingAndersen 1990: 46).

Die Beschreibung dieser unterschiedlichen Politiktypen bzw. Ausdifferenzierungen des Wohlfahrtsstaates ist dem Modell von Pfenning und Bahle sehr ähnlich und unterscheidet sich vor allem in der dritten Kategorie. Hier untergliedern Pfenning und Bahle, während Esping-Andersen es als einen Typus im weiteren sozialpolitischen Sinn versteht. Diese Kategorisierung verschiedener Wohlfahrtsstaatsregime war nicht unbedingt neu und kann auch nicht alle nationalen Wohlfahrtsunterschiede erläutern. Sie ist aber dennoch sehr aufschlussreich, „weil hier die Diskussion der Sozialsysteme über die enge Grenzen der Höhe der Sozialausgaben allein hinausgeführt, und die Aufmerksamkeit auf den größeren institutionellen Komplex gerichtet wird, in dem die sozialen Probleme verarbeitet werden“ (Myles 1998: 342). Durch Esping-Andersens Arbeit wird das Rollenverhältnis von Markt, Staat und Familie verdeutlicht.

Vor Esping-Andersens Ansatz hatte es bereits diverse Analysemodelle wohlfahrtsstaatlichen Handelns gegeben. Hierbei bilden z.B. die Finanzierungsarten den Ausgangspunkt verschiedener Kategorisierungen, wie das Beveridge-System (Fokus: Steuerfinanzierung) und das Bismarck-System (Fokus: Sozialversicherungssystem). Die liberalen und sozialdemokratischen Typen sind beide tendenziell am Beveridge System orientiert, auch wenn sie sich in der De-Kommmodifizierungsskala genau gegenüberstehen. Konservative Systeme wie z.B. das deutsche Wohlfahrtsregime sind als Bismarck-Modell eingerichtet.

Ähnlichkeit haben die Regimetypen von Esping-Andersen auch mit den sozialstaatlichen Grundmodellen, die Tittmuss 1958 unterschied, und die für lange Zeit großen Einfluss auf wohlfahrtsstaatliches Denken hatten. Tittmuss 
unterscheidet nicht zwischen Idealtypen sondern zwischen Modellen. Er benennt „erstens das residuale Modell einer bedürftigkeitsgeprüften Sozialhilfe, zweitens das Sozialversicherungsmodell und drittens das Modell der universellen sozialen Bürgerrechte. Jeder entwickelte Sozialstaat wird als eine spezielle Konfiguration dieser drei Grundblöcke beschrieben. Folgt man der Logik und Terminologie von Esping-Andersen, so gruppiert sich der liberale Typ in erster Linie um den Block der residualen und bedürftigkeitsgeprüften Sozialhilfe, der konservative Typ um den Block der Sozialversicherung und im sozialdemokratischen Typ dominierten die Prinzipen der Universalität und der sozialen Bürgerrechte“ (Lammert 2005: 14f).

Durch diese Ausführungen wird der inhaltliche Zusammenhang/der Hintergrund, deutlich vor dem die Theorien Esping-Andersens verstanden werden müssen. In einem (angepassten) Schaubild von Lammert wird dieser Zusammenhang schematisch dargestellt. 
Tabelle 8: Charakteristiken der unterschiedlichen Wohlfahrtsregimes (Lammert 2005: 13), Bearbeitung Willenborg (2008)

\begin{tabular}{|c|c|c|c|c|}
\hline & \multicolumn{4}{|c|}{ Wohlfahrtsregime } \\
\hline Wurzeln/Ursprung & Beveridge & & & Bismarck \\
\hline Regimetyp (dominante & Liberal & Sozialdem. & Kons & vativ \\
\hline Genereller Charakter & Residual & Institutionell & Residual & Institutionell \\
\hline Hauptziele & $\begin{array}{l}\text { Verminderung } \\
\text { von Armut }\end{array}$ & $\begin{array}{c}\text { Abschaffung der } \\
\text { Armut, } \\
\text { Größere } \\
\text { Einkommensgl., } \\
\text { Vollbeschäftigung }\end{array}$ & $\begin{array}{c}\text { Verminderung von } \\
\text { Armut }\end{array}$ & Einkommenserhaltung \\
\hline Programmschwerpunkt & Sozialhilfe & $\begin{array}{l}\text { Soziale } \\
\text { Bürgerrechte }\end{array}$ & Sozialversicherung & Sozialversicherung \\
\hline $\begin{array}{l}\text { Reichweite sozialer } \\
\text { Leistungen }\end{array}$ & Begrenzt & Umfassend & Begrenzt & Begrenzt \\
\hline $\begin{array}{l}\text { Höhe der öffentlichen } \\
\text { Ausgaben ( } \% \text { des BIP) }\end{array}$ & Niedrig & Hoch & Hoch & Hoch \\
\hline $\begin{array}{c}\text { Privater } \\
\text { Wohlfahrtssektor }\end{array}$ & Signifikant & Gering & Gering & Gering \\
\hline Prototyp & $\begin{array}{l}\text { Angelsächsische } \\
\text { Länder }\end{array}$ & $\begin{array}{l}\text { Skandinavische } \\
\text { Länder }\end{array}$ & Mittelmeerländer & $\begin{array}{c}\text { Kontinentaleuropäische } \\
\text { Länder }\end{array}$ \\
\hline
\end{tabular}


Die ursprüngliche Dreiteilung der Regime ist in der wissenschaftlichen Forschung inzwischen um zwei weitere Typen erweitert. Hierbei handelt es sich um rudimentäre Wohlfahrtsregime, wie sie mit geringen Dekommodifizierungniveaus vor allem im mediterranen Raum auftreten (z.B. Portugal und Italien) und postsozialistischen Wohlfahrtsstaaten, die in den transformatorischen politischen Systemen Osteuropas vorherrschen (z.B. Polen)“ (vergl. http://de.wikipedia.org/wiki/Wohlfahrtsstaat, aufgerufen am 17.04.2008). Diese beiden Regimetypen werden hier allerdings nicht vertieft.

Ein weiterer in diesem Zusammenhang entscheidender Begriff lautet DeKommodifizierung. Gemeint ist hiermit die direkte Unabhängigkeit privater Wohlfahrt vom Funktionieren des Marktes durch ein Eingreifen des Staates. Es geht hierbei um eine Abkopplung Sozialer Sicherheit vom Funktionieren des Arbeitsmarktes, was dazu führt, dass sowohl Arbeitnehmer als auch die Empfänger von Sozialleistungen weniger abhängig sind von den Schwankungen des Marktes. „Zu De-Kommodifizierung kommt es, wenn ein Dienst als ein Recht angesehen wird, und wenn eine Person sich ein Leben leisten kann, ohne auf den Markt angewiesen zu sein“ (vergl. Esping-Andersen 1990: 21f). Der Bürger hat einen Anspruch auf Sozialleistungen und kann im Falle der typischen Risiken des Lebens (wie z.B. Elternschaft) auf Sozialleistungen zurückgreifen.

Die Höhe dieser Unabhängigkeit ist eine wichtige Eigenschaft der zuvor umschriebenen drei Wohlfahrtsstaatstypen. Die Einteilung in Wohlfahrtstaatsregimes ist maßgeblich bestimmt durch den Grad der DeKommodifizierung. Esping-Andersen verwendet in seiner 18-Länder Analyse drei Indikatoren: den allgemeinen Zugang zu den Einkommensersätzen, die Höhe des gezahlten Betrages und den Schutz gegen die sozialen Grundrisiken wie z.B. Krankheit und Alter. Dem Regimetyp entsprechend werden private Aufgaben durch Staat, Markt und Familie ausgeführt. Durch das Verhältnis dieser Aufgabenteilung ergibt sich der De-Kommodifizierungsgrad. Würde man die drei Wohlfahrtsstaatsregimes auf einer Skala darstellen, bekämen die sozialdemokratischen Regime einen Platz am oberen Ende, während die konservativen Regime eine Mittelposition einnähmen. Die DeKommodifizierung der liberalen Regime ist laut Esping-Andersen am wenigsten entwickelt und daher im unteren Drittel der Skala einzuordnen. 
Von den drei genannten Regimetypen hebt der Autor insbesondere den sozialdemokratischen Typus hervor, der im Gegensatz zum konservativen Regimetyp nicht erst alle Kräfte der eigenen Familie anwendet, sondern ganz direkt auf eine Kombination von Markthandeln und Eingreifen durch die Familie setzt. „Das Resultat ist ein Wohlfahrtsstaat der direkte Transfers an die Kinder garantiert und direkte Verantwortung in der Kindespflege, bei der Pflege der Älteren und Hilfsbedürftigen übernimmt. Er hat sich dementsprechend einem aufwändigen sozialen System unterworfen, nicht nur um die Bedürfnisse der Familie zu unterstützen sondern auch, um es Frauen zu ermöglichen sich anders als im Haushalt zu betätigen" (vergl. Esping-Andersen 1990: 28). Obwohl er den sozialdemokratischen Regimetyp als Idealregime interpretiert, sieht er die hohen Kosten öffentlicher Ausgaben als deutliches Problem dieses Regimetyps. Dieses Preisniveau sei eines der Argumente dafür, dass dieses Regime nicht für jeden Wohlfahrtsstaat ein gleich realistisches Zukunftsmodell darstelle. Alle ,drei Welten der Wohlfahrt' werden laut Esping-Andersen auch in der postindustriellen Phase unterschiedliche Reformpfade wählen. „Während das liberale Regime unter Druck gerate, die Löhne in niedrig qualifizierten Sektoren abzusenken und auf diese Weise Armutsfallen riskiere, könne das sozialdemokratische Regime auf Dauer seine Vollbeschäftigung nicht erhalten, und das konservativ-kontinentale Regime sei im Zirkel ,welfare without work' gefangen - einer wachsenden Gruppe von Ausgeschlossenen des Arbeitsmarktes stehe eine sinkende Zahl von Insidern gegenüber" (Lütz 2004: 19).

Die Rolle der Frauen und des Arbeitsmarktregimes

Der Autor unterstreicht den Zusammenhang zwischen dem Wohlfahrtsstaatsregime und der Rolle der Frauen. Während in einigen Ländern Frauen bewusst dem Arbeitsmarkt ferngehalten wurden, seien es vor allem die skandinavischen Länder in denen die Einrichtung des Wohlfahrtsstaates einen positiven Beitrag zur bezahlten Arbeitsmarktteilnahme von Frauen geleistet habe (vergl. Esping-Andersen 1990: 159). Seinem Ermessen nach hat eine stille Revolution stattgefunden, in welcher die Mechanismen des Wohlfahrtsstaates das Funktionieren des freien Marktes untermauerten. Soziale Politik versteht er als einen „säubernden Mechanismus des Arbeitsmarktes“ (Esping-Andersen 1990: 160). Es gehe in der sozialen Politik nicht nur um die Garantie von 
Vollerwerbstätigkeit, sondern auch um die Absicherung der Verteilungsgerechtigkeit. Jeder Wohlfahrtsstaat, also jedes Wohlfahrtsstaatsregime, gehe mit einem entsprechenden Arbeitsmarktregime einher. Als Beweis hierfür sieht er seinen Vergleich dreier Länder (entsprechend der drei Regimes also die USA, Deutschland und Großbritannien). Aus der Analyse verschiedener Arbeitsmarktinstrumente zieht er den Schluss, dass es zwischen allgemeiner sozialer Politik und Arbeitsmarktpolitik einen Interaktionseffekt gibt. Frauen, die z.B. Unterstützung bei der Erfüllung familiärer Aufgaben erhalten (z.B. in der Form von geeigneter Infrastruktur) seien eher geneigt die übrige Familienarbeit zu kombinieren mit bezahlter Arbeit (vergl. Esping-Andersen 1990: 149).

Obwohl er entscheidende Ziele der emanzipatorischen Bewegung durch seinen Ansatz unterstützt, hat er diverse Kritiken aus ihren Reihen entgegen nehmen müssen. In seinem Theorieansatz sehe Esping-Andersen Emanzipation lediglich als eine Folge staatlicher Bedürfnisse am Arbeitsmarkt. Auch aus der kulturellen/anthropologischen Sicht wird seine Theorie kritisiert. Die Rolle der Frau in der Gesellschaft sei in starkem Maße abhängig von den kulturellen Gegebenheiten eines Landes. Hierauf werde in seinem Ansatz wenig Rücksicht genommen. Auf diese Kritiken ist der Autor in seinen späteren Werken näher eingegangen.

\subsubsection{2 'Welfare States in Transition - National Adaptations in Global Economies'} (1996)

Das zweite Buch, dessen analytische Betrachtung an dieser Stelle erfolgt, zeigt deutliche Parallelen mit der ersten Arbeit. Die theoretischen Ansätze sind hier weiterentwickelt vor dem Hintergrund des Wohlfahrtsstaats in der Krise. Die Analyse dieses Werkes erfolgt anhand der in diesem Buch zentral stehenden Themen: ,Wohlfahrtsstaatskrise und Diagnose', ,die Rolle der Frauen', ,Demographie', und ,Neue Risiken - die Armutsfalle'.

\section{Wohlfahrtsstaatskrise und Diagnose}

$\mathrm{Zu}$ Beginn des zweiten Buches unterscheidet Esping-Andersen verschiedene Diagnosen wohlfahrtsstaatlicher Krisen und ordnet ihnen entsprechende Kausalitäten $\mathrm{zu}$, wie z.B. einen durch den Wohlfahrtsstaat wenig flexibel 
gewordenen Markt, die Gesellschaftsalterung und das Funktionieren eines neuen globalen Marktes (vergl. Esping-Andersen 1996: 1f). Er ist der Meinung, dass der Keynianismus der Nachkriegszeit seine Wirkung nicht mehr zeige. Aufgrund der soziokulturellen Entwicklungen (z.B. weniger Geburten und eine verminderte Ehestabilität) komme es $\mathrm{zu}$ neuen Herausforderungen für den Wohlfahrtsstaat. Hierbei, so der Autor, werden wiederum "nationalinstitutionelle Traditionen“ (Esping-Andersen 1996: 6) sichtbar.

Neoliberalen Instrumenten, wie z.B. der Privatisierung großer Teile des Staatsbetriebes steht Esping-Andersen kritisch gegenüber. Positiver äußert er sich gegenüber dem Dritten Weg und dem Instrument der „Sozialinvestition“ (Esping-Andersen 1996: 14). Er verdeutlicht, dass die vermeidliche Krise nicht Schuld des Wohlfahrtsstaates sondern einer nicht funktionierenden Wirtschaft sei. Der Wohlfahrtsstaat werde hierdurch in Mitleidenschaft gezogen, da er aus verhältnismäßig geringen Einkünften die Kosten vieler Bedürftiger decken müsse (vergl. Esping-Andersen 1996: 33).

Der Autor kommt in seiner Arbeit zu dem Schluss, dass die Wohlfahrtsstaaten ihre egalitären Prinzipien auf eine radikale Weise überdenken müssen. Dabei sei es aus seiner Perspektive nicht entscheidend, ob es sich um rechte oder linke Regierungen handele. Gründe für diese Neuorientierung sehe er erstens im stets heterogeneren Gesellschaftsaufbau und zweitens in den wachsenden Einkommensunterschieden (vergl. Esping-Andersen 1996: 264).

Um die Bürger für wirtschaftliche Umstrukturierungen zu wappnen, hält der Autor lebenslanges Lernen und Strategien zur Sozialinvestition für nützlich (vergl. Esping-Andersen 1996: 259f). „Die Philosophie hinter der Sozialinvestitionsstrategie ist nicht revolutionär. Es war seit Jahrzehnten Teil im Paket des schwedischen sozialdemokratischen Modells; es ist die offizielle Rhetorik der Regierung Clinton; und es trägt die Hauptstrategie mit der die Asiatischen Tiger auf die globalen Herausforderungen reagieren“ (EspingAndersen 1996: 260).

Die Rolle der Frauen

Während direkt nach dem Zweiten Weltkrieg in den meisten, von ihm untersuchten, Ländern die Hausfrauenehe stark dominierte, können schon seit den frühen 1970er Jahre drei unterschiedliche wohlfahrtstaatlichen Antworten 
auf wirtschaftliche und soziale Veränderungen identifiziert werden. Vor allem die Investitionen der skandinavischen Länder, die es Frauen ermöglichen bezahlte Arbeit und Familie zu kombinieren, findet Esping-Andersen wegweisend. „Der Zweiverdiener Haushalt ist jetzt die Norm; und im Vergleich mit wo auch immer, ist die Armutsrate von durch Frauen ernährten Familien unbedeutend“ (Esping-Andersen 1996: 13).

Nachdem die Rolle der Frau in seinem vorigen Werk relativ stark kritisiert wurde, hat er seine theoretischen Ausführungen zur Rolle der Frau in diesem Buch deutlich erweitert. De-Familisierung bzw. Familisierung sind Begriffe, die er in diesem zweiten Werk zur Verdeutlichung des Zusammenhangs zwischen familiärer Aufgabenteilung und Arbeitsmarktteilnahme verwendete. Mit diesem Begriffspaar ist der Grad der Zuweisung von Reproduktionsaufgaben an Familien bzw. ihrer Entlastung durch öffentliche oder private Dienstleistungsangebote umschrieben. De-Familisierung bildet auf diese Weise die Voraussetzung für die Arbeitsmarktteilhabe von Frauen bzw. Müttern (vergl. auch Orloff 1993) (vergl. Dingeldey 2004: 113f). In einigen Ländern ist die Betreuung der Kinder und die Versorgung älterer Menschen außerhäuslich organisiert (also de-familialisiert), und eine entsprechende Infrastruktur gilt als selbstverständlich, während in anderen Ländern familiäre Aufgaben nur in sehr marginaler Weise als Staatsaufgaben verstanden werden. „Frauen wollen bezahlte Arbeit und größere wirtschaftliche Unabhängigkeit; die Familie ist wahrscheinlich flexibeler, und weniger wahrscheinlich arm, falls sie sich auf zwei Einkommen verlassen kann“ (Esping-Andersen 1996: 26f). Familisierung wird aus Sicht Esping-Andersens stets mehr der Vergangenheit angehören. Familienpolitik der Zukunft baue auf De-Familisierung.

Kremer kritisiert die Theorie Esping-Andersens und fügt ihr eine kulturelle Dimension hinzu. Sie beruft sich unter anderem auf die OESO Studie ,Babies and Bosses' (2002) in der soziale Normen als ein wichtiger Faktor im Bezug auf die Frauenerwerbstätigkeit gesehen werden (vergl. Kremer 2007: 61). Warum es in den unterschiedlichen Ländern verschiedene Traditionen im Bezug auf Frauenerwerbstätigkeit gibt beschäftigt auch Pfau-Effinger. Der Wohlfahrtsstaatsansatz von Esping-Andersen biete keine zufrieden stellende Antwort, da er nicht ausreichend verdeutlicht, warum in einigen Staaten momentan die weibliche Erwerbstätigkeit schnell steigt, während sie in anderen, vergleichbaren, Staaten auf einem niedrigen Niveau verharrt (vergl. Kremer 
2007: 62f). Eine mögliche Antwort bietet Hakim. Ihrer Theorie zufolge gibt es in der Gesellschaft 20\% Frauen die ganzzeitlich bei ihrer Familie bleiben wollen, 20\% wollen ganzzeitlich einer außerhäuslichen Beschäftigung nachgehen, und die übrigen 60\% wollen Familie und Beruf kombinieren (vergl. Kremer 2007: 68).

\section{Demographie}

Als Ursache für die schnelle Alterung der Gesellschaft sieht der Autor vor allem die niedrigen Geburtenzahlen. Diese ständen allerdings nicht in einem negativen Kausalverband mit hohen Erwerbszahlen von Frauen. Im Gegenteil, so behauptet er, denn bis auf wenige Ausnahmen seien es vor allem Länder mit einer hohen Frauenerwerbsbeteiligung, die auch eine relativ hohe Fruchtbarkeitsrate aufweisen. Diese Erkenntnis war revolutionär, da bis zu dem Zeitpunkt noch von der traditionell negativen Kausalität zwischen dem Beschäftigungsgrad der Frauen und der nationalen Fertilitätsrate ausgegangen wurde. Bei der Entscheidung zur Familiengründung hält der Autor den Wohlfahrtsstaat für sehr entscheidend, da dieser junge Familien durch soziale Dienstleistungen unterstützen kann.

„Die Befreiung der Familie von ihren Betreuungspflichten und die Individualisierung von Kindheit und Alter sollen wie so oft mit Blick auf Schweden, Dänemark oder Finnland gleich mehreren politischen Zielen dienen: der Erhöhung der Zahl erwerbstätiger Frauen, der Verwirklichung des ,Kinderwunsches', konkret: der Steigerung der Geburten, insgesamt dem Umbau des Wohlfahrtsstaats in Zeiten neuer ökonomischer Herausforderungen“ (vergl. Esping-Andersen 2002) (http://www.wiso.unikoeln.de/soziologie/altesDesign/02_stud/2003-04/os/1296_ostner, aufgerufen am 07.05.2008).

Neue Risiken - die Armutsfalle

Risikoreich werde es, laut Esping-Andersen, wenn sich die gesellschaftliche Situation im Land verändere, ohne dass sich das gesellschaftliche Muster anpasse. In den meisten Ländern sicherte der Nachkriegsstaat eine ununterbrochene berufliche Karriere, der sich am Ende einige pensionsberechtigte Lebensjahre anschlossen. Als relativer Luxus wurde gesehen, dass Frauen sich nicht an der (oft körperlich schweren) Arbeit 
beteiligen mussten und sich um den Haushalt und die Versorgung der Kinder kümmern konnten. Diese Situation habe sich allerdings verändert. Die Lebensläufe seien vielfältiger geworden, und die größte Herausforderung für den Wohlfahrtsstaat sei die Verteilung wirtschaftlicher Risiken. Ursache für diese Verteilung seien die wirtschaftliche Umstrukturierung, die Veränderungen am Arbeitsmarkt (durch welche reiche Länder einfache Arbeit an Niedriglohnländer verlieren) und die Entstehung neuer Familienformen als Folge hoher Scheidungsraten bzw. allein stehender Eltern (vergl. EspingAndersen 1996: 131).

Esping-Andersen unterscheidet drei Formen von sozialen Risiken, erstens die Lebenszyklusrisiken, zweitens intergenerationelle Risiken und drittens Klassenrisiken (vergl. Esping-Andersen 1999: 40ff). Folgende Instrumente hält er zur Risikobekämpfung geeignet: „Workfare, Lohnzuschüsse, Einkommenszuschüsse und Sozialinvestition“ (Esping-Andersen 1996: 132).

Im weiteren Text beruht sich der Autor auf unter anderem Keynes und Beveridge, die schon zu ihrer Zeit betonten, dass der soziale Wohlstand auf den Fundamenten eines funktionierenden Arbeitsmarktes gebaut sei. Je mehr Menschen in den Arbeitsprozess eingebettet wären, desto kleiner fallen die sozialen Lasten für den einzelnen Teilnehmer aus.

Auch andere Wissenschaftler, wie z.B. Opielka unterstreichen die hohen Kosten für Familien in modernen Wohlfahrtsstaaten. Er unterscheidet zwischen vier Formen der Teilung familiärer Kosten und knüpft hiermit an die Theorien Esping-Andersens an. Dem liberalen, sozialistisch-etatistischen (sozialdemokratischen) und konservativen Varianten fügt er die garantistische Form hinzu. Diese „setzt auf die Optionserweiterung aller Familienakteure und vermeidet die sozialpolitische Normalisierung biographischer Muster, die unter den Bedingungen der Pluralisierung der Lebensformen zur Exklusion zunehmender Bevölkerungsteile führt. Sie kombiniert deshalb die Dienstleistungsstrategie mit grundrechtsförmigen Geldtransfers" (Opielka 2004: 109f). 


\subsubsection{3 'Why We Need a New Welfare State' (2002)}

Bei diesem Buch handelt es sich um eine Koproduktion von Esping-Andersen und anderen Autoren, welche im Zusammenhang mit der Lissabon-Konferenz publiziert wurde. In diesem Werk bieten die Autoren kein Rezept für eine allgemein gültige Familienpolitik, sondern entwerfen sie einen Ansatz mit Voraussetzungen für einen Neuentwurf von Familienpolitik aus heutiger Sicht (vergl. Esping-Andersen u.a. 2002: 24). Die Autoren verwenden das Prinzip des Lebenszyklusrahmens. Hierdurch wird das Leben eines Einzelnen in einem größeren Zusammenhang gesehen und nicht die Familienphase als temporäres Abstraktum. Die verschiedenen Ereignisse und Risiken eines Lebens werden so miteinander verbunden (vergl. Esping-Andersen u.a. 2002: 7). Die beschreibenden Analyse dieses Werkes erfolgt anhand der Themen: ,Aktiver (aktivierender) Wohlfahrtsstaat', ,Demographie', ,Entscheidende Lebensphase: Kindheit', ,Neue Risiken - die Armutsfalle' und ,die Rolle von Frauen und Männern'.

\section{Aktiver (aktivierender) Wohlfahrtsstaat}

Über den Neo-Liberalismus Thatchers und den Dritten Weg Blairs äußern sich Esping-Andersen und seine Co-Autoren kritisch. Der Ansatz Thatchers wird inhaltlich abgelehnt und als kurzsichtig umschrieben. Den Theorien Blairs wirft man keinen falschen Ansatz aber dafür Undeutlichkeit und Allgemeinheit vor. „Der Dritte Weg bleibt frustrierend undeutlich und allgemein, aber falls man versucht die verschiedenen Ziele mit den wenigen konkreten Maßnahmen die letztendlich eingeführt wurden zusammen zu puzzeln, erscheinen die Umrisse einer basalen Architektur" (vergl. Esping-Andersen u.a. 2002: 4). Sie bevorzugen den Term aktiver/aktivierender Wohlfahrtsstaat zur Bezeichnung der von ihnen favorisierten Form eines sozialen Investitionsstaates (vergl. Esping-Andersen u.a. 2002: X).

Ganz im Sinne des Aktivierenden Staates halten die Autoren die Teilnahme von Männern und Frauen am Arbeitsmarkt für entscheidend. Als problematisch erfahren sie die Beschäftigungsunterstützung geringer Ausgebildeter, und sie stellen fest, dass Einkommensunterstützung für die Gruppe der Langzeitarbeitslosen nötig sei. Die übrigen Bürger können nach einem Verlust ihrer Arbeit durch Maßnahmen wie ,Make work pay' und ,Aktivierung' wieder 
in den Arbeitsprozess eingebunden werden (vergl. Esping-Andersen u.a. 2002: 46).

Im Zusammenhang mit der Entwicklung des Aktivierenden Staates und der Bedeutung der zivilen Gesellschaft, wird vor allem die Rolle der Familie betont. Diese Renaissance der Familien nach einer Periode der unbedingten Individualisierung findet er nicht so verwunderlich. „Individualisierung, verstanden als Erweiterung individueller Handlungsoptionen, und Familiensolidariät ergeben keineswegs einen Widerspruch. Ohnehin haben, worauf Bertram (1997) aufmerksam macht, Individualisierungsprozesse kaum etwas mit dem Wandel von Ehe und Familie zu tun, wie sie selbst die notwendige Voraussetzung für die moderne, auf freier Wahl der Partner gegründete Form von Ehe und Familie sind. Damit die voluntaristische Solidarität der Familienmitglieder - Durkheim differenzierte bereits den kooperativen Individualismus vom utilitaristischen Individualismus - nicht überfordert wird, darf die Gesellschaft die Familien vor allem in den kritischen Phasen ihrer Entwicklung nicht allein lassen. Das ist der Grund für den Zusammenhang von Familien und Familienpolitik heute" (Opielka 2004: 138).

Welche politischen Maßnahmen für die einzelnen Länder letztendlich zutreffend sind, hängt vom entsprechenden Regimetyp ab. Den drei Wohlfahrtsregimes können verschiedene Probleme zugeordnet werden. Hemerijk schreibt z.B., dass das größte Problem des sozialdemokratischen (skandinavischen) Systems die hohe Steuerlast und das relativ geringe Ausmaß der gesellschaftlichen Dienstleistung in der Privatwirtschaft sei. Das Problem des liberalen (angelsächsischen) Systems seien Armut und soziale Ungleichheit sowie zu teure und schwer zugängliche Kinderbetreuung. Das Problem des konservativen (kontinentalen) Regimes bleibe ein Mangel an Arbeitsplätzen, auch für einfache Arbeiten im Haushalt (vergl. Hemerijk 2007: 124).

Die Autoren des dritten Werkes unterscheiden zwischen einer ,Servicestrategie' und einer ,Transferstrategie' (vergl. Esping-Andersen u.a. 2002: 61f). Der Begriff ,Servicestrategie' umschreibt das Anbieten von Dienstleistungen zur Unterstützung von Familien, während bei der ,Transferstrategie' Geldleistungen direkt an die Familien überwiesen werden. Auf den ersten Blick, so scheint es, ist das Anbieten von Diensten teurer als ein direkter monetärer Transfer in Richtung Eltern. Allerdings betonen die Autoren, dass es sich hierbei um zwei 
unabhängige Instrumente handele mit unterschiedlichen sekundären Effekten. Durch die Investition in Tagesbetreuung werde es möglich, dass Eltern weiterhin einer bezahlten Arbeit nachgehen und somit höhere Steuereinkommen erwirtschaften. Gesamtwirtschaftlich gesehen sei diese Strategie sowohl kurzfristig als auch langfristig wirtschaftlicher.

Unter der Bezeichnung Wohlfahrtspluralismus kann das Wechselspiel zwischen Staat, Markt und Familie verstanden werden. Diese drei Akteure sind aufeinander angewiesen und stehen in einer Abhängigkeitsbeziehung zueinander. Der Staat ist in vielerlei Hinsicht abhängig von der Kraft der Familien. Gleichzeitig sind allerdings Familien angewiesen auf staatliche Transfers in Form monetärer Mittel und entsprechender Infrastruktur. Entscheidend ist der richtige Mix dieser Instrumente, der abhängig sein sollte von den wohlfahrtsstaatlichen Möglichkeiten und den entsprechenden Bedürfnissen der Familien.

\section{Demographie}

Man spricht von einer ,Kinderdifferenz' (vergl. Esping-Andersen u.a. 2002: 63), da die europäischen Bürger sich im Durchschnitt 2,4 Kinder wünschten, diese Anzahl aber bei weitem nicht realisieren. Vor allem im südlichen Europa und in Mitteleuropa sei die Differenz am höchsten. In den skandinavischen Ländern werden im Verhältnis relativ viele Kinder geboren während gleichzeitig auch viele Frauen einer bezahlten Beschäftigung nachgehen. Esping-Andersen und die übrigen Co-Autoren beschreiben ihre Existenz als eine Revolution demographischen und familiären Verhaltens. Eine entscheidende Ursache hierfür seien die erfolgreichen weiblichen Emanzipationsbestrebungen nach wirtschaftlicher Unabhängigkeit. Diese gehen einher mit abgeschlossenen Ausbildungen und lebenslangen Karrieren. Geheiratet werde nicht mehr aus wirtschaftlicher Notwendigkeit oder gesellschaftlicher Verpflichtung, sondern aus Liebe. Hierdurch komme es zur Entstehung „von neuen und weniger stabilen Haushalten und Familienarrangements. Ein normales Kind wird mit zunehmender Wahrscheinlichkeit seine Kindheit nicht mit sowohl der Mutter als dem Vater verbringen. All dieses spiegelt eine größere individuelle Entscheidungsfreiheit wieder, aber auch Unsicherheiten und Risiken“ (EspingAndersen u.a. 2002: 2). 
Als Hauptziel der Familienpolitik der Nachkriegszeit interpretieren die Autoren die Absicherung der Hausfrauenehe sowie die (finanzielle) Unterstützung von Großfamilien. Aufgrund gesellschaftlicher und finanzieller Veränderungen wie z.B. der Teilnahme von Frauen am Arbeitsmarkt, der Brüchigkeit von Familienstrukturen, Jugendarbeitslosigkeit und hoher Immobilienpreise werden junge Menschen an der Familiengründung gehindert. Solange Staaten es nicht schaffen diese Probleme zu lösen, werden sie mit niedriger Fertilität konfrontiert. Die Realisierung der erwünschten Kinderzahl sei dann nicht möglich (vergl. Esping-Andersen u.a. 2002: 19f). Dieser Analyse fügt Brinkgreve hinzu, dass die Erziehung von Kindern in vorindustriellen Gesellschaften nicht nur weniger kostete, sondern sich auch noch bezahlbar machte, dass die Kinder im Haushalt oder auf dem Hof entscheidende Hilfe leisten konnten und auch zur materiellen Absicherung im Alter wichtig waren. In der heutigen Gesellschaft seien Kinder für Eltern aus einer emotionalen Perspektive sehr wichtig. Finanziell gesehen formen sie allerdings eine erhebliche Belastung. Gesellschaftlich seien Kinder auch materiell von großem Wert. Sie sind entscheidend für die Zukunft des Wohlfahrtsstaates, für die Erfüllung der Aufgaben am Arbeitsmarkt usw. (vergl. Brinkgreve/Velde 2006: 214f).

Als Gründe für die niedrige Fertilität sehen die Co-Autoren neben der Probleme Jugendlicher am Arbeits- und Wohnungsmarkt vor allem das Fehlen bezahlbarer Kinderbetreuungsstätten (vergl. Esping-Andersen u.a. 2002: 186). Opielka schreibt über den demographischen Wandel, dass „der ,natürliche” Reproduktionsprozess in Familien abnimmt, hat mehrere, vor allem soziokulturelle Gründe, insbesondere den Wandel der Geschlechterrolle. Während Frauen noch immer voranging Familienaufgaben übernehmen, passte sich ihr Bildungs- und Beschäftigungshintergrund immer mehr demjenigen der Männer an und überholte ihn teilweise (...), was infolge ihrer immer erfolgreicheren Integration in den Arbeitsmarkt zu einer Situation multipler Verpflichtungen führte“ (Opielka 2004: 108f).

Vor allem zwei Formen von Mutterschaft seien schwer mit einer Erwerbstätigkeit $\mathrm{zu}$ verbinden und bedürfen daher einer besonderen Unterstützung durch den Staat. Das seien erstens die Mütter mit Kindern im Kleinkindalter und zweitens Mütter mit mehr als zwei Kindern (vergl. EspingAndersen u.a. 2002: 75). Teilzeitarbeit könne hierbei eine wichtige Hilfe bieten. 
In skandinavischen Ländern bekommen gut ausgebildete Frauen ähnlich viele Kinder wie Frauen ohne einen erweiterten Bildungsweg.

\section{Entscheidende Lebensphase: Kindheit}

Durch die wirtschaftlichen Bedingungen leben Familien heute in einem grundlegend veränderten Zusammenhang. „Lebenschancen werden stets abhängiger von dem kulturellen, sozialen und kognitivem Kapital, dass Bürger sich aneignen können. Die entscheidende Lebensphase ist die Kindheit, und die entscheidende Angelegenheit ist das Zusammenspiel zwischen elterlichen und gesellschaftlichen Investitionen in die Entwicklung der Kinder" (EspingAndersen u.a. 2002: 26f). Durch eine positive kognitive Entwicklung und das Erlernen sozialer Fähigkeiten würden während der Kindheit die Weichen gestellt, um ein postindustrieller Gewinner zu werden. Daher finden die Autoren es auch von zunehmender Bedeutung allen Kindern gleiche Ausbildungschancen zu bieten. „Eine soziale Investitionsstrategie, die sich auf Kinder richtet muss das Mittelstück jeder Politik für soziale Inklusion sein“ (Esping-Andersen u.a. 2002: 30). Durch Sozialinvestitionen müssten Bürger unterstützt werden, um sich gesellschaftlichen Veränderungen sowie den Entwicklungen am Arbeitsmarkt anzupassen. Esping-Andersen fügt dem hinzu, dass diese Form der Investition vor allem erfolgreich sein kann, wenn schon in der frühen Kindheit die entsprechenden Weichen gestellt wurden. „Da inzwischen gut bekannt ist, dass das Können und die Motivation zu lernen in erster Linie abhängig ist von wirtschaftlichen und sozialen Konditionen in der Kindheit, werden Politiken die darauf zielen das Wohlbefinden von Kindern zu schützen als eine Investition gesehen eventuell mit und vielleicht noch dringender als Investitionen in Bildung" (Esping-Andersen u.a. 2002: 9). Das Thema Chancengleichheit und die Förderung frühkindlicher Erziehung gehören für Esping-Andersen zusammen - und seit langem preist er den dänischen Sozialstaat als überlegenes Modell. In Dänemark habe die Kinderbetreuung zu einem Abbau des sozialen Ungleichgewichts geführt" (Niejahr, http://images.zeit.de/text/2003/41/Esping-Andersen aufgerufen am 17.04.2008).

Neben einer guten Gesundheit und einer finanziellen Basis halten die Autoren es für entscheidend, dass die primären Mechanismen der Kinder gut stimuliert werden durch z.B. regelmäßiges Vorlesen. Hierzu könne der Staat die Eltern 
nicht zwingen. Allerdings kann er Eltern dadurch unterstützen, indem er ihnen das Recht auf einen stressarmen Arbeitsplatz, oder hochwertige Kinderbetreuung anbietet (vergl. Esping-Andersen u.a. 2002: 49). In einem später erschienen Text schreibt Esping-Andersen zu diesem Thema, dass zwei Faktoren für die Entwicklung von Kindern wichtig seien:

- $\quad$ Erstens, Fremdbetreuung von Kindern im ersten Lebensjahr kann negativ für die spätere Entwicklung des Kindes sein und

- Zweitens, falls die Qualität der Tagesbetreuung von Kindern (ab einem Jahr also) gut ist, kann sie sich positiv auf Schulresultate von Kindern auswirken. Das gilt insbesondere für ,weniger privilegierte' Kinder (vergl. Esping-Andersen 2008a: 29).

Neue Risiken - die Armutsfalle

Im weiteren Text thematisieren die Co-Autoren die Erblichkeit von Armut. Gemeint ist hiermit, dass Kinder aus armen Familien einem verhältnismäßig größerem Risiko ausgesetzt sind, um auch in Armut zu enden. Eben aus diesem Grund sehen die Autoren die Bekämpfung von Kinderarmut als eines der wichtigsten Ziele gegenwärtiger Familienpolitik (vergl. Esping-Andersen u.a. 2002: 54). Hierzu sei es wichtig, dass politisches Handeln sich in erster Linie auf Armutsprävention richte. Wenn eventuelle Maßnahmen nicht den gewünschten Erfolg mit sich bringen bzw. sich dennoch Armut entwickelt hat, müssen als zweiter Schritt Aktivierungsmaßnahmen eingeleitet werden (vergl. Engelen/Hemerijk/Trommel 2007: 21), denn für ein Ansteigen der Kinderarmut werden gewöhnlich zwei Gründe „angeführt: die Zunahme von Alleinerziehenden-Haushalten und die häufig prekäre Erwerbsintegration von Alleinerziehenden (Klocke/Hurrelmann: 2001, Kränzl-Nagl u.a.: 2003)“ (Opielka 2004: 80).

Vor dem Hintergrund der Chancengleichheit finden die Co-Autoren es essentiell, dass es dem Staat gelingt, den Zusammenhang zwischen den kognitiven Fähigkeiten von Eltern und Kindern zu verringern. Folgende drei Punkte können hierbei behilflich sein:

- „Sicherung eines adäquaten Familieneinkommens, 
- Hilfen, die den direkten Einfluss der Eltern auf die kognitive Entwicklung der Kinder schwächen und

- Verbesserung der Arbeitssituation/Arbeitsbedingungen der arbeitenden Mütter" (Esping-Andersen u.a. 2002: 56).

Theoretisch baut dieser Ansatz auf Rawls Gerechtigkeitsverständnis auf, das er ausdrücklich „auf Termen wie Lebensprospekte oder Erwartungen des Wohlbefindens" basiert (vergl. Esping-Andersen u.a. 2002: XVI). Auch im Hinblick auf die Teilung von Verantwortlichkeiten „zwischen dem Staat, dem Markt und der Familie“ (vergl. Esping-Andersen u.a. 2002: 13) wird auf die Gerechtigkeitstheorien verwiesen.

„Martin Kronauer (2002) analysierte, dass dieser Prozess der ,Exklusion' seine Ursache in den Umbrüchen der Erwerbsarbeit, dem Brüchig werden sozialstaatlicher ,Inklusion' und der Schwächung familiärer Bindungen hat: ,Das Draußen der Ausgrenzung liegt nicht im gesellschaftlichen Jenseits sondern ist aufs Engste mit dem Drinnen verschränkt' (ebd., S. 235). Die Forderung der meisten Ökonomen und vieler Sozialpolitiker zielt auf einen ,Niedriglohnsektor', womit für die dort Tätigen die Erwerbsarbeit allein zur gesellschaftlichen Teilhabe nicht reicht. Das Ergebnis ist Armut - mit oder ohne Arbeit -, jedenfalls dann wenn die unterstützenden Einkommenssubventionen nicht ,armutsfest' sind“ (Opielka 2004: 73).

Die Rolle von Frauen und Männern

Emanzipationspolitik wird als Teil eines gesamtgesellschaftspolitischen Ansatzes verstanden, der vor allem mit Arbeitsmarktpolitik und der Bekämpfung von Armut verbunden sei. Hieraus ergeben sich zwei Schlüsse. Erstens sei es für die Wohlfahrt von Familien und der Wohlfahrt der Gesellschaft essentiell die Ressourcen von Frauen besser zu verwenden, und zweitens sollte man aus diesem Grund Emanzipationspolitik als Gesellschaftspolitik interpretieren (vergl. Esping-Andersen u.a. 2002: 67). „Die Steuerung von Reproduktionsaufgaben bzw. familialer Erwerbsmuster hat daher den Charakter einer Querschnittspolitik. Relevant sind dabei das Politikfeld der Familienpolitik selbst, die Arbeitsmarktpolitik (einschließlich der Regulierung sozialer Sicherung z.B. in Ableitung vom Erwerbsstatus), aber auch die Bildungspolitik, die kollektiven Arbeitsbeziehungen, die Arbeitszeitgestaltung, 
bis hin zur Infrastrukturplanung der Kommunen usw. (...) Um in einer solchen hoch komplexen Beeinflussungsstruktur überhaupt eine Steuerungswirkung auf familiale Entscheidungen erzeugen zu können ist eine Art Harmonisierung bzw. Koordination der Anreizstruktur über die verschiedenen Politikfelder hinweg Voraussetzung (Dingeldey 2000: 36f)" (Dingeldey 2004: 113). In der Lissabon-Strategie haben die Europäischen Staaten bezüglich des Beschäftigungsgrades gemeinsame Ziele vereinbart. Die allgemeine Arbeitsteilnahme muss im Jahre 2010 70\% betragen. Für Frauen gilt eine Mindestgrenze von 60\% und für ältere Arbeitnehmer 50\% (vergl. Hemerijk 2007: 123).

Um diese Ziele zu erreichen sei auch die Rolle der Männer bzw. Väter entscheidend. Dieser Punkt ist in den früheren Arbeiten Esping-Andersens weniger beleuchtet. Auffallend an seinen Theorien ist (nicht nur) die Angleichung des weiblichen Lebenslaufes an den männlichen. Durch berufliche Qualifikationen und vergleichbare Tätigkeiten konnte sich der weibliche Lebenslauf dem männlichen angleichen obgleich es Grenzen gäbe, vor allem in dem Moment in dem zwei oder mehr Kinder geboren werden. Um diesem Effekt einigermaßen entgegen zu steuern sei es daher nötig, dass Väter eine bezahlte Elternzeit (ohne Plafond) nehmen könnten (vergl. Esping-Andersen u.a. 2002: 93f). Nicht nur in den skandinavischen Ländern, sondern auch in stets mehr europäischen Ländern ist in den vergangenen Jahren eine nicht übertragbare Elternkomponente für die Väter eingeführt worden.

\subsubsection{4 'The Incomplete Revolution - Adapting to Women's New Roles' (2009)}

5In seinem 2009 erschienen Buch bestätigt der Autor größtenteils die bereits dargestellten Sichtweisen hinsichtlich der Themen ,Demographie' und ,Neue Risiken' und bietet vor allem im Bereich der Rolle der Frau in der Familienpolitik, der Chancengleichheit für Kinder und der Seniorenpolitik gedankliche Erweiterungen. Obwohl Esping-Andersen nachvollziehen kann, dass Staaten Lösungen suchen, um familienpolitische Maßnahmen effizienter 
$\mathrm{zu}$ gestalten, äußert er sich wiederum kritisch gegenüber Liberalisierungsansätzen im Bezug auf Dienste wie z.B. Kinderbetreuung und stellt vor allem die Langzeitfolgen dieser Maßnahmen in Frage. Er erwarte von Regierungsseite eine aktive Verantwortungsübernahme in Richtung eines sozialen Investitionsstaates. Die beschreibende Analyse dieses Werkes erfolgt anhand der Themen: ,die Rolle der Frau in der Familienpolitik' und ,Chancengleichheit für alle Kinder.

Familienpolitik und die veränderte Rolle der Frau

Esping-Andersen weist auf das demographische Defizit vieler westlicher Wohlfahrtsstaaten und der von ihm bereits thematisierten ,Kinderdifferenz'. Diese Differenz sei kleiner in Staaten in den Frauen Familie und Beruf gut vereinbaren könnten ${ }^{6}$. Im Zusammenhang mit der für Wohlfahrtsstaaten relevanten demographischen Nachhaltigkeit unterstreicht der Autor darum die Bedeutung dieses Gleichgewichts für den Wohlfahrtsstaat. Die ,Investitionskosten' der Eltern (insbesondere aber der Mütter) müssten durch den Staat auf ein niedriges Niveau zurückgefahren werden. Insbesondere qualitiativ hochwertige und bezahlbare Kinderbetreuung sei hierfür ein wichtiges Instrument. Er warnt an dieser Stelle vor der Liberalisierung im Bereich der Kinderbetreuung und argumentiert am Beispiel der USA, dass durch einen freien Kinderbetreuungsmarkt auf lange Sicht die Ungleichheiten zwischen den Familien zunähmen. Die bestehende Gleichstellungsinbalance könne durch eine Familienpolitik ausgeglichen werden in der Frauen im ersten Jahr nach der Geburt einen bezahlten Mutterschutz aufnehmen könnten, danach für kurze Zeit als Teilzeitbeschäftigte arbeiten, um auf lange Sicht wieder vollständig auf den Arbeitsmarkt zurückzukehren. Er warnt vor zu kurzen Familienurlauben, da hierdurch bei vielen Frauen das Risiko bestehe, nicht oder nur begrenzt zur bezahlten Form der Beschäftigung zurückzukehren.

\footnotetext{
6 Der Titel dieses Werkes ,The Incomplete Revolution - Adapting to Women's New Roles unterstreicht vor allem das Thema Vereinbarkeit von Familie und Beruf für Frauen. In den meisten europäischen Ländern seien zwar immer mehr Frauen auf dem Arbeitsmarkt aktiv, aber immer noch würden Mütter in vielen Ländern mit hohen ,Investitionskosten' für ihre Kinder konfrontiert, da die Unterstützung familialer Leistungen noch nicht ausgewogen sei. So lange Staaten dieses Gleichgewicht nicht herstellen - so Esping-Andersen - werden die Geburtenzahlen auf einem (verhältnismäßig) niedrigem Niveau verharren.
} 
Durch bezahlte und unübertragbare Elternurlaube für Väter könnten auch sie sich an die Sorge für das neugeborene Kind gewöhnen. Wichtig für eine dauerhafte Teilnahme der Väter an der Erziehung ihrer Kinder sei allerdings auch ein gutes Einkommen der Mütter (verbessert die Verhandlungsfähigkeit der Mütter), eine nicht unrealistisch hohe Betreuungszeit (also keine familiäre Vollversorgung der Kinder ohne den Einsatz von außerhäuslicher Betreuung) und letztendlich die Begrenzung der Arbeitszeit zum Tagesende hin (Väter die regelmäßig bis $20 \mathrm{Uhr}$ an ihrem Arbeitsplatz bleiben könnten nur ungenügend zur Sorge für die Kinder beitragen). Wichtig sei es hierbei auch alle Arbeitgeber $\mathrm{zu}$ erreichen und einer geschlechtsspezifischen Segregation (Frauen im öffentlichen Dienst und Männer in der freien Wirtschaft) vorzubeugen.

Er sei sich der Tatsache bewusst, dass die konsequente Teilnahme der Frauen auf dem Arbeitsmarkt auch die Ungleichheit zwischen den Familien vergrößern könne, denn ein gut ausgebildetes Zweiverdienerpaar verfüge nicht nur finanziell über andere Möglichkeiten als eine begrenzt ausgebildete alleinstehende Mutter (vergl. Esping-Andersen 2009: 59f). Das von ihm favorisierte frauenfreundliche familienpolitische Modell führe zu erheblichen Kosten, könnte aber essentielle gesellschaftliche Ungleichheiten korrigieren und habe mehr Chancengleichheit zwischen Kindern zur Folge.

Esping-Andersen könne verstehen, dass Staaten die Finanzierungsverantwortung teilen wollten, bietet aber keine konkreten Alternativen. Einerseits schreibt er, dass die Debatte zum Thema Privatisierung regelmäßig die Gegner gegeneinander ausspiele. „Die Wahrheit liegt im Detail und nicht in der Ideologie. Die Menükarte der Privatisierung ist groß und reicht von einem rein kommerziellen System zu Quasimärkten in einer öffentlichen Umgebung. Innerhalb dieser Bandbreite finden wir eine Überfülle von Möglichkeiten“ (Esping-Andersen 2009: 105). Im selben Atemzug fügt er dieser Differenzierung allerdings hinzu, dass sich unabhängig vom gewählten Menü makroökonomisch nicht viel verändern werde. Er weise vor allem auf Langzeiteffekte, die z.B. die Privatisierung der Kinderbetreuung mit sich bringen könnte. Es bestände hierdurch das Risiko, dass die Segregation zwischen chancenreichen und -armen Kindern vergrößert werde. Die Vergrößerung dieses Unterschiedes sei aber nicht nur aus sozialer Sicht problematisch sondern vor allem auch wirtschaftlich unvorteilhaft. Er rufe die Wohlfahrtsstaaten daher auf, bei ihren politischen Entscheidungen nicht nur die 
jährliche Ausgabenseite zu betrachten, sondern auch mögliche Langzeiteffekte in Betracht zu ziehen. Wirtschaftlich entscheidend sei letztendlich nicht der öffentliche Haushalt, sondern das Bruttoinlandsprodukt.

Öffentliche Leistungen für Familien seien in Dänemark im Verhältnis zu den Vereinigten Staaten $(6 \% / 1,1 \%)$ sehr hoch. Würde man - vor dem Hintergrund der Effekte der unterschiedlichen Maßnahmen aufeinander - über soziale Sicherheit im breiten Sinne des Wortes sprechen wären die Finanzierungsmengen schon deutlich weniger groß (34\%/16\%). Bedenke man zusätzlich, dass ein Großteil der ausgezahlten Leistungen durch Steuern direkt wieder zurückverdient würde und subtrahiere man auch noch die öffentlichen Ausgleichsleistungen, die vor allem in liberalen Staaten wie den USA gezahlt werden würden käme man auf einen Nettoindex von $26 \%$ des Bruttoinlandproduktes in Dänemark gegenüber 25\% des Bruttoinlandproduktes in den USA. Die Kostendifferenz zwischen diesen beiden Ansätzen sei letztendlich also gering. Viel erheblichere Unterschiede gäbe es allerdings hinsichtlich der Frage wer diese Leistungen zu zahlen habe, wem die Mittel zuteil kämen und was die Langzeitfolgen dieser Investitionen seien.

Der Autor unterstreicht, dass zusätzliche Serviceleistungen für Familien auf lange Sicht unabdingbar seien, um ein Gleistellungsgleichgewicht zu erreichen. Blieben diese Leistungen weiterhin von den Familien selbst abhängig, werde es entweder eine anhaltende, niedrige Fertilität oder aber kleine Familieneinkommen zur Folge haben.

\section{Chancengleichheit für alle Kinder}

Aus wohlfahrtsstaatlicher Sicht halte Esping-Andersen die Chancengleichheit von Kindern für entscheidend, denn sie führe zu sozialer Gerechtigkeit und sei außerdem sehr effizient. Durch demographische Effekte wie z.B. die Homogenisierung der Partnerwahl (also eine Partnersuche innerhalb derselben sozialwirtschaftlichen Gruppe), Immigration und zunehmender Partnerschaftsinstabilität mit einer dementsprechend steigenden Anzahl alleinerziehender Eltern (die wiederum über weniger Geld und Zeit verfügen als Familien mit zwei Elternteilen) werde die Chancengleichheit der Kinder negativ beeinflusst. Gleichzeitig herrsche in der Entwicklungspsychologie inzwischen ein Konsens darüber, „dass die entscheidenden kognitiven und Verhaltensgrundierungen, um zu Lernen in der frühen Kindheit verankert sind. 
Die Entwicklung im Vorschulalter ist grundlegend für die Möglichkeiten und Motivation von Kindern“ (Esping-Andersen 2009: 113). In vielen Ländern würden Kinder in dieser Phase vor allem zu Hause erzogen. Daher spielen die privaten Hintergründe eine entscheidende Rolle für die Entwicklungschancen von Kindern. Man könne drei Familieneffekte unterscheiden: den „finanziellen Effekt, de(n) Zeitinvestitionseffekt und de(n) Lernkultureffekt. Ein interessanter Aspekt in diesem Zusammenhang ist die Tatsache, dass die drei Faktoren nicht unbedingt gemeinsam auftreten müssten: die Reichen investieren nicht notwendigerweise die meiste Zeit bzw. Förderung in ihre Kinder" (EspingAndersen 2009: 122). Obwohl die Bedeutung der vorschulischen Phase inzwischen bekannt sein dürfte, würden Staaten immer noch verhältnismäßig viel in die tertiäre Ausbildung und wenig in die vorprimäre Förderung investieren. Für eine soziale Investitionsstrategie mit dem Ziel der Chancengleichheit für Kinder halte der Autor es daher für wichtig, den Einkommenseffekt zu reduzieren und Armut zu beschränken. Man müsse Familien neben dem klassischen Leistungsausgleich ein Minimumeinkommen garantieren. Zweitens sollte man die Lernumgebung von Kindern homogenisieren. Hiervon könnten vor allem die sozialschwachen Familien profitieren. Und drittens sei die Teilnahme der Mütter am Arbeitsmarkt auch für die Kinder entscheidend. Um diese Teilnahme realisieren zu können, sei dann wiederum eine anerkannte, homogenisierte und bezahlbare Form der Kinderbetreuung ab dem ersten Lebensjahr wichtig.

\subsubsection{Familienpolitischer Ansatz Esping-Andersens}

Im letzten Paragraphen dieses Unterkapitels wird eine zusammenfassende Evaluierung der beschriebenen und analysierten Bücher geboten. Ein zusammenhängender familienpolitischer Ansatz Esping-Andersens entsteht aus der Zusammenfassung der wichtigsten Elemente der Evaluierung. Hierbei werden auch die Interpretationen anderer Wissenschaftler hinzugezogen.

Im Allgemeinen fällt eine deutliche Entwicklung des theoretischen Rahmens Esping-Andersens auf. In den 1980er Jahren lagen seine quantitativen Forschungsschwerpunkte vor allem in der direkten Wohlfahrtsstaatsanalyse. Er verglich Sozialsysteme anhand quantitativer Daten über sozialstaatliche Ausgaben bzw. Investitionen. Durch seinen fundierten und systematischen 
Ansatz erfolgen sorgfältige internationale Vergleiche. Diese halfen zur Interpretation der Rollenverteilung der drei Wohlfahrtsproduzenten Staat, Markt und Familie.

Als De-Kommodifizierung bezeichnet er den Grad der Unabhängigkeit des Bürgers von seinem Staat, und er differenziert zwischen einem liberalen, einem konservativen und einem sozialdemokratischen Regimetyp. Das sozialdemokratische Regime sieht er als eine Art Idealmodell, im Hinblick auf die Lösung der wohlfahrtsstaatlichen Krise. In diesem Regime sind die gesellschaftlichen Umstände relativ klein und ist die Chance auf Armut verhältnismäßig niedrig. Einen entscheidenden Faktor spielt die Rolle der Frauen. Schon sehr früh wird im sozialdemokratischen System der Übergang von der Hausfrauenehe zum Zweiverdienermodell realisiert. Dieser Übergang führe zur wirtschaftlichen Selbstständigkeit von Frauen.

Esping-Andersen befasst sich mit der Frage nach den Ursachen der Wohlfahrtsstaatskrise. Einen wichtigen Grund für diese Krise sieht er im wenig flexiblen europäischen Markt einerseits und dem Funktionieren neuer globaler Märkte andererseits. Nicht in allen Ländern sei der wirtschaftliche Übergang gleich gut gelungen. Dies sei eine der wichtigsten Ursachen für die wohlfahrtsstaatliche Krise dieser Zeit. Außerdem befasst sich der Autor seit dieser Zeit schwerpunktmäßig mit dem demographischen Wandel. Neben der gesellschaftlichen Alterung sieht er vor allem in der niedrigen Fertilität die Ursache wohlfahrtsstaatlicher Probleme. Sein Ideal ist das Zweiverdienermodell mit entsprechender Unterstützung der Infrastruktur für Kinder. Die Begriffe Familialisierung und De-Familialisierung deuten - in diesem Zusammenhang auf den Grad der Verteilung familiärer Aufgaben zwischen den bereits unterschiedenen Wohlfahrtsstaatsautoren. Esping-Andersen identifiziert neue Risiken die zu gesellschaftlichem Abstieg bzw. Armut führen, wie z.B. die stark gestiegenen Scheidungsraten. Da allerdings nicht alle Momente im Leben gleich große Risiken mit sich bringen, betone er die Möglichkeiten des Lebenszyklusrahmens. Dem politischen Leitbild des Aktivierenden Staates entsprechend unterstützt er Instrumente wie z.B. Sozialinvestition zum Aufbau bzw. zur Stärkung individueller Kräfte und Fähigkeiten. Lebenslanges Lernen hält er für eine der wichtigsten Voraussetzungen für die individuelle Risikobeherrschung am Arbeitsmarkt. 
Inklusionsarbeit und die Aktivierung von Langzeitarbeitslosen und niedrig Ausgebildeten betont Esping-Andersen auch in seinem dritten Werk. Er und seine Co-Autoren schreiben über die Renaissance der Familie und unterstreichen ihre Kräfte und Fähigkeiten. Die demographische Entwicklung Europas hält er nicht nur für gesellschaftlich risikovoll, sondern er betont das europäische ,Kinderdefizit'. Hierbei handele es sich um die Differenz zwischen der Anzahl an Kindern die Menschen gerne bekommen würden und die Anzahl der letztendlich realisierten Kinder. Neben eventueller persönlicher Enttäuschungen, die dieses Defizit mit sich bringe, habe die niedrige Fertilität auch gesamtgesellschaftlich einen starken Einfluss, da einerseits die Zahl der potentiellen Arbeitnehmer in der Zukunft niedriger werde und andererseits der graue Druck bzw. der Anspruch auf wohlfahrtsstaatliche Leistungen durch Senioren zunehme. Ein Grund für die niedrige Fertilität sei der Arbeitsmarkt, der mit Zeitverträgen und unsicheren Verhältnissen vor allem für jüngere Menschen problematische Folgen habe. Weiterhin seien die stark gestiegen Wohnraumkosten und vor allem das Fehlen geeigneter Betreuungsmöglichkeiten ein Hindernis zur Familiengründung. Insbesondere Mehrkindfamilien hätten es schwer. Generell fehle es Familien an Zeit. Daher findet er das Angebot zur Teilzeit auch wichtig für eine familienfreundliche Gesellschaft. Deutlich wird in diesem Werk auch sein Erziehungsideal. Die Kindheit sieht er als die alles entscheidende Phase des Lebens. Hier werden Lebenschancen verteilt. Daher ruft er vor allem zur Sozialinvestition in junge Kinder auf. Hierdurch könne auch bei eventuellen Problemen zu späteren Zeitpunkten im Leben bessere Inklusionsarbeit geleistet werden. Durch geeignete Formen der Kindererziehung mit qualifizierten Fachkräften, die sich um die Kinder kümmern, könne das Risiko auf soziokulturelle Vorsortierung verkleinert werden. Die Armutsfalle sieht er als eines der großen Risiken seiner Zeit. Vor allem wenn Kinder in Armut aufwachsen und keine Chancengleichheit haben werde normale gesellschaftliche Teilnahme schwer realisierbar. Um Familien gut zu unterstützen sei ein Wohlfahrtsmix aus finanziellen Transfers und entsprechenden Dienstleistungen wichtig. Weiterhin betont er, dass die Karriere von Frauen im Zweiverdienermodell nicht nur wirtschaftliche Selbstständigkeit fördere, sondern auch das Armutsrisiko verkleinere. Er hebt geschlechtsneutrale Werte hervor und hält eine aktive Beteiligung der Väter bei der Erziehung ihrer Kinder für sowohl Väter als auch Kinder für entscheidend. 
Im jüngsten der hier bearbeiteten Werke Esping-Andersens formuliert der Autor familienpolitische Anforderungen für den Wohlfahrtsstaat. Obwohl er durchaus Alternativen zur Ausführungsverantwortung bzw. dem gewählten Policy-Mix sehe, betont der Autor wiederum das sozialdemokratische System als ideal im Hinblick auf Familienpolitik. Aus aktivierender Sicht müssen seine diesbezüglichen Kostenrechnungen als einseitig bezeichnet werden, und bietet er neben inhaltlich relevanten Erneuerungen keine politisch-administrativen Verbesserungsvorschläge. Frauen müssten im Verhältnis zu den Männern gleich gut bezahlt werden und im Jahr nach der Geburt eines Kindes Recht auf eine bezahlte Elternzeit erhalten. Nach diesem Jahr sei eventuell eine Phase der Teilzeitbeschäftigung übergangsweise nötig bis es letztendlich wieder zur Vollbeschäftigung der Frauen käme. Männer können durch einen gut eingerichteten Familienurlaub (also bezahlt und nicht übertragbar) ihre Frauen unterstützen und frühzeitig ein enges Verhältnis zu ihren Kindern aufbauen. Langfristig sei es für Männer wichtig, dass die Betreuungszeiten nicht unrealistisch hoch seien (also keine familiäre Vollbetreuung) und der Arbeitstag pünktlich beendet werde. Denn nur so könne an der abendlichen Versorgung der Kinder aktiv teilgenommen werden. Die Chancengleichheit der Kinder betont Esping-Andersen insbesondere. Um diese $\mathrm{zu}$ erreichen, sei ein angemessenes Haushaltseinkommen, entsprechende Zeitinvestition durch die Eltern und der Lernkultureffekt in der Familie wichtig. Frühzeitige gesellschaftliche Segregation und Chancenungleichheit könnten bekämpft werden durch eine homogenisierte Form der Kinderbetreuung für Kinder ab dem ersten Lebensjahr.

Aus der Evaluierung seiner Werke wird deutlich, welche Themen für den anstehenden internationalen Politikvergleich von Bedeutung sind, und welche Inhalte für den Gesamtrahmen bzw. das hier zu entwickelnde familienpolitische Idealmodell des Aktivierenden Staates weniger hilfreich sind. Daher erfolgt an dieser Stelle eine Selektion der Kernthemen, die in der weiteren Arbeit behandelt werden. Schematisch lassen sich diese Themen wie folgt darstellen: 
Tabelle 9: Kernthemen Esping-Andersens

\begin{tabular}{|c|c|c|c|c|}
\hline & Werk 1 & Werk 2 & Werk 3 & Werk 4 \\
\hline Kernthema 1 & $\begin{array}{l}\text { Wohlfahrtsstaats- } \\
\text { ansatz }\end{array}$ & Krise Wohlfahrtsstaat & Akt. Staat & Akt. Staat \\
\hline Kernthema 2 & Rolle der Frau & Rolle der Frau & $\begin{array}{l}\text { Rolle Frau und } \\
\text { Mann }\end{array}$ & $\begin{array}{l}\text { Rolle Frau in der } \\
\text { Familienpolitik }\end{array}$ \\
\hline Kernthema 3 & & Demographie & Demographie & Demographie \\
\hline Kernthema 4 & & Neue Risiken & Neue Risiken & Neue Risiken \\
\hline Kernthema 5 & & & Kindheit & $\begin{array}{l}\text { Chancengleichheit } \\
\text { für alle Kinder }\end{array}$ \\
\hline
\end{tabular}

Im folgenden Paragraphen werden die Ziele und Instrumente dieser Kernthemen mit Hilfe des familienpolitischen Modells Kaufmanns weiter erörtert.

\subsection{Zusammenführung der Resultate vergleichender Wohlfahrtsforschung mit den allgemeinen familienpolitischen Erkenntnissen}

\subsubsection{Einleitung und Beschreibung der Arbeitsschritte}

Esping-Andersens Wohlfahrtsstaatsansatz umfasst verschiedene sozialpolitische Themenbereiche, und er betont die integrative Wirkung sozialer Politiken. Etwas weniger deutlich ist der Unterschied zwischen Ziel- und Instrumentenkategorien seines familienpolitischen Ansatzes und der Frage nach der Ausführungs- bzw. Finanzierungsverantwortung. In der politischen 
Praxis wird allerdings relativ deutlich zwischen Zielsetzungen und Instrumenten unterschieden. Üblicherweise werden politische Ziele wie z.B. ,Verringerung der Anzahl der Kinder, die aus Gründen der Armut nicht am gesellschaftlichen Leben teilhaben können mit 50\%' zu Beginn einer Legislaturperiode oder zu Beginn der Laufzeit eines politischen Programmes gesetzt. Die Ausführung der vorgenommenen Instrumente bzw. die Anpassung des Instrumentenmixes sind Themen täglicher politischer Handlungen. Hierbei geht es immer wieder um die Finanzierungs- bzw. Ausführungsverantwortung.

Für den hier angestrebten politischen Vergleich ist die Unterscheidung zwischen Zielen, Instrumenten und entsprechenden Verantwortungen von großer Bedeutung. Auf eine gründliche Differenzierung ebendieser wird im Idealmodell besonderer Wert gelegt. Als Grundstruktur dieses Modells dienen die klassischen Kategorisierungen von Kaufmann. Diese werden mit den sich entwickelnden Kernpunkten Esping-Andersens zusammengefügt. Das Modell Kaufmanns dient hierbei vor allem als strukturierendes Grundgerüst in dem Zielsetzungen und Instrumente auf einer analytischen Ebene voneinander unterschieden werden können. Aufgebaut wird hierbei auf den Ergebnissen aus dem ersten Unterkapitel dieses Kapitels. Dort wird unterschieden zwischen:

- Bevölkerungspolitischen Zielsetzungen (Bev.pol. Z.),

- Emanzipatorischen Zielsetzungen (Emanz. Z.),

- Sozialpolitischen Zielsetzungen (Soz.pol. Z.),

- Familieninstitutionellen Zielsetzungen (Fam.inst. Z.) und

- Auf das Kindeswohl gerichtete Zielsetzungen (Kw.Z.).

Im empirischen Teil dieser Dissertation wird der nationalstaatliche Vergleich auch einen Fokus auf das verwendete Instrumentarium erfahren. Kaufmann unterscheidet hier zwischen:

- Instrumenten, die den rechtlichen Status der Familienmitglieder betreffen (R. I.),

- Instrumenten, die die ökonomische Situation der Familienhaushalte betreffen (Ökon. M.),

- Instrumenten, die die ökologischen Bedingungen des Familienlebens betreffen (Ökol. M.) und 
- Instrumenten zur Förderung oder Wiederherstellung von Kompetenzen der Familienangehörigen (I.K.).

Werden nun die Zielsetzungen auf eine X-Achse und die Maßnahmen bzw. Instrumente auf eine Y-Achse sortiert, ergibt sich folgendes Grundmodell.

Tabelle 10: Politische Zielsetzungen und Instrumente (Kaufmann, 2002)

\begin{tabular}{|c|c|c|c|c|c|}
\hline & Bev.pol.z. & Emanz. Z. & Soz.pol. Z. & Fam.inst. Z. & Kw.Z. \\
\hline R. I. & & & & & \\
\hline Ökon. & & & & & \\
\hline Ökol. I & & & & & \\
\hline I. K. & & & & & \\
\hline
\end{tabular}

Nicht alle Instrumente müssen in jedem Fall zur Erreichung aller Zielsetzungen geeignet sein. Familieninstitutionelle Zielsetzungen werden z.B. wahrscheinlich relativ selten durch ökonomische Instrumente unterstützt. Theoretisch möglich wäre es allerdings.

Die Zielsetzungen Esping-Andersens müssen nicht unbedingt mit den von Kaufmann unterschiedenen Zielsetzungen und Instrumenten übereinstimmen. In den folgenden zwei Unterkapiteln wird daher zuerst verdeutlicht welche der Zielsetzungen und Instrumente des hier aufgenommenen Basismodells aufgrund der analysierten Schriften Esping-Andersens als entscheidend bewertet werden können. Hierzu sind die Ergebnisse aus der beschreibenden Zusammenfassung im ersten Abschnitt dieses Kapitels (siehe Tabelle 9) verwendet. Die in den folgenden Abschnitten analysierten Zielsetzungen und Instrumente erfahren eine weitere Einfüllung und werden am Ende dieses Unterkapitels zusammengefasst. Im Folgenden liegt der Schwerpunkt auf den Zielsetzungen und Instrumenten des Aktivierenden Staates sowie von Bandemer formuliert. Es wird untersucht inwieweit das Basismodell Esping- 
Andersens den Kriterien Aktivierender Politik entspricht, und an welcher Stelle es einiger Anpassungen bzw. Erweiterungen bedarf.

\subsubsection{Zielsetzungen}

\subsubsection{Bevölkerungspolitische Zielsetzungen}

Vor allem seit der zweiten Arbeit Esping-Andersens, die er Mitte der neunziger Jahre verfasste, kommt der bevölkerungspolitischen Zielsetzung eine große Aufmerksamkeit zu. Sowohl in diesem, als auch in dem letzten, hier untersuchten, Werk identifizierte er wohlfahrtstaatliche Probleme, die durch den gesellschaftlichen Alterungsprozess verursacht werden. Hierzu zählt er z.B. das Ungleichgewicht zwischen wohlfahrtsstaatlichen Investitionen in jüngere bzw. ältere Bürger und die zukünftig kleiner werdenden Generationen von Arbeitnehmern. Er analysiert die Gründe, die zur Kinderdifferenz führen und argumentiert, dass in europäischen Ländern in denen die Kombination von Arbeit und Familien gut organisiert und unterstützt sei bzw. passende Infrastrukturen für Familien vorhanden seien, verhältnismäßig mehr Kinder geboren werden. Vor allem Mütter sehr junger Kinder bzw. Mehrkindfamilien bedürfen einer besonderen Form der Unterstützung.

Aus wohlfahrtsstaatlicher Sicht sei eine gleichmäßige Verteilung der bestehenden Ressourcen und Mittel entscheidend. Altert die Gesellschaft in einem relativ hohen Tempo aufgrund niedriger Geburtenzahlen, die den normalen Alterungsprozess der Gesellschaft noch verstärken, habe das eine verhältnismäßig große Zahl von Rentenempfängern und eine niedrige Zahl von jungen Arbeitnehmern zur Folge. Hierdurch werde das wohlfahrtsstaatliche Gleichgewicht bedroht, und könnten bestehende Regelungen auf Dauer nicht weiterbestehen. Abhängig vom entsprechenden Steuer- bzw Rentensystem sei dieses Risiko unterschiedlich groß. Werden keine eingreifenden Maßnahmen getroffen drohe der jüngeren Generation aus wohlfahrtsstaatlicher Sicht eine verhältnismäßig ungünstige Ausgangssituation. Neben diesem gesamtgesellschaftlichen Problem sei der individuelle Wunsch nach Kindern ein grundsätzliches Recht, dem entgegengekommen werden sollte. 


\subsubsection{Emanzipatorische Zielsetzungen}

Esping-Andersen thematisiert die Rolle der Frauen in allen hier untersuchten Werken. Der Fokus liegt auf der weiblichen Arbeitsmarktteilnahme. Das Zweiverdienermodell sei gesellschaftlich wertvoll, da es hierdurch zu einer besseren Verwendung gesellschaftlicher Kapazitäten komme. Für Frauen selbst führe es vor allem $z u$ wirtschaftlicher Unabhängigkeit und einem geringeren Risiko auf familiäre Armut. Daher nennt der Autor Gleichstellungspolitik auch Gesellschaftspolitik. Er unterstreicht die Vorteile des dänischen Arbeitsmarktmodells. Nachdem diese Sichtweise sowohl aus emanzipatorischer wie auch aus anthropologischer Sicht kritisiert wurde, spielt der Begriff der DeFamilialisierung für Esping-Andersen eine wichtige Rolle, da er die Arbeitsmarktteilnahme beider Elternteile erst ermögliche.

Da der Anpassung des weiblichen Lebenslaufes an den männlichen natürliche Grenzen gestellt seien, halte er auch die Anpassung des männlichen Lebenslaufes an den weiblichen für wichtig. In seinen späteren Werken bekommt die Rolle des Mannes eine größere Bedeutung als in den früheren Schriften.

\subsubsection{Sozialpolitische Zielsetzungen}

Sozialpolitisch konzentriert Esping-Andersen sich vor allem auf verschiedene Armutsrisiken wie Risiken zwischen den Generationen, erbliche Risiken, Klassenrisiken usw.. Diesen Risiken gelte es durch geeignete (hauptsächlich) präventive Maßnahmen zu begegnen. Ein geeigneter Arbeitsmarkt sei hierfür von großer Bedeutung. Daher legt der Autor die Betonung auf lebenslanges Lernen. Sozialpolitik versteht er als Zusammenwirken verschiedener Politikfelder wie z.B. Arbeitsmarktpolitik, Bildungspolitik und Emanzipationspolitik. Nur durch einen integralen Ansatz dieser Politikfelder können Zielsetzungen erreicht werden. Umgekehrt könnten nicht gut aufeinander abgestimmte Ziele auch einen negativen Einfluss aufeinander ausüben. Vor allem erbliche Armut findet Esping-Andersen risikoreich. Hierdurch verringere sich die Chance auf eine Rückkehr aus der Armut. Entscheidend sei der präventive Umgang mit Armutsrisiken. Armut müsse nicht kleiner werden, sondern sich am besten gar nicht erst etablieren. 


\subsubsection{Familieninstitutionelle Zielsetzungen}

Esping-Andersen konzentriert sich in seinen Zielen und Instrumenten auf die Individuen in der Familie und will ihre Lebensweise unterstützen. Wichtig ist in seinem Denken die Rolle, die diese Individuen, ob als Familie oder nicht, in der Gesellschaft einnehmen. In den hier untersuchten Arbeiten ist vor allem im funktionalen Sinne über die Familie geschrieben. Den Schutz des Familienbegriffs als solches hat er in den hier untersuchten Werken nicht aufgenommen.

\subsubsection{Auf das Kindeswohl gerichtete Zielsetzungen}

Ein besonderes familiäres Merkmal ist die Abhängigkeit der Angehörigen voneinander. Dies gilt insbesondere für Kinder. In der Regel sind sie noch nicht im Stande ein selbstständiges Leben zu führen. Ihnen gilt daher in der Familienpolitik eine besondere Aufmerksamkeit. Dieses Bewusstsein für das Wohlbefinden der Kinder hat vor allem in den letzten Jahren an politischer Bedeutung gewonnen.

Das Wohl des Kindes ist einer der Kernpunkte, der sich in Esping-Andersens Werken im Laufe der Zeit entwickelt hat. Vor allem in der dritten Arbeit wird der Kindheit, aus Sicht des Lebensphasenmodells entscheidende Aufmerksamkeit gewidmet. Sie sei die wichtigste Periode menschlichen Lebens, da hier die Basis gelegt werde für die in Zukunft relevanten Fähigkeiten. Während der Kindheit könne man eine soziokulturelle Vorsortierung verhindern bzw. begrenzen. In vielen Ländern seien heute schon bei der Einschulung so unterschiedliche Weichenstellungen erfolgt, dass einige Kinder einen Vorsprung haben, der nur noch schwierig oder nicht eingeholt werden könne.

Wichtige Voraussetzung zum Entgegenwirken von Segregation sei einerseits eine geeignete Infrastruktur in Form einer homogenisierten, hochwertigen Kinderbetreuungslandschaft. Gemeint ist hiermit eine zugängliche und bezahlbare staatliche Betreuung von Kindern durch gut ausgebildetes Personal. Weiterhin brauchen Mütter und Väter Zeit und Ruhe in den Momenten, in denen sie selber die Erziehung der Kinder in die Hände nehmen. Vor allem das erste Lebensjahr von Kindern bedürfe eines besonderen Schutzes. In der Zeit sei 
es wichtig eine stabile Eltern-Kind Beziehung aufzubauen. Öffentliche Kinderbetreuung in größeren Gruppen sei für ganz kleine Kinder ungeeignet. Wichtig sei vor allem eine hochwertige und gleichwertige Früherziehung von Kleinkindern aller Bevölkerungsschichten.

\subsubsection{Zwischenfazit}

Von den fünf durch Kaufmann unterschiedenen Zielsetzungen sind in diesem Zusammenhang vor allem die bevölkerungspolitischen/demographischen Zielsetzungen, Ziele im Bezug auf Emanzipation und Sozialpolitik wie letztendlich auf das Kindeswohl gerichtete Zielsetzungen wichtig. Die Rolle der Frau spielte in allen Werken eine große Rolle und wurde im dritten Werk um die Rolle des Mannes erweitert. Die demographische Zielsetzung hat der Autor seit der Mitte der neunziger Jahre favorisiert. Das Kindeswohl erfährt in seinen letzten Werken eine besondere Rolle. Familieninstitutionelle Zielsetzungen sind durch Esping-Andersen nicht weiter vertieft. Sie werden daher im hier zu entwickelnden Idealmodell nicht aufgenommen. Die sozialpolitischen Zielsetzungen weisen eine große Schnittmenge mit den emanzipatorischen Zielen und den Zielsetzungen für Kinder auf. Sie werden daher im Rahmen der beiden Zielkategorien ,Emanzipation von Frauen' und ,Kindeswohl' bearbeitet und erscheinen im Folgenden nicht als gesonderte Kategorie.

\subsubsection{Instrumente}

Wie zu Beginn des Kapitels verdeutlicht, erfolgt in diesem Paragraphen die Instrumentenanalyse. Per Instrument bzw. Maßnahme wird den beschriebenen Zielsetzungen entsprechend eine Sortierung vorgenommen. Eine schematische Verdeutlichung aller Ziele und Instrumente erfolgt im letzten Unterkapitel. Während die Instrumente im laufenden Text größtenteils auf analytischem Niveau bleiben, sind in der Anlage einige Beispiele aufgenommen.

\subsubsection{Instrumente, die den rechtlichen Status der Familienmitglieder betreffen}

In den hier analysierten Arbeiten Esping-Andersens spielen Instrumente, die den rechtlichen Status der Familienmitglieder betreffen keine oder nur im sehr allgemeinen Sinne eine Rolle. In keinem der in der beschreibenden Analyse 
festgestellten Kernpunkte Esping-Andersens wird der rechtliche Status der Familienmitglieder ausdrücklich thematisiert.

\subsubsection{Instrumente, die die ökonomische Situation der Familienhaushalte betreffen}

Im Hinblick auf die Erreichung demographischer Zielsetzungen nennt EspingAndersen Instrumente, die die ökonomische Situation der Familienhaushalte betreffen. Neben den bereits genannten Möglichkeiten zur weiblichen Teilnahme am Arbeitsmarkt nennt er auch die Möglichkeit zur Arbeit in Teilzeit. $\mathrm{Zu}$ bestimmten Momenten des Lebenslaufs ist das Zweiverdienermodell nicht realistisch. Um den Anschluss an den regulären Arbeitsmarkt nicht zu verlieren, sei es zu solchen Zeitpunkten besser in Teilzeit zu arbeiten bzw. sehr kurzzeitig (am besten mit staatlicher Unterstützung) den Arbeitsmarkt $\mathrm{zu}$ verlassen. Weiterhin nennt er die stark gestiegenen Wohnungspreise als ein Hindernis zur Erfüllung der Wunschkinderzahl. Hierfür müsse Menschen in der Familienphase finanzielle Unterstützung geboten werden.

Ökonomische Instrumente zur Erreichung emanzipatorischer Zielsetzungen seien hauptsächlich auf die Arbeitsmarktteilnahme von Frauen und der Erreichung des Zweiverdienermodells mit einem vergleichbaren Einkommensniveau gerichtet. Durch das Beziehen gleicher Positionen am Arbeitsmarkt erreichen Frauen eher den Grad der wirtschaftlichen Selbstständigkeit. Das Armutsrisiko alleinerziehender Mütter bzw. größerer Familien werde hierdurch kleiner. Zwei traditionellere Instrumente in diesem Zusammenhang seien die Lohnzuschüsse bzw. Einkommenszuschüsse, falls die Entlohnung einer Vollzeitstelle zu gering sei. Aktuellere Instrumente in diesem Bereich, seien die des Workfares bzw. der Sozialinvestition. Hierbei handele es sich um Instrumente, bei denen finanzielle Unterstützung geboten wird, mit dem Ziel des Erreichens einer wirtschaftlichen Unabhängigkeit.

Zur Unterstützung des Kindeswohls nennt Esping-Andersen zwei ökonomische Perspektiven bzw. Instrumente. Grundsätzlich betont er, dass in der Kindheit nicht allein die Basis der individuellen Zukunft sondern auch die Basis der gesellschaftlichen Zukunft begründet sei. Daher findet er, dass die Investitionen in dieser Lebensphase auch höher sein müssten als bisher üblich. Die 
Erblichkeit von Armut betrachtet er als besonders problematisch. Hierdurch komme es schnell zu einer Ansammlung von Problemen für die oft keine Lösungen mehr gefunden werden können.

\subsubsection{Instrumente, die die ökologischen Bedingungen des Familienlebens betreffen}

Esping-Andersen nennt verschiedene ökologische Bedingungen zur emanzipatorischen Zielerreichung. Um eine Teilnahme am Arbeitsmarkt zu ermöglichen, sei eine reguläre, bezahlbare Kinderbetreuung Grundvoraussetzung für viele Frauen. Nicht immer sei das reguläre Angebot zur Kinderbetreuung ausreichend. Krankheitsfälle erfordern additionale Maßnahmen bzw. Regelungen. Auch für die Pflege älterer Menschen seien passende ökologischen Bedingungen wichtig. Weiterhin sei die Verbesserung der Arbeitssituation/Arbeitsbedingungen arbeitender Mütter eine wichtige Maßnahme. Falls Mütter zu viel Stress erfahren könne das negative Folgen für die Erziehung der Kinder haben.

Ökologische Bedingungen seien auch für die Wohlfahrt der Kinder von großer Bedeutung. Ist z.B. die Bezahlbarkeit einer regulären Kinderbetreuung ein emanzipatorisches Argument, spiele aus Sicht des Kindeswohls vor allem der qualitative Standard der Betreuung eine wichtige Rolle. Frühkindliche Entwicklung könne man stärken und fördern durch gut ausgebildetes Personal. Die Verstaatlichung dieser Ausbildungen könne dem Risiko einer frühzeitigen soziokulturellen Vorsortierung entgegen wirken.

\subsubsection{Instrumente zur Förderung oder Wiederherstellung von Kompetenzen der Familienangehörigen}

Zur Erreichung der genannten emanzipatorischen Zielsetzungen seien diverse Maßnahmen relevant, die der Förderung bzw. Wiederherstellung von Kompetenzen der Familienangehörigen dienen. Zwei Maßnahmen, die bereits im Rahmen der ökonomischen Instrumente genannt wurden, aber auch aus sozialpolitischer Sicht von großer Bedeutung seien: ,Workfare' und ,Sozialinvestition'. Den Anschluss an den Arbeitsmarkt zu verlieren sei eines der neuen Risiken. Falls dieser Anschluss nicht mehr vorhanden ist, müsse eine Sozialinvestition erfolgen, um die erneute Eingliederung in den Arbeitsmarkt zu 
ermöglichen. Neben dieser beiden eher kurativen Maßnahmen betont EspingAndersen, dass vor allem auch präventive Maßnahmen wie ,lebenslanges Lernen' eine gute Absicherung vor den verschiedenen Lebensrisiken bilden.

Auch aus Sicht der Kindeswohlfahrt nennt er einige Maßnahmen zur Förderung kindlicher Fähigkeiten. Hierbei handelt es sich in erster Linie um die Stärkung primärer Mechanismen. Durch regelmäßiges Vorlesen z.B. können diese Mechanismen gefördert werden. Wichtig sei weiterhin die frühkindliche Ausbildung außerhalb des Elternhauses. Durch eine für alle Bevölkerungsschichten zugängliche qualitativ hochwertige Ausbildung könne der direkte kognitive Einfluss von Eltern auf Kinder relativiert und frühzeitiger Benachteiligung begegnet werden.

\subsubsection{Zwischenfazit}

Alle vier der hier genannten Instrumente dienen der Erreichung der im vorherigen Paragraphen benannten Zielsetzungen. Einige Maßnahmen sind zur Erreichung aller Ziele relevant und werden besonders betont, wie z.B. die ökonomischen und ökologischen Instrumente. Aber auch die übrigen Kategorien hält Esping-Andersen für entscheidend, um einen passenden PolicyMix zu realisieren. Während bei den Zielsetzungen auffiel, dass eine der Kategorien Kaufmanns nicht besetzt wurde, ist bei den Instrumenten eine Hinzufügung nötig. Zeit-Instrumente hält Esping-Andersen für relevant sowohl im Hinblick auf die Erreichung demographischer Zielsetzungen, als auch aus emanzipatorischer Perspektive (bezahlte Elternzeit für Väter und Mütter, Teilzeit, Lebenszeitmodelle, Arbeitszeiten) und aus Sicht der Kindeswohlfahrt (bezahlter Elternurlaub im ersten Lebensjahr, und spätere Zeitinvestitionen).

\subsubsection{Zusammenführung des Modells}

In den beiden vorherigen Paragraphen haben sich die Zielsetzungen und Instrumente der festgestellten Kernpunkten Esping-Andersens herauskristallisiert. Schematisch ergibt sich folgendes Modell: 
Tabelle 11: Politische Zielsetzungen und Instrumente (Kaufmann) eingefüllt mit Kernpunkten von Esping-Andersen

\begin{tabular}{|c|c|c|c|c|c|}
\hline & Bev.pol. Z & Emanz. Z. & Soz.pol. Z. & Fam.inst. Z. & Kw.Z. \\
\hline R. I. & keine & Keine & keine & keine & Keine \\
\hline Ökon. I. & $\mathrm{x}$ & $\mathrm{x}$ & $\mathrm{x}$ & keine & $\mathrm{x}$ \\
\hline Ökol. I. & $\mathrm{x}$ & $\mathrm{x}$ & $\mathrm{x}$ & keine & $\mathrm{x}$ \\
\hline I. K. & keine & $\mathrm{x}$ & $\mathrm{x}$ & keine & $\mathrm{x}$ \\
\hline
\end{tabular}

Familial-insititutionelle Zielsetzungen spielten in den hier verwendeten Kernpunkten Esping-Andersens keine Rolle und die sozialpolitischen Zielsetzungen werden - wie bereits erläutert - gemeinsam mit den emanzipatorischen Zielen bzw. den Zielsetzungen für die Kinder bearbeitet. Sie werden daher auch nicht $\mathrm{im} \mathrm{zu}$ erstellenden Idealmodell Aktivierender Familienpolitik aufgenommen. Ähnliches gilt für die rechtlichen Instrumente. Auch sie werden in den Kernpunkten ungenügend verwendet und daher nicht ins Idealmodell übertragen. Weiterhin wurde festgestellt, dass verschiedene Zeitpolitiken ein wichtiges Instrument in den genannten Kernpunkten formen. Aus diesem Grund wird dieses Instrument dem Modell hinzugefügt. Es ergibt sich folgendes Modell: 
Tabelle 12: Familienpolitisches Basismodell unter Verwendung der Theorien von EspingAndersen und Kaufmann

\begin{tabular}{|c|c|c|c|}
\hline & Bev.pol. Z. & Emanz. Z. & Kw.z. \\
\hline Ökon. I. & $\mathrm{x}$ & $\mathrm{x}$ & $\mathrm{x}$ \\
\hline Ökol. I. & $\mathrm{x}$ & $\mathrm{x}$ & $\mathrm{x}$ \\
\hline I. K. & keine & $\mathrm{x}$ & $\mathrm{x}$ \\
\hline Z.I. & $\mathrm{x}$ & $\mathrm{x}$ & \\
\hline
\end{tabular}

Dieses Basismodell bietet eine Übersicht der von Esping-Andersen favorisierten Ziele und Instrumente. Außer dem ersten Kernpunkt seiner Arbeit (allgemeiner Wohlfahrtsansatz) konnten alle Themen zugeordnet werden. Das der allgemeine Wohlfahrtsansatz keinen eigenständigen Platz in der Tabelle einnimmt ist allerdings nicht verwunderlich, da hier vor allem ein allgemeingültiger Ansatz vorgestellt wird. Unterschieden wurde zwischen den drei verschiedenen Wohlfahrtsstaatsregimes. Diese von ihm auch als Wohlfahrtsregime bezeichneten Gruppen sind abhängig von den Investitionen bzw. den Instrumenten, so wie sie in der Tabelle dargestellt werden. Da die Erstellung des Idealmodells Aktivierender Familienpolitik Ziel dieses Kapitels ist, wird das bereits erstellte Basismodell im folgenden Unterkapitel um die Zielsetzungen und Instrumente des Aktivierenden Staates, und die im ersten Kapitel festgestellten Instrumente Aktivierender Politik, erweitert. 


\subsection{Zusammenführung mit Resultaten Aktivierender Staat}

\subsubsection{Einleitung und Beschreibung der Arbeitsschritte}

Die Konturen des familienpolitischen Modells Esping-Andersen sind in einem für einen politischen Vergleich anwendbaren Modell verdeutlicht (Tabelle 12). Obwohl der Autor mit seinen wohlfahrtsstaatlichen Theorien das sozialdemokratische Modell favorisiert und nicht nach einer konsequenten Verwendung des aktivierenden politischen Prinzips strebt, knüpft er aus verwaltungswissenschaftlicher Sicht an das Leitbild Aktivierender Staat an bzw hebt er mögliche Chancen dieses Leitbildes hervor. Die Konturen des familienpolitischen Modells Esping-Andersens werden aus diesem Grund erweitert um die entscheidenden Prinzipien Aktivierender Politik. Bei der Erweiterung des festgestellten Modells Esping-Andersens Familienpolitik wird wiederum zwischen Zielsetzungen und Instrumenten unterschieden. Die schematische Zusammenführung erfolgt im letzten Abschnitt dieses Unterkapitels und endet mit einer Beschreibung des Idealmodells.

\subsubsection{Zielsetzungen}

\subsubsection{Zielsetzung Aktivierender Staat}

Staatliches Handeln wird über Perioden hinweg durch politische Leitbilder beeinflusst. Diese Leitbilder sind eine Art gemeinsamer Nenner oder Rahmen diverser staatlicher Politiken. Hintergrund für die Verwendung eines Leitbildes sind gesellschaftliche oder finanzielle Trends und Entwicklungen. Eingreifende Maßnahmen in diversen Bereichen sind erforderlich, um neue Herausforderungen $\mathrm{zu}$ meistern und den wohlfahrtsstaatlichen Charakter $\mathrm{zu}$ bewahren. Werden nach einem Jahrzehnt oder länger neue Probleme und Entwicklungen deutlich, ist eine Weiterentwicklung des bestehenden Leitbildes wahrscheinlich. Wie im ersten Kapitel bereits beschrieben, spricht Jann daher von den ,langen Wellen der Leitbildentwicklung'. Er unterscheidet zwischen dem demokratischen Rechtsstaat, dem Aktiven Staat, dem Schlanken Staat und dem Aktivierenden Staat. Das letztgenannte Leitbild wird durch Politik und 
Verwaltung in Europa seit Mitte der neunziger Jahre verwendet. Während das vorherige Leitbild des Schlanken Staates vor allem wirtschaftliche Zielsetzungen kannte (erhebliche Kürzungen von Haushalten und Orientierung an betrieblichem Management zur Steigerung der Effizienz öffentlichen Handelns) kennt der Aktivierende Staat weitergehende Grundsätze. Statt eines auf Einsparungen begrenzten Fokus geht es um eine neue und prinzipielle Aufgabendiskussion von Staat und Gesellschaft. Die Frage nach Verantwortlichkeiten spielt eine wichtige Rolle bei der immerwährenden Staatsmodernisierung. Nicht mehr der Staat führt alle Ausgaben aus oder ist verantwortlich für jede von ihm verwendete Form von Outsourcing. Koproduktion und Verantwortungsteilung sind im Aktivierenden Staat Richtlinien staatlichen Handelns. Bandemer unterscheidet vier Indikatoren aktivierender Politiken. Diese lauten: gesellschaftlicher Dialog, neue Verantwortungsteilung, Koproduktion und Leistungsaktivierung. Diese Indikatoren verdeutlichen zwei verschiedene Formen von Zielsetzungen. Erstens handelt es sich hierbei um die eher inhaltlichen Zielsetzungen wie die Bewahrung der wohlfahrtsstaatlichen Prinzipien Gerechtigkeit und Chancengleichheit, und zweitens um Zielsetzungen die Antwort geben auf die Frage wie wohlfahrtsstaatliche Prinzipien geschützt bzw. verbessert werden können. Der Begriff, neue Verantwortungsteilung' z.B. knüpft an die Frage nach der Idealtypologie sozialer Risikoverteilung, während ein Begriff wie ,Leistungsaktivierung' eher deutet auf die betriebswirtschaftlich beeinflusste Umsetzungsstrategie festgesetzter Zielsetzungen und daher $\mathrm{zu}$ den Instrumenten gezählt werden kann.

Unter Zielsetzungen wird in diesem Paragraphen die inhaltliche Sichtweise verstanden. Gemeint ist hiermit die Frage nach der Gewährung wohlfahrtsstaatlicher Prinzipien und Verantwortlichkeiten. Wie im vorherigen Kapitel verdeutlicht, sind es vor allem Rawls und Dworkin die diesen Gedanken aufgegriffen haben in der Gerechtigkeitstheorie bzw. der Erweiterung dieser Theorie mit dem Prinzip der Pflichten bzw. Verantwortlichkeiten. Schmid verdeutlicht dieses Dilemma sehr gut in seiner Unterscheidung nach akteurrelativierten Risikomanagementkapazitäten. Risiken werden durch ihn anhand der Kriterien ,selbstverschuldet' bzw. ,durch externe Umstände verursacht' unterteilt. Hinzugefügt wird dieser Unterteilung die Frage, ob der Ausgleich der Risiken durch das Individuum bzw. das Kollektiv geschehen 
kann/muss. Zusammengefasst ist das wichtigste Ziel des Aktivierenden Staates die Gewährleistung von Gerechtigkeit und Chancengleichheit durch eine neue Verantwortungsteilung, bei der von individuellen Möglichkeiten und eigener Verantwortlichkeit ausgegangen wird. Im folgenden Paragraphen sind die vier familienpolitischen Zielsetzungen des Modells Esping-Andersens um diesen Begriff der neuen Verantwortungsteilung erweitert.

\subsubsection{Neue Verantwortungsteilung und familienpolitische Zielsetzungen}

Ziel des Aktivierenden Staates ist die Realisierung der ,Neuen Verantwortungsteilung'. Gemeint ist hiermit dass der traditionelle Unterschied zwischen Leistungsempfänger (Bürger) und Leistungserbringer (Staat) überwunden werden muss. Der Staat bleibt verantwortlich für die Leistungserbringung, er kann aber auch entsprechende Gegenleistungen erwarten. Der Staat übernimmt in jedem Fall die Gewährleistungsverantwortung. Pro Aufgabenbereich muss entschieden werden, ob auch die Finanzierungsverantwortung bzw. die Vollzugsverantwortung durch den Staat getragen werden sollte. Neben dem Staat können aus der Sicht dieses Leitbildes auch der Markt, die Familie und der Dritte Sektor Leistungserbringer sein. Diese Interpretation der Aufgabenverteilung wird im Folgenden zusammengeführt mit den Zielen des familienpolitischen Grundmodells.

Demographie

Als wichtigste demographische bzw. bevölkerungspolitische Zielsetzung sieht Esping-Andersen einen demographisch stabilen Gesellschaftaufbau um Generationskonflikte im Bezug auf den Arbeitsmarkt, die Renten, die Pflege älterer Menschen usw. zu vermeiden. Menschen haben ein Recht ihren Kinderwunsch zu realisieren und werden durch die bestehenden Verhältnisse daran gehindert ihre Wunschkinderzahl zu realisieren.

Im Bezug auf die demographischen bzw. bevölkerungspolitischen Zielsetzungen sieht Esping-Andersen keine geteilte Verantwortlichkeit. Er unterstreicht den ursprünglichen Kinderwunsch junger Menschen. Die Tatsache, dass dieser Wunsch nicht realisiert wird, begründet er vor allem mit dem Argument der unzureichenden Organisation von Familie und Beruf. Der 
Staat sei verantwortlich für eine gute Infrastruktur zur Kinderbetreuung und familienfreundlichen Arbeitsbedingungen. Entsprechend der Theorie des Aktivierenden Staates können die Voraussetzungen für Familiengründung bzw. für die Erweiterung der Familie aber auch Verantwortung des Staates, des Marktes, des Dritten Sektors und der Bürger selbst sein. Unternehmen können z.B. an der Kinderbetreuung mitbezahlen bzw. diese organisieren, und es ist eine individuelle Entscheidung die Geburt von Kindern nicht $\mathrm{zu}$ lange aufzuschieben. Hierauf geht der Autor nur begrenzt ein.

Emanzipation

Als wichtigste emanzipatorische Zielsetzungen versteht Esping-Andersen die Realisierung der wirtschaftlichen Selbstständigkeit von Frauen, bzw. ihrer durchgängigen Arbeitsmarktteilnahme. Er sieht das Zweiverdienermodell als logische Konsequenz der emanzipatorischen Kräfte sowie der Investitionen in Bildung. Die De-Familisierung, also die Erfüllung häuslicher Aufgaben wie Betreuung der Kinder und/oder Pflege der Eltern sieht er als eine Grundvoraussetzung für die unbeschränkte Teilnahme von Frauen am Arbeitsmarkt.

Bei den emanzipatorischen Zielsetzungen fällt auf, dass der Autor (in erster Linie) den Familien (mehr Beteiligung von Frauen am Arbeitsmarkt und Einführung eines Vaterschaftsurlaubs) und dem Staat (mehr Kinderbetreuung) Verantwortung zuordnet. In diesem Bereich beschreibt er eine geteilte Verantwortung. Diese wird allerdings nicht ganz konsequent durchgeführt, da dem Markt und dem Dritten Sektor verhältnismäßig wenig Verantwortung zukommen.

Kindeswohl

Im Bereich des Kindeswohls sieht er die Basis wohlfahrtstaatlichen Funktionierens. Durch eine hochwertige und gleichwertige Früherziehung von Kleinkindern aller Bevölkerungsschichten müssen Lebenschancen optimal genutzt werden. Einer gesellschaftlichen Vorsortierung könne somit präventiv begegnet werden.

Im Kindesbereich betont der Autor die Verantwortungsteilung zwischen Staat und Familie. Im ersten Jahr liegt die Verantwortlichkeit für die Pflege der Kinder aufgrund sozialpsychologischer Erkenntnisse zwar noch in den Händen 
der Eltern, aber schon nach dem ersten Lebensjahr sei es der Staat, der genau wie im schulischen Bereich verantwortlich sei ist für die homogenisierte Erziehung bzw. Betreuung von Kindern. Auf diese Weise können soziale Unterschiede verringert bzw. einer sozialen Segregation begegnet werden.

\subsubsection{Zwischenfazit}

Ursprünglich unterschied der Autor zwischen drei wohlfahrtsstaatlichen Modellen, dem konservativen-, dem liberalen- und dem sozialdemokratischen Modell. Als Idealregime identifizierte er den sozialdemokratischen Typus. Dementsprechend trägt das auf seinen Kernpunkten aufbauende Modell der Familienpolitik einen sozialdemokratischen Charakter. Seit der Entwicklung dieser Idealmodelle im Laufe der achtziger Jahre haben sich allerdings die sozialdemokratischen Prinzipien, genau wie die liberalen und die konservativen, stark gewandelt. Im Leitbild des Aktivierenden Staates, das von vielen politischen Parteien favorisiert wurde, ist diese veränderte politischadministrative Handlungsweise verdeutlicht. Die neue Aufgabenteilung wurde zu einem grundlegenden Prinzip, das auch in der Sozialdemokratie zu einem Mentalitätsumschwung führte. Die Typologie Esping-Andersen ist daher in einigen Punkten um das hier hantierte Grundprinzip des Aktivierenden Staates erweitert worden.

Vor allem im demographischen Bereich sieht Esping-Andersen die Verantwortung beim Staat, während auch in der Gesellschaft selbst Raum für Kinder entstehen müsste bzw. Menschen auch selbst für die Realisierung ihres Kinderwunsches verantwortlich sind. Im Bereich der emanzipatorischen Zielsetzungen sieht der Autor die Verantwortlichkeiten vor allem bei den Frauen und dem Staat. Hier bedarf es einer Ausweitung um die Männer, den Markt und den Dritten Sektor. Für die Erreichung der Zielsetzungen im Bereich der Kindeswohlfahrt identifiziert der Autor zwei Verantwortliche. Für das erste Lebensjahr des Kindes sieht er die Verantwortlichkeit vor allem bei den Müttern und in zunehmendem Maße auch bei den Vätern. Nach dieser Zeit ist es der Staat, dem ein Großteil der Betreuungs- und Erziehungsaufgaben zukommt. Die Verantwortlichkeit des Marktes und des Dritten Sektors werden von EspingAndersen nicht (oder nur in sehr geringem Maße) betont. 


\subsubsection{Instrumente}

\subsubsection{Instrumente des Aktivierenden Staates}

Wie im Kapitel über den Aktivierenden Staat beschrieben, ist das Prinzip der Leistungskette entscheidend für die Verwendung aktivierender Instrumente. In dieser Wertschöpfungskette wird analysiert, wem Aufgabenverantwortung zugeteilt werden kann. Zwischen den Stufen dieser Kette kommt es zu einem permanenten Wechselspiel. Prinzipiell geht es bei diesem Wechselspiel um die Frage, ob die Ausführungsverantwortung staatlich ist, oder ob diese Verantwortung durch einen anderen Akteur getragen werden sollte. Entscheidungen hierüber werden in erster Linie auf der Basis eines breiten Dialogs getroffen. Hierdurch ergibt sich der effizienteste Policy-Mix.

Die drei hier zu betrachtenden aktivierenden Instrumente sind: Privatisierung, Öffentliche-Private Partnerschaft (ÖPP)/Public Private Partnership (PPP) und Sozialinvestition. In der letztgenannten Kategorie gibt es Instrumente mit dem Ziel einer Integration bzw. Reintegration einerseits (Fördern und Fordern) und der Unterstützung von freiwilligem Engagement andererseits (Freiwilligkeit und Engagement). Eine Erläuterung dieser Instrumente ist in den folgenden Paragraphen aufgenommen. Danach kommt es zu einer Zusammenführung mit den Instrumenten des familienpolitischen Modells. Die konkreten Maßnahmen sind als Anlage aufgenommen.

\section{Privatisierung}

Dieses Instrument, das in den letzten Jahren vielfältig kritisiert wurde, gehört zwar traditionell eher zum Instrumentarium des Schlanken Staates, kann aber auch für den Aktivierenden Staat von Bedeutung sein. Man unterscheidet zwischen direkten Pfaden zur Steigerung privater Finanzierung (z.B. durch Steuermittel) und den indirekten Pfaden zur Steigerung privater Finanzierung (z.B. Gutscheinsysteme).

Bei den von Esping-Andersen favorisierten Instrumenten zur Unterstützung der Familien ist nur wenig über Möglichkeiten zur Privatisierung geschrieben. In seinem jüngsten Werk (vergl. Esping-Andersen 2009: 104f) äußert er sich 
kritisch gegenüber der Liberalisierung des Kinderbetreuungsmarktes in den Vereinigten Staaten. Die Verantwortlichkeit für familiäre Aufgaben sieht der Autor in sehr starkem Maße beim Staat und in zweiter Instanz bei den Familien (vor allem bei den Frauen). Wie bereits angegeben sind in den vergangenen Jahren Privatisierungen (ehemals öffentlicher Leistungen) oft kritisiert worden. Andererseits sollte man nicht vergessen, dass es auch viele erfolgreiche Privatisierungsbeispiele gibt bzw. sich einige Dienste - unabhängig davon ob sie privatisiert oder nicht privatisiert sind - vermutlich generell im Zentrum öffentlicher Kritik bewegen werden.

\section{Öffentlich-Private Partnerschaft (ÖPP)}

Budäus unterscheidet zwischen drei Formen von ÖPP's. Für die hier vorliegende Forschungsarbeit ist allerdings nur die dritte Form einer ÖPP entscheidend. Es handelt sich hierbei um eine Form der Kooperation, die dem Gedanken der Corporate Social Responsibility entspricht. Durch die Verteilung bestimmter gesellschaftlicher Aufgaben entstehen neue Märkt von denen private Unternehmen profitieren können. In der Regel kennen ÖPP's erhebliche Finanzierungsrahmen. Unterschieden werden in der Regel Tauschmodelle (Leistung und Gegenleistung) und Poolmodelle (gemeinsame Verwendung von Ressourcen). Durch die Kontinuität dieser Partnerschaft können auf die Dauer erhebliche Gewinne für alle Beteiligten erzielt werden.

Bei den von Esping-Andersen favorisierten Maßnahmen zur Unterstützung der Familien ist nicht oder nur wenig über ÖPP's geschrieben. Die Verantwortlichkeit familiärer Aufgaben sieht der Autor, wie bereits erläutert, in sehr starkem Maße beim Staat und bei den Familien.

\section{Sozialinvestition}

In diesem Fall betrifft es eine Gruppe von Instrumenten, die auf die Zivilgesellschaft gerichtet ist. Einerseits geht es darum Individuen zu stärken und (wieder) in den Arbeitsprozess einzubinden. Dann spricht man von Integration bzw. Reintegration. Andererseits handelt es sich um Maßnahmen zur Förderung persönlichen Engagements und freiwilliger Arbeit. Hintergrund dieser Familie von Instrumenten ist die temporäre Unterstützung von Individuen oder Gruppen von Menschen mit dem Ziel der Aktivierung individueller bzw. gemeinsamer Kräfte. 
Fördern und Fordern

Nicht mehr der Anspruch auf soziale Leistungen sondern der Gedanke der sozialen Investition ist sehr charakteristisch für den Aktivierenden Staat. Personen, die nicht am Arbeitsmarkt teilnehmen können, werden durch staatliche Investitionen (z.B. Bildungsinvestitionen) wieder in Richtung soziale Selbstständigkeit bewegt. Sie werden aktiviert. Falls die Sozialinvestition nicht gelingt, gibt es eine Kompensation auf verhältnismäßig niedrigem Niveau.

Dieses Instrument des Förderns und Forderns nimmt in den familienpolitischen Theorien Esping-Andersens eine sehr zentrale Rolle ein. Klassische Instrumente des Aktivierenden Staates wie z.B. Workfare bezeichnet er als besonders wichtig. Die beste Prävention gegen Armut ist die Teilnahme von Männern und Frauen auf dem bezahlten Arbeitsmarkt. Falls dieser durch Umstände verlassen wurde, und Menschen ins soziale Abseits rutschen, kann die Maßnahme des ,lebenslangen Lernens' Abhilfe schaffen. Wie erfolgreich allerdings Reintegrationsmaßnahmen sind, ist abhängig von der erfahrenen Ausbildung und Erziehung. Schon im frühsten Kindesalter wird die Basis für einen erfolgreichen Lebenslauf gelegt. Neben dieser Ausbildung trägt der Staat aus Sicht Esping-Andersens auch Verantwortung für die kontinuierliche Teilnahme während des weiteren Lebenslaufes. In unterschiedlichen Momenten des Lebens kann man den Anschluss an den Arbeitsmarkt verlieren. Aus diesem Grund favorisiert Esping-Andersen ein Lebenslaufmodell, in dem Menschen in risikoreichen Übergangsmomenten staatliche Unterstützung erfahren.

Freiwilligkeit und Engagement

Durch die Förderung bürgerschaftlichen Engagements wird freiwilliger Einsatz belohnt. Oft handelt es sich hierbei um Investition in neue bzw. innovative Projekte. Auch die Verbände, die in großem Rahmen Gebrauch von freiwilligem Engagement machen, sowie weitere Organisationen des Dritten Sektors, spielen in diesem Zusammenhang eine wichtige Rolle.

Bei den von Esping-Andersen favorisierten Instrumenten zur Unterstützung der Familien ist nicht oder nur wenig über Maßnahmen zur Unterstützung von freiwilligem Engagement geschrieben. Aufgaben außerhalb des Arbeitsmarktes sind soviel wie möglich durch den Staat zu erfüllen. Eine Ausnahme hierin sind vielleicht die ersten Monate nach der Geburt des Kindes. Hier, so fügt er in 
seinen späteren Werken hinzu, kann eine Fremdbetreuung vielleicht sogar schädlich sein. Der Autor unterstreicht die bedeutende Rolle der DeFamilialisierung. Familiäre Aufgaben, die Frauen bzw. Eltern daran hindern am Arbeitsmarkt teilzunehmen, müssten ausgelagert werden. Weiteren Formen von Freiwilligem Engagement kommt in seinen Texten keine oder eine nur unbedeutende Rolle zu.

\subsubsection{Zwischenfazit}

In diesem Paragraphen werden die Instrumente aus dem familienpolitischen Modell Esping-Andersens erweitert um die Instrumente des Aktivierenden Staates (Privatisierung, ÖPP und Sozialinvestition).

Privatisierung wurde durch Esping-Andersen nicht oder nur in sehr beschränktem Maße favorisiert. Auch die Möglichkeiten von ÖPP's betont er nicht oder nur in sehr beschränktem Maße. Neben der Familie und dem Staat sieht er den Markt als dritten Akteur. Arbeitgeber sollten Mütter bzw. Eltern unterstützen durch Maßnahmen wie die Möglichkeit zur Arbeit in Teilzeit etc..

Gegenüber den Möglichkeiten aus dem Bereich der Sozialinvestition verhält Esping-Andersen sich ambivalent. Die Maßnahmen zur Förderung der Integration bzw. Reintegration in den Arbeitsmarkt sieht er als entscheidendes Mittel zur Prävention sozialer Ausgrenzung und Armut. Seine Theorien richten sich maßgeblich auf die konsequente Teilnahme möglichst großer Gruppen am Arbeitsmarkt. Hierfür muss während der Kindheit die geeignete Basis gelegt werden. Im späteren Lebenslauf kann es zu Situationen kommen, in denen die Teilnahme am Arbeitsmarkt erschwert wird. Daher sollten präventive oder kurative Maßnahmen getroffen werden, um den Bürger auf schwierige Momente vorzubereiten.

Das zweite Instrument aus dem Bereich der Sozialinvestition wird von ihm nicht hervorgehoben und der Dritte Sektor nicht als Akteur eingebunden. Freiwillige Aufgabenerfüllung z.B. im familiären Bereich können einen negativen Einfluss haben auf die Teilnahme am Arbeitsmarkt. DeFamilialisierung sei darum auch entscheidend. $\mathrm{Zu}$ anderen Formen sozialen Engagements hat der Autor sich nicht oder nur weniger deutlich geäußert. 
Nicht viele der Instrumente Esping-Andersens entsprechen den Instrumenten des Aktivierenden Staates. Ein Beispiel ist die frühkindliche Betreuung. Ab ungefähr dem ersten Lebensjahr des Kindes sieht er hierin eine staatliche Aufgabe. Verantwortungseilung in diesem Bereich hält er letztendlich für nicht effizient und inhaltlich weniger wertvoll. Gleichzeitig gibt er zu bekennen, dass die Kosten der Dienstleistungen hoch sind. Vor allem im Instrument zur Integration bzw. Reintegration auf den Arbeitsmarkt (Fördern und Fordern) wird sein Bezug zum Aktivierungsprinzip deutlich.

Das Ziel des Aktivierenden Staates ist Verantwortungsteilung nicht nur aus Gründen der Effizienz sondern auch vor dem kommunitaristischen Hintergrund des Modells bzw. der Betonung der Eigenverantwortung aus ethischer Sicht. Im Bereich der Kleinkindbetreuung könnte der Markt mehr Verantwortung übernehmen. Schließlich profitieren die Unternehmen einerseits durch die Zunahme der weiblichen Teilnahme am Arbeitsmarkt und andererseits von einer jungen und vitalen Bevölkerung jetzt und in Zukunft. Außerdem entsteht hierdurch ein Bewusstsein des Arbeitgebers für die Belange seines Arbeitnehmers.

Aus dieser Analyse kann der Schluss gezogen werden, dass von den drei Pfeilen der hier folgenden Tabelle Esping-Andersen vor allem der dritte Pfeil betont wird. Der Logik des Aktivierenden Staates entsprechend, wäre allerdings eine Verantwortungsteilung zwischen allen Akteuren erforderlich. 
Tabelle 13: Aktivierender Staat - Aktive Gesellschaft (Olk 2000: 115), Bearbeitung Willenborg (2008)

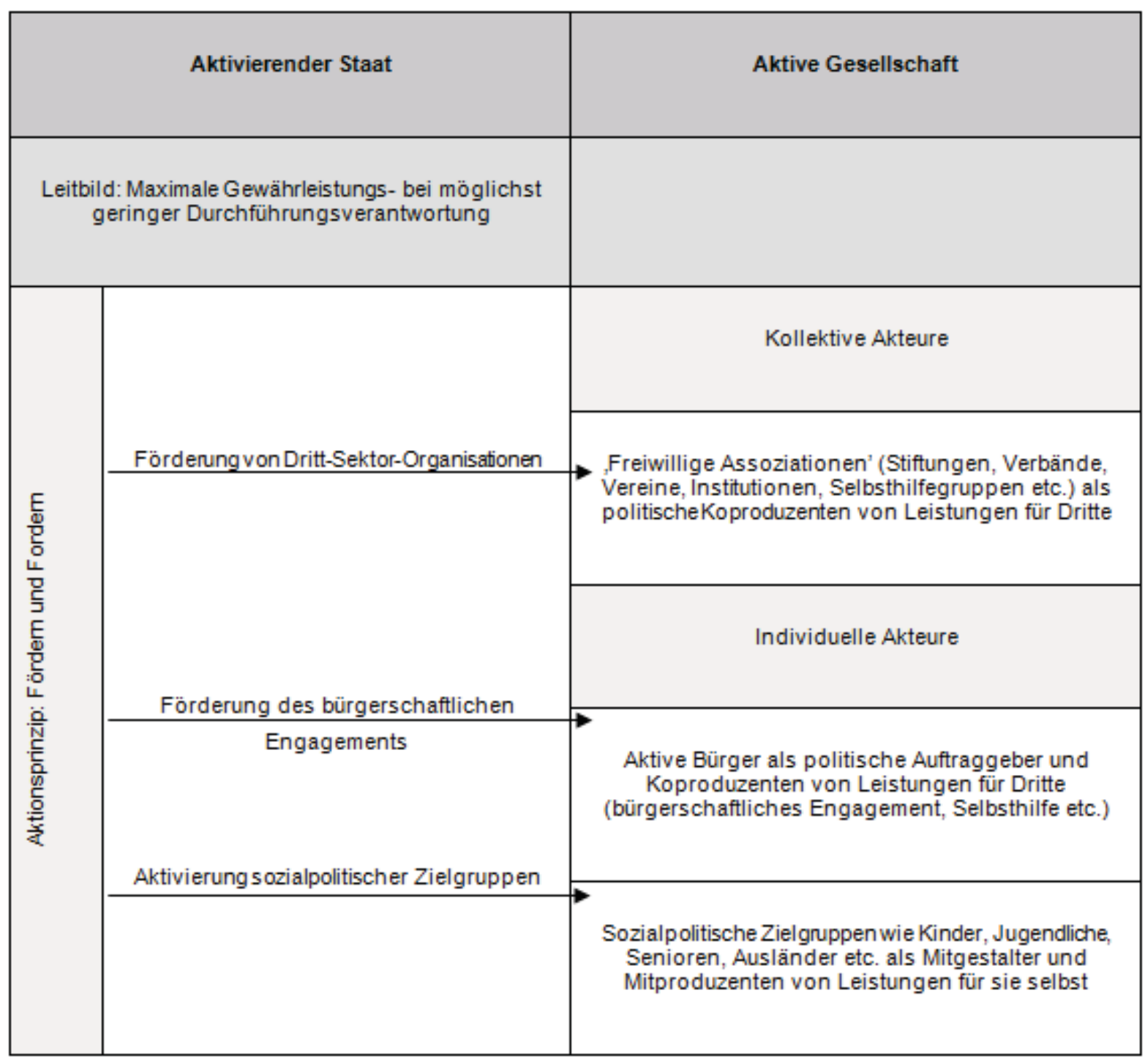

Im letzten Unterkapitel werden die wichtigsten Erkenntnisse aus den vorherigen Abschnitten zusammengefasst. Zudem erfolgt die Schilderung des Modells Aktivierender Familienpolitik. Dieses Idealmodell wird im weiteren Dissertationstext, sowohl für die Empirie als auch für die Analyse, verwendet. 


\subsection{Das Idealmodell Aktivierender Familienpolitik}

Basis des Idealmodells bildet die analytische Unterscheidung familienpolitischer Ziele und Instrumente von Kaufmann (Tabelle 10). Nicht alle Instrumente bzw. Maßnahmen dienen der Unterstützung der von Kaufmann unterschiedenen Ziele. Eine vollständige Füllung dieser Tabelle wird in der politischen Praxis eher selten anzutreffen sein. Die Tabelle verdeutlicht allerdings die politischen Schwerpunkte.

Die Kernpunkte, der in dieser Arbeit verwendeten Werke Esping-Andersens, sind in Tabelle 11 eingefügt und haben nicht nur Schwerpunkte verdeutlicht, sondern führten auch zu einigen Anpassungen des ursprünglichen Modells (Tabelle 12). Im folgenden Analyseschritt wird dieses Grundmodell mit den Eigenschaften des Aktivierenden Staates, so wie im ersten Kapitel des Dissertationsvorhabens beschrieben und in Tabelle 14 schematisch dargestellt, zusammengefügt. Das bedeutet bei den Zielsetzungen eine Erweiterung um den Begriff ,Neue Verantwortungsteilung', welcher für alle Zielsetzungen relevant ist. Die Instrumentenkategorien sind um die Begriffe Privatisierung, ÖPP und Sozialinvestition erweitert.

Tabelle 14: Idealmodell Aktivierender Familienpolitik

\begin{tabular}{|c|c|c|c|c|}
\hline & & Bev.pol.z. & Emanz. Z. & Kw.Z. \\
\hline & & \multicolumn{3}{|c|}{ Neue Verantwortungsteilung (zwischen Zivilgesellschaft, Markt und Staat) } \\
\hline Ökon. I. & \multirow{4}{*}{$\begin{array}{c}\text { Privatisierung, } \\
\text { ÖPP, } \\
\text { Sozialinvestition }\end{array}$} & & & \\
\hline Ökol. I. & & & & \\
\hline I. K. & & & & \\
\hline Z.I. & & & & \\
\hline
\end{tabular}


$\mathrm{Zu}$ Beginn dieses Kapitels wird unterschieden zwischen institutionellem und residualem Charakter verschiedener Systeme (Tabelle 8). Die Möglichkeiten des residualen Charakters spielen bei Esping-Andersen nur eine begrenzte Rolle. Er hat das Modell bzw. die Ziele und Instrumente des Aktivierenden Staates nicht konsequent verwendet. Chancen, die das Modell des Aktivierenden Staates dem familienpolitischen Bereich bietet werden hierdurch nicht vollständig genutzt. Durch eine effizientere Teilung von Verantwortlichkeiten, bietet das Leitbild des Aktivierenden Staates vor allem für die Finanzierbarkeit und damit auch für die Nachhaltigkeit von Familienpolitik entscheidende Möglichkeiten.

Zusammenfassend kann festgestellt werden, dass die Frage nach der Verantwortungsteilung und der hieraus resultierenden Instrumente durch Esping-Andersen nur teilweise beantwortet wurde. Im Idealmodell sind die Fragen ,Welche Zielsetzungen müssen verfolgt werden?', ,Welche Instrumente können hierbei hilfreich sein?', ,Wer ist verantwortlich für die zu erreichende Zielsetzung?' und ,Wie können die Instrumente am effizientesten ausgeführt werden?' dem familienpolitischen Basismodell hinzugefügt. Das Modell der Leistungskette kommt hierdurch zum Tragen.

Dieser Paragraph formt den Übergang zwischen den theoretischen Kapiteln und dem empirisch-analytischen Teil der Arbeit. In der Empirie steht die Beschreibung der Familienpolitiken Deutschlands und der Niederlande im Mittelpunkt. Hierbei wird zwischen den politisch gewählten Zielsetzungen und Instrumenten unterschieden. Ein Vergleich der empirischen Ergebnisse mit dem Idealmodell Aktivierender Familienpolitik rundet den analytischen Teil der Arbeit ab. Aus den politischen Bewegungen ergeben sich Parallelen und Unterschiede der beiden Länder. Dadurch können Antworten auf die Frage gefunden werden, ob bzw. wie der Aktivierende Staat die Familienpolitiken beider Länder beeinflusste, in welchem Land die Möglichkeiten des Leitbildes optimal verwendet wurden, und wo es eventuell noch Anpassungsmöglichkeiten gäbe. Außerdem wird deutlich, ob die beiden Staaten noch ihrem ursprünglich vorgesehenen Regimetyp zugerechnet werden können. 


\section{Verwendung familienpolitischer Zielsetzungen und Instrumente in Deutschland}

\subsection{Beschreibung der Arbeitsweise}

In diesem Kapitel erfolgt die Darstellung der familienpolitischen Zielsetzungen bzw. Instrumente der rot-grünen Koalitionen unter Schröder und der Koalitionen unter Merkel auf der Grundlage der entsprechenden Koalitionsvereinbarungen und Regierungserklärungen, sowie der Stellungnahmen der Bundesregierung zu den Familienberichten und Kinderund Jugendberichten die zwischen dem Antritt der ersten Regierung unter Schröder und der zweiten Regierung unter Merkel verfasst wurden. Auch die Zielsetzungen und Instrumente der Schwarz-Gelben Koalition unter Merkel so wie sie zum Zeitpunkt des Schreibens vorlagen - werden beschrieben. Für eine abschließende Bewertung dieser Politik ist es allerdings noch zu früh. Die Darstellung der niederländischen familienpolitischen Zielsetzungen und Instrumente erfolgt im vierten Kapitel.

Wie bereits bei der Entwicklung des Modells Aktivierender Familienpolitik dargestellt, erfolgt die empirische Beschreibung der familienpolitischen Ziele in dieser Arbeit anhand bevölkerungspolitischer, emanzipatorischer und auf das Kindeswohl konzentrierte Zielsetzungen. Die Instrumente werden, entsprechend des Modells Aktivierender Familienpolitik unterschieden nach Maßnahmen die die ökonomische Situation und ökologische Bedingungen der Familien betreffen, die zur Förderung oder Wiederherstellung von Kompetenzen der Familienangehörigen eingesetzt werden bzw. die den Eltern Zeit für Pflege und Erziehung ermöglichen. Bei der Beschreibung der Zielsetzungen und Instrumente werden auch die in den Dokumenten unterschiedenen Verantwortlichkeiten bzw. die Art der Umsetzung der Instrumente (Sozialinvestition, Öffentlich-Private Partnerschaft oder Privatisierung) berücksichtigt. Diese ergeben sich aus der breiteren Umschreibung bzw. dem politischen Kontext. Daher erfolgt die Beschreibung 
von Zielsetzungen und Instrumenten sowohl ausführlich in Textform (für die Bewertung von Verantwortlichkeiten bzw. Art der Umsetzung) als auch tabellarisch. Pro Zielsetzung und Instrument ist die Fundstelle des entsprechenden Dokuments bzw. ein kurzer Textauszug in die Darstellung aufgenommen. Die Analyse des hier beschriebenen Materials erfolgt (gemeinsam mit der Analyse der niederländischen Politik) im fünften Kapitel des Dissertationsvorhabens.

In allen Dokumenten wurden diverse aktivierende Ziele und Instrumente benannt. Die hierbei hantierten Umschreibungen sind allerdings nicht immer gleich konkret, was $\mathrm{zu}$ etwas unterschiedlichen Formulierungen von Zielsetzungen und Instrumenten führt. Um für die Darstellung und in der folgenden Analyse verwendet werden $\mathrm{zu}$ können müssen sowohl die Zielsetzungen als auch die Instrumente als solche erkennbar sein. Für die verwendeten Zielsetzungen gilt z.B., dass es in einigen Fällen ganz konkrete Richtzahlen in Prozenten und Jahrzahlen gibt, während in anderen Fällen eher an etwas undeutlicheren Leitbildern festgehalten wird. In der hier erstellten Darstellung sind sowohl die konkreten als auch die weniger konkreten Zielsetzungen aufgenommen.

Auch die Definition von Instrumenten erfolgte in den untersuchten Texten verschiedenartig. In einigen Fällen werden Maßnahmen bis auf das Mikroniveau umschrieben, während in anderen Fällen eher auf Makroniveau gearbeitet wurde. In der hier erstellten Darstellung ist, soweit wie für die Analyse nötig, auf Mikroniveau gearbeitet. Auch wenn der Schwerpunkt auf den neuen Instrumenten liegt, sind wo für den Zusammenhang erforderlich auch alte Instrumente mit in die Darstellung aufgenommen. Einige Instrumente bzw. Instrumentenbündel sind für verschiedene Kategorien relevant und daher gegebenenfalls mehrmals aufgenommen. Vor allem bei den Maßnahmen zur Unterstützung der Kompetenzen der unterschiedlichen Familienmitglieder fiel auf, dass in den verwendeten Dokumenten neben einiger größerer Maßnahmen auch viel kleine und sehr kleine Instrumente genannt und beschrieben wurden. Da es bei dieser Arbeit um die Art der Umsetzung des Aktivierungsprinzips im Allgemeinen geht und nicht um die lückenlose Darstellung aller initiierten Programme bzw. Instrumenten, ist bei der Auswahl der hier dargestellten Instrumente vor allem auf die Relevanz im Bezug auf die Fragestellung geachtet und weniger auf das Ziel der lückenlosen Darstellung aller Maßnahmen. 


\subsection{Familienpolitische Zielsetzungen}

\subsubsection{Bevölkerungspolitische Zielsetzungen}

7In der Stellungnahme der Bundesregierung zum Zwölften Kinder- und Jugendbericht wird das Ziel der Familienfreundlichkeit betont. Es müssten die entsprechenden Rahmenbedingungen weiter ausgebaut werden, um den Bedürfnissen verschiedener Familien gerecht zu werden. „Aufgabe der Politik ist es (...) Chancengerechtigkeit herzustellen und gute gesellschaftliche Rahmenbedingungen zu schaffen" (Bundesministerium für Familie, Senioren, Frauen und Jugend 2005: 3). Entscheidend zur Verbesserung der Familienfreundlichkeit in Deutschland erachte die Bundesregierung die Nachhaltigkeit der Familienpolitik. „Die Indikatoren für Nachhaltigkeit sind dabei Geburtenrate, Vereinbarkeit, Armutsrisiko, Bildungsniveau und Erziehungskompetenz (...) Eine erfolgreiche nachhaltige Familienpolitik besteht daher aus einem Mix aus Zeitressourcen, Infrastruktur, monetärer und sozialer Unterstützung. Die Bundesregierung hat sich zum Ziel gesetzt Deutschland bis zum Jahr $2010 \mathrm{zu}$ einem der kinder- und familienfreundlichsten Länder Europas zu machen“ (Bundesministerium für Familie, Senioren, Frauen und Jugend 2005: 11).

Auch im Koalitionsvertrag vom 11.11.2005 wird die demographische Zielsetzung wieder aufgegriffen und stellt die neue Koalition fest, dass zu dem Zeitpunkt zu wenig Kinder in Deutschland geboren werden. Da eine "Gesellschaft ohne Kinder" (Bundesregierung 2005a: 17) auch ohne Zukunft sei, wolle die Bundesregierung "mehr Kinder in den Familien und mehr Kinder in der Gesellschaft" (Bundesregierung 2005a: 17). Die Familie sehe sie als den Ort an dem Kinder Verantwortung zu übernehmen lernen. Insbesondere gilt die Sorge der Bundesregierung der Bevölkerungsentwicklung im östlichen Teil des Landes. Daher wolle sie „die Regionen in Ostdeutschland dabei unterstützen

7 In der Stellungnahme auf den Neunten Jugendbericht, den Zehnten Kinder- und Jugendbericht, der Koalitionsvereinbarung und Regierungserklärung von 1998, der Stellungnahme auf den Sechsten Familienbericht, der Stellungnahme auf den Elften Kinderund Jugendbericht und in der Koalitionsvereinbarung sowie der Regierungserklärung der zweiten Schröder Regierung wurden keine konkreten Zielsetzungen im Hinblick auf die demographische Entwicklung gefunden. 
den demographischen Wandel aktiv zu gestalten“ (Bundesregierung 2005a: 92). Außerdem betont die Regierung die Bedeutung des Zusammenhangs der verschiedenen Generationen und damit der gesamten Gesellschaft. Es gehe ihr um die Realisierung einer "ganzheitlichen“ (Bundesregierung 2005a: 111) Politik. Da Familien wichtige gesellschaftliche Aufgaben übernehmen, sollen sie gestärkt werden in ihrer Leistungsfähigkeit aber auch in ihrer Eigenverantwortung, „angefangen von traditionellen Familien bis hin $\mathrm{zu}$ Patchwork-, Stief- und Einelternfamilien" (Bundesregierung 2005a: 111). Menschen in Deutschland wollen, aus Sicht der Bundesregierung, sowohl eine berufliche Karriere als auch eine eigene Familie. Die Koalition werde daher unterstützen, dass „Frauen und Männer ihre Lebensvorstellungen verwirklichen können" (Bundesregierung 2005a: 111) und die Regierung nicht ein bestimmtes Lebensmodell vorschreibe. Heutige Lebensweisen und Politiken sollten allerdings nicht zu Kosten künftiger Generationen gehen. „Geringer werdende finanzielle Spielräume machen es notwendig, dass der Staat seine Investiven und konsumtiven Aufgaben daraufhin überprüft, ob sie dem Prinzip der Nachhaltigkeit Rechnung tragen“ (Bundesregierung 2005a: 112). Dieses Konzept der nachhaltigen Familienpolitik komme aus Sichtweise der Regierung gut zum Ausdruck in den zwischen Bund, Länder und Kommunen geschlossenen ,Allianzen für Familie، (Bundesregierung 2005a: 116). Neben der demographischen Situation Deutschlands sei auch die Entwicklung im europäischen Raum Thema der Stellungnahme. Gefahren der Destabilisierung durch "gegenläufige demographische Entwicklungen“ (Bundesregierung 2005a: 162) wolle die Regierung durch entsprechende Maßnahmen begegnen.

In der Regierungserklärung vom 30.11.2005 betonte die Kanzlerin erneut, dass es ihr nicht um das Vorschreiben von Lebensentwürfen durch den Staat ginge. Ihr Ziel sei es, "dass der Staat gute Rahmenbedingungen schafft” (Bundesregierung 2005b: 6), um $\mathrm{zu}$ erreichen, dass mehr Menschen in Deutschland sich für mehr Kinder entscheiden „und auch entscheiden können” (Bundesregierung 2005b: 6).

Relativ kurze Zeit später unterstreicht die Bundesregierung, in der Stellungnahme zum Siebten Familienbericht die hohe Position der Familie auf der Agenda dieser Regierung. Der Bericht sei dann auch auf Basis vielfältiger Kommunikationen und Dialogen mit verschiedensten gesellschaftlichen Akteuren erstellt. Ziel der Regierung sei es Familien zu unterstützen, mit 
zunehmend auch indirekten Transfers wie „Zeit, eine unterstützende Infrastruktur und Einkommen" (Bundesministerium für Familie, Senioren, Frauen und Jugend 2006: 24), so „dass Lebensentwürfe mit Kindern realisiert werden können“ (Bundesministerium für Familie, Senioren, Frauen und Jugend 2006: 24) und Familien in der Lage seien ihr eigenes Dasein selbstständig zu finanzieren. Auch die Unterstützung des Zusammenhaltes zwischen den Generationen sehe die Regierung als ein entscheidendes Ziel, und sie erhoffe sich hierdurch neue Netze zu schaffen, „um die Vorteile der früheren Großfamilien in moderne Sozialstrukturen übertragen $\mathrm{zu}$ können“ (Bundesministerium für Familie, Senioren, Frauen und Jugend 2006: 24). Durch „eine bessere Koordination von familiären und beruflichen Anforderungen“ (Bundesministerium für Familie, Senioren, Frauen und Jugend 2006: 25) könnte die Entscheidung für Kinder zunehmen. Der Begriff der Nachhaltigkeit sei für diese Regierung auch im Bereich der Familienpolitik von großer Bedeutung und die Regierung verstehe ihre Rolle „als Impulsgeber und Motor" (Bundesministerium für Familie, Senioren, Frauen und Jugend 2006: 25). Hierbei erfahre sie Unterstützung aus der Gesellschaft. Gemeinsam mit wichtigen Vertretern gesellschaftlicher Gruppen wolle sie sich einsetzen, „Deutschland bis zum Jahr $2010 \mathrm{zu}$ den familienfreundlichsten Ländern Europas" (Bundesministerium für Familie, Senioren, Frauen und Jugend 2006: 25) gehören $\mathrm{zu}$ lassen. Aus dem Grunde sei ein gut funktionierender gesellschaftlicher Zusammenhang notwendig. Eine „neue Integration zwischen Familien, Gemeinden, Arbeitsorten und Nachbarschaft" könne nur gelingen, „wenn auch die Kooperation und Integration von professionellen, freiwilligen und familialen Unterstützungsleistungen neu bestimmt werden. Der Ausbau von Infrastrukturen für Familien hat dabei für die Bundesregierung Priorität" (Bundesministerium für Familie, Senioren, Frauen und Jugend 2006: 25). Menschen scheinen vor allem Familien zu gründen, wenn es mit dem Beruf zu kombinieren sei. Daher wolle die Bundesregierung „Ansatzpunkte für eine lebenslaufbezogene Zeitpolitik für Familien“ (Bundesministerium für Familie, Senioren, Frauen und Jugend 2006: 31) näher untersuchen. Sie erwarte hierdurch einen positiven Beitrag zur Vereinbarkeit von Familie und Beruf leisten zu können. Vor allem im Zusammenhang mit den geschlossenen ,Allianzen für Familie' und dem freiwilligen Einsatz der deutschen Wirtschaft wolle die Bundesregierung Familienfreundlichkeit weiter ausbauen. Dieser Ausbau von Familienfreundlichkeit solle im Hinblick auf die hohe 
Kinderlosenrate von Akademikerinnen auch an den Universitäten stattfinden. Ein geeignetes Instrument sehe die Bundesregierung in der Teilnahme der Universitäten an den ,Lokalen Bündnissen für Familie' (Bundesministerium für Familie, Senioren, Frauen und Jugend 2006: 32). Um das Prinzip der Nachhaltigkeit noch besser nutzen $\mathrm{zu}$ können, sollten verschiedene familienpolitische Einzelmaßnahmen sich besser als bisher im gemeinsamen Zusammenhang auf den Lebenslauf junger Familien richten „und vor allem Eltern in den ersten Jahren des Familienaufbaus fördern“ (Bundesministerium für Familie, Senioren, Frauen und Jugend 2006: 32).

Das Ziel der nachhaltigen Familienpolitik unterstrich die Regierung auch in der Stellungnahme zum Dreizehnten Kinder- und Jugendbericht. Zur Zielerreichung sei die Abstimmung zwischen den verschiedenen Politikinstrumenten wichtig (vergl. Bundesministerium für Familie, Senioren, Frauen und Jugend 2009: 7). Die nicht aufzuhaltenden demographischen Veränderungen Deutschlands würden sich in den kommenden Jahren in den verschiedensten gesellschaftlichen Bereichen bemerkbar machen. Daher stimmt die Regierung eine interministerielle Koordination $\mathrm{zu}$ den sich hieraus ergebenden Fragen an. „Die Chancen des demographischen Wandels sollen verstärkt in der Öffentlichkeitsarbeit der Bundesregierung mit der Veröffentlichung eines ,Berichts der Bundesregierung zur demographischen Lage und künftigen Entwicklung des Landes' im Jahre 2011 berücksichtigt werden" (Bundesministerium für Familie, Senioren, Frauen und Jugend 2009: 73).

Auch der Koalitionsvertrag der Schwarz-Gelben Regierung unter Merkel vom 26.10.2009 kennt eine Vielzahl demographischer Zielsetzungen. Stärken wolle die neue Bundesregierung die wirtschaftliche und soziale Leistungsfähigkeit der Familien, da die Familien das Fundament der Gesellschaft seien, die für den Zusammenhalt zwischen den Generationen im Zusammenhalt mit dem demographischen Wandels besonders wichtig sei (vergl. Bundesregierung 2009a: 7). Wie ihre Vorgänger betone auch diese Regierung die Verantwortung, die in den Familien selber getragen werde. Zur demographischen Nachhaltigkeit der Gesellschaft halte die Regierung nicht nur die Geburtenzahlen für relevant, sondern setze sie sich auch konkrete verwaltungstechnische Umbaumaßnahmen zum Ziel. Sie wolle daher eine solide Haushalts- und Finanzpolitik mit dem Ziel einer finanziellen Konsolidierung führen. 
Außerdem solle die Handlungsfähigkeit des Staates gewährleistet bleiben. „Die Sicherung der Tragfähigkeit der öffentlichen Finanzen ist vor allem vor dem Hintergrund der demographischen Entwicklung eine zentrale Herausforderung der kommenden Legislaturperiode“ (Bundesregierung 2009a: 19). Auch im Zusammenhang mit der nachhaltigen Stadtentwicklung sei der demographische Wandel entscheidend (vergl. Bundesregierung 2009a: 41). Während im ersten Teil des Koalitionsvertrags vor allem der Umbau politischer Felder bzw. der politisch-administrativen Verwaltung im Mittelpunkt steht, wiederholt sich im zweiten Teil der Aufruf der ersten Regierung unter Merkel, um mehr Kinder zu bekommen. Um dieses Ziel $\mathrm{zu}$ erreichen wolle sie die verschiedenen familienpolitischen Bereiche noch stärker fördern (vergl. Bundesregierung 2009a: 67). Familienfreundlichkeit solle daher ein Markenzeichen sein, das für alle Städte, Gemeinden und Unternehmen gelte. Außerdem wiederholt die Regierung das bereits von den Vorgängern im Amt angekündigte Ziel einer besseren Koordinierung demographischer Fragen (vergl. Bundesregierung 2009a: 73).

In der Regierungserklärung vom 10.11.2009 unterstreicht die Kanzlerin noch einmal die große Bedeutung der demographischen Transformationsprozesse. Einerseits gehe es hierbei um Umbaumaßnahmen im bestehenden System der sozialen Sicherheit und andererseits ginge es um die Stärkung der Familien und mehr Familienfreundlichkeit im Allgemeinen (vergl. Bundesregierung 2oogb: 8).

Tabelle 15: Chronologisches Übersichtstableau der demographischen Zielsetzungen in Deutschland

\begin{tabular}{|l|l|l|}
\hline Jahr & Dokument & Zielsetzung \\
\hline 2005 & $\begin{array}{l}\text { Stellungnahme Zwölfter Kinder- } \\
\text { und Jugendbericht }\end{array}$ & $\begin{array}{l}\text { Führen einer nachhaltigen Familienpolitik, } \\
\text { Deutschland bis zum Jahr 2010 zu einem der kinder- } \\
\text { und familienfreundlichsten Länder Europas machen }\end{array}$ \\
\hline 2005 & $\begin{array}{l}\text { Koalitionsvertrag der Großen } \\
\text { Koalition unter Merkel }\end{array}$ & $\begin{array}{l}\text { Mehr Kinder in den Familien und mehr Kinder in der } \\
\text { Gesellschaft, } \\
\text { Aktive Gestaltung des demographischen Wandels, } \\
\text { Führung einer ganzheitlichen Politik, um } \\
\end{array}$ \\
& $\begin{array}{l}\text { Eigenverantwortung der Generationen zu stärken, } \\
\text { Bessere Rahmenbedingungen für die Vereinbarkeit } \\
\text { von Beruf und Familie schaffen, } \\
\text { Nachhaltigkeit der Familienpolitik fördern, } \\
\end{array}$ & $\begin{array}{l}\text { Die demographische Situation Europas fördern } \\
\text { Mehr Kinder in den Familien und mehr Kinder in der }\end{array}$ \\
\hline 2005 & Regierungserklärung der & \\
\hline
\end{tabular}




\begin{tabular}{|c|c|c|}
\hline & Großen Koalition unter Merkel & Gesellschaft \\
\hline 2007 & $\begin{array}{l}\text { Stellungnahme Siebter } \\
\text { Familienbericht }\end{array}$ & $\begin{array}{l}\text { Familien mehr als bisher mit indirekten Transfers } \\
\text { (Infrastruktur) unterstützen, } \\
\text { Lebensentwürfe mit Kindern realisieren, } \\
\text { Zusammenhang zwischen Generationen durch } \\
\text { Übersetzung der Funktionen von Großfamilien } \\
\text { fördern, } \\
\text { Bessere Vereinbarkeit von Beruf und Familie } \\
\text { realisieren, } \\
\text { Nachhaltigkeit der Familienpolitik fördern, } \\
\text { Deutschland zu einem der familienfreundlichsten } \\
\text { Länder Europas entwickeln, } \\
\text { Ungewollte Kinderlosigkeit von Akademikern } \\
\text { bekämpfen, } \\
\text { Besserer Zusammenhang in der Familienpolitik durch } \\
\text { Lebenslaufansatz mit Fokus auf junge Eltern }\end{array}$ \\
\hline 2009 & $\begin{array}{l}\text { Stellungnahme Dreizehnter } \\
\text { Kinder- und Jugendbericht }\end{array}$ & $\begin{array}{l}\text { Nachhaltigkeit der Familienpolitik durch ein } \\
\text { wirkungsorientiert abgestimmtes Instrumentarium, } \\
\text { Koordination der Beschäftigung mit demographischen } \\
\text { Fragen und Publikation eines interministeriellen } \\
\text { Berichts }\end{array}$ \\
\hline 2009 & $\begin{array}{l}\text { Koalitionsvertrag der Schwarz- } \\
\text { Gelben Koalition unter Merkel }\end{array}$ & $\begin{array}{l}\text { Stärkung der wirtschaftlichen und sozialen } \\
\text { Leistungsfähigkeit der Familien, } \\
\text { Sicherung der Tragfähigkeit öffentlicher Finanzen vor } \\
\text { dem Hintergrund der demographischen Entwicklung, } \\
\text { Bewältigung des demographischen und } \\
\text { wirtschaftsstrukturellen Wandels in der } \\
\text { Stadtentwicklung, } \\
\text { Mehr Geburten durch mehr Kinder- und } \\
\text { Familienfreundlichkeit, } \\
\text { Koordination der Beschäftigung mit demographischen } \\
\text { Fragen und Veröffentlichung eines entsprechenden } \\
\text { Berichts }\end{array}$ \\
\hline 2009 & $\begin{array}{l}\text { Regierungserklärung der } \\
\text { Schwarz-Gelben Koalition unter } \\
\text { Merkel }\end{array}$ & $\begin{array}{l}\text { Antworten auf die Veränderungen des Altersaufbaus } \\
\text { der Gesellschaft finden }\end{array}$ \\
\hline
\end{tabular}




\subsubsection{Emanzipatorische Zielsetzungen}

In ihrer Koalitionsvereinbarung vom 20.10.1998 unterstreicht die Rot-Grüne Koalition die Bedeutung der Themen „Gleichberechtigung von Frauen” (Bundesregierung 1998a: 5f) sowie Verbesserung der Kinderfreundlichkeit in der Gesellschaft. Als gemeinsame Ziele sähen die Regierungsparteien: „eine zukunftsorientierte Bildung und Ausbildung für alle Jugendlichen sichern und Chancengleichheit herstellen (...) (und) die Gleichstellung von Frauen in Arbeit und Gesellschaft entscheidend voran(zu)bringen“ (Bundesregierung 1998a: 5f). Frauen und Familien wolle die Regierung stärken, nicht nur wirtschaftlich, sondern auch im Sozialen solle sich die Situation verbessern. Daher sei es nötig, die Verknüpfung der verschiedenen politischen Ebenen und Fachbereiche voranzutreiben und „die Familien sowohl über wirtschaftliche Maßnahmen als auch über den Ausbau der sozialen Infrastruktur (zu) entlasten” (Bundesregierung 1998a: 34). Die Gleichstellung von Mann und Frau verstehe die Regierung als eines ihrer großen Reformprojekte. „Ein Bündnis für Arbeit muß zugunsten von Frauen und Männern gleichsam wirken“ (Bundesregierung 1998a: 35$)$.

In der Regierungserklärung vom 10.11.1998 unterstreicht Bundeskanzler Schröder die Emanzipation der Frauen und ihre Teilnahme am „Erwerbsleben” (Bundesregierung 1998b: 14) erneut. Die qualitative und quantitative Verbesserung der Infrastrukturen für Kinder halte er aus diesem Grund für wichtig.

In der Stellungnahme der Bundesregierung zum Sechsten Familienbericht, betont die Bundesregierung, dass „die Rahmenbedingungen für Familien unter dem Aspekt von Chancengleichheit, Gerechtigkeit und Solidarität“ (Bundesministerium für Familie, Senioren, Frauen und Jugend 2000: 12) nicht nur für deutsche, sondern für alle Familien in Deutschland verbessert werden sollten. Aufmerksamkeit gelte insbesondere dem Thema Vereinbarkeit von Familie und Beruf, das vor allem zu Lasten der Frauen ausgetragen werde. Daher sei aus „familien- und frauenpolitischer Sicht (...) der Stärkung der Fähigkeiten (empowerment) von Frauen und Müttern verstärkte Aufmerksamkeit zu widmen“ (Bundesministerium für Familie, Senioren, Frauen und Jugend 2000: 17). 
Im Elften Kinder- und Jugendbericht unterstreicht die Bundesregierung die zukünftig strukturierte Herangehensweise, um Entwicklungen im Bereich der Chancengleichheit von Männern und Frauen besser hervorzuheben. „Bei allen Maßnahmen ist es Ziel der Politik der Bundesregierung, künftig die Grundlagen einer geschlechterübergreifenden und vom Geschlecht unabhängigen Chancengleichheit $\mathrm{zu}$ schaffen (...) Die hierzu von der Bundesregierung genutzte Strategie des Gender Mainstreaming soll - wie in allen Politikfeldern - auch in der Kinder- und Jugendhilfe handlungsleitend sein."

Die schon in der vorherigen Koalitionsvereinbarung betonte Bedeutung des Themas Emanzipation, wiederholt die zweite Rot-Grüne Koalition in ihrer Koalitionsvereinbarung vom 16.10.2002. Sie mache an dieser Stelle deutlich, dass die „Durchsetzung der tatsächlichen Gleichberechtigung von Frauen und Männern (...) durchgängiges Leitprinzip der Bundesregierung in jedem Ressort” (Bundesregierung 2002: 61) sei. Um diesen Gedanken auch in der Arbeitswelt zu manifestieren und die Rolle der Frau am Arbeitsplatz zu verbessern, werde Gleichstellung zu einem der wichtigen Themen des Bündnisses für Arbeit. Durch diese und andere Maßnahmen soll es gelingen das auf europäischer Ebene vereinbarte Ziel zu erreichen und „die Frauenerwerbsquote auf über 60\% zu steigern“ (Bundesregierung 2002: 61).

${ }^{8}$ Zum Ende ihrer Regierungszeit betont Rot-Grün in der Stellungnahme zum 12. Kinder- und Jugendbericht, dass sie Familien und damit meint sie Väter und Mütter kein bestimmtes Familien- bzw. Betreuungsmodell vorschreiben wolle. „Die Bundesregierung wird aber die Voraussetzungen dafür verbessern, dass Vater oder Mutter sich nicht durch ökonomische Gründe für die eine und gegen die andere Lösung entscheiden müssen“ (Bundesministerium für Familie, Senioren, Frauen und Jugend 2005: 17f).

Ihre Nachfolger im Amt weisen im Koalitionsvertrag vom 11.11.2005 darauf, dass die Vereinbarkeit von Wissenschaft und Familie ihr besonders wichtig sei. „Es bleibt ein zentrales Anliegen dieser Bundesregierung Frauen in Lehre und 
Forschung bessere Karrierechancen $\mathrm{zu}$ eröffnen“. Aber auch für übrige Sektoren, in denen Kinderlosigkeit von Frauen weniger häufig vorkommt als im wissenschaftlichen Bereich sei das Ziel der Regierung die Vereinbarkeit diverser Lebensvorstellungen im Bezug auf Familie und Beruf. Daher müsse "Gleichstellungspolitik (...) sich an den Erwartungen und Lebensentwürfen von Männern und Frauen in allen Lebensbereichen orientieren" (Bundesregierung 2005a: 111). Die neue Regierung halte Arbeit, welche zur finanziellen Unabhängigkeit von Frauen führe, neben „Aufstiegsmöglichkeiten für Frauen” (Bundesregierung 2005a: 111f), für besonders wichtig. Wie auch die zweite RotGrüne Koalition setzt die Große Koalition unter Merkel sich für das international verabredete Ziel einer Frauenerwerbsquote von minimal 60\% ein. Auch die europäische Verpflichtung "gleicher Lohn für gleiche und gleichwertige Arbeit" wolle die Regierung vorantreiben. Vor allem im Hinblick auf die Selbstständigkeit von Frauen werde sie Bewegung erzeugen und „die unterdurchschnittliche Rate von selbstständigen Frauen (...) auf europäischen Durchschnitt anheben“ (Bundesregierung 2005a: 120). Insbesondere den Frauen ausländischer Herkunft gelte die Aufmerksamkeit der Bundesregierung. Ihr Ziel sei es die Instrumente konsequent einzurichten, dass Gleichberechtigung für Frauen ausländischer Herkunft z.B. im Bezug auf das politische, wirtschaftliche und kulturelle Leben realisiert werden können.

${ }^{9}$ Wie aus der Stellungnahme, die diese Große Koalition zum Siebten Familienbericht erstellte deutlich wurde, verwendete sie den Begriff Gleichberechtigung breiter als zuvor, und schließt nicht nur Männer und Frauen sondern auch alle Kinder mit ein. Da deutlich geworden sei, dass in Deutschland Chancengleichheit in Ausbildung und Beruf immer weniger die Regel werde, sehe die Regierung auch Kinder als neue Fokusgruppe im Hinblick auf Gleichberechtigung (vergl. Bundesministerium für Familie, Senioren, Frauen und Jugend 2006: 23). Die Vereinbarkeit von Familie und Beruf für sowohl Männer als auch Frauen erscheine der Regierung nicht nur aus Gründen der Gerechtigkeit wichtig. „Bessere Möglichkeiten für die Erwerbstätigkeit beider Elternteile erweitern finanzielle Spielräume und 
vermindern Armutsrisiken von Familien. Um dieses Ziel zu realisieren, wolle die Koalition die Kräfte der verschiedenen gesellschaftlichen Akteure mobilisieren, denn eine "gute Balance von Familie und Arbeitswelt liegt im gemeinsamen Interesse und in gemeinsamer Verantwortung von Politik und Wirtschaft" (Bundesministerium für Familie, Senioren, Frauen und Jugend 2006: 31).

Kurz vor dem Ende der ersten Koalition unter Merkel weist die Regierung in der Stellungnahme zum Dreizehnten Kinder- und Jugendbericht im Zusammenhang mit der Armutsprävention von Familien darauf hin, dass in Familien in denen beide Elternteile arbeiten (unabhängig von der Frage ob es eine Vollzeit- oder Teilzeitbeschäftigung ist) Armut praktisch keine Rolle spiele (Bundesministerium für Familie, Senioren, Frauen und Jugend 2009: 7).

Auch die Schwarz-Gelbe Koalition unter Merkel betont in ihrem Koalitionsvertrag vom 26.10.2009 verschiedene emanzipatorische Zielsetzungen. Im Allgemeinen gilt für alle hier festgestellten Regelungen, dass mehr als zuvor genderneutral formuliert wurde. Konkret betone die Regierung sie wolle die Erreichung von Wahlfreiheit im Bezug der Vereinbarkeit zwischen Familie und Beruf vergrößern. „Eltern sollen die Wahlfreiheit haben, Familienleben und Erwerbstätigkeit nach ihren Wünschen zu gestalten. Alle, die Kinder erziehen, erbringen eine Leistung für die ganze Gesellschaft und verdienen daher deren besondere Anerkennung. Förderinstrumente sollen direkt in der Lebenswelt von Familien ansetzen“ (Bundesregierung 2009a: 67). Da es für Männer in der Vergangenheit oft keine Entscheidungsmöglichkeit für Familie zu geben schien, während immer mehr Männer sich neben des Berufs mehr Zeit für Familie wünschten, betonte die Regierung: „Diese Koalition will gleiche Chancen für Frauen und Männer im Beruf wie im Familienleben verwirklichen" (Bundesregierung 2009a: 67). Dabei unterstreiche sie insbesondere, dass dieses Ziel für alle Lebensphasen gelte. Aus diesem Grund erarbeite sie „einen Rahmenplan zur gleichberechtigten Teilnahme von Frauen und Männern in allen Phasen des Lebensverlaufs" (Bundesregierung 2009a: 73). Auch für mehr Frauen in Führungspositionen wolle sich die Regierung verstärkt einsetzen. Aus diesem Anlass werden das Bundesgleichstellungsgesetz sowie das Bundesgremienbesetzungsgesetz im Hinblick auf eine möglich effektivere Gestaltung geprüft. „Der Anteil von Frauen in Führungspositionen in der Wirtschaft und im öffentlichen Dienst soll maßgeblich erhöht werden. Dazu 
wird ein Stufenplan, insbesondere zur Erhöhung des Anteils von Frauen in Vorständen und Aufsichtsräten vorgelegt“ (Bundesregierung 2009a: 74). Neben der Rolle von Frauen betone die Regierung auch die Anpassung der Männerrolle in Familie und Beruf und ihrer aktiven Unterstützung seitens der Bundesregierung. „Wir wollen eine eigenständige Jungen- und Männerpolitik entwickeln und bereits bestehende Projekte für Jungen und junge Männer fortführen und intensivieren. Damit eröffnen wir ihnen auch in erzieherischen und pflegerischen Berufen erweiterte Perspektiven“ (Bundesregierung 2009a: 74). Eine letzte Zielsetzung im emanzipatorischen Bereich gilt den Alleinerziehenden. Hierbei geht es um die praktische Unterstützung von Familien mit nur einem Elternteil. „Wir wollen die Rahmenbedingungen für Alleinerziehende durch ein Maßnahmenpaket verbessern. Dieses soll insbesondere in verlässlichen Netzwerkstrukturen für Alleinerziehende lückenlos, flexibel und niedrigschwellig bereitgestellt werden“ (Bundesregierung 2009a: 69) ${ }^{10}$.

Tabelle 16: Chronologisches Übersichtstableau der emanzipatorischen Zielsetzungen in Deutschland

\begin{tabular}{|l|l|l|}
\hline Jahr & Dokument & Zielsetzung \\
\hline 1998 & $\begin{array}{l}\text { Koalitionsvereinbarung der } \\
\text { ersten Rot-Grünen Koalition }\end{array}$ & $\begin{array}{l}\text { Zukunftsorientierte Bildung und Ausbildung für alle } \\
\text { Jugendlichen sichern und Chancengleichheit } \\
\text { herstellen, } \\
\text { Gleichstellung von Frauen in Arbeit und Gesellschaft } \\
\text { entscheidend voranbringen }\end{array}$ \\
\hline $\mathbf{1 9 9 8}$ & $\begin{array}{l}\text { Regierungserklärung der ersten } \\
\text { Rot-Grünen Koalition }\end{array}$ & $\begin{array}{l}\text { Gleichstellung von Frauen in Arbeit und Gesellschaft } \\
\text { entscheidend voranbringen }\end{array}$ \\
\hline 2000 & $\begin{array}{l}\text { Stellungnahme Sechster } \\
\text { Familienbericht }\end{array}$ & $\begin{array}{l}\text { Gleichstellung von Frauen in Arbeit und Gesellschaft } \\
\text { entscheidend voranbringen }\end{array}$ \\
\hline 2002 & $\begin{array}{l}\text { Stellungnahme Elfter Kinder- } \\
\text { und Jugendbericht }\end{array}$ & $\begin{array}{l}\text { Chancengleichheit durch die Strategie des Gender } \\
\text { Mainstreamings }\end{array}$ \\
\hline 2002 & $\begin{array}{l}\text { Koalitionsvertrag der zweiten } \\
\text { Rot-Grünen Koalition }\end{array}$ & $\begin{array}{l}\text { Durchsetzung der tatsächlichen Gleichberechtigung } \\
\text { von Frauen und Männern (ist) durchgängiges } \\
\text { Leitprinzip der Bundesregierung in jedem Ressort, } \\
\text { Frauenerwerbsquote auf über 6o\% steigern }\end{array}$ \\
\hline 2005 & Stellungnahme Zwölfter Kinder- & Verbesserung der Möglichkeiten zur Einfüllung \\
\hline & & \\
\hline
\end{tabular}




\begin{tabular}{|l|l|l|}
\hline & und Jugendbericht & eigener Wünsche in Bezug auf Vater- bzw. \\
Mutterschaft
\end{tabular}

\subsubsection{Auf das Kindeswohl gerichtete Zielsetzungen}

In der Koalitionsvereinbarung vom 20.10.1998 wird das "Recht auf Bildung“ (Bundesregierung 1998a: 25) als eine entscheidende Zielsetzung genannt. Eine Bildungsreform, die dazu führe die Chancengleichheit aller Kinder zu fördern erscheine der Regierung notwendig. Durch bessere Förderung von Bildung und Ausbildung müsse es gelingen die Jugendarbeitslosigkeit zu verringern. „Wir wollen, dass alle Jugendlichen, die länger als sechs Monate arbeitslos sind einen Ausbildungsplatz oder einen Arbeitsplatz erhalten“ (Bundesregierung 1998a: 
33). Neben den Investitionen in Bildung und Ausbildung sehe die Regierung in der Stärkung der Kinderrechte ein wichtiges Ziel. „Wir werden wirksame Konzepte zum Schutz der Kinder vor sexueller Gewalt und vor Missbrauch durch Vertrauenspersonen entwickeln“ (Bundesregierung 1998a: 33). Auch die Verbesserung der sozialen Lage von Familien sei eine wichtige Zielsetzung der Regierung. Dieses Ziel verfolge sie in Zusammenarbeit mit verschiedenen Akteuren. „Die Familienpolitik der Bundes-, Landes- und der kommunalen Ebene muß besser verzahnt werden, um die Effizienz der familienpolitischen Maßnahmen zu steigern. Der Dialog mit den Verbänden und gesellschaftlichen Gruppen wird wieder aufgebaut. Dazu werden wir Initiativen ergreifen“ (Bundesregierung 1998a: 34).

"Die Regierung wiederholt die Zielsetzung der Bekämpfung der Jugendarbeitslosigkeit in ihrer Stellungnahme zum Sechsten Familienbericht und bezeichnet es als eine "Schwerpunktsaufgabe“ (Bundesministerium für Familie, Senioren, Frauen und Jugend 2000: 22). Zur Erreichung der Zielsetzung fände die Regierung vor allem die Arbeit des Bündnisses für Arbeit, Ausbildung und Wettbewerbsfähigkeit entscheidend.

Kurz vor dem Ende ihrer ersten Regierungsperiode betont die Rot-Grüne Koalition in der Stellungnahme auf den Elften Kinder- und Jugendbericht die Zielsetzung, die sie im Programm „Chancen im Wandel“ (Bundesministerium für Familie, Senioren, Frauen und Jugend 2002: 4) setze. Ihr gehe es einerseits darum Jugendpolitik als Querschnittspolitik zu verstehen, um Synergieeffekte mit positiven Auswirkungen auf die Leistungen für Jugendliche $\mathrm{zu}$ erzielen. Andererseits wolle sie „eine aktivierende Jugendpolitik, die die Stärken der Jugendlichen fördert, aber die Jugendlichen auch zur aktiven Beteiligung an der Gestaltung" (Bundesministerium für Familie, Senioren, Frauen und Jugend 2002: 4) der Gesellschaft aufrufe. Zu erreichen versuche sie dieses Ziel durch den Einsatz einer breiten Allianz zwischen Jugend und Politik. Hierdurch erhoffe sich die Regierung eine nachhaltige Politik für die Jugend. Auch die Chancengleichheit der Jugendlichen sehe die Regierung als eine wichtige Zielsetzung. „Mit ihrer Politik zur Verbesserung der Lebenslagen geht sie dabei 
über Detailveränderungen hinaus und nimmt (sie) ganzheitliche Lebensabschnitte und typische Lebenssituationen ins Blickfeld“ (Bundesministerium für Familie, Senioren, Frauen und Jugend 2002: 5). Da es auch in der Jugendpolitik vermehrt $\mathrm{zu}$ internationalen Vergleichen komme, wolle die Bundesregierung aktiv mitarbeiten bei der Erarbeitung der Maßstäbe, die zu diesen Vergleichen herangezogen würden (vergl. Bundesministerium für Familie, Senioren, Frauen und Jugend 2002: 6). Als eine weitere Zielsetzung sehe die Regierung den Ausbau der Möglichkeiten zur Kinderbetreuung (vergl. Bundesministerium für Familie, Senioren, Frauen und Jugend 2002: 15). Diese müsse ab jetzt eine besondere Rolle spielen.

Die zweite Rot-Grüne Koalition unterstreicht in ihrer Koalitionsvereinbarung vom 16.10.2002 die Chancengleichheit von Kindern, unabhängig von ihrem Wohnort, als eines ihrer Ziele. „Dazu brauchen wir zügig nationale Bildungsstandards. Deren Einhaltung soll durch eine unabhängige wissenschaftliche Einrichtung (Evaluationsagentur) überprüft werden“ (Bundesregierung 2002: 31). Weiterhin sehe sie eine quantitative und qualitative Verbesserung von Ausbildungsplätzen als ein wichtiges Anliegen, dass unter anderem auch zu einer Verbesserung von Chancengleichheiten von Männer und Frauen am Arbeitsmarkt führen könnte. „Die Sicherung eines ausreichenden Ausbildungsplatzangebots für alle Jugendlichen hat für uns Priorität (...) Unser Anliegen ist es, den Anteil von Frauen in den technischen und naturwissenschaftlichen Berufen deutlich zu steigern" (Bundesregierung 2002: 31). Die Regierung rufe daher die verschiedenen gesellschaftlichen Akteure auf sich für eine Verringerung von Jugendarbeitslosigkeit einzusetzen (vergl. Bundesregierung 2002: 31).

In der Regierungserklärung, die der Koalitionsvereinbarung am 14.03.2003 folgte, wurden die Verringerung der Jugendarbeitslosigkeit und die Förderung von Chancen junger Menschen als wichtige Eckpunkte genannt. Das Ziel der geteilten Verantwortung und des Förderns und Forderns wurde hierbei unterstrichen. „Diesem Recht - das muss genauso klar festgestellt werden entspricht allerdings die Pflicht, zumutbare Angebote auch anzunehmen“ (Bundesregierung 2003: 11).

Zum Ende ihrer Amtszeit bezeichnet die zweite Rot-Grüne Regierung in der Stellungnahme auf den 12. Kinder- und Jugendbericht den konsequenten 
Ausbau für das „öffentliche System von Betreuung, Bildung und Erziehung im Interesse der Kinder und Jugendlichen weiter zu entwickeln" als eine ihrer entscheidendsten Zielsetzungen (vergl. Bundesministerium für Familie, Senioren, Frauen und Jugend 2005: 3f). Durch dieses System öffentlicher Betreuung würden Eltern in ihrer Erziehungskompetenz gestärkt und unterstützt. Der Einsatz diene unter anderem der Prävention problematischer Entwicklungsdefizite von Kindern und Jugendlichen (vergl. Bundesministerium für Familie, Senioren, Frauen und Jugend 2005: 30). Mit diesem Gesamtsystem seien ausdrücklich alle Einrichtungen von der Kindertagesstätte bis zur Ganztagsschule gemeint. Insbesondere gelte die Aufmerksamkeit dem Ausbau der Kinderbetreuung von „Kinder(n) unter drei Jahren sowie der Ganztagsplätze für Kinder im Kindergartenalter" (vergl. Bundesministerium für Familie, Senioren, Frauen und Jugend 2005: 18) einerseits und die Qualitätssicherung in der Kindertagespflege andererseits (vergl. Bundesministerium für Familie, Senioren, Frauen und Jugend 2005: 21). Als Verantwortliche für diese Zielsetzung sehe die Bundesregierung alle in diesem Bereich entscheidenden Akteure. Neben dem Aufbau dieses Gesamtsystems öffentlicher Förderung und der quantitativen Verbesserung des Angebotes sei auch die qualitative Verbesserung der Förderung Zielsetzung der Bundesregierung. „Das Ziel, Gesundheitsförderung in Kindertagesstätten und Schulen, insbesondere im Hinblick auf gesundes Ernährungs- und Bewegungsverhalten, die sprachlichen und motorischen Fähigkeiten und die Fähigkeit zur Stressbewältigung zu stärken ist ein wichtiger Bestandteil des nationalen Aktionsplans ,Für ein kindgerechtes Deutschland 2005-2010'“ (Bundesministerium für Familie, Senioren, Frauen und Jugend 2005: 8). Wichtig finde die Bundesregierung die Einführung einheitlicher Zielsetzungen der pädagogischen Frühförderung. Sie halte die Einführung eines externen und von Trägern und Finanzgebern unabhängiges und „bundesweit wirksames Qualitätssicherungssystem für erstrebenswert" (Bundesministerium für Familie, Senioren, Frauen und Jugend 2005: 22f). Durch „die Verknüpfung der unterschiedlichen Bildungsorte und Lernwelten" (Bundesministerium für Familie, Senioren, Frauen und Jugend 2005: 25) strebe die Regierung dem Ziel einer umfassenden Förderung der kindlichen und jugendlichen Kompetenzen nach. Neben Zielsetzungen die sich auf die Betreuungsangebote richten, stelle sich die Regierung auch Zielsetzungen im Hinblick auf Bildung und Ausbildung. „Die Präsidentin der Kultusministerkonferenz wird sich im Rahmen ihrer Möglichkeiten dafür 
einsetzen, dass die Länder ihre Anstrengungen mit dem Ziel verstärken, die Zahl der Schulabgänger ohne Schulabschluss bis zum Ende des Jahrzehnts deutlich zu verringern“ (Bundesministerium für Familie, Senioren, Frauen und Jugend 2005: 9). Da in Deutschland die Herkunft eines Kindes für die Bildungschancen sehr entscheidend sei, benenne die Bundesregierung die Förderung von Entwicklungsbedingungen von Kindern „aus benachteiligten Familien oder aus Familien mit Migrationshintergrund“ (Bundesministerium für Familie, Senioren, Frauen und Jugend 2005: 24) als einen besonderen Schwerpunkt ihrer Arbeit. Um die Realisierung verschiedener Vorhaben auch langfristig zu garantieren und eine nachhaltige Politik zu führen, wolle die Regierung die Verbesserung der ressortübergreifenden Kooperation und Einbindung verantwortlicher Gruppen und Parteien realisieren (vergl. Bundesministerium für Familie, Senioren, Frauen und Jugend 2005: 34).

Auch in der Koalitionsvereinbarung der Großen Koalition unter Merkel werden am 11.11.2005 die Ausbildungs- und Beschäftigungschancen junger Menschen als ein wichtiges Anliegen der neuen Regierung betont. Die Chancen von Jugendlichen auf dem Arbeitsmarkt sollten deutlich verbessert werden. Ziel sei es "dass in Zukunft kein Jugendlicher länger als drei Monate arbeitslos ist" (Bundesregierung 2005a: 28). Bei der Durchsetzung dieser Zielsetzungen seien ergänzend $\mathrm{zu}$ den „Arbeitsmarktakteuren die Träger der Kinder- und Jugendhilfe" (Bundesregierung 2005a: 123) gefordert. Auch die Förderung von Chancengleichheit aller Kinder, unabhängig ihrer Herkunft bzw. dem Ausbildungsniveau ihrer Eltern, sehe die Regierung als ein wichtiges Ziel (vergl. Bundesregierung 2005a: 111). Die Stärkung von Kinderrechten und Teilhabe von Kindern an gesellschaftlichen Prozessen wolle die Regiering fördern und sie „stärker in die Öffentlichkeit transportieren“ (Bundesregierung 2005a: 121). Außerdem wolle die Regierung der Chancengleichheit von Migrantenkindern eine besondere Aufmerksamkeit zuteil werden lassen. Hierbei werde sie die Kapazitäten der verschiedenen Akteure in diesem Bereich vernetzen und besser nutzen. Insbesondere halte die Regierung „die Einbeziehung der Eltern durch schulische und außerschulische Bildungs- und Betreuungseinrichtungen (...) für Migrantenkinder“ (Bundesregierung 2005a: 139) für wichtig.

In der Regierungserklärung, die der Koalitionsvereinbarung am 30.11.2005 folgte, betont die neue Regierung vor allem auch die Eigenverantwortung von Eltern bis zum 25. Lebensjahr ihrer Kinder. „Deshalb werden wir die 
Regelungen so ändern, dass Kinder unter 25 Jahren zunächst einmal von ihren Eltern unterhalten werden“ (Bundesregierung 2005b: 7).

Kurze Zeit später, in der Stellungnahme der Bundesregierung auf den Siebten Familienbericht, unterstreicht die Bundesregierung neben der Chancengleichheit von Frauen insbesondere die Chancengleichheit von Kindern. „Für die Kommission wie für die Bundesregierung sind gleiche Chancen der Geschlechter und gute Entwicklungschancen für alle Kinder gleichermaßen wesentliche Lebensziele“ (Bundesministerium für Familie, Senioren, Frauen und Jugend 2006: 23).

Zum Ende ihrer Amtszeit konzentriert sich die Regierung, in der Stellungnahme auf den Dreizehnten Kinder- und Jugendbericht (aufgrund des vorliegenden Schwerpunktberichts mit dem Thema Gesundheit) vor allem auf Ziele zur Prävention von Krankheiten und Verbesserung des Wohlbefindens von Kindern und Jugendlichen. Generell gehe es ihr darum die Rahmenbedingungen für das Aufwachsen der kommenden Generationen zu verbessern. „Dazu gehört auch das soziale, psychische und physische Wohlbefinden von Kindern und Jugendlichen. Die bestmögliche Förderung der Gesundheit ist dabei ein zentrales Anliegen der Bundesregierung“" (Bundesministerium für Familie, Senioren, Frauen und Jugend 2009: 4). Aufgrund der Tatsache, dass die Erziehung von Kindern in erster Linie die Verantwortung der Eltern selbst sei, appelliere die Regierung vor allem an die Lebensweise der Eltern und ihre Fähigkeiten zur Förderung ihrer Kinder. „Die Bundesregierung will Eltern unter Beachtung ihres Erziehungsrechts dazu motivieren und - wo erforderlich - auch befähigen die Vorbedingungen einer gesunden Lebensführung für die physische und psychische Gesundheit, für das Wohlbefinden ihrer Kinder frühzeitig zu erkennen“ (Bundesministerium für Familie, Senioren, Frauen und Jugend 2009: 6). In einigen Familien bedürfe es - aufgrund der schwachen Lebensumstände - besonderer Hilfen. Nicht alle Familien könnten ihrer Eigenverantwortung gerecht werden. „Aus diesem Grund richtet die Bundesregierung ihre Anstrengungen darauf, gerade diejenigen Familien besonders zu unterstützen, in denen die gesundheitliche Förderung der Kinder nicht optimal verläuft. Sie tut dies mit einer sinnvollen Kombination aus gezielter finanzieller Förderung und der Verbesserung von Strukturen“ (Bundesministerium für Familie, Senioren, Frauen und Jugend 2009: 6). Um möglichen späteren Gefahren oder Fehlentwicklungen 
vorzubeugen, halte die Regierung es wichtig, um so viel und so früh wie möglich in die Prävention von Krankheiten $\mathrm{zu}$ investieren (vergl. Bundesministerium für Familie, Senioren, Frauen und Jugend 2009: 9). Vor dem Hintergrund der frühen Gesundheitsförderung und der Prävention von Krankheiten sei vor allem die Verbesserung des direkten Lebensumfelds von Kindern und Jugendlichen wichtig (vergl. Bundesministerium für Familie, Senioren, Frauen und Jugend 2009: 12). Nur mit niedrigschwelligen Angeboten in den Kindertagesstätten, Schulen usw. könnten Präventionsziele erreicht werden. Kindern und Jugendlichen mit Behinderungen jeglicher Art (auch Legasthenie und Dyskalkulie) stehe eine besondere Art der Förderung zu. Sie dürften nicht zum Opfer der Aufspaltung von Verantwortlichkeiten in Schule und Jugendhilfe werden. „Aus Sicht der Bundesregierung sollte das Verhältnis von Kinder- und Jugendhilfe und Schule als weitere Schnittstelle im Rahmen einer Überprüfung der für die Förderung junger Menschen mit Behinderung verantwortlichen Leistungssysteme besondere Aufmerksamkeit erfahren“ (Bundesministerium für Familie, Senioren, Frauen und Jugend 2009: 16f). Zuletzt betont die Regierung, dass Gesundheit und Wohlbefinden von Kindern die Basis formen für die übrige Entwicklung von Kindern. „Insofern stellt der 13. Kinder- und Jugendbericht auch eine Erweiterung des 12. Kinder- und Jugendberichts dar. Während dort Bildung (...) weitgehend losgelöst von Körpererfahrung und Leiblichkeit, von Wohlbefinden und Gesundheit diskutiert wurde, betont der 13. Kinder- und Jugendbericht, dass der eigene (gesunde) Körper nicht nur eine Voraussetzung für gelingende Bildungsprozesse darstellt, sondern dass der eigene Körper selbst ein Medium für Bildungsprozesse darstellt" (Bundesministerium für Familie, Senioren, Frauen und Jugend 2009: 17).

Die Verbesserung der Chancengleichheit von Kindern wird auch im Koalitionsvertrag der Schwarz-Gelben Koalition von Merkel (26.10.2009) als ein wichtiges politisches Ziel definiert. Es gehe ihr darum Stärken zu erkennen, Benachteiligungen $\mathrm{zu}$ verhindern und Kinderarmut zu bekämpfen. Hierfür müsse auch die entsprechende Infrastruktur zur Verfügung stehen. „Wir wollen mehr Chancengerechtigkeit am Start, Durchlässigkeit und faire Aufstiegschancen für alle ermöglichen. Wir wollen Deutschland zur Bildungsrepublik machen, mit den besten Kindertagesstätten, den besten Schulen und Berufsschulen sowie den besten Hochschulen und 
Forschungseinrichtungen“ (Bundesregierung 2009a: 59). Chancengleichheit von Kindern und Jugendlichen durch entsprechende Bildungsmöglichkeiten sei der Regierung ein besonderes Anliegen. Und das gelte nicht allein für Kinder sondern auch für Jugendliche und junge Erwachsene. Vor allem im Hochschulbereich solle diese Zielsetzung spürbar werden. „Wir setzen uns zum Ziel, die Studienanfängerquote weiter $\mathrm{zu}$ steigern. Künftig sollen mehr Studienanfänger über die berufliche Bildung an die Hochschule kommen. Insbesondere müssen wir dafür Sorge tragen, dass mehr Studierende ihr Studium auch erfolgreich beenden“ (Bundesregierung 2009a: 61). Genau wie ihre Vorgänger im Amt betont auch diese Regierung erneut die Eigenverantwortung der Eltern. Erziehung von Kindern sei in erster Linie eine private Aufgabe und in zweiter Linie Verantwortung der vielen Bildungs- und Jugendhilfeeinrichtungen. „Wir wollen Eltern, Betreuungseinrichtungen, Schulen und Einrichtungen der Jugendarbeit in ihrer werteorientierten Erziehungsverantwortung bestärken“ (Bundesregierung 2009a: 71). Zur Erkennung der Potentiale von Kindern und Jugendlichen trügen auch eine eigenständige Jugendpolitik, eine starke Jugendhilfe sowie eine gut funktionierende Jugendhilfe bei. Hierbei gelte es auch auf kulturelle Eigenschaften, sowie auf die Zusammenarbeit mit der übrigen Infrastruktur für Jugendliche zu achten (vergl. Bundesregierung 2009a: 71). Besonderen Wert lege die Regierung auf die Chancen von Kindern und Jugendlichen mit Migrationshintergründen. Noch zu häufig werden Talente von Kindern dieser Zielgruppe nicht gut erkannt bzw. genutzt. „Zu viele junge Migranten scheitern in Schule und Berufsausbildung. Die Länder haben sich im Nationalen Integrationsplan und der gemeinsamen Qualifizierungsinitiative verpflichtet die Zahl der Schulabbrecher mit Migrationshintergrund bis zum Schuljahr 20122013 auf den Gesamtschnitt aller Schüler zu reduzieren“ (Bundesregierung 2009a: 76). Als eine weitere Zielsetzung in der Kategorie der Kindeswohlfahrt nennt die Schwarz-Gelbe Koalition unter Merkel den von der Vorgängerregierung im Zusammenhang mit der Stellungnahme zum Dreizehnten Kinder- und Jugendbericht unterstrichenen Präventionsansatz. Sie greift ihn hier allerdings breiter auf und bezieht sich nicht nur auf die Gesundheit von Kindern und Jugendlichen. „Unsere Präventionsstrategie wird Vorhandenes bewerten und aufeinander abstimmen, nationale und internationale Erfahrungen und Erkenntnisse analysieren sowie auf bewährten 
Programmen und Strukturen aufbauen, diese weiterentwickeln und sie in die Fläche bringen“ (Bundesregierung 2009a: 85).

Auch in der wenige Wochen später folgenden Regierungserklärung vom 10.11.2009 nannte die Kanzlerin zwei Punkte, die im Rahmen dieser Zielkategorie von Belang sind. Erstens unterstreiche sie die Vergrößerung der Chancengleichheit für Kinder. Sie verweist auf wissenschaftliche Befunde aus denen hervorgehe, dass es in Deutschland große Unterschiede im Bezug auf die Chancengleichheit von Kindern gäbe. $\mathrm{Da}$ allerdings die Haupterziehungsverantwortung bei den Familien läge, wolle die neue Regierung diese besonders unterstützen. „Es ist ein Gebot der Gerechtigkeit, dass jeder die Chance erhält, im Berufsleben Erfolg zu haben. Schaffen werden wir dies aber nur, wenn die Politikbereiche zusammenwirken: für die Unterstützung von Familien, die Bildung, die Integration, die Arbeitsmarktpolitik und die sozialen Sicherungssysteme. Dabei kommt zweifelsohne den Familien die größte Aufgabe zu. Familien müssen deshalb besonders unterstützt werden“ (Bundesregierung 2009b: 9). Im Zusammenhang mit der Chancengleichheit der jüngeren Generationen steht auch das zweite Ziel, das die Kanzlerin in diesem Zusammenhang hervorhebt. Es ginge ihr um die Möglichkeiten zum Sozialaufstieg für alle Kinder durch die entsprechenden Bildungseinrichtungen. Da Bildung in Deutschland zur Kompetenz der Bundesländer gehört rufe sie diese auf, ihrer Verantwortung gerecht zu werden (vergl. Bundesregierung 2009b: 9). 
Tabelle 17: Chronologisches Übersichtstableau der auf das Kindeswohl gerichteten Zielsetzungen in Deutschland

\begin{tabular}{|c|c|c|}
\hline Jahr & Dokument & Zielsetzung \\
\hline 1998 & $\begin{array}{l}\text { Koalitionsvereinbarung der } \\
\text { ersten Rot-Grünen Koalition }\end{array}$ & $\begin{array}{l}\text { Recht auf Bildung für alle Kinder und Jugendliche } \\
\text { umsetzen, } \\
\text { Verringerung der Jugendarbeitslosigkeit, } \\
\text { Verstärkung von Kinderrechten, } \\
\text { Verbesserung der sozialen Lage in der Kinder } \\
\text { aufwachsen und Verkleinerung der Chance auf Armut }\end{array}$ \\
\hline 2000 & $\begin{array}{l}\text { Stellungnahme Sechster } \\
\text { Familienbericht }\end{array}$ & Verringerung der Jugendarbeitslosigkeit \\
\hline 2002 & $\begin{array}{l}\text { Stellungnahme Elfter Kinder- } \\
\text { und Jugendbericht }\end{array}$ & $\begin{array}{l}\text { Synergieeffekte mit positiven Auswirkungen auf } \\
\text { Leistungen für Jugendliche erzielen, } \\
\text { Führung einer Aktivierenden Jugendpolitik, die fördert } \\
\text { und fordert, } \\
\text { Verbesserung der Chancengleichheit von } \\
\text { Jugendlichen, } \\
\text { Mitarbeit an der Erstellung internationaler Maßstäben } \\
\text { zum Vergleich von Jugendpolitiken, } \\
\text { Ausbau der sozialen Infrastruktur, mit Name in der } \\
\text { Kinderbetreuung }\end{array}$ \\
\hline 2002 & $\begin{array}{l}\text { Koalitionsvereinbarung der } \\
\text { zweiten Rot-Grünen Koalition }\end{array}$ & $\begin{array}{l}\text { Wohnortunabhängige Verbesserung der } \\
\text { Chancengleichheit von Jugendlichen, } \\
\text { Quantitative und qualitative Verbesserung von } \\
\text { Ausbildungsplätzen, } \\
\text { Verringerung der Jugendarbeitslosigkeit }\end{array}$ \\
\hline 2003 & $\begin{array}{l}\text { Regierungserklärung der } \\
\text { zweiten Rot-Grünen Koalition }\end{array}$ & $\begin{array}{l}\text { Quantitative und qualitative Verbesserung von } \\
\text { Ausbildungsplätzen }\end{array}$ \\
\hline 2005 & $\begin{array}{l}\text { Stellungnahme Zwölfter Kinder- } \\
\text { und Jugendbericht }\end{array}$ & $\begin{array}{l}\text { Konsequenter Ausbau des öffentlichen Systems der } \\
\text { Kinderbetreuung von Kindertagesstätten bis zu } \\
\text { Ganztagsschulen, } \\
\text { Qualitative Verbesserung des Angebotes öffentlicher } \\
\text { Kinderbetreuung und Einführung eines bundesweit } \\
\text { wirksamen Qualitätssicherungssystems, } \\
\text { Umfassende Förderung der kindlichen und } \\
\text { jugendlichen Kompetenzen, } \\
\text { Deutliche Verringerung der Zahl der Schulabgänger } \\
\text { ohne Schulabschluss bis zum Ende des Jahrzehnts, } \\
\text { Mehr Chancengleichheit durch eine besondere } \\
\text { Förderung von Entwicklungsbedingungen von Kindern } \\
\text { aus benachteiligten Familien oder aus Familien mit } \\
\text { Migrationshintergrund, } \\
\text { Verbesserung der ressortübergreifenden Kooperation } \\
\text { und Einbindung verantwortlicher Gruppen und } \\
\text { Parteien }\end{array}$ \\
\hline
\end{tabular}




\begin{tabular}{|c|c|c|}
\hline 2005 & $\begin{array}{l}\text { Koalitionsvertrag der Großen } \\
\text { Koalition unter Merkel }\end{array}$ & $\begin{array}{l}\text { Quantitative und qualitative Verbesserung von } \\
\text { Ausbildungsplätzen, } \\
\text { Verringerung der Jugendarbeitslosigkeit, auf maximal } \\
\text { drei Monate pro Person, } \\
\text { Verbesserung der Chancengleichheit aller Kinder, } \\
\text { Stärkung von Kinderrechten und Teilhabe von Kindern } \\
\text { an gesellschaftlichen Prozessen, } \\
\text { Gewaltbereitschaft und Fremdenfeindlichkeit junger } \\
\text { Menschen bekämpfen, } \\
\text { Verbesserung der Chancengleichheit von } \\
\text { Migrantenkindern }\end{array}$ \\
\hline 2005 & $\begin{array}{l}\text { Regierungserklärung der } \\
\text { Großen Koalition unter Merkel }\end{array}$ & $\begin{array}{l}\text { Prinzipielle Eigenverantwortung von Eltern bis zum } 25 \text {. } \\
\text { Lebensjahr ihrer Kinder }\end{array}$ \\
\hline 2007 & $\begin{array}{l}\text { Stellungnahme Siebter } \\
\text { Familienbericht }\end{array}$ & Verbesserung der Chancengleichheit aller Kinder \\
\hline 2009 & $\begin{array}{l}\text { Stellungnahme Dreizehnter } \\
\text { Kinder- und Jugendbericht }\end{array}$ & $\begin{array}{l}\text { Verbesserung der Rahmenbedingungen des } \\
\text { Aufwachsens, } \\
\text { Motivierung einer gesunden Lebensweise von Eltern, } \\
\text { Unterstützung gesundheitlicher Förderung von } \\
\text { Kindern in schwierigen Lebensbedingungen, } \\
\text { Frühsignalisierung durch den Ausbau Früher Hilfen, } \\
\text { Verankerung von Gesundheitsförderung im direkten } \\
\text { Lebensumfeld von Kindern, } \\
\text { Verbesserung der Förderung behinderter Kinder und } \\
\text { Jugendlicher, } \\
\text { Verbesserung der Einbindung von Gesundheit und } \\
\text { Wohlbefinden in der Praxis der Kinder- und } \\
\text { Jugendhilfe }\end{array}$ \\
\hline 2009 & $\begin{array}{l}\text { Koalitionsvertrag der Schwarz- } \\
\text { Gelben Koalition unter Merkel }\end{array}$ & $\begin{array}{l}\text { Vergrößerung der Chancengleichheit für Kinder, } \\
\text { Steigerung von Studienanfängerquoten, } \\
\text { Stärkung der Erziehungsverantwortung von Eltern, } \\
\text { Schulen und Einrichtungen der Jugendarbeit, } \\
\text { Verbesserung der Kinder- und Jugendhilfe, } \\
\text { Verringerung der Schulabbrecherquote von Schülern } \\
\text { mit Migrationshintergrund, } \\
\text { Verbesserung der Prävention für Kinder und } \\
\text { Jugendliche }\end{array}$ \\
\hline 2009 & $\begin{array}{l}\text { Regierungserklärung der } \\
\text { Schwarz-Gelben Koalition unter } \\
\text { Merkel }\end{array}$ & $\begin{array}{l}\text { Vergrößerung der Chancengleichheit für Kinder, } \\
\text { Verbesserung der Schulausbildungen }\end{array}$ \\
\hline
\end{tabular}




\subsection{Familienpolitische Instrumente in Deutschland}

\subsubsection{Instrumente, die die ökonomische Situation der Familienhaushalte betreffen}

Im Koalitionsvertrag vom 20. Oktober 1998 hebt die erste Rot-Grüne Regierung hervor, dass sie als Schritt zur Bekämpfung von Kinderarmut „regelmäßig einen Armuts- und Reichtumsbericht erstellen" werde (Bundesregierung 1998a: 30). Die angekündigte Steuerreform und die Anpassung des Kindergeldes führten, laut Bundesregierung, zu einer erheblichen Entlastung der Familien. „Eine durchschnittliche Familie mit zwei Kindern wird um rund 2700 DM im Jahr entlastet. Das Kindergeld für das erste und zweite Kind wird 1999 auf 250 DM und im Jahr 2002 auf 260 DM im Monat angehoben“ (Bundesregierung 1998a: 34). Außerdem betont die neue Regierung, sie wolle das Erziehungsgeld umbauen $\mathrm{zu}$ einem Elterngeld und einen Elternurlaub einführen. Diese Instrumente werden allerdings erst später konkretisiert und daher an dieser Stelle nicht weiter ausgeführt.

In der dem Koalitionsvertrag folgenden Regierungserklärung vom 10. November 1998 unterstreicht und erklärt die Regierung die vorgenommenen Veränderungen im Bezug auf die Einkommenssteuer und das Kindergeld. „Die Einkommenssteuersätze werden nachhaltig gesenkt, das Kindergeld wird erhöht" (Bundesregierung 1998b: 3). Neben der Steuerreform nennt der Bundeskanzler in der Regierungserklärung auch die Rentenreform, die vor allem zu Gunsten der Frauen durchgeführt werde, die wegen ihrer Mutterschaft geringere (oder keine) Einkünfte auf dem Arbeitsmarkt erhielten. „So werden wir bei der Rentenreform selbstverständlich die Zunahme der so genannten unsteten Erwerbsläufe angemessen berücksichtigen. Insbesondere Frauen dürfen eben nicht dafür bestraft werden, dass sie ihr Leben flexibel gestalten, dass Phasen der Kindererziehung, der Erwerbsarbeit und des Lernens einander abwechseln“ (Bundesregierung 1998b: 13).

Die Ausführung der angekündigten Kindergelderhöhung beschreibt die Bundesregierung circa anderthalb Jahre später in der Stellungnahme zum Sechsten Familienbericht (vergl. Bundesministerium für Familie, Senioren, Frauen und Jugend 2000: 12). Sie betont die Einführung des 
Betreuungsfreibetrages, der im Familienlastenausgleich neben dem Kinderfreibetrag eingeführt werde. Weiterhin werde im „Rahmen des Steuerentlastungsgesetzes (...) der Eingangssteuersatz gesenkt: dies entlastet gerade Familien mit geringem und mittleren Einkommen. Gegenwärtig wird die Zweite Reformstufe des Familienlastenausgleichs, wie vom Bundesverfassungsgericht für das Jahr 2002 gefordert, vorbereitet" (Bundesministerium für Familie, Senioren, Frauen und Jugend 2000: 12). Ein weiterer Schwerpunkt dieses Familienberichts ist die Reform des Bundeserziehungsgesetzes. Durch diese Reform werde eine stärkere Beteiligung der Väter an der Kindeserziehung angestrebt. Sie gelte für Geburten ab dem 1. Januar 2001. „Die Reform erhöht unter anderem die Einkommensgrenzen und die Kinderzuschläge für den Bezug von Erziehungsgeld ab dem siebten Lebensmonat (je nach Kinderzahl um rund 10 bis 24 Prozent). Die Eltern können den Erziehungsurlaub (seitdem: ,Elternzeit') von insgesamt drei Jahren künftig auch gemeinsam nehmen. Sie haben während der Elternzeit einen grundsätzlichen Anspruch auf Verringerung ihrer Arbeitszeit - sowohl Väter als auch Mütter dürfen gleichzeitig bis zu jeweils 30 Wochenstunden erwerbstätig sein“ (Bundesministerium für Familie, Senioren, Frauen und Jugend 2000: 13). Eine besondere Aufmerksamkeit wird an dieser Stelle auch dem Risiko von Familien und Armut gewidmet. Neben der bereits eher angekündigten Einführung von Armuts- und Reichtumsberichten wolle die Koalition additionelle flankierende Maßnahmen treffen, um Armut von Familien zu bekämpfen.

Auch in der Stellungnahme zum Elften Kinder- und Jugendbericht, welche die erste Rot-Grüne Koalition zum Ende ihrer Regierungszeit dem Bundestag vorlegt wird ausführlich auf die Neuregelungen des zweiten Gesetzes zur Familienförderung, das zum 1. Januar 2002 in Kraft trat, eingegangen. Genannt wird an dieser Stelle die erneute Erhöhung des Kindergeldes. Außerdem wird der Kinderfreibetrag für das allgemein sächliche Existenzminimum den aktuellen Lebensverhältnissen angepasst (vergl. Bundesministerium für Familie, Senioren, Frauen und Jugend 2002: 14). Erhöht werde auch der zum 1. Januar 2000 eingeführte Betreuungsfreibetrag für den Erziehungsbedarf eines Kindes. Da dieser unabhängig vom Familienstand sei, werde parallel der „bisher auf allein Erziehenden beschränkte(n) Haushaltsfreibetrag“ (Bundesministerium für Familie, Senioren, Frauen und Jugend 2002: 14) abgebaut. Abgeschafft 
werde die Altersgrenze dieses Freibetrages (vorher 16 Jahre). „Für volljährige Kinder tritt an die Stelle des Betreuungs- und Erziehungsbedarfs der Ausbildungsbedarf“ (Bundesministerium für Familie, Senioren, Frauen und Jugend 2002: 14). „Für Kinder unter 14 Jahren wird ergänzend zum Familienleistungsausgleich ein Abzug für nachgewiesene erwerbsbedingte Betreuungskosten von 1500 Euro zugelassen, soweit diese Betreuungskosten den Betrag des bisherigen Betreuungsfreibetrages von 1548 Euro übersteigen“ (Bundesministerium für Familie, Senioren, Frauen und Jugend 2002: 14). Auch für volljährige Kinder, die sich in einer Ausbildung befänden und auswärts untergebracht seien, könnten Eltern einen Freibetrag erhalten. „Der Sonderausgabenabzug für Aufwendungen für ein hauswirtschaftliches Beschäftigungsverhältnis entfällt $\mathrm{ab}$ 2002, da die steuerliche Förderung dieser Beschäftigungsverhältnisse nicht $\mathrm{zu}$ den erhofften arbeitsmarktpolitischen Wirkungen geführt hat" (Bundesministerium für Familie, Senioren, Frauen und Jugend 2002: 14).

${ }^{12}$ In der Koalitionsvereinbarung der zweiten Rot-Grünen Koalition vom 16. Oktober 2002 betont die neue Bundesregierung die positiven Folgen der Steuerreform für Familien. Sie hebt hierbei insbesondere den Ausbau der „steuerliche(n) Abzugsfähigkeit von Betreuungskosten“ (Bundesregierung 2002: 30) hervor. Die Regierung verspreche dem Auftrag des Bundesverfassungsgerichts gerecht $\mathrm{zu}$ werden und bis Ende 2004 „die Erziehung von Kindern bei der Bemessung der Beiträge zur sozialen

12 Während des Bundestagswahlkampfes 2002 wurde das Gutachten der Kommission 'Moderne Dienstleistungen am Arbeitsmarkt' (Hartz-Kommission) vorgelegt. Aufgrund der Vorschläge dieser Kommission wurden erhebliche arbeitsmarkt- und sozialpolitische Reformen durchgeführt. Insgesamt kam es zu vier Umsetzungsschritten (Hartz I, II, III und IV). Zu den wichtigsten Reformzielen zählten die effizientere Gestaltung der Arbeitsmarktpolitik und die Reform der staatlichen Arbeitsvermittlung. Die Arbeitslosenzahl von 4 Millionen Menschen sollte um die Hälfte reduziert werden. Das aktivierende Schlagwort des ,Förderns und Forderns‘ wurde von großen Teilen der Bevölkerung mit den Hartz-Reformen in Verbindung gebracht. Da diese Reformen zu viel öffentlicher Kritik führten, wird auch das Leitbild ,Aktivierender Staat` (vor allem im Zusammenhang mit der deutschen Arbeitsmarktpolitik) oft negativ bewertet.

Die Hartz-Reformen sind für das Leben vieler Familien in Deutschland, bzw. von Menschen die eine Familie gründen möchten von großer Bedeutung. In der politischen Praxis der untersuchten Periode werden diese Reformen allerdings nicht im Rahmen der Familienpolitik sondern im Zusammenhang mit der Arbeitsmarktpolitik verwendet. Sie wurden daher im Rahmen dieser Untersuchung auch nicht analysiert. Eine Ausnahme ist der Kinderzuschlag. Durch den Kinderzuschlag werden vor allem Familien mit geringerem Einkommen gefördet. Der Unterschied zum Arbeitslosengeld II vergrößert sich durch diese Maßnahme und der Arbeitsanreiz steigt. Im Fazit dieser Arbeit werden - im Zusammenhang mit der Behandlung des Leitbildes ,Aktivierender Staat ${ }^{-}$ auch die Hartz-Reformen thematisiert. 
Pflegeversicherung zu berücksichtigen“" (Bundesregierung 2002: 51). Um die Lohnunterschiede zwischen den Geschlechtern zu verringern und mehr Chancengleichheit herzustellen, werden Maßnahmen getroffen wie z.B. die Neustrukturierung des Bundesangestelltentarifs und solle ein Bericht zur Bewertung von Arbeit ausgeführt werden. $\mathrm{Da}$ die bestehenden Bundesprogramme zur Förderung von Unternehmensgründungen bisher vorrangig auf Branchen die schwerpunktmäßig von Männern favorisiert werden gerichtet seien, wolle die neue Bundesregierung in Zukunft diese finanziellen Förderungen auch auf „den Bereich der Dienstleistungen, der vor allem von Frauen bevorzugt wird“ (Bundesregierung 2002: 61), ausbreiten.

${ }^{13}$ In der Stellungnahme der Bundesregierung auf den zwölften Kinder- und Jugendbericht kündigt die zweite Rot-Grüne Koalition kurz vor dem Ende ihrer Regierungsperiode an, sie werde überprüfen, wie „das Bundeserziehungsgeld, ähnlich wie in anderen europäischen Staaten, haushaltsneutral zu einem Elterngeld“ (Bundesministerium für Familie, Senioren, Frauen und Jugend 2005: 12f) entwickelt werden könne. Diese Maßnahme werde die Entscheidung zur Familiengründung erleichtern und Vätern reale Optionen bieten, um Zeit für Erziehung zu nehmen.

Die erste Koalition unter Merkel betont die Teilung der Verantwortlichkeiten und unterstreicht im Koalitionsvertrag vom 11. November 2005 sie halte Öffentlich-Private Partnerschaften für ein geeignetes Mittel zur Unterstützung finanzieller Maßnahmen. „Mit dem Gesetz zur Beschleunigung der Umsetzung von Öffentlich-Privaten Partnerschaften wurden in der 15. Legislaturperiode die gesetzlichen Rahmenbedingungen bereits verbessert. Mit einer Novellierung des Gesetzes soll dieser Weg nun fortgesetzt und weitere Hemmnisse abgebaut werden“ (Bundesministerium für Familie, Senioren, Frauen und Jugend 2005a: 21). Die unterschiedlichen bestehenden Programme und Maßnahmen zur Lohnergänzung „vom ergänzenden Arbeitslosengeld II über das Einstiegsgeld bis hin zum Kinderzuschlag“ (Bundesregierung 2005a: 32) wolle die neue Regierung in einer „Familienkasse“ (Bundesregierung 2005a: 118) bündeln und zusammenfassen, um mehr Transparenz über diese Finanzleistungen zu 
schaffen und eine Grundlage $\mathrm{zu}$ bieten für einen effizienteren Einsatz finanzieller Mittel zu Gunsten der Familien. Einführen wolle die Bundesregierung das bereits von ihren Vorgängern besprochene Elterngeld, mit dem ab 2007 eine einkommensabhängige Leistung für Eltern von Neugeborenen geschaffen werde. „Für ein Jahr erhält ein Elternteil $67 \%$ des letzten Nettoeinkommens bis $\mathrm{zu} 1800$ Euro monatlich“ (Bundesregierung 2005a: 79). Dieses Elterngeld richte sich an die Anspruchsberechtigten im Sinne des vorherigen Bundeserziehungsgeldgesetzes. Die Regierung weise darauf hin, dass die bisherigen Regelungen zur Elternzeit erhalten blieben und betone insbesondere: „Die zwölf Monate des Bezugszeitraums können zwischen den Eltern aufgeteilt werden. Zwei Monate bleiben dem Vater, zwei Monate der Mutter reserviert“ (Bundesregierung 2005a: 117). Beschäftigungsmöglichkeiten in privaten Haushalten wolle die neue Bundesregierung stärker unterstützen. Durch eine entsprechende Investierung wolle sie im Jahr 2006 „haushaltsnahe Dienstleistungen“ (Bundesregierung 2005a: 79) fördern. Weiterhin werde die Bundesregierung die Förderung von Familien mit Kindern verbessern. Die „Kinderzulage für die ab 1.1.2008 geborenen Kinder (wird) von dann 185 Euro auf 300 Euro jährlich erhöht“ (Bundesregierung 2005a: 97). Eine finanzielle Unterstützung erhalten die Eltern außerdem für die Kosten der verschiedenen Formen von Kinderbetreuung denn die Regierung habe sich zu Ziel gesetzt „die bereits in einigen Ländern vorgesehene bzw. umgesetzte Gebührenbefreiung der Eltern im letzten Kindergartenjahr bundesweit (...) (zu) realisieren“ (Bundesregierung 2005a: 113). Außerdem werde die Regierung Kinderbetreuungskosten bei der Einkommenssteuer berücksichtigen. Durch den Einsatz dieser Maßnahme wolle sie mit der "geminderten steuerlichen Leistungsfähigkeit von erwerbstätigen Eltern Rechnung“ (Bundesregierung 2005a: 113) tragen.

${ }^{14}$ In der Stellungnahme der Bundesregierung zum Siebten Familienbericht betont die Große Koalition unter Merkel die Möglichkeiten zum Ausbau der Beschäftigungsmöglichkeiten in Privathaushalten. Wie bereits angekündigt sehe sie Chancen in der Entwicklung dieses Sektors. „Deswegen hat die 
Bundesregierung die steuerliche Berücksichtigung von Aufwendungen für Dienstleitungen zur Betreuung von Kindern erheblich ausgebaut und die Spielräume für eine steuerliche Berücksichtigung privater Aufwendungen für haushaltsnahe Dienstleistungen deutlich erweitert" (Bundesministerium für Familie, Senioren, Frauen und Jugend 2006: 29). Durch das Gesetz zur Förderung von Wachstum und Beschäftigung werde die Inanspruchnahme von Betreuungsleistungen unterstützt. „Künftig können die meisten Eltern mit Kindern bis 14 Jahren zwei Drittel ihrer Aufwendungen, bis zu 4000 Euro jährlich je Kind, für die Betreuung ihrer Kinder steuerlich geltend machen. Mit dieser verbesserten steuerlichen Berücksichtigung von Kinderbetreuungskosten werde insgesamt die Nachfrage nach Betreuungsdienstleistungen gestärkt. Dies wirke sich nicht nur auf die Tagesmütter und insbesondere Kinderfrauen in Privathaushalten aus, sondern auch auf die Arbeitsplätze von Erzieherinnen und Erziehern in öffentlichen oder von freien Trägern betriebenen Kindertagesstätten“ (Bundesministerium für Familie, Senioren, Frauen und Jugend 2006: 29). Diese Regelung gelte rückwirkend zum 1. Januar 2006. Wie bereits in der Koalitionsvereinbarung verdeutlicht, werde in dieser Stellungnahme erneut die Einführung des Elterngeldes zum 1. Januar 2007 beschrieben. Auch der Kinderzuschlag, der bereits in der Koalitionsvereinbarung angekündigt sei, werde an dieser Stelle erneut erläutert. Die Regierung wolle die Armut von Familien mit allein erziehenden Eltern besser bekämpfen. Hierfür habe das „Bundesministerium für Familie, Senioren, Frauen und Jugend (...) mit dem Deutschen Jugendinstitut München ein integriertes Handlungskonzept entwickelt, in dem Wege für Alleinerziehende aufgezeigt werden, wie sie durch intensive Beratung und Betreuung durch die Jobcenter der Arbeitsgemeinschaften (ARGE) oder durch die so genannten Optionskommunen mit passgenauen Qualifizierungsmöglichkeiten, in Verbindung mit flexibler Kinderbetreuung und Unterstützungsnetzen in den Stadtquartieren in Erwerbstätigkeit integriert und von Leistungen der Grundsicherung für Arbeitssuchende unabhängig werden können“ (Bundesministerium für Familie, Senioren, Frauen und Jugend 2006: 34). Der Zugang zu den entsprechenden Informationen über finanzielle Maßnahmen zur Förderung von Familien wolle die Bundesregierung verbessern. Aus diesem Grund kündige sie an dieser Stelle einen zentralen Internet-Service (Familienwegweiser) an (vergl. Bundesministerium für Familie, Senioren, Frauen und Jugend 2006: 35). 
Kurz vor dem Ende ihrer gemeinsamen Regierungsphase reagierte die Große Koalition in ihrer Stellungnahme zum Dreizehnten Kinder- und Jugendbericht auf die Bedeutung des Wohlstands in den Familien für die Entwicklung der Kinder. Sie unterstreiche daher die finanziellen Leistungen die sie umsetzte, um die wirtschaftliche Stabilität von Familien zu verbessern und Armut von Kindern zu reduzieren. „Maßgebliche Beiträge haben die Einführung des Elterngeldes im Jahr 2007 sowie die Erhöhung und frühere und stärkere Staffelung des Kindergeldes zu Beginn 2009 geleistet“ (Bundesministerium für Familie, Senioren, Frauen und Jugend 2009: 6). Im Zusammenhang mit der Armutsreduzierung betone die Regierung weiterhin, dass sie aus internationalen Erfahrungen gelernt hätte, wie wichtig diese Investition in ein System finanzieller Leistungen und steuerlicher Maßnahmen neben der Bereitstellung einer geeigneten Infrastruktur für Familien seien. Insbesondere verwies die Regierung in diesem Zusammenhang auf die Einführung des Kinderzuschlags für Geringverdiener. „Durch gezielte Reformschritte wurde der Kinderzuschlag 2008 als ein maßgebliches Instrument zur Armutsreduktion mit speziellem Zuschnitt insbesondere auf Mehrkindfamilien mit geringem bzw. nur einem Einkommen gestärkt. Im Zusammenhang mit den ab 2009 geltenden Verbesserungen beim Wohngeld werden rund 150.000 Kinder mehr erreicht als bisher" (Bundesministerium für Familie, Senioren, Frauen und Jugend 2009: 7). Weiterhin weise die Regierung auf die zusätzlichen Leistungen, mit denen sie Familien mit geringerem Haushaltseinkommen bzw. einer im Verhältnis zum Einkommen größeren Anzahl von Kindern unterstützen wolle. „Sie (diese Familien) erhalten für die Schule zukünftig längstens bis zum Erwerb der Hochschulreife bzw. um höhere Schulabschlüsse zu erlangen zusätzlich einen pauschalen Betrag in Höhe von 100 Euro jährlich“ (Bundesministerium für Familie, Senioren, Frauen und Jugend 2009: 7). Auch Familien mit Sozialhilfe bzw. der Grundsicherung für Arbeitssuchenden und mit Kindern zwischen sechs und dreizehn Jahren würden von der Regierung zusätzlich unterstützt. Für diese Kinder „werden vom 1. Juli 2009 bis zum 31. Dezember 2011 die Regelsätze von 60 auf 70 Prozent der maßgebenden Regelleistung erhöht“ (Bundesministerium für Familie, Senioren, Frauen und Jugend 2009: 7). Nicht nur die Familien mit einem geringeren Haushaltseinkommen, sondern alle Familien wolle die Regierung im Jahr 2009 durch einen Kinderbonus (Einmalzahlung von 100 Euro) entlasten (vergl. Bundesministerium für Familie, Senioren, Frauen und Jugend 2009: 7). Armut 
sei vor allem für Familien mit alleinerziehenden Eltern ein großes Risiko, da die Vereinbarkeit von Beruf und Familien oft nur schwer möglich sei, bzw. das Haushaltseinkommen des Alleinverdieners in diesen Familien zu gering ausfalle. Oft seien diese Familien von Leistungen verschiedener Verwaltungsbereiche abhängig. „Vor diesem Hintergrund kooperieren das Bundesfamilienministerium und das Bundesministerium für Arbeit und Soziales mit der Bundesagentur für Arbeit im Rahmen einer strategischen Partnerschaft ,Perspektiven Alleinerziehende“ (Bundesministerium für Familie, Senioren, Frauen und Jugend 2009: 7).

Im Koalitionsvertrag der Schwarz-Gelben Koalition unter Merkel vom 26.10.2009 kündige die neue Regierung trotz der wirtschaftlich prekären Situation weitere finanzielle Entlastungen der Familien an. „Der Kinderfreibetrag wird in einem ersten Schritt zum 1.1.2010 auf 7008,- Euro und das Kindergeld um je 20,- Euro erhöht“ (Bundesregierung 2009a: 10). Die Modernisierung des Steuerrechts und die Vereinfachung bzw. Entbürokratisierung der Regelungen seien der Regierung wichtige Anliegen. Das betreffe auch die Familien. „Wir werden insbesondere: (...) ein schlüssiges und verständliches Konzept der steuerlichen Berücksichtigung von Aufwendungen für Familien und Kinder und im Haushalt, die steuerliche Abzugsfähigkeit von Ausbildungskosten neu ordnen (...) (Bundesregierung 2009a: 12). Aber auch im Hinblick auf die Erbschaftssteuer solle es - durch die Einführung eines Sofortprogramms - zu familiengerechterer Lösungen kommen und werde „die Steuerbelastung für Geschwister und Geschwisterkinder durch einen neuen Steuertarif von 15 bis 43 Prozent“ (Bundesregierung 2009a: 13) gesenkt. Die Wahlfreiheit zwischen der Betreuung von Kleinkindern (bis zu drei Jahren) zu Hause wolle die Regierung neben der Möglichkeit zur Betreuung in einer Kindertageseinrichtung unterstützen. Daher „soll ab dem Jahr 2013 ein Betreuungsgeld in Höhe von 150,- Euro, gegebenenfalls als Gutschein, für Kinder unter drei Jahren als Bundesleistung eingeführt werden“ (Bundesregierung 2009a: 68). Auch das Thema der Anerkennung von Erziehungsleistungen in der Alterssicherung greift diese Regierung auf. Der Gedanke kann als Teil der Realisierung des Prinzips der Wahlfreiheit zwischen Erziehung und Vereinbarkeit von Familie und Beruf interpretiert werden. „Wir werden im Rahmen der finanziellen Möglichkeiten prüfen, wie wir die familienpolitische Komponente stärken und deshalb 
Erziehungsleistungen in der Alterssicherung noch besser berücksichtigen können“ (Bundesregierung 2009a: 84). An die Verantwortung von Eltern, Verwandten und Paten appelliere die neue Regierung insbesondere im Bereich der finanziellen Vorsorge für die Ausbildung der Kinder. „Deshalb werden wir jedem neu geborenen Kind beispielsweise ein Zukunftskonto mit einem Startguthaben von 150 Euro einrichten und Einzahlungen bis zur Volljährigkeit mit einer Prämie unterstützen“ (Bundesregierung 2009a: 60). Die Förderung von Bildung sei eines der Hauptanliegen der neuen Regierung. Sie unterstreiche neben der Rolle der Schulen vor allem die Chancen der Schüler und Studenten im universitären Bereich. Talente gelte es besonders zu fördern. „Das bisherige Büchergeld der Begabtenförderungswerke wird auf 300 Euro angehoben und bleibt von der BAföG-Anrechnung befreit“ (Bundesregierung 2009a: 60). Familien würden in Deutschland mit sehr unterschiedlichen Maßnahmen unterstützt. Obwohl deutlich sei, dass ein Instrumentenmix zur Erreichung der verschiedenen familienpolitischen Zielsetzungen unabdingbar sei, wolle die Regierung die wissenschaftliche Evaluierung familienpolitischer Leistungen fortsetzen. „Ziel sind konkrete Handlungsempfehlungen, um Leistungen wirksamer und effizienter zu gestalten und zu bündeln. Weiterhin werden wir prüfen, wie die Leistungen im Unterhaltsrecht, Steuerrecht, Sozialrecht und Familienrecht harmonisiert werden können und entsprechende Schritte einleiten“ (Bundesregierung 2009a: 69). Lohnunterschiede zwischen Männern und Frauen wolle diese Regierung bekämpfen. „Wir werden in der Wirtschaft dafür werben, das beratungsunterstützte Lohntestverfahren Bogib-D einzusetzen. Hiermit sollen Entgeltunterschiede und deren Ursachen festgestellt werden“ (Bundesregierung 2009a: 74). Aber auch für den öffentlichen Dienst werde die neue Regierung darauf achten, dass es zu mehr Frauen- und Familienfreundlichkeit komme. Für Arbeit in Jugendfreiwilligendienstleistungen bzw. der Wehr- und Zivildienstzeit wolle die Regierung den Kindergeldbezug anpassen. „Der Kindergeldbezug in Zeiten geregelter und ungeregelter Jugendfreiwilligendienste wird vereinheitlicht, ein Kindergeldbezug während der Wehr- und Zivildienstzeit (...) geprüft“ (Bundesregierung 2009a: 80). Die Auszahlung des Kindergeldes bzw. steuerfinanzierte familienpolitische Leistungen wolle man modernisieren. „Wir werden prüfen, ob die von den Familienkassen durchgeführte Auszahlung des Kindergeldes einer anderen Stelle übertragen werden kann. Darüber hinaus 
wird geprüft, ob weitere steuerfinanzierte familienpolitische Leistungen zusammengefasst werden können“ (Bundesregierung 2009a: 83).

In ihrer Regierungserklärung vom 10.11.2009 unterstreicht die neue Regierung erneut die Bedeutung der Prävention von Kinderarmut und die Rolle, die der Kinderzuschlag in diesem Zusammenhang spielen könne. Die Regierung werde darum „den Kinderzuschlag weiterentwickeln, weil niemand wegen seiner Kinder in staatliche Abhängigkeit geraten sollte" (Bundesregierung 2009b: 7). Außerdem betonte die Kanzlerin in der Regierungserklärung die Wahlfreiheit von Eltern zwischen der selbstständigen Betreuung von Kindern bzw. der Betreuung in Kindertagesstätten. Wahlfreiheit setze Wahlmöglichkeit voraus und daher werde die Regierung neben des Ausbaus der Kinderbetreuung auch für die unter Dreijährigen eine finanzielle Regelung schaffen, denn zur „Wahlfreiheit im umfassenden Sinne gehört auch, dass wir für Eltern, die ihre Kinder zu Hause erziehen, ab 2013 ein Betreuungsgeld, gegebenenfalls auch als Gutschein, einführen wollen“ (Bundesregierung 2009b: 9).

Tabelle 18: Chronologisches Übersichtstableau der ökonomischen Instrumente in Deutschland

\begin{tabular}{|c|c|c|}
\hline Jahr & Dokument & Instrument \\
\hline 1998 & $\begin{array}{l}\text { Koalitionsvereinbarung der } \\
\text { ersten Rot-Grünen Koalition }\end{array}$ & $\begin{array}{l}\text { Regelmäßige Erstellung von Armuts- und } \\
\text { Reichtumsberichten, } \\
\text { Entlastung von Familien durch eine Steuerreform, } \\
\text { Erhöhung des Kindergeldes }\end{array}$ \\
\hline 1998 & $\begin{array}{l}\text { Regierungserklärung der ersten } \\
\text { Rot-Grünen Koalition }\end{array}$ & $\begin{array}{l}\text { Entlastung von Familien durch eine Steuerreform, } \\
\text { Erhöhung des Kindergeldes, } \\
\text { Besondere Berücksichtigung der unsteten } \\
\text { Erwerbsläufe bei der Rentenreform }\end{array}$ \\
\hline 2000 & $\begin{array}{l}\text { Stellungnahme Sechster } \\
\text { Familienbericht }\end{array}$ & $\begin{array}{l}\text { Entlastung von Familien durch eine Steuerreform, } \\
\text { Erhöhung des Kindergeldes, } \\
\text { Einführung des Betreuungsfreibetrages, } \\
\text { Reform des Bundeserziehungsgesetzes (Einführung } \\
\text { der Elternzeit), } \\
\text { Regelmäßige Erstellung von Armuts- und } \\
\text { Reichtumsberichten }\end{array}$ \\
\hline 2002 & $\begin{array}{l}\text { Stellungnahme Elfter Kinder- } \\
\text { und Jugendbericht }\end{array}$ & $\begin{array}{l}\text { Erhöhung des Kindergeldes, } \\
\text { Erhöhung des Kinderfreibetrages, } \\
\text { Erhöhung des Betreuungsfreibetrages (und } \\
\text { Abschaffung des Haushaltsfreibetrags und der } \\
\text { Altersgrenze für den Betreuungsfreibetrag), } \\
\text { Abzug von erwerbsbedingten Betreuungskosten im } \\
\text { Rahmen des Familienleistungsausgleichs, }\end{array}$ \\
\hline
\end{tabular}




\begin{tabular}{|c|c|c|}
\hline & & $\begin{array}{l}\text { Einführung eines Freibetrags von Auszubildenden, } \\
\text { die außer Haus wohnen, } \\
\text { Abschaffung des Sonderausgabenabzugs für } \\
\text { Aufwendungen für ein hauswirtschaftliches } \\
\text { Beschäftigungsverhältnis }\end{array}$ \\
\hline 2002 & $\begin{array}{l}\text { Koalitionsvereinbarung der } \\
\text { zweiten Rot-Grünen Koalition }\end{array}$ & $\begin{array}{l}\text { Ausbau der steuerlichen Abzugsfähigkeit von } \\
\text { Betreuungskosten, } \\
\text { Berücksichtigung von Erziehungszeiten in den } \\
\text { Beiträgen zur sozialen Pflegeversicherung, } \\
\text { Neustrukturierung Bundesangestelltentarif und } \\
\text { Fertigstellung eines Berichts zur Bewertung von } \\
\text { Arbeit, } \\
\text { Ausbreitung von finanziellen } \\
\text { Unterstützungsmaßnahmen zur Förderung von } \\
\text { Unternehmensgründungen im Bereich der } \\
\text { Dienstleistungen }\end{array}$ \\
\hline 2005 & $\begin{array}{l}\text { Stellungnahme Zwölfter Kinder- } \\
\text { und Jugendbericht }\end{array}$ & Ankündigung des neuen Elterngeldes \\
\hline 2005 & $\begin{array}{l}\text { Koalitionsvertrag der Großen } \\
\text { Koalition unter Merkel }\end{array}$ & $\begin{array}{l}\text { Abbau von Hemmnissen zur Behinderung von } \\
\text { Öffentlich-Privaten Partnerschaften, } \\
\text { Bündelung und Zusammenfassung verschiedener } \\
\text { finanzieller Förderinstrumente in einer } \\
\text { Familienkasse, } \\
\text { Einführung des neuen Elterngeldes, } \\
\text { Förderung haushaltsnaher Dienstleistungen, } \\
\text { Erhöhung der Kinderzulage, } \\
\text { Vorgenommene Realisierung der bundesweiten } \\
\text { Gebührenbefreiung von Eltern mit Kindern im letzten } \\
\text { Kindergartenjahr, } \\
\text { Berücksichtigung von Kinderbetreuungskosten bei } \\
\text { der Einkommenssteuer }\end{array}$ \\
\hline 2007 & $\begin{array}{l}\text { Stellungnahme Siebter } \\
\text { Familienbericht }\end{array}$ & $\begin{array}{l}\text { Erweiterung steuerlicher Berücksichtigungen für } \\
\text { haushaltsnahe Dienstleistungen, } \\
\text { Einführung des Elterngeldes, } \\
\text { Einführung des Kinderzuschlags (2008), } \\
\text { Ausarbeitung Handlungskonzept für } \\
\text { Alleinerziehende, } \\
\text { Einführung des Internetinstruments } \\
\text {,Familienwegweiser' }\end{array}$ \\
\hline 2009 & $\begin{array}{l}\text { Stellungnahme Dreizehnter } \\
\text { Kinder- und Jugendbericht }\end{array}$ & $\begin{array}{l}\text { Einführung des Elterngeldes und frühere und stärkere } \\
\text { Staffelung des Kindergeldes, } \\
\text { Einführung des Kinderzuschlags (2008), } \\
\text { Auszahlung eines Pauschalbetrags für höhere } \\
\text { Schulabschlüsse von Kindern aus } \\
\text { Geringverdienerfamilien, }\end{array}$ \\
\hline
\end{tabular}




\begin{tabular}{|c|c|c|}
\hline & & $\begin{array}{l}\text { Einführung zusätzlicher Hilfen für } \\
\text { Geringverdienerfamilien im Rahmen des } \\
\text { Konjunkturprogramms II, } \\
\text { Einmalzahlung eines Kinderbonus für alle } \\
\text { Kindergeldberechtigten, } \\
\text { Einführung der strategischen Partnerschaft } \\
\text { „Perspektiven Alleinerziehende‘ }\end{array}$ \\
\hline 2009 & $\begin{array}{l}\text { Koalitionsvertrag der Schwarz- } \\
\text { Gelben Koalition unter Merkel }\end{array}$ & $\begin{array}{l}\text { Vorgenommene Erhöhung des Kindergeldes und des } \\
\text { Kinderfreibetrages, } \\
\text { Geplante Vereinfachung des Systems steuerlicher } \\
\text { Aufwendungen für Familien, } \\
\text { Geplante Entbürokratisierung der Regelungen bei der } \\
\text { Erbschaftssteuer, } \\
\text { Vorgenommene Einführung eines Betreuungsgeldes } \\
\text { für Kinder unter drei Jahren, } \\
\text { Prüfung einer möglichen Stärkung von } \\
\text { Erziehungsleistungen in der Alterssicherung, } \\
\text { Geplante Einrichtung eines Zukunftskontos für } \\
\text { Kinder, } \\
\text { Geplante Anhebung des Büchergeldes der } \\
\text { Begabtenförderungswerke, } \\
\text { Fortsetzung der wissenschaftlichen Evaluation } \\
\text { familienbezogener Leistungen, } \\
\text { Vorgenommene Einsetzung des Lohntestverfahrens } \\
\text { für Frauen, } \\
\text { Geplante Vereinheitlichung des Kindergeldbezuges in } \\
\text { Zeiten geregelter und ungeregelter } \\
\text { Jugendfreiwilligendienste, } \\
\text { Prüfung einer möglichen Veränderung der } \\
\text { Auszahlung finanzieller Leistungen für Familien }\end{array}$ \\
\hline 2009 & $\begin{array}{l}\text { Regierungserklärung der } \\
\text { Schwarz-Gelben Koalition unter } \\
\text { Merkel }\end{array}$ & $\begin{array}{l}\text { Geplante Weiterentwicklung des Kinderzuschlags, } \\
\text { Vorgenommene Einführung eines Betreuungsgeldes } \\
\text { für Kinder unter drei Jahren }\end{array}$ \\
\hline
\end{tabular}




\subsubsection{Instrumente, die die ökologischen Bedingungen des Familienlebens betreffen}

In der Koalitionsvereinbarung der ersten Rot-Grünen Koalition vom 20. Oktober 1998 betont die neu gewählte Bundesregierung bessere Rahmenbedingungen für den Ausbau von „Kinderbetreuungsangeboten aller Altersgruppen“ (Bundesregierung 1998a: 34) schaffen $\mathrm{zu}$ wollen. Vom Ausbau der Kindertagesbetreuung würden vor allem die Frauen profitieren und ihre Rolle auf dem Arbeitsmarkt verstärkt werden. Den Schutz von Frauen und Kindern vor Gewalt wolle die neue Bundesregierung gewährleisten. Hierzu sei nicht nur der weitere Ausbau von Frauenhäusern und Schutzwohnungen von großer Bedeutung. Auch wolle die neue Regierung "für von Gewalt bedrohte bzw. betroffene Frauen und ihre Kinder die vereinfachte Wohnungszuweisung" regeln (Bundesregierung 1998a: 35f). Die entsprechenden Täter wolle die RotGrüne Koalition nicht nur konsequent verfolgen sondern auch bestrafen.

${ }^{15}$ In der Stellungnahme der Bundesregierung zum Sechsten Familienbericht hebt die Rot-Grüne Koalition den Ausbau der Kinderbetreuung hervor. „Hier sind noch erhebliche Anstrengungen erforderlich, bis ein angemessenes Angebot an Tageseinrichtungen für Kinder und an Ganztagsschulen erreicht ist. Dabei sind in erster Linie die Länder und Kommunen angesprochen. Die Bundesregierung arbeitet ihrerseits an Vorschlägen zur Verbesserung des Angebots an Tagesbetreuung" (Bundesministerium für Familie, Senioren, Frauen und Jugend 2000: 13). Ein weiterer Schwerpunkt der Maßnahmen ziele auf die Verbesserung des sozialen Lebensumfeldes in Umgebungen mit hoher Zuwanderungsrate. „Unter anderem für diese Stadt- und Ortsteile ist in Ergänzung der bisherigen Städtebauförderung das neue Bund-Länder Gemeinschaftsprogramm ,Stadtteile mit besonderem Entwicklungsbedarf - die soziale Stadt' geschaffen worden, mit dem in den Wohnquartieren durch Bündelung und koordiniertem Einsatz aller stadtentwicklungspolitisch bedeutsamen Ressourcen und Maßnahmen zusätzliche Möglichkeiten zur 
Integration von Zuwanderfamilien geschaffen werden“ (Bundesministerium für Familie, Senioren, Frauen und Jugend 2000: 26).

Die erste Rot-Grüne Regierung unterstreicht auch in der Stellungnahme auf den Elften Kinder- und Jugendbericht das Projekt „Stadtteile mit besonderem Entwicklungsbedarf - die soziale Stadt" als eine wichtige politische Maßnahme. Die Regierung wolle in diesem Projekt verschiedene Handlungsfelder verknüpfen, um Familien in den entsprechenden Quartieren und Stadteilen optimal zu fördern. „Dabei soll eine möglichst breite Mitwirkung der Bevölkerung und der Akteure in den Stadtteilen sichergestellt werden und dies eine verlässliche Grundlage für die Aktivierung privater Potenziale sein“ (Bundesministerium für Familie, Senioren, Frauen und Jugend 2002: 5f). Um die Vereinbarkeit von Familie und Beruf besser zu unterstützen, wolle die Regierung „Dokumentationen und Analysen der Kinderbetreuungsangebote, insbesondere über Krippen- und Hortplätze“ (Bundesministerium für Familie, Senioren, Frauen und Jugend 2002: 8) anfertigen lassen. Außerdem stärke sie Familien durch „höhere Einkommensgrenzen und Leistungsverbesserungen bei Erziehungsgeld, Wohngeld und BAföG“ (Bundesministerium für Familie, Senioren, Frauen und Jugend 2002: 14).

Die zweite Rot-Grüne Koalition unterstreicht in ihrem Koalitionsvertrag (11.11.2005) die Bedeutung der Qualitätsverbesserung von vorschulischen Kinderbetreuungseinrichtungen in Ostdeutschland. „Hier müssen die aufgestockten Mittel dazu verwendet werden, die Qualität und die Ausstattung der bestehenden Einrichtungen und Schulen $\mathrm{zu}$ verbessern und/oder die Elternbeiträge $\mathrm{zu}$ senken“ (Bundesregierung 2002: 25). In den westlichen Bundesländern, die zum Teil an einem erheblichen Mangel an Betreuungsplätzen leiden, wolle die Bundesregierung vor allem den quantitativen Ausbau von Betreuungsplätzen vorantreiben, so dass der Betreuungsgrad von Kindern unter drei Jahren auf $20 \%$ liege. „Wir werden 10.000 zusätzliche Ganztagsschulen aufbauen und die Betreuung der Kinder unter drei Jahren qualitativ und quantitativ deutlich verbessern. Durch den Aufbau von 10.000 zusätzlichen Ganztagsschulen und die sinkenden Kinderzahlen ständen in den nächsten Jahren 500.000 Kindergarten- und Hortplätze zur Verfügung. Die Bundesregierung erwarte von Ländern und Kommunen, die freiwerdenden Plätze nicht abzubauen, sondern in Betreuungsangebote für unter Dreijährige und Ganztagsplätze im Kindergarten 
umzuwandeln“ (Bundesregierung 2002: 29). Selbst stelle die Bundesregierung mit dem „Programm ,Zukunft Bildung und Betreuung' im Zeitraum von 2003 bis 2007 vier Milliarden (Euro) für die Einrichtung von Ganztagsschulen zur Verfügung“ (Bundesregierung 2002: 30f). In allen Bundesländern wolle die Regierung die qualitative Verbesserung der Kinderbetreuung und Vereinbarkeit von Beruf und Familie realisieren. „Wir werden alle an Fragen der Kinderbetreuung Beteiligten - Bund, Länder, Kommunen, Freie Träger, Unternehmen - zu einem Gipfel für Bildung und Betreuung einladen, auf dem wir gemeinsam Vereinbarungen über die bedarfsgerechte und qualitativ hochwertige Kinderbetreuung treffen werden. Um den Fortzug junger Menschen aus verschiedenen Regionen zu bremsen, werde die Regierung das Lebensumfeld junger Familien verbessern (durch u.a. den Wettbewerb ,Die Jugend bleibt') (vergl. Bundesregierung 2002: 26). Außerdem wolle die Regierung auch den Grad des Wohneigentums von Familien steigern. „Wir werden die Alt- und Neubauförderung angleichen und auf Familien mit Kindern konzentrieren. Eigenheimförderung und Altersvorsorge werden besser miteinander verzahnt“ (Bundesregierung 2002: 59).

${ }^{16}$ In der Stellungnahme der Bundesregierung zum 12. Kinder- und Jugendbericht betont die zweite Rot-Grüne Koalition kurz vor dem Ende ihrer Regierungsperiode die Bedeutung des Investitionsprogramms ,Zukunft Bildung und Betreuung' (IZBB) sowie das Gesetz zum qualitätsorientierten und bedarfsgerechten Ausbau der Tagesbetreuung für Kinder (Tagesbetreuungsausbaugesetz - TAG) (vergl. Bundesministerium für Familie, Senioren, Frauen und Jugend 2005: 4). In den Kindertagesstätten sehe die Bundesregierung die Möglichkeit zur Bündelung verschiedener familienorientierter Angebote. Hierdurch erhoffe sie die Schaffung sozialer Anlaufstellen. „Gemeint sind Einrichtungen, die über die Betreuung, Erziehung und Bildung der Kinder hinaus weitere familienorientierte Angebote und Dienste, wie etwa Bildungs- und Beratungsangebote oder Treffpunkte integrieren. Die Bundesregierung verfolgt das Ziel, nach dem Vorbild der englischen ,Early Excellence Centres’ Kindertageseinrichtungen zu Eltern-Kind- 
Zentren unter Berücksichtigung bereits vorhandener einschlägiger Aktivitäten von Ländern und Kommunen zu entwickeln, in denen Bildung, Beratung und Betreuung innovativ verbunden werden“ (Bundesministerium für Familie, Senioren, Frauen und Jugend 2005: 15). Eine Gebührenfreiheit für die Betreuungseinrichtungen von Kindern wolle sie nicht direkt verordnen, da dies die Möglichkeiten der kommunalen Haushalte übersteige. Allerdings wolle die „Bundesregierung sich gemeinsam mit Ländern und Kommunen für eine schrittweise Gebührenfreiheit einsetzen“ (Bundesministerium für Familie, Senioren, Frauen und Jugend 2005: 20). Den Ausbau der Kinderbetreuung werde die Bundesregierung qualitativ unterstützen. „Durch die bundesweite Informationskampange ,KINDER KRIEGEN mehr...!' sollen Eltern über die Möglichkeiten für eine gute Betreuung und frühe Förderung informiert" (Bundesministerium für Familie, Senioren, Frauen und Jugend 2005: 20) werden. Die qualitative Verbesserung der Kindertagespflege sei eine weitere Maßnahme, denn die Bundesregierung sehe hier wichtige Chancen zur Anfüllung der Infrastruktur der Kindertagesbetreuungsstätten. Mit einem Online-Handbuch wolle sie am Ausbau beteiligte Verantwortungsträger über Qualitätsstandards informieren (vergl. Bundesministerium für Familie, Senioren, Frauen und Jugend 2005: 21). Den Aufbau der Infrastruktur zur Kinderbetreuung unterstütze die Regierung außerdem durch weitere Forderungen an die am kindlichen Entwicklungsprozess beteiligten Akteure. „Das TAG ist der erste Teil einer Reform des Kinder- und Jugendhilfegesetzes, der zweite Teil - das Gesetz zur Weiterentwicklung der Kinder- und Jugendhilfe (KICK) - (...) ergänzt das TAG an für eine gelingende Kooperation der am Entwicklungsprozess des Kindes beteiligten Akteure entscheidenden Stellen“ (Bundesministerium für Familie, Senioren, Frauen und Jugend 2005: 33f) wie z.B. die Verpflichtung der Tageseinrichtungen, um mit Schulen oder anderen Einrichtungen der Familienbildung zu kooperieren.

In der Koalitionsvereinbarung der Großen Koalition unter Merkel betont die Bundesregierung sie wolle die aktivierenden Qualitäten der traditionellen Großfamilien nutzen und auf die Lebensweise heutiger Familien übersetzen. Dies gelinge der Bundesregierung durch die Einführung von Mehrgenerationenhäusern. In diesen Häusern könne Erziehung und sozialer Zusammenhang zwischen den Generationen unterstützt werden. „Die Mehrgenerationenfamilie bietet die Chance Verantwortung füreinander $\mathrm{zu}$ 
übernehmen. Wir werden Modelle entwickeln, wie mehrere Generationen zusammen leben und Verantwortung füreinander übernehmen können“ (Bundesregierung 2005a: 17). Deshalb stelle die Bundesregierung in dieser Legislaturperiode eine Start-Up Förderung für die Schaffung eines Mehrgenerationenhauses in jedem Landkreis und in jeder kreisfreien Stadt in Deutschland bereit. Zum Ausbau der Kinderbetreuungsinfrastruktur führe die neue Regierung einerseits das von der zweiten Rot-Grünen Regierung initiierte „Investitionsprogramm ,Zukunft, Bildung und Betreuung' zum Bau von Ganztagsschulen“ (Bundesregierung 2005a: 41) fort. Andererseits wolle sie auch die Infrastruktur zur Tagesbetreuung kleiner Kinder ausbauen. „Die Koalitionspartner stehen $\mathrm{zu}$ dem mit dem Tagesbetreuungsausbaugesetz verankerten Ausbau der Betreuungsangebote für unter dreijährige Kinder als einer unabweisbaren gesellschaftspolitischen Aufgabe, für die auch der Bund Verantwortung trägt. Bis zum Jahr 2010 entstehen 230.000 zusätzliche Betreuungsplätze“ (Bundesregierung 2005a: 112). Falls mehr als 10\% der Kommunen das geforderte Angebot 2010 nicht leisten könnten drohe die Regierung mit einem Rechtsanspruch für Kinder ab dem zweiten Lebensjahr. Aus Kostengründen sollten Kinder, so die Bundesregierung, nicht von der Kinderbetreuung fernbleiben dürfen. „Wir wollen deshalb mit den Ländern gemeinsam nach Wegen suchen, die bereits in einigen Ländern vorgesehene bzw. umgesetzte Gebührenbefreiung der Eltern im letzten Kindergartenjahr bundesweit zu realisieren“ (Bundesregierung 2005a: 113). Die Bestrebungen der vorigen Bundesregierung, um Kräfte auf kommunaler Ebene besser zu stärken und zu nutzen, werden darum weiter unterstützt. „Die Initiative ,Lokale Bündnisse' operiert in den Kommunen. Familienfreundlichkeit ist ein Standortfaktor, der nicht nur die Lebensqualität sondern auch die Wertschöpfungs- und Wirtschaftskraft von Kommunen und Regionen erhöht. In den Lokalen Bündnissen arbeiten Kommunen, Kammern, Verbände, Wirtschaft und soziale Organisationen erfolgreich zusammen, um attraktive Lebensbedingungen für Familien zu schaffen (...) Die Zahl von heute 200 bestehenden Bündnissen und weiteren 200 in Gründung begriffenen Standorten soll weiter steigen“ (Bundesregierung 2005a: 116).

In der Regierungserklärung dieser Großen Koalition (30.11.2005) wird die Bedeutung des selbst genutzten Wohneigentums für die Altersvorsorge betont. Die Bundesregierung wolle ,auch die kapitalgedeckte Altersversorgung für 
junge Familien deutlich verbessern und das selbst genutzte Wohneigentum in die Altersversorgung integrieren" (Bundesregierung 2005b: 5). Weiterhin werden an dieser Stelle die bereits in der Koalitionsvereinbarung genannten Maßnahmen zum Ausbau der Kindertagesbetreuung von unter Dreijährigen und der Mehrgenerationenhäuser erneut hervorgehoben.

Nur kurze Zeit nach dem Regierungsantritt betont die Regierung in ihrer Stellungnahme zum Siebten Familienbericht die Bedeutung des Projekts zur Errichtung von Mehrgenerationenhäuern. „Erforderlich ist ein neuer gemeinwesenorientierter Ansatz der Förderung, Unterstützung und Hilfe für Familien durch die Ansiedlung von Angeboten aus einer Hand und unter einem Dach. Diesem Gedanken tragen verschiedene neue Angebotsformen, insbesondere aber das in 2006 umzusetzende Bundesmodellprojekt zur Einrichtung von Mehrgenerationenhäusern, Rechnung. Für die Gewährleistung einer guten Infrastruktur gibt es eine gemeinsame Verantwortung von Bund, Ländern, Kommunen, der Wirtschaft und anderen gesellschaftlichen Akteuren (Bundesministerium für Familie, Senioren, Frauen und Jugend 2006: 25). Hingewiesen wird auch auf das Tagesbetreuungsausbaugesetz (TAG), den eventuellen Rechtsanspruch auf Betreuung der Zweijährigen, das Ganztagsschulenprogramm und das - inzwischen bestehende - Angebot eines Online-Handbuchs zur Qualitätssicherung in der Tagesbetreuung. Zum Ausbau der Kinderbetreuung ist aus Sicht der Bundesregierung auch das freiwillige Engagement von Unternehmen wichtig. „Der Ausbau der betrieblichen und betrieblich unterstützten Kinderbetreuung ist ein Schwerpunkt im Rahmen der ,Allianz für die Familie'. Die vielfältigen Möglichkeiten reichen vom klassischen Betriebskindergarten über Kinderbetreuungszuschüsse bis hin zu Belegplätzen in öffentlichen Kindertagesstätten. Innovative Lösungen ergeben sich durch die Kooperation von Unternehmen mit Partnern aus anderen gesellschaftlichen Bereichen in lokalen Bündnissen für Familie“ (Bundesministerium für Familie, Senioren, Frauen und Jugend 2006: 26). Die Bundesregierung betone in dieser Stellungnahme die Verantwortungsteilung zwischen den verschiedenen gesellschaftlichen Akteuren. „Der ,ermöglichende' Staat behält die öffentliche Verantwortung, ohne alles selbst tun zu wollen oder zu müssen. Viele Ziele lassen sich durch die Kooperation sich ergänzender (lokaler) Partner erreichen. Die Bundesregierung setze deshalb auf strategische lokale Partnerschaften und unterstützt deren Gründung. Aus diesem Grund habe das Bundesministerium 
für Familie, Senioren, Frauen und Jugend 2004 die Initiative ,Lokale Bündnisse für Familie' ins Leben gerufen“ (Bundesministerium für Familie, Senioren, Frauen und Jugend 2006: 29).

In der Stellungnahme zum Dreizehnten Kinder- und Jugendbericht, die die Große Koalition kurz vor dem Ende ihrer Regierungszeit an den Bundestag schickte, werden die Erweiterung des bereits bestehenden Bund-LänderProgramms ,Soziale Stadt' und die Möglichkeit zur Finanzierung gesundheitsbezogener Projekte betont (vergl. Bundesministerium für Familie, Senioren, Frauen und Jugend 2009: 4, 12). Außerdem weise die Regierung auf die Entwicklungen im Bereich der Kindertagesbetreuung im Zusammenhang mit dem Inkrafttreten des Kinderförderungsgesetzes. „Bis 2013 soll für 35 Prozent der Kinder in Deutschland ein Betreuungsplatz in einer Kindertagesstätte oder in der Tagespflege zur Verfügung stehen. Ab dem 1. August 2013 hat jedes Kind einen Rechtsanspruch auf einen Betreuungsplatz“ (Bundesministerium für Familie, Senioren, Frauen und Jugend 2009: 5). Mit einer großen Summe unterstütze die Bundesregierung im Zusammenhang mit dem konjunkturpolitischen Maßnahmenpaket II die Bildungsinfrastruktur von Kindern und Jugendlichen (vergl. Bundesministerium für Familie, Senioren, Frauen und Jugend 2009: 5). Zuletzt wiederholt sie die besondere Bedeutung der - während ihrer Koalitionsperiode gestarteten - Einführung bzw. des Ausbaus der Mehrgenerationenhäuser, denn die „MGH verbinden bürgerschaftliches Engagement, Selbsthilfe und professionelle Unterstützung. Sie schaffen mit ihren Angeboten eine niedrigschwellige Infrastruktur, auf die alle Generationen vor Ort zurückgreifen können“ (Bundesministerium für Familie, Senioren, Frauen und Jugend 2009: 8).

Im Koalitionsvertrag der Schwarz-Gelben Koalition unter Merkel wird die Verbesserung des qualitativen und quantitativen Ausbaus der Kinderbetreuung in seiner Trägervielfalt unterstrichen. Besser vernetzt werden sollen diese Einrichtung mit der übrigen Familieninfrastruktur. „Wir werden darauf hinwirken, dass sich Bund und Länder auf gemeinsame Eckpunkte der frühkindlichen Bildung, insbesondere auch der Sprachförderung, einigen. Wir begrüßen eine freiwillige Zertifizierung der Einrichtungen bei wissenschaftlicher Begleitung. Um qualifiziertes Personal zu gewinnen, wird eine Verbesserung der Rahmenbedingungen angestrebt“ (Bundesregierung 2ooga: 68). 
In der zum Koalitionsvertrag gehörenden Regierungserklärung betont die Bundeskanzlerin am 10.11.2009 sie wolle die Wahlfreiheit für Eltern im Bezug auf die Vereinbarkeit von Beruf und Familie weiter verbessern. Sie nenne insbesondere die Fortsetzung der bereits beschlossenen Einrichtung von 230.000 zusätzlichen Betreuungsplätzen für unter Dreijährige bis zum Jahr 2010. „Wahlfreiheit setzt Wahlmöglichkeit voraus. Deshalb führen wir weiter, was begonnen wurde, nämlich den Ausbau der Kinderbetreuung auch für die unter Dreijährigen, eine Verbesserung sowohl im Umfang als auch in der Qualität“ (Bundesregierung 2009b: 9).

Tabelle 19: Chronologisches Übersichtstableau der ökologischen Instrumente in Deutschland

\begin{tabular}{|c|c|c|}
\hline Jahr & Dokument & Instrument \\
\hline 1998 & $\begin{array}{l}\text { Koalitionsvereinbarung der } \\
\text { ersten Rot-Grünen Koalition }\end{array}$ & $\begin{array}{l}\text { Ausbau von Kinderbetreuungsangeboten aller } \\
\text { Altersgruppen, } \\
\text { Vereinfachte Wohnungszuweisung für von Gewalt } \\
\text { bedrohte bzw. betroffene Frauen und ihre Kinder }\end{array}$ \\
\hline 2000 & $\begin{array}{l}\text { Stellungnahme Sechster } \\
\text { Familienbericht }\end{array}$ & $\begin{array}{l}\text { Vorschläge zur Verbesserung des Angebots an } \\
\text { Tagesbetreuung, } \\
\text { Durchführung des Projekts ,Stadtteile mit } \\
\text { besonderem Entwicklungsbedarf - die soziale Stadt' }\end{array}$ \\
\hline 2002 & $\begin{array}{l}\text { Stellungnahme Elfter Kinder- } \\
\text { und Jugendbericht }\end{array}$ & $\begin{array}{l}\text { Durchführung des Projekts ,Stadtteile mit } \\
\text { besonderem Entwicklungsbedarf - die soziale Stadt', } \\
\text { Anfertigung von Dokumentationen und Analysen der } \\
\text { Kinderbetreuungsangebote, insbesondere über } \\
\text { Krippen- und Hortplätze, } \\
\text { Hantierung höherer Einkommensgrenzen und } \\
\text { Leistungsverbesserungen bei Erziehungsgeld, } \\
\text { Wohngeld und BaföG }\end{array}$ \\
\hline 2002 & $\begin{array}{l}\text { Koalitionsvereinbarung der } \\
\text { zweiten Rot-Grünen Koalition }\end{array}$ & $\begin{array}{l}\text { Qualität und Ausstattung der bestehenden } \\
\text { Infrastruktur für Kinder in Ostdeutschland verbessern } \\
\text { und/oder die Elternbeiträge senken, } \\
\text { Ausbau von Ganztagsschulen und } \\
\text { Kinderbetreuungseinrichtungen für unter Dreijährige } \\
\text { in Westdeutschland durch das Programm ,Zukunft } \\
\text { Bildung und Betreuung', } \\
\text { Durchführung eines Gipfels für Bildung und } \\
\text { Betreuung, } \\
\text { Durchführung des Wettbewerbs ,Die Jugend bleibt', } \\
\text { Bessere Gestaltung der Förderung von } \\
\text { Wohnungseigentum von Familien }\end{array}$ \\
\hline 2005 & $\begin{array}{l}\text { Stellungnahme Zwölfter Kinder- } \\
\text { und Jugendbericht }\end{array}$ & $\begin{array}{l}\text { Fortführung des Investitionsprogramms ,Zukunft, } \\
\text { Bildung und Betreuung' zum Bau von }\end{array}$ \\
\hline
\end{tabular}




\begin{tabular}{|c|c|c|}
\hline & & $\begin{array}{l}\text { Ganztagsschulen, } \\
\text { Aufbau von 230.000 zusätzlichen Betreuungsplätzen } \\
\text { für unter Dreijährige bis zum Jahr 2010, } \\
\text { Entwicklung von Kindertageseinrichtungen zu Eltern- } \\
\text { Kind-Zentren nach dem Vorbild der englischen ,Early } \\
\text { Excellence Centres', } \\
\text { Einführung eines Mehrgenerationenhauses in jedem } \\
\text { Landkreis und in jeder kreisfreien Stadt in } \\
\text { Deutschland, } \\
\text { Einsatz für eine schrittweise Gebührenfreiheit von } \\
\text { Kinderbetreuung, } \\
\text { Durchführung der bundesweiten } \\
\text { Informationskampagne ,KINDER KRIEGEN mehr...!, } \\
\text { Entwicklung eines Online - Handbuchs für den } \\
\text { qualitätsorientierten Ausbau der Kindertagespflege, } \\
\text { Inkrafttreten des Gesetz zur Weiterentwicklung der } \\
\text { Kinder- und Jugendhilfe (KICK) }\end{array}$ \\
\hline 2005 & $\begin{array}{l}\text { Koalitionsvertrag der Großen } \\
\text { Koalition unter Merkel }\end{array}$ & $\begin{array}{l}\text { Einführung eines Mehrgenerationenhauses in jedem } \\
\text { Landkreis und in jeder kreisfreien Stadt in } \\
\text { Deutschland, } \\
\text { Fortführung des Investitionsprogramm ,Zukunft, } \\
\text { Bildung und Betreuung' zum Bau von } \\
\text { Ganztagsschulen, } \\
\text { Aufbau von 230.0oo zusätzlichen Betreuungsplätzen } \\
\text { für unter Dreijährige bis zum Jahr 2o10, } \\
\text { Gebührenbefreiung der Eltern im letzten } \\
\text { Kindergartenjahr bundesweit realisieren, } \\
\text { Ausbau der Initiative ,Lokale Bündnisse' }\end{array}$ \\
\hline 2005 & $\begin{array}{l}\text { Regierungserklärung der } \\
\text { Großen Koalition unter Merkel }\end{array}$ & $\begin{array}{l}\text { Integration des selbst genutzten Wohneigentums in } \\
\text { die Altersversorgung, } \\
\text { Aufbau von } 230.000 \text { zusätzlichen Betreuungsplätzen } \\
\text { für unter Dreijährige bis zum Jahr 2010, } \\
\text { Einführung eines Mehrgenerationenhauses in jedem } \\
\text { Landkreis und in jeder kreisfreien Stadt in } \\
\text { Deutschland }\end{array}$ \\
\hline 2007 & $\begin{array}{l}\text { Stellungnahme Siebter } \\
\text { Familienbericht }\end{array}$ & $\begin{array}{l}\text { Einführung von Mehrgenerationenhäusern in } \\
\text { gemeinsamer Verantwortung von Bund, Ländern, } \\
\text { Kommunen, der Wirtschaft und anderen } \\
\text { gesellschaftlichen Akteuren, } \\
\text { Aufbau von 230.0oo zusätzlichen Betreuungsplätzen } \\
\text { für unter Dreijährige bis zum Jahr 2010, } \\
\text { Fortführung des Investitionsprogramms ,Zukunft, } \\
\text { Bildung und Betreuung' zum Bau von } \\
\text { Ganztagsschulen, } \\
\text { Entwicklung eines Online - Handbuchs für den } \\
\text { qualitätsorientierten Ausbau der Kindertagespflege, }\end{array}$ \\
\hline
\end{tabular}




\begin{tabular}{|l|l|l|}
\hline \multirow{2}{*}{2009} & Stellungnahme Dreizehnter & Entwicklung des Programms ,Allianz für die Familie' \\
& Kinder- und Jugendbericht & Stweiterung des Bund-Länder-Programms ,Soziale \\
& & Inkrafttreten des Kinderförderungsgesetz, \\
& & Förderung des Zukunftsinvestitionsgesetzes im \\
& Rahmen des Maßnahmenpakets II, \\
& Einführung von Mehrgenerationenhäusern in \\
& gemeinsamer Verantwortung von Bund, Ländern, \\
& & $\begin{array}{l}\text { Kommunen, der Wirtschaft und anderen } \\
\text { gesellschaftlichen Akteuren }\end{array}$ \\
\hline 2009 & Koalitionsvertrag der Schwarz- & Verbesserung der Trägervielfalt und der \\
& Gelben Koalition unter Merkel & Qualifikationen in der Kindertagesbetreuung \\
\hline 2009 & Regierungserklärung der & Fortsetzung des Aufbaus von 230.ooo zusätzlichen \\
& Schwarz-Gelben Koalition unter & Betreuungsplätzen für unter Dreijährige bis zum Jahr \\
& Merkel & 2010 \\
\hline
\end{tabular}




\subsubsection{Instrumente zur Förderung oder Wiederherstellung von Kompetenzen der Familienangehörigen}

In der Koalitionsvereinbarung der ersten Rot-Grünen Koalition wird betont, dass die Bekämpfung von Arbeitslosigkeit und Jugendarbeitslosigkeit nicht nur eine Sache der Regierung sei, sondern dass es sich um ein gesellschaftliches Anliegen handele, für das es viele Verantwortliche gäbe. „Zur Bekämpfung der Arbeitslosigkeit wird die neue Bundesregierung alle gesellschaftlichen Kräfte mobilisieren. Wir wollen ein Bündnis für Arbeit und Ausbildung“ (Bundesregierung 1998a: 8). Gemeinsam mit Unternehmen und Gewerkschaften wolle die Bundesregierung konkrete Maßnahmen vereinbaren, um Arbeitslosigkeit abzubauen. Als ersten Schritt nach ihrem Amtsantritt werde die neue Regierung ein Sofortprogramm starten mit dem sie 100.000 Jugendliche so schnell wie möglich in Ausbildung und Beschäftigung bringen wolle. „Zu dem Sofortprogramm gehört auch die Möglichkeit, Schulabschlüsse nachzuholen. Alle Jugendlichen, die länger als sechs Monate arbeitslos sind, sollen einen Ausbildungsplatz, einen Arbeitsplatz oder eine Fördermaßnahme erhalten“ (Bundesregierung 1998a: 8). Auch die Qualität der Ausbildungen wolle die neue Bundesregierung modernisieren und verbessern und eine neue Ausbildungsoffensive starten. „Wir werden die Benachteiligtenprogramme bedarfs- und zielgerecht weiterentwickeln, damit auch lernschwächere junge Menschen die Chance auf eine qualifizierte Berufsausbildung und damit auf eine berufliche Zukunft erhalten“ (Bundesregierung 1998a: 25). Lebenslanges Lernen erscheine der Regierung stets notwendiger. Um diese Entwicklung zu unterstützen wolle sie den „Ausbau und (die) Verankerung der Weiterbildung als vierte Säule des Bildungssystems" (Bundesregierung 1998a: 25) vorantreiben. Auch im Bereich der Hochschulen sehe die neue Bundesregierung einige Maßnahmen vor. So werde sie ab 1999 eine Reform der Ausbildungsförderung durchführen, um die Ausbildungsförderung grundlegend $\mathrm{zu}$ verbessern, das Hochschulrahmengesetz weiterzuentwickeln und dabei die Erhebung von Studiengebühren auszuschließen. Sie wolle die Hochschulen zu Zukunftswerkstätten umbauen, um sie attraktiver $\mathrm{zu}$ gestalten (vergl. Bundesregierung 1998a: 25). Da die Regierung gesellschaftliches Engagement stärken und nutzen wolle, kündigt sie an, sie werde rechtliche und 
institutionelle Hindernisse zur Selbsthilfe abbauen, Zugänge zum sozialen Engagement erleichtern und Freiwilligendienste ausbauen und absichern (vergl. Bundesregierung 1998a: 32). Zur Stärkung gesellschaftlicher Kompetenzen von Kindern und Jugendlichen werde die Bundesregierung über den Bereich der kontinuierlichen Maßnahmen hinaus noch extra Schwerpunkte setzen wie z.B. die Förderung der politischen Partizipation (vergl. Bundesregierung 1998a: 33). Insbesondere wolle die erste Rot-Grüne Koalition auch die gleichberechtigte Teilhabe von Frauen in Beruf und Gesellschaft fördern. Hierzu starte sie ab 1999 das Aktionsprogramm ,Frau und Beruf, denn nur „wenn sich das geistige Potential und die Kreativität der Frauen in Wirtschaft und Gesellschaft entfalten können, wird unser Land die Herausforderungen der Zukunft bestehen“ (Bundesregierung 1998a: 35). Zu den Maßnahmen dieses Aktionsplans zähle die neue Regierung die Einführung eines effektiven Gleichstellungsgesetzes, die gleichberechtigte Teilhabe von Frauen an der Arbeitsförderung, Unterstützung von Existenzgründungen von Frauen, Ausbau der Teilzeitmöglichkeiten etc..

In der den Koalitionsvertrag begleitenden Regierungserklärung der ersten RotGrünen Koalition werden die Bedeutung des neuen Sofortprogramms zur Beschäftigung und Ausbildung von Jugendlichen, sowie das Bündnis für Arbeit und Ausbildung erneut unterstrichen. Hinzugefügt wird das Streben nach einem europäischen Beschäftigungspakt. „In ihm sollen ausdrücklich verbindliche Ziele zum Abbau der Jugend- und Langzeitarbeitslosigkeit, sowie zur Überwindung der Diskriminierung von Frauen auf dem Arbeitsmarkt aufgenommen werden“" (Bundesregierung 1998b: 21).

Die Regierung betont in ihrer Stellungnahme zum Sechsten Familienbericht die sie circa anderthalb Jahre nach ihrem Regierungsantritt an den Bundestag schickte - die große Bedeutung der Vereinbarkeit von Familie und Beruf. Erneut hebe sie das 1999 initiierte Programm ,Frau und Beruf hervor, „das Schritt für Schritt umgesetzt wird und bereits nach einem Jahr gute Erfolge zeigte“ (Bundesministerium für Familie, Senioren, Frauen und Jugend 2000: 13). Die Stärkung ausländischer Frauen und Mädchen unterstütze die Bundesregierung in zweierlei Hinsicht. Einerseits solle anhand eines Forschungsberichts zur Integration in die deutsche Gesellschaft verdeutlicht werden, wo Defizite bzw. Chancen seien. Außerdem würden in Modellprogrammen „Projekte und Trägereinrichtungen, die an (...) spezifischen Konfliktlagen von Kindern und Jugendlichen, insbesondere von Mädchen ausländischer Herkunft“ 
(Bundesministerium für Familie, Senioren, Frauen und Jugend 2000: 19) anknüpfen, unterstützt. Die Bundesregierung schildert die ersten Erfahrungen mit dem Sofortprogramm für Ausbildung und Beschäftigung und kündigt weitere Maßnahmen an. „Der Anteil der jungen Ausländerinnen und Ausländer an den verschiedenen Maßnahmen des Sofortprogramms betrug 1999 rund 13 Prozent. Um diesen Anteil weiter zu steigern, ist in den ab Januar 2000 geltenden Richtlinien festgeschrieben worden, dass ausländische Jugendliche ohne Ausbildung und Arbeitsplatz entsprechend ihrem Anteil an der Jugendarbeitslosigkeit $\mathrm{zu}$ berücksichtigen sind“ (Bundesministerium für Familie, Senioren, Frauen und Jugend 2000: 22). An dieser Stelle nennt die Regierung das von ihr initiierte Bündnis für Arbeit, Ausbildung und Wettbewerbsfähigkeit. Im Rahmen dieses Bündnisses „wurden weit reichende Aktivitäten und Leitlinien zur Sicherung eines ausreichenden Ausbildungsplatzangebotes insbesondere in den neuen Ländern, zur Förderung von Jugendlichen mit schlechteren Startchancen, zur Früherkennung von neuem Qualifikationsbedarf sowie zur strukturellen Weiterentwicklung der dualen Berufsausbildung und der beruflichen Weiterbildung vereinbart“ (Bundesministerium für Familie, Senioren, Frauen und Jugend 2000: 24). Einer besonderen Aufmerksamkeit bedürfe, aus Sicht der Bundesregierung, die Situation ausländischer Auszubildender. „Um insbesondere neuen Projekten den Einstieg in die Arbeit zu erleichtern sowie den Erfahrungsaustausch zwischen den einzelnen Initiativen zu optimieren, wurde im Juli 1999 die ,Koordinierungsstelle Ausbildung in ausländischen Unternehmen' (KAUSA) (...) mit dem Ziel eingerichtet, in den nächsten drei Jahren durch eine zentrale Informations- und Koordinierungsarbeit die Anzahl der Ausbildungsplätze in Unternehmen mit Inhabern ausländischer Herkunft zu erhöhen“ (Bundesministerium für Familie, Senioren, Frauen und Jugend 2000: 24). Neben der bereits genannten Programme hat das Bundesministerium für Familie, Senioren, Frauen und Jugend „das Programm ,Entwicklung und Chancen junger Menschen in sozialen Brennpunkten (E\&C)' ins Leben gerufen“ (Bundesministerium für Familie, Senioren, Frauen und Jugend 2000: 24). Zielgruppe dieses Programms seien junge Zuwanderer. Innerhalb eines Jahres solle ihnen anhand maßgeschneiderter Trainingsmaßnahmen fehlende soziale und berufliche Schlüsselqualifikationen vermittelt werden. Als essentiell für die Integration in die deutsche Gesellschaft bzw. dem deutschen Arbeitsmarkt halte die Regierung die entsprechenden Sprachkenntnisse. Sie lege deshalb auf 
Sprachförderungsmaßnahmen großes Gewicht und arbeite „an einem gruppenübergreifenden Gesamtkonzept für ein völlig neues System der Sprachförderung mit der Leitidee, in Zukunft alle Zuwanderer mit einer dauerhaften Aufenthaltsperspektive gleich zu behandeln“ (Bundesministerium für Familie, Senioren, Frauen und Jugend 2000: 25).

In ihrer Stellungnahme auf den Elften Kinder- und Jugendbericht beschreibt die erste Rot-Gründe Koalition die weitere Intensivierung der bestehenden Förderungsmaßnahmen in sozialen Brennpunkten und Projekte zur Förderung freiwilligen Engagements. Genannt werden die Reform der Ausbildungsförderung und die im Bündnis für Arbeit, Ausbildung und Wettbewerbsfähigkeit beschlossenen Grundsätze zur Modernisierung der Ausbildung. Im Bereich der Kleinkindentwicklung und -betreuung unterstütze die Bundesregierung Berufe in der Kindertagesbetreuung durch z.B. das Projekt „Zum Bildungsauftrag von Kindertageseinrichtungen' (...) Es wurden Materialien für Erzieherinnen entwickelt, mit denen sie im Rahmen des Situationsansatzes die Bildungsprozesse von Kindern ermöglichen, unterstützen und herausfordern können“ (Bundesministerium für Familie, Senioren, Frauen und Jugend 2000: 16). Auch Eltern werden durch verschiedene Projekte in ihrer Erziehungsfähigkeit unterstützt. Basierend auf den Erfahrungen aus dem Sofortprogramm der Bundesregierung für Beschäftigung und Ausbildung habe die Bundesregierung beschlossen „erfolgreiche Elemente des Sofortprogramms (...) ab 2004 in das Arbeitsförderungsrecht“ (Bundesministerium für Familie, Senioren, Frauen und Jugend 2000: 17) aufzunehmen. Für besonders benachteiligte Kinder und Jugendliche unterstützt die Bundesregierung Programme wie z.B. ,Arbeitsweltbezogene Jugendsozialarbeit' und ,Freiwilliges Soziales Trainingsjahr' um „individuelle(n) Bedürfnisse(n) und Kompetenzen von besonders benachteiligten Jugendlichen" (Bundesministerium für Familie, Senioren, Frauen und Jugend 2000: 17) gerecht zu werden. Auch im Bereich des Umgangs mit neuen Technologien unterstütze die Bundesregierung diverse Programme wie z.B. ,Jugendarbeit ans Netz' und ,Benachteiligte ans Netz'.

In der Koalitionserklärung der zweiten Rot-Grünen Koalition (16.10.2002) spielt die Stärkung von Kompetenzen der Jugendlichen eine Rolle. Das JUMP-PlusProgramm werde initiiert, um "Jugendlichen nach der Erstausbildung eine Brücke in den Arbeitsmarkt" (Bundesregierung 2002: 26) zu bauen. Im östlichen Teil des Landes wolle man dieses Programm durch Zusatzmodule 
erweitern, um Jugendlichen, die in Teilzeit arbeiten bei möglichen extra Qualifizierungen zu unterstützen. Die Stärkung von Bildung und die Qualität der Bildung werde die zweite Rot-Grüne Koalition durch verschiedene Maßnahmen vorantreiben. So solle z.B. eine unabhängige Expertenkommission alle zwei Jahre einen Bildungsbericht vorlegen und „Empfehlungen für die Weiterentwicklung des Bildungswesens erarbeiten“ (Bundesregierung 2002: 31). Neben dieser direkt an der Praxisarbeit orientierten Maßnahme wolle die Regierung auch die Bildungsforschung unterstützen. Daher richte sie die Stiftung ,Bildung und Erziehung' ein. Diese Organisation werde eine Grundlage sein „für einen „breiten bildungspolitischen Dialog (...) (der) auch bundesweit innovative Programme, Initiativen und Projekte fördern soll“ (Bundesregierung 2002: 31). Weil der Frauenanteil im Hochschulbereich zu niedrig sei, wolle die Regierung die Rolle von Frauen in Lehre und Forschung stärken. Sie werde die Frauenfreundlichkeit von Hochschulen verbessern durch z.B. die Etablierung weiterer Frauenstudiengänge und „eines bundesweiten Netzwerkes für internationale Frauenstudiengänge“ (Bundesregierung 2002: 33). Außerdem wolle die Bundesregierung Frauen im Berufsbildungsbereich insbesondere fördern, um hierdurch ein breiteres Berufsbildungsspektrum von Frauen zu erreichen. So soll z.B. das „Programm ,Innovation und Arbeitsplätze Im Informationszeitalter des 21. Jahrhundert'“ eingesetzt werden, um den Frauenanteil in IT-Berufen $\mathrm{zu}$ erweitern“ (Bundesregierung 2002: 33). Konsequenter als zuvor wolle die Bundesregierung den 3. Artikel des Grundgesetzes umsetzen. Durch Gender Mainstreaming und Gender Budgeting soll Frauenförderung in Zukunft besser verankert werden. Die Regierung werde „ein Gender-Kompetenzzentrum aufbauen, das die Einführung von Gender Mainstreaming in alle Politikbereiche unterstützt" (Bundesregierung 2002: 62f). Zusätzlich wolle die Regierung das Bundesgremienbesetzungsgesetz überarbeiten und die Repräsentanz von Frauen in Gremien des Bundes gewährleisten. Eine weitere Zielgruppe dieser Regierung seien die Jugendlichen, die sie insbesondere durch eine Allianz mit Kindern und Jugendlichen zum Mitgestalten gewinnen wolle. Mit der Bundesinitiative ,Jugend ans Netz' werde die Bundesregierung „den bereits vorhandenen Internetzugängen in der Schule auch Internetzugänge in allen Einrichtungen der Jugendhilfe schaffen“ (Bundesregierung 2002: 57f). 
${ }^{17}$ In der Stellungnahme auf den 12. Kinder- und Jugendbericht betont die zweite Rot-Grüne Koalition die Bedeutung der modernen Kinder- und Jugendhilfe für die Entwicklung von jungen Menschen. Auch das neu verabschiedete Gesetz zur Weiterentwicklung der Kinder- und Jugendhilfe (KICK) wird in diesem Zusammenhang begrüßt. Durch eine bessere Verzahnung der verschiedenen Aufgabenbereiche müssten Übergänge von Kindergarten in die Schule besser gesichert werden. „Das TAG ist der erste Teil einer Reform des Kinder- und Jugendhilfegesetzes, der zweite Teil- das Gesetz zur Weiterentwicklung der Kinder- und Jugendhilfe (KICK) (...) ist auch in Bezug auf den Ausbau der Tagebetreuung ein großer Erfolg. Es ergänzt das TAG an für eine gelingende Kooperation der am Entwicklungsprozess des Kindes beteiligten Akteure entscheidenden Stellen " (Bundesministerium für Familie, Senioren, Frauen und Jugend 2005: 33f). Die weitere Modernisierung und Vernetzung des bestehenden Angebots in der Jugendhilfe halte die Regierung für entscheidend und wolle sie fördern durch Programme wie ,E\&C - Entwicklung und Chancen junger Menschen in sozialen Brennpunkten'. Das Prinzip des Angebots aus einer Hand müsse aus Sicht der Bundesregierung auch im Bereich der Kindertagesbetreuung realisiert werden (vergl. Bundesministerium für Familie, Senioren, Frauen und Jugend 2005: 15). Die Bedeutung von Erziehung durch Eltern werde durch die Regierung auf verschiedene Arten unterstützt. Das Recht auf eine gewaltfreie Erziehung wurde in den betreffenden Gesetzen verankert und gelte somit als Leitbild in der Erziehung. Weiterhin betone die Regierung die Initiative ,Verantwortung Erziehung', die sie gemeinsam mit den beiden großen Kirchen durchführe. „Ziel ist die gemeinsame Erarbeitung eines ,Erziehungskontraktes', bei dem es um Elternkompetenz und die werteorientierte Erziehung in Institutionen und durch Tageseltern geht" (Bundesministerium für Familie, Senioren, Frauen und Jugend 2006: 14). Auch die Kompetenzen im Umgang mit Medien und neuen Technologien wolle die Bundesregierung unterstützen durch z.B. die Kampagne ,SCHAUH HIN! Was deine Kinder machen' (vergl. Bundesministerium für Familie, Senioren, Frauen und Jugend 2005: 15f). Das Bundesministerium für Familie, Senioren, Frauen 
und Jugend fördere diese Kampagne gemeinsam mit dem Magazin HÖRZU, dem Telekommunikationsunternehmen ARCOR, der ARD und dem ZDF. Die Regierung verstehe diese Zusammenarbeit als „ein gelungenes Projekt einer so genannten Public Private Partnership, die die gesellschaftliche und gemeinschaftliche Verantwortung für das Aufwachsen von Kindern sowie für die Bedingungen von Erziehung aufgreift" (Bundesministerium für Familie, Senioren, Frauen und Jugend 2005: 15f). Ein weiteres Projekt in diesem Bereich ist die Etablierung der „Initiative D 21 ein Internetportal für die in den Tageseinrichtungen tätigen Fachkräfte“ (Bundesministerium für Familie, Senioren, Frauen und Jugend 2005: 24). Auch dieses Projekt führe die Regierung gemeinsam mit Partnerunternehmen durch. Eine qualitative Verbesserung des Bildungssystems erhoffe sich die Bundesregierung aus der Erstellung eines Bildungsberichts (vergl. Bundesministerium für Familie, Senioren, Frauen und Jugend 2005: 25). Die Sprachförderung von besonders benachteiligten Kindern und Jugendlichen sei der Bundesregierung ein großes Anliegen. Als entscheidend für die passende Förderung bewerte die Bundesregierung daher das "5-jährige Modellprogramm ,Förderung von Kindern und Jugendlichen mit Migrationshintergründen - FörMig' der BundLänder-Kommission für Bildungsplanung und Forschungsförderung (BLK)“ (Bundesministerium für Familie, Senioren, Frauen und Jugend 2005: 26).

In der Koalitionsvereinbarung der Großen Koalition unter Merkel betont die neue Regierung sie werde „den ,Nationalen Pakt für Ausbildung und Fachkräftenachwuchs' in Deutschland fortführen. Konkret wolle sie jährlich 30.000 neue Ausbildungsplätze sowie 25.000 betriebliche Einstiegsqualifizierungen schaffen. Hilfsbedürftige junge Menschen wolle die Bundesregierung bei der Einführung in den Arbeitsmarkt in besonderem Maße unterstützen. „Das neue System der Grundsicherung für Arbeitsuchende sieht eine konsequente Aktivierung insbesondere junger hilfsbedürftiger erwerbsfähiger Menschen vor. Diesen Jugendlichen wird ein persönlicher Ansprechpartner und Arbeitsvermittler zur Seite gestellt. Dieser soll künftig flächendeckend höchstens 75 Jugendliche betreuen und kann so im direkten Kontakt ihre Integration gezielt verbessern“ (Bundesregierung 2005a: 28f). Für die Erziehung im breiteren Sinn wolle die Regierung verschiedene Projekte initiieren. So soll z.B. gemeinsam mit den beiden großen Kirchen und den Wohlfahrtsverbänden werteorientierte Erziehung gefördert werden durch die 
Initiative ,Verantwortung Erziehung' (vergl. Bundesregierung 2005a: 116). Mehr berufsorientierte Erziehung solle z.B. realisiert werden durch die Umsetzung des durch die EU initiierten ,Pakts für die Jugend' in dem regionale und nationale Kinder- und Jugendangebote gefördert und die finanzielle Ausstattung des EU-Programms ,Jugend in Aktion' unterstützt werden. Auch sei das Berufswahlspektrum von Mädchen und Jungen ein Anliegen dieser Regierung. Dieses Spektrum solle erweitert und Geschlechtsstereotypen überwunden werden. Der neu initiierte ,Girls Day' sei eine der von der Bundesregierung genannte Maßnahme aus diesem Bereich. Zur Verstärkung der Gleichstellung von Frauen und Männern wolle die Bundesregierung einen ,Bericht zur Gleichstellung von Frauen und Männern' vorlegen. In einer nachfolgenden Regierungserklärung sollen die Fortschritte und Defizite der Gleichstellungspolitik verdeutlicht werden. „Dieser Bericht ersetzt auch alle durch das BMFSFJ bislang abzugebenden Detailberichte der Bundesregierung zu den Themen der Frauen- und Gleichstellungspolitik“ (Bundesregierung 2005a: 119).

${ }^{18}$ Kurze Zeit später betont die Große Koalition in der Stellungnahme auf den Siebten Familienbericht die Bedeutung der Vermittlung von Werten in der Erziehung (vergl. Bundesministerium für Familie, Senioren, Frauen und Jugend 2006: 28). Daher wolle sie gemeinsam mit den beiden großen Kirchen und weiteren Experten aus der Praxis ein ,Bündnis für Erziehung' initiieren. Mit den entsprechenden Dialogen erhoffe sich die Regierung die Verbesserung der Rahmenbedingungen des Aufwachsens.

In der Stellungnahme der Bundesregierung zum Dreizehnten Kinder- und Jugendbericht, den die Große Koalition kurz vor dem Ende ihrer Regierungsperiode an den Bundestag schickte, steht die gesundheitliche Entwicklung von Kindern im weitesten Sinne im Mittelpunkt der Betrachtungen. Da die Eltern die Erziehungsverantwortung für ihre Kinder trügen, hierbei allerdings unter Umständen Hilfe bräuchten, wolle die Regierung gemeinsam mit den übrigen Einrichtungen wie z.B. Schulen und 
Kindertagesstätten durch die Bundesinitiative für gesundes Ernährungs- und Bewegungsverhalten ,IN FORM‘ Eltern befähigen bzw. aktivieren, um die physische und psychische Wohlfahrt ihrer Kinder erkennen zu können (vergl. Bundesministerium für Familie, Senioren, Frauen und Jugend 2009: 5). Vor allem in sozial benachteiligten Familien sei die Früherkennung und Befähigung der Eltern wichtig. Gesundheitsprobleme gingen oft mit übrigen sozialen Problemen einher. Aus diesem Grund wolle die Regierung die verschiedenen Akteure, die den Familien zur Verfügung ständen zur besseren Kooperation verhelfen. „Ein gutes Beispiel ist der Kooperationsverbund ,Gesundheitsförderung bei sozial Benachteiligten' der von der BZgA im Auftrag des Bundesgesundheitsministeriums initiiert wurde. Mit ihm ist es gelungen, auf Bundesebene die relevanten Akteure der Gesundheitsförderung mit dem Ziel der Verbesserung gesundheitlicher Chancen zusammenzuführen“ (Bundesministerium für Familie, Senioren, Frauen und Jugend 2009: 8). Ein weiteres Instrument zur Förderung der Entwicklung junger Kinder sei die Hinzufügung einer weiteren Früherkennungsuntersuchung. „Im Rahmen der Früherkennungsuntersuchungen für Kinder wurde zum 1. Juli 2008 eine zusätzliche Untersuchung U 7a vom 34. bis zum 36. Lebensmonat als Leistung der gesetzlichen Krankenversicherung eingeführt" (Bundesministerium für Familie, Senioren, Frauen und Jugend 2009: 8). Aufgrund der großen Bedeutung der frühkindlichen Entwicklung und der Möglichkeiten, die sich für die Kinder durch eine Frühförderung ergäben, wolle die Regierung Eltern und Kinder in diesem Bereich noch stärker unterstützen. Familien mit Migrationshintergrund sollten hiervon in besonderem Maße profitieren. „Mit dem Anstoß zum Ausbau Früher Hilfen durch das Aktionsprogramm ,Frühe Hilfen für Eltern und Kinder und soziale Frühwarnsysteme' verfolgt die Bundesregierung vor allem das Ziel, mittels selektiver Prävention einen Beitrag für einen verbesserten Schutz von Kindern insbesondere von der vorgeburtlichen Zeit bis zum Alter von etwa drei Jahren zu leisten“ (Bundesministerium für Familie, Senioren, Frauen und Jugend 2009: 9).

Auch im Koalitionsvertrag der Schwarz-Gelben Koalition unter Merkel vom 26.10.2009 ist eine große Vielzahl von Instrumenten zur Wiederherstellung bzw. Förderung der Kompetenzen verschiedener Familienangehöriger aufgenommen. Die neue Regierung betone, dass sie Bildung als eine gesamtstaatliche Aufgabe sehe und wolle daher mit allen Verantwortlichen der 
Bildungskette eng zusammenarbeiten. Sie investiere in Bildung und Forschung und fordere eine bessere Anerkennung von Abschlüssen und Teilleistungen. „Wir werden vor Ort Bildungsbündnisse aller relevanten Akteure - Kinder- und Jugendhilfen, Eltern, Schulen, Arbeitsförderung sowie Zivilgesellschaft fördern die sich mit diesem Ziel zusammenschließen. Wir werden ihre Arbeit unterstützen, indem jedes Bündnis ein Kontingent z.B. von Bildungsschecks zur Weitergabe an benachteiligte Kinder und Jugendliche erhält" (Bundesregierung 2009a: 59). Chancengleichheit von Kindern wolle die neue Regierung verstärken. $\mathrm{Zu}$ früh werde in Deutschland über die Zukunft von Kindern entschieden. Die Integration verschiedener Bevölkerungsgruppen sei daher nur schwer möglich bzw. unmöglich. Als einen Schlüssel zur besseren Integration sehe die Regierung die Sprachfähigkeit der Kinder. Sie wolle Eltern hierbei in ihrer Erziehungsverantwortung unterstützen durch verschiedene Maßnahmen wie z.B. den Ausbau der Bildungseinrichtungen und Kampagnen wie ,Deutsch lernen - Deutschland kennen lernen“ (vergl. Bundesregierung 2009a: 59f). Wichtig für die Entwicklung und Betreuung von Kindern seien auch die Kompetenzen des Betreuungspersonals. Insbesondere werde die Regierung hierbei auch auf die Betreuung in der Tagespflege achten. „Um die Attraktivität der Kindertagespflege zu erhöhen, wollen wir die Qualifikationen der Tagespflegepersonen weiterentwickeln und schärfen. Die Anrechenbarkeit der erworbenen Qualifikation auf die Ausbildung in pädagogischen Berufen soll erreicht werden“ (Bundesregierung 2009a: 68). Zur Realisierung von Chancengleichheit gehöre auch die Möglichkeit zum Bildungsaufstieg. Gerade im weiterführenden Bildungsbereich könne es hierbei aber unter Umständen zu Problemen kommen. Daher wolle die neue Regierung ein nationales Stipendienprogramm ins Leben rufen mit dem BaföG-Freistellungen finanziert werden sollen. „Der Bildungsaufstieg darf an finanziellen Hürden nicht scheitern. Deshalb wollen wir mit dem Dreiklang aus BaföG, Bildungsdarlehen und Stipendien jungen Menschen ein Studium ermöglichen“ (Bundesregierung 2009a: 60). Auch das BaföG selbst wolle die Regierung weiterentwickeln. Zu einem lebenslangen Lernen gehören auch nach dem dreißigsten Lebensjahr noch Möglichkeiten zur Finanzierung ebendessen. „Die Möglichkeit, Bildungskredite über das 30. Lebensjahr hinaus zu verlässlichen Konditionen zu erhalten, werden wir ausbauen“ (Bundesregierung 2009a: 60). Noch zu oft kämen hochbegabte Schüler und Schülerinnen nicht zu ihrem Recht. Hierdurch würden bestehende Kompetenzen ungenügend genutzt und könnten diese 
Kinder sich nicht optimal entwickeln (vergl. Bundesregierung 2009a: 61). Insgesamt zeige sich die Regierung zufrieden mit der Umsetzung des BolognaProzesses. Da viele Ziele inzwischen erreicht seien, gelte es nun zu evaluieren und eventuelle Verbesserungsmöglichkeiten vorzunehmen. „Gemeinsam mit den Ländern und Hochschulen werden wir ein ,Bologna-Qualitäts- und Mobilitätspaket' schnüren, das die Studienreform zügig voranbringt und die Qualität des Studiums und die Mobilität der Studierenden weiter verbessert" (Bundesregierung 2009a: 61). Aber auch die Schüler, die den Weg des dualen Ausbildungssystems gehen, werde die Regierung unterstützen. Sie betone hierbei aber auch die eigene Verantwortlichkeit des Schülers. „Jugendliche mit Ausbildungsrisiken müssen frühzeitig erkannt und gefördert werden. Deshalb bauen wir die frühe Berufsorientierung in den Schulen aus. Unser besonderes Augenmerk gilt Jugendlichen mit Migrationshintergrund sowie denjenigen, die sich bereits längere Zeit vergeblich um eine Lehrstelle bemüht haben“ (Bundesregierung 2009a: 62). Zur Erreichung der Chancengleichheit sei neben den sprachlichen Fähigkeiten von Kindern der allgemeine Schutz von Kindern wichtig. Hierbei spielen vor allem präventive Maßnahmen zur Elternbildung eine wichtige Rolle. „Mit dem Nationalen Zentrum Frühe Hilfen soll der Ausund Aufbau Früher Hilfen und die Initiativen für einen aktiven Kinderschutz in Deutschland intensiviert werden“ (Bundesregierung 2009a: 69). Neue Medien seien Teil der Alltagskultur geworden und daher müssten die Fähigkeiten von Kindern diesbezüglich deutlich gestärkt werden (vergl. Bundesregierung 2009a: 71f). Wie bereits in den Zielsetzungen verdeutlicht, wolle die neue Regierung außerdem die Chancengleichheit von Frauen im Beruf in allen Lebensphasen fördern. Dieses gelte vor allem auch vor dem Hintergrund der hochwertigen Ausbildungen und Qualifizierungen, die Frauen inzwischen in der Regel erworben haben und vorrangig auch für die Gruppe der alleinerziehenden Frauen. „Das Aktionsprogramm ,Perspektive Wiedereinstieg wird in Partnerschaft mit der Bundesagentur für Arbeit fortgeführt und ausgebaut. Es wird geprüft, inwieweit sich die lokalen Modelle des Programms, die sich als zielführend erwiesen haben, in die Fläche übertragen und verstetigt werden können. Dabei ist die Situation Alleinerziehender in besonderer Weise zu berücksichtigen“ (Bundesregierung 2009a: 73). Aber nicht nur Wiedereinsteiger wolle die Regierung in besonderem Maße fördern. Gerade in wirtschaftlich unsicheren Zeiten sei die Unterstützung verschiedener Bevölkerungsgruppen diesbezüglich relevant. Der Einstieg in das Berufsleben sei für die Gruppe der 
Migranten und Migrantinnen oft schwer. „Begleitung und Beratung können jungen Menschen eine unverzichtbare Unterstützung beim Ausbildungserfolg sein. Ein neues Instrument des ganzheitlichen Integrationscoachings (...) dient der Integration in Ausbildung bzw. in den ersten Arbeitsmarkt und zur Stabilisierung dieser Integration“ (Bundesregierung 2009a: 76). Ein anderes Programm, welches diese Regierung im Bereich der gesellschaftlichen Integration von Migrantinnen einsetzen wolle sei das Programm ,Integration durch Sport' (vergl. Bundesregierung 2009a: 77). Für diese Zielgruppe wäre die Basis einer soliden Ausbildung sehr wichtig. Hierdurch ermöglicht sich oft erst die öffentliche und gesellschaftliche Teilnahme dieser Frauen. „Dafür brauchen wir eine Bildungs- und Ausbildungsoffensive für Migrantinnen. Auch auf die Aufklärung über Menschenrechte, Bürgerrechte und Sozialrechte und auf die Sensibilisierung für die Gleichberechtigung von Frauen und Männern muss großes Augenmerk gelegt werden“ (Bundesregierung 2009a: 77).

In der Regierungserklärung vom 10.11.2009 unterstreicht diese Regierung erneut den Schwerpunkt der Themen Bildung und Ausbildung. Chancengleichheit solle hierdurch gefördert und Kapazitäten besser genutzt werden (vergl. Bundesregierung 2009b: 9). Zu oft sei in Deutschland die Höhe des Ausbildungsgrads der Kinder direkt abhängig von der Höhe der elterlichen Ausbildung. Um diese schulischen Aufstiegschancen zu erhöhen, wolle die Regierung talentierte Schüler und Studenten besser unterstützen. „Mit den Ländern gemeinsam bauen wir ein nationales Stipendienprogramm für 10 Prozent der Studierenden auf“ (Bundesregierung 2009b: 9). Ein erfolgreicher Start sei für die spätere Chancengleichheit der Kinder wichtig. Für entscheidend halte die Regierung daher die sprachlichen Fähigkeiten der Kinder. „Jedes Kind soll vor dem Schulbeginn eine Sprachförderung erhalten, wenn das notwendig ist. Ich sage mit Nachdruck: Auch die Integration der Zuwanderer und ihrer Kinder führt zuerst und vorneweg über Sprache und Ausbildung“ (Bundesregierung 2009b: 9). 
Tabelle 20: Chronologisches Übersichtstableau der Instrumente zur Wiederherstellung oder Förderung der Kompetenzen verschiedener Familienangehörigen in Deutschland

\begin{tabular}{|c|c|c|}
\hline Jahr & Dokument & Instrument \\
\hline 1998 & $\begin{array}{l}\text { Koalitionsvereinbarung der } \\
\text { ersten Rot-Grünen } \\
\text { Koalition }\end{array}$ & $\begin{array}{l}\text { Gründung eines Bündnisses für Arbeit und } \\
\text { Ausbildung, } \\
\text { Verabschiedung eines Sofortprogramms für die } \\
\text { Ausbildung und Beschäftigung von Jugendlichen, } \\
\text { Start einer Ausbildungsoffensive, } \\
\text { Ausbau und Verankerung der Weiterbildung als vierte } \\
\text { Säule des Bildungssystems, } \\
\text { Reform der Ausbildungsförderung, } \\
\text { Weiterentwicklung des Hochschulrahmengesetzes, } \\
\text { Umbau der Hochschulen zu Zukunftswerkstätten, } \\
\text { Diverse Maßnahmen zur Unterstützung sozialen } \\
\text { Engagements, } \\
\text { Erweiterung der Maßnahmen des Kinder- und } \\
\text { Jugendplans, } \\
\text { Einführung des Aktionsplans ,Frau und Beruf }\end{array}$ \\
\hline 1998 & $\begin{array}{l}\text { Regierungserklärung der } \\
\text { ersten Rot-Grünen } \\
\text { Koalition }\end{array}$ & $\begin{array}{l}\text { Verabschiedung eines Sofortprogramms für die } \\
\text { Ausbildung und Beschäftigung von Jugendlichen, } \\
\text { Investierung in das Bündnis für Arbeit und } \\
\text { Ausbildung, } \\
\text { Gründung eines europäischen Beschäftigungspakts }\end{array}$ \\
\hline 2000 & $\begin{array}{l}\text { Stellungnahme Sechster } \\
\text { Familienbericht }\end{array}$ & $\begin{array}{l}\text { Einführung des Aktionsplans ,Frau und Beruf, } \\
\text { Durchführung einer Gesetzesnovelle zur Ächtung von } \\
\text { Gewalt in der Erziehung mit einer begleitenden } \\
\text { Informationskampagne, } \\
\text { Unterstützung von Modellprojekten zur Stärkung der } \\
\text { Rolle ausländischer Mädchen und Frauen, } \\
\text { Weiterentwicklung des Sofortprogramms für } \\
\text { Ausbildung und Beschäftigung von Jugendlichen, } \\
\text { Praxisnahes Weiterbildungscurriculum zur } \\
\text { Entwicklung von Handlungsstrategien für die } \\
\text { Bekämpfung von Fremdenfeindlichkeit und zur } \\
\text { Vorbereitung der Umsetzung von Konzepten der } \\
\text { Gewaltprävention, } \\
\text {,Bündnis für Arbeit und Ausbildung und Aufbau der } \\
\text { Koordinierungsstelle Ausbildung in ausländischen } \\
\text { Unternehmen' (KAUSA), } \\
\text { Einführung des Programms ,Entwicklung und } \\
\text { Chancen junger Menschen in sozialen Brennpunkten } \\
\text { (E\&C), } \\
\text { Erarbeitung eines gruppenübergreifenden } \\
\text { Gesamtkonzepts für ein neues System der } \\
\text { Sprachförderung }\end{array}$ \\
\hline
\end{tabular}




\begin{tabular}{|c|c|c|}
\hline 2002 & $\begin{array}{l}\text { Stellungnahme Elfter } \\
\text { Kinder- und Jugendbericht }\end{array}$ & $\begin{array}{l}\text { Intensivierung von Förderungsmaßnahmen in sozialen } \\
\text { Brennpunkten, } \\
\text { Intensivierung von Förderungsmaßnahmen zur } \\
\text { Förderung freiwilligen Engagements, } \\
\text { Reform der Ausbildungsförderung, } \\
\text { Im Rahmen des Bündnis für Arbeit, Ausbildung und } \\
\text { Wettbewerbsfähigkeit beschlossene Grundsätze zur } \\
\text { Modernisierung der Ausbildung, } \\
\text { Unterstützung von Fortbildungen für Berufe in der } \\
\text { Kindertagesbetreuung, } \\
\text { Unterstützung der Erziehungsfähigkeiten von Eltern, } \\
\text { Aufnahme erfolgreicher Elemente des } \\
\text { Sofortprogramms,JUMP' in das } \\
\text { Arbeitsförderungsrecht, } \\
\text { Unterstützung von Programmen für besonders } \\
\text { benachteiligte Kinder und Jugendliche, } \\
\text { Unterstützung von Jugendlichen im Bereich der neuen } \\
\text { Technologien }\end{array}$ \\
\hline 2002 & $\begin{array}{l}\text { Koalitionsvereinbarung der } \\
\text { zweiten Rot-Grünen } \\
\text { Koalition }\end{array}$ & $\begin{array}{l}\text { Einführung des ,JUMP-Plus-Programms', } \\
\text { Einführung eines zweijährigen Bildungsberichts, } \\
\text { Aufbau der Stiftung ,Bildung und Erziehung', } \\
\text { Etablierung weiterer Frauenstudiengänge und eines } \\
\text { bundesweiten Netzwerkes für internationale } \\
\text { Frauenstudiengänge, } \\
\text { Durchführung des Programms ,Innovation und } \\
\text { Arbeitsplätze im Informationszeitalter des 21. } \\
\text { Jahrhundert', } \\
\text { Anwendung von Gender Mainstreaming und Gender } \\
\text { Budgeting, sowie Aufbau eines Gender- } \\
\text { Kompetenzzentrums, } \\
\text { Überarbeitung des Bundesgremienbesetzungsgesetz, } \\
\text { Einführung einer Allianz mit Kindern und } \\
\text { Jugendlichen, } \\
\text { Durchführung der Bundesinitiative ,Jugend ans Netz' }\end{array}$ \\
\hline 2005 & $\begin{array}{l}\text { Stellungnahme Zwölfter } \\
\text { Kinder- und Jugendbericht }\end{array}$ & $\begin{array}{l}\text { Zustimmung zum Gesetz zur Weiterentwicklung der } \\
\text { Kinder- und Jugendhilfe (KICK), } \\
\text { Durchführung des Programms ,E\&C - Entwicklung } \\
\text { und Chancen junger Menschen in sozialen } \\
\text { Brennpunkten', } \\
\text { Weiterentwicklung von Kindertageseinrichtungen zu } \\
\text { Eltern-Kind-Zentren, } \\
\text { Fortführung des Dialogs ,Verantwortung Erziehung' } \\
\text { mit dem Ziel eines gemeinsamen } \\
\text { Erziehungskontraktes, } \\
\text { Durchführung der Kampagne ,SCHAUH HIN! Was } \\
\text { deine Kinder machen', }\end{array}$ \\
\hline
\end{tabular}




\begin{tabular}{|c|c|c|}
\hline & & $\begin{array}{l}\text { Etablierung der Initiative D 21, ein Internetportal für } \\
\text { die in den Tageseinrichtungen tätigen Fachkräfte, } \\
\text { Erstellung eines Bildungsberichts, } \\
\text { Durchführung des Modellprogramms ,Förderung von } \\
\text { Kindern und Jugendlichen mit } \\
\text { Migrationshintergründen' }\end{array}$ \\
\hline 2005 & $\begin{array}{l}\text { Koalitionsvertrag der } \\
\text { Großen Koalition unter } \\
\text { Merkel }\end{array}$ & $\begin{array}{l}\text { Fortführung des ,Nationalen Pakts für Ausbildung und } \\
\text { Fachkräftenachwuchs', } \\
\text { Einführung von persönlichen Ansprechpartnern und } \\
\text { Arbeitsvermittler für Jugendliche, } \\
\text { Umsetzung des ,Nationalen Aktionsplans für ein } \\
\text { kindgerechtes Deutschland', } \\
\text { Durchführung des Projekts ,Frühe Förderung für } \\
\text { gefährdete Kinder - Prävention durch Frühförderung', } \\
\text { Durchführung der Initiative, ,Verantwortung } \\
\text { Erziehung', } \\
\text { Unterstützung des europäischen Pakts für die Jugend } \\
\text { und des europäischen Programms, Jugend in Aktion', } \\
\text { Durchführung des ,Girls Day', } \\
\text { Einführung eines Berichts zur Gleichstellung von } \\
\text { Frauen und Männern mit zugehöriger } \\
\text { Regierungserklärung }\end{array}$ \\
\hline 2007 & $\begin{array}{l}\text { Stellungnahme Siebter } \\
\text { Familienbericht }\end{array}$ & Fortführung des Dialogs ,Verantwortung Erziehung' \\
\hline 2009 & $\begin{array}{l}\text { Stellungnahme Dreizehnter } \\
\text { Kinder- und Jugendbericht }\end{array}$ & $\begin{array}{l}\text { Ausführung des Nationalen Aktionsplans zur } \\
\text { Prävention von Fehlernährung, Bewegungsmangel, } \\
\text { Übergewicht und damit zusammenhängenden } \\
\text { Krankheiten ,IN FORM‘, } \\
\text { Initiierung des Kooperationsverbundes } \\
\text {,Gesundheitsförderung bei sozial Benachteiligten', } \\
\text { Einführung einer zusätzlichen } \\
\text { Früherkennungsuntersuchung vom 34. bis zum } 36 \text {. } \\
\text { Lebensmonat, } \\
\text { Ausführung des Aktionsprogramms ,Frühe Hilfen für } \\
\text { Eltern und Kinder und soziale Frühwarnsysteme‘ }\end{array}$ \\
\hline 2009 & $\begin{array}{l}\text { Koalitionsvertrag der } \\
\text { Schwarz-Gelben Koalition } \\
\text { unter Merkel }\end{array}$ & $\begin{array}{l}\text { Gründung einer Bildungspartnerschaft mit örtlichen } \\
\text { Bildungsbündnissen, } \\
\text { Unterstützung verbindlicher Sprachstandstests, } \\
\text { Verbesserungen der Qualifikationen von } \\
\text { Tagespflegepersonal, } \\
\text { Einführung eines Stipendiatenprogramms, } \\
\text { Weiterentwicklung des BAföGs, } \\
\text { Verstärkung der Hochbegabtenförderung, } \\
\text { Initiierung des ,Bologna-Qualitäts-und } \\
\text { Mobilitätspaktes', } \\
\text { Ausbau der frühen Berufsorientierung in den Schulen, }\end{array}$ \\
\hline
\end{tabular}




\begin{tabular}{|c|c|c|}
\hline & & $\begin{array}{l}\text { Ausbau früher Hilfen für aktiven Kinderschutz, } \\
\text { Initiierung eines Nationalen Aktionsplans zur } \\
\text { Verbesserung des Jugendschutzes, } \\
\text { Stärkung der Medienkompetenz von Kindern durch } \\
\text { unterschiedliche Programme, } \\
\text { Ausbau des Aktionsprogramms, ,Perspektive } \\
\text { Wiedereinstieg, } \\
\text { Einsetzung eines neuen Instruments zur Förderung } \\
\text { des ganzheitlichen Integrationscoachings, } \\
\text { Durchführung des Programms ,Integration durch } \\
\text { Sport', } \\
\text { Initiierung einer Bildungs- und Ausbildungsoffensive } \\
\text { für Migrantinnen }\end{array}$ \\
\hline 2009 & $\begin{array}{l}\text { Regierungserklärung der } \\
\text { Schwarz-Gelben Koalition } \\
\text { unter Merkel }\end{array}$ & $\begin{array}{l}\text { Schaffung von } 275.000 \text { neuen Studienplätzen, } \\
\text { Aufbau eines nationalen Stipendienprogramms, } \\
\text { Verstärkung der Sprachförderungsmaßnahmen für } \\
\text { Kinder }\end{array}$ \\
\hline
\end{tabular}




\subsubsection{Zeit-Instrumente}

In der Koalitionsvereinbarung der ersten Rot-Grünen Koalition (20.10.1998) wird die gleichberechtigte Teilhabe der Frauen in Beruf und Gesellschaft besonders betont. Die Regierung kündigt in diesem Zusammenhang die Durchführung des Aktionsprogramms ,Frau und Beruf an. Zu diesem Programm zähle die Regierung eine Reihe von Instrumenten. Im Bereich der zeitpolitischen Instrumente seien insbesondere der Einsatz für flexiblere Arbeitszeiten und bessere Bedingungen zur Teilzeit durch ein effektives Gleichstellungsgesetz, die Förderung von Dienstleistungsagenturen und die „Verbesserung der Rahmenbedingungen zur Schaffung von mehr Kinderbetreuungseinrichtungen“ (Bundesregierung 1998a: 35) zu nennen.

In der dazugehörigen Regierungserklärung der ersten Rot-Grünen Koalition werden die gleichberechtigte Teilhabe der Frauen in Beruf und Gesellschaft, sowie die Durchführung des Aktionsprogramms ,Frau und Beruf betont (vergl. Bundesregierung 1998b: 14).

Circa anderthalb Jahre später erläutert dieselbe Regierung in ihrer Stellungnahme zum Sechsten Familienbericht die zum 1. Januar 2001 in Kraft tretende Reform des Bundeserziehungsgesetzes. „Die Eltern können den Erziehungsurlaub (künftig ,Elternzeit') von insgesamt drei Jahren künftig auch gemeinsam nehmen. Sie haben während der Elternzeit einen grundsätzlichen Anspruch auf Verringerung ihrer Arbeitszeit - sowohl Väter als auch Mütter dürfen gleichzeitig bis $\mathrm{zu}$ jeweils 30 Wochenstunden erwerbstätig sein“ (Bundesministerium für Familie, Senioren, Frauen und Jugend 2000: 13).

Während der zweiten Rot-Grünen Regierungsphase wird in der Stellungnahme auf den Elften Kinder- und Jugendbericht die „Verbesserung der Vereinbarkeit von Familie und Erwerbstätigkeit, z.B. durch Elternzeit und Teilzeit-Anspruch betont" (Bundesministerium für Familie, Senioren, Frauen und Jugend 2002: 14). 
${ }^{19}$ Außerdem fügt sie in der Stellungnahme zum Zwölften Kinder- und Jugendbericht hinzu, dass für die Balance von Familie und Beruf der Wiedereinstieg in das Berufsleben nach der Elternzeit und eine angemessene Kinderbetreuung wichtig seien. „Neben der Förderung von Kindertagesstätten bedeutet das auch, die Tagespflege und die betrieblich unterstützte Kinderbetreuung auszubauen“ (Bundesministerium für Familie, Senioren, Frauen und Jugend 2005: 21).

In der Koalitionsvereinbarung der Großen Koalition unter Merkel wird am 11.11.2005 die Bedeutung der Vereinbarkeit von Familie und Beruf erneut hervorgehoben. Die „Offensive ,Familienbewusste Arbeitswelt' soll daher das Thema Elternschaft, auch und besonders von Führungskräften aufgreifen und konkrete Vorschläge für eine bessere Balance von Familie und Arbeitswelt in Unternehmen verankern“. Außerdem werde im Rahmen des Unternehmensprogramms unter anderem „die Verbesserung der Wiedereinstiegssituation während und nach der Elternzeit sowie eine familienbewusste Personalpolitik“ (vergl. Bundesregierung 2005a: 116) besonders gefördert.

${ }^{20}$ In ihrer Stellungnahme auf den Siebten Familienbericht kündigt dieselbe Große Koalition diverse zeitpolitische Maßnahmen an, denn die Bundesregierung wolle Familien bzw. Menschen die eine Familie gründen wollen mit einer besseren Koordination öffentlicher und betrieblicher Zeitpolitiken unterstützen. Da die Lebensspanne in der Kinderwünsche in Deutschland realisiert werden können momentan zu beschränkt sei, ziele die Regierung darauf diese Spanne zu erweitern. „Damit Kinderwünsche nicht immer weiter aufgeschoben werden und letztendlich auf Elternschaft verzichtet wird, müssen Infrastrukturangebote entwickelt werden, die eine Familiengründung begleiten bzw. unterstützen, die in die Ausbildungszeit fällt oder erst nach einer intensiven Vollzeitbeschäftigung stattfindet" (Bundesministerium für Familie, Senioren, Frauen und Jugend 2006: 30). In

19 In der Koalitionsvereinbarung der zweiten Rot-Grünen Regierung, sowie in der entsprechenden Regierungserklärung wurden keine zeitpolitischen Maßnahmen festgestellt. 20 In der Regierungserklärung der ersten Großen Koalition unter Merkel wurden keine zeitpolitischen Maßnahmen festgestellt. 
einer breiten Zusammenarbeit mit hochrangigen Partnern aus verschiedenen Gesellschaftsbereichen habe die Bundesregierung die ,Allianz für die Familie' gefördert. „Im Rahmen der ,Allianz für die Familie’ würden seit 2003 vielfältige Projekte und Maßnahmen durchgeführt, um Beschäftigten mit Kindern oder pflegebedürftigen Angehörigen die zeitliche Balance zwischen Familie und Arbeitswelt zu erleichtern. Die Allianz ziele darauf betrieblich geförderte Kinderbetreuung zu stärken, Betriebsnähe und Chancen zum Wiedereinstieg nach der Elternzeit zu verbessern und familienorientierte Maßnahmen stärker in betriebliche Vereinbarungen zu verankern. Hierzu verwendete „Maßnahmen reichen von flexiblen Arbeitszeitmodellen über familienfreundliche Regelungen in Betriebsvereinbarungen und Tarifverträgen bis hin zur Anpassung der Öffnungszeiten von Kindertagesstätten an die zeitlichen Bedarfe berufstätiger Eltern" (Bundesministerium für Familie, Senioren, Frauen und Jugend 2006: 31). Mit zwei Aktionsprogrammen, erstens den ,Lokalen Bündnissen für Familie' und zweitens dem ,Unternehmensprogramm Erfolgsfaktor Familie' setze sich die Bundesregierung gemeinsam mit Verantwortlichen z.B. aus dem Bereich der Kommunen und Unternehmen für eine familienfreundliche Zeitpolitik ein. Weiterhin nennt die Regierung in diesem Bereich den Unternehmenswettbewerb des BMFSFJ und das Audit berufundfamilie $₫$ der Hertie-Stiftung als familienunterstützende Instrumente. Insbesondere für kleine und mittlere Unternehmen sei eine familienfreundliche Gestaltung der Arbeitswelt oft eine besondere Herausforderung. Um hierbei besondere Unterstützung zu bieten „wurde von der Bertelsmann Stiftung im Rahmen ihrer Kooperation mit dem BMFSFJ das Internetportal ,Mittelstand und Familie' aufgebaut'“ (Bundesministerium für Familie, Senioren, Frauen und Jugend 2006: 31). Die im Koalitionsvertrag vorgesehene Einführung eines Pflegeurlaubs für Arbeitnehmerinnen und Arbeitnehmer ziele außerdem darauf, auch die Pflege von Familienangehörigen mit einer Berufstätigkeit besser vereinbaren zu können (vergl. Bundesministerium für Familie, Senioren, Frauen und Jugend 2006: 30). Als entscheidend für die Zeitpolitik bewertet die Regierung den Umbau des bestehenden Erziehungsgeldes in ein Elterngeld. „Das Elterngeld das die Bundesregierung 2007 einführen will - soll 67 Prozent des vorherigen Nettoeinkommens (maximal 1800 Euro pro Monat) des betreuenden Elternteils ersetzen. Für nicht oder gering erwerbstätige Eltern sei eine Mindestleistung vorgesehen. Das Elterngeld soll für 12 Monate gezahlt werden, die zwischen den Eltern aufgeteilt werden können, wobei je zwei Monate für den Vater und für die 
Mutter reserviert sein sollen. Der Teilzeitanspruch nach dem Teilzeit- und Befristungsgesetz (TzBfG) sowie der Anspruch auf eine maximal dreijährige Elternzeit mit Teilzeitanspruch und Jobgarantie soll erhalten bleiben“ (Bundesministerium für Familie, Senioren, Frauen und Jugend 2006: 32f).

${ }^{21}$ Im Koalitionsvertrag der Schwarz-Gelben Koalition unter Merkel (26.10.2009) werden die Notwendigkeit der zeitlichen Unterstützung von Familien und die hierdurch zunehmende Flexibilität der Eltern unterstrichen. In diesem Zusammenhang wolle die Regierung den Einsatz von Au-Pair-Diensten unterstützen. „Wir wollen die Au-Pair-Beschäftigung attraktiver gestalten. Wir werden die Anhebung der Altersgrenzen und die Möglichkeit einer Verlängerung des Aufenthalts prüfen“ (Bundesregierung 2009a: 68). Die bestehenden Rahmenbedingungen für eine familiengerechte Arbeitswelt gelte es weiterhin $\mathrm{zu}$ verbessern. Hierbei denke die Regierung beispielsweise an flexible Arbeitszeitmodelle und Möglichkeiten für Auszeiten vom Beruf, um sich z.B. der Familienarbeit widmen zu können. „Um die Vereinbarkeit von Familie und Beruf zu einem festen Bestandteil einer modernen und nachhaltigen Personalpolitik in den Unternehmen zu machen, werden wir die bisherige Initiative zu einer großen Kampagne erweitern“ (Bundesregierung 2009a: 68). Ein weiteres Vornehmen sei die Entwicklung des eingeführten Elterngeldes bzw. der Elternzeit. Vor allem die Flexibilität, die Möglichkeit diese Maßnahmen auch über einen längeren Zeitraum zu dehnen und die praktischere Einrichtung seien hierbei wichtige Ziele. „Wir wollen eine Weiterentwicklung, Flexibilität und Entbürokratisierung des Elterngeldes, gerade auch in Hinblick auf die Einkommensermittlung. Die Partnermonate sollen gestärkt und ein Teilelterngeld bis zu 28 Monaten eingeführt werden“ (Bundesregierung 2009a: 68). ${ }^{22}$ 
Tabelle 21: Chronologisches Übersichtstableau der Zeit-Instrumente in Deutschland

\begin{tabular}{|c|c|c|}
\hline Jahr & Dokument & Instrument \\
\hline 1998 & $\begin{array}{l}\text { Koalitionsvereinbarung der } \\
\text { ersten Rot-Grünen Koalition }\end{array}$ & $\begin{array}{l}\text { Durchführung des Aktionsprogramms ,Frau und } \\
\text { Beruf }\end{array}$ \\
\hline 1998 & $\begin{array}{l}\text { Regierungserklärung der ersten } \\
\text { Rot-Grünen Koalition }\end{array}$ & $\begin{array}{l}\text { Durchführung des Aktionsprogramms, Frau und } \\
\text { Beruf }\end{array}$ \\
\hline 2000 & $\begin{array}{l}\text { Stellungnahme Sechster } \\
\text { Familienbericht }\end{array}$ & Reform des Bundeserziehungsgesetzes \\
\hline 2002 & $\begin{array}{l}\text { Stellungnahme Elfter Kinder- } \\
\text { und Jugendbericht }\end{array}$ & Reform des Bundeserziehungsgesetzes \\
\hline 2005 & $\begin{array}{l}\text { Stellungnahme Zwölfter Kinder- } \\
\text { und Jugendbericht }\end{array}$ & $\begin{array}{l}\text { Förderung des Wiedereinstiegs nach der Elternzeit, } \\
\text { Ausbau der Kinderbetreuung }\end{array}$ \\
\hline 2005 & $\begin{array}{l}\text { Koalitionsvertrag der Großen } \\
\text { Koalition unter Merkel }\end{array}$ & $\begin{array}{l}\text { Durchführung der Offensive ,Familienbewusste } \\
\text { Arbeitswelt', } \\
\text { Durchführung des Unternehmensprogramms mit } \\
\text { u.a. dem Schwerpunkt Wiedereinstiegssituation von } \\
\text { Eltern }\end{array}$ \\
\hline 2007 & $\begin{array}{l}\text { Stellungnahme Siebter } \\
\text { Familienbericht }\end{array}$ & $\begin{array}{l}\text { Gründung der ,Allianz für die Familie', } \\
\text { Durchführung des Aktionsprogramms ,Lokale } \\
\text { Bündnisse für Familien', } \\
\text { Durchführung des Aktionsprogramms ,Erfolgsfaktor } \\
\text { Familie', } \\
\text { Entwicklung von Infrastruktur, um die Lebensspanne } \\
\text { in der Kinderwünsche realisiert werden zu erweitern, } \\
\text { Weiterführung des Unternehmenswettbewerbs und } \\
\text { des Audits ,berufundfamilie@', } \\
\text { Aufbau des Internetportals ,Mittelstand und Familie', } \\
\text { Einführung eines Pflegeurlaubs für } \\
\text { Arbeitnehmerinnen und Arbeitnehmer, } \\
\text { Einführung des Elterngeldes }\end{array}$ \\
\hline 2009 & $\begin{array}{l}\text { Koalitionsvertrag der Schwarz- } \\
\text { Gelben Koalition unter Merkel }\end{array}$ & $\begin{array}{l}\text { Attraktivere Gestaltung von Au-Pair-Beschäftigung, } \\
\text { Führung einer großen Kampagne zur Verbesserung } \\
\text { der Vereinbarkeit von Familie und Beruf, } \\
\text { Weiterentwicklung, Flexibilität und } \\
\text { Entbürokratisierung von Elterngeld/-zeit }\end{array}$ \\
\hline
\end{tabular}




\section{$4 \quad$ Verwendung familienpolitischer Zielsetzungen und Instrumente in den Niederlanden}

\subsection{Beschreibung der Arbeitsweise}

Erörtert werden in diesem Kapitel die familienpolitischen Zielsetzungen und Instrumente der beiden so genannten violetten Koalitionen unter der Leitung von Ministerpräsident Kok und (wie bereits zuvor erläutert) drei der vier großen Koalitionen unter der Leitung von Ministerpräsident Balkenende. Grundlage sind die entsprechenden Koalitionsvereinbarungen mit anschließenden Regierungserklärungen sowie die Familienberichte ,Notitie Gezin' (hier: Notiz zum Thema Familie), ,Nota Gezin' (hier: Bericht zum Thema Familie) und ,Nota Gezin 2008' (hier: Bericht zum Thema Familie 2008) sowie das ,Programma voor Jeugd en Gezin 2007-2011' (hier: Jugend- und Familienprogramm 2007-2011). Die letztgenannten Dokumente sind im untersuchten Zeitraum durch das niederländische Ministerium für Gesundheit, Wohlfahrt und Sport bzw. das Ministerium für Jugend und Familie als zusammenhängende familien- bzw. jugendpolitische Berichterstattung (inklusive der Stellungnahme), der Tweede(n) Kamer (hier: Bundestag) vorgelegt. Verwaltungstechnisch bzw. für die zu führende Politik haben diese Berichte einen etwas anderen Stellenwert als die Koalitionsvereinbarungen. Für die im folgenden Kapitel zu tätigende Analyse sind sie aber dennoch sehr wertvoll, da sie die Familienpolitik zusammenhängend darstellen und neue, inhaltliche Entwicklungen aufgreifen und erläutern. Auch die verwendeten Regierungserklärungen sind weniger bedeutend als die Koalitionsvereinbarungen. Da die Regierungen allerdings an dieser Stelle die wichtigsten Punkte aus dem Koalitionsvertrag hervorheben, sind diese Dokumente bei der Analyse hilfreich, um die Schwerpunktsetzung bzw. die Umsetzung der Politiken besser zu verstehen und sie im Hinblick auf das Modell Aktivierender Familienpolitik zu beurteilen.

In Deutschland hat die Publikation von Familienberichten Tradition und einen bestimmten Stellenwert. Sie wird begleitet von einer Stellungnahme der 
Regierung. In diesem Dokument nimmt die Regierung Stellung zu den Ergebnissen des Berichts. In den Niederlanden gibt es keine institutionalisierte Form der Familienberichterstattung (bzw. institutionalisierte Berichterstattung $\mathrm{zu}$ den Themenbereichen Jugend, Frauen und Ältere Menschen) und können Berichte dieser Art sowohl von der Regierung beantragt, als auch durch die verantwortlichen Minister selbstständig angekündigt werden. Daher ist die Abgrenzung der für die Analyse verwendbaren Dokumente etwas diskutabler. Berichte wie z.B. der Jugendkulturbericht 2009 (mit dem Titel: Onze Jeugd van Tegenwoordig) oder die unterschiedlichen Emanzipationsberichte grenzen an das für diese Arbeit relevante Forschungsfeld. Sie werden an dieser Stelle allerdings nicht berücksichtigt, da es erstens aus deutscher Sicht (noch) kein direktes Pendant gibt, sie zweitens teilweise sehr technisch detailliert sind und damit den Rahmen dieser Arbeit sprengen würden und sie drittens für den eigentlichen Forschungszweck wenig relevant sind. In der untersuchten Periode hat es in den Niederlanden die drei bereits genannten Familienberichte sowie ein Jugend- und Familienprogramm gegeben. Weitere Dokumente, wie z.B. zu den Themen Jugend, Frauen und Ältere Menschen wurden für diese Arbeit aufgrund des begrenzten Zusammenhangs mit den übrigen familienpolitischen Schwerpunkten und der teilweise sehr spezifischen Themenerarbeitung nicht verwendet.

Wichtig ist $\mathrm{zu}$ erwähnen, dass es erst seit der vierten Regierung unter Balkenende einen Minister für Jugend und Familie gibt. Er verfügt über einen kleinen eigenen Beamtenapparat, kann aber als so genannter Programmminister außerdem zugreifen auf Mitarbeiter des Ministeriums für Soziale Angelegenheiten und Arbeit, des Justizministeriums, des Ministeriums für Unterricht, Forschung und Kultur, des Gesundheitsministeriums und des Programmministeriums Wohnen, (Stadt)Viertel und Integration. Diese neue Form der Zusammenarbeit ist Teil der gegenwärtigen Modernisierungsstrategien der niederländischen Regierung. Ausgangspunkt ist eine effizientere, ressortübergreifende Zusammenarbeit. Inwiefern diese Struktur beibehalten wird, ist zum Zeitpunkt des Schreibens noch undeutlich. Vor der vierten Regierung unter Balkenende wurde das Thema Familie von den Staatssekretären im Gesundheitsministerium koordiniert.

Genau wie im vorigen Kapitel werden auch auf der niederländischen Seite die bevölkerungspolitischen, emanzipatorischen und auf die Entwicklung der 
Kinder gerichteten Zielsetzungen der Regierung sowie die ökologischen und ökonomischen Maßnahmen, und Instrumente die die Kompetenzen bzw. den Zeitrahmen der Familienmitglieder unterstützen, untersucht. Hierbei stehen wiederum die beschriebenen Verantwortlichkeiten und die Art der Umsetzung der Instrumente (Sozialinvestition, Öffentlich-Private Partnerschaft oder Privatisierung) im Mittelpunkt, welche sich aus dem Kontext der Instrumente bzw. dem politischen Kontext ergeben. Daher erfolgt die Beschreibung von Zielen und Instrumenten sowohl ausführlich in Textform (für die Bewertung von Verantwortlichkeiten bzw. die Art der Umsetzung) als auch punktuell in einer Tabelle mit Zeitleiste am Ende der Beschreibung eines entsprechenden Ziels bzw. Instrumentes. Die Analyse der hier beschriebenen Politiken erfolgt (gemeinsam mit der Analyse der deutschen Politik) im fünften Kapitel des Dissertationsvorhabens.

In der untersuchten Periode wurde eine Vielzahl familienpolitischer Zielsetzungen und Instrumente benannt. Genau wie an deutscher Seite gilt auch in diesem Kapitel, dass die hierbei verwendeten Umschreibungen nicht immer gleich konkret sind und sich daher teilweise etwas unterschiedliche Formulierungen ergeben können. Um für die Darstellung und in der folgenden Analyse verwendet werden zu können, müssen sowohl die Zielsetzungen als auch die Instrumente als solche erkennbar sein. Für die erörterten Ziele gilt z.B., dass es in einigen Fällen ganz konkrete Richtzahlen in Prozenten und Jahrzahlen gibt, während in anderen Fällen eher an etwas undeutlicheren Leitbildern festgehalten wird. In der hier erstellten Darstellung wurden sowohl die konkreten als auch die weniger konkreten Zielsetzungen aufgenommen. Auch für die Definition von Instrumenten verwendeten die unterschiedlichen Regierungen verschiedene Begrifflichkeiten. In einigen Fällen wurden Instrumente bis auf das Mikroniveau heruntergebrochen, während andere Umschreibungen eher auf Makroniveau erfolgten. In der hier erstellten Darstellung ist, soweit für die Analyse notwendig, auf Mikroniveau gearbeitet. Auch wenn der Schwerpunkt auf den neuen Instrumenten liegt, sind, wo für den Zusammenhang erforderlich, auch alte Instrumente mit in die Darstellung aufgenommen. Instrumente bzw. Instrumentenbündel die für verschiedene Kategorien relevant sind, werden daher gegebenenfalls mehrmals genannt. 


\subsection{Familienpolitische Zielsetzungen in den Niederlanden}

\subsubsection{Bevölkerungspolitische Zielsetzungen}

${ }^{23}$ Erst im Koalitionsvertrag der vierten Koalition unter Balkenende vom 07.02.2007 bekommt der Themenbereich Jugend und Familie eine besondere Stellung. Man wolle eine gezielt familienfreundliche Politik führen. „Eine familienfreundliche Politik trägt ihren Teil dazu bei, dass Kinder ab jungem Alter Selbstvertrauen, Durchsetzungsvermögen und Verantwortlichkeitsgefühl mitbekommen“ (Ministerie van Algemene Zaken 2007a: 23). Die Regierung unterstreicht in diesem Dokument mehrmals den besonderen Wert der Familie als Ort an dem Kinder erzogen und die essentiellen Werte und Normen an die folgende Generation vorgelebt und übertragen werden. Eltern haben in diesem Zusammenhang eine besondere Verantwortung und müssen daher über genügend „Zeit, Mittel und Fähigkeiten“ (Ministerie van Algemene Zaken 2007a: 29) verfügen.

In der kurze Zeit später folgenden Regierungserklärung der vierten Koalition unter Balkenende erklärt die Regierung am 01.03.2007 die Haltung des Gleichgewichts zwischen der Ablösung von Staatsschulden einerseits und neuen Investierungen, um die gesellschaftlichen Kräfte zu stärken, andererseits, als eines ihrer wichtigen Ziele. „Die Rendite dieser Investitionen helfen später beim Auffangen der Folgen des demographischen Wandels" (Ministerie van Algemene Zaken 2007b: 8).

Der zu Regierungsbeginn der vierten Koalition neu ernannte Minister für Jugend und Familie unterstreicht in seinem Programm, welches er wenige

23 Sowohl in der Koalitionsvereinbarung als auch in der Regierungserklärung der ersten Violetten Koalition aus dem Jahr 1994, dem Familienbericht aus dem Jahr 1996 und der Koalitionsvereibarung und Regierungserklärung der zweiten Violetten Koalition aus dem Jahr 1998 wurden keine konkreten Zielsetzungen im Hinblick auf die demographische Entwicklung (entsprechend der hier hantierten Definition) gefunden. Andere Themen, wie z.B. die gleichgeschlechtliche Ehe, bzw. die Integration von Zuwanderern wurden in diesen Dokumenten durchaus behandelt. Sie sind an dieser Stelle allerdings nicht weiter ausgeführt, da sie für die hier zu bearbeitende Fragestellung nicht relevant sind. Im Koalitonsvertrag und der Regierungserklärung der ersten Koalition unter Balkenende aus dem Jahr 2002, dem Koalitionsvertrag und der Regierungserklärung der zweiten Koalition unter Balkenende aus dem Jahre 2003 und dem Familienbericht aus dem Jahre 2006 wurden keine konkreten Zielsetzungen im Hinblick auf die demographische Entwicklung gefunden. 
Wochen nach dem Regierungsantritt an die Tweede Kamer (hier: Bundestag) schickte, dass vor allem in Familien Werte und Normen an Kinder übermittelt werden. Familien formen die Basis der Entwicklungsmöglichkeiten von Kindern. „Familien haben eine wichtige gesellschaftliche Rolle: wenn Kinder in ihrer Familie das gute Gepäck für die Zukunft erhalten, hat das einen positiven Einfluss auf die gesamte Gesellschaft“ (Jeugd en Gezin 2007: 8).

Im Familienbericht mit dem Titel ,Die Stärke der Familie، (im Niederländischen: De Kracht van het Gezin) erläutert der Minister im Jahr 2008 die familienpolitischen Ziele und Instrumente der vierten Koalition unter Balkenende. In diesem Bericht wird angeknüpft an die Prinzipien des Koalitionsvertrags, denn „das Kabinett will die Niederlande familienfreundlicher gestalten“ (Jeugd en Gezin 2008: 3), da der soziale Zusammenhang im Allgemeinen und das Funktionieren der Gesellschaft in den Familien beginne. Verschiedene gesellschaftliche Modernisierungsprozesse der vergangenen Jahre hätten für Unsicherheit und Unzufriedenheit in der Gesellschaft gesorgt. Da die gesellschaftlichen Netzwerke weniger stabil seien als in der Vergangenheit, finde die Regierung vor allem den Rückhalt in den Familien wichtig. Dieser Rückhalt sei für die gesellschaftliche Stabilität entscheidend, könne aber nicht nur durch die Regierung organisiert werden. Daher wolle die Regierung eine Politik führen, die die „Kraft von Familien verstärkt, zur Balance zwischen Familie und Beruf beiträgt, Familien Boden unter den Füßen gibt und einen Beitrag zur sozialen Stabilität leistet“ (Jeugd en Gezin 2008: 5). Der niederländische Rat für Gesundheit und Pflege habe in einer Forschungsreihe zum Thema Aufschub von Schwangerschaften das hohe Alter der Erstgebärenden kritisiert und darauf hingewiesen, dass die Gesellschaft unzulänglich über die Risiken später Schwangerschaften informiert sei. Der Fortschrittsglaube in Fruchbarkeitstechniken sei unrealistisch groß. Außerdem betone der Rat, die hohen gesellschaftlichen Hürden der Elternschaft und bekäme die Kombination von Familie und Beruf keine zufrieden stellende Unterstützung. Durch die Regierung wird im Familienbericht daher ausdrücklich betont, dass Menschen zwar selbst bestimmen müssen, wann und wie viel Kinder sie bekommen wollen, dass jedoch „Hindernisse so weit wie möglich ausgeräumt werden müssen, z.B. durch die Verbesserung der Vereinbarkeit von Familie und Beruf, und dass die Bürger Zugang haben 
müssen $\mathrm{zu}($ (..) Informationen über medizinische Risiken später Elternschaft“ (Jeugd en Gezin 2008: 44).

Tabelle 22: Chronologisches Übersichtstableau der demographischen Zielsetzungen in den Niederlanden

\begin{tabular}{|c|c|c|}
\hline Jahr & Dokument & Zielsetzung \\
\hline 2007 & $\begin{array}{l}\text { Koalitionsvereinbarung } \\
\text { Balkenende IV }\end{array}$ & $\begin{array}{l}\text { Führung einer familienfreundlichen Politik, } \\
\text { Eltern müssen über genügend Zeit, Mittel und } \\
\text { Fähigkeiten verfügen }\end{array}$ \\
\hline 2007 & $\begin{array}{l}\text { Regierungserklärung } \\
\text { Balkenende IV }\end{array}$ & $\begin{array}{l}\text { Gutes Gleichgewicht zwischen Ablösung von } \\
\text { Staatsschulden und neuen Investitionen, um Folgen } \\
\text { des demographischen Wandels auffangen zu können }\end{array}$ \\
\hline 2007 & $\begin{array}{l}\text { Programm für Jugend und } \\
\text { Familie, 2007-2011 }\end{array}$ & Positive Unterstützung von Familien \\
\hline 2008 & Familienbericht 2008 & $\begin{array}{l}\text { Vergrößerung der Familienfreundlichkeit, } \\
\text { Verstärkung der Kraft von Familien, } \\
\text { Unterstützung von selbst gewünschten } \\
\text { Familiengründungen und Informationen über den } \\
\text { Aufschub von Schwangerschaften }\end{array}$ \\
\hline
\end{tabular}

\subsubsection{Emanzipatorische Zielsetzungen}

In der Koalitionsvereinbarung der ersten Violetten Koalition vom 13.08.1994 wird auf das Bedürfnis vieler Bürger nach mehr Flexibilität in beruflichen Lebensläufen hingewiesen. Da die heutige Gesetzeslage den Bedürfnissen der Frauen nur unzureichend entspreche, wolle die Regierung gemeinsam mit den Sozialpartnern nach möglichen Lösungen suchen, denn das „Stimulieren einer größeren Flexibilität und Variation in den Arbeitsmustern während der gesamten Lebensphase ist gleichwohl Verantwortung der sozialen Partner wie auch der Regierung. Das Kabinett nimmt sich vor, um im Dialog mit den Sozialpartnern zu untersuchen, auf welche Weise diese Entwicklung gefördert werden kann“ (Ministerie van Algemene Zaken 1994a: 9). Die Regierung betont ausdrücklich, dass die traditionelle Hausfrauenfamilie mit dem auf sie zugeschnittenen System der sozialen Sicherheit dem Muster eines „emanzipierten und wirtschaftlich selbstständigen Bürger(s)“ (Ministerie van Algemene Zaken 1994a: 46) weichen solle. Dieses Ziel werde allerdings nur unter der Bedingung verfolgt, dass es nicht zu größeren sozialen Ungleichheiten 
führe. Entsprechend erfodere Emanzipationspolitik einen zusammenhängenden Ansatz für die Vereinbarkeit von Beruf und Familie, in dem die temporäre Erfüllung von Pflegeleistungen ermöglicht werde und „in der Männer und Frauen gleiche Rechte haben auf eine wirtschaftliche Selbstständigkeit“ (Ministerie van Algemene Zaken 1994a: 46).

In der kurze Zeit später folgenden Regierungserklärung wird am 31.08.1994 das Ziel der wirtschaftlichen Selbstständigkeit von Männern und Frauen und das Ende der traditionellen Hausfrauenehe erneut genannt. Allerdings warnt die Regierung davor, dass in den kommenden Jahren noch stets eine "gewisse Spannung bleibe zwischen dem Streben nach Emanzipation und Individualisierung und der Art auf die bestehende Regelungen in der Gesellschaft ihre Grundlage bekommen haben“ (Ministerie van Algemene Zaken 1994b: 4).

In einer umfangreichen Notiz zum Thema Familie, welche die Staatssekretärin des Ministeriums für Gesundheit, Wohlfahrt und Sport im Jahre 1996 an die Tweede Kamer (hier: Bundestag) schickte, wird die Zielsetzung der Regierung bekräftigt, jedem Bürger, unabhängig vom Geschlecht oder Familienstand, durch Emanzipationspolitik die Möglichkeit zu bieten, ein selbstständiges Bestehen aufzubauen „in einer Gesellschaft, in der Frauen und Männer gleiche Rechte, Chancen, Freiheiten und Verantwortlichkeiten haben" (Ministerie van Volksgezondheid, Welzijn en Sport 1996: 13). Auch Menschen mit Erziehungsverantwortung sollten in der Lage sein, die Sorge für die Kinder mit einer Teilnahme am Berufsleben kombinieren zu können. Erziehung sei nicht nur Sache der Frauen. Daher habe die Regierung ihre Emanzipationspolitik ausgedehnt auf „Frauen und Männer, denen es wichtig ist bezahlte Arbeit mit anderen Aktivitäten verbinden zu können vor allem mit der Betreuung und Erziehung ihrer Kinder. Diese Entwicklung verläuft Hand in Hand mit dem Durchbrechen traditioneller Meinungen über die Rollen, welche Männer und Frauen spielen zu haben“ (Ministerie van Volksgezondheid, Welzijn en Sport 1996: 13).

Die zweite Violette Regierung unterstreicht in ihrer Koalitionsvereinbarung vom 18.07.1998 das Ziel einer besseren Vereinbarkeit von Familie und Beruf, denn „so können bisher verborgene Talente von Frauen im Erwerbsleben und Männern bei der Erziehung besser zu ihrem Recht kommen" (Ministerie van 
Algemene Zaken 1998a: 23). Die Regierung wolle Vorurteile im Bezug auf geschlechtsspezifische und kulturelle Leitbilder durchbrechen. „Ziel ist daher, ein Gleichgewicht zwischen Fürsorge, Arbeit, gesellschaftlicher Betätigung und Freizeit für Männer und Frauen zu erreichen" (Ministerie van Algemene Zaken 1998a: 65). Bei der Förderung von Emanzipation und gleichberechtigter Teilnahme an Arbeit und Familie richte sich die Regierung insbesondere an Frauen mit Migrationshintergrund. „Sie werden häufig konfrontiert mit verschiedenen Erwartungshaltungen. Die Rolle dieser Mädchen kann von großer Bedeutung sein bei der Gestaltung sozialen Zusammenhangs in einer multikulturellen Gesellschaft“ (Ministerie van Algemene Zaken 1998a: 65f).

In der dazugehörigen Regierungserklärung der zweiten Violetten Koalition vom 25.08.1998 deutet Kok auf die große Bedeutung, welche seine Regierung der Vereinbarkeit von Familie und Beruf zumesse (vergl. Ministerie van Algemene Zaken 1998b: 2). Da die Regierung dieses Ziel durch diverse Maßnahmen unterstützen wolle, ernennt er für diese Regierungsperiode einen zweiten Staatssekretär für Soziale Angelegenheiten und Arbeit. Außerdem bekräftigt er die Zielsetzung seiner Regierung hinsichtlich "moderner Emanzipationspolitik” (Ministerie van Algemene Zaken 1998b: 9). In allen denkbaren Bereichen sollten die Bürger das Recht und die Möglichkeit bekommen, sich entsprechend ihrer individuellen Wünsche entfalten zu können.

Die genannten Hintergründe für Emanzipationspolitik ändern sich deutlich im Koalitionsvertrag der ersten Koalition unter Balkenende (26.07.2002). Hier wird die Teilnahme von Frauen auf dem Arbeitsmarkt weniger aus Gründen der Gleichheit und Gerechtigkeit gefordert, sondern eher aus sozialwirtschaftlichen Beweggründen. „Unterstützung der Arbeitsteilnahme ist sowohl wirtschaftlich nötig als auch sozial wünschenswert" (Ministerie van Algemene Zaken 2002a: 2). Diese Regierung unterscheide zwischen Lebensphasen, in denen unterschiedliche Zielsetzungen wie z.B. Beruf und Erziehung Vorrang bekommen können, denn die „Bedürfnisse und Wünsche von Menschen zum Thema Arbeit, Kindererziehung, Familie oder anderem Lebensverband und Lehre/Studium kann pro Lebensphase unterschiedlich sein" (Ministerie van Algemene Zaken 2002a: 17). Das Lebensphasenmodell wolle die Regierung durch passende Instrumente unterstützen. 
In der Regierungserklärung der ersten Koalition unter Balkenende vom 03.07.2002 wird erneut unterstrichen, dass „es sowohl wirtschaftlich als auch sozial wünschenswert ist, dass die Teilnahme am Arbeitsmarkt vergrößert wird“ (Ministerie van Algemene Zaken 2002b: 9). Frauen werden aus diesem Grund aufgerufen, um sich aktiv am Arbeitsmarkt zu beteiligen.

${ }^{24} \mathrm{Im}$ Familienbericht, den die Staatssekretärin des Ministeriums für Gesundheit, Wohlfahrt und Sport im Jahre 2006 an die Tweede Kamer (hier: Bundestag) schickte, wird auf den Unterschied zwischen der Rolle des Vaters und der Mutter größtenteils verzichtet. Im Bezug auf die Vereinbarkeit von Familie und Beruf betont die Regierung, sie wolle beide Eltern durch entsprechende Rahmenbedingungen unterstützen, da „Eltern ihre besondere Rolle als Erzieher in Kombination mit der aktiven gesellschaftlichen Teilnahme verbinden können“ (Ministerie van Volksgezondheid, Welzijn en Sport 2006: 2).

Im darauf folgenden Jahr wird in der Koalitionsvereinbarung der Regierung Balkenende IV am 07.02.2007 hervorgehoben, dass die unterschiedlichen Schwerpunkte der sozialen Politiken miteinander verwoben seien und daher auch zusammenhängend gefördert werden sollen. Ein funktionierender gesellschaftlicher Bereich sei für soziale Kohäsion entscheidend. „Jeder muss hierfür Verantwortung übernehmen. An erster Stelle Eltern und Erziehungsberechtigte“ (Ministerie van Algemene Zaken 2007a: 9). Die soziale Kohäsion könne vor allem durch eine Teilnahme am Arbeitsmarkt vergrößert werden. Hierzu gehörten nach Ansicht der Regierung vor allem auch Frauen, die nach einer Erziehungsphase wieder ins Berufsleben zurückkehren wollen. Wichtig seien die Frauen in der Lebensphase zwischen 35 und 40 Jahren. Zu dem Zeitpunkt müsse nicht der Anschluss bzw. Wiedereinstieg in den Arbeitsmarkt verpasst werden. „Wir wollen jedem eine gerechte Chance auf einen Arbeitsplatz bieten. Dieses ist eine gemeinsame Aufgabe des Kabinetts mit den Sozialpartnern“ (Ministerie van Algemene Zaken 2007a: 23). Kritisiert wird in diesem Koalitionsvertrag außerdem, dass die Löhne von Frauen immer 
noch niedriger sind als die von Männern. „Das Kabinett setzt sich dafür ein, diese Ungleichheit zu beenden" (Ministerie van Algemene Zaken 2007a: 23). Als eine weitere Ungerechtigkeit beurteilt das Kabinett die Tatsache, dass immer noch verhältnismäßig wenige Frauen in Spitzenfunktionen arbeiten. Darum „spricht die Regierung Arbeitgeber auf ihre Anstrengungen an, um mehr Frauen in Spitzenfunktionen zu bringen" (Ministerie van Algemene Zaken 2007a: 30). Insbesondere im Unterrichtssektor sei es wichtig, dass mehr Frauen hohe Funktionen besetzten, da von hier aus ein wichtiges Signal ausgehe.

Am 01.03.2007 folgt die Regierungserklärung der vierten Koalition unter Balkenende. Die aktive Teilnahme am Arbeitsmarkt stärke die Gesellschaft und die Förderung dieser Partizipation sei ein Hauptanliegen dieser Regierung. Insbesondere werde angestrebt die Teilnahme von Frauen am Arbeitsmarkt zu verbessern (vergl. Ministerie van Algemene Zaken 2007b: 4). Weiterhin fordert die Regierung eine gleichmäßige Entwicklung der Löhne. „Menschen in einer verletzlichen Position und Haushalte mit Kinder wird dabei eine besondere Aufmerksamkeit gewidmet“ (Ministerie van Algemene Zaken 2007b: 6).

Nachdem in den ersten beiden hier untersuchten Dokumenten dieser Regierung die Arbeitsmarktteilnahme aller Bürger einen hohen Stellenwert hatte, benannte der Minister für Jugend und Familie in seinem Programm für Jugend und Familie, 2007-2011, das er 2008 an die ,Tweede Kamer' (hier: Bundestag) schickte, vor allem Ziele, die das Zusammenleben mit Kindern und Jugendlichen betreffen. Er unterstrich in diesem Zusammenhang, dass die Arbeitsmarktteilnahme von Vätern und Müttern nicht so weit reichen dürfe, dass Kindern nicht mehr die Aufmerksamkeit erhalten, die sie bräuchten. „Es ist sehr wichtig, dass Eltern auf ein gutes Gleichgewicht zwischen dem Familienleben und der Teilnahme am Arbeitsmarkt achten (...) Die Politik der Regierung unterstützt das Finden eines guten Gleichgewichts, insbesondere in der Phase, in der junge Kinder (im Haushalt) wohnen“ (Jeugd en Gezin 2007: 7).

Diese Vereinbarkeit von Familie und Beruf ist auch in seinem Familienbericht aus dem Jahre 2008 ein wichtiges Thema. Betont werde, dass die meisten Frauen Arbeit und Kindererziehung kombinieren wollen, aber im ersten Lebensjahr vor allem die Kindererziehung vorziehen. Die Regierung verstehe es daher als ihre Aufgabe „um, (...) die entsprechenden Rahmenbedingungen zu 
schaffen und, was die Verantwortlichkeiten/Kompetenzen anderer Parteien betrifft, gemeint sind vor allem Arbeitgeber und Sozialpartner, sie hierzu einzuladen und zu stimulieren“ (Jeugd en Gezin 2008: 35). Neben der Rolle der Mütter wird in diesem Bericht ausdrücklich die Rolle der Väter angesprochen. Ziel sei es auch, um Väter zu stimulieren, eine aktive Rolle bei der Pflege und in der Erziehung ihrer Kinder zu spielen. "Um ihre Erziehungsaufgaben gut wahrnehmen zu können, ist es nötig, dass Eltern hierzu genügend Zeit zur Verfügung steht“ (Jeugd en Gezin 2008: 9). Hierbei sei vor allem die erste Lebensphase von Kindern entscheidend. „Zeit und Aufmerksamkeit sind in dieser Lebensphase wichtig. Darum unterstützen Regierung und Arbeitgeber Mütter und Väter durch die Verwendung diverser Urlaubsformen“ (Jeugd en Gezin 2008: 35f).

Tabelle 23: Chronologisches Übersichtstableau der emanzipatorischen Zielsetzungen in den Niederlanden

\begin{tabular}{|c|c|c|}
\hline Jahr & Dokument & Zielsetzung \\
\hline 1994 & Koalitionsvertrag Kok I & $\begin{array}{l}\text { Stimulieren einer größeren Flexibilität und Variation } \\
\text { in den Arbeitsmustern während der gesamten } \\
\text { Lebensphase, } \\
\text { Falls es nicht zu größeren sozialen Ungleichheiten } \\
\text { führt, soll die traditionelle Hausfrauenfamilie dem } \\
\text { Muster eines emanzipierten und wirtschaftlich } \\
\text { selbstständigen Bürger(s) weichen, } \\
\text { Männer und Frauen haben das gleiche Rechte auf } \\
\text { wirtschaftliche Selbstständigkeit }\end{array}$ \\
\hline 1994 & Regierungserklärung Kok I & $\begin{array}{l}\text { Unterstützung von Emanzipation und } \\
\text { Individualisierung }\end{array}$ \\
\hline 1996 & Familienbericht 1996 & $\begin{array}{l}\text { Unterstützung wirtschaftlicher Selbstständigkeit von } \\
\text { Männern und Frauen, } \\
\text { Beendung der traditionellen Rollenmuster und Aufruf } \\
\text { zur Vereinbarkeit von Familie und Beruf für Männer } \\
\text { und Frauen }\end{array}$ \\
\hline 1998 & Koalitionsvertrag Kok II & $\begin{array}{l}\text { Unterstützung einer besseren Vereinbarkeit von Beruf } \\
\text { und Familie für Männer und Frauen, } \\
\text { Unterstützung von Mädchen und Frauen mit } \\
\text { Migrationshintergrund mit dem Ziel einer besseren } \\
\text { Vereinbarkeit von Beruf und Familie }\end{array}$ \\
\hline 1998 & Regierungserklärung Kok II & $\begin{array}{l}\text { Bessere Unterstützung der Vereinbarkeit von Beruf } \\
\text { und Familie, } \\
\text { Möglichkeit zur freien Entfaltung von Frauen und } \\
\text { Männern entsprechend individueller Wünsche }\end{array}$ \\
\hline 2002 & Koalitionsvertrag Balkenende I & Vergrößerung der Arbeitsteilnahme von Frauen aus \\
\hline
\end{tabular}




\begin{tabular}{|c|c|c|}
\hline & & $\begin{array}{l}\text { sozialwirtschaftlichen Gründen, } \\
\text { Unterstützung in Form eines Lebensphasenmodells }\end{array}$ \\
\hline 2002 & $\begin{array}{l}\text { Regierungserklärung } \\
\text { Balkenende I }\end{array}$ & $\begin{array}{l}\text { Vergrößerung der Arbeitsteilnahme von Frauen aus } \\
\text { sozialwirtschaftlichen Gründen }\end{array}$ \\
\hline 2006 & Familienbericht 2006 & $\begin{array}{l}\text { Unterstützung der Eltern bei der Vereinbarkeit von } \\
\text { Beruf und Familie durch entsprechende } \\
\text { Rahmenbedingungen }\end{array}$ \\
\hline 2007 & Koalitionsvertrag Balkenende IV & $\begin{array}{l}\text { Verbesserung der sozialen Kohäsion durch einen } \\
\text { funktionierenden sozialen Bereich, } \\
\text { Verbesserung der Chance auf einen Arbeitsplatz für } \\
\text { Frauen, die nach einer Erziehungsphase (wieder) } \\
\text { beruflich aktiv werden wollen, } \\
\text { Forderung nach gleichen Löhnen für Frauen und } \\
\text { Männer, } \\
\text { Mehr Frauen in Topfunktionen }\end{array}$ \\
\hline 2007 & $\begin{array}{l}\text { Regierungserklärung } \\
\text { Balkenende IV }\end{array}$ & $\begin{array}{l}\text { Verbesserung der sozialen Kohäsion durch } \\
\text { funktionierenden sozialen Bereich, } \\
\text { Forderung nach einer gleichmäßigen Lohnentwicklung } \\
\text { für Menschen in prekären Lebensumständen und } \\
\text { Haushalten mit Kindern }\end{array}$ \\
\hline 2007 & $\begin{array}{l}\text { Programm für Jugend und } \\
\text { Familie, 2007-2011 }\end{array}$ & $\begin{array}{l}\text { Unterstützung einer guten Vereinbarkeit von Familie } \\
\text { und Beruf }\end{array}$ \\
\hline 2008 & Familienbericht 2008 & $\begin{array}{l}\text { Unterstützung der Vereinbarkeit von Familie und } \\
\text { Beruf entsprechend dem Wunsch junger Mütter, } \\
\text { Väter und Mütter müssen über genügend Zeit zur } \\
\text { Erziehung verfügen, } \\
\text { Spezielle Unterstützung der Vereinbarkeit von Familie } \\
\text { und Beruf in der ersten Lebensphase von Kindern }\end{array}$ \\
\hline
\end{tabular}

\subsubsection{Auf das Kindeswohl gerichtete Zielsetzungen}

Im Koalitionsvertrag der ersten Violetten Koalition vom 13.08.1994 wird die Bedeutung der praktischen Erfahrung von Schülern und Studenten unterstrichen. Besser als zuvor sollten sie in Ausbildung und Studium auf eine aktive Teilnahme am Arbeitsmarkt vorbereitet werden. „Dieses Ziel erfordert gemeinsame Anstrengungen und Verantwortung von Ausbildungsstätten und Wirtschaft" (Ministerie van Algemene Zaken 1994a: 8). Auch das zweite Ziel dieser Regierung knüpft an eine aktive Teilnahme auf dem Arbeitsmarkt an. Die Politiker betonen, dass viele Schüler mit sozialen Problemen und zerstörten Beziehungen zu kämpfen haben und hierdurch eine aktive Teilnahme am Arbeitsmarkt letztendlich nicht möglich sei. Daher müsse der „Prävention und 
Frühsignalisierung von problematischen Situationen systematische Aufmerksamkeit zuteil kommen“ (Ministerie van Algemene Zaken 1994a: 43). Ganz deutlich hebt die Regierung die entscheidende Rolle von Schulen in Erziehung und Integration von Kindern und Jugendlichen hervor, denn Schulen bereiteten Kinder und Jugendliche auf eine aktive gesellschaftliche Teilnahme vor. Die Anstrengungen, die Schulen im Bereich Lehre und Erziehung leisten müssten, seien abhängig von der lokalen Bevölkerung, bzw. dem Standort der Schule. Es sei darum auch weniger sinnvoll, Schulen zentral zu steuern. Indem sie Eigenverantwortung übernehmen, könnten die Schulen den gestellten Anforderungen besser gerecht werden. „Sie (die Schulen) werden daher vorbereitet, in sich verändernden und verschiedenartigen Situationen selbstständig handeln zu können. Daneben wird das Netzwerk von verwaltungsund pädagogischen Hilfsstrukturen im Schulwesen insofern vereinfacht, dass es konkreter auf die aktuellen und praktischen Problemen von Schulen gerichtet ist" (Ministerie van Algemene Zaken 1994a: 48). Auch im Hinblick auf die individuellen Studienkredite (hier: BAföG-Kosten) habe das Kabinett eine prinzipielle Entscheidung getroffen, „bei der die Studenten selbst Verantwortung für Studien- und Lebensunterhaltskosten treffen“ (Ministerie van Algemene Zaken 1994a: 52). Im Rahmen der Neugestaltung des Darlehens für Studienkosten sei es der Regierung wichtig, dass Studenten diese Darlehen zu akzeptablen Konditionen zurückzahlen könnten, und dass realistische Studienbedingungen (auch für Studenten aus ärmeren Haushalten) gewährleistet blieben.

In der dazugehörigen Regierungserklärung hebt die Regierung am 31.08.1994 hervor, sie wolle die Probleme von und mit Jugendlichen, die infolge verschiedener gesellschaftlicher Entwicklungen zunähmen, stärker bekämpfen. Es sei vor allem ein harter Kern Jugendlicher, der für unverhältnismäßig große gesellschaftliche Probleme sorge. „Eine zusammenhängende Politik auf dem Gebiet der Jugendhilfe und des Jugendschutzes im Zusammenhang mit schneller korrigierenden strafrechtlichen Auftreten, ist daher dringend erforderlich“ (Ministerie van Algemene Zaken 1994b: 9). Neben der Schulen, denen die Regierung schon im Koalitionsvertrag viel Freiraum für Eigenverantwortung biete, wolle man auch die Möglichkeiten zur Verwendung von Kinderbetreuung stärken. „Die Politik zur Vergrößerung der Kinderbetreuungskapazitäten wird fortgesetzt. Mit den Sozialpartnern wird sich 
über eine Zunahme der Anzahl betrieblicher Kinderbetreuungsplätze beraten“ (Ministerie van Algemene Zaken 1994b: 13).

In der umfangreichen Notiz zum Thema Familie, den die Staatssekretärin des Ministeriums für Gesundheit, Wohlfahrt und Sport im Jahre 1996 an die Tweede Kamer (hier: Bundestag) schickte, betont sie, dass es die Erziehung von Kindern sei, die dem Themenbereich Familie zu einen besonderen Stellenwert verhelfe. „Falls die Kernfunktionen der Familie nicht erfüllt werden, und es hierdurch $\mathrm{zu}$ einer Bedrohung oder Antastung der Kinderrechte komme, müssten Schutz und Unterstützung der Kinder sowie eine Wahrnehmung ihrer Bedürfnisse durch die Politik“ gesichert sein (Ministerie van Volksgezondheid, Welzijn en Sport 1996: 8). In diesem Zusammenhang wird auch die Chancengleichheit von Kindern unterstrichen. Nicht in allen Familien seien die Grundbedingungen für eine gelungene Erziehung vorhanden. „Wo es ohne Förderung $\mathrm{zu}$ großer Ungleichheit von Entwicklungsmöglichkeiten von Familien oder einzelnen Familienmitgliedern kommt, liegt die Verantwortlichkeit bei der Regierung, um diese (Lebens) Chancen zu vergrößern“ (Ministerie van Volksgezondheid, Welzijn en Sport 1996: 8). Die Verbesserung der Chancengleichheit gelte auch als Zielsetzung im Hinblick auf die Schulen, denn sozialwirtschaftliche Ungleichheiten in der Familie könnten die Resultate von Schülern negativ beeinflussen. „Schwerpunkte in der Schulpolitik sind darum: Bekämpfung von schulischen Rückständen von Schülern aus einer sozial benachteiligten Umgebung oder mit einem anderen kulturellem Hintergrund sowie die Betreuung von Schülern mit sozialemotionalen Problemen, Verhaltensstörungen und Lernschwächen im Unterricht“ (Ministerie van Volksgezondheid, Welzijn en Sport 1996: 16). Hieran anknüpfend solle auch der Jugendschutz Probleme von Kindern und Jugendlichen mehr als zuvor im Zusammenhang mit den bestehenden Familienhintergründen einbeziehen. „Durch die Teilnahme eines Sozialarbeiters (am regulären Familienleben) können die Familien an einer besseren Wahrnehmung einer oder mehrerer Familienaufgaben arbeiten“ (Ministerie van Volksgezondheid, Welzijn en Sport 1996: 17).

Im Koalitionsvertrag der zweiten Violetten Koalition vom 18.07.1998 wird, wie auch schon während der ersten Violetten Regierung, betont wie wichtig es sei Kindern und Jugendlichen $\mathrm{zu}$ lernen Eigenverantwortung $\mathrm{zu}$ übernehmen. Hierdurch vergrößere sich die Chance auf eine aktive gesellschaftliche 
Teilnahme auch in ihrem späteren Leben. Man wolle daher sowohl die präventive als auch die kurative Jugendhilfe stärken. „Das Ziel ist eine Situation, in der alle Kinder in den Niederlanden die Möglichkeit bekommen, sich in einer zuwendungsvollen, sicheren Umgebung zu entwickeln, in der Probleme frühzeitig signalisiert und angepackt werden“ (Ministerie van Algemene Zaken 1998a: 67).

In der Regierungserklärung der zweiten Violetten Koalition vom 25.08.1998 wird, wie auch schon im Familienbericht von 1996, die Bekämpfung der Jugendkriminalität als Ziel genannt (vergl. Ministerie van Algemene Zaken 1998b: 4). Stärker als zuvor hob man an dieser Stelle die verschiedenen Betreuungsmöglichkeiten von Kindern und Jugendlichen hervor, denn der soziale Zusammenhang in der Gesellschaft beginne schon in der Jugend. Hierdurch müsse vorgebeugt werden, „dass Gruppen sich von der Gesellschaft abwenden oder, was noch schlimmer ist, sich gegen die Gesellschaft kehren. Durch eine gemeinsame Kräftebündelung von Eltern, den Jugendlichen selbst und dem Bildungssystem wird diese negative Entwicklung gebremst" (Ministerie van Algemene Zaken 1998b: 14). Auch betont die Regierung die Bedeutung von Betreuungsmöglichkeiten für Kleinkinder. Schon bei sehr jungen Kindern sei es wichtig, gesellschaftlichen Segregationsbewegungen entgegen zu steuern (vergl. Ministerie van Algemene Zaken 1998b: 15).

Einige Jahre später spielt im Koalitionsvertrag der ersten Koalition unter Balkenende (03.07.2002) wiederum die Schule als Ort der Vorbereitung von Kindern auf gesellschaftliche Integration und Übernahme von Verantwortung eine Rolle. Als Gegenreaktion auf das Wachsen der Schulen während der violetten Regierungen wolle diese Regierung sich an kleineren Maßstäben orientieren, um Orte der Geborgenheit für Schüler zu erzeugen. „Fusionen und die Bildung immer größerer Schulen werden aus diesem Grund gebremst“ (Ministerie van Algemene Zaken 2002a: 12). Inhaltlich werde allerdings festgehalten an der während der violetten Koalitionen eingesetzten Selbstregulierung von Schulen. „Dabei wird den Schulen mehr Raum gegeben bei der Umsetzung des ,Studienhauses' (niederländisches Reformkonzept zur aktiven Lernbeteiligung), der Gestaltung der Grundausbildung und der Klassenverkleinerung. Auch für finanzielle Fragen übernehmen die Schulen selbst Verantwortung" (Ministerie van Algemene Zaken 2002a: 12). Bildungsinstitute müssen sich auf ihre eigentlichen Aufgaben konzentrieren 
können und sollen so wenig wie möglich mit zusätzlicher Verwaltungsarbeit belastet werden. „Aus diesem Grund werden „Verwaltungslasten, die die Schulen belasten, kritisch auf ihren Nutzen und ihre Notwendigkeit überprüft" (Ministerie van Algemene Zaken 2002a: 12). Wenn möglich, sollten Schulen verselbstständigt werden. Die Integration von Kindern mit verschiedenen kulturellen Hintergründen sei der Regierung ein wichtiges Anliegen. „Im Bezug auf die Förderung von Kohäsion und gesellschaftlicher Integration wird im Sekundarunterricht der gesellschaftlichen Orientierung, in der die verschiedenen Lebensanschauungen eine Rolle spielen, eine besondere Aufmerksamkeit gewidmet“ (Ministerie van Algemene Zaken 2002a: 12).

In ihrer Regierungserklärung betont diese Regierung am 26.07.2002, dass die Regierung Verantwortung teilen wolle, gleichzeitig aber auch Grenzen stellen müsse. Das gelte insbesondere für die Wohlfahrt der Kinder. „Die verantwortlichen Instanzen müssen Kinder mit Problemen frühzeitig erkennen und adäquate Lösungen erarbeiten“ (Ministerie van Algemene Zaken 2002b: 6).

Die zweite Koalition unter Balkenende greift in ihrem Koalitionsvertrag vom 16.05.2003 das Thema der Früherkennung kindlicher Entwicklungsprobleme erneut auf. Die Regierung unterstreicht die Eigenverantwortung der Eltern hinsichtlich der Erziehung ihrer Kinder, hebt aber erneut die Rolle der Früherkennung von Problemen hervor. „Es muss ein Jugendhilfesystem geben, in dem in Problemsituationen frühzeitig Unterstützung geboten und adäquat eingegriffen wird, um ernsthafte Beschädigungen zu verhüten“ (Ministerie van Algemene Zaken 2003a: 6).

In ihrer Regierungserklärung räumt die neue Koalition (11.06.2003) der Bekämpfung der Jugendarbeitslosigkeit eine hohe Priorität ein. Gemeinsam mit den Sozialpartnern wolle die Regierung hieran arbeiten, „so dass Jugendliche eine Perspektive haben und dem Arbeitsmarkt keine Generation verloren geht“ (Ministerie van Algemene Zaken 2003b: 7). Wie schon im Koalitionsvertrag verdeutlicht, wolle die Regierung die Erziehung von Kindern und Jugendlichen stärken. Sie hebe daher erneut die Eigenverantwortung der Eltern hervor, unterstreiche aber auch, dass „Einrichtungen der Jugendhilfe nicht aus Mangel an gegenseitiger Abstimmung zulassen dürfen, dass die Probleme in einer Familie ausufern. Die Regierung will daher Verbesserungen in der Jugendhilfe einen kräftigen Impuls geben“ (Ministerie van Algemene Zaken 2003b: 10). 
Im Familienbericht 2006, den die Staatssekretärin des Ministeriums für Gesundheit, Wohlfahrt und Sport im Jahre 2006 an die Tweede Kamer (hier: Bundestag) schickte, wird betont, dass ein Eingreifen „in Situationen, welche die Rechte und Entwicklungsmöglichkeiten des Kindes bedrohen" ein wichtiges Ziel der Familienpolitik sei (Ministerie van Volksgezondheid, Welzijn en Sport 2006: 2). Die Belange des Kindes stehen hierbei zentral, und falls Eltern sich nicht kooperativ verhalten, würden sie stärker als zuvor zur Zusammenarbeit mit den Einrichtungen in der Jugendhilfe gezwungen. Die Situation in den Familien sei von außen oft nur schwer zu beurteilen. Um aber der Entwicklung der Kinder soweit wie möglich gerecht $\mathrm{zu}$ werden, solle die öffentliche Verantwortung so nah wie möglich bei den Familien angesiedelt werden. „Das Kabinett findet, dass die Verantwortung zuerst den Gemeinden zukommt. Sie können die Bedürfnisse von Eltern und Jugendlichen am besten übersehen und sorgen für mehr Zusammenhang im Wust der Einrichtungen und Organisationen (auf diesem Gebiet). Die lokale Regiefunktion bildet auch den Leitgedanken im neuen Gesetz zur Gesellschaftlichen Unterstützung, dass in 2007 in Kraft treten wird“ (Ministerie van Volksgezondheid, Welzijn en Sport 2006: 11). Aber auch die Prävention jugendlicher Entwicklungsprobleme sei der Regierung ein wichtiges Anliegen. Hierbei wolle sie die Eltern in ihrer Erziehungsverantwortung unterstützen. „Erziehungsunterstützung muss normaler und sozial akzeptiert werden: es muss selbstverständlich und nicht außergewöhnlich sein, wenn Eltern zum Beispiel bei ihrem ersten Kind an Erziehungskursen teilnehmen“ (Ministerie van Volksgezondheid, Welzijn en Sport 2006: 12). Da Probleme in den Familien oft an den Schulen sichtbar werden, wolle die Regierung ein Erziehungsnetzwerk zwischen den primären und weiterführenden Unterrichtsformen aufbauen (vergl. Ministerie van Volksgezondheid, Welzijn en Sport 2006: 18). Schulen seien nicht in erster Linie Orte, an denen sich Jugendprobleme manifestieren, sondern vor allem Orte, an denen gesellschaftliche Werte und Normen auf jüngere Generationen übertragen werden. „Die Förderung von bürgerschaftlichem Engagement und sozialer Integration wird ein ausdrücklich gesetzlich festgelegtes Ziel für primäre und sekundäre Schulen“ (Ministerie van Volksgezondheid, Welzijn en Sport 2006: 22). Außerdem wolle die Regierung das Entstehen von ,Breiten (Übergreifenden) Schulen', in denen Aufgaben der Kinderbetreuung und Jugendpflege mit anderen gesellschaftlichen Angeboten verbunden werden, fördern. Die Kompetenz liege in diesem Bereich bei den Gemeinden. „Das 
Kabinett unterstützt das Vorhaben der Gemeinden, im Jahr 2010 über mindestens 1200 so genannte ,Breite Schulen' in den Niederlanden zu verfügen“ (Ministerie van Volksgezondheid, Welzijn en Sport 2006: 17).

Kurze Zeit nach diesem Familienbericht ist im Koalitionsvertrag der vierten Koalition unter Balkenende (07.02.2007) das Verlassen der Schule ohne Abschluss ein wichtiges politisches Thema. Erfolgreiche Schulabschlüsse werden als notwendiger Einstieg in die aktive gesellschaftliche Teilnahme gesehen. Daher wolle man bis zum Jahr 2012 die Anzahl der Schulabgänger ohne erfolgreichen Abschluss halbieren. Um dieses Ziel zu verwirklichen, "wird der offensive Angriff fortgesetzt und die Zusammenarbeit zwischen Regierung, Eltern, Schulen, Wirtschaft (...), Sozialarbeit, Jugendhilfe, Gemeinden und Polizei verstärkt“. (Ministerie van Algemene Zaken 2007a: 7). Neben dieser eher quantitativen Zielsetzung erklärt die Regierung auch die generelle Förderung von Talenten junger Menschen zu einem wichtigen Ziel für den individuellen Menschen einerseits und der Gesellschaft andererseits (vergl. Ministerie van Algemene Zaken 2007a: 18). Die wachsende Jugendkriminalität halte die Regierung für unakzeptabel, und sie wolle ihr daher auf zweierlei Arten begegnen. Erstens „durch eine Ausweitung der Strafmaßnahmen“ (Ministerie van Algemene Zaken 2007a: 35) und zweitens durch die breite Zusammenarbeit zur Prävention kriminellen Verhaltens (vergl. Ministerie van Algemene Zaken 2007a: 33).

Die Koalition wiederholt in ihrer Regierungserklärung am 01.03.2007 die Zielsetzung hinsichtlich der Schulabschlüsse und betont. „Das Kabinett will gemeinsam mit Schulen, Lehrern und anderen Beteiligten den Anfall auf den Schulausfall einsetzen“ (Ministerie van Algemene Zaken 2007b: 4). Ihre Erziehung präge Kinder ihr ganzes Leben. Daher erkläre die Regierung eine geeignete Erziehung von Kindern als entscheidendes Kriterium und unterstreiche sie, wie schon in Dokumenten der Vorgängerregierung angekündigt, sie wolle „Eltern, wo nötig, unterstützen in ihrer Erziehungsaufgabe“ (Ministerie van Algemene Zaken 2007b: 7). In diesem Zusammenhang werde auch ein weiteres Ziel wiederholt, welches die Regierung als Priorität bezeichne. „Jedes Kind verdient gerechte Chancen, unabhängig von dem Ort an dem seine Wiege steht. Diesen Chancen für Kinder werde das Kabinett viel Aufmerksamkeit widmen (Ministerie van Algemene Zaken 2007b: 7). 
Der neu ernannte Minister für Jugend und Familie unterstreicht in seinem Programm, das er 2007 an die Tweede Kamer (hier: Bundestag) schickte, dass es Ziel der Jugend- und Familienpolitik sein müsse, allen Kindern Chancengleichheit $\mathrm{zu}$ bieten. Er verdeutlicht diesen Begriff durch die Benennung von fünf Bedingungen, die bei der Erziehung von Kindern erfüllt werden sollten. Ziel der Regierung sei „ein Land in dem Kinder gesund und sicher aufwachsen können, in dem sie ihre Talente entwickeln können und Freude haben, in dem sie lernen, ihren Beitrag an der Gesellschaft zu leisten und gut auf die Zukunft vorbereitet sind: Kurzum, wo alle Kinder alle Chancen bekommen“ (Jeugd en Gezin 2007: 3). Den meisten Kindern in den Niederlanden gehe es gut. Sie haben keine Entwicklungsschwierigkeiten. Allerdings gäbe es noch immer einen harten Kern von Kindern, die viele Probleme hätten. „Dem müssen wir mit allen Verantwortlichen - Eltern, Professionals, und dem Kabinett - alle Aufmerksamkeit schenken. Unverbindlichkeit ist dabei nicht mehr denkbar; jeder muss Verantwortung übernehmen“ (Jeugd en Gezin 2007: 4). Um zu einer erfolgreichen Teilung der Verantwortlichkeiten zu kommen, sei eine interdisziplinäre Zusammenarbeit zwischen den verschiedenen Einrichtungen der Jugendhilfe notwendig. Mit Pflege- und Beratungsteams (NL: ZAT's), in denen die verschiedenen Einrichtungen aktiv zusammenarbeiten, habe man sehr gute Erfahrungen gemacht. Daher rufe das Kabinett „die Gemeinden auf, um zu einer 100prozentigen Abdeckung dieser Erziehungs- und Beratungsteams für alle Altersgruppen zu kommen“ (Jeugd en Gezin 2007: 17). Für das Wohlergehen von Kindern halte die Regierung auch die Lebensumweltbedingungen von Kindern für sehr entscheidend. Unter einer kinderfreundlichen Umgebung verstehe sie eine saubere, gesunde und sichere Umgebung mit passenden Angeboten zur Freizeitgestaltung. Bei der Einrichtung der Lebensumwelt wolle sie Kinder einbeziehen. Ziel sei, dass „im Jahr 2011 jede Gemeinde ein Mitspracherecht u.a. zum Thema kinderfreundliche Wohn- und Lebensumwelt für Kinder und Jugendliche“ kenne (Jeugd en Gezin 2007: 24). Da die Wartezeiten in der Jugendhilfe zu diesem Zeitpunkt als problematisch angesehen werden, habe das Kabinett den Jugendhilfesektor aufgefordert, neue Normen im Hinblick auf maximale Wartezeiten im Bereich der Jugendhilfe zu entwickeln. Bis dahin „bleiben die Wartezeiten in der Jugendfürsorge innerhalb der hierfür verabredeten Normen“ (Jeugd en Gezin 2007: 30). Weiterhin benennt die Regierung Ziele im Hinblick auf Kinder und Jugendliche ohne 
Ausbildung oder Abschlusszeugnis. Um sie vor einer gesellschaftlichen Ausgrenzung bzw. einem Absturz zu bewahren, wolle „das Kabinett ihnen durch die nationale Einrichtung von Kampussen im Jahr 2011, die auf Schulung und Begleitung in Richtung Arbeit gerichtet sind, eine Perspektive bieten“ (Jeugd en Gezin 2007: 34).

Im Familienbericht 2008, den der neu ernannte Minister für Jugend und Familie an die Tweede Kamer (hier: Bundestag) schickte, spielt das Thema Konflikte zwischen Eltern und Folgen von Trennungen eine wichtige Rolle. In der Jugendhilfe werde der große Einfluss, den die elterlichen Beziehungen auf das Wohlbefinden der Kinder haben, stets deutlicher. Daher sehe die Regierung es als ihr Ziel, „in Fähigkeiten zu investieren, um die Qualität der Beziehung zwischen den Eltern zu verbessern“ (Jeugd en Gezin 2008: 7). Wie auch im Programm zum Thema Jugend und Familie unterstreicht der Minister erneut, dass es normal sei, in der Erziehung von Kindern vor offenen Fragen zu stehen. „Da der soziale Kontext von Familien sich verändert hat (...) fehle es Eltern hierdurch in zunehmendem Maße an einem sozialem Netzwerk, auf das sie zurückgreifen können“" (Jeugd en Gezin 2008: 8). Aus diesem Grund sollen Fragen der Erziehung auch eine öffentliche Unterstützung erhalten. Als ein weiteres Ziel wird in diesem Bericht die Bekämpfung von sozialer Ausgrenzung infolge von Kinderarmut benannt. „Armut kann (gesellschaftlicher) Teilhabe im Wege stehen, wodurch das Risiko auf soziale Isolation steigt" (Jeugd en Gezin 2008: 9). Da allerdings die gesellschaftliche Teilhabe zur Realisierung der Chancengleichheit von Kindern essentiell sei, müsse diese Entwicklung bekämpft werden. 
Tabelle 24: Chronologisches Übersichtstableau der auf das Kindeswohl gerichteten Zielsetzungen in den Niederlanden

\begin{tabular}{|c|c|c|}
\hline Jahr & Dokument & Zielsetzung \\
\hline 1994 & Koalitionsvertrag Kok I & $\begin{array}{l}\text { Bessere Vorbereitung von Auszubildenden und Studenten } \\
\text { auf eine aktive Teilnahme am Arbeitsmarkt, } \\
\text { Systematische Aufmerksamkeit für Prävention und } \\
\text { Frühsignalisierung von problematischen Situationen, } \\
\text { Stärkung von Eigenverantwortung im Schulwesen, } \\
\text { Stärkung finanzieller Eigenverantwortung von Studenten }\end{array}$ \\
\hline 1994 & Regierungserklärung Kok I & $\begin{array}{l}\text { Einführung einer zusammenhängenden Politik im } \\
\text { Bereich Jugendhilfe und Jugendschutz sowie schnelleres } \\
\text { strafrechtliches Auftreten, } \\
\text { Fortsetzung der Politik zur Kinderbetreuung und Ausbau } \\
\text { der betrieblichen Kinderbetreuungsmöglichkeiten }\end{array}$ \\
\hline 1996 & Familienbericht 1996 & $\begin{array}{l}\text { Gewährung von Kinderrechten durch die Politik, } \\
\text { Verkleinerung der Ungleichheiten zur } \\
\text { Entwicklungsmöglichkeit für Familien oder } \\
\text { Familienmitglieder, } \\
\text { Besondere Unterstützung von Kindern und Schülern mit } \\
\text { schwächerem Lernhintergrund, } \\
\text { Besondere Unterstützung in schwachen Familien durch } \\
\text { den Einsatz von Sozialarbeitern, } \\
\text { Bekämpfung von Jugendkriminalität }\end{array}$ \\
\hline 1998 & Koalitionsvertrag Kok II & $\begin{array}{l}\text { Verstärkung von sowohl präventiver als auch kurativer } \\
\text { Jugendhilfe mit dem Ziel einer Chancengleichheit für alle } \\
\text { Kinder }\end{array}$ \\
\hline 1998 & Regierungserklärung Kok II & $\begin{array}{l}\text { Bekämpfung von Jugendkriminalität, } \\
\text { Bessere Betreuung von Kindern und Jugendlichen vor } \\
\text { dem Hintergrund einer gesellschaftlichen Verstärkung, } \\
\text { Bessere Förderung von Kleinkindern, um frühzeitige } \\
\text { Segregation zu verhindern }\end{array}$ \\
\hline 2002 & $\begin{array}{l}\text { Koalitionsvertrag Balkenende } \\
\text { I }\end{array}$ & $\begin{array}{l}\text { Begrenzung von Schulgrößen, } \\
\text { Verstärkung der Eigenverantwortung der Schulen, } \\
\text { Begrenzung der Verwaltungslasten und } \\
\text { Verselbstständigung von Schulen, } \\
\text { Förderung von kultureller Integration im } \\
\text { Sekundarunterricht }\end{array}$ \\
\hline 2002 & $\begin{array}{l}\text { Regierungserklärung } \\
\text { Balkenende I }\end{array}$ & $\begin{array}{l}\text { Bessere Frühsignalisierung von Entwicklungsproblemen } \\
\text { bei Kindern }\end{array}$ \\
\hline 2003 & $\begin{array}{l}\text { Koalitionsvertrag Balkenende } \\
\text { II }\end{array}$ & Verbesserung frühzeitiger Erziehungshilfen \\
\hline 2003 & $\begin{array}{l}\text { Regierungserklärung } \\
\text { Balkenende II }\end{array}$ & $\begin{array}{l}\text { Gemeinsame Bekämpfung der Jugendarbeitslosigkeit mit } \\
\text { den Sozialpartnern, } \\
\text { Verbesserung der Arbeit in den Einrichtungen der }\end{array}$ \\
\hline
\end{tabular}




\begin{tabular}{|c|c|c|}
\hline & & Jugendhilfe \\
\hline 2006 & Familienbericht 2006 & $\begin{array}{l}\text { Eingriff in Situationen, welche die Rechte und } \\
\text { Entwicklungsmöglichkeiten von Kindern bedrohen, } \\
\text { Unterstützung der Regiefunktion im Bereich der } \\
\text { Jugendhilfen auf lokaler Ebene, } \\
\text { Verbreitung von Erziehungsunterstützung für Eltern, } \\
\text { Realisierung eines übergreifenden Pflegenetzwerks an } \\
\text { Schulen, } \\
\text { Förderung von Bürgerschaft und sozialer Integration an } \\
\text { Schulen, } \\
\text { Realisierung von } 1200 \text {,Breiten Schulen' bis zum Jahr } 2010\end{array}$ \\
\hline 2007 & $\begin{array}{l}\text { Koalitionsvertrag Balkenende } \\
\text { IV }\end{array}$ & $\begin{array}{l}\text { Halbierung der Schulabgänger ohne Abschlusszeugnis bis } \\
\text { zum Jahre 2012, } \\
\text { Förderung der Talente durch Wahlfreiheit von Eltern und } \\
\text { Schülern, } \\
\text { Bekämpfung von Jugendkriminalität durch strengere } \\
\text { Straf- und gezielte Präventionsmaßnahmen }\end{array}$ \\
\hline 2007 & $\begin{array}{l}\text { Regierungserklärung } \\
\text { Balkenende IV }\end{array}$ & $\begin{array}{l}\text { Verringerung des Schulausfalls, } \\
\text { Bereitstellung von Erziehungshilfen für Eltern, } \\
\text { Förderung von Chancengleichheit von Kindern }\end{array}$ \\
\hline 2007 & $\begin{array}{l}\text { Programm für Jugend und } \\
\text { Familie, 2007-2011 }\end{array}$ & $\begin{array}{l}\text { Förderung von Chancengleichheit von Kindern durch die } \\
\text { Erfüllung von fünf Bedingungen in ihrer Erziehung, } \\
\text { Verbindliche Bekämpfung des harten Kerns der } \\
\text { Jugendproblematik, } \\
\text { Aufbau einer 10o-prozentigen Deckung von lokalen Pflege- } \\
\text { und Beratungsteams für alle Altersgruppen, } \\
\text { Realisierung eines Mitspracherechts für Kinder und } \\
\text { Jugendliche bis zum Jahre } 2011 \text { u.a. zum Thema } \\
\text { kinderfreundliche Wohn- und Lebensumwelt in allen } \\
\text { Gemeinden, } \\
\text { Wartezeiten in der Jugendfürsorge bleiben innerhalb der } \\
\text { hierfür verabredeten Normen, } \\
\text { Einführung von Kampussen für perspektivlose } \\
\text { Jugendliche im Jahr 2oll, } \\
\text { 10\% Verminderung der Jugendkriminalität }\end{array}$ \\
\hline 2008 & Familienbericht 2008 & $\begin{array}{l}\text { Unterstützung der Erziehungskapazitäten von Eltern mit } \\
\text { Beziehungsproblemen bzw. im Falle einer Scheidung, } \\
\text { Allgemeine Unterstützung bei Erziehungsfragen von } \\
\text { Eltern, } \\
\text { Bekämpfung von sozialer Ausgrenzung infolge von } \\
\text { Kinderarmut }\end{array}$ \\
\hline
\end{tabular}




\subsection{Familienpolitische Instrumente in den Niederlanden}

\subsubsection{Instrumente, die die ökonomische Situation der Familienhaushalte betreffen}

Im Koalitionsvertrag der ersten Violetten Koalition vom 13.08.1994 kommt die neue Verantwortungsteilung der Regierung sehr deutlich zur Sprache im Zusammenhang mit den vorgenommenen Einsparungen im Bereich des Kindergeldes und der Witwen- bzw. Waisenrente. „Während die Arbeitnehmerversicherungen vor allem um eine neue Verantwortungsteilung zwischen Regierung, Arbeitgebern und Arbeitnehmern fragen, eignen sich das Kindergeldgesetz und das Allgemeine Witwen- bzw. Waisengesetz für neue Abwägungen im Hinblick auf die Verantwortungsteilung zwischen der Gemeinschaft der Bürger einerseits und den individuellen Bürgern bzw. Familien und anderen Lebensformen andererseits" (Ministerie van Algemene Zaken 1994a: 17). Das Kindergeld werde vor dem Hintergrund der sozialen Sicherheit für das erste Kind erhöht. Die Höhe des Kindergeldes sollte aber für jedes dritte und weitere Kind nicht höher sein als für zweite Kinder und das Alter der Kinder entscheidend für die Höhe des Kindergeldes. Zur Kompensierung von z.B. Krankenversicherungskosten werde man in Zukunft quartalsweise finanzielle Zuschläge auszahlen (vergl. Ministerie van Algemene Zaken 1994a: 17). Im Bezug auf Veränderungen im Bereich der Witwen- und Waisenrente habe die Vorgängerregierung einen Gesetzesentwurf beschlossen, der aber letztendlich nicht durchgesetzt werden konnte. Die neue Regierung betone, dass im Gegensatz zur Vergangenheit heute „viele Frauen finanziell nicht oder weniger abhängig von ihrem Ehegatten sind. Für die Deckung des Risikos um Witwe (oder Witwer) zu werden, sind eine Vielzahl privater Versicherungsmöglichkeiten geschaffen“ (Ministerie van Algemene Zaken 1994a: 18f). Daher diene die neue Regierung einen Vorschlag zur Neuregelung der Gesetzeslage ein, bei dem es keine Rechte mehr auf eine Witwenrente für kinderlose Witwen gäbe, und die Höhe der Renten von Witwen mit Kindern einkommensabhängig gestaltet werde. Neben der vielen Einsparungen, welche die neue Regierung als erforderlich sehe, richtete sie sich aber insbesondere auf 
die Kaufkraft finanziell schwächerer Gruppen. Aus diesem Grund wolle die Regierung „eine Kindertabelle für die individuellen Mietzuschüsse zum 1. Januar 1995" einführen. Hierdurch werde sie Wohnlasten von Familien mit Kindern verringern. Den Gedanken der Verantwortungseilung verwende die Regierung auch im Studienbereich. Ein Studium müsse in der Regel auf eine vierjährige Dauer angelegt sein, und Studenten würden durch eine entsprechende BAföG-Regelung stimuliert, um innerhalb dieser Periode ihr Studium erfolgreich zu beenden. „Die Rückzahlpflicht wird vom Studientempo und der Rückzahlungskapazität abhängig gemacht“" (Ministerie van Algemene Zaken 1994a: 52).

In der Regierungserklärung der ersten Violetten Koalition vom 31.08.1994 werden die vorgenommenen Einsparungen im Bereich des Kindergeldes und der Witwen- und Waisenrente, wie bereits im Koalitionsvertrag angekündigt, unterstrichen und der hinter liegende Gedanke der neuen Verantwortungsteilung verdeutlicht. „Die soziale Sicherheit muss sich den gesellschaftlichen Veränderungen durch Emanzipation und Individualisierung besser anpassen. Das bedeutet weniger kollektive Regelungen für dynamische und selbstständige Menschen. Gleichzeitig wird soziale Sicherheit gewährt bleiben müssen, um Arbeitnehmer, von denen mehr Flexibilität erwartet wird, hierzu die Gelegenheit zu bieten, und muss für Menschen, für die es keine andere Möglichkeit gibt, bleibender sozialer Schutz geboten werden“ (Ministerie van Algemene Zaken 1994b: 19).

In der umfangreichen Notiz zum Thema Familie, welche die Staatssekretärin des Ministeriums für Gesundheit, Wohlfahrt und Sport im Jahre 1996 an die Tweede Kamer (hier: Bundestag) schickte, wird betont, dass Schulen Hilfe aus der direkten Umgebung bräuchten, um Probleme mit Schülern und Eltern lösen zu können. Um ihrer Verantwortung in dem Punkt gerecht zu werden, müssten die Schulen daher in der Lage sein, besser als bisher mit den lokalen Einrichtungen der Jugendhilfe zusammenzuarbeiten. „Die Gemeinden erhalten hierzu die Kompetenzen und finanziellen Mittel. Da die Verantwortung für die präventive Jugendhilfe auch dezentralisiert und auf die Gemeinden übertragen wurde, kann auf lokalem Niveau Abstimmung zwischen diesen Sektoren erreicht werden“ (Ministerie van Volksgezondheid, Welzijn en Sport 1996: 12). Um die finanzielle Selbstständigkeit von Frauen und Familien zu fördern, werde auf den Ausbau der Kinderbetreuung besonderer Wert gelegt. „Zum 1. 
Januar 1996 ist die Abgabenverminderung für Kinderbetreuung eingeführt, eine finanzielle Stimulierung für Arbeitgeber, die Kinderbetreuungsplätze für ihre Arbeitnehmer mieten. Außerdem sind für die zeitliche Unterstützung während des Übergangs von sozialer Unterstützung nach Arbeit oder Schulung 85 Millionen für die Bereitstellung von Kinderbetreuung für alleinstehende Sozialhilfeempfänger mit Kindern reserviert" (Ministerie van Volksgezondheid, Welzijn en Sport 1996: 15). Weiterhin wolle die Regierung insbesondere die Gruppe der Eltern mit behinderten Kindern fördern. Ziel sei es diese Kinder soviel wie eben möglich in ihrer direkten Umgebung zu unterstützen, und sie am regulären Unterricht teilnehmen zu lassen. Daher werde an der „Einführung einer lehrlinggebundenen Finanzierung für Schüler mit Behinderungen im Primär- und Sekundarunterricht" gearbeitet (Ministerie van Volksgezondheid, Welzijn en Sport 1996: 19).

Die Nachfolgeregierung unterstreicht in ihrem Koalitionsvertrag vom 18.07.1998 ihr Ziel, die aktive Teilnahme der Bevölkerung am Arbeitsmarkt zu fördern. Hierzu sei es nötig den Ausbau und die Inanspruchnahme von Kinderbetreuung voranzutreiben. Daher „werden im Bereich der Steuern Maßnahmen getroffen, die die Schwelle für Eltern erniedrigen können, bzw. Arbeitgebern eine Stimulans geben, um in Tarifverträgen ihren Mitarbeitern Möglichkeiten zur Kinderbetreuung anzubieten“ (Ministerie van Algemene Zaken, 1998a: 10). Die Förderung einer geeigneten Schulausbildung von Kindern aus sozialschwachen Familien sei der Regierung ein besonderes Anliegen. Daher werde das „Budget für die Regelung der Unterstützung in Studienkosten (...) erhöht, um die Beihilfe von Eltern mit einem relativ niedrigen Einkommen den steigenden Preisen für Bücher, Lehrmittel und übrigen Studienkosten anzupassen“ (Ministerie van Algemene Zaken 1998a: 12).

${ }^{25} \mathrm{Im}$ Koalitionsvertrag der ersten Koalition unter Balkenende vom 03.07.2002 wird das Ziel einer aktiven Teilnahme möglichst großer Teile der Bevölkerung am Arbeitsmarkt unterstrichen. Die Regierung wolle ein Gleichgewicht im Reichshaushalt durch unter anderem „eine Verschiebung der steuerlichen 
Stimulans der Frage nach Arbeit in Richtung Förderung des Arbeitsangebotes“ (Ministerie van Algemene Zaken 2002a: 2) herstellen. Dementsprechend unterstütze sie Maßnahmen, die eine Teilnahme am Arbeitsmarkt fördern. Konkret wolle sie Familien fördern durch die Einführung einer Lebenslaufvergünstigung, die es Menschen erleichtern solle, z.B. eine zeitlich begrenzte Erziehungsperiode von Kindern im Rahmen des bestehenden Arbeitsverhältnisses zu ermöglichen. Das Prinzip dieser Lebenslaufregelung baue auf einer finanziellen Unterstützung von strukturellen Sparmaßnahmen von Arbeitnehmern und einer finanziellen Unterstützung von staatlicher Seite auf. Außerdem wolle die Regierung durch die Einführung einer einkommensbedingten Ermäßigung für Kinder die finanzielle Situation von Familien stärken. Im Zusammenhang mit der bevorstehenden Krankenversicherungsreform unterstütze die Koalition die Einführung einer einkommensbedingten Pflegezulage neben eines Reichsbeitrags für die Prämie der Krankheitskosten für Kinder (vergl. Ministerie van Algemene Zaken 2002a: 18).

In ihrer Regierungserklärung wiederholt die Regierung am 26.07.2002 ihr Anliegen, die Arbeitsmarktteilnahme durch einen größtmöglichen Teil der Bevölkerung zu stimulieren. Durch entsprechende Maßnahmen wie z.B. die neu einzuführende Lebenslaufvergünstigung, werde es Arbeitnehmern ermöglicht, zum Beispiel für Lebensphasen, in denen Pflege eines Familienmitgliedes erforderlich sei, Zeit und Geld zur Verfügung zu haben (Ministerie van Algemene Zaken 2002b: 8). Solidarität zwischen finanziell stärkeren und schwächeren Mitgliedern der Gesellschaft sei der Regierung ein Anliegen. Diese Solidarität gelte vor allem auch für Familien mit Kindern. Sie „profitieren von der Einführung einer einkommensbedingten Steuerermäßigung für Kinder“ (Ministerie van Algemene Zaken 2002b: 8).

Relativ kurze Zeit später unterstreicht die neue Koalition Balkenende II in ihrem Koalitionsvertrag vom 16.05.2003 (wie bereits im Koalitionsvertrag der ersten Koalition unter Balkenende) sie werde die aktive Arbeitsmarktteilnahme durch finanzielle Regelungen stimulieren. Hierzu wolle sie die einkommensabhängigen Regelungen weiter straffen (durch die Bündelung von Regelungen und die Uniformierung der Einkommensbegriffe z.B. im Familienbereich). „In kürzester Zeit wird die heutige Steuerermäßigung für Familien mit Kindern einkommensabhängig gestaffelt; diese 
(Steuerermäßigung) wird mit Eingang des Jahres 2004 erhöht“ (Ministerie van Algemene Zaken 2003a: 4). Schulen spielen im Hinblick auf die Erziehung und Integration von Kindern mit verschiedenartigen Hintergründen eine wichtige Rolle. Daher wolle die Regierung die finanzielle Eigenverantwortung von Schulen stärken. „Schuletats sollen soviel wie möglich gebündelt und (auch von den Gemeinden) dezentralisiert werden in die Richtung der Schulen“ (Ministerie van Algemene Zaken 2003a: 5).

${ }^{26} \mathrm{Im}$ Familienbericht 2006, welchen die Staatssekretärin des Ministeriums für Gesundheit, Wohlfahrt und Sport im Jahre 2006 an die Tweede Kamer (hier: Bundestag) schickte, unterstreicht die Regierung die Rolle der Gemeinden bei der Erziehungsunterstützung von Risikofamilien. „Die Regierung hat ab 2004 fünfzig Gemeinden extra Geld gegeben für das Finden und die Unterstützung von Risikofamilien (...) Ziel ist es, sich frühzeitiger ein Bild von Risikofamilien $\mathrm{zu}$ formen, ihnen zu helfen und zu sehen, ob lokale Hilfsmaßnahmen in der Praxis auch funktionieren“ (Ministerie van Volksgezondheid, Welzijn en Sport 2006: 10). Aufgrund der wirtschaftlich prekären Situation habe die Regierung Familien finanziell unterstützt. Die Regierung weise an dieser Stelle auf die Erhöhung der Summe der einkommensbedingten Steuerermäßigung für Familien mit Kindern, die kostenlose Krankenversicherung von Kindern bis zu 18 Jahren im neuen Gesundheitsversicherungssystem und die Befreiung von Schulgeldern in der Sekundarstufe bzw. für 16- und 17-jährige Auszubildende (Ministerie van Volksgezondheid, Welzijn en Sport 2006: 24f). Auch die Vereinbarkeit von Familie und Beruf wolle die Regierung unterstützen. Sie habe daher Maßnahmen in zwei Bereichen getroffen. Erstens bekomme „der weniger verdienende Teil des Elternpaars und alleinstehende Eltern (...) eine extra Steuerermäßigung: die ergänzende Kombinationsermäßigung“ (Ministerie van Volksgezondheid, Welzijn en Sport 2006: 24f). Außerdem habe die Regierung stark investiert in den Ausbau der Kinderbetreuung für Arbeitnehmer. Seit dem Jahre 1990, also fünfzehn Jahre zuvor, sei das Angebot in der Kinderbetreuung um ein zehnfaches gestiegen, von 20.000 auf 200.000 Plätze. Hierauf sei auch gesetzlich reagiert. „Seit dem 1. Januar 2005 gilt das Kinderbetreuungsgesetz. 
Dieses Gesetz garantiert eine finanzielle Zugänglichkeit für vor allem die niedrigeren Einkommensgruppen und liberalisiert den Kinderbetreuungssektor. Das Gesetz geht von einer dreigliedrigen Finanzierung aus, die zu gleichen Teilen durch die Regierung, die Arbeitgeber und die Eltern (einkommensabhängig) finanziert wird. Arbeitnehmer, die Arbeit und Erziehung kombinieren, können die Kinderbetreuungszulage in Anspruch nehmen“ (Ministerie van Volksgezondheid, Welzijn en Sport 2006: 29). In erster Linie gehe die Regierung hierbei von einer freiwilligen Beteiligung der Arbeitgeber an dieser gesetzlichen Regelung zur Kinderbetreuung aus. Falls aus Evaluierungen allerdings deutlich werde, dass nicht wenigstens $90 \%$ der Arbeitgeber sich an diesen Kosten beteiligen, werde der Arbeitgeberanteil in den Betreuungskosten als Pflichtanteil gesetzlich verankert. Im Falle von Arbeitslosigkeit behalten Arbeitnehmer sechs Monate Recht auf die Kinderbetreuungszulage. In diesem Zusammenhang unterstreiche die Regierung, dass das Recht auf Arbeitslosengeld in den Niederlanden an diverse Reintegrationsmaßnahmen gekoppelt sei. Nicht immer halte die Regierung die vorgesehene, zügige Reintegration für realistisch. „Eine temporäre Befreiung (von den Reintegrationsmaßnahmen) kann in Frage kommen, falls (Pflege)Eltern entsprechend intensive notsächliche Betreuung für ihr krankes oder behindertes Kind oder Partner übernehmen“ (Ministerie van Volksgezondheid, Welzijn en Sport 2006: 31).

Im Koalitionsvertrag der vierten Großen Koalition unter Balkenende vom 07.02.2007 verspricht die Regierung den Eltern von schulpflichtigen Kindern, "Schulbücher im Sekundarunterricht werden gratis“ (Ministerie van Algemene Zaken 2007a: 18). Wie in allen Koalitionsverträgen unter Balkenende, wird auch an dieser Stelle die aktive Teilnahme aller Bürger in Beruf und Gesellschaft als ein wichtiges Ziel unterstrichen, welches diese Regierung mit diversen Maßnahmen unterstützen wolle. Drei der an dieser Stelle genannten Maßnahmen seien direkt an Familien gerichtet. Hierbei gehe es erstens um die Übertragbarkeit der allgemeinen Abgabenermäßigung, welche „gleichmäßig in zwanzig Jahren mit 5\%-Punkten jährlich verringert wird. Diese Maßnahme betrifft alle nach 1971 Geborenen, die keine Kinder in der Alterskategorie von o bis 6 Jahren haben“ (Ministerie van Algemene Zaken 2007a: 25). Eine weitere Maßnahme in diesem Bereich betreffe die Einführung der einkommensabhängigen Arbeitsermäßigung. Diese solle anstelle der 
bestehenden Arbeitsermäßigung eingeführt werden, um die Arbeitsmarktteilnahme von Geringverdienern zu unterstützen. „Für Haushalte mit Kindern in denen beide Partner arbeiten, kommt auf die einkommensabhängige Arbeitsermäßigung eine einkommensabhängige Kombinationsermäßigung" (Ministerie van Algemene Zaken 2007a: 25). Als dritte Maßnahme in diesem Bereich unterstreiche die Regierung, sie wolle Familien, in denen beide Eltern verdienen, einen besseren Zugang zu Steuerermäßigungen für informelle Kinderbetreuung bieten (Ministerie van Algemene Zaken 2007a: 25). Familienfreundlichkeit wolle die Regierung stärken. Neben Zeit und Fähigkeiten bräuchten Eltern vor allem auch finanzielle Mittel, um Kindern eine angemessene Erziehung zu vermitteln. Familien müssten entsprechend der Anzahl ihrer Kinder und ihres Einkommens hierin unterstützt werden. Daher "gibt es ab Beginn 2008 ein einkommensabhängiges kindgebundenes Budget, in welchem der heutige Kinderzuschlag aufgeht und an welches in verschiedenen Phasen additionale Mittel hinzugefügt werden sollen“ (Ministerie van Algemene Zaken 2007a: 29). Auch die Situation von Alleinerziehenden mit chronisch kranken Kindern wolle die Regierung stärken. Eine geeignete finanzielle Regelung müsse sie aber noch erarbeiten. Aufgrund des Aktivierungsprinzips in der Arbeitsmarktpolitik seien auch alleinstehende Sozialhilfeempfänger mit Kindern bewerbungspflichtig. Diese Pflicht werde die Regierung abschaffen für alleinstehende Sozialhilfeempfänger mit Kindern bis zum Alter von 5 (max. 6) Jahren. „Es wird eine Schulungspflicht für diese Gruppe alleinstehender Eltern geben, um nach der Freistellung (von der Bewerbungsverpflichtung) einen Beruf $\mathrm{zu}$ finden. Es soll eine Regelung geschaffen werden, die das Arbeiten in Teilzeit für bewerbungspflichtige, alleinstehende Eltern mit Kindern finanziell reizvoll macht" (Ministerie van Algemene Zaken 2007a: 29).

${ }^{27}$ Der neuernannte Minister für Jugend und Familie unterstreicht in dem Programm, das er 2007 an die Tweede Kamer (hier: Bundestag) schickte, noch einmal die Bedeutung der Einführung des kindgebundenen Budgets. „Hiermit können finanzielle Probleme und Hindernisse, die sie (die Eltern) daran 
hindern ihre Kinder angemessen zu erziehen, erleichtert oder weggenommen werden" (Jeugd en Gezin 2007: 5). Außerdem betone die Regierung, dass Familien geholfen werde bei der Sanierung ihrer Schuldenproblematik. Sie werden nicht „von den Grundversorgungen wie Gas, Wasser, Licht abgeschlossen oder aus dem Haus gesetzt ohne, dass vorhergehend eine Lösung für die Kinder gefunden wird" (Jeugd en Gezin 2007: 5). Weiterhin hebe die Regierung hervor, sie wolle sich noch beraten, ob weitergehende finanzielle Maßnahmen für Familien nötig seien.

Im Familienbericht 2008, welchen der neuernannte Minister für Jugend und Familie bereits kurze Zeit später an die Tweede Kamer (hier: Bundestag) schickte, weise die Regierung auf die Gesetzesvorlage zu der im Koalitionsvertrag angekündigten Abschaffung der Bewerbungspflicht für alleinstehende Sozialhilfeempfänger mit Kindern bis zum Alter von max. 6 Jahren und auf die Einführung einer Schulungsverpflichtung für diese Kategorie (vergl. Jeugd en Gezin 2008: 9). Auch verdeutliche die Regierung in diesem Bericht die Einführung des einkommensabhängigen kindgebundenen Budgets, in welchem der Kinderzuschlag aufgehe und an welchen ab 2010 auch die Rückerstattung der Schulkosten gekoppelt werde. „Um Familien mit niedrigem und mittlerem Einkommen neben des Kindergeldes extra zu unterstützen, ist die Kinderermäßigung 2008 umgeformt in einen Kinderzuschlag (...) Dieser Kinderzuschlag wird 2009 ein kindgebundenes Budget: ein Betrag pro Kind (...) 2010 wird das kindgebundene Budget ausgebreitet mit einer Schulkostenerstattung" (Jeugd en Gezin 2008: 10). Außerdem werde ab 2009 das kindgebundene Budget für Familien mit mehr als einem Kind, pro Kind erhöht (Jeugd en Gezin 2008: 12). Soziale Teilhabe von Kindern sei der Regierung ein wichtiges Anliegen, da schon im jüngsten Alter entscheidende Weichen für die Zukunft von Kindern gestellt werden. Aus diesem Grund empfangen die Gemeinden in den Jahren 2008 und $2009 € 40$ Millionen „um sozialen Ausschluss von Kindern zu bekämpfen. Hierdurch werde es den Kindern ermöglicht, an Sportvereinigungen oder kulturellen Aktivitäten teilzunehmen“ (Jeugd en Gezin 2008: 10).

Vor demselben Hintergrund habe das Kabinett auch beschlossen, dass „Museen frei zugänglich werden für Kinder bis zum Alter von 12 Jahren“ (Jeugd en Gezin 2008: 13). Wie auch bereits im Koalitionsvertrag genannt, wolle die Regierung an dieser Stelle insbesondere die Einführung einer einkommensabhängigen 
Kombinationsermäßigung für Eltern mit Kindern unterstreichen. „Das Kabinett stimuliert die Arbeitsmarktteilnahme beider Eltern. Darum wird die einkommensabhängige Kombinationsermäßigung (...) eingeführt, und ab dem Jahr 2009 die Übertragbarkeit der allgemeinen Abgabenermäßigung schrittweise abgeschafft. Eltern mit jungen Kindern will das Kabinett die Wahl zwischen Arbeit und Erziehung oder eine Kombination beider überlassen. Darum bleibt es bei der Übertragbarkeit der Abgabenermäßigung für junge Familien mit Kindern bis fünf Jahren“ (Jeugd en Gezin 2008: 37). Im Zusammenhang mit der im Koalitionsvertrag angekündigten Regelung, die das Arbeiten in Teilzeit für bewerbungspflichtige, alleinstehende Eltern mit Kindern finanziell reizvoll machen solle, betone die Regierung, dass sie ab Beginn 2009 ein entsprechendes Experiment starten wolle, um $\mathrm{zu}$ untersuchen welche Regelungen hierfür geeignet seien.

Tabelle 25: Chronologisches Übersichtstableau der ökonomischen Instrumente in den Niederlanden

\begin{tabular}{|c|c|c|}
\hline Jahr & Dokument & Instrument \\
\hline 1994 & Koalitionsvertrag Kok I & $\begin{array}{l}\text { Verantwortungsteilung durch finanzielle Kürzungen im } \\
\text { Bereich des Kindergeldes, } \\
\text { Verantwortungsteilung durch finanzielle Kürzungen im } \\
\text { Bereich der Witwen- und Waisenrente, } \\
\text { Einführung einer Kindertabelle für individuelle Mietzuschüsse, } \\
\text { Einführung der vierjährigen Studienzeit mit entsprechender } \\
\text { BAföG-Regelung }\end{array}$ \\
\hline 1994 & $\begin{array}{l}\text { Regierungserklärung } \\
\text { Kok I }\end{array}$ & $\begin{array}{l}\text { Verantwortungsteilung durch finanzielle Kürzungen im } \\
\text { Bereich des Kindergeldes, } \\
\text { Verantwortungsteilung durch finanzielle Kürzungen im } \\
\text { Bereich der Witwen- und Waisenrente }\end{array}$ \\
\hline 1996 & Familienbericht 1996 & $\begin{array}{l}\text { Übertragung der finanziellen Verantwortung von Gemeinden } \\
\text { für Einrichtungen im Bereich der Jugendhilfe, } \\
\text { Finanzielle Förderung der Kinderbetreuung durch die } \\
\text { Einführung der Abgabenverminderung für Arbeitgeber und die } \\
\text { Übergangsregelung für alleinstehende Sozialhilfeempfänger } \\
\text { mit Kindern, } \\
\text { Ausarbeitung einer lehrlinggebundenen Finanzierung für } \\
\text { Schüler mit Behinderungen im Primär- und } \\
\text { Sekundarunterricht }\end{array}$ \\
\hline 1998 & Koalitionsvertrag Kok II & $\begin{array}{l}\text { Einführung von Steuerbegünstigungen für } \\
\text { Kinderbetreuungsmaßnahmen, } \\
\text { Beitrag zur Schulkostenerstattung für sozialschwache Familien }\end{array}$ \\
\hline 2002 & $\begin{array}{l}\text { Koalitionsvertrag } \\
\text { Balkenende I }\end{array}$ & $\begin{array}{l}\text { Einführung einer Lebenslaufvergünstigung, } \\
\text { Einführung einer einkommensbedingten Steuerermäßigung }\end{array}$ \\
\hline
\end{tabular}




\begin{tabular}{|c|c|c|}
\hline & & $\begin{array}{l}\text { für Familien mit Kindern, } \\
\text { Einführung einer einkommensbedingten Pflegezulage neben } \\
\text { eines Reichsbeitrags für die Prämie der Krankheitskosten für } \\
\text { Kinder }\end{array}$ \\
\hline 2002 & $\begin{array}{l}\text { Regierungserklärung } \\
\text { Balkenende I }\end{array}$ & $\begin{array}{l}\text { Einführung einer Lebenslaufvergünstigung, } \\
\text { Einführung einer einkommensbedingten Steuerermäßigung } \\
\text { für Familien mit Kindern }\end{array}$ \\
\hline 2003 & $\begin{array}{l}\text { Koalitionsvertrag } \\
\text { Balkenende II }\end{array}$ & $\begin{array}{l}\text { Realisierung einer einkommensabhängigen Steuerermäßigung } \\
\text { für Familien mit Kindern, } \\
\text { Bündelung und Dezentralisierung von Schulhaushalten }\end{array}$ \\
\hline 2006 & Familienbericht 2006 & $\begin{array}{l}\text { Finanzielle Unterstützung der Gemeinden in Sachen } \\
\text { Erziehungsunterstützung von Risikofamilien, } \\
\text { Erhöhung des Betrags der einkommensabhängigen } \\
\text { Steuerermäßigung für Familien mit Kindern, } \\
\text { Befreiung von Kindern bis zu } 18 \text { Jahren von den Kosten der } \\
\text { Gesundheitsversicherung, } \\
\text { Befreiung von Schulgeldern in der Sekundarstufe bzw. für 16- } \\
\text { und 17-jährige Auszubildende, } \\
\text { Einführung der ergänzenden Kombinationsermäßigung, } \\
\text { Einführung des Kinderbetreuungsgesetzes und der } \\
\text { Kinderbetreuungszulage, } \\
\text { Temporäre Befreiung von Reintegrationsmaßnahmen von } \\
\text { arbeitslosen Eltern mit einem kranken oder behinderten Kind } \\
\text { oder Partner }\end{array}$ \\
\hline 2007 & $\begin{array}{l}\text { Koalitionsvertrag } \\
\text { Balkenende IV }\end{array}$ & $\begin{array}{l}\text { Erstattung der Kosten für Schulbücher im Sekundarunterricht, } \\
\text { Schrittweise Verringerung der Möglichkeit zur Übertragung } \\
\text { der allgemeinen Abgabenermäßigung für Eltern, die nach } 1971 \\
\text { geboren wurden und keine Kinder in der Alterskategorie von o } \\
\text { bis } 6 \text { Jahren haben, } \\
\text { Einführung einer einkommensabhängigen Arbeitsermäßigung } \\
\text { und einer additionalen einkommensabhängigen } \\
\text { Kombinationsermäßigung für Eltern mit Kindern, } \\
\text { Steuerermäßigung zur Deckung der Kosten informeller } \\
\text { Kinderbetreuung von Eltern mit doppeltem Einkommen, } \\
\text { Einführung eines einkommensabhängigem kindgebundenen } \\
\text { Budgets, in welchem der Kinderzuschlag aufgeht, } \\
\text { Abschaffung einer Bewerbungspflicht für alleinstehende } \\
\text { Sozialhilfeempfänger mit Kindern bis zum Alter von } 5 \text { (max. 6) } \\
\text { Jahren und Einführung einer Schulungspflicht für diese } \\
\text { Kategorie }\end{array}$ \\
\hline 2007 & $\begin{array}{l}\text { Programm für Jugend } \\
\text { und Familie, 2007-2011 }\end{array}$ & $\begin{array}{l}\text { Einführung eines einkommensabhängigem kindgebundenen } \\
\text { Budgets, in welchem der Kinderzuschlag aufgeht, } \\
\text { Hilfe bei der Schuldenproblematik von Familien }\end{array}$ \\
\hline 2008 & Familienbericht 2008 & $\begin{array}{l}\text { Abschaffung einer Bewerbungspflicht für alleinstehende } \\
\text { Sozialhilfeempfänger mit Kindern bis zum Alter von max. } 6\end{array}$ \\
\hline
\end{tabular}


Jahren und Einführung einer Schulungspflicht für diese Kategorie,

Einführung eines einkommensabhängigem kindgebundenen

Budgets, in welchem der Kinderzuschlag bzw. die

Kinderermäßigung aufgeht,

Kopplung der Schulkostenerstattung an das kindgebundene Budget,

Schrittweise Erhöhung des kindgebundenen Budgets ab 2009, Investierung von $2 \mathrm{x} € 40$ Millionen in den Jahren 2008 und 2009, um die gesellschaftliche Teilnahme von Kindern zu fördern,

Vorgenommene Befreiung von Eintrittsgeldern für Museen für Kinder bis zu 12 Jahren,

Einführung einer einkommensabhängigen Arbeitsermäßigung und einer additionalen einkommensabhängigen Kombinationsermäßigung für Eltern mit Kindern, Schrittweise Verringerung der Möglichkeit zur Übertragung der allgemeinen Abgabenermäßigung für Eltern, die nach 1971 geboren wurden und keine Kinder in der Alterskategorie von 0 bis 6 Jahren haben

\subsubsection{Instrumente, die die ökologischen Bedingungen des Familienlebens betreffen}

Im Koalitionsvertrag der ersten Violetten Koalition vom 13.08.1994 wird die demographische Entwicklung der Niederlande thematisiert. Da die Bevölkerung wachse bzw. aufgrund der zunehmenden Individualisierung verhältnismäßig mehr Platz gebraucht werde, entstehe Bedarf an mehr Wohnraum. „Die aktuellsten demographischen Prognosen lassen einen höheren Bedarf an Wohnraum sehen. Darum hat man sich dafür entschieden den Bau von Sozialbauwohnungen mit 15.000 Wohnungen zu steigern“ (Ministerie van Algemene Zaken 1994a: 69).

${ }^{28}$ In dieser umfangreichen Notiz zum Thema Familie, welche die Staatssekretärin des Ministeriums für Gesundheit, Wohlfahrt und Sport im Jahre 1996 an die Tweede Kamer (hier: Bundestag) schickte, wird betont, dass 
Sicherheit an Schulen eine wichtige Voraussetzung sei. „Kinder können nur gut lernen, wenn die Schule für sie eine sichere Umgebung bietet. Das gilt vor allem für Kinder, die zu Hause keine Geborgenheit finden. Mit der Kampagne ,Die sichere Schule' sei gestartet, um die Sicherheit in und um Schulen zu vergrößern und Schulen zu helfen Sicherheitsprobleme zu thematisieren und an zu packen“ (Ministerie van Volksgezondheid, Welzijn en Sport 1996: 16).

Im Koalitionsvertrag der zweiten Violetten Koalition vom 18.07.1998 ist die Arbeitsmarktteilnahme von Frauen ein wichtiges politisches Thema. Die Betreuung von Kindern bzw. jungen Kindern sei in den Niederlanden bisher unüblich und daher gäbe es auch nur weniger geeignete als gewünschte Betreuungsmöglichkeiten. „Um die Arbeitsmarktteilnahme zu vergrößern, wird der Kinderbetreuung ein finanzieller Impuls gegeben. Dieser Impuls nimmt konkret Form an, durch die Ausbreitung der Kapazität der Kinderbetreuungseinrichtungen. So wird man der wachsenden Nachfrage von Kinderbetreuungsplätzen entgegenkommen" (Ministerie van Algemene Zaken 1998a: 10). Insgesamt strebe man mit dem neuen Gesetz ,Basiseinrichtung von Kinderbetreuungsplätzen' nach einer Verdopplung der Auffangkapazität. Hierbei solle auch ein Akzent auf die außerschulische Betreuung älterer Kinder gelegt werden. Die soziale Infrastruktur von Städten bedürfe einer besonderen Aufmerksamkeit. Von der Verstärkung dieser sozialen Infrastruktur erwarte man auch positive wirtschaftliche Effekte und eine zunehmende Sicherheit (vergl. Ministerie van Algemene Zaken 1998a: 13). Um die soziale Kohäsion in bedrohten Stadtvierteln $\mathrm{zu}$ verstärken seien verschiedene Maßnahmen erforderlich. Ein Beispiel forme die breite Nachbarschaftsschule bzw. Fensterschule (diese Einrichtung ist auch unter dem Namen ,Breite Schule' bekannt). Sie „kann als Fangnetz für Kinder in Problemsituationen einen wichtigen Beitrag zur sozialen Kohäsion in einem Viertel liefern“ (Ministerie van Algemene Zaken 1998a: 58). Da Schulen insgesamt einen sehr wichtigen Beitrag in den Bereichen Erziehung und soziale Integration leisten, wolle man die Qualität der Schulbedingungen verbessern. Hierfür sei vor allem auch eine quantitative Anpassung nötig. „Die Verkleinerung der Klassen im Schulsystem beginnt 1999 mit der Anfüllung genügender Klassenräume. Über den Gemeindefonds wird den Gemeinden die Möglichkeit geboten, um das Bauvolumen, das der Richtungsvorgabe 1 Lehrer auf 20 Schüler entspricht in den ersten vier Schulklassen effizient einzurichten“ (Ministerie van Algemene 
Zaken 1998a: 59). Ziel der Regierung sei es, dass in Zukunft soviel Kinder wie eben möglich ab dem vierten Lebensjahr zur Schule gehen. Außerdem seien auch im Sekundarbereich Erneuerungen in den Schulen erforderlich. Hierbei gehe es allerdings weniger um eine Erweiterung der Einrichtungen als um Renovierungsmaßnahmen (vergl. Ministerie van Algemene Zaken 1998a: 59).

29Einige Jahre später kommt es dann zur Gründung einer neuen Einrichtung. Im Familienbericht, den die Staatssekretärin des Ministeriums für Gesundheit, Wohlfahrt und Sport im Jahre 2006 an die Tweede Kamer (hier: Bundestag) schickte, spielen Erziehungsfragen von Eltern eine wichtige Rolle. Aufgrund der schnell veränderten gesellschaftlichen Verhältnisse hätten junge Eltern konkrete Fragen zum Thema Erziehung, auf die sie bisher oft keine Antworten finden könnten. Darum wolle das Kabinett, „dass Gemeinden die Regie übernehmen, um pro Viertel oder Gemeinde einen zentralen Punkt zu formen, für alle Fragen von Familien zum Thema Erziehen und Aufwachsen, ein Zentrum für Jugend und Familie. Die Jugendgesundheitsfürsorge muss hierin den Mittelpunkt formen“ (Ministerie van Volksgezondheid, Welzijn en Sport 2006: 12). Nicht nur weitergeführt, sondern auch intensiviert werde durch die Regierung Balkenende II in die ,Breiten Schulen'. Die Große Koalition unterstreiche, dass sie auch die Zusammenarbeit mit den Eltern verbessere. „Die ,Breite Schule’ ist ein Rollenmodell für die Zusammenarbeit zwischen Organisationen der verschiedenen Disziplinen mit dem Hauptziel: mehr Entwicklungsmöglichkeiten für Kinder, aus einem gemeinsamen, pädagogischen Gesamtkonzept. In der ,Breiten Schule' wird ein zusammenhängendes Angebot von schulischen und außerschulischen Aktivitäten angeboten. Die Entwicklung von mehr ,Breiten Schulen' ist eine Aufgabe der Gemeinden. Das Kabinett unterstützt das Vorhaben der Gemeinden, um 2010 in den Niederlanden über mindestens 1200 ,Breite Schulen' zu verfügen“ (Ministerie van Volksgezondheid, Welzijn en Sport 2006: 17). Aber nicht nur diese Form der Betreuung halte die Regierung für förderungswürdig. $\mathrm{Um} \mathrm{zu}$ einer guten Alternative zur häuslichen

29 In der Regierungserklärung der zweiten Violetten Koalition, aus dem Jahr 1998, sowie der ersten beiden Koali-tionsverträge und Regierungserklärungen der ersten beiden Koalitionen unter Balkenende wurden keine konkre-ten ökologischen Instrumente gefunden 
Kinderbetreuung $\mathrm{zu}$ kommen, sei es wichtig, dass es zwischen den verschiedenen Aufenthaltsorten der Kinder wie z.B. Schulen, Betreuungs- und Freizeiteinrichtungen zu einer guten Abstimmung komme. Wenn Eltern das Gefühl hätten, ihre Kinder werden außer Haus eben gut betreut wie durch sie selbst, falle ihnen die Teilnahme am Arbeitsmarkt leichter. Daher wolle man investieren in schlüssige Tagesangebote in denen Kinderbetreuung, Schule und Freizeitangebote ineinander übergehen. „Die Tagesangebote helfen Eltern um Arbeit und Erziehung besser miteinander zu kombinieren; sie bieten Kindern Sicherheit, Kontinuität in der Erziehung und ein gutes pädagogisches Klima. Das Kabinett hat für die Jahre 2006, 2007 und 2008 extra Mittel zur Verfügung gestellt um diese Tagesangebote $\mathrm{zu}$ stimulieren“ (Ministerie van Volksgezondheid, Welzijn en Sport 2006: 30). Teil dieser Tagesangebote sei auch die neue gesetzliche Regelung, die ab dem 1. Januar 2007 gelten solle, und in der "Schulen verpflichtet werden entweder vor oder nach Schulbeginn Kinderbetreuung anzubieten (zwischen 7.30 Uhr und 18.30 Uhr), oder Einrichtungen anzubieten, in denen andere Parteien diese Betreuungsaufgaben ausführen und entsprechende Rahmenbedingungen hierbei $\mathrm{zu}$ nennen“ (Ministerie van Volksgezondheid, Welzijn en Sport 2006: 31).

Schon kurze Zeit nach der Publikation dieses Familienberichts wird im Koalitionsvertrag der vierten Koalition unter Balkenende am 07.02.2007 auf die demographischen Entwicklungen in einigen ländlichen Regionen reagiert. Durch Begrenzungen in der Gesetzeslage, zum Beispiel im Hinblick auf die Wohnungsbauentwicklung, gäbe es ein demographisches Ungleichgewicht mit dementsprechenden Folgen. Daher betone die Regierung, sie wolle die Versorgung „von genügend Einrichtungen in kleinen Landgemeinden unterstützen. Im Hinblick auf den Erhalt einer vitalen Dorfstruktur wird es Landgemeinden ermöglicht, für die eigene Bevölkerung $\mathrm{zu}$ bauen. Das Wohnungsbaugesetz wird dahingehend angepasst" (Ministerie van Algemene Zaken 2007a: 21). Einen starken Akzent lege diese Regierung außerdem auf die Revitalisierung problematischer Stadtviertel. Die Regierung wolle daher schon bald einen zusammenhängenden Plan vorstellen, aus dem hervorgehe, wie sie innerhalb einer Periode von acht bis zehn Jahren Probleme dieser Stadtbereiche, wie z.B. Jugendarbeitslosigkeit und Schulausfall, durch Stadtverbesserungsmaßnahmen bekämpfen werde. Um dieses Ziel zu erreichen, wolle man mit den Wohnungsbaugesellschaften zusammenarbeiten 
und Verabredungen zur Lösung sozialer Probleme in diesen Vierteln treffen (vergl. Ministerie van Algemene Zaken 2007a: 31). Um den demographischen Entwicklungen weiterhin gerecht werden zu können, werde man außerdem die Wohnungsbauproduktion steigern. Hierbei solle insbesondere auch auf die Bevölkerungszusammenstellung der unterschiedlichen Gebiete geachtet werden. „Um zu einer gleichgewichtigen Wohnungsentwicklung und Bevölkerungszusammenstellung in einem zusammenhängenden städtischen Bereich zu kommen, kann es wünschenswert sein, dass Randgemeinden mehr für niedrigere und mittlere Einkommensgruppen bauen. Wo das der Fall ist, wird eine Anweisungsbefugnis entwickelt für die Situationen in denen Randgemeinden nicht mitarbeiten wollen“ (Ministerie van Algemene Zaken 2007a: 32). Außerdem solle das generationenbeständige Bauen in Zukunft besser unterstützt werden, da ältere Menschen so lang wie eben möglich in ihrem eigenen Viertel wohnen bleiben wollten. Daher wolle man die Wohngebiete "generationsbeständig (...) gestalten und in den (entsprechenden) Stadtvierteln die Einrichtung von Servicepunkten für Wohlsein und Pflege fördern. Auch eine größere Variation im Wohnungsangebot (mit Name ambulante Pflege) kann dazu beitragen, dass ältere Menschen länger in ihrem eigenen Viertel wohnen bleiben. Wohnungsbaugesellschaften werden auf diese gesellschaftliche Aufgabe angesprochen“ (Ministerie van Algemene Zaken 2007a: 32).

${ }^{30}$ Zusätzlich zu den Äußerungen im Koalitionsvertrag unterstreicht der neuernannte Minister für Jugend und Familie in seinem Programm, das er 2007 an die Tweede Kamer (hier: Bundestag) schickte, die sorgfältige Vorgehensweise im Falle von andauernden Mietschulden von Familien mit Kindern. Das Gemeinwohl der Kinder und eine angemessene Erziehung seien wichtig, und ihre Sicherheit dürfe nicht gefährdet werden (vergl. Jeugd en Gezin 2007: 24).

In seinem Familienbericht, den der neuernannte Minister für Jugend und Familie im Jahr darauf an die Tweede Kamer (hier: Bundestag) schickte, wird 
erneut betont man wolle Familien Erziehungshilfen in unmittelbarer Nähe ihres Wohnortes bieten. „Das Kabinett will bis zum Jahr 2011 ein national deckendes Netzwerk der Jugend- und Familienzentren (NL: CJG) einrichten“ (vergl. Jeugd en Gezin 2008: 10). Man wolle außerdem die Familien- und Kinderfreundlichkeit von Gemeinden im Allgemeinen vergrößern. In Folge eines noch zu erstellenden Berichts zur Jugendkultur werde man in diesem Bereich ein entsprechendes Projekt starten. Unerwartet stark sei weiterhin die Inanspruchnahme der Kinderbetreuung gestiegen. Daher sei es nötig geworden, in diesem Bereich stärker $\mathrm{zu}$ investieren als ursprünglich geplant. Eine besondere Stellung im Bereich der Kinderbetreuung nehme die Betreuung durch Tageseltern ein, welche sich einer wachsenden Beliebtheit erfreue. „Betreuung durch Tageseltern bleibt möglich in Form eines kleinen Netzwerks zur Kinderbetreuung. Ab 2010 gibt es zwei Formen der Betreuung durch Gasteltern: eine professionelle Form bei den Gasteltern zu Hause und eine einfachere Form, für die es weniger Qualitätsanforderungen (und auch weniger Fördermittel) geben wird“ (Jeugd en Gezin 2008: 12).

Wiederum betone die Regierung die große Rolle der ,Breiten Schulen', in denen wichtige Betreuungs- und Erziehungsaufgaben geleistet würden. Darum werde die Anzahl dieser Einrichtungen auch vergrößert (Jeugd en Gezin 2008: 38). 
Tabelle 26: Chronologisches Übersichtstableau der ökologischen Instrumente in den Niederlanden

\begin{tabular}{|c|c|c|}
\hline Jahr & Dokument & Instrument \\
\hline 1994 & Koalitionsvertrag Kok I & Steigerung des Baus von Sozialbauwohnungen \\
\hline 1996 & Familienbericht 1996 & Durchführung der Kampagne ,Die sichere Schule’ \\
\hline 1998 & Koalitionsvertrag Kok II & $\begin{array}{l}\text { Ausbreitung der Kapazitäten der verschiedenen Formen von } \\
\text { Kinderbetreuung durch das Gesetz ,Basiseinrichtung von } \\
\text { Kinderbetreuungsplätzen“, } \\
\text { Investitionen in die soziale Infrastruktur von Städten, } \\
\text { Unterstützung des Prinzips der breiten Nachbarschaftsschule } \\
\text { bzw. Fensterschule (hier: ,Breite Schule'), } \\
\text { Neubau von Klassenräumen für die ersten vier Schuljahre, } \\
\text { Renovierung der Schulen im Sekundarbereich }\end{array}$ \\
\hline 2006 & Familienbericht 2006 & $\begin{array}{l}\text { Einführung der Zentren für Jugend und Familie, } \\
\text { Realisierung von mindestens } 1200 \text {,Breiten Schulen’ bis zum } \\
\text { Jahr 2010, } \\
\text { Stimulierung von Tagesarrangements aus Betreuung, Schule } \\
\text { und Freizeitmöglichkeiten, } \\
\text { Einführung der Betreuungsverpflichtung von Schulen } \\
\text { zwischen 7.30 Uhr und 18.30 Uhr }\end{array}$ \\
\hline 2007 & $\begin{array}{l}\text { Koalitionsvertrag } \\
\text { Balkenende IV }\end{array}$ & $\begin{array}{l}\text { Ermöglichung des Baus neuen Wohnraums in } \\
\text { Landgemeinden, } \\
\text { Steigerung der Wohnungsbauproduktion und Förderung einer } \\
\text { gleichgewichtigen Wohnungsentwicklung im städtischen } \\
\text { Bereich, } \\
\text { Unterstützung des generationenfreundlichen Bauens in } \\
\text { Stadtvierteln }\end{array}$ \\
\hline 2007 & $\begin{array}{l}\text { Programm für Jugend } \\
\text { und Familie, 2007-2011 }\end{array}$ & $\begin{array}{l}\text { Prävention von Hausaussetzungen von Familien mit Kindern } \\
\text { bzw. sorgfältige Abhandlung dessen unter besonderem Schutz } \\
\text { der Kinder }\end{array}$ \\
\hline 2008 & Familienbericht 2008 & $\begin{array}{l}\text { Einführung der Zentren für Jugend und Familie, } \\
\text { Intensivierung der Kinderbetreuung und Behalt von Betreuung } \\
\text { durch Tageseltern, } \\
\text { Vergrößerung der Anzahl ,Breiter Schulen' }\end{array}$ \\
\hline
\end{tabular}

\subsubsection{Instrumente zur Förderung oder Wiederherstellung von Kompetenzen der Familienangehörigen}

Im Koalitionsvertrag der ersten Violetten Koalition vom 13.08.1994 unterstreicht die Regierung das Ziel der Frühwarnung bzw. Prävention im Bereich der Jugendhilfe. Da zu viele Jugendliche mit Problemen in der Schule oder zu Hause kämpfen, sei eine besondere Unterstützung ihrer Kompetenzen 
erforderlich. „Prävention und Frühsignalisierung von Problemsituationen bekommt systematisch Aufmerksamkeit. Die gesellschaftliche Perspektive wird durch praktischen Berufsunterricht und aktivierende Arbeitsmarktpolitik verbessert mit dem Jugendarbeitsgarantieplan als Schlussstein“ (Ministerie van Algemene Zaken 1994a: 43). Da Schulen in der Erziehung und Integration von Kindern und Jugendlichen eine wichtige Rolle spielen betone die Regierung, sie wolle die Rolle der Schulen durch die Zunahme der Eigenverantwortlichkeit stärken. „Schulen bekommen mehr Handlungsfreiheit durch eine Verminderung der politischen Richtlinien und einen globaleren Haushalt (...) Primäre Unterrichtsaufgaben, so wie die pädagogische Einrichtung und die Personalpolitik werden durch die Schulen selbst erfüllt. Rahmenbedingungen, sowie die Unterhaltung der Schulgebäude (...) werden dezentralisiert und auf die Gemeinden übertragen“ (Ministerie van Algemene Zaken 1994a: 48). Vor dem Hintergrund eines erfolgreichen Übergangs von der Schule auf den Arbeitsmarkt, sei der Berufsschulunterricht wichtig. Verschiedene berufsbildende Schulformen wolle man daher in regionalen Unterrichtszentren (ROC's) zusammenfassen. Neben dieser Schulreform sei es auch nötig, Schüler im Sekundarbereich durch eine Profilwahl ihrer Schulfächer besser auf den Unterricht an den berufsbildenden Schulen vorzubereiten (Ministerie van Algemene Zaken 1994a: 49).

In der dazugehörigen Regierungserklärung unterstreicht die neue Regierung am 31.08.1994 die bereits im Koalitionsvertrag angekündigten Dezentralisierungsvorhaben im Hinblick auf Verantwortlichkeiten und Aufgaben im Schulbereich. „Weil die Schulorganisation mehr Möglichkeiten zur Schaffung eines eigenen Profils bekommt, werden die Kreativität und Professionalität der Schulleitung und der Lehrer im Mittelpunkt stehen“ (Ministerie van Algemene Zaken 1994b: 11). Weiterhin wiederholt die Regierung, sie wolle den Anschluss des Sekundarunterrichts auf die Berufsbildung verbessern und zielgerichtete Profilierungen in der Fächerkombination des individuellen Schülers ermöglichen. Die Stärkung des Praxisbezugs in Schulen sei für die spätere Teilnahme am Arbeitsmarkt wichtig und werde darum in Zukunft zusätzlich gestärkt (vergl. Ministerie van Algemene Zaken 1994b: 11f).

In der umfangreichen Notiz zum Thema Familie, welche die Staatssekretärin des Ministeriums für Gesundheit, Wohlfahrt und Sport im Jahre 1996 an die Tweede Kamer (hier: Bundestag) schickte, wird, wie schon zuvor im 
Koalitionsvertrag und der Regierungserklärung der Regierung, auf die besondere Bedeutung aktivierender Arbeitsmarktmaßnahmen gewiesen. Dies gelte vor allem auch im Bereich der Schulen und Jugendpolitik. Um auch Schülern mit einer Lernschwäche berufliche Perspektiven zu ermöglichen, werde im Sekundarunterricht „für Schüler, für die ein erfolgreicher Schulabschluss nicht möglich ist, der arbeitsmarktgerichtete Lehrweg eingeführt" (Ministerie van Volksgezondheid, Welzijn en Sport 1996: 16). Neben der Schule sei vor allem die Erziehung in den Familien wichtig für das Wohlergehen der Schüler. Die Jugendarbeit bedürfe daher einiger qualitativer Verbesserungen, in denen auch der individuellen Situation einzelner Familienhaushalte Rechnung getragen werde. Daher erfolgt die Entwicklung eines nachfragegesteuerten Präventionsangebotes. „Weiterhin wird die so genannte Home-Start-Methode erprobt. Freiwillige Helfer unterstützen hierbei Eltern mit jungen Kindern (...) so lange bis die Eltern wieder auf eigenen Beinen stehen können“ (Ministerie van Volksgezondheid, Welzijn en Sport 1996: 17). Auch die Betreuung von Kindern, die nicht bei ihren eigenen Eltern bleiben könnten, wolle man durch das Innovationsprogramm für Pflegeeltern unterstützen. „Hierdurch soll ein Impuls zur Qualitätsverbesserung, an die inhaltliche Erneuerung der Arbeit und an die Verstärkung der Position von Pflegeeltern gegeben werden“ (Ministerie van Volksgezondheid, Welzijn en Sport 1996: 18). Insbesondere wolle die Regierung die Rolle der Familie mit behinderten Kindern fördern. Hierzu sei teilweise eine Begleitung in den Haushalten und teilweise Betreuung in Einrichtungen der Behindertenfürsorge erforderlich. Stärker als zuvor wolle die Regierung die Integration behinderter Menschen in ein normales Lebensumfeld stimulieren. Daher „wird an der Einführung einer lehrlinggebundenen Finanzierung für behinderte Schüler in Primär- und Sekundarstufen gearbeitet. Durch diese Finanzierungsform können diese Schüler besser innerhalb des regulären Unterrichts betreut werden. Der Ausgangspunkt hierbei ist: die Pflege folgt dem Schüler und nicht umgekehrt“ (Ministerie van Volksgezondheid, Welzijn en Sport 1996: 19).

Im Koalitionsvertrag der zweiten Violetten Koalition unterstreicht die Regierung am 18.07.1998 die Bedeutung der Schulen für die Erziehung der Kinder. Aus diesem Grund wolle sie im Schulbereich erhebliche Investitionen tätigen. Vor allem in den ersten Schuljahren bräuchten Kinder viel Aufmerksamkeit und Lehrer genügend Zeit. Daher wolle man zu einer Verringerung der 
Klassenstärken übergehen. „Die kleineren Klassen ermöglichen mehr Maßarbeit im Unterricht der jüngsten Schüler. Ein guter Start ist eine wichtige Rüstung gegen spätere Unterrichtsrückstände“ (Ministerie van Algemene Zaken 1998a: 10). Auch wolle man das Inventar in den Schulen verbessern. Hierbei sei vor allem auch die schulische Einrichtung im IT-Bereich gemeint. Sie bilde eine Voraussetzung für den späteren Anschluss ins Berufsleben (vergl. Ministerie van Algemene Zaken 1998a: 10). Wie bereits während der ersten Legislaturperiode dieser Regierung, wird auch an dieser Stelle die Autonomie der Schulen betont. Infolge dieser zugenommenen Selbstständigkeit und vor allem auch im Zusammenhang mit den großen Investitionen, die in diesem Bereich getätigt seien, halte die Regierung es für wichtig, dass Schulen auch Rechenschaft über ihr Handeln ablegen. „Die öffentliche Verantwortung über die Qualität des Unterrichts wird verstärkt. Die Schule wird gegenüber Eltern und Regierung Rechenschaft ablegen müssen über die zur Verfügung stehenden Mittel und erreichten Resultate" (Ministerie van Algemene Zaken 1998a: 58). Auch die Integration der Schüler mit Migrationshintergründen sei eine wichtige Aufgabe der Schulen. In vielen Fällen gelänge Schülern mit Migrationshintergrund dann auch der erfolgreiche Übergang von der Schule ins Berufsleben. In den Fällen, in denen der Übergang nicht gelänge, lägen die Hintergründe oft im sprachlichen Bereich. Sprachliche Frühförderung wolle man daher unterstützen (vergl. Ministerie van Algemene Zaken 1998a: 64). Als verbindendes Element zwischen den verschiedenen Bevölkerungsgruppen verstehe die Regierung den Sport. Neben dem Topsport wolle sie daher vor allem auch den Breitensport fördern. „Die Rolle der Gemeinden wird verstärkt, um Sport besser mit anderen Bereichen, sowie Jugendpolitik, Schulpolitik, Seniorenpolitik und Integrationspolitik zu verknüpfen“ (Ministerie van Algemene Zaken 1998a: 67). Ein weiterer Maßnahmenschwerpunkt sei die präventive und kurative Jugendpolitik. Ausgangspunkt hierbei sollten vor allem die Bedürfnisse der Kinder und Jugendlichen sein. Möglichkeiten zur Entwicklung von Kindern und Jugendlichen werden durch verschiedene Maßnahmen gefördert. Wichtig sei das neu zu erstellende Jugendfürsorgegesetz „mit einer eindeutigen Ansteuerung und Finanzierung (...) Das Büro für Jugendfürsorge, welches eine zentrale Rolle in der Jugendhilfe einnimmt, wird eine unabhängige Rechtsperson unter eigener Obrigkeit mit eigener Finanzierung. Funktionäre des Büros für Jugendfürsorge arbeiten intensiv mit den Schulen in ihrer Region zusammen: so formen sie die notwendige 
Verwendung familienpolitischer Zielsetzungen ... in den Niederlanden

Verbindung zwischen Unterricht, Jugendfürsorge und Jugendgesundheitsfürsorge“ (Ministerie van Algemene Zaken 1998a: 67f).

In der kurze Zeit später folgenden Regierungserklärung der zweiten Violetten Koalition (25.08.1998) unterstreicht die Regierung die bereits im Koalitionsvertrag beschlossene Verringerung der Klassenstärken in den ersten Schuljahren. Hierdurch wolle man individuelle Schüler besser fördern. „Auch die vorgenommene Verkleinerung der Schulklassen, vor allem in den ersten Jahren der Grundschule, zielt auf mehr Aufmerksamkeit für individuelle Schüler und ihre Schwächen, Möglichkeiten und Talenten“ (Ministerie van Algemene Zaken 1998b: 15).

Im Koalitionsvertrag der ersten Koalition unter Balkenende thematisiert diese neue Regierung am 03.07.2002 dann die Verantwortlichkeiten im Schulbereich. Im Zuge der Dezentralisierung vieler Aufgaben während der violetten Koalitionen sei die Rolle der Schulen größer geworden. Dieses dürfe sich aber nicht negativ auf die Qualität der Schulen auswirken. „Die Regierung ist hauptverantwortlich für die Bestimmung der Rahmenrichtlinien innerhalb welcher sich die Schulen bewegen können. Sie stellt die Endbedingungen und das Kerncurriculum jeder Schulform fest und garantiert unabhängige Qualitätsaufsicht“ (Ministerie van Algemene Zaken 2002a: 12).

${ }^{3}$ Im Familienbericht 2006 den die Staatssekretärin des Ministeriums für Gesundheit, Wohlfahrt und Sport an die Tweede Kamer (hier: Bundestag) schickte, wird die Zusammenarbeit zwischen den verschiedenen Einrichtungen in der Jugendwohlfahrt thematisiert. In diesem Zusammenhang habe die Regierung eine Expertenkommission mit dem Namen ,Operatie JONG' (hier: Operation (Kommission) für junge Menschen) (Ministerie van Volksgezondheid, Welzijn en Sport 2006: 10) eingerichtet. Die Regierung werde auf das noch ausstehende Gutachten mit einem Kabinettsstandpunkt reagieren. Deutlich sei aber in jedem Fall, dass man Problemen von Kindern und

3IIn der Regierungserklärung der ersten Koalitionen unter Balkenende, aus dem Jahr 2002, dem Koalitionsvertrag und der Regierungserklärung der zweiten Koalition unter Balkenende aus dem Jahr 2003 wurden keine konkreten Instrumente zur Förderung oder Wiederherstellung der Kompetenzen Familienangehöriger gefunden. 
Jugendlichen in Zukunft professioneller als zuvor begegnen wolle. „Gutes und schnelles Austauschen der Informationen ist entscheidend für die Unterstützung der Fürsorge. Ein wichtiger Schritt hierin ist die Einführung des elektronischen Kinderdossiers (...) Speziell für die Signalisierung von Risiken (der Vorbote von Problemen) wird es daneben noch den so genannten Verweisindex geben“ (Ministerie van Volksgezondheid, Welzijn en Sport 2006: 11). Um allerdings Probleme und Risiken nicht nur nachträglich zu bekämpfen, sondern auch $\mathrm{zu}$ vermeiden, unterstreiche die Regierung die Rolle von Präventionsmaßnahmen. „Jede Gemeinde empfängt ab dem 1. Januar 2006 anhand der zeitlichen Regelung für spezifische Unterstützung in der Jugendgesundheitspflege (...) 50 Euro pro Säugling, für eine bessere präventive Jugendpolitik. Das Kabinett rät Gemeinden dieses Geld für effektive Methoden der Erziehungsunterstützung einzusetzen“ (Ministerie van Volksgezondheid, Welzijn en Sport 2006: 11). Auch wolle die Regierung Eltern und Kindern mit Erziehungsfragen bzw. Entwicklungsschwierigkeiten helfen. Die Verwendung von Hilfen in diesem Bereich solle viel selbstverständlicher werden und auf lokaler Ebene allen Eltern und Kindern zur Verfügung stehen (vergl. Ministerie van Volksgezondheid, Welzijn en Sport 2006: 12). Da die Inanspruchnahme der Einrichtungen in der Jugendfürsorge gestiegen sei, habe die Regierung zusätzliche Mittel investiert. Die adäquate Hilfe von jungen Menschen und ihren Eltern sei der Regierung ein wichtiges Anliegen. Daher wolle sie investieren, um die verschiedenen Formen der Fürsorge zu unterstützen und die vorhandenen Wartelisten wegzuarbeiten (Ministerie van Volksgezondheid, Welzijn en Sport 2006: 14). Weiterhin hebe die Regierung den Einfluss von Scheidungen auf alle Betroffenen hervor. Die Belange von Kindern zu schützen verstehe sie als ihr Ziel und als Grund dafür sich im Bereich von Ehescheidungen stärker als zuvor zu engagieren. „Darin bekommt die Schlichtung eine stets größere Rolle. Daneben ist der so genannte ,Elternschaftsplan' der sehr wahrscheinlich eingeführt wird, eine wichtige Stimulans für Eltern, um gute Regelungen hinsichtlich der Rolle der Kinder nach der Scheidung zu treffen. Der Elternschaftsplan wird zum Pflichtteil des Scheidungsantrags" (Ministerie van Volksgezondheid, Welzijn en Sport 2006: 15). Was die Alimente betreffe, wolle die Regierung zu einer Gesetzesänderung kommen, um mehr Deutlichkeit im Hinblick auf die finanzielle Unterstützung zu garantieren. Ein anderer Punkt, der zuvor bereits angesprochen wurde und an dieser Stelle als Überleitung zum Thema Integration von Familien mit 
Migrationshintergrund dient, sind die sprachlichen Fähigkeiten von Kindern. „Die Vor- und Frühschulische Ausbildung (...) kann junge Kinder von 2 bis 6 Jahren helfen, um den sprachlichen Rückstand aufzuholen. Das geschieht über die Kindertagesstätten, Kindergärten und Schulen. Hierzu gibt es gut strukturierte didaktische Programme“ (Ministerie van Volksgezondheid, Welzijn en Sport 2006: 19). Neben der sprachlichen Frühförderung von Kindern unterstreiche die Regierung die weitere Integration von Familien mit Migrationshintergrund. Sie sei der Regierung ein wichtiges Anliegen, welches sie durch eine Vielzahl von Programmen unterstütze. Hierbei handele es sich z.B. um eine Plattform zur Förderung der Verbesserung der Zusammenarbeit zwischen Eltern mit Migrationshintergrund und Schulen, um die Radikalisierung von Jugendlichen mit Migrationshintergrund zu bekämpfen, um die Rolle von Frauen mit Migrationshintergrund zu stärken etc. (Ministerie van Volksgezondheid, Welzijn en Sport 2006: 22). Auch im Bereich der audiovisuellen Medien käme es darauf an, die Kompetenzen von Eltern und Kindern zu stärken. Durch die neuen Entwicklungen im Bereich multimedialer Medien könne es Familien schwer fallen zu beurteilen welche Medien für Kinder geeignet bzw. ungeeignet seien. „Die Kammer (hier: Bundestag) hat das Kabinett gefragt, um in diesem Bereich weitere Aktionen zur Unterstützung der Familien zu fördern und das Kabinett hat bereits Maßnahmen getroffen, unter anderem die Einführung eines Systems zur Selbstregulierung audiovisueller Produkte wie Kinofilme, Videofilme, DVDs und Fernsehprogramme. Dieser so genannte ,Kijkwijzer' (hier: Einschaltanzeiger) warnt Eltern, Kinder und Jüngere über mögliche schädliche Folgen dieser Produkte" (Ministerie van Volksgezondheid, Welzijn en Sport 2006: 22f).

Wenige Monate nach der Veröffentlichung des Familienberichts wird im Koalitionsvertrag der vierten Koalition unter Balkenende am 07.02.2007 ein besonderer Schwerpunkt auf die Unterstützung der Kompetenzen von Kindern und Schulen in Bildungseinrichtungen gelegt. Wie schon die Vorgängerkoalitionen betone auch die vierte Regierung unter Balkenende, sie wolle die Schulen in ihrer Eigenverantwortung unterstützen. Allerdings sehe sie auch, dass es nötig sei individuelle Schulen durch einige zentral zu steuernde Maßnahmen zu helfen. Um Segregation an Schulen zu vermindern, wolle sie daher mit Fixmomenten für die Schulanmeldung und mit Lossystemen arbeiten. Die Organisation von Schulen in kleinem Maßstab wolle die Regierung 
unterstützen durch Abschaffung des ,Fusionsanreizes' (Maßnahme zur Unterstützung von Schulfusionen). Kosten für Schulbücher im Bereich der Sekundarstufen werde sie übernehmen und Familien hierdurch unterstützen. (Ministerie van Algemene Zaken 2007a: 18). Ein besonderer Schwerpunkt im Schulbereich sei die Bekämpfung des Schulabbruchs. Die Regierung wolle das hierfür eingesetzte Stimulierungsprogramm finanziell unterstützen, weise aber auch auf die Verantwortungsteilung in diesem Bereich. „Hier liegt auch eine große gesellschaftliche Verantwortung für die Privatwirtschaft: Unternehmen werden genügend Ausbildungsplätze anbieten müssen. Der Berufsschulunterricht wird für den Anschluss an die Berufspraxis sorgen“ (Ministerie van Algemene Zaken 2007a: 19). Wie im familienpolitischen Dokument der Vorgängerregierung bereits unterstrichen, lege auch diese Regierung einen wichtigen Schwerpunkt im Bereich der frühkindlichen Sprachförderung. Dies gelte für Kinder mit und ohne Migrationshintergrund. „Kinder, bei denen im Alter von drei Jahren durch die Beratungsstelle (für junge Eltern), oder anderweitig, ein sprachlicher Rückstand festgestellt wird, werden in den Kindertagesstätten/Kindergärten, vor- und frühschulische Bildung (Gruppe o) und gesonderten (Schalter)Klassen auf das erforderliche Niveau gebracht. Die Eltern dieser Kinder werden hierbei direkt eingebunden über die erweiterte Lehrpflicht“ (Ministerie van Algemene Zaken 2007a: 28). Außerdem nennt die Regierung einige Fördermaßnahmen zur Entwicklung von Kindern. So solle z.B. die bereits im Familienbericht der Vorgängerregierung angekündigte Einführung der Zentren für Jugend und Familie nun auch ausgeführt werden (Ministerie van Algemene Zaken 2007a: 29). Kinder, die in Problemfamilien aufwachsen, wolle man in ihrer Entwicklung frühzeitig unterstützen. Da Eltern allerdings nicht immer freiwillig ihrer Erziehungspflicht nachkämen, seien im Falle ernst zu nehmender Bedrohungen in der Kindesentwicklung verpflichtete Erziehungsunterstützungsmaßnahmen notwendig. „Eltern werden gesetzlich haftbar für den Schaden, den sie ihren minderjährigen Kindern anrichten“ (Ministerie van Algemene Zaken 2007a: 29). Die Regierung unterstreicht an dieser Stelle auch zwei Maßnahmen, welche im kurz zuvor publizierten Familienbericht bereits angekündigt, aber aufgrund des Regierungswechsels noch nicht ausgeführt wurden, nämlich die Einführung des Elternschaftsplans bzw. des Gesetzes zur Beförderung fortgesetzter Elternschaft (und sorgfältige Scheidung) und die Einführung des Elektronischen Kinderdossiers (inkl. Verweisindex) (Ministerie van Algemene Zaken 2007a: 29). Ein letzter Punkt an 
dieser Stelle ist die Förderung gesellschaftlicher Teilnahme bzw. Aufgabenwahrnehmung von Jugendlichen. „Junge Menschen werden während ihrer Schulzeit ein dreimonatiges gesellschaftliches Praktikum absolvieren, so dass sie die (breitere) Gesellschaft besser kennen lernen“ (Ministerie van Algemene Zaken 2007a: 29). Sie sollen hierdurch auf künftige Aufgaben in Arbeit und Gesellschaft vorbereitet werden.

${ }^{32}$ Der Minister für Jugend und Familie unterstreicht in seinem Programm, welches er 2007 an die Tweede Kamer (hier: Bundestag) schickte, die entscheidende Rolle der Erziehungsunterstützung (bzw. Unterstützung beim Aufwachsen) für Familien. Aufgrund diverser gesellschaftlicher Entwicklungen falle Eltern die Erziehung ihrer Kinder heute schwerer als zuvor und bräuchten sie Hilfe bei der Erziehung. Aufbauend auf den vorherigen Äußerungen zu diesem Thema, verspreche die Regierung an dieser Stelle, dass es „im Jahr 2011 ein flächendeckendes Netzwerk von Zentren für Jugend und Familie“ (Jeugd en Gezin 2007: 5) gäbe. Die Zusammenarbeit der verschiedenen Einrichtungen im Bereich der Jugendfürsorge sei bisher unzureichend und müsse daher dringend professionalisiert werden. Familien könne man nicht zumuten, dass Prozesse in der Jugendfürsorge nicht aufeinander abgestimmt seien. Risiken, die eventuelle Fehlkommunikationen bzw. fehlende Kommunikation in der Jugendfürsorge mit sich bringen, seien nicht tragbar. „Die Lösung der Probleme in einer Familie erfolgt durch den Ansatz ,Eine Familie, ein Plan'. Ein Gesamtplan für alle Probleme in einer Familie, mit einem Ansprechpartner für die Familie und die Hilfeleistenden“ (Jeugd en Gezin 2007: 5). Neben einer Professionalisierung in der Organisation und Arbeitsweise der Jugendfürsorge sei auch die Stimulierung der eigenen Kompetenzen der Familie entscheidend. Fürsorge müsse in der Regel nur begrenzt geleistet werden. Nach der Hilfeleistung sei es wichtig, dass Familien wieder selbstständig und in eigener Verantwortung leben können. „Die Verwendung des Instruments einer ,Eigenen Kraft Konferenz', bei der untersucht wird wie das eigene Netzwerk (Familie, Freunde, Nachbarn) die Familien unterstützen kann" (Jeugd en Gezin 2007: 5) solle in der Hilfeleistung eher Regel als Ausnahme sein. Die Folgen von Ehescheidungen für Kinder halte 
die Regierung für sehr relevant, und die Durchführung der bereits vorgenommenen Gesetzesänderung wolle sie weiter ausführen (vergl. Jeugd en Gezin 2007: 5). Falls es Eltern trotz aller Hilfestellungen nicht gelinge dauerhaft für die eigenen Kinder Verantwortung zu übernehmen, sei es (als letztes Glied in dieser Kette) in einigen Fällen nötig, Kinder permanent außerhalb der eigenen Familie $\mathrm{zu}$ betreuen. Vor allem die Aufnahme von Kindern in Pflegefamilien wolle man unterstützen. „Betreuung in Pflegefamilien bietet eine Alternative für Kinder, die nicht bei den eigenen Eltern aufwachsen können und ist daher eine adäquate Form der Jugendfürsorge. Es darf keine finanziellen Behinderungen geben, um Pflegemutter oder Pflegevater zu werden" (Jeugd en Gezin 2007: 6). Die Prävention von Erziehungsproblemen bzw. von Problemen beim Aufwachsen, so unterstreiche die Regierung, sei wichtig, um spätere, oft viel komplexere Probleme in der Jugendfürsorge $\mathrm{zu}$ vermeiden. „Eine gedeihliche Erziehung eines Kindes vor dem vierten Lebensjahr ist die beste Prävention gegen viele Probleme im späteren Alter. Für jedes Kind wird in den ersten vier Jahren nach der Geburt ein Test bezüglich der Erziehungs- und Wachstumsprobleme durch die Jugendgesundheitsfürsorge vorgenommen und wird, wo nötig, Fürsorge geboten“ (Jeugd en Gezin 2007: 6). Um die Professionalität des Jugendhilfesektors voran $\mathrm{zu}$ treiben und mögliche Jugendschutzrisiken auszuschließen, sei, wie bereits durch die Vorgängerregierung betont, die Einführung eines elektronischen Kinderdossiers (inkl. Verweisindex) nötig. Die Regierung unterstreicht, dass sie dieses Instrument und seinen Einsatz vorantreiben wolle. „Jeder Experte, der mit Kindern arbeitet, muss Informationen teilen können und über die bereits festgestellten, für die Gesundheit und Sicherheit relevanten Daten über das Kind oder die Familie verfügen. Das Elektronische Kinderdossier und der Verweisindex helfen den zuständigen Experten dabei. Bis zum vierten Jahr besitzt die Jugendgesundheitsfürsorge alle relevanten Informationen. Ab dem vierten oder fünften Jahr geht das Kind täglich zur Schule und wird die Schule zur Bezugsperson, die als erste Probleme signalisiert" (Jeugd en Gezin 2007: 6). Für die gesunde Erziehung von Kindern sei die Teilnahme an Sportaktivitäten einerseits, aber auch genügend Bewegung im täglichen Lebensablauf andererseits eine wichtige Voraussetzung. Außerdem trage die Teilnahme von Kindern und Jugendlichen am Breitensport positiv zur Integration bei. Eine Intensivierung von Sport- und Bewegungsmaßnahmen im Jugendbereich, durch eine Reihe von Maßnahmen, sei daher von der Regierung geplant (Jeugd 
en Gezin 2007: 21). Das bereits im Koalitionsvertrag dieser Regierung genannte gesellschaftliche Praktikum für Sekundarschüler wolle die Regierung in den kommenden Jahren schrittweise realisieren (vergl. Jeugd en Gezin 2007: 23). Im Jugendfürsorgegesetz sei die Zahlung eines eigenen Beitrags in der Pflegeversicherung Pflicht für Jugendliche, die über ein eigenes Einkommen verfügen. Hierdurch werde einerseits die Inanspruchnahme der Jugendfürsorge bzw. die Teilnahme am Arbeitsmarkt behindert. Man wolle diese Regelung aus diesem Grunde abschaffen und hierdurch die Aktivierung von diesen Jugendlichen erreichen (Jeugd en Gezin 2007: 23). Auch vorzeitiges Schulverlassen, ein Thema das von den Vorgängerregierungen unter Balkenende bereits an verschiedenen Stellen thematisiert wurde, ist an dieser Stelle erneut bearbeitet. Wiederum werden einige Präventionsmaßnahmen genannt, die frühzeitigem Schulverlassen entgegen wirken könnten. Diese Maßnahmen seien aber noch nicht ausreichend genug. „Darum fördert das Kabinett auch die Einführung einer Lehr- Arbeitsverpflichtung für junge Menschen bis zum Alter von 27 Jahren in Kombination mit der Möglichkeit der Einbehaltung einer eventuellen Sozialhilfe“ (Jeugd en Gezin 2007: 34). Neben der Kategorie ,Jugendlicher mit Problemen' gäbe es, so unterstreiche die Regierung, noch eine sehr kleine Prozentzahl von Jugendlichen, bei der übliche Hilfeunterstützungen oder Lehrtrajekte nicht helfen. Um zu vorkommen, dass diese Jugendlichen aussichtslos am Rande der Gesellschaft verharren, sei für sie eine besondere Unterstützungsmaßnahme notwendig. „Jungen Menschen ohne Aussicht auf eine Ausbildung oder einen Arbeitsplatz, die mit den gebräuchlichen Hilfsmaßnahmen nicht erreicht werden können, will das Kabinett darum eine Perspektive bieten durch die Einführung von Kampussen, die sich richten auf: Wiedererziehung, Schulung und Begleitung zum Arbeitsmarkt“ (Jeugd en Gezin 2007: 35f).

Im Familienbericht 2008, den der neu ernannte Minister für Jugend und Familie im ersten Jahr nach dem Regierungsantritt an die Tweede Kamer (hier: Bundestag) schickte, unterstreicht die Regierung den positiven Zusammenhang zwischen Erziehungsproblemen bzw. Problemen des Aufwachsens und Ehescheidungen von Eltern. Da die Regierung Kinder in diesem Zusammenhang schützen wolle, werde sie neben dem bereits erwähnten Elternschaftsplan, der bereits in Vorbereitung sei, eine Skala an Maßnahmen treffen, um Kinder vor andauernden Konflikten ihrer Eltern zu schützen. So 
wolle sie z.B. Programme entwickeln, die jungen Eltern (auf freiwilliger Basis) helfen könnten Konflikte auszutragen. Falls eine Scheidung unvermeidlich geworden sei, müsse es nicht nur gute Informationen für Eltern geben, sondern sollten Kinder frühzeitig in den Prozess der Scheidungsabwicklung einbezogen werden und sollten Eltern lernen, sich durch ein Hinzuziehen von Mediatoren, besser in die Rolle ihrer Kinder zu versetzen (Jeugd en Gezin 2008: 13f). Wie bereits seit dem Familienbericht aus dem Jahr 2006 in jedem der untersuchten Dokumente thematisiert, ist auch an dieser Stelle der Ausbau der Jugend- und Familienzentren beschrieben (vergl. Jeugd en Gezin 2008: 10). Neben des Ausbaus dieser Jugend- und Familienzentren wolle man auch die inhaltliche Debatte über Erziehungsfragen stimulieren. Da es an verschiedenen Orten bereits lokale Initiativen in diesem Bereich gäbe, werde man bei der nationalen Debatte zum Thema Erziehung soviel wie möglich anschließen an diesen Prozessen. „Zur Stimulierung dieser Debatte wird 2009 ein Startsignal für eine Estafette lokaler Erziehungsdebatten gegeben. Professionals, Eltern, junge Menschen und die soziale Umgebung der Familien können so gemeinsam nach Antworten, auf aktuelle Probleme beim Aufwachsen und Fragen der Erziehung, suchen“ (Jeugd en Gezin 2008: 10). Insbesondere auf die Erziehung zwischen zwei Kulturen lege die Regierung besonderen Wert. In regionalen akademischen Werkstätten wolle sie daher gemeinsam mit Eltern und Freiwilligen mit Migrationshintergründen an möglichen Lösungen, für Erziehungsfragen von Kindern und Eltern mit Migrationshintergründen, arbeiten. Außerdem, so betone die Regierung, werde die extra Dimension interkulturellen Erziehens in das Curriculum der Ausbildungsberufe in der Sozialhilfe aufgenommen (Jeugd en Gezin 2008: 13, 44f). 
Tabelle 27: Chronologisches Übersichtstableau der Instrumente zur Förderung oder Wiederherstellung der Kompetenzen Familienangehöriger in den Niederlanden

\begin{tabular}{|c|c|c|}
\hline Jahr & Dokument & Instrument \\
\hline 1994 & Koalitionsvertrag Kok I & $\begin{array}{l}\text { Intensivierung von Prävention und Frühsignalisierung im } \\
\text { Bereich der Jugendhilfe, } \\
\text { Dezentralisierung der Aufgaben im Schulbereich auf } \\
\text { Schulen und Gemeinden, } \\
\text { Reform des Berufsschulunterrichts und bessere } \\
\text { Vorbereitung auf Berufsausbildung an den Sekundarstufen }\end{array}$ \\
\hline 1994 & $\begin{array}{l}\text { Regierungserklärung Kok } \\
\text { I }\end{array}$ & $\begin{array}{l}\text { Dezentralisierung der Aufgaben im Schulbereich auf } \\
\text { Schulen und Gemeinden, } \\
\text { Reform des Berufsschulunterrichts und bessere } \\
\text { Vorbereitung auf Berufsausbildung an den Sekundarstufen }\end{array}$ \\
\hline 1996 & Familienbericht 1996 & $\begin{array}{l}\text { Einführung des arbeitsmarktgerichteten Lehrwegs, } \\
\text { Verbesserung der Jugendhilfe, } \\
\text { Qualitätsverbesserung Pflegeelternschaft, } \\
\text { Integration behinderter Kinder in den regulären Unterricht }\end{array}$ \\
\hline 1998 & Koalitionsvertrag Kok II & $\begin{array}{l}\text { Verkleinerung der Klassengrößen in den ersten Schuljahren, } \\
\text { Verbesserung des Schulinventars (Möbel, ICT etc.), } \\
\text { Fortsetzung der Autonomisierung im Schulbereich, } \\
\text { Verbesserung sprachlicher Fähigkeiten von Kindern mit } \\
\text { Migrationshintergrund, } \\
\text { Investition in Sportmaßnahmen, } \\
\text { Verbesserung der Jugendhilfe und Einführung eines neuen } \\
\text { Gesetzes zur Jugendfürsorge }\end{array}$ \\
\hline 1998 & $\begin{array}{l}\text { Regierungserklärung Kok } \\
\text { II }\end{array}$ & Verkleinerung der Klassengrößen in den ersten Schuljahren \\
\hline 2002 & $\begin{array}{l}\text { Koalitionsvertrag } \\
\text { Balkenende I }\end{array}$ & Anpassung der Qualitätskontrolle in Schulen \\
\hline 2006 & Familienbericht 2006 & $\begin{array}{l}\text { Einführung des elektronischen Kinderdossiers und des } \\
\text { Verweisindexes, } \\
\text { Zeitliche Regelung spezifischer Unterstützung in der } \\
\text { Jugendgesundheitspflege, } \\
\text { Einführung eines Zentrums für Jugend und Familie in jeder } \\
\text { Gemeinde bzw. jedem Stadtteil, } \\
\text { Bereitstellung von additionellen Mitteln für die } \\
\text { Jugendfürsorge, } \\
\text { Vorschlag zur Einführung des Elternschaftsplans im Falle } \\
\text { einer Scheidung und Änderung im Alimentationsgesetz, } \\
\text { Intensivierung des Früh- und Vorschulischen } \\
\text { Sprachprogramms, } \\
\text { Einführung eines Selbstregulierungsprogramms im Bereich } \\
\text { audiovisueller Produkte, } \\
\text { Einführung des ,Kijkwijzers' (Einschaltanzeigers) }\end{array}$ \\
\hline
\end{tabular}




\begin{tabular}{|c|c|c|}
\hline 2007 & $\begin{array}{l}\text { Koalitionsvertrag } \\
\text { Balkenende IV }\end{array}$ & $\begin{array}{l}\text { Einführung von Fixmomenten zur Schulanmeldung und } \\
\text { Möglichkeit zur Hantierung von Lossystemen, } \\
\text { Abschaffung des Fusionsanreizes für Schulen, } \\
\text { Einführung von gratis Schulbüchern im } \\
\text { Sekundarschulbereich, } \\
\text { Finanzielle Unterstützung des Programms zur Bekämpfung } \\
\text { des Schulabbruchs, } \\
\text { Intensivierung der frühkindlichen Sprachförderung, } \\
\text { Weitere Ausführung der Einführung der Zentren für Jugend } \\
\text { und Familie, } \\
\text { Vornehmen zur Einführung einer gesetzlicher } \\
\text { Erziehungsverpflichtung von Eltern, } \\
\text { Einführung des Gesetzes zur Beforderung fortgesetzter } \\
\text { Elternschaft (Elternschaftsplan), } \\
\text { Einführung des elektronischen Kinderdossiers (inkl. } \\
\text { Verweisindex), } \\
\text { Einführung des dreimonatigen gesellschaftlichen } \\
\text { Praktikums für Schüler }\end{array}$ \\
\hline 2007 & $\begin{array}{l}\text { Programm für Jugend und } \\
\text { Familie, 2007-2011 }\end{array}$ & $\begin{array}{l}\text { Weitere Ausführung der Einführung der Zentren für Jugend } \\
\text { und Familie, } \\
\text { Ausführung der Jugendhilfe anhand des Prinzips ,Eine } \\
\text { Familie, ein Plan', } \\
\text { Einführung der ,Auf eigene Kraft Konferenzen', } \\
\text { Einführung des Gesetzes zur Beförderung fortgesetzter } \\
\text { Elternschaft (Elternschaftsplan), } \\
\text { Unterstützung von Pflegefamilien, } \\
\text { Vornehmen der Einführung einer Erziehungsrisikenanalyse } \\
\text { für Kinder unter vier Jahren, } \\
\text { Einführung des elektronischen Kinderdossiers (inkl. } \\
\text { Verweisindex), } \\
\text { Intensivierung von Sport- und Bewegungsmaßnahmen im } \\
\text { Jugendbereich, } \\
\text { Durchführung des dreimonatigen gesellschaftlichen } \\
\text { Praktikums für Schüler, } \\
\text { Abschaffung des eigenen Beitrags in der Pflegeversicherung } \\
\text { für Jugendliche mit eigenem Einkommen, } \\
\text { Einführung der Schulungs- und Arbeitsverpflichtung bis zu } \\
27 \text { Jahren, } \\
\text { Einführung von Kampussen zur Wiedererziehung, Schulung } \\
\text { und Hinführung zum Arbeitsmarkt }\end{array}$ \\
\hline 2008 & Familienbericht 2008 & $\begin{array}{l}\text { Verabschiedung eines Maßnahmenpaketes im Bereich } \\
\text { Scheidungs- und Umgangsrecht, } \\
\text { Einführung von Erziehungsdebatten, } \\
\text { Ausbau Jugend- und Familienzentren, } \\
\text { Verwendung akademischer Arbeitsplätze }\end{array}$ \\
\hline
\end{tabular}




\subsubsection{Zeit-Instrumente}

33In der umfangreichen Notiz zum Thema Familie, die die Staatssekretärin des Ministeriums für Gesundheit, Wohlfahrt und Sport im Jahre 1996 an die Tweede Kamer (hier: Bundestag) schickte, umschreibt die Regierung unter anderem auch Maßnahmen zur Vereinbarkeit von Familie und Beruf. Sie unterstreiche in diesem Zusammenhang den Erziehungsurlaub, die Verlängerung und Flexibilisierung des Schwangerschafts- und Entbindungsurlaubs, die Stimulierungsregel zur Kinderbetreuung, die Beförderung von Teilzeitarbeit usw.. Diese Maßnahmen unterstützen Eltern bei der Arbeitsmarktteilnahme, böten ihnen aber auch genügend Familienzeit. „Ob beide Eltern arbeiten wollen und wie viel, bestimmen sie im Prinzip selbst (...) Um auch in Zukunft optimale Konditionen zur Vereinbarkeit von Familie und Beruf zu schaffen, seien Veränderungen in der Arbeitsorganisation nötig. Hierbei geht es um ein gemeinsames Interesse und daher auch um eine gemeinsame Verantwortung aller hierbei beteiligten Parteien, Regierung, Arbeitgeber und Arbeitnehmer" (Ministerie van Volksgezondheid, Welzijn en Sport 1996: 13). Die Erziehungszeit sei bisher so eingerichtet, dass Arbeitnehmer in den ersten vier Lebensjahren ihrer Kinder Erziehungsurlaub nehmen dürften, wodurch sie ihre Arbeitszeit auf minimal 20 Wochenstunden und für die Dauer von maximal sechs Monate verringern könnten (Ministerie van Volksgezondheid, Welzijn en Sport 1996: 14). Ein wichtiges Kriterium zur Vereinbarkeit von Familie und Beruf sei die Möglichkeit zur Teilzeitbeschäftigung. Dieses Modell der Arbeitszeiteinteilung passe gut zur niederländischen Kultur und habe in den Niederlanden traditionell eine wichtige Rolle gespielt. „Darum werden Teilzeitstellen stimuliert, so wie in dem kürzlich durch das Parlament angenommenen Gesetzesvorschlag, der von einem Verbot, auf unterschiedliche Behandlung bei den Arbeitsbedingungen aufgrund der Differenz der Arbeitswochenstunden (Vollzeitstelle Teilzeitstelle), ausgeht“ (Ministerie van Volksgezondheid, Welzijn en Sport 1996: 14). Auch das Arbeitszeitengesetz, welches im Jahre 1995 verabschiedet sei, unterstütze die Möglichkeit von Arbeitnehmern, um im normalen 
Tagesablauf genügend Zeit für die Familie zu reservieren. Einerseits werde in diesem Gesetz davon ausgegangen, dass der Arbeitnehmer mögliche Änderungswünsche bezüglich seiner Arbeitszeit frühzeitig an den Arbeitgeber melde. Andererseits müsse der Arbeitgeber „hinsichtlich der Arbeits- und Ruhezeiten Rücksicht nehmen auf die persönlichen Umstände der Arbeitnehmer" (Ministerie van Volksgezondheid, Welzijn en Sport 1996: 15).

Im Koalitionsvertrag der zweiten Violetten Koalition, vom 18.07.1998, wird die Einführung des Rahmengesetzes Arbeit und Pflege ausführlich thematisiert. In diesem Gesetz werden die bestehenden Regelungen im Bezug auf die Vereinbarkeit zwischen Beruf und Pflege Angehöriger (bzw. Erziehung der Kinder) zusammengefasst und modernisiert. Auch das Recht auf Teilzeitarbeit, sowie die Bedingungen für Arbeitgeber und Arbeitnehmer, werden in diesem Gesetz verankert und sollen zum 1. Januar 1999 geltend sein. „Die bestehenden gesetzlichen Beurlaubungsmöglichkeiten werden aufeinander abgestimmt und gebündelt. Außerdem wird ein Rahmen geschaffen indem das Sparen für die Beurlaubung (in Zeit und/oder Geld) aufgrund der Tarifverabredungen oder individuelle Übereinkommen gefördert wird. Individuelle Arbeitnehmer können die gesparte Urlaubszeit, mit Rücksicht auf den Inhalt ihrer Arbeitsbedingungen flexibel aufnehmen“ (Ministerie van Algemene Zaken 1998a: 23). Zusätzlich zu dieser gesetzlichen Regelung wolle die Regierung Stimulierungsmaßnahmen zur Tageseinteilung fördern. „Hierbei geht es unter anderem um die Förderung passender Arbeitszeiten, Erweiterung der Schulöffnungszeiten und die Unterstützung des Arbeitspools und des Unternehmertums in der persönlichen Dienstverleihung“ (Ministerie van Algemene Zaken 1998a: 23).

In der darauf folgenden Regierungserklärung dieser zweiten Regierung unter Kok unterstreichen die Politiker am 25.08.1998 ihr Vorhaben zur Bündelung, Abstimmung und Ausdehnung der Beurlaubungsmöglichkeiten zur Vereinbarkeit von Beruf und Betreuung von Angehörigen (bzw. Erziehung von Kindern). Das hierin verankerte Recht auf Teilzeitarbeit betone die Regierung insbesondere. „Noch in diesem Jahr wird (...) eine gesetzliche Regelung bezüglich eines klausulierten Rechts auf Teilzeitarbeit vorbereitet" (Ministerie van Algemene Zaken 1998b: 9).

Der Lebensphasenansatz wird wenige Jahre später im Koalitionsvertrag der ersten Großen Koalition unter Balkenende am 03.07.2002 unterstrichen. Es 
gäbe im Lauf einer beruflichen Karriere Momente an denen private Betreuung und Erziehung zentral ständen, während es zu anderen Zeitpunkten möglich sei, um z.B. mehr Zeit in die berufliche Karriere $\mathrm{zu}$ investieren. „Um Arbeitnehmern mehr Möglichkeiten zur Vereinbarkeit von Arbeit und anderen Aktivitäten zu bieten, wird eine Lebenslaufregelung eingeführt“ wodurch „ein Umschlag vom Rentensparen in Richtung eines Sparens, das sich auf ein längeres, produktiveres und gesunderes Arbeitsleben richtet, stimuliert werde“ (Ministerie van Algemene Zaken 2002a: 18).

${ }^{34}$ Auch die zweite Koalition unter Balkenende betont in ihrem Koalitionsvertrag vom 16.05.2003 die Einführung der Lebenslaufregelung. Die Realisierung dieser Maßnahme habe nach dem schnellen Fall der ersten Regierung unter Balkenende noch nicht stattgefunden. Im Vergleich zum Koalitionsvertrag der Vorgängerregierung werden allerdings erste Konkretisierungen der neuen ZeitMaßnahme verdeutlicht. Sie werde laut Koalitionsvertrag als eine vergleichbare Wahloption neben dem Sparlohn angeboten (vergl. Ministerie van Algemene Zaken 2003a: 4).

35Im Familienbericht 2006 der Staatssekretärin des Ministeriums für Gesundheit, Wohlfahrt und Sport wird die Einführung dieser Lebenslaufregelung (geltend seit dem 1. Januar 2006) umfassend beschrieben. Diese Regelung, die seit der Regierungserklärung der ersten Koalition unter Balkenende mehrmals angekündigt sei, ergänze längere (unbezahlte) Beurlaubungszeiten um Finanzierungsmöglichkeiten. Hierdurch könnten mehr Eltern als bisher es sich finanziell auch leisten, um die bestehenden Beurlaubungsmöglichkeiten zur Pflege und Erziehung auch tatsächlich in Anspruch zu nehmen. „Arbeitnehmer erhalten durch die Lebenslaufregelung steuerliche Vorteile, wenn sie für eine Periode unbezahlter Beurlaubung sparen. Arbeitnehmer mit Kindern unter 8 Jahren, die im Rahmen der Lebenslaufregelung sparen und ihren (unbezahlten) Erziehungsurlaub aufnehmen, haben dadurch, dass sie eine Steuerermäßigung von 50\% des

34 In der Regierungserklärung der ersten Koalition unter Balkenende, aus dem Jahr 2002, wurden keine konkreten ZeitInstrumente gefunden. 35 In der Regierungserklärung der zweiten Koalition unter Balkenende, aus dem Jahr 2003, wurden keine konkreten ZeitInstrumente gefunden. 
gesetzlichen Minimallohns anwenden können (maximal $€ 6_{3} 6$ pro Monat) einen größeren steuerlichen Vorteil. Die Regierung sorgt durch die Lebenslaufregelung für eine Grundfinanzierung der Erziehungszeit“" (Ministerie van Volksgezondheid, Welzijn en Sport 2006: 28). Insbesondere hebe die Regierung an dieser Stelle hervor, dass die Partner in den Familien selbst entscheiden, wer von den Eltern die Möglichkeit zur Beurlaubung verwenden wolle. Hierdurch fördere die Regierung die Gleichstellung der Geschlechter. Interessant sei in diesem Zusammenhang auch die neue Finanzierungsmöglichkeit, des zum 1. Januar 2005 eingeführten unbezahlten, langen Pflegeurlaubs. Für eine Periode von maximal sechs Wochen kann ein Arbeitnehmer beurlaubt werden, um sein lebensbedrohlich erkranktes Kind, Partner oder Elternteil zu pflegen. „Ein Arbeitnehmer kann das Guthaben seiner Lebenslaufregelung verwenden, um die Beurlaubungsperiode zu finanzieren“ (Ministerie van Volksgezondheid, Welzijn en Sport 2006: 29). Wie auch im Themenbereich der Beurlaubungen, der Kinderbetreuung und der Arbeitszeiten bereits verdeutlicht, sei Flexibilität auch für die weitere Infrastruktur der Familien (wie z.B. Ärzte, Einkaufsmöglichkeiten etc.) wichtig. Diese Flexibilität solle durch geeignete Maßnahmen gefördert werden. Das "Projekt Tageseinteilung/ESF-3 und Probeprojekte bei Gemeinden und anderen Sektoren richten sich auf das 'Ausdehnen' der Tagesgrenzen von 9 bis $17 \mathrm{Uhr}$ nach 7 bis 19 Uhr. Keine längeren Wochenarbeitszeiten, wohl aber mehr Flexibilität, um die Lebensqualität der Familien zu verbessern“ (Ministerie van Volksgezondheid, Welzijn en Sport 2006: 30f).

Auch im Koalitionsvertrag der vierten Koalition unter Balkenende vom 07.02.2007 wird angekündigt, man wolle Eltern bei der Vereinbarkeit von Familie und Beruf besser unterstützen. Familien bräuchten Zeit, (finanzielle) Mittel und Fähigkeiten zur Erziehung ihrer Kinder. „Das gesetzliche Recht auf einen Erziehungsurlaub wird von 13 auf 26 Wochen pro Arbeitnehmer verlängert und ist nicht übertragbar" (Ministerie van Algemene Zaken 2007a: 29). Gemeinsam verfügen Eltern seit dieser Zeit über das Recht auf einen Erziehungsurlaub von 52 Wochen. Außerdem werde durch die Regierung das Ziel der aktiven Teilnahme möglichst große Teile der Bevölkerung am Arbeitsmarkt erneut unterstrichen. Ein Anliegen sei der Regierung daher die Lebenslaufregelung entsprechend der Bedürfnisse der Eltern anzupassen. „Lehrrechte für Schulungseinrichtungen und das Sparen für ein Einkommen 
während eines erweiterten Erziehungsurlaubs werden an die bestehende Lebenslaufregelung gekoppelt“ (Ministerie van Algemene Zaken 2007a: 24f).

${ }^{36} \mathrm{Im}$ Familienbericht 2008, den der neu ernannte Minister für Jugend und Familie an die Tweede Kamer (hier: Bundestag) schickte, unterstreicht die Regierung den bereits im Koalitionsvertrag genannten Dreiklang von Zeit, (finanzieller) Mittel und Fähigkeiten, den Eltern zur Erziehung ihrer Kinder bräuchten. Die bereits durch diese Regierung angekündigte Verlängerung der Erziehungszeit werde zusätzlich unterstützt durch die Entkopplung der Erziehungsurlaubssteuerermäßigung von der Lebenslaufregelung. Da nur ein kleiner Teil der Bevölkerung an der Lebenslaufregelung teilnehme, sei auch die steuerliche Unterstützungsmaßnahme bisher nur von sehr wenigen Eltern in Anspruch genommen. Ab dem 1. Januar 2009 werde es allerdings für alle Eltern, die den Erziehungsurlaub aufnehmen möglich, um bis zu 50\% des Minimallohns, in Form einer Steuerermäßigung, zu empfangen. Hierdurch wolle man den Erziehungsurlaub finanziell attraktiver gestalten. Außerdem wolle die Regierung die bestehenden Aufnahmemodelle des Erziehungsurlaubs weiter entwickeln mit dem Ziel einer größeren Flexibilität der Aufnahme durch die Eltern. „Die Möglichkeiten, um die Vereinbarkeit von Familie und Beruf weiter $\mathrm{zu}$ vereinfachen, unter anderem durch die Flexibilisierung des Erziehungsurlaubs werden in einer Notiz über die Modernisierung des gesetzlichen Systems für Arbeitszeiten und Beurlaubung weiter erörtert“ (Jeugd en Gezin 2008: 9, 11f). Zu mehr Unterstützung für selbstständige Eltern sei es durch die Einführung des Schwangerschafts- und Entbindungsurlaubs von 16 Wochen für Selbstständige gekommen. Sie haben seit dieser Zeit das gleiche Recht wie Arbeitnehmerinnen. Neben der Finanzierung des Erziehungsurlaubs sei aber auch die Rolle der Arbeitgeber entscheidend für das Ausmaß der Zeit, die Eltern mit ihren Kindern verbringen könnten. „Das Kabinett will gemeinsam mit den Sozialpartnern und anderen Beteiligten untersuchen, wie die bestehenden Initiativen, sowie flexible Arbeitszeiten, Teilzeitarbeit und Heimarbeit von andern Betrieben nachgeahmt und das Konzept ,Familienfreundlicher Arbeitgeber' weiterentwickelt und verbreitet werden kann. 
Als Anstoß hierzu findet im Frühjahr 2009 eine Konferenz statt (...) und stehe das Thema auf der Agenda der Frühjahrs- und Herbstbesprechungen (mit den Sozialpartnern)“ (Jeugd en Gezin 2008: 9). Wie bereits im Familienbericht 2006 wird an dieser Stelle auch die Infrastruktur der Familien als ein wichtiger Bestandteil im Bereich der Zeitmaßnahmen interpretiert. Die Flexibilität der Öffnungs- bzw. Besuchszeiten entlaste Familien sehr. Daher wolle die Regierung auf diesem Weg weiterarbeiten und Familien in diesem Bereich der Zeitpolitik besser unterstützen. „Der Minister für Unterricht, Kultur und Wissenschaft hat mit einigen Provinzen und Gemeinden Verwaltungsabkommen über Zeitpolitik gemacht und den ,Schalter 7-7' eingerichtet“ (Jeugd en Gezin 2008: 12). Ziel sei es entsprechende Dienstleistungen für Familien zwischen 7 und 19 Uhr anzubieten. 
Tabelle 28: Chronologisches Übersichtstableau der Zeit-Instrumente in den Niederlanden

\begin{tabular}{|c|c|c|}
\hline Jahr & Dokument & Instrument \\
\hline 1996 & Familienbericht 1996 & $\begin{array}{l}\text { Schrittweise Anpassung der Instrumente zur } \\
\text { Vereinbarkeit von Familie und Beruf, } \\
\text { Vorbereitungen zur Ausbreitung und Flexibilisierung } \\
\text { der Pflegeurlaube, } \\
\text { Stimulierung von Teilzeitarbeitsplätzen, } \\
\text { Einführung des Arbeitszeitengesetzes zur } \\
\text { Unterstützung der Vereinbarkeit von Familie und } \\
\text { Beruf }\end{array}$ \\
\hline 1998 & Koalitionsvertrag Kok II & $\begin{array}{l}\text { Einführung des Rahmenrechts Arbeit und Pflege, } \\
\text { Stimulierungsmaßnahme zur Tageseinteilung }\end{array}$ \\
\hline 1998 & Regierungserklärung Kok II & Einführung des Rahmenrechts Arbeit und Pflege \\
\hline 2002 & Koalitionsvertrag Balkenende I & Ankündigung der Einführung der Lebenslaufregelung \\
\hline 2003 & Koalitionsvertrag Balkenende II & Ankündigung der Einführung der Lebenslaufregelung \\
\hline 2006 & Familienbericht 2006 & $\begin{array}{l}\text { Einführung der Lebenslaufregelung, } \\
\text { Möglichkeit der Finanzierung des unbezahlten } \\
\text { langwierigen Pflegeurlaubs im Rahmen der } \\
\text { Lebenslaufregelung, } \\
\text { Stimulierung flexibeler Tagesarrangements }\end{array}$ \\
\hline 2007 & Koalitionsvertrag Balkenende IV & $\begin{array}{l}\text { Verlängerung des Erziehungsurlaubs von } 13 \text { auf } 26 \\
\text { Wochen, } \\
\text { Ausweitung und Anpassung der Lebenslaufregelung }\end{array}$ \\
\hline 2008 & Familienbericht 2008 & $\begin{array}{l}\text { Kopplung der Erziehungsurlaubssteuerermäßigung } \\
\text { an den Erziehungsurlaub (anstelle einer Kopplung an } \\
\text { die Lebenslaufregelung an der nur ein sehr kleiner } \\
\text { Teil der Bevölkerung teilnimmt), } \\
\text { Weitere Flexibilisierungsvorhaben im Bereich des } \\
\text { Erziehungsurlaubs, } \\
\text { Einführung eines Schwangerschafts- und } \\
\text { Entbindungsurlaubs von } 16 \text { Wochen für Selbständige, } \\
\text { Stimulierungsmaßnahmen im Bereich } \\
\text { familienfreundlicher Unternehmen, } \\
\text { Stimulierungsmaßnahmen im Bereich } \\
\text { familienfreundlicher Kommunen }\end{array}$ \\
\hline
\end{tabular}




\section{Analyse}

\subsection{Beschreibung der Arbeitsweise}

Während in den vorherigen zwei Kapiteln die politischen Zielsetzungen und Instrumente Deutschlands und der Niederlande getrennt voneinander behandelt wurden, erfolgt in dieser Analyse - aufbauend hierauf - eine vergleichende Zusammenführung mit einem analytischen Vergleich der Parallelen und Differenzen. Alle relevanten Zielsetzungen und Instrumente werden im Zusammenhang mit den bereits erläuterten Ausgangspunkten und dem Idealmodell Aktivierender Familiepolitik betrachtet. Bewertet wird hierbei nicht nur die konsequente Anwendung von Bestandteilen des Modells Aktivierender Familienpolitik, sondern auch die Berücksichtigung des Prinzips der Aktivierung eigener Verantwortung beim Politikentwurf und die Verwendung des aktivierenden Instrumentariums. Die entsprechenden Textfragmente der untersuchten Dokumente bilden hierfür die Basis. Auf diese Weise wird deutlich wie die beiden Länder den Aktivierenden Staat in konkrete Politiken umgesetzt haben und an welchen Stellen sich Divergenzen und Konvergenzen ergeben.

Deutschland und die Niederlande kennen einen ähnlichen familienpolitischen Hintergrund und haben beide Aktivierungsstrategien im familienpolitischen Bereich verwendet. In dieser vergleichenden Analyse liegt der Fokus allerdings auf den Besonderheiten der Politiken beider Länder. Wo unterscheiden sich Deutschland und die Niederlande in ihrer Herangehensweise, und was könnten hierfür die Gründe sein? Wie Kremer 2007 in ihrer Arbeit darstellte, lässt Esping-Andersen kulturelle Aspekte in seiner Makroanalyse der europäischen Wohlfahrtsstaaten weitgehend außer Acht. Für das Funktionieren einzelner Politiken sind diese kulturellen Aspekte allerdings essentiell. In diesem fünften Kapitel stehen - bei allen Parallelen zwischen den Ländern - dann auch die familienpolitischen Differenzen zentral. 
Gründe für diese Differenzen kann es viele geben. Hinweise werden in der Analyse beschrieben, aber nicht abschließend analysiert. Das Zwischenfazit, welches dieses fünfte Kapitel abrundet, fasst die entscheidenden Konvergenzen und Divergenzen zusammen und gibt Ausschluss darüber, welches Land dem Idealmodell am nächsten kommt. Hier wird auch an das Prinzip der Wertschöpfungskette angeknüpft.

Im Fazit, welches sich diesem fünften Kapitel anschließt, folgt letztendlich die abschließende Bewertung der Forschungsfragen unter Berücksichtigung der Rolle des Systemebenenansatzes von Esping-Andersen und können theoretische Schlussfolgerungen gezogen werden. Anhand der Analyseresultate werden Lücken und Defizite des Idealmodells bzw. der Theorien von Esping-Andersen an der Stelle thematisiert und das Wirken des Aktivierenden Staates im familienpolitischen Bereich beurteilt. An der Stelle kommt es zur Beantwortung der Frage, ob der Aktivierende Staat weisend ist in Richtung eines europäischen Sozialmodells.

\subsection{Analyse der familienpolitischen Zielsetzungen}

\subsubsection{Bevölkerungspolitische Zielsetzungen}

Die demographischen Zielsetzungen bilden eine der drei Kategorien von Zielsetzungen im Idealmodell Aktivierender Familienpolitik. Wie bereits bei der Definition der Zielsetzungen erläutert, werden hier unter demographischen Zielen vor allem die natürlichen Bevölkerungsentwicklungen verstanden und nicht die Entwicklungen im Bereich der Migration. Esping-Andersen betont in seinen Ausführungen über den aktivierenden Wohlfahrtsstaat die Relevanz ausgewogener Geburtenraten. Eine nachhaltige Bevölkerungsentwicklung stehe im Zusammenhang mit der Haltbarkeit der wohlfahrtsstaatlichen Infrastruktur bzw. der Leistungen, die den Bürgern des Staates zugute kommen. Diese Nachhaltigkeit halte er für wichtig, da das Verhältnis zwischen dem produzierenden und dem empfangenden Teil der Gesellschaft ausgewogen sein müsse. Außerdem betone er die Bedeutung des Bevölkerungsaufbaus im Hinblick auf die Konkurrenzposition mit anderen Ländern. Um zu einer 
größeren Eigenverantwortung der Generationen zu kommen, müsse ihr auch aus demographischer Sicht die Möglichkeit hierzu geboten werden. Wenn dieses Gleichgewicht nicht vorhanden ist, kann die Verantwortungsteilung zwischen den Generationen ins Wanken geraten.

\subsubsection{Analyse der bevölkerungspolitischen Zielsetzungen in Deutschland}

An deutscher Seite ist das demographische Ziel vor allem seit der Stellungnahme auf den Zwölften Kinder- und Jugendberichts inhaltlich im Sinne des Idealmodells Aktivierender Familienpolitik besetzt ${ }^{37}$. Die zweite RotGrüne Regierung betonte die Bedeutung von Elternschaft für den Einzelnen einerseits, und im Sinne einer stabilen Bevölkerungsentwicklung andererseits. Dies sei für die Leistungen der sozialen Sicherheit und das Wohlfahrtsniveau der Bevölkerung von großer Bedeutung. Entscheidend für die Zielerreichung im aktivierenden Sinne sei die Verantwortungsteilung. Neben der öffentlichen Verantwortung, z.B. im Bereich der Kinderbetreuung strebte diese Regierung nach einer Aktivierung der Arbeitswelt und der Familien (bzw. Verwandtschaft). Im Zusammenhang mit der Arbeitswelt werden Arbeitgeber bereits seit der RotGrünen Regierungszeit auf die Bedeutung der Kombination van Beruf und Familie gewiesen. Unternehmen bekommen Unterstützung bei der Wahrnehmung dieser Verantwortung (siehe Analyse der Instrumente).

Die Große Koalition unter Merkel hat im Großen und Ganzen den kurz vor ihrer Regierungszeit von der Rot-Grünen Regierung eingeschlagenen Pfad der demographischen Nachhaltigkeit weiter bewandert. Im privaten Bereich wird vor allem seit der Großen Koalition unter Merkel auf die Strukturvorteile von Großfamilien gewiesen. Gemeint war hiermit nicht, dass alle Menschen Großfamilien gründen sollten, wohl aber wollte die Regierung verdeutlichen, dass die Aufgabenteilung, so wie sie in Großfamilien wahrgenommen wird, für Familien im allgemeinen Sinne eine geeignete Lösung zur Wahrnehmung verschiedener Aufgaben sein kann.

37 Auch vor dieser Zeit gab es demographische Zielsetzungen in der deutschen Familienpolitik. Sie wurden allerdings weniger deutlich als ebensolche definiert. 
Auch die Schwarz-Gelbe Koalition scheint das Prinzip der demographischen Nachhaltigkeit und geteilten Verantwortung in ihrer politischen Arbeitsweise fortführen $\mathrm{zu}$ wollen. Sie betont neben den Zielsetzungen ihrer Vorgängerregierungen aber auch in verstärktem Maße die Bedeutung des Umbaus verschiedenster Politikbereiche, um der Nachhaltigkeit auch kurzfristig gerecht werden zu können.

\subsubsection{Analyse der bevölkerungspolitischen Zielsetzungen in den Niederlanden}

In den Niederlanden ist die demographische Zielsetzung im hier untersuchten Zeitraum nicht im Sinne des Idealmodells Aktivierender Familienpolitik besetzt. Entscheidungen im Bezug auf Familiengründungen werden ausschließlich als Privatangelegenheit angesehen. Die Politik kann helfen Barrieren für Familien zu nehmen, sieht es allerdings nicht als Ziel die Geburtenzahlen positiv zu beeinflussen. Den demographischen Wandel interpretierte z.B. die vierte Regierung unter Balkenende als eine Tatsache, auf die sie reagiert, die sie aber im Gegensatz zur deutschen Regierung nicht aktiv beeinflussen will. In diesem Zusammenhang muss auf die rasante Bevölkerungsentwicklung der Niederlande innerhalb der zweiten Hälfte des zwanzigsten Jahrhunderts gewiesen werden ${ }^{3}$. Die heutige Regierung bereitet sich auf die Folgen des demographischen Wandels vor und betont in diesem Zusammenhang die Bedeutung eines ausgeglichenen Haushaltes und eines Gleichgewichtes zwischen Ausgaben und Einnahmen, um auf eventuelle finanzielle Folgen des demographischen Wandels finanziell reagieren zu können.

\footnotetext{
38 Zur Zeit der Ölkrise und nach der Publikation des Buches "The population bomb" von Paul Ehrlich gab es in den Niederlanden vehemente Diskussionen über die Begrenzung des nationalen Bevölkerungswachstums. Die Arbeit einer wissenschaftlichen Kommission, die über Möglichkeiten zur Geburtenreduzierung informieren sollte, wurde allerdings eingeholt durch schnell sinkende Fruchtbarkeitsziffern in den siebziger Jahren. Zum Zeitpunkt des Schreibens gibt es in den Niederlanden noch immer eine Stiftung, die sich eine Bevölkerungsreduzierung zum Ziel setzt. Sie nennt sich ,De Club van Tien Miljoen' und will, wie der Name verdeutlicht, die niederländische Bevölkerung auf zehn Millionen Einwohner reduzieren.
} 


\subsubsection{Analytischer Vergleich der bevölkerungspolitischen Politikdifferenzen}

Aus dem Vergleich der Politiken beider Länder kann festgestellt werden, dass demographische Ziele in Deutschland im gesamten untersuchten Zeitraum eine Rolle spielten. Parallel zu dieser Arbeit wurde deutlich, dass es auch vor dieser Zeit bevölkerungspolitische Zielsetzungen gab. Verändert hat sich allerdings die Art der Diskussion über demographische Ziele und die Formulierung ebendieser. Seit der Stellungnahme des Zwölften Kinder- und Jugendberichts wurden sie in Deutschland stets mehr im Sinne des Aktivierenden Modells verfolgt. In den Niederlanden wurden demographische Entwicklungen - im hier formulierten Sinne - während der untersuchten Periode (aber auch zuvor) von den meisten Regierungen nicht als politisches Ziel besetzt. „Die niederländische Regierung hat sich von alters her vom Führen einer Bevölkerungspolitik fern gehalten. Diese stand nach dem zweiten Weltkrieg in einem schiefen Licht (...) Weiterhin gab es, mit einer jungen Bevölkerung, jahrzehntelang auch keinen Grund, um hierüber nachzudenken (...) Erst jetzt sieht man für die Regierung eine Rolle weggelegt bei der Unterstützung der Möglichkeiten von Menschen, um Kinder zu bekommen und sie zu erziehen“ (Wetenschappelijke Raad voor het Regeringsbeleid 2006: 174f). Wie bereits festgestellt, haben erst die Politiker des vierten Kabinetts unter Balkenende die Veränderung des Bevölkerungsaufbaus thematisiert, und das Fazit gezogen, dass man diesen Wandel nicht beeinflussen, aber durchaus auf ihn reagieren müsste.

Nicht nur die inhaltliche Besetzung des Themas sondern auch die Art der Umsetzung, im Sinne von geteilten Verantwortlichkeiten, wurde in Deutschland konsequenter aktivierend betrieben als in den Niederlanden. Die verschiedenen Regierungen teilten die Verantwortlichkeiten zwischen den Familien, den Arbeitgebern, der Regierung usw., während in den Niederlanden im demographischen Bereich höchstens über die Unterstützung privater Wünsche durch die Regierung geschrieben wurde.

\subsubsection{Emanzipatorische Zielsetzungen}

Die zweite Zielsetzung im Idealmodell Aktivierender Familienpolitik gilt der Emanzipation von Frauen. Wie bereits verdeutlicht, ist hiermit vor allem die Chancengleichheit von Frauen in Ausbildung und Beruf gemeint. Übrige 
Zielsetzungen in diesem Bereich (Gewalt gegen Frauen usw.) werden aufgrund des wohlfahrtsstaatlichen Schwerpunkts dieser Arbeit nicht analysiert. Die aktive Teilnahme von Frauen auf dem Arbeitsmarkt ist aus wohlfahrtsstaatlicher Sicht im Sinne staatlicher Einnahmen und Ausgaben wünschenswert. Zwar führt eine vergleichbare Arbeitsmarktteilnahme von Frauen und Männern zur DeFamilialisierung und somit zu höheren staatlichen Kosten. Gleichzeitig kann sie aber auch eine höhere Produktivität und größere Einnahmen zur Folge haben. Außerdem wird als eines der Vorteile wirtschaftlicher Unabhängigkeit von Männern und Frauen gesehen, dass die Armutsrisiken von Frauen im Allgemeinen geringer sind.

\subsubsection{Analyse der emanzipatorischen Zielsetzungen in Deutschland}

An deutscher Seite ist die emanzipatorische Zielsetzung seit der ersten RotGrünen Koalition inhaltlich besetzt. Aus den Texten wurde deutlich, dass es sich hierbei um einen inhaltlichen Strom handelt, für den bereits in der Phase vor der hier untersuchten Periode eine entscheidende Richtungsvorgabe stattfand. Die Perspektive der Zielsetzungen war in der ersten - hier untersuchten Phase stark mit dem prinzipiellen Recht auf gleiche Entfaltung verbunden.

Dieser Ansatz änderte sich. Durch Gender Mainstreaming rief man zur Verantwortungsteilung auf, und im Gleichschritt mit den europäischen Zielsetzungen in diesem Bereich setzte sich die zweite Rot-Grüne Koalition konkrete quantitative Ziele im Hinblick auf die Beschäftigung von Frauen. Hierdurch wurde die Chancengleichheit von Frauen konkretisiert und quantifiziert, und es fand eine entscheidende Richtungsweisung statt.

Die Emanzipationspolitik der Großen Koalition wich von der Politik ihrer beiden Vorgängerregierungen in dem Sinne ab, dass sie weniger ein individuelles Ziel an sich formte, sondern in stärkerem Maße mit den allgemeinen familienpolitischen Maßnahmen verbunden war. Hiermit entspricht sie dem Prinzip Esping-Andersens, der sich für ein Zusammenwirken dieser Politikfelder ausspricht. Die Vereinbarkeit von Familie und Beruf im Zusammenhang mit dem Lebenslaufmodell als breiten Politikansatz kommt in dem Sinne dem Modell der Aktivierenden Familienpolitik sehr nah. Auch die zunehmende Aufmerksamkeit auf den Vater als Akteur im Bereich der 
Aufgabenteilung von Eltern ist dem Idealmodell Aktivierender Familienpolitik entsprechend.

Für die Schwarz-Gelbe Koalition unter Merkel gilt im Hinblick auf die emanzipatorischen Zielsetzungen Chancengleichheit und Wahlfreiheit von Frauen und Männern im Hinblick auf die Arbeitsmarktteilnahme und die Karriereperspektiven einerseits und der Möglichkeit für Familienzeit andererseits. Zur ursprünglich einseitigen Unterstützung der Frauen in Richtung Arbeitsmarktteilnahme kommt eine aktive Förderung von Männern in Rollen wie Erziehung und Pflege.

Insgesamt ist das Prinzip der Verantwortungsteilung in den hier untersuchten deutschen Dokumenten mehr oder weniger konsequent als Ausgangspunkt verwendet. Ihre eigene Rolle sah die Regierung in der Bereitstellung entsprechender Rahmenbedingungen. Auch von den Arbeitgebern erwartete die Regierung die Bereitstellung von positiven Rahmenbedingungen zur Vereinbarkeit von Beruf und Familie. Von der Zivilgesellschaft und den Familien selbst forderte sie eine aktive Unterstützung der Frauen.

\subsubsection{Analyse der emanzipatorischen Zielsetzungen in den Niederlanden}

In den Niederlanden hat es in der untersuchten Periode eine Vielzahl emanzipatorischer Zielsetzungen gegeben. Die Aktivierung von Frauen auf dem Arbeitsmarkt war allen Regierungen ein wichtiges Ziel und kam dem Idealmodell Aktivierender Familienpolitik sehr nah. Inhaltlich fallen insbesondere die Zielsetzungen der ersten Violetten Koalition und der ersten Regierung unter Balkenende auf. Während der ersten Violetten Koalition wurde eine radikale Abschaffung der Hausfrauenehe gefordert. Finanzielle Selbstständigkeit von Männern und Frauen war ein wichtiges Ziel.

Das Ziel der finanziellen Selbstständigkeit von Frauen bauten die Politiker in der ersten Regierungszeit unter Balkenende weiter aus. Die Regierung förderte die Arbeitsmarktteilnahme von Frauen auch aus sozialwirtschaftlicher Sicht. Für die Erhaltung des Wohlfahrtsstaatsniveaus sei diese Teilnahme unabdingbar.

In der zweiten Violetten Regierungen bzw. der zweiten und vierten Regierung unter Balkenende waren die emanzipatorischen Zielsetzungen vor allem auf die Vereinbarkeit von Familie und Beruf gerichtet. Im Kabinett Balkenende IV 
wurde diese Vereinbarkeit besonders betont und die Rolle der Väter in der Erziehung, ganz im Sinne des Idealmodells Aktivierender Familienpolitik, stärker hervorgehoben.

Verantwortung für die emanzipatorische Zielsetzung sahen die violetten Kabinette und auch die ersten Regierungen unter Balkenende vor allem bei der Regierung und den Familien selbst. Die Teilung der Verantwortlichkeiten mit den Arbeitgebern erfolgte erst zu einem späteren Zeitpunkt. Vor allem das vierte Kabinett unter Balkenende unterstreicht diese Verantwortungsteilung.

\subsubsection{Analytischer Vergleich der emanzipatorischen Politikdifferenzen}

Aufgrund der Analyse wird deutlich, dass in beiden Ländern emanzipatorische Ziele entsprechend des Idealmodells verfolgt wurden. In den Niederlanden betonte man seit der ersten Violetten Koalition die wirtschaftliche Selbstständigkeit von Frauen. Seit der ersten Regierung unter Balkenende propagierte die Regierung die Arbeitsmarktteilnahme aus sozialwirtschaftlicher Sicht. In Deutschland kamen diese Zielsetzungen erst später und vor allem weniger vehement zum Ausdruck. Die Vereinbarkeit mit dem Familienleben stand hier während der untersuchten Periode immer im Vordergrund, während das für die Niederlande erst seit dem vierten Kabinett unter Balkenende gilt.

Die Aktivierung der Väter in der Erziehung setzten sich beide Länder erst relativ spät zum Ziel. Vor allem die vierte Regierung unter Balkenende und beide Koalitionen unter Merkel haben dieses Thema inhaltlich deutlich besetzt. Verantwortungsteilung war in beiden Ländern ein wichtiges Prinzip bei der Definition der emanzipatorischen Ziele. Während in Deutschland ein Schwerpunkt auf der Verantwortung von Staat und Arbeitgebern liegt, wurden in den Niederlanden vor allem die Aktivierung der Frauen und ihre eigene Verantwortung diesbezüglich betont. Auch im Hinblick auf die Väter unterstreichen die niederländischen Politiker die Eigenverantwortung der Familien. In Deutschland hingegen wurde die Aktivierung von Müttern für den Arbeitsmarkt bzw. Väter für Erziehung und Hausarbeit stärker von der Regierung unterstützt und hat man weniger auf die Eigenverantwortung der Eltern gewiesen. 


\subsubsection{Auf das Kindeswohl gerichtete Zielsetzungen}

Das Kindeswohl, bzw. die Entwicklung von Kindern ist die dritte der Zielsetzungen im Idealmodell Aktivierender Familienpolitik. Als entscheidend gilt hier vor allem die Entwicklung von kleinen Kindern, bzw. Säuglingen. An dieser Stelle im Lebenslauf werden für die Lebenschancen von Kindern entscheidende Weichen gestellt. Segregation von Kindern im Sinne schulischer Leistungen, Aussichten auf dem Arbeitsmarkt und Vermeidung von Armutsrisiken können $\mathrm{zu}$ diesem Zeitpunkt am erfolgreichsten verhindert werden. Individuelle Förderungen von Kindern $\mathrm{zu}$ einem späteren Zeitpunkt sind viel komplexer und bieten weniger Aussicht auf Erfolg. Aus wohlfahrtsstaatlicher Sicht sind sie daher auch weniger erstrebenswert. Sie führen zu größeren Risiken und höheren staatlichen Kosten. Wie bereits erläutert, kann eine hochwertige und für alle Kinder zugängliche Kinderbetreuungslandschaft Segregation entgegenwirken. Schlüsselwörter sind die Zugänglichkeit und Finanzierbarkeit der staatlichen Einrichtungen, sowie die Betreuung durch gut ausgebildetes Personal.

Für die Zeit, in denen Mütter und Väter eigenständig ihre Kinder betreuen brauchen sie Zeit und Ruhe. Vor allem das erste Lebensjahr von Kindern bedarf eines besonderen Schutzes, da einerseits das Kind noch sehr klein ist und andererseits zu diesem Zeitpunkt das Fundament für eine stabile Eltern-Kind Beziehung gelegt wird (vergl. Esping-Andersen 2002, 2009).

\subsubsection{Analyse der auf das Kindeswohl gerichteten Zielsetzungen in Deutschland}

Die erste Rot-Grüne Regierung hat die auf das Kindeswohl gerichtete Zielsetzung des Idealmodells inhaltlich besetzt. Sie wollte Bildungschancen für alle Kinder vergrößern, Armut verkleinern und Kinderrechte verstärken. Den speziellen Fokus auf Kinder in der ersten Lebensfase nahm sie weniger konkret in ihre Zielsetzungen auf. Insbesondere folgte die Regierung dem aktivierenden Muster im Bereich der Jugendarbeitslosigkeit. Seit dem Elften Kinder- und Jugendbericht aus dem Jahre 2002 wird auch der Ausbau der sozialen Einrichtungen (insbesondere zur Kinderbetreuung) als Ziel besetzt.

Nicht im Koalitionsvertrag der zweiten Rot-Grünen Koalition, wohl aber in der Stellungnahme auf den Zwölften Kinder- und Jugendbericht werden deutliche 
Ziele im Sinne aktivierender Familienpolitik benannt. Seit diesem Moment entsprechen die Zielsetzungen der deutschen Familienpolitik in diesem Bereich mehr und mehr dem Idealmodell Aktivierender Familienpolitik (konsequenter Ausbau des öffentlichen Systems der Kinderbetreuung von Kindertagesstätten bis zu Ganztagsschulen, qualitative Verbesserung des Angebots öffentlicher Kinderbetreuung, Einführung eines bundesweit wirksamen Qualitätssicherungssystems und eine umfassende Förderung kindlicher und jugendlicher Kompetenzen). Wie ihre Vorgänger legte auch diese Regierung einen besonderen Fokus auf die Kinder sozialschwacher Bevölkerungsgruppen, fügte dem aber hinzu, dass sie das Ziel einer Reduzierung der Schulabbrecherquote mit 10\% verfolge. Erreichen wollte sie ihre Ziele durch eine Verbesserung der ressortübergreifenden Kooperation und Einbindung verantwortlicher Gruppen und Parteien.

Die Große Koalition unter Merkel unterstrich in diesem Bereich die Zielsetzungen der zweiten Rot-Grünen Koalition und fügte dem hinzu, dass sie prinzipiell von einer Eigenverantwortung der Eltern bis zum 25. Lebensjahr ihrer Kinder ausgehe. Auch im 13. Kinder- und Jugendbericht, in dem die Große Koalition die besondere Bedeutung der Gesundheit und des Wohlbefindens von Kindern unterstrich, betonte sie die Verantwortung der Familien. Sie wolle den Familien hierbei entgegenkommen. Daher weise die Regierung auch insbesondere auf die verschiedenen Einrichtungen für Kinder (Kindertagesstätten, Schulen, Einrichtungen der Jugendhilfe usw.).

Die Schwarz-Gelbe Koalition unter Merkel betonte das Ziel der Kindeswohlfahrt in besonderem Maße. Chancengleichheit für Kinder und Investitionen für Schulen und Universitäten halte diese Regierung für wichtig. Im Gegensatz zu ihren Vorgängern im Amt betont die Regierung an dieser Stelle allerdings nicht die besondere Bedeutung des Kleinkindalters, um diese Chancengleichheit zu erreichen. Zur weiteren Gestaltung und Umsetzung dieser Ziele kann allerdings zum Zeitpunkt des Schreibens dieser Arbeit noch kein Urteil gefällt werden.

\subsubsection{Analyse der auf das Kindeswohl gerichteten Zielsetzungen in den Niederlanden}

Wie bei den emanzipatorischen Zielsetzungen hat es auch bezüglich des Kindeswohls in den Niederlanden eine große Vielzahl von Zielsetzungen 
gegeben. Vor allem die erste Violette Koalition benannte $\mathrm{zu}$ Beginn ihrer Regierungsperiode Zielsetzungen, die von adäquater Kinderbetreuung von Kleinkindern bis zur Ausbildung bzw. Studium inhaltlich im Sinne des Idealmodells Aktivierender Familienpolitik sind. Auch spielten in zunehmendem Maße (vor allem seit der Veröffentlichung des Familienberichts von 1996) die Rechte von Kindern eine wichtige Rolle in der niederländischen Familienpolitik. Kindern gelte das Recht auf eine gute Entwicklung mit gleichen Chancen für alle Kinder, ungeachtet ihres sozialen Hintergrundes bzw. ihrer Lernfähigkeiten. Wie bei den emanzipatorischen Zielen wurde auch hinsichtlich des Kindeswohls deutlich auf die Eigenverantwortung der Bürger gewiesen.

Die zweite Violette Regierung setzte den aktivierenden Ansatz ihrer Vorgänger fort. Sie zielte auf eine bessere Betreuung von Kindern und auf eine stärkere Förderung von Kleinkindern, um Segregation zu vermeiden. Falls es zu einem späteren Zeitpunkt zu Problemen komme, sollte die präventive wie auch kurative Jugendhilfe die Chancengleichheit aller Kinder fördern. Bei der Ausgestaltung ihrer Zielsetzungen hat die zweite Violette Regierung die Teilung der Verantwortlichkeiten allerdings nicht sehr deutlich definiert.

$\mathrm{Zu}$ einem Bruch mit den konsequent aktivierenden Zielsetzungen ihrer Vorgänger kommt es im Koalitionsvertrag der ersten Koalition unter Balkenende. Weniger die direkte Kindeswohlfahrt als Systemdiskussionen über Schulgrößen und Selbstverwaltung beherrschten das Bild dieser Koalition. Die zweite Koalition unter Balkenende fügte den Zielsetzungen im Bereich der Erziehungshilfen hinzu. Im Familienbericht von 2006 erfolgt eine weitere Trendwende in der niederländischen Familienpolitik. Das Recht des Kindes auf eine gesunde Erziehung spielt ab dem Zeitpunkt eine wichtige Rolle. Die Regierung versprach einzugreifen in Situationen in denen diesem Prinzip nicht gerecht werde. Außerdem betonte sie das Angebot im Bereich elterlicher Erziehungsfragen.

Die vierte Regierung unter Balkenende hat sich inhaltlich an der Politik des Familienberichts 2006 orientiert und hinzugefügt, sie werde vor allem im Bereich des Schulabbruches einsetzen (Halbierung bis zum Jahre 2012), um die Chancengleichheit von Kindern optimal zu fördern. Realisieren werde sie dieses Ziel gemeinsam mit Schulen, Lehrern und anderen Beteiligten. Mehr noch als im Koalitionsvertrag seiner Regierung, hat sich der neu eingesetzte Minister für 
Jugend und Familie in seinen Zielsetzungen am Idealmodell Aktivierender Familienpolitik orientiert. Ein großer Teil seiner Aufgaben war der Erfüllung des Umstrukturierungskatalogs der Jugendpolitik im Allgemeinen gewidmet. Dem fügte er wichtige Ziele im Bereich der Entwicklungsmöglichkeiten von Kindern hinzu. Die Chancengleichheit wollte er fördern und Kinder sollten im Sinne politischer Teilhabe Aktivierung erfahren. Ausgrenzung von sozialer Teilhabe aufgrund von Armut galt es zu vermindern und die Fähigkeiten von Eltern, um ernste Beziehungsprobleme zu lösen, sollten gestärkt werden.

\subsubsection{Analytischer Vergleich der Kindeswohl-Politikdifferenzen}

Aufgrund der Analyse wird deutlich, dass in beiden Ländern die Förderung des Kindeswohls im Sinne des Idealmodells auf unterschiedliche Weise unterstützt wurde. Während in Deutschland vor allem durch den Einfluss des Elften und Zwölften Kinder- und Jugendberichts eine zunehmende und stets deutlichere und konsequentere Ausrichtung am Idealmodell Aktivierender Familienpolitik stattfand (sowohl inhaltlich als auch im Sinne der Verantwortungsteilung) war die Verwendung der niederländischen Zielsetzungen aus der Perspektive des Idealmodells innerhalb der untersuchten Periode unstetig. Durch die erste Violette Regierung wurde aus Sicht des Idealmodells ein konsequenter Start gemacht. Die erste und zweite Regierung unter Balkenende haben sich im Bereich des Kindeswohls allerdings nicht oder nur in geringem Maße an den Zielsetzungen des Idealmodells orientiert. Der inhaltliche Schwerpunkt lag in dieser Zeit vor allem auf Systemdiskussionen im Bereich der Schulpolitik bzw. Jugendhilfe. Während es allerdings bei den Schulen aus technischer Sicht zu geteilten Verantwortlichkeiten im Sinne des Idealmodells kam, hat im Bereich der Jugendhilfe der Ausbau eines dezentralisierten und bürokratischen Hilfssystems stattgefunden.

Im vierten Kabinett unter Balkenende kam es zu einer neuen Kursänderung. Einerseits unterstützte die Regierung den starken Ausbau eines formellen Jugendhilfesystems, andererseits betonte sie aber auch die eigenen Stärken von Familien (im Sinne der Verantwortungsteilung des aktivierenden Leitbildes) und die Bedeutung von Chancengleichheit für alle Kinder ab jungem Alter, so wie im Idealmodell vorgesehen. Nach einer Periode verschiedener 
Perspektivenwechsel werden seit dieser Zeit Zielsetzungen wieder entsprechend des Aktivierenden Idealmodells formuliert.

Wie sich in Deutschland die Förderung des Kindeswohls unter der SchwarzGelben Regierung weiterentwickelt ist noch nicht ganz deutlich. Festgestellt werden muss allerdings, dass der Fokus der neueren Regierungsdokumente auf dem Bereich der Ausbildung in Schulen und Hochschulen liegt. Inwiefern die Kleinkindentwicklung dementsprechend weniger Gewicht bekommt, ist noch fraglich.

\subsection{Analyse der familienpolitischen Instrumente}

Den Kern dieses Kapitels formt die Instrumentenanalyse. Es werden, wie bereits bei den Zielen auch die Instrumente sowohl im Allgemeinen als auch im Zusammenhang zueinander analysiert. Hieraus wird deutlich inwiefern die Aktivierungsbestrebungen der verschiedenen Regierungen im Sinne des Idealmodells Aktivierender Familienpolitik erfolgten. Aufgrund der hier verwendeten Dokumentenanalyse umschließt die Analyse einen Großteil aller Zielsetzungen und Instrumente. Wie bereits angedeutet kommt es an dieser Stelle allerdings nicht zur Analyse aller vorgenommenen Maßnahmen. Erstens würde das den Rahmen dieser Arbeit sprengen und zweitens sind nicht alle Instrumente (ausreichend) in den verwendeten Quellen umschrieben, wodurch eine Analyse aller Maßnahmen nicht möglich ist. Für das eigentliche Ziel dieser Arbeit, der Forschung nach der Umsetzung des Leitbildes ,Aktivierender Staat', gibt es allerdings genügend Beispiele und ist die hundertprozentige Vollständigkeit des Instrumentariums nicht relevant.

Bei der Einrichtung der Instrumente wird die Umsetzung des Prinzips der geteilten Verantwortlichkeit noch deutlicher als bei den bereits analysierten Zielsetzungen. Es werden im Idealmodell vier Instrumente unterschieden:

- Ökonomische Instrumente,

- Ökologische Instrumente,

- Instrumente zur Förderung oder Wiederherstellung von Kompetenzen der Familienangehörigen und 
- Zeit- Instrumente.

Neben dieser inhaltlichen Zusammensetzung des für die aktivierende Familienpolitik relevanten Instrumentenmixes ist auch die Frage der Ausführung der Instrumente relevant. $\mathrm{Zu}$ einer geteilten Verantwortlichkeit würde es nicht passen, wenn der Staat die Ausführungsverantwortung für alle Instrumente in eigenen Händen hält. Ihm kommt im Idealmodell Aktivierender Familienpolitik vor allem die Gewährleistungsverantwortung zu. Diesem Gedanken entsprechend sind Öffentlich-Private Zusammenarbeit, Sozialinvestition und eventuell auch Privatisierungsmaßnahmen geeignete Instrumente im Idealmodell Aktivierender Familienpolitik.

Im Vergleich $\mathrm{zu}$ der Zielsetzungsanalyse ist die Instrumentenanalyse ausführlicher, weil sie einerseits wichtige Hinweise auf die Art der Umsetzung des Aktivierungsprinzips und andererseits interessante Beispiele für eine erfolgreiche Verwendung von Aktivierungsstrategien bietet. Theoretisch kann man unterscheiden zwischen Instrumenten und den Zielen, denen sie dienen. In der politischen Praxis wird dieser Unterschied oft weniger deutlich. Einige Instrumente können verschiedenen Zielen dienen. An dieser Stelle soll dann auch nicht pro Instrument beschrieben werden welcher Zielsetzung es dient, sondern geht es in erster Linie um die Anwendung aktivierender Maßnahmen im Allgemeinen.

\subsection{1 Ökonomische Situation der Familienhaushalte}

$\mathrm{Zu}$ den ökonomischen Instrumenten zählen Maßnahmen, die die wirtschaftliche Situation von Familien beeinflussen. Aufwachsen in Armut kann für die Entwicklung von Kindern ein Risikofaktor sein, und die Erblichkeit von Armut ist aus verschiedenen Gründen besonders problematisch. In dieser Situation kann es schnell zu Problemen kommen, für die gute Hilfe schwer oder nicht mehr möglich ist. Zwei traditionelle ökonomische Instrumente sind Lohnzuschüsse bzw. Einkommenszuschüsse, um das Familieneinkommen aufzustocken. Opielka differenziert zwischen drei Formen der Geldleistungen für Familien:

- „Geldleistungen an Eltern zum Lebensunterhalt der Kinder (v.a. Kindergeld). Sie dienen dem Ausgleich sozialer Ungleichheiten und folgen 
entweder dem Bedarfsprinzip oder sind (wie in Deutschland) universalistisch. Diese Leistungen sind in Ländern mit starkem Familienernährermodell ausgeprägter (der Wohlfahrtsstaat übernimmt quasi die Ernährerrolle), in den skandinavischen und liberalen Ländern gering.

- Geldleistungen für ein Recht auf Sorge (,Zeit für Kinder’). Hierzu gehören das Erziehungsgeld (und ein Erziehungsgehalt), aber auch Mutterschutz, Elternurlaub und Geldleistungen für familiäre Pflege. Dabei handelt es sich um neue soziale Rechte (Knijn/Kremer 1997). Diese Leistungen werden seit Mitte der 1980er Jahre in den meisten Wohlfahrtsstaaten ausgeweitet, besonders stark in den skandinavischen Ländern (v.a. Norwegen), teils als Lohnersatz, aber auch in Deutschland und Frankreich. Sie entsprechen einem garantistischen Sozialpolitiktyp.

- Geldleistungen (und Rechtsansprüche) um Sorge (care) zu erhalten, beispielsweise als Anrecht auf eine öffentliche Kinderbetreuung oder auf Pflegeleistungen. Auch diese Rechte werden zunehmend ausgeweitet, teils als unmittelbarer Anspruch auf Dienstleistungen, teils in Form von ,Gutscheinen' (voucher). Insbesondere Gutscheine lassen sich als ein ,Mix' aus konservativen, liberalen und sozialistisch-sozialdemokratischen Regimekonzepten, insoweit als auch als garantistisch interpretieren“ (Opielka 2004: 115f).

Die zweite und dritte Kategorie werden in dieser Arbeit im Rahmen der ökologischen Instrumente bzw. Zeit-Instrumente analysiert. Es bleiben an dieser Stelle die Geldleistungen zur Unterstützung des Lebensunterhalts der Kinder. Aus der Sicht des Idealmodells Aktivierender Familienpolitik muss man diese Kategorie der ökonomischen Instrumente allerdings erweitern. Neben der durch Opielka unterschiedenen finanziellen Formen der Unterstützung durch den Staat deckt an erster Stelle das Haushaltseinkommen die ökonomische Situation der Familie. Um Aktivierung im Sinne von Arbeitsmarktteilnahme zu erreichen, muss Arbeit lohnend sein und wird außerdem (wo möglich) neben der Arbeitsmarktteilnahme von Vätern auch von einer beruflichen Tätigkeit der Mütter ausgegangen. Diese Arbeitsmarktteilnahme kann der Staat durch steuerliche Maßnahmen fördern und somit Aktivierung ermöglichen. 
Die Politiksteuerung in diesem Bereich ist also zweigliedrig. Einerseits gibt es die direkte Bezuschussung von Familien durch z.B. das Kindergeld. Diese Maßnahme gehört zum klassischen Instrumentarium der Familienpolitik und geht aus von dem Prinzip der Unterstützung von Eltern, da diese durch die Erziehung ihrer Kinder eine gesamtgesellschaftlich relevante Tätigkeit ausüben, die mit öffentlichen Mitteln unterstützt wird. Das zweite Finanzierungsprinzip ist weniger pauschal, sondern abhängig von der Frage der Arbeitsmarktteilnahme. Hierbei betrifft es steuerliche Begünstigungen. Nicht immer leisten beide Eltern einen wirtschaftlichen Beitrag zum Haushaltseinkommen, bzw. gibt es zwei Elternteile. Auch ist es illusorisch davon auszugehen, dass (vor allem) alle Mütter am Arbeitsmarkt teilnehmen wollen. Wie im theoretischen Teil dieser Arbeit bereits dargestellt unterscheidet Hakim (vergl. Kremer 2007: 68) zwischen Frauen, die in Vollzeit am Arbeitsmarkt teilnehmen (wollen) bzw. sich in Vollzeit um die Kinder kümmern (wollen) von der übergroßen Gruppe von Frauen, die Arbeitsmarktteilnahme mit Erziehungsaufgaben kombinieren. Das Idealmodell geht daher auch nicht von einem kompletten Zweiverdienermodell aus, sondern berücksichtigt im Bezug auf die Arbeitsteilnahme die persönlichen Wünsche der Eltern und unterscheidet zwischen verschiedenen Lebensphasen in denen sich die Bedürfnisse der Eltern verändern.

\subsubsection{Analyse der ökonomischen Instrumente in Deutschland}

Die erste Rot-Grüne Koalition hat Familien durch eine begrenzte Anzahl ökonomischer Instrumente unterstützt. Hierbei lag der Schwerpunkt vor allem auf der Beistellung der bestehenden Instrumente wie z.B. das Kindergeld und den Kinderfreibetrag, welche erhöht bzw. angepasst wurden. Die Steuerreform aus dem Jahr 2000 ermöglichte es Steuerabzüge für nachgewiesene, erwerbsbedingte Betreuungskosten $\mathrm{zu}$ erhalten (siehe auch ökologische Instrumente). Dieses kann als eine Maßnahme zur Aktivierung im Sinne der Arbeitsmarktteilnahme beider Elternteile gedeutet werden. Unabhängig hiervon wird es Eltern ermöglicht einen Freibetrag für den Ausbildungsbedarf ihrer (volljährigen) Kinder zu erhalten.

In der zweiten Regierungsperiode unter Schröder wurden nur wenig familienpolitische, ökonomische Instrumente in den hier verwendeten 
Dokumenten angetroffen. Wie bereits erwähnt lag in dieser Regierungsperiode der sozialpolitische Schwerpunkt auf den so genannten Hartz-Reformen (die nicht zum inhaltlichen Fokus dieser Arbeit gehören). Im Zusammenhang mit diesen Reformen wurde auch der (befristete) Kinderzuschlag eingeführt, durch den die Arbeitsmarktteilnahme von Familien mit einem niedrigen Einkommen stimuliert werden sollte. Gleichberechtigung von Frauen verstand die Regierung als ein wichtiges Thema. Maßnahmen, wie z.B. eine geschlechterfreundlichere Neugestaltung des Bundesangestelltentarifvertrags bzw. die Förderung von Unternehmensgründungen in frauenfreundlichen Branchen wie dem Dienstleistungssektor, erreichten in ihrer Wirkung allerdings nur eine begrenzte Anzahl der Familien.

Während der Großen Koalition unter Merkel wurden einige ökonomische Instrumente eingesetzt bzw. erneuert. Die Einführung des unbefristeten Kinderzuschlags ist durchaus aktivierend, denn sie erhöht den Anreiz, um am Arbeitsmarkt teilzunehmen. Die Bündelung verschiedener Maßnahmen zur Lohnergänzung in einer Familienkasse und auch die Einführung eines Familienwegweisers mögen $\mathrm{zu}$ mehr Transparenz der ökonomischen Leistungen geführt haben, sind aber im Sinne des hier verwendeten Modells Aktivierender Familienpolitik praktisch ohne Mehrwert. Die Erhöhung der Kinderzulage bietet vielen Familien durchaus einen finanziellen Spielraum, ist aber im Sinne aktivierender Familienpolitik nur von begrenzter Bedeutung, da ihr Aktivierungsbezug gering ist. Nicht uninteressant im Sinne des hier verwendeten Modells ist allerdings die Staffelung des Kindergeldes, die 2009 vorgenommen wurde. Auch die Auszahlung des Pauschalbetrags, um Kindern aus ärmeren Familien den Weg $\mathrm{zu}$ besseren Bildungsabschlüssen $\mathrm{zu}$ ermöglichen bzw. die Einführung zusätzlicher Hilfen für Geringverdienerfamilien im Rahmen des Konjunkturprogramms II ist aus aktivierender Sicht hilfreich, um die Zukunftschancen der Kinder aus finanziell schwächeren Familien zu stärken.

Die Schwarz-Gelbe Koalition unter Merkel scheint den am Ende der vorherigen Regierungsperiode eingeschlagenen Weg der Intensivierung im Bereich der ökonomischen Instrumente fortsetzen $\mathrm{zu}$ wollen. Inwiefern sie dieses Versprechen auch einlösen wird kann zum Zeitpunkt des Schreibens noch nicht abschließend beurteilt werden. Vorgeschlagen hat die neue Regierung neben der Erhöhung des Kindergeldes und der Weiterentwicklung des Kinderzuschlags 
auch die Anhebung des Kinderfreibetrages. Dieses zweite Instrument könnte einen positiven Effekt auf die Arbeitsmarktteilnahme von Müttern haben und somit zur weiteren Aktivierung führen. Auch wolle die Regierung verstärkt investieren in die Ausbildungsfinanzierung von Kindern. Hierdurch wolle sie die private Verantwortung stimulieren und Eltern und Verwandte aktivieren, um für Ausbildungskosten der Kinder selber zu sparen. Auffallend sind auch die Intensivierungen, die diese Regierung in die Möglichkeit zur Eigenbetreuung von Kindern investieren wolle. So beginnt sie eine erneute Diskussion um die Anerkennung von Erziehungszeiten im Rentensystem und schlägt sie die Einführung eines Betreuungsgeldes vor, wodurch sie die Wahlfreiheit der Eltern zwischen Fremd- und Eigenbetreuung vergrößern wolle. In wiefern diese Maßnahme aktivierend sein kann, ist abhängig von der definitiven Einrichtung des Instrumentes und kann zum Zeitpunkt des Schreibens noch nicht abschließend beurteilt werden.

Tabelle 29: Aktivierende ökonomische Instrumente in Deutschland

\begin{tabular}{|c|c|}
\hline Pauschal & Einkommensabhängig \\
\hline $\begin{array}{c}\text { Einrichtung eines Zukunftskontos für Kinder } \\
\text { Einführung des Betreuungsgeldes (abhängig von der } \\
\text { definitiven Formgebung des Instrumentes) }\end{array}$ & $\begin{array}{c}\text { Anpassung des Kinderfreibetrags, } \\
\text { Einführung des Betreuungsfreibetrags, } \\
\text { Einführung des Kinderzuschlags }\end{array}$ \\
\hline
\end{tabular}

\subsubsection{Analyse der ökonomischen Instrumente in den Niederlanden}

In der niederländischen Familienpolitik hat es in der untersuchten Periode eine Vielzahl ökonomischer Maßnahmen gegeben. Nicht in allen Fällen waren es Maßnahmen, die zu einer kritiklosen Erhöhung der Leistungen für alle Familien führten. So wurde z.B. durch die erste Violette Regierung das Kindergeld für jedes erste Kind zwar erhöht, die Höhe des Kindergeldes für jedes dritte und weitere Kind ist aber nicht mehr höher als für zweite Kinder und orientiert es sich am Alter der Kinder. Auch im Bereich der Witwenrenten kam es zu Sparmaßnahmen, die Aktivierungselemente enthielten, da sie nicht mehr prinzipiell die Ehe belohnten. 
Nachdem die zweite Violette Regierungsperiode keine nennenswerten ökonomischen Maßnahmen traf, kam es während der ersten Koalition unter Balkenende zur Einführung der Lebenslaufregelung, welche auf dem Prinzip einer finanziellen Unterstützung struktureller Sparmaßnahmen des Arbeitnehmers und einer finanziellen Unterstützung von staatlicher Seite aufbaute. Um die Kaufkraft von Familien zu verstärken, hat die Regierung die einkommensbedingte Ermäßigung für Kinder eingeführt. Im Zusammenhang mit der Krankenversicherungsreform kam es zur Bildung einer einkommensbedingten Pflegezulage neben dem Reichsbeitrag für die Prämie der Krankheitskosten für Kinder. Armutsrisiken sollten hierdurch verringert werden.

Auch während der zweiten Regierung unter Balkenende wurde der eingeschlagene Weg der Stimulierung der Arbeitsmarktteilnahme möglichst großer Teile der Bevölkerung durch den Ausbau der einkommensabhängigen Regelungen konsequent weiter gegangen. Die bereits bestehende Steuerermäßigung für Familien mit Kindern wurde einkommensabhängiger und die Teilnahme am Arbeitsmarkt hierdurch lohnender. Außerdem bekommen der weniger verdienende Teil des Elternpaars und alleinstehende Eltern seitdem eine extra Steuerermäßigung: die ergänzende Kombinationsermäßigung. Gemeinden werden seit dem Jahr 2004 finanziell unterstützt, um die dort sesshaften Risikofamilien vor sozialer Exklusion und Armut zu bewahren. Eines der Grundprinzipien des Leitbildes Aktivierender Staat ist die Kopplung des Rechts auf Arbeitslosengeld an Reintegrationsmaßnahmen. In den Niederlanden wurde diese Kopplung während der ersten Violetten Regierung vorgenommen. Da diese Reintegration nicht immer so problemlos wie geplant verlief, wollte die zweite Regierung unter Balkenende es Eltern ermöglichen, im Falle der Pflege eines pflegebedürftigen Kindes bzw. eines pflegebedürftigen Partners, eine temporäre Befreiung von dieser Verpflichtung zu erhalten.

Den Trend zur Förderung der Arbeitsmarktteilnahme durch den Ausbau der einkommensabhängigen Regelungen hat die vierte Regierung unter Balkenende fortgesetzt. Vor diesem Hintergrund beschloss sie den Abbau der Übertragbarkeit der allgemeinen Abgabenermäßigung, welche vor allem die Hausfrauenehe förderte. Nur für sehr junge Familien mit Kindern blieb die Übertragbarkeit der Abgabenermäßigung erhalten. Außerdem kam es zur 
Einführung der einkommensabhängigen Arbeitsermäßigung, die die bestehende Arbeitsermäßigung ersetzt und der Unterstützung der Arbeitsmarktteilnahme von Geringverdienern dient. Wenn beide Elternteile arbeiten, wird dieser Betrag durch eine einkommensabhängige Kombinationsermäßigung ergänzt. Wie schon zur Zeit der zweiten Regierung unter Balkenende stand die Bewerbungspflicht von Sozialhilfeempfängern mit Kindern zur Diskussion. Umgeformt wurde diese Pflicht dann auch von der vierten Regierung unter Balkenende für alleinstehende Sozialhilfeempfänger mit Kindern bis zum Alter von 5 (max. 6) Jahren in eine Schulungspflicht, wodurch es nach der Freistellung von der Bewerbungsverpflichtung für Eltern einfacher sein sollte einen Beruf $\mathrm{zu}$ finden. Auch die Unterstützung der Gemeinden, um es Kindern aus einkommensschwachen Haushalten zu ermöglichen an Sportvereinigungen oder kulturellen Aktivitäten teilzunehmen, wurde intensiviert mit dem Ziel der Armutsbekämpfung und Aktivierung von Kindern aus sozialschwachen Gruppen. Ein besonderes Instrument, das abwich von der Politik der Vorgängerregierungen unter Balkenende, war die Einführung des Kindgebundenen Budgets. Um diese einkommensabhängige Maßnahme schaffen zu können (und hierdurch vor allem Familien mit niedrigerem bis mittlerem Einkommen zu fördern) formte die Regierung die Kinderermäßigung $2008 \mathrm{um}$ in einen Kinderzuschlag, bevor sie ihn 2009 als ein kindgebundenes Budget, also einen Betrag pro Kind auszahlen konnte. Im Jahr 2010 wird das Kindgebundene Budget mit einer Schulkostenerstattung ausgebreitet. Neben dieser Umformung wurde das Instrument für Familien mit mehr als einem Kind im Jahre 2009 pro Kind erhöht. Armutsrisiken von Familien mit einer größeren Anzahl von Kindern wurden hierdurch kleiner und die Grundsicherung des Kindeswohls größer. 
Tabelle 30: Aktivierende ökonomische Instrumente in den Niederlanden

\begin{tabular}{|c|c|}
\hline \multicolumn{1}{|c|}{ Pauschal } & Einkommensabhängig \\
\hline $\begin{array}{c}\text { Umgestaltung des Kindergeldes, } \\
\text { Investition zur Förderung der Teilnahme von Kindern aus } \\
\text { sozialwirtschaftlich schwachen Familien an Sport und } \\
\text { Kultur, } \\
\begin{array}{c}\text { Befreiung von Kindern bis zu 18 Jahren von den Kosten } \\
\text { der Gesundheitsversicherung }\end{array}\end{array}$ & $\begin{array}{c}\text { Einführung der Lebenslaufvergünstigung, } \\
\text { Einführung der einkommensabhängigen } \\
\text { Steuerermäßigung für Familien mit Kindern, } \\
\begin{array}{c}\text { Einführung der ergänzenden Kombinationsermäßigung, } \\
\text { Schrittweise Abschaffung der Möglichkeit zur Übertragung } \\
\text { der allgemeinen Abgabenermäßigung für Eltern, } \\
\text { Einführung einer einkommensabhängigen } \\
\text { Arbeitsermäßigung und einer zusätzlichen }\end{array} \\
\text { einkommensabhängigen Kombinationsermäßigung für } \\
\text { Eltern mit Kindern, }\end{array}$ \\
$\begin{array}{c}\text { Einführung des einkommensabhängigen Kindgebundenen } \\
\text { Budgets, in welchem der Kinderzuschlag aufgeht }\end{array}$ \\
\hline
\end{tabular}

\subsubsection{Analytischer Vergleich der ökonomischen Politikdifferenzen}

Für eine lange Zeit wurde sowohl in Deutschland als auch in den Niederlanden die Hausfrauenehe sehr stark unterstützt. „Es war ein Standardmodell und für die Ausnahmen konnten relativ leicht anfüllende Maßnahmen getroffen werden. Das Kindergeld hielt das Nettoeinkommen großer Familien auf Niveau, die Witwen- und Waisenrechte und die Sozialhilfe sorgten für das Einkommen von Familien ohne Ernährer und über die Steuern wurden zu große Unterschiede der Familieneinkommen kompensiert" (Knijn 2007: 64). Um die verwendeten Instrumente $\mathrm{zu}$ analysieren, werden an dieser Stelle zwei Finanzierungsmöglichkeiten unterschieden. Durch die Pauschale in Form eines Kindergeldes können Mindeststandards für die Kindererziehung gewährleistet werden. Aus Sicht des Kindes bzw. dem Schutz des Kindes vor Armut können Finanzierungspauschalen wichtig sein. Das zweite Finanzierungsprinzip allerdings fördert gesellschaftliche Aktivierung in Form der Arbeitsmarktteilnahme und dem Ziel wirtschaftlicher Selbstständigkeit und Eigenverantwortung möglichst vieler Eltern.

Im Vergleich der beiden Länder fällt auf, dass in den Niederlanden ein familienpolitischer Schwerpunkt im Bereich der ökonomischen Instrumente (im hier definierten Sinne) gesetzt wurde. Auch in Deutschland kam es zur Investierung von erheblichen Mitteln in die Familienpolitik. Diese Mittel sind 
aber zum großen Teil gebunden an die ökologischen Instrumente und an ZeitInstrumente. Im Sinne der hier verwendeten Definition ökonomischer Instrumente hat es in der deutschen Familienpolitik nicht nur wenige Maßnahmen gegeben, sondern entsprachen diese auch eher traditionelleren Unterstützungsmaßnahmen als aktivierenden Förderinstrumenten. $\mathrm{Zu}$ den Ausnahmen zählte z.B. die Steuerreform aus dem Jahr 2000. Hierdurch wurde es möglich Steuerabzüge $\mathrm{zu}$ erhalten für nachgewiesene, erwerbsbedingte Betreuungskosten (siehe auch ökologische Instrumente). Auch der Kinderzuschlag mit dem Chancengleichheit für Kinder aus Haushalten mit einem niedrigen Einkommen gefördert werden soll, kann langfristig zu Aktivierung führen. Inwiefern die Bestrebungen der Schwarz-Gelben Koalition unter Merkel zu mehr Aktivierung führen werden ist noch nicht abschließend $\mathrm{zu}$ beurteilen.

In den Niederlanden wurde das ökonomische Instrument also viel deutlicher im Sinne des Idealmodells Aktivierender Familienpolitik verwendet als in Deutschland. Während die violetten Regierungen vor allem bestehende Regelungen sanierten und pauschale Unterstützungen - wie z.B. das Kindergeld - frühzeitig und sehr konsequent staffelten, haben die Koalitionen unter Balkenende sich vor allem auf den Ausbau der einkommensabhängigen Regelungen konzentriert. Hierdurch wurde eine gemeinsame aktive Teilnahme am Arbeitsmarkt für beide Elternteile lukrativer. Verwendet haben die Politiker eine Vielzahl steuerlicher Instrumente, die Abgaben von Familien entsprechend ihrer Teilnahme am Arbeitsmarkt höher oder niedriger ausfielen ließen. Seit dem vierten Kabinett unter Balkenende gibt es in den Niederlanden das Kindgebundene Budget. Dieses Instrument fördert Familien entsprechend der Anzahl ihrer Kinder und des Haushaltseinkommens. Das Armutsrisiko von Mehrkindfamilien wird hierdurch verringert.

\subsection{2 Ökologische Situation der Familienhaushalte}

Durch die Verwendung verschiedener ökologischer Instrumente können die Wohn- und Lebensumfeldbedingungen von Familien verbessert werden. Bei einem sicheren und angenehmen Lebensumfeld steigt die Chance auf gesellschaftliche Teilnahme. Außerdem kann die Teilnahme von Frauen am Arbeitsmarkt durch eine passende Infrastruktur in Form von 
Kinderbetreuungsplätzen verschiedenster Art gefördert werden (von der Kleinkindbetreuung über die Betreuung in Kindergärten bis zur außerschulischen Betreuung). Um eine Teilnahme am Arbeitsmarkt zu ermöglichen, ist eine reguläre und bezahlbare Kinderbetreuung eine Grundvoraussetzung für viele Frauen. Aus Sicht des Kindeswohls spielt vor allem die Qualität der Betreuung eine wichtige Rolle. Gut ausgebildetes Personal kann die frühkindliche Entwicklung stärken und fördern. Die Verstaatlichung dieser Ausbildungen kann dem Risiko einer frühzeitigen, soziokulturellen Vorsortierung entgegenwirken. Aus der Perspektive des Idealmodells Aktivierender Familienpolitik muss diese Verstaatlichung der Ausbildungen nicht zur Folge haben, dass der Staat sie (vergleichbar mit dem System öffentlicher Schulen) auch selbst anbietet. Wie bereits erläutert, ist es Aufgabe des Staates, um seiner Gewährleistungsverantwortung gerecht $\mathrm{zu}$ werden und für ein qualitativ hochwertiges Angebot zur Kinderbetreuung sorgen, um die Aktivierung der Gesellschaft voran zu treiben. Nicht immer ist das reguläre Angebot zur Kinderbetreuung ausreichend. Im Falle von Krankheit z.B. sind zusätzliche Maßnahmen bzw. Regelungen erforderlich. Auch für die Pflege älterer Menschen sind passende ökologische Bedingungen wichtig. In diesem Zusammenhang ist der Ausbau des gesellschaftlichen Dienstleistungssektors entscheidend.

Die Instrumente in diesem Bereich erstrecken sich insgesamt auf die Situation der Betreuungsmöglichkeiten von Kindern und Schülern, die Bereitstellung von Möglichkeiten zur Erziehungshilfe bzw. zur gesellschaftlichen Teilhabe sowie (im begrenzten Sinne) auf das Wohnumfeld. Nicht aufgenommen sind an dieser Stelle Instrumente zum Schutz von Familienmitgliedern vor Gewalt (Frauenhäuser, Männerhäuser usw.). Für die hier zu untersuchenden Fragestellung sind diese Instrumente nicht relevant.

\subsubsection{Analyse der ökologischen Instrumente in Deutschland}

Nachdem es unter der Vorgängerregierung 1996 zu einem Rechtsanspruch auf einen (halbtägigen) Kindergartenplatz für alle Kinder (die das dritte Lebensjahr vollendet haben) kam, hat es seit der ersten Rot-Grünen Koalition bis zur Großen Koalition unter Merkel in Deutschland einen stetigen Ausbau ökologischer Instrumente gegeben. Hierbei gab es einerseits Anstrengungen 
zum Ausbau der Kinderbetreuungsmöglichkeiten in Kindertagesstätten und Schulen und andererseits zur Unterstützung der Infrastruktur. Beide Kategorien sind durchaus im Sinne des Aktivierenden Modells. Allerdings wurde innerhalb der ersten Kategorie (Ausbau der Betreuung) während der ersten Rot-Grünen Koalition nur sehr zaghaft investiert. Ein Beispiel ist die Einführung des Betreuungsfreibetrages neben dem Kinderfreibetrag innerhalb des Verrechnungssystems des Familienleistungsausgleichs, wodurch eine bessere Teilnahme von Müttern am Arbeitsmarkt ermöglicht werden sollte. Weiterhin gab es Bestrebungen der Bundesregierung, um Länder und Kommunen an ihre Ausführungsverantwortung hinsichtlich des Ausbaus der Kinderbetreuung zu erinnern. Die zweite Kategorie (Verbesserung der Infrastruktur) wurde - in Zusammenarbeit mit den Bundesländern - durch das Investitionsprogramm zur Integration von Zuwanderfamilien stärker gefördert.

Die zweite Rot-Grüne Koalition hat dem quantitativen und qualitativen Ausbau des Betreuungsangebotes vom Kleinkind bis zum Schüler durch das Programm ,Zukunft Bildung und Betreuung' und vor allem durch die Gesetze zum qualitätsorientierten und bedarfsgerechten Ausbau der Tagesbetreuung für Kinder (TAG) und zur Weiterentwicklung der Kinder- und Jugendhilfe (KICK) kräftige Impulse gegeben. Diese wurden im Zusammenhang mit der intensiven gesellschaftlichen Debatte über die demographische Entwicklung Deutschlands getroffen. Die Basis dieses Ausbaus bzw. dieser Weiterentwicklung wurde allerdings bereits 1990/1991 in der grundlegenden Überarbeitung und Neugestaltung des Sozialgesetzbuches Kinder- und Jugendhilfe gelegt. Der Ausbau der Tagesbetreuung kann also als eine Weiterentwicklung dieses Gesetzes gesehen werden. In der zweiten hier unterschiedenen Kategorie, die sich auf infrastrukturelle Maßnahmen des Wohnens richtet, wurden Instrumente wie z.B. der Wettbewerb ,Die Jugend bleibt' und die Stimulierung von Wohneigentum von Familien verwendet.

Die Große Koalition führte den Aktivierungsgedanken im Bereich der ökologischen Instrumente durch die Fortführung des Investitionsprogramms Zukunft, Bildung und Betreuung und den Ausbau der betrieblichen und betrieblich unterstützten Kinderbetreuung als eines der Schwerpunkte der mit den Unternehmen geschlossenen ,Allianz für die Familie' (siehe auch ZeitInstrumente) weiter aus. Außerdem gestaltete sie die Betreuungsinfrastruktur finanziell attraktiver, indem sie Eltern von den Kindergartengebühren im letzten 
Kindergartenjahr befreite und die Betreuungsleistungen für Kinder unter 14 Jahren bis $\mathrm{zu}$ einer bestimmten Höhe steuerlich absetzbar machte. Eine besondere Form der Aktivierung nahm diese Regierung in der zweiten Kategorie vor. Sie stimulierte gesellschaftliches Engagement und gleichzeitig die direkte Förderung von Kindern und Familien durch die Einführung von Mehrgenerationenhäusern und ,Lokalen Bündnissen für Familie'. Hiermit knüpfte sie an die Erfahrungen und Netzwerke der bereits seit vielen Jahren bestehenden Mütterzentren an. Die ,Lokalen Bündnisse für Familie' sind ein besonderes Beispiel für die Konkretisierung von Aktivierung in der hier untersuchten Definition und der Teilung gesellschaftlicher Verantwortung mit einer Ausführungsverantwortung auf lokalem Niveau. Außerdem führte diese Regierung mit der Umsetzung des Kinderförderungsgesetzes den Rechtsanspruch auf einen Betreuungsplatz für jedes Kind ein. Als Reaktion auf die wirtschaftliche Situation im Jahr 2009 investierte die Regierung in das Zukunftsinvestitionsgesetz von dem die Infrastruktur für Kinder und Jugendliche im breiten Sinne profitierte.

Die Schwarz-Gelbe Koalition unter Merkel versprach die Fortführung bzw. Ausführung der durch die Vorgängerregierung versprochenen Infrastrukturmaßnahmen. Sie wolle hierbei vor allem auch auf eine qualitative Verbesserung der Angebote achten. Neuerungen versprach die Regierung in diesem Bereich allerdings keine. 
Tabelle 31: Aktivierende ökologische Instrumente in Deutschland

\begin{tabular}{|c|c|}
\hline Betreuung & Infrastruktur \\
\hline $\begin{array}{c}\begin{array}{c}\text { Einführung des Betreuungsfreibetrages im } \\
\text { Familienlastenausgleich, }\end{array} \\
\text { Durchführung des Programms Zukunft Bildung und } \\
\text { Betreuung, } \\
\text { Verabschiedung des Gesetzes zum qualitätsorientierten } \\
\text { und bedarfsgerechten Ausbau der Tagesbetreuung für } \\
\text { Kinder (TAG), } \\
\text { Verabschiedung des Gesetzes zur Weiterentwicklung de } \\
\text { Kinder- und Jugendhilfe (KICK), } \\
\text { Befreiung der Eltern von der Kindergartengebühr im } \\
\text { letzten Kindergartenjahr, } \\
\text { Steuerliche Absetzbarkeit der Betreuungskosten bis zum } \\
\text { 14. Lebensjahr, } \\
\text { Verabschiedung des Kinderförderungsgesetzes }\end{array}$ & $\begin{array}{l}\text { Durchführung des Investitionsprogramms zur } \\
\text { Verbesserung der Integration von Zuwandererfamilien, } \\
\text { Einführung des Wettbewerbs ,Die Jugend bleibt', } \\
\text { Stimulierung von Wohneigentum für junge Familien, } \\
\text { Einführung von Mehrgenerationenhäusern, } \\
\text { Aufrichtung von ,Lokalen Bündnissen für Familie', } \\
\text { Intensivierung des Zukunftsinvestitionsgesetzes }\end{array}$ \\
\hline
\end{tabular}

\subsubsection{Analyse der ökologischen Instrumente in den Niederlanden}

Genau wie in Deutschland wurden auch in den Niederlanden ökologische Instrumente zum Ausbau der Betreuungseinrichtungen von Kindern und der Infrastruktur von Familien im Sinne des Idealmodells Aktivierender Familienpolitik verwendet. So hat die erste Violette Regierung die Abgabenverminderung für die Kinderbetreuung eingeführt. Arbeitgeber wurden hierdurch finanziell stimuliert, um Kinderbetreuungsplätze für ihre Arbeitnehmer zu mieten. Außerdem unterstützte die Regierung alleinstehende Sozialhilfeempfänger während der Reintegration in den Arbeitsprozess mit einer finanziellen Unterstützung für die Kinderbetreuung. Neben dem Ausbau der Kinderbetreuung wollte die Regierung in der in dieser Arbeit unterschiedenen zweiten Kategorie (Infrastruktur) Eltern durch individuelle Mietzuschüsse für finanzschwache Familien in Form einer Kindertabelle fördern.

Während der zweiten Violetten Regierung wurde das niederländische Prinzip zur Finanzierung der Kinderbetreuung noch deutlicher. Die Regierung förderte den Ausbau und die Inanspruchnahme von Kinderbetreuung durch steuerliche 
Vergünstigungen, die Eltern zur aktiven Teilnahme auf dem Arbeitsmarkt bewegen bzw. Arbeitgeber motivieren sollten, den Mitarbeitern in ihren Tarifverträgen weitere Möglichkeiten zur Kinderbetreuung anzubieten. Im neuen Gesetz ,Basiseinrichtung von Kinderbetreuungsplätzen' legte sie das Ziel der Verdopplung der Betreuungskapazitäten fest, und richtete sich die Regierung dabei auch auf die Betreuung älterer Kinder. Insbesondere unterstützte sie die Betreuung von Kindern in Wohnumgebungen mit einer sozialschwachen Infrastruktur. Ein Schwerpunkt in der zweiten ökologischen Kategorie war der Ausbau der Breiten Schulen. Parallel zum bestehenden Schulhaushalt sind in dieser Einrichtung gesellschaftliche Funktionen angesiedelt, die hauptsächlich von dort angestellten Personen ausgeführt werden. Obwohl diese Einrichtung keinen generationenübergreifenden Anspruch hat, weist sie Parallelen zum deutschen Mehrgenerationenhaus auf.

Nach einer konsequenten Aktivierungspolitik durch ökologische Instrumente während der zwei violetten Koalitionen, kam dieser Aktivierungsdrang zunächst zum Stillstand und wurde er erst wieder nach Beendigung der ersten Koalition, also während der zweiten Regierung unter Balkenende, wieder aufgenommen, z.B. durch die Verabschiedung des Kinderbetreuungsgesetzes. Darin sind die Liberalisierung der Kinderbetreuung und das Prinzip der dreigeteilten Finanzierung (Regierung, Arbeitgeber und Eltern) festgelegt. Auch im Schulbereich hat man die eingeleitete Verantwortungsteilung durch die Dezentralisierung der Schulhaushalte fortgesetzt. In Folge des konsequenten Ausbaus der Betreuungseinrichtungen entstand ein Betreuungsflickenteppich, den die Regierung schlüssiger gestalten wollte. Aus diesem Grund wurden Schulen aufgefordert, zwischen 7.30 und 18.30 Uhr Kinderbetreuung anzubieten. Außerdem wurden Familien von den Schulgeldern in der Sekundarstufe bzw. von den Kosten für 16- und 17-jährige Auszubildende befreit. Durch den weiteren Ausbau der ,Breiten Schulen' wollte die zweite Regierung Balkenende die Infrastruktur stärken. Da sie die Förderung von Kindern ab dem jüngsten Alter - ganz im Sinne des Idealmodells Aktivierender Familienpolitik für ein wichtiges Ziel hielt, bot diese Regierung außerdem eine zentrale Anlaufstelle in jeder Gemeinde bzw. jedem Stadtteil an, in dem Eltern mit Fragen zum Thema Erziehung Hilfe erhielten.

Den Ausbau der Kinderbetreuung setzte auch die vierte Regierung unter Balkenende fort. Aufgrund der stark gestiegenen Inanspruchnahme der 
Kinderbetreuung waren zusätzlich zu den geplanten Investitionen weitere Mittel der Regierung notwendig, und mussten auch die Eltern und die Arbeitgeber eine höhere finanzielle Beihilfe leisten. Außerdem hat die Regierung die Steuerermäßigungen für informelle Kinderbetreuung wieder einschränken müssen. Deutlich aktivierende Akzente setzte diese Regierung im Bezug auf das Wohnumfeld von Familien. Aufgrund der demographischen Veränderungen in den ländlichen Regionen kündigte sie an, das Wohnungsbaugesetz dahingehend $\mathrm{zu}$ verändern, dass es ländlichen Gemeinden ermöglicht wird ausreichend Wohnraum für die eigene Bevölkerung bereit zu stellen. Durch eine gesteigerte Wohnungsbauproduktion in problematischen Stadtvierteln wollte sie eine gleichmäßigere Einkommensverteilung erreichen und soziale Brennpunkte durch Stadterneuerungsmaßnahmen verbessern. Hinsichtlich dieser beiden Aufgaben unterstrich die Regierung insbesondere auch die Ausführungsverantwortung der Wohnungsbaugesellschaften. Weitere Maßnahmen betrafen den Ausbau und der qualitativen Verbesserung der ,Breiten Schulen' und der Zentren für Jugend und Familie. In einem Jugendund Familienmonitor sollten Jugend- und Familienfreundlichkeit von Gemeinden sichtbar gemacht werden.

Tabelle 32: Aktivierende ökologische Instrumente in den Niederlanden

\begin{tabular}{|c|c|}
\hline Betreuung & Infrastruktur \\
\hline $\begin{array}{l}\text { Abgabenverminderung für die Kinderbetreuung, } \\
\text { Unterstützung bei den Betreuungskosten von Kindern } \\
\text { alleinstehender Elternteile die an } \\
\text { Reintegrationsmaßnahmen teilnehmen, } \\
\text { Einführung steuerlicher Vergünstigungen, die eine } \\
\text { Teilnahme am Arbeitsmarkt ermöglichen, } \\
\text { Verabschiedung des Gesetzes ,Basiseinrichtungen } \\
\text { Kinderbetreuungsplätzen, } \\
\text { Verabschiedung des Kinderbetreuungsgesetzes, } \\
\text { Unterstützung informeller Kinderbetreuung durch } \\
\text { steuerliche Vorteile }\end{array}$ & $\begin{array}{c}\begin{array}{c}\text { Einführung der Kindertabelle für individuelle } \\
\text { Mietzuschüsse, }\end{array} \\
\text { Einführung der ,Breiten Schule', } \\
\text { Dezentralisierung der Schulhaushalte, } \\
\text { Kinderbetreuung von } 7.30 \text { Uhr bis } 18.30 \text { Uhr durch } \\
\text { Schulen ermöglicht, } \\
\text { Befreiung von Schulgeldern in der Sekundarstufe bzw. be } \\
16-17 \text { jährigen Auszubildenden, } \\
\text { Einführung der Zentren für Jugend und Familie, } \\
\text { Änderung des Wohnungsbaugesetzes, um in } \\
\text { Landgemeinden mehr Wohnraum zu schaffen, } \\
\text { Verbesserung des Wohnungsangebotes in } \\
\text { problematischen Stadtvierteln, } \\
\text { Durchführung von Stadtverbesserungsmaßnahmen in } \\
\text { problematischen Stadtvierteln, } \\
\text { Einführung des Jugend- und Familienmonitors }\end{array}$ \\
\hline
\end{tabular}




\subsubsection{Analytischer Vergleich der ökologischen Politikdifferenzen}

Zusammenfassend kann man sagen, dass zwar in beiden Ländern sowohl der Ausbau der Kinderbetreuung als auch die Verbesserung der Infrastruktur von Familien im Sinne des Aktivierenden Modells vorangetrieben wurde, sie hierzu allerdings ganz unterschiedliche Herangehensweisen verwendeten. „Die Bereitstellung von Kinderbetreuungseinrichtungen erfolgt in den Niederlanden größtenteils privatwirtschaftlich, wobei die Kommunen ihren eigenen oder gemeinnützigen Einrichtungen finanzielle Mittel zuweisen. Die von den privaten Anbietern erwirtschafteten Überschüsse werden oftmals nicht ausgeschüttet, sondern fließen den Aufgaben ihrer Einrichtungen zu, so dass die Tätigkeit dieser Anbieter als nicht-erwerbswirtschaftlich zu charakterisieren ist“ (OECD 2002: 88). Aus dem Instrumentarium des aktivierenden Modells ist an niederländischer Seite also relativ konsequent zur Privatisierung der Aufgaben gegriffen worden. In Deutschland hingegen hat man beim Ausbau der Kinderbetreuung auf unterschiedliche Modelle gesetzt. Teilweise gab es Privatisierungen, teilweise eher Öffentlich-Private Partnerschaften usw..

Charakteristisch für das niederländische System ist die konsequente Verantwortungsteilung zwischen Staat, Arbeitgebern und Eltern. „An der Finanzierung der Betreuungskosten sind neben dem Staat und den Eltern grundsätzlich auch die Arbeitgeber beteiligt. Rund 65\% der Tarifverträge enthalten entsprechende Klauseln zur Kinderbetreuung. In den Fällen, in denen der Arbeitgeber sich nicht an der Finanzierung beteiligt, werden kommunale Zuschüsse gezahlt“ (OECD 2002: 91). In Deutschland wird der Ausbau der Betreuungseinrichtungen $\mathrm{zu}$ einem verhältnismäßig großen Anteil aus öffentlichen Mitteln bezahlt. Das ist ein Ansatz der eher dem von EspingAndersen als sozialdemokratisch bezeichnetem System als dem Idealmodell der Aktivierenden Familienpolitik entspricht. Charakteristisch für die Niederlande ist die schon frühzeitig eingeführte, konsequente steuerliche Bevorteilung, die Eltern durch die Möglichkeit zum Abzug der Betreuungskosten von den Steuern (bzw. im Fall alleinstehender Sozialhilfeempfänger als Zuschuss) erhalten. Ähnliches wurde in Deutschland zwar auch ansatzweise durch die erste RotGrüne Regierung ins Leben gerufen, dann aber erst von der Großen Koalition erneut aufgegriffen. 
Beim Ausbau der übrigen Infrastruktur für Familien hat Deutschland vor allem durch die Einführung von Mehrgenerationenhäusern und der Einrichtung der ,Lokalen Bündnisse für Familien' sehr im Sinne des Idealmodells Aktivierender Familienpolitik gehandelt. Durch die Mehrgenerationenhäuser wird gesellschaftlicher Zusammenhalt vor Ort und durch verschiedene Generationen stimuliert. Die Lokalen Bündnisse können als Instrument zur Selbsthilfe interpretiert werden. Bei beiden Instrumenten handelt es sich um Sozialinvestitionen, die $\mathrm{zu}$ einer Aktivierung innerhalb des direkten Lebensumfelds von Familien führen können. In den Niederlanden ist in diesem Bereich die Aktivierung weniger konsequent als in anderen Bereichen ausgefallen. Familien wurden durch eine Reihe von ökologischen Instrumente unterstützt. Interessant ist vor allem die Einführung der ,Breiten Schulen', die das Angebot von Schulen um außerschulische Aktivitäten erweiterten. Hervorzuheben ist auch die Dezentralisierung der Schulhaushalte, durch die Schulen ihrer Ausführungsverantwortung besser gerecht werden können. Erwähnenswert sind weiterhin die Bestrebungen, gemeinsam mit den Wohnungsbaugesellschaften in einer Art Öffentlich-Privater Partnerschaft problematische Stadtteile nicht nur $\mathrm{zu}$ verbessern, sondern auch die Bevölkerungszusammenstellung durch gezielten Wohnungsbau zu verändern. Auffallend ist allerdings, dass die Schwarz-Gelbe Regierung unter Merkel den sehr ambitionierten Plänen und Ideen der Vorgängerregierung praktisch keine Neuerungen hinzufügte, sondern vor allem auf den weiteren Ausbau der bereits anwesenden Instrumente verwies.

Inhaltlich aktivierend ist der Auf- und Ausbau der Zentren für Jugend und Familie. Allerdings wählte man in diesem Zusammenhang einen eher aktiven Ansatz mit Ausführungsverantwortung für die Kommunen anstelle eines aktivierenden Modells mit einer breiteren Verantwortungsteilung. Im Gegensatz zum deutschen Beispiel wird in diesem Bereich nicht in Allianzen gearbeitet, vergleichsweise wenig Verwendung von gesellschaftlichen Kräften gemacht und zur Professionalisierung im Sinne klassischer Bürokratie übergegangen. 


\subsubsection{Förderung oder Wiederherstellung von Kompetenzen der Familienangehörigen}

Wie bereits verdeutlicht, geht das Idealmodell Aktivierender Familienpolitik davon aus, dass in der frühen Kindheit soziale Weichen für die kommenden Lebensphasen gestellt werden, die zu einem späteren Zeitpunkt nur noch schwer korrigierbar sind. Die frühkindliche Förderung nimmt dabei einen wichtigen Stellenwert ein. Chancen von Kindern auf eine erfolgreiche Teilnahme in der Gesellschaft und am Arbeitsmarkt sind zum großen Teil von dieser Phase abhängig. Instrumente im Bezug auf die frühkindliche Entwicklung richten sich in erster Linie auf die Stärkung primärer Mechanismen. Durch regelmäßiges Vorlesen z.B. können diese Mechanismen gefördert werden. Wichtig ist auch die frühkindliche Ausbildung außerhalb des Elternhauses. Durch eine für alle Bevölkerungsschichten zugängliche qualitativ hochwertige Form der Betreuung bzw. Ausbildung kann der direkte kognitive Einfluss von Eltern auf Kinder relativiert und frühzeitiger Benachteiligung begegnet werden.

Für ältere Kinder bzw. Jugendliche ist vor allem die weitere Ausbildung und Eingliederung in den Arbeitsmarkt von großer Bedeutung. Wie in der Empirie verdeutlicht, ist der Umgang mit Gewalt und Rechtsradikalismus für viele Jugendliche ein besonderes Problem. Diese Tatsache ist allerdings für die hier $\mathrm{zu}$ bearbeitende Fragestellung nicht relevant und wird an dieser Stelle daher auch nicht bearbeitet.

Die Selbstentwicklung der Eltern ist aus zweierlei Perspektiven interessant. Da die Erziehung von Kindern eine wichtige gesellschaftliche Aufgabe darstellt, die gleichzeitig aber auch einen hohen Einsatz erfordert, will der Staat Eltern für eventuelle Nachteile, die sie gegenüber kinderlosen Menschen haben, kompensieren. Ferner ist für die Entwicklung der Kinder wichtig, dass auch ihre Eltern über relevante Kompetenzen verfügen, denn hierdurch steigen die Aussichten der Folgegeneration auf eine erfolgreiche Teilnahme an Arbeit und Gesellschaft. Bei den Eltern handelt es sich neben klassischen Förderungsmaßnahmen oft um eine Wiederherstellung von Kompetenzen, da sie durch Haushalt und Erziehung eventuell für einige Jahre aus dem Berufsleben ausgeschieden waren bzw. nur in einem sehr geringen Rahmen hieran teilnahmen. Zwei Maßnahmen, die bereits im Rahmen der 
ökonomischen Instrumente genannt wurden, aber auch an dieser Stelle von großer Bedeutung sind, heißen ,Workfare' und ,Sozialinvestition'. Neben dieser beiden eher kurativen Instrumente betont Esping-Andersen, dass vor allem auch präventive Instrumente wie ,lebenslanges Lernen' eine gute Absicherung vor den verschiedenen Lebensrisiken bilden.

\subsubsection{Analyse der Kompetenzen-Instrumente in Deutschland}

Alle deutschen Regierungen verwendeten im untersuchten Zeitraum Instrumente zur Förderung oder Wiederherstellung von Kompetenzen der Familienangehörigen. Schematisch lassen sich die verwendeten Instrumente in drei Kategorien unterteilen, denn in der Regel richteten sich die Maßnahmen dieser Kategorie auf Kinder, Jugendliche und Frauen. Nur in sehr begrenzten Fällen umfasst diese Kategorie auch die Unterstützung von Vätern. War dies der Fall (z.B. im Zusammenhang mit Trennungen bzw. Ehescheidungen) dann richteten sich die Zielsetzungen immer auch gleichzeitig auf die Mütter. Daher sind in der dritten Kategorie (in Klammern) auch die Eltern aufgenommen.

Ganz im Sinne des Aktivierenden Staates setzte die erste Rot-Grüne Regierung einen Schwerpunkt im Bereich der Aktivierung Jugendlicher. Beispiele hierfür sind die Bekämpfung von Jugendarbeitslosigkeit durch das Reformprogramm JUMP, die Ausbildungsoffensive für Jugendlichen sowie das Bündnis für Arbeit, Ausbildung und Wettbewerbsfähigkeit, in dem Aktivitäten und Leitlinien zur Sicherung eines angemessenen Ausbildungsangebotes vereinbart wurden. Um ausländische Jugendliche besser zu berücksichtigen, initiierte die Regierung das Programm ,Entwicklung und Chancen junger Menschen in sozialen Brennpunkten (E\&C)', das sich auf die Integration junger Zuwanderer durch passende Ausbildungsprogramme konzentriert, und richtete sie die ,Koordinierungsstelle Ausbildung in ausländischen Unternehmen' (KAUSA) ein. Akademische Ausbildungen wurden durch eine Reform der Ausbildungsförderung und das Vornehmen zum Umbau der Hochschulen in Zukunftswerkstätten, in denen an kreativen Lösungen für gesellschaftliche Veränderungsprozesse gearbeitet wird, unterstützt. Die Aktivierung von Frauen für den Arbeitsmarkt war dieser Regierung ein Anliegen, das sie nicht nur durch das Programm ,Frau und Beruf (mit verschiedenen Maßnahmen zur Förderung der Arbeitsmarktteilnahme), sondern auch - genau wie die Bekämpfung der 
Jugendarbeitslosigkeit - durch einen europäischen Beschäftigungspakt fördern wollte. Im Bereich der Kinder hat die erste Rot-Grüne Koalition keine nennenswerten aktivierenden Instrumente verwendet. Orientiert hat sich diese Regierung vor allem am allgemeinen Leitbild Aktivierender Staat und dem Ziel einer möglichst großen Arbeitsmarktteilnahme.

Die zweite Rot-Grüne Koalition setzte die Bekämpfung der Jugendarbeitslosigkeit fort, z.B. durch die Verabschiedung des JUMP-PlusProgramms, der Einführung des in regelmäßigen Abständen erscheinenden Bildungsberichts und der Einrichtung der Stiftung Bildung und Erziehung. Um gesellschaftlich erfolgreich aktiv zu sein, ist die Beherrschung der Sprache eine wichtige Voraussetzung, die von der Regierung z.B. durch das Modellprogramm ,Förderung von Kindern und Jugendlichen mit Migrationshintergründen' unterstützt wurde. An dieselbe Zielgruppe richtete sich auch die Kampagne ,SCHAUH HIN! Was deine Kinder machen', zur Förderung eines aufgeklärten Umgangs mit Medien. Dieses Projekt ist eine der wenigen Öffentlich-Privaten Partnerschaften im familienpolitischen Bereich. Im Gegensatz zur ersten Regierung unter Schröder spielten während der zweiten Regierungsphase auch die Erziehung und Frühförderung von Kindern eine stets wichtigere Rolle. Diese Entwicklung fand zum einen Ausdruck im Gesetz zur Weiterentwicklung der Kinder- und Jugendhilfe (KICK), das auf eine bessere Kooperation der am Entwicklungsprozess des Kindes beteiligten Akteure zielt, und zum anderen in der Unterstützung der privaten Erziehung in der gemeinsam mit den großen Kirchen geförderte Initiative ,Verantwortung Erziehung'. Nur wenige Instrumente der zweiten Rot-Grünen Regierung zielten auf die Aktivierung von Frauen. Durch ein konsequentes Gender Mainstreaming und Gender Budgeting wollte die Regierung den Frauenanteil in unterrepräsentierten Berufszweigen fördern.

Das Instrumentarium der Großen Koalition war in dieser Kategorie des Modells Aktivierender Familienpolitik verhältnismäßig gering. Sie führte den Nationalen Pakt für Ausbildung und Fachkräftenachwuchs weiter, der bereits von der RotGrünen Koalition ins Leben gerufen wurde. Im Sinne des Idealmodells Aktivierender Familienpolitik unterstützte sie nicht nur die bereits bestehende ,Initiative Erziehung', sondern gründete sie mit entsprechenden Partnern das ,Bündnis für Erziehung', um ein breiteres soziales Engagement anzuschieben. Zur Förderung der Emanzipation von Mädchen und Frauen initiierte die 
Regierung einen jährlichen ,Girls Day' und den ,Bericht zur Gleichstellung von Frauen und Männern', der ähnlich wie die Familienberichte die Entwicklung der Gleichstellung von Frauen beschreibt und bewertet. Zum Ende ihrer Regierungszeit unterstrich die Koalition die Bedeutung von Gesundheit für die Entwicklungsmöglichkeiten von Kindern. Durch additionale Maßnahmen zur Früherkennung bzw. Prävention von Krankheiten werde Kindern zu einer gesundheitlich verbesserten Basis geholfen.

Die Schwarz-Gelbe Koalition scheint ein besonderes Gewicht auf die Entwicklung von Fähigkeiten bzw. Kompetenzen legen $\mathrm{zu}$ wollen. Chancengleichheit und die Förderung ebendieser wird als Basis verschiedener Instrumente gesehen. Frühkindliche Entwicklung, sprachliche Kenntnisse von Kleinkindern aber auch Chancen im Bereich von Ausbildung und Studium gelte es zu fördern. Hierbei wird das Aktivierungsprinzip besonders deutlich. Vom Kleinkindalter an wird auf Chancengleicheit und Entwicklungsmöglichkeiten gesetzt. An verschiedenen Stellen verweist die neue Regierung auch auf die hierfür notwendige Verantwortungsteilung. Ein konkretes Beispiel hierfür sind die neu zu initiierenden örtlichen Bildungsbündnisse. Inwiefern es der neuen Koalition auch gelingt dieses aktivierende Prinzip umzusetzen ist zum Zeitpunkt des Schreibens noch nicht deutlich.

Tabelle 33: Aktivierende Instrumente zur Förderung oder Wiederherstellung von Kompetenzen von Familienangehörigen in Deutschland

\begin{tabular}{|c|c|}
\hline Kinder & $\begin{array}{l}\text { Verabschiedung des Modellprogramms ,Förderung von Kindern und } \\
\text { Jugendlichen mit Migrationshintergründen', } \\
\text { Durchführung der Kampagne ,SCHAUH HIN! Was deine Kinder machen', } \\
\text { Verabschiedung des Gesetzes zur Weiterentwicklung der Kinder- und } \\
\text { Jugendhilfe (KICK), } \\
\text { Förderung der Initiative ,Verantwortung Erziehung', } \\
\text { Gründung des Bündnis für Erziehung, } \\
\text { Ausführung des Aktionsplans, ,IN FORM‘, } \\
\text { Einführung einer zusätzlichen Früherkennungsuntersuchung, } \\
\text { Ausführung des Aktionsprogramms ,Frühe Hilfen für Eltern und Kinder und } \\
\text { soziale Frühwarnsysteme, } \\
\text { Vorgenommene Gründung einer Bildungspartnerschaft mit örtlichen } \\
\text { Bildungsbündnissen, } \\
\text { Geplante Unterstützung verbindlicher Sprachstandstests und entsprechende } \\
\text { Förderhilfen, } \\
\text { Geplanter Ausbau Früher Hilfen für aktiven Kinderschutz }\end{array}$ \\
\hline & Durchführung des Reformprogramms JUMP, \\
\hline
\end{tabular}




\begin{tabular}{|c|c|}
\hline Jugendliche & $\begin{array}{l}\text { Führung einer Ausbildungsoffensive, } \\
\text { Aufrichtung des Bündnis für Arbeit, Ausbildung und Wettbewerbsfähigkeit, } \\
\text { Initiierung des Programms ,Entwicklung und Chancen junger Menschen in } \\
\text { sozialen Brennpunkten', } \\
\text { Einrichtung der Koordinierungsstelle Ausbildung in ausländischen } \\
\text { Unternehmen (KAUSA), } \\
\text { Durchführung der Reform der Ausbildungsförderung, } \\
\text { Verabschiedung Europäischer Beschäftigungspakt, } \\
\text { Verabschiedung des JUMP-Plus Programms, } \\
\text { Einführung von Bildungsberichten und der Stiftung Bildung und Erziehung, } \\
\text { Verabschiedung des Modellprogramms ,Förderung von Kindern und } \\
\text { Jugendlichen mit Migrationshintergründen', } \\
\text { Durchführung der Kampagne ,SCHAUH HIN! Was deine Kinder machen', } \\
\text { Verabschiedung des Gesetzes zur Weiterentwicklung der Kinder- und } \\
\text { Jugendhilfe (KICK), } \\
\text { Vorgenommene Einführung eines Stipendantenprogramms und } \\
\text { Weiterentwicklung des BAföGs, } \\
\text { Geplante Schaffung von 275.○oo neuen Studienplätzen, } \\
\text { Geplante Verstärkung der Hochbegabtenförderung, } \\
\text { Vorgenommene Initiierung des ,Bologna-Qualitäts- und Mobilitätspaktes, } \\
\text { Geplanter Ausbau der frühen Berufsorientierung in den Schulen }\end{array}$ \\
\hline Frauen (Eltern) & $\begin{array}{l}\text { Verabschiedung des Maßnahmenprogramms ,Frau und Beruf, } \\
\text { Verabschiedung Europäischer Beschäftigungspakt, } \\
\text { Einführung Gender Mainstreaming und Gender Budgeting, } \\
\text { Einführung ,Girls Day', } \\
\text { Einführung des ,Berichts zur Gleichstellung von Frauen und Männern, } \\
\text { Geplanter Ausbau des Aktionsprogramms ,Perspektive Wiedereinstieg‘, } \\
\text { Geplante Einsetzung eines neuen Instruments zur Förderung des } \\
\text { ganzheitlichen Integrationscoachings, } \\
\text { Vorgenommene Durchführung des Programms ,Integration durch Sport‘, } \\
\text { Geplante Initiierung einer Bildungs- und Ausbildungsoffensive für } \\
\text { Migrantinnen }\end{array}$ \\
\hline
\end{tabular}

\subsubsection{Analyse der Kompetenzen-Instrumente in den Niederlanden}

Genau wie auf deutscher Seite wurden auch durch die niederländischen Regierungen im untersuchten Zeitraum Instrumente zur Förderung oder Wiederherstellung von Kompetenzen der Familienangehörigen verwendet. Wie bei der Analyse der deutschen Instrumente ergibt sich in den Niederlanden eine Dreiteilung. Im Unterschied zu Deutschland kommt es allerdings häufiger vor, dass sich die Regierung an die Eltern und nicht nur an die Frauen richtet. 
Aktivierung durch die Förderung von Kompetenzen hat es während der Regierungszeit vor allem für Jugendliche und Kinder gegeben. Die erste Violette Koalition intensivierte den praktischen Berufsunterricht und führte eine aktivierende Arbeitsmarktpolitik mit Jugendarbeitsgarantieplan. Um Schüler besser auf den späteren Arbeitsmarkt vorzubereiten, wurde neben der Profilwahl im Sekundarschulbereich für lernschwache Schüler außerdem der arbeitsmarktgerichtete Lernweg eingeführt. Durch entsprechende Strukturreformen fasste man berufsbildende Schulformen in regionalen Zentren zusammen und allgemeinbildenden Schulen bot man - durch die Dezentralisierung der öffentlichen Verantwortung - eine größere Handlungsfreiheit bei der Wahrnehmung von Unterrichtsaufgaben. Die Aktivierung eigener Kräfte stimulierte die Regierung auch im akademischen Bereich durch eine Neustrukturierung der Studienordnungen und eine Begrenzung der Studienfinanzierung (hier: BAföG-Regelung) auf eine vierjährige Laufzeit. Bei den Kindern richtete diese Regierung ihre aktivierenden Instrumente insbesondere auf zwei Gruppen: Kinder in Pflegefamilien wurden durch individuelle nachfragegesteuerte Präventionsangebote unterstützt und behinderte Kinder sollten durch die Einführung einer schülergebundenen Finanzierung in Primär- und Sekundarstufen besser in ihr reguläres Lebensumfeld integriert werden.

Auch die zweite Regierung Kok setzte einen bildungspolitischen Schwerpunkt. Eine Reduzierung der Klassengrößen und des regulären Schulbesuchs von Kindern ab dem vierten Lebensjahr zielten auf eine bessere Frühförderung ab. Die Chancengleichheit von Kindern aus sozialschwachen Familien wollte die Regierung durch eine Erhöhung der Beihilfe für einkommensschwache Eltern vergrößern. Eventuelle Sprachdefizite, die zu Integrationsproblemen führen könnten, sollten durch passende schulische Programme behoben werden. Mit dem Ziel einer umfassenderen Integration dieser Kinder förderte die Regierung auch den Breitensport. Um Probleme von Menschen frühzeitig zu erkennen und ihnen entgegen zu wirken, wurde von der zweiten Violetten Koalition ein Jugendfürsorgegesetz verabschiedet. Die hierdurch entstandenen Büros für Jugendfürsorge sollen Verbindungen zwischen den Erziehungsstrukturen der Schulen und den professionellen Kräften der Jugendfürsorge und der Jugendgesundheitsfürsorge schaffen. 
Während der ersten Regierungszeit unter Balkenende kam es kaum zur Verwendung aktivierender Instrumente im sinne des Idealmodells Aktivierender Familienpolitik. Eine Ausnahme bildeten die Instrumente im Zusammenhang mit der neuen Gewährleistungsverantwortung im Schulbereich. Um die Qualitätsentwicklung der Schulen und somit die Kompetenzen der Schüler zu fördern, führte die Regierung Rahmenrichtlinien an denen die Schulen sich orientieren müssen, Endbedingungen denen der Kenntnisstand der Schüler nach Beendung der entsprechenden Schulform zu entsprechen hat und das Kerncurriculum (global umschriebene Themenauswahl) für die verschiedenen Schulformen ein.

Während ihre Vorgänger vor allem den Schulbereich in den Mittelpunkt der Reformbestrebungen stellten, richtete die zweite Koalition unter Balkenende sich stark auf die Jugendhilfe. Sie legte hierzu den Grundstein mit der Berufung der Regierungskommission ,Operatie JONG'. Auf deren Rat hin führte die Regierung das Elektronische Kinddossier und den Risikoindex ein Instrumente, die das Teilen von Informationen zwischen den verschiedenen Einrichtungen in der Jugendhilfe nicht nur ermöglichten, sondern die Mitarbeiter auch auffordern, Verantwortung im Sinne einer harmonischen Entwicklung von Kindern zu tragen. Zusätzlich hierzu will die Regierung durch die Einführung der lokalen Zentren für Jugend und Familie Eltern aktivieren und sie bei der Erziehung ihrer Kinder unterstützen (siehe auch ökologische Instrumente). Um bestehende Wartelisten in der Jugendhilfe abzubauen, wurden zusätzliche Investitionen getätigt. Mit dem Beschluss zur Einführung des Elternschaftsplans nach dem Ende ihrer Beziehung wollte die Regierung Eltern motivieren, Verantwortung in der Erziehung besser gerecht zu werden. Auch im Bereich der audiovisuellen Medien wurden Eltern zur Eigenverantwortung aufgerufen und durch die Einführung des Systems zur Selbstregulierung audiovisueller Produkte (Kijkwijzer) aktiviert.

Die starke Betonung der Rolle von Schulen ist auch ein wichtiges Thema während der vierten Regierungsperiode unter Balkenende. Im Vergleich zu den violetten Regierungen und der ersten Regierung unter Balkenende 
dezentralisiert sie allerdings nicht weiterhin die Aufgaben von Schulen, sondern tritt sie ordnend auf, durch z.B. die Einführung von Fixmomenten bzw. Lossystemen ${ }^{39}$, die Abschaffung des ,Fusionsanreizes' und die Finanzierung der Schulbücher im Sekundarunterricht. Erfolgreiche gesellschaftliche Teilnahme ist ohne einen Schulabschluss schwer. Aus diesem Grund legte die Regierung nicht nur ein Stimulierungsprogramm zur Bekämpfung des Schulabbruchs auf, sondern auch eine Lehr- Arbeitsverpflichtung für junge Menschen bis zum Alter von 27 Jahren. Zur Aktivierung der gesellschaftlichen Teilnahme bzw. Aufgabenwahrnehmung von Jugendlichen rief man - ganz im Sinne des Aktivierenden Modells - ein dreimonatiges gesellschaftliches Schulpraktikum ins Leben. Im Jugendfürsorgegesetz, das man während der zweiten Regierung unter Kok verabschiedete, war die Zahlung eines Beitrags in die Pflegeversicherung vorgesehen, wenn Jugendliche über ein eigenes Einkommen verfügen. Diese Vorschrift behinderte die Aktivierung Jugendlicher am Arbeitsmarkt und wurde deshalb während der vierten Koalition unter Balkenende abgeschafft. Durch die Aufrichtung akademischer Werkstätten und die Intensivierung der Instrumente im Breitensport wollte die Regierung Integration und Aktivierung von Kindern mit Migrationshintergrund unterstützen. Ganz im Sinne des Idealmodells Aktivierender Familienpolitik unterstreicht die Regierung die Bedeutung der Entwicklung von Kindern in den ersten Lebensjahren und führte sie eine ausgebreitete Kontrolleuntersuchung von Kindern $4^{\circ}$ ein. Auch das Prinzip ,Eine Familie - Ein Plan' und das Instrument ,Eigene Kraft Konferenz' entsprachen dem Idealmodell. Durch diese Maßnahmen sollten Familien ganzheitlich unterstützt und aktiviert werden, um ihr eigenes Leben selbstständig führen $\mathrm{zu}$ können, anstelle eine permanente Abhängigkeit von Fürsorge $\mathrm{zu}$ entwickeln. In Fällen in denen die herkömmlichen pädagogischen Hilfen nicht greifen, wird seit dieser Zeit konträr zum Prinzip des Modells Aktivierender Familienpolitik - der Jugendliche aus seinem normalen Lebensumfeld geholt und auf einem Kampus erzogen und zum Arbeitsmarkt begleitet. Ganz im Sinne des Idealmodells sind

39 Anstelle einer Zulassungsbeschränkung durch Verwendung eines Numerus Clausus, wurde eine Studienplatz Lotterie verwendet. 40 Hierbei werden, vier Jahre nach ihrer Geburt, die Gesundheit und Entwicklung von Kindern untersucht. 


\section{hingegen die Instrumente Elektronisches Kinddossier und Verweisindex, Kurse zum Umgang mit Konflikten sowie die Teilnahme an Debatten zum Thema Erziehung, mit denen die Regierung Eltern unterstützen wollte.}

Tabelle 34: Aktivierende Instrumente zur Förderung oder Wiederherstellung von Kompetenzen von Familienangehörigen in den Niederlanden

\begin{tabular}{|c|c|}
\hline Kinder & $\begin{array}{l}\text { Einführung eines individuellen Präventionsgebots für Kinder in } \\
\text { Pflegefamilien, } \\
\text { Einführung der lehrlinggebundenen Finanzierung für behinderte Kinder im } \\
\text { Primär- und Sekundarschulbereich, } \\
\text { Bessere Frühförderung durch Reduzierung der Klassenstärken und } \\
\text { Schulbeginn ab dem vierten Lebensjahr, } \\
\text { Erhöhung der Schulbeihilfe für einkommensschwache Eltern, } \\
\text { Einführung intensiver Sprachprogramme, } \\
\text { Förderung des Breitensports, } \\
\text { Einführung des Jugendfürsorgegesetzes und der Büros für Jugendfürsorge, } \\
\text { Einführung der Rahmenrichtlinien, der Endbedingungen und des } \\
\text { Kerncurriculums, } \\
\text { Einführung des Elektronischen Kinddossiers und des Verweisindexes, } \\
\text { Tätigung von extra Investitionen zum Abbau von Wartelisten in der } \\
\text { Jugendhilfe, } \\
\text { Einführung der Zentren für Jugend und Familie, } \\
\text { Einführung ordnender Instrumente im Schulbereich wie z.B. die Einführung } \\
\text { von Fixmomenten bzw. Lossystemen, die Abschaffung des ,Fusionsanreizes' } \\
\text { und die Finanzierung der Schulbücher im Sekundarunterricht, } \\
\text { Einführung der zusätzlichen Vorsorgeuntersuchung bei vierjährigen Kindern }\end{array}$ \\
\hline Jugendliche & $\begin{array}{l}\text { Intensivierung des praktischen Berufsschulunterrichts, } \\
\text { Aktivierende Arbeitsmarktpolitik mit Jugendarbeitsgarantieplan, } \\
\text { Einführung der Profilwahl im Sekundarschulbereich, } \\
\text { Einführung des arbeitsmarktgerichteten Lehrweges, } \\
\text { Zusammenfassung der Berufsbildenden Schulen in regionalen Zentren, } \\
\text { Dezentralisierung der öffentlichen Verantwortung im Schulbereich, } \\
\text { Neustrukturierung akademischer Ausbildungen und BAföG Regelungen, } \\
\text { Einführung des Jugendfürsorgegesetzes und der Büros für Jugendfürsorge, } \\
\text { Einführung der Rahmenrichtlinien, der Endbedingungen und des } \\
\text { Kerncurriculums, } \\
\text { Einführung des Elektronischen Kinddossiers und des Verweisindexes, } \\
\text { Extra Investitionen zum Abbau von Wartelisten in der Jugendhilfe, } \\
\text { Einführung der Zentren für Jugend und Familie, } \\
\text { Einführung ordnender Maßnahmen im Schulbereich wie z.B. die Einführung } \\
\text { von Fixmomenten bzw. Lossystemen, die Abschaffung des ,Fusionsanreizes' } \\
\text { und die Finanzierung der Schulbücher im Sekundarunterricht, }\end{array}$ \\
\hline
\end{tabular}




\begin{tabular}{|l|l|}
\hline & $\begin{array}{l}\text { Stimulierungsprogramm zur Bekämpfung des Schulabbruchs und } \\
\text { Einführung einer Lehr- Arbeitsverpflichtung für junge Menschen bis zum } \\
\text { Alter von 27 Jahren, } \\
\text { Abschaffung des eigenen Pflegebeitrags für die Krankenversicherung im } \\
\text { Rahmen des Jugendfürsorgegesetzes, } \\
\text { Einführung akademischer Arbeitsplätze und Intensivierung des Breitensports, } \\
\text { Einführung der Kampusse zur (Wieder)Erziehung von Jugendlichen }\end{array}$ \\
\hline Frauen/Eltern & $\begin{array}{l}\text { Einführung des Elternschaftsplans, } \\
\text { Einführung des Systems zur Selbstregulierung audiovisueller Produkte } \\
\text { (Kijkwijzer), } \\
\text { Einführung des Prinzips ,Eine Familie - Ein Plan' und ,Eigene Kraft } \\
\text { Konferenzen', } \\
\text { Kursangebot zur Konflikthantierung von Eltern und Möglichkeit zur } \\
\text { Teilnahme an Debatten zum Thema Erziehung }\end{array}$ \\
\hline
\end{tabular}

\subsubsection{Analytischer Vergleich der Politikdifferenzen im Bereich der Förderung oder Wiederherstellung von Kompetenzen der Familienangehörigen}

Zusammenfassend kann man feststellen, dass es in beiden Ländern einen starken Fokus auf die Kompetenzen Jugendlicher gegeben hat. Den zweiten Förderschwerpunkt bilden die Kompetenzen von Kindern. Vor allem in der jüngeren Vergangenheit haben die Regierungen in diesem Bereich verstärkt investiert. Auffallend ist auch der starke bildungspolitische Fokus durch den niederländischen Zentralstaat. Da Bildung in Deutschland Ländersache ist, hat es in den hier verwendeten deutschen Dokumenten nur sehr wenig vergleichbares Material gegeben. Aus den Texten der aktuellen Regierung ergibt sich allerdings eine vorgenommene Kursänderung in diesem Bereich. Stärker als zuvor scheint die Bundesregierung ihrer Verantwortlichkeiten im Bereich Bildung nachkommen $\mathrm{zu}$ wollen bzw. andere verantwortliche Parteien $\mathrm{zu}$ aktivieren, um ihrer Verantwortung gerecht zu werden.

Bei der näheren Analyse der deutschen Politik fällt auf, dass im untersuchten Zeitraum insbesondere die Unterstützung von Jugendlichen - vor allem in Hinblick auf die Chancen am Arbeitsmarkt - eine Zielsetzung war, die durch viele Instrumente unterstützt wurde. Diese Zielvorgabe stand vor allem während der ersten Rot-Grünen Koalition im Mittelpunkt und verlor im Laufe der folgenden Koalitionen an Aktualität. 
Zur Förderung von Kindern im Sinne des Modells Aktivierender Familienpolitik sind die genannten Instrumente der zweiten Rot-Grünen Regierung interessant. Von ihren Vorgängern wurden die Kompetenzen von Kindern nur wenig gefördert, und die Große Koalition baute vor allem aus, was die zweite Regierung Schröder bereits aufbaute. Viele Pläne im Bezug auf die Förderung von Kindern mit dem Ziel einer größeren Chancengleichheit hat die SchwarzGelbe Regierung. Inwiefern es ihr gelingen wird die Zielsetzung im Bereich der Sprachförderung usw. zu erreichen kann allerdings noch nicht beurteilt werden.

Auffallend ist die relativ geringe Bedeutung der Frauenförderung bzw. Förderung von Eltern. Das Modell Aktivierender Familienpolitik fand hier kaum Anwendung.

Insgesamt ist das auf deutscher Seite verwendete Instrumentarium jedoch eindeutig aktivierend. Viele Initiativen werden gemeinsam mit anderen Beteiligten ausgeführt, es kommt sogar zur Bildung von Bündnissen und einer Öffentlich-Privaten Partnerschaft. Abgesehen von der Bildungspolitik hat auf niederländischer Seite ein ähnlicher inhaltlicher Prozess stattgefunden. In der ersten Phase stand die Aktivierung von Jugendlichen im Hinblick auf den Arbeitsmarkt im Mittelpunkt der Reformbestrebungen. Erst danach gewann die frühkindliche Entwicklung stets mehr an politischer Bedeutung. Auffallend ist genau wie an deutscher Seite - der relativ begrenzte Fokus auf die Aktivierung der Kompetenzen von Frauen bzw. Eltern. Teilweise begründet sich dieser Fokus durch die starke Ausrichtung auf diese Zielgruppe in den anderen hier untersuchten Instrumentenbereichen. Anders als in Deutschland ist in den Niederlanden aber ein deutlicher Trend in Bezug auf die gemeinsame Aktivierung der Kompetenzen von Eltern festzustellen. Dieser Trend zeigt sich vor allem bei den Themengebieten Konfliktbeherrschung/Scheidung und Erziehung.

Obwohl die inhaltliche Anwendung der niederländischen Instrumente im Großen und Ganzen dem Idealmodell Aktivierender Familienpolitik entspricht, ist die konkrete Ausgestaltung nur teilweise aktivierend. Die Aktivierung von Schulen ist ein Bereich, in dem man an niederländischer Seite die Eigenverantwortung zunächst extrem stark betonte und letztendlich - durch die Einführung gewisser Regulierungen - wieder ordnender auftrat. Ein aktivierendes Gleichgewicht scheint sich hier noch nicht ergeben zu haben. 
Hinsichtlich der Erziehung wird deutlich stärker auf die gesellschaftlichen Kräfte gesetzt. Die so genannten ,Eigene Kraft Konferenzen' und Erziehungsdebatten sind interessante Beispiele. Im Bereich der Jugendhilfe allerdings wurde - wie bereits festgestellt - relativ bürokratisch vorgegangen. Sowohl die Büros für Jugendfürsorge als auch die Zentren für Jugend und Familie sind in ihrer Konzeption klassische öffentliche Einrichtungen mit professionellen Angestellten und wenig Raum für zivilgesellschaftliche Aktivierung. Mit diesen Organisationen haben die dritte und vierte Regierung unter Balkenende neue Hilfsinstanzen geschaffen bzw. aufgebaut, die nicht auf bürgerschaftliches Engagement zurückgreifen und auch inhaltlich neben den bereits bestehenden Büros für die Jugendpflege nicht aktivierend, sondern eher im aktiven Sinne positioniert sind. Daher entsprechen sie eher dem Leitbild des Aktiven Staates als dem Leitbild des Aktivierenden Staates.

\subsubsection{Zeit-Instrumente}

Zur Wahrnehmung verschiedener gesellschaftlicher Aufgaben wie Arbeit, Betreuung und Pflege oder aber gesellschaftliches Engagement ist eine Balance zwischen den verschiedenen Optionen wichtig. Da diese Balance leicht vom einen oder anderen Faktor (z.B. Beruf bzw. Familie) zerstört wird, kann öffentliche Unterstützung der verschiedenen Familienmitglieder bei der Aktivierung helfen. Gekoppelt an die Möglichkeit, um sich Zeit für Arbeit, Fürsorge und Erziehung oder andere Aktivitäten zu nehmen ist oft die Frage der Finanzierung. Neben dem Recht auf Zeit wird es durch die Finanzierungsmöglichkeiten dieser Form der Beurlaubung oft erst möglich, um diese Zeit auch für den ihr zugeschriebenen Zweck anzuwenden. Entsprechend werden in der folgenden Analyse Zeit- und Finanzierungsaspekte unterschieden.

Da die Familienpolitik in den untersuchten Länder in der Vergangenheit von der Hausfrauenehe ausging, kam es nicht oder nur in sehr begrenztem Rahmen zur Verwendung von Zeit-Instrumenten. Dies hat sich allerdings in beiden Ländern geändert, und vor allem während der hier untersuchten Periode wurden die entsprechenden Weichen dazu gestellt. Aktivierung der Eigenverantwortung im Bezug auf Arbeit, Erziehung und Gesellschaftsteilnahme ist ein grundlegendes Prinzip des Idealmodells Aktivierender Familienpolitik. Um Eltern, bzw. 
Menschen, die eine Familie gründen wollen, die Möglichkeit zur Wahrnehmung der Eigenverantwortung $\mathrm{zu}$ bieten, müssen sie über Zeit bzw. über entsprechende Zeitarrangements verfügen können.

Grundvoraussetzung für die Gründung einer Familie ist nicht nur aus wohlfahrtsstaatlicher Sicht, sondern auch aus Sicht der meisten jungen Europäer eine (mehr oder weniger) erfolgreiche Teilnahme am Arbeitsmarkt. Dem aktivierenden Modell entsprechend, wird im Falle einer Familiengründung von einem doppelten Haushaltseinkommen ausgegangen. Hierbei muss hinzugefügt werden, dass auch der Arbeitsmarktteilnahme Grenzen gesetzt sind. Einige Frauen bzw. Männer wollen nicht am Arbeitsmarkt teilnehmen und einige Menschen können keinen Beruf ausüben. In den meisten Fällen wird allerdings von einer Arbeitsmarktteilnahme ausgegangen. Ab dem Moment der Schwangerschaft bzw. der Geburt, erfährt die Berufstätigkeit Konkurrenz durch das Kind, das Betreuung bzw. Erziehung braucht. Das Idealmodell Aktivierender Familienpolitik geht von einer Erziehung durch alle Elternteile aus. Für den Faktor Arbeit bedeutet dies, dass weniger Zeit zur Verfügung steht und Eltern entweder Urlaub zur Betreuung des Kindes nehmen, in Teilzeit arbeiten, oder den bezahlten Arbeitsmarkt (zeitweise) verlassen, um sich der Erziehung von Kindern (oder anderen gesellschaftlichen Aufgaben) $\mathrm{zu}$ widmen. Unterschiedliche Zeit-Maßnahmen sind hierzu denkbar.

\subsubsection{Analyse der Zeit-Instrumente in Deutschland}

Ganz im Sinne des Aktivierenden Staates setzte die erste Rot-Grüne Regierung nach dem Ende der christlich-liberalen Regierungsperiode unter Kohl die Reform des Bundeserziehungsgesetzes durch. Um für beide Elternteile die Vereinbarkeit von Familie und Beruf $\mathrm{zu}$ verbessern, erhöhte sie die Einkommensgrenzen und Kinderzuschläge für den Bezug von Erziehungsgeld ab dem siebten Monat und ermöglichte es Eltern, den Erziehungsurlaub/die Elternzeit von insgesamt drei Jahren auch gemeinsam zu nehmen. Neben diesem Recht auf Urlaub konnten sie während der Elternzeit auch auf eine Verringerung der Arbeitszeit zugreifen. „Schon unter der christlich-liberalen Koalition wurden in den 199oer Jahren als Reaktion auf kulturelle Veränderungen verstärkt Schritte in Richtung einer Individualisierung der Ansprüche auf familienpolitische Leistungen und damit eine stärkere 
Unabhängigkeit der Mütter von einem männlichen Versorger unternommen (Erziehungszeiten in der Rentenversicherung usf.). Die Rot-Grüne Koalition hat seit 1998 weitere Schritte in Richtung einer 'kulturellen Modernisierung der Versorgerehe' unternommen. Seit dieser Zeit gilt, dass ,Elternzeit' (früher: Erziehungsurlaub) und Erziehungsgeldanspruch mit Teilzeitbeschäftigung von bis zu 30 Stunden verbunden werden können, ein Jahr der Elternzeit bis zum Ende des achten Lebensjahres des Kindes aufgeschoben werden kann, Väter und Mütter gleichzeitig Elternzeit in Anspruch nehmen dürfen und ein Rechtsanspruch auf Teilzeitarbeit in Betrieben mit mindestens 15 Beschäftigen eingeräumt wird. Elternschaft tritt damit einen weiteren Schritt aus der allein privaten Sphäre in den gesellschaftlichen Verantwortungszusammenhang“ (Opielka 2004: 128f). Neben der Reform des Bundeserziehungsgesetzes wollte die erste Rot-Grüne Regierung die aktive Teilnahme von Frauen am Arbeitsmarkt weiterhin unterstützen durch das Aktionsprogramm ,Frau und Beruf (flexiblere Arbeitszeiten, bessere Teilzeit etc.).

Während der zweiten Rot-Grünen Koalition entstand eine Debatte über eine erneute Änderung des Bundeserziehungsgesetzes. An konkreten Instrumenten konnte allerdings nur die Berücksichtigung der Bemessung der Erziehungszeit bei den Beiträgen zur sozialen Pflegeversicherung festgestellt werden. Dieses Instrument beinhaltet zwar keine direkte Form der Aktivierung, verringert allerdings das Armutsrisiko von alten Menschen, vergrößert die gesellschaftlichen Chancen älterer Menschen und belohnt die Erziehung von Kindern finanziell.

Die bereits während der zweiten Rot-Grünen Koalition geführte Debatte über eine Reform des Bundeserziehungsgesetzes konnte von der Großen Koalition umgesetzt werden. Parallel zur Elternzeit wurde das 12-monatige Elterngeld eingeführt. Die Ansprüche auf Teilzeitarbeit und eine maximal dreijährige (unbezahlte) Elternzeit blieben parallel $\mathrm{zu}$ dieser Reform aber erhalten. Eingeführt wurde außerdem ein Pflegeurlaub mit dem Ziel der Vereinbarkeit von Beruf und Pflege. Im Bereich der Zeit-Instrumente hat gesellschaftliche Aktivierung während der Regierungszeit der ersten großen Koalition eine bedeutende Rolle gespielt. Dabei arbeitete man intensiv mit Unternehmen und Kommunen zusammen. Die Offensive ,Familienbewusste Arbeitswelt' appellierte z.B. an die Verantwortung von Führungskräften gegenüber ihren Arbeitnehmern mit Kindern. Das ,Unternehmensprogramm Erfolgsfaktor 
Familie' richtete sich auf die Flexibilität der Lebensläufe und förderte z.B. die Verbesserung des Wiedereinstiegs von Eltern. Sehr deutlich wird das Aktivierungsprinzip auch in der ,Allianz für die Familie', in der gesellschaftliche Partner auf die Verankerung familienorientierter Maßnahmen in betrieblichen Vereinbarungen (z.B. flexible Arbeitszeitmodelle und die Anpassung der Öffnungszeiten von Kindertagesstätten an Arbeitszeiten) zielen. Gemeinsam mit einigen Stiftungen richtete sich die Regierung mit dem Audit berufundfamilie $₫$ und dem Internetportal ,Mittelstand und Familie' insbesondere auf die Familienfreundlichkeit von mittelständischen Betrieben. Da sich die Produktionsprozesse in den vergangenen Jahrzehnten verändert haben, ist es wichtig zu realisieren wie in einigen Sektoren der Einfluss des Berufslebens auf das Privatleben des Arbeitnehmers zugenommen hat. Die Zeitsouveränität von Familien wurde hierdurch begrenzt. Um das Verantwortungsbewusstsein von Betrieben $\mathrm{zu}$ stärken, führte die Regierung dann auch einen Unternehmenswettbewerb durch, mit dem sie Beispiele für Familienfreundlichkeit in der Arbeitswelt aufzeigte.

Die Schwarz-Gelbe Koalition unter Merkel fügte der Politik ihrer Vorgängerregierung bis zum Zeitpunkt des Schreibens nur wenige Neuerungen zu. Eine Verbesserung der Au-Pair Regelung ist aus aktivierender Sicht wenig relevant (da voraussichtlich nur ein sehr begrenzter Teil der Eltern hiervan profitieren kann). Wohl aber kann der Ausbau der Initiative für familienfreundlichere Rahmenbedingungen in der Arbeitswelt zu einer großen Kampagne aus aktivierender Sicht als positiv beurteilt werden. Inwiefern dies auch für die Umgestaltung der Elternzeit und des Elterngeldes gilt ist zum Zeitpunkt des Schreibens nicht ganz deutlich. Prinzipiell ist die Betreuung von sehr kleinen Kindern bzw. Säuglingen durch ihre Eltern aus Sicht des hier verwendeten Idealmodells wichtig. Ob dieses Prinzip unter einer Flexiblisierung der Regelungen leiden wird, kann zu diesem Zeitpunkt noch nicht festgestellt werden. 
Tabelle 35: Aktivierende Zeit-Instrumente in Deutschland

\begin{tabular}{|c|c|}
\hline Zeit & Mittel \\
\hline $\begin{array}{l}\text { Erziehungsurlaub/Elternzeit von insgesamt drei } \\
\text { Jahren kann gemeinsam genommen werden, } \\
\text { Recht auf Teilzeit während Elternzeit, } \\
\text { Durchführung des Aktionsprogramms ,Frau und } \\
\text { Beruf (flexiblere Arbeitszeiten, bessere Teilzeit } \\
\text { etc.), } \\
\text { Einführung des Pflegeurlaubs, } \\
\text { Durchführung der Offensive ,Familienbewusste } \\
\text { Arbeitswelt', } \\
\text { Durchführung des ,Unternehmensprogramms } \\
\text { Erfolgsfaktor Familie', } \\
\text { Initiierung der Allianz für die Familie, } \\
\text { Initiierung des Audits berufundfamilie@, } \\
\text { Initiierung des Internetportals ,Mittelstand und } \\
\text { Familie', } \\
\text { Durchführung des Unternehmenswettbewerbs, } \\
\text { Weiterentwicklung der Initiative für bessere } \\
\text { Rahmenbedingungen für Familien zu einer } \\
\text { großen Kampagne }\end{array}$ & $\begin{array}{l}\text { Erhöhung der Einkommensgrenzen und } \\
\text { Kinderzuschläge für den Bezug von } \\
\text { Erziehungsgeld ab dem siebten Monat, } \\
\text { Berücksichtigung der Erziehungszeit bei den } \\
\text { Beiträgen zur sozialen Pflegeversicherung, } \\
\text { Einführung des 12-monatigen Elterngeldes, } \\
\text { Vorgenommene Einführung des Teilelterngeldes }\end{array}$ \\
\hline
\end{tabular}

\subsubsection{Analyse der Zeit-Instrumente in den Niederlanden}

Die violetten Koalitionen haben die Bedeutung des Faktors Zeit im Zusammenhang mit den Aktivierungsbestrebungen frühzeitig erkannt und entsprechende Instrumente im Arbeitszeitengesetz (mit dem Recht auf Teilzeitarbeit und der Festlegung der Arbeitszeiten) und im Gesetz Arbeit und Pflege (das den Schwangerschafts- und Entbindungsurlaub, den Erziehungsurlaub und einige andere Pflegeurlaube regelt) verankert. Im Zusammenhang mit der weiteren (z.B. finanziellen) Ausgestaltung und Verwendung dieser Gesetze appelliert die Regierung an die Verantwortung der Unternehmen.

In entsprechenden Programmen beschloss die zweite Violette Koalition die Erweiterung der Schulöffnungszeiten und andere Maßnahmen, die junge Familien in ihrem Zeit-Management unterstützen. 
Während der ersten beiden Kabinette unter Balkenende kommt es zur Ankündigung bzw. Einführung der Lebenslaufregelung. Diese Regelung geht von der Aktivierung eigener Verantwortlichkeiten aus, da es ein Sparsystem ist, das Arbeitnehmer dazu motivieren soll, um für mögliche Urlaubszeiten (im Sinne des Gesetzes Arbeit und Pflege) selbst zu sorgen. Durch diese Regelung können Arbeitnehmer einen Teil ihres Bruttolohns sparen. Wenn sie zu einem späteren Zeitpunkt dann ihren (unbezahlten) Erziehungsurlaub aufnehmen wollen, erhalten sie einen größeren steuerlichen Vorteil in Form einer Steuerermäßigung von 50\% des gesetzlichen Mindestlohns. Neben dieser Gesetzesänderung werden von der Regierung Projekte gefördert, die Eltern in der Vereinbarkeit von Familie und Beruf unterstützen sollen. Hervorzuheben ist das Projekt ,Tageseinteilung/ESF-3'. Es zielt auf Aktivierung durch die Ausdehnung der Tagesarbeitsgrenzen von 9 bis 17 Uhr auf 7 bis 19 Uhr.

Auch für die vierte Koalition unter Balkenende spielte das Zeit-Instrument eine besondere Rolle. Bei der Einführung der Maßnahmen achtete man sehr auf die Verantwortungsteilung zwischen Staat, Eltern und Arbeitgeber. So wurde z.B. der Erziehungsurlaub von 13 auf 26 (nicht übertragbare) Wochen pro Partner verlängert. Festgehalten hat die Regierung allerdings an der Finanzierung dieses Erziehungsurlaubs durch den Arbeitgeber bzw. die Eltern selbst. Neu war auch das Recht für selbstständig tätige Schwangere auf einen Schwangerschafts- und Entbindungsurlaub von 16 Wochen. Da die Erwartungen an die Lebenslaufregelung sich nicht erfüllen zu schienen, passte die Regierung die Regelungen an. Lehrrechte für Schulungseinrichtungen wurden an die Regelung gekoppelt, während die Erziehungsurlaubssteuerermäßigung entkoppelt wurde, und es für Eltern seitdem möglich ist, bis zu 50\% des Mindestlohns in Form einer Steuerermäßigung zu empfangen. Um eigene Verantwortung im Bereich Arbeit und Pflege zu übernehmen, ist Flexibilität für Eltern eine wichtige Voraussetzung. Aus diesem Grund überarbeitete die Regierung das Rahmengesetz zur Arbeit und Pflege, bietet sie Eltern eine größere Flexibilität bei der Einrichtung ihrer Pflegeurlaube und flexibilisierte sie die Infrastruktur durch Programme wie den ,Schalter 7-7'. Um Unternehmen und ihre Verbände $\mathrm{zu}$ mehr Verantwortung für Familienfreundlichkeit $\mathrm{zu}$ aktivieren, nahm die Regierung familienfreundliche Arbeitsbedingungen als potentiellen Bestandteil ihrer traditionellen Frühjahrs- und Herbstbesprechungen (mit den Sozialpartnern) auf. 
Tabelle 36: Aktivierende Zeit-Instrumente in den Niederlanden

\begin{tabular}{|c|c|}
\hline Zeit & Mittel \\
\hline $\begin{array}{l}\text { Verabschiedung des Gesetzes Arbeit und Pflege, } \\
\text { Verabschiedung des Arbeitszeitengesetzes, } \\
\text { Unterstützung durch Zeitprogramme, } \\
\text { Durchführung des Projekts } \\
\text {,Tageseinteilung/ESF-3', } \\
\text { Verlängerung des Erziehungsurlaubs von } 13 \text { auf } \\
26 \text { Wochen, } \\
\text { Flexibilisierung des Rahmengesetzes Arbeit und } \\
\text { Pflege, } \\
\text { Durchführung von Programmen zur } \\
\text { Flexibilisierung der Infrastruktur von Eltern wie } \\
\text { z.B. den ,Schalter 7-7', } \\
\text { Aufnahme familienfreundlicher } \\
\text { Arbeitsbedingungen als Bestandteil der } \\
\text { Frühjahrs- und Herbstbesprechungen }\end{array}$ & $\begin{array}{l}\text { Einführung der Lebenslaufregelung, } \\
\text { Einführung des Rechts auf Schwangerschafts- und } \\
\text { Entbindungsurlaub von } 16 \text { Wochen für } \\
\text { Selbstständige, } \\
\text { Kopplung von Lehrrechten für } \\
\text { Schulungseinrichtungen an die } \\
\text { Lebenslaufregelung, } \\
\text { Entkopplung der } \\
\text { Erziehungsurlaubssteuerermäßigung von der } \\
\text { Lebenslaufregelung }\end{array}$ \\
\hline
\end{tabular}

\subsubsection{Analytischer Vergleich der Zeitpolitikdifferenzen}

Wenn man die Zeit-Instrumente dieser beiden Länder vergleicht, kann man zusammenfassend feststellen, dass diese Kategorie in beiden Ländern im Laufe der Jahre stets mehr an Bedeutung gewann. Um Verantwortung für Beruf, Erziehung und übrige gesellschaftliche Aufgaben übernehmen zu können, brauchen Eltern Zeit und Flexibilität ohne allzu große Einkommensverluste. Zeit ist also vor allem auch in Kombination mit finanziellen Mitteln relevant. Dieses Bewusstsein ist inzwischen in den Politiken beider Länder verankert. Unterschiedlich sind allerdings die Aktivierungspfade, die beide Länder eingeschlagen haben. In Deutschland können die Zeit-Rechte von Eltern mit jungen Kindern als hoch bezeichnet werden. Fraglich ist allerdings inwiefern z.B. die dreijährige Erziehungszeit der Aktivierung gesellschaftlicher Potentiale dient. In den Niederlanden ist diese Erziehungszeit deutlich begrenzter. Außerdem wird sie verhältnismäßig wenig bzw. in Teilzeit genutzt. Auch nimmt die deutsche Regierung mit z.B. dem Elterngeld die Ausführungsverantwortung an sich und trägt dabei - vergleichbar mit den sozialdemokratischen, skandinavischen Ländern - hohe finanzielle Lasten. Die niederländische Regierung übernimmt in diesem Bereich nur begrenzte Kosten 
selbst und ruft vor allem Arbeitgeber und Arbeitnehmer auf, um für die Finanzierung von pflegebedingten Urlaubszeiten $\mathrm{zu}$ sparen. Inwiefern dieser Versuch z.B. in Form der Lebenslaufregelung erfolgreich ist, bleibt fraglich. Auf jeden Fall hat die niederländische Regierung durch die Entkopplung der Erziehungsurlaubssteuerermäßigung von der Lebenslaufregelung in diesem Bereich aus aktivierender Sicht einen Schritt zurück gemacht.

Ein interessantes Beispiel im Sinne des Idealmodells Aktivierender Familienpolitik ist die Aktivierung der deutschen Arbeitswelt für das Thema Familienfreundlichkeit. Die deutsche Familienpolitik weist eine Vielzahl von Programmen zur Förderung des Bewusstseins für Familienpolitik auf, die gemeinsam von Kommunen, Unternehmen und Stiftungen getragen werden. Hierbei ist Sprache von einer Sozialinvestition. In den Allianzen für Familien wird das Prinzip der Verantwortungsteilung zwischen der Regierung und den Unternehmen im Land deutlich. In den Niederlanden ist diese Art der Verantwortungsteilung auf freiwilliger Basis nicht oder nur sehr begrenzt zu verzeichnen. Im Gegensatz zu den ökologischen Instrumenten sind hier die Betriebe auch nicht durch eine gesetzliche Regelung zur Mitfinanzierung gezwungen. Die wichtigsten Instrumente der niederländischen Politik zur Kombination von Familie und Beruf sind das Recht auf Teilzeitarbeit und die große Flexibilität, die die Eltern genießen.

\subsection{Zwischenfazit}

Konvergenzen und Divergenzen zwischen den analysierten Zielsetzungen und Instrumente und dem Idealmodell sind Gegenstand der Betrachtungen des Zwischenfazits. Anhand einer diesbezüglichen Diskussion kommt es zur Beantwortung der Frage, an welchen Stellen sich die beiden Länder dem Idealmodell nähern und welche Abweichungen es gibt. Die abschließende Bewertung der Forschungsfragen unter Berücksichtigung der Theorien EspingAndersens, sowie die theoretischen Schlussfolgerungen schließen sich im Fazit dieser Arbeit an.

Wie bereits im theoretischen Kader beschrieben, kennt das Idealmodell Aktivierender Familienpolitik drei Zielsetzungen. Innerhalb dieser Bereiche geht es um eine neue Verantwortungsteilung zwischen Zivilgesellschaft, Markt 
und Staat. Den Zielsetzungen gegenüber stehen vier Instrumente, die sich neben unterschiedlichen inhaltlichen Schwerpunkten der aktivierenden Instrumente Privatisierung, Öffentlich-Private Partnerschaften und Sozialinvestition bedienen.

Tabelle 37: Idealmodell Aktivierender Familienpolitik

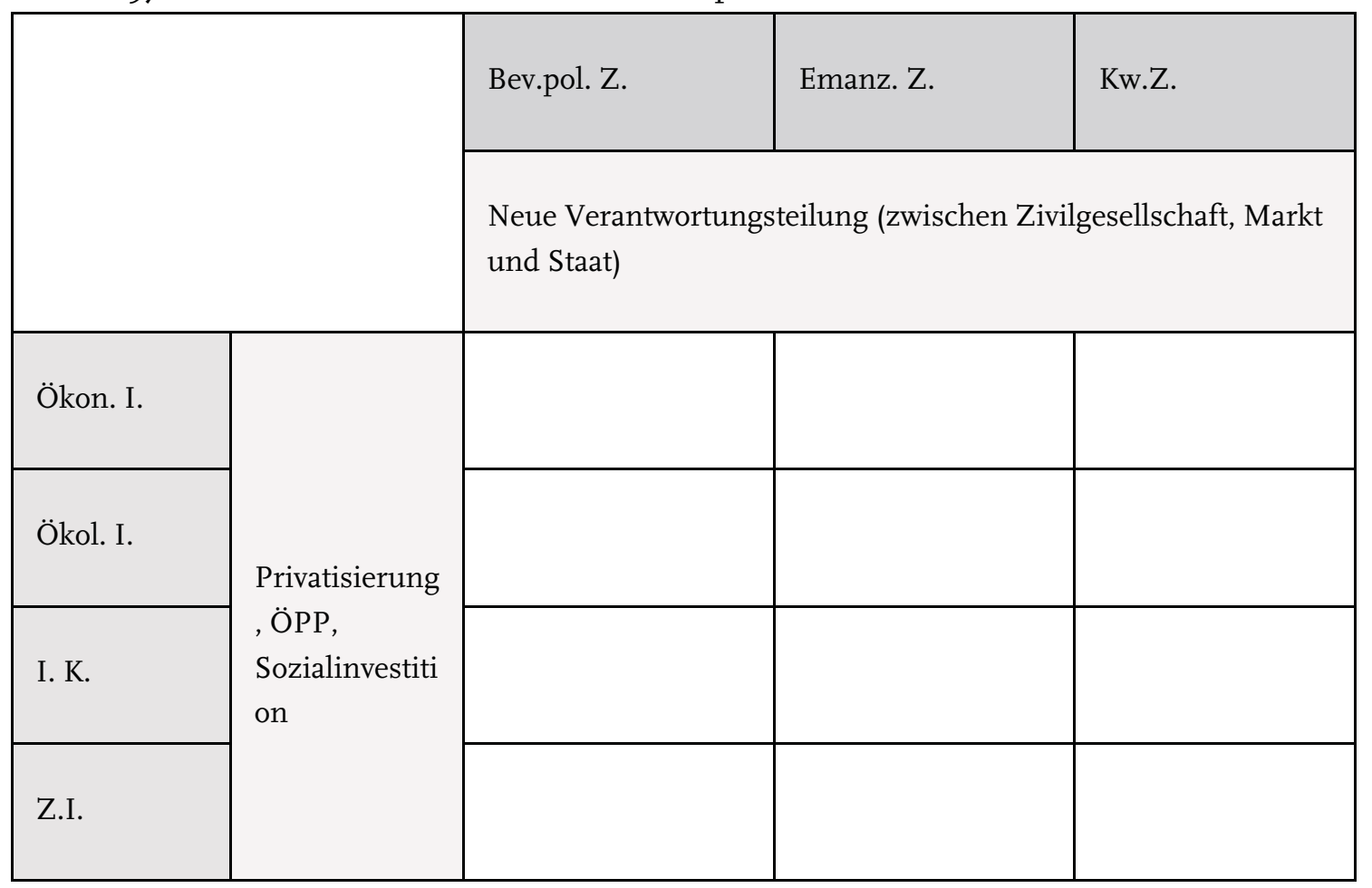

In der vergleichenden Analyse sind alle in den untersuchten Dokumenten vorhandenen Zielsetzungen und Instrumente bearbeitet und die aktivierenden Bestrebungen beider Länder hervorgehoben. In den folgenden zwei Unterkapiteln ist zusammenfassend beschrieben inwiefern die Politiken inhaltlich dem Modell entsprechen, welche aktivierenden Instrumente die Länder verwendeten und welche Gründe es für die politischen Entscheidungen gegeben haben konnte. Nicht aufgenommen ist die Frage inwiefern die Regierungen mit ihren Bestrebungen erfolgreich waren. Dieses wäre eventuell Forschungsmaterial für eine weitere Arbeit zu diesem Thema.

\subsubsection{Zwischenfazit Zielsetzungen}

Nicht alle Zielsetzungen des Idealmodells Aktivierender Familienpolitik ließen sich in den Politiken der beiden Länder finden. Demographische Zielsetzungen - entsprechend der hier verwendeten Definition - waren in beiden Ländern zu 
Beginn der Forschungsperiode kein politisches Thema. Gründe hierfür mag es viele geben. Für Deutschland war es sicherlich die Tabuisierung dieses Themas aufgrund der Erfahrungen in der Nazizeit, denn der demographische Wandel hatte schon lange vor der untersuchten Periode $\mathrm{zu}$ einer extrem niedrigen Geburtsrate geführt. Durch die intensive demographische Debatte, die während der zweiten Rot-Grünen Koalition einsetzte, konnte dieses Tabu gebrochen werden und wurden bevölkerungspolitische Zielsetzungen genannt. Seitdem bemühen sich die deutschen Regierungen um demographische Nachhaltigkeit im Sinne des Idealmodells Aktivierender Familienpolitik. Die Regierungen betonen den starken privaten Charakter dieses Themas und verweisen auf die Verantwortung verschiedener privater Gesellschaftsbereiche. In den Niederlanden wurden - im untersuchten Zeitraum - praktisch keine demographischen Ziele formuliert. Wahrscheinlich sind die im europäischen Vergleich relativ hohe Fertilitätsrate und das schnelle Bevölkerungswachstum in der zweiten Hälfte des zwanzigsten Jahrhundert relevante Gründe hierfür.

Allerdings spielt - genau wie in Deutschland bis zu Beginn der zweiten RotGrünen Regierung - die Tabuisierung dieses Themas eine nicht unwichtige Rolle. Aber auch in den Niederlanden nimmt die Anzahl der älteren Menschen im totalen Bevölkerungsaufbau zu und bekommen Menschen weniger Kinder, als sie ursprünglich beabsichtigten. So wird dieses Thema durch die vierte Koalition unter Balkenende besetzt und $\mathrm{zu}$ reaktivem Handeln aufgerufen (Konsequenzen des demographischen Wandels in gute Bahnen leiten). Einen Aufruf, vergleichbar mit dem der Bundesregierung „mehr Kinder in den Familien und mehr Kinder in der Gesellschaft“ (Bundesregierung 2005a: 17), gab es in den Niederlanden nicht.

Bei den emanzipatorischen Zielsetzungen folgten die beiden Länder einen unterschiedlichen Weg. In den Niederlanden wurde zu Beginn der untersuchten Periode das Ende der Hausfrauenehe (Ministerie van Algemene Zaken 1994: 9) propagiert. Wirtschaftliche Selbstständigkeit von Frauen ist seither ein wichtiges emanzipatorisches Ziel. Zur Erreichung dieser Zielsetzung übernimmt die Regierung Gewährleistungsverantwortung im Sinne des rechtlichen Rahmens, verweist aber ansonsten vor allem auf die Verantwortung der Frauen selbst. Entsprechend des Idealmodells Aktivierender Familienpolitik behandelt sie dieses Ziel seit der vierten Koalition unter Balkenende im Zusammenhang mit den übrigen familienpolitischen Zielsetzungen. In Deutschland hingegen waren 
die emanzipatorischen Zielsetzungen etwas weniger vehement, aber wie in den Niederlanden inhaltlich permanent besetzt. Entsprechend dem Idealmodell wurde Emanzipation - stärker als in den Niederlanden - im untersuchten Zeitraum im Zusammenhang mit familienpolitischen Zielsetzungen behandelt. Der Grund hierfür mag in der Tatsache liegen, dass diese Themenbereiche in Deutschland einem Ministerium unterliegen, während sie in den Niederlanden bis zum vierten Kabinett unter Balkenende zwischen verschiedenen Ressorts aufgeteilt waren. Weniger als in den Niederlanden sah diese Regierung die Verantwortung allerdings als Aufgabe der Regierung und vor allem der Arbeitgeber. In beiden Ländern wurde das Ziel der Emanzipation von Männern bzw. Vätern erst in den jüngeren Dokumenten gefunden. Vor allem die Große Koalition unter Merkel und die vierte Koalition unter Balkenende äußerten sich dazu gleichermaßen deutlich, und ihre Forderung entsprach durchaus dem Idealmodell Aktivierender Familienpolitik. Unterschiedlich allerdings war wiederum die Verantwortungsteilung. In Deutschland sah man die Verantwortung bei der Regierung und den Arbeitgebern, während die niederländische Regierung vor allem die Väter selbst zu aktivieren versuchte.

Wie schon im theoretischen Teil dieser Arbeit beschrieben, hat sich der Fokus auf die Entwicklung des Kindeswohls vor allem in den letzten zwei bis drei Jahrzehnten immer stärker entwickelt. In beiden hier untersuchten Ländern wurde die Zielsetzung durchaus verfolgt, die gesetzten Schwerpunkte waren allerdings sehr unterschiedlich. In Deutschland standen $\mathrm{zu}$ Beginn der untersuchten Periode - entsprechend des allgemeinen Aktivierungsmodells vor allem die Jugend und ihr Anschluss an den Arbeitsmarkt im Zentrum der politischen Aufmerksamkeit. Seit dem Elften Kinder- und Jugendbericht allerdings nahmen die Entwicklung des jungen Kindes und die geteilte Verantwortung - im Sinne des Idealmodells Aktivierender Familienpolitik einen zentralen Platz ein. In den Niederlanden waren die inhaltliche Besetzung des Ziels, sowie die Art der Umsetzung unstetig. Die violetten Koalitionen haben ab Mitte der Neunziger Jahre die Zielsetzungen sowohl inhaltlich als auch in der Umsetzung entsprechend des Idealmodells Aktivierender Familienpolitik verfolgt. In den ersten zwei Koalitionen unter Balkenende lag der Fokus allerdings auf Systemdiskussionen. Dabei wurden unterschiedliche Wege beschritten. Während der Schulbereich weiterhin das Ideal der geteilten Verantwortung mit einer starken Rolle der Schulen selbst verfolgte, wurde im 
Bereich der Jugendhilfe ein eher aktives System mit einer starken öffentlichen Verantwortung (vor allem auf der Ebene der Kommunalverwaltung) und einer eher geringen aktivierenden Wirkung geschaffen. Grund hierfür waren sicherlich Missstände in Familien, denen mehrere Kinder zum Opfer fielen und die den politischen Druck sehr erhöhten.

Zusammenfassend kann man feststellen, dass die Zielsetzungen an deutscher Seite erst später einsetzten als an niederländischer Seite, danach aber inhaltlich verhältnismäßig konsequent im Sinne des Idealbildes Aktivierender Familienpolitik verwendet wurden. Diese Entwicklung hat sich vor allem seit der Publikation des Elften Kinder- und Jugendberichts kontinuierlich fortgesetzt. In den Niederlanden war das demographische Ziel - wie bereits festgestellt praktisch nicht vorhanden. Bei den Zielsetzungen der übrigen Kategorien ist zur Regierungszeit der beiden violetten Koalitionen eine kontinuierliche Weiterentwicklung im Sinne des Aktivierenden Familienpolitischen Idealmodells $\mathrm{zu}$ beobachten. Diese Regierungen besetzten die familienpolitischen Ziele inhaltlich im Sinne des Aktivierenden Idealmodells. Die Zielsetzungen der ersten beiden Regierungen unter Balkenende sind aus Sicht des hier untersuchten Idealmodells allerdings verhältnismäßig unstetig und inkonsequent besetzt. Erst die Politik der vierten Regierung unter Balkenende entspricht wieder stärker dem Idealmodell Aktivierender Familienpolitik und ähnelt in ihrem Ansatz der Großen Koalition in Deutschland.

Die Verantwortung hat man auf deutscher Seite (vor allem in den älteren Dokumenten) insgesamt etwas weniger der Bevölkerung selbst zugesprochen, während in den Niederlanden die Verantwortung beinahe strukturell auf die Gesellschaft übertragen wurde.

\subsubsection{Zwischenfazit Instrumente}

Während die deutsche Politik ihre Zielsetzungen stets mehr im Sinne des Aktivierenden Idealmodells formulierte, gab es bei den Instrumenten einige deutliche Divergenzen. Obwohl auch die deutsche Politik im untersuchten Zeitraum erhebliche Mittel in die Familienpolitik investierte, wurden in der ökonomischen Kategorie - mit einer Ausnahme während der ersten Rot-Grünen 
Koalition - so gut wie keine aktivierenden Instrumente angetroffen. In regelmäßigen Abständen hat allerdings das Bundesverfassungsgericht in die familienpolitische Praxis eingegriffen. „Zu Beginn des dritten Jahrtausends lässt sich ohne Übertreibung sagen, dass alle den FLA (...) bestimmenden Eckwerte wie die Freistellung des sachlichen Existenzminimums sowie des Erziehungsund Betreuungsbedarfs für Kinder von der Besteuerung, die Höhe zu berücksichtigender minimaler Aufwendungen, die Bindung des Gesetzgebers an bestimmte Instrumente (Steuerfreibeträge) sowie die Berücksichtigung von Erziehungsleistungen im Rahmen der Sozialversicherungen vom Bundesverfassungsgericht formuliert wurden" (Gerlach, 2010: 155). Das regelmäßige Eingreifen des Bundesverfassungsgerichts in die Familienpolitik hat unterschiedliche Hintergründe und wird an dieser Stelle nicht näher erörtert. Deutlich ist allerdings, dass es in der Kategorie der ökonomischen Instrumente noch ein erhebliches Aktivierungspotential gibt, denn sowohl das Ehegattensplitting, welches kinderlose Paare gegenüber Familien besser stellt, als auch das Kindergeld sind in ihrer heutigen Einrichtung aus aktivierender Sicht ineffizient gestaltet. In den Niederlanden war die finanzielle Selbstständigkeit der Familien ein wichtiges Ziel der Aktivierungsbestrebungen. Vor allem die Teilnahme beider Partner am Arbeitsmarkt haben die niederländischen Regierungen inhaltlich im Sinne des Aktivierenden Idealmodells gefördert. Auffallend ist, dass die Regierungen hierbei kreative einkommensabhängige und steuerliche Maßnahmen getroffen haben. Diese Form aktivierender Instrumente war ursprünglich nicht im Idealmodell Aktivierender Familienpolitik aufgenommen. Sie ist allerdings durchaus kompatibel mit den Ausgangspunkten des Modells und wird daher im Fazit dieser Arbeit ausführlicher diskutiert.

Der Ausbau der Kinderbetreuung und die Verbesserung der Infrastruktur für Familien wurden von beiden Ländern relativ spät, dafür aber umso konsequenter in Richtung einer aktivierenden Familienpolitik geführt. An niederländischer Seite wird hierbei das Prinzip der geteilten Verantwortung in der Wertschöpfungskette sehr deutlich. Die Gewährleistungsverantwortung durch quantitativen Ausbau der Infrastruktur und allgemeiner Qualitätskontrolle unterliegt dem Staat. Die Finanzierungsverantwortung liegt in gleichen Teilen bei der Regierung, den Eltern und den Arbeitgebern. Die Ausführungsverantwortung ist privatisiert und kann von einer Vielzahl 
formeller und informeller Träger ausgeführt werden. Entsprechend hat der Ausbau der Kinderbetreuung in den Niederlanden in den letzten Jahren in einem so schnellen Tempo stattgefunden, dass die öffentlichen Mittel, die zur Finanzierung vorgesehen waren, letztendlich nicht ausreichten. In Deutschland war der Ausbau der Kinderbetreuung selbst weniger aktivierend als in den Niederlanden. Sowohl die Gewährleistungsverantwortung als auch die Finanzierungsverantwortung lagen hauptsächlich bei der öffentlichen Verwaltung. Für die Ausführungsverantwortung wurde in Deutschland eine Vielzahl von Instrumenten verwendet (Privatisierungen, Öffentlich-Private Partnerschaften usw.).

Der Ausbau der familienpolitischen Infrastruktur in Deutschland entspricht im Großen und Ganzen dem Idealmodell Aktivierender Familienpolitik. Interessante Sozialinvestitionsbeispiele sind die ,Mehrgenerationenhäuser' und die ,Lokalen Bündnisse für Familien'. Durch eine mehrjährige Startinvestition kommt es zur Aktivierung generationenübergreifenden, gesellschaftlichen Engagements im direkten Lebensumfeld der Familien. Die Ausführungsverantwortung liegt bei diesen Beispielen sehr nahe bei den Familien selbst, da die Personalzusammenstellung aus Festangestellten und Ehrenamtlichen besteht. In den Niederlanden wurde dieser Bereich weniger konsequent ausgebaut. „Breite Schulen' entwickelten ein Angebot außerschulischer Aktivitäten, verwendeten aber in geringerem Maße den ehrenamtlichen Einsatz. Die Einrichtung der Zentren für Jugend und Familien entspricht nicht nur inhaltlich weniger dem Aktivierungsgedanken, da hier vor allem problemorientiert gearbeitet wird. Auch die Einrichtung dieser Zentren wurde eher aktiv durch die dominierende Rolle des Staates betrieben. Interessant sind aus der Sicht des Aktivierenden Idealmodells allerdings die Bestrebungen der Regierung, in Öffentlich-Privaten Partnerschaften, gemeinsam mit den großen Wohnungsbaugesellschaften problematische Stadtteile zu verbessern.

In der Kategorie zur Förderung der Kompetenzen von Kindern, Jugendlichen und Frauen bzw. Eltern hat man sich in beiden Ländern zu Beginn der untersuchten Periode im Sinne des allgemeinen Aktivierungsmodells auf die Kompetenzen der Jugendlichen im Hinblick auf den Arbeitsmarkt gerichtet. In beiden Ländern wurde dabei entsprechend dem Idealmodell in gemeinsamer Verantwortung mit den Arbeitgebern und in den Niederlanden auch in 
Zusammenarbeit mit den Schulen vorgegangen. Die Frühförderung von Kindern spielte auf deutscher Seite erst seit der Initiierung verschiedener Maßnahmen der zweiten Rot-Grünen Koalition eine Rolle im Sinne des Aktivierenden Idealmodells. In den Niederlanden gab es diesen Aktivierungsschwerpunkt bereits seit der zweiten Violetten Koalition. Mit Ausnahme der Jugendhilfepolitik wurden auch an niederländischer Seite überwiegend aktivierende Instrumente eingesetzt. Vor allem die Verantwortungsteilung in der Schulpolitik hat sehr konsequent stattgefunden. Die Unterstützung der Kompetenzen von Frauen bzw. Eltern kann in Deutschland als aktivierend bezeichnet werden, war aber - im Verhältnis zu den niederländischen Initiativen in diesem Bereich - eher begrenzt. Aktivierung der Eltern spielte in den Niederlanden eine wichtige Rolle. Ein interessantes Beispiel sind die ,Eigene Kraft Konferenzen', bei denen Eltern in Problemlagen im Rahmen einer begrenzten Sozialinvestition lernen, auf ihr eigenes Umfeld zurückzugreifen, um selbstständig Problemlösungsfähigkeit zu erlernen.

In der letzten Instrumentenkategorie des hier verwendeten Idealmodells entsprechen beide Länder inhaltlich dem Idealmodell. In den Niederlanden wurde Zeitpolitik seit der ersten Violetten Koalition als wichtiges Instrument zur Förderung der Familien betrachtet, während sie in Deutschland vor allem seit dem zwölften Kinder- und Jugendbericht einen hohen Stellenwert erlangte. Um die theoretisch für Eltern bereitstehende Zeit auch nutzbar zu machen, ist finanzielle Unterstützung wichtig. In Deutschland hat bei der Einführung des Elterngeldes der Staat die Finanzierungsverantwortung übernommen, während die niederländische Regierung - durch die Lebenslaufregelung - Eltern zum Sparen aktivieren wollte. Diese Form der Aktivierung entspricht theoretisch dem Idealmodell. Es kam allerdings nicht zu einer breiten Anwendung dieser Regelung in der Bevölkerung. Als Konsequenz wurde die Erziehungsurlaubssteuerermäßigung wieder von der Lebenslaufregelung entkoppelt. Das Problem der Lebenslaufregelung ist nicht so sehr ihr aktivierender Charakter, sondern eher die komplexe Ausführung der Regelung und die geringen Erträge, die der Sparer letztendlich erhält.

Ein interessantes Beispiel im Rahmen einer geteilten Ausführungsverantwortung sind die deutschen Allianzen für die Familien, in denen zur Zusammenarbeit aktiviert wird. „Deshalb scheint es (...) auch ein großer Fortschritt zu sein, wenn z.B. die vom Familienministerium ausgerufene 
,Allianz für die Familie' auch an Unternehmen und Kammern appelliert und die geförderten ,Lokalen Bündnisse für Familien' ganz selbstverständlich neben Elterngruppen, Gewerkschaften u.a. auch Unternehmen als Beteiligte gewinnen wollen. Das Problem bei einer neuen Form der Einbeziehung von Wirtschaftsvertretern in öffentliche Planungsprozesse lässt sich etwa so umschreiben: Wie kann ich mehr Vertreter der Wirtschaft beteiligen, ohne gleichzeitig nicht-wirtschaftliche Entscheidungskriterien abzuwerten? Fazit: Aktivierung der Zivilgesellschaft bedeutet auch, die Wirtschaft nicht länger als einen von öffentlichen Angelegenheiten besser fernzuhaltenden Privatsektor zu betrachten, sondern angemessene (...) Formen zu finden, sie einzubeziehen nicht nur im Rahmen der traditionellen Abstimmung mit Kammern und Verbandsvertretern, sondern im direkten Kontakt mit dem einzelnen Unternehmen als potentiellen ,Corporate Citizen'“ (Evers 2004: 8f). Ein Äquivalent zu dieser Zusammenarbeit in Allianzen gibt es in den Niederlanden nicht.

\subsubsection{Von der Analyse zum Fazit}

Zusammenfassend muss festgestellt werden, dass die deutsche Politik im Sinne der definierten Zielsetzungen inhaltlich mehr den Zielsetzungen des Aktivierenden Idealmodells entspricht. Im Hinblick auf die Verantwortungsteilung sind die Instrumente allerdings nicht in allen Bereichen gleichermaßen aktivierend. Vor allem die Kategorie ökonomischer Instrumente, die Arbeit lohnend macht und Eltern eine stabile Teilnahme am Arbeitsmarkt ermöglicht, wurde in den Niederlanden konsequenter aktivierend eingerichtet. Bei den ökologischen Instrumenten ist das niederländische System zum Ausbau der Kinderbetreuung als aktivierendes Element hervorzuheben, während in Deutschland die Bereitstellung der übrigen Infrastruktur dem Idealmodell Aktivierender Familienpolitik entspricht. Maßnahmen zur Wiederherstellung oder Förderung von Kompetenzen sind in beiden Ländern für die Zielgruppen Jugendliche und Kinder vorhanden. In den Niederlanden werden auch die Kompetenzen der Eltern stark gefördert. Deutsche Maßnahmen konnten in diesem Bereich nur begrenzt festgestellt werden. Zeit-Instrumente wurden in beiden Ländern eingesetzt. Beim Elterngeld trägt die deutsche Regierung die Finanzierungsverantwortung, während die niederländische Regierung Eltern 
aktivieren will, um für zusätzliche Urlaubszeiten selbst zu sparen. In der Praxis muss man feststellen, dass vor allem Teilzeitarbeit ein von den Niederländern stark favorisiertes Instrument ist. In Allianzen wird an deutscher Seite gemeinsame Verantwortung getragen, um die Zeiteinteilung von Familien zu verbessern.

Obwohl nicht alle Ziele und Instrumente der deutschen und niederländischen Familienpolitik analysiert werden konnten, wurden dennoch die unterschiedlichen Aktivierungsbestrebungen der beiden Länder bzw. die Konvergenzen und Divergenzen zum Idealmodell Aktivierender Familienpolitik deutlich. Unklar ist allerdings noch, welche Auswirkungen die politische Praxis im Sinne der im theoretischen Rahmen dieser Arbeit bearbeiteten Theorien hatte. Welchen wohlfahrtstaatlichen Weg haben diese beiden Länder beschritten? Welche Rolle spielten die betreffenden Politiker hierbei und inwiefern kann Aktivierung für die Familienpolitik als ein Ideal gelten? Diese und andere theoretische Fragen werden im Fazit dieser Arbeit abschließend beantwortet. Dabei wird auch verdeutlicht, zu welchen Fragen es noch weiteren Forschungsbedarf gibt. 


\section{$6 \quad$ Fazit}

\subsection{Aufgreifen der Fragestellungen}

Im Zentrum dieser Untersuchung steht die Frage nach der Art der Umsetzung des politisch-administrativen Leitbildes Aktivierender Staat in der Familienpolitik Deutschlands und der Niederlande. In der vergleichenden Analyse wurde eine Vielzahl aktivierender, familienpolitischer Zielsetzungen und Instrumente Deutschlands und der Niederlande auf Mikroniveau bearbeitet. Hinsichtlich der Zielsetzungen sind die Konvergenzen und Divergenzen innerhalb der drei unterschiedenen Kategorien - unter Verwendung des Begriffs Verantwortungsteilung - herausgearbeitet und bei den Instrumenten unter Verwendung des Prinzips der Koproduktion in Form von Privatisierungen, Öffentlich-Privater Zusammenarbeit und Sozialinvestitionen konkretisiert.

Das Leitbild des Aktivierenden Staates war in den letzten zwei Jahrzehnten Thema vieler politischer Grundsatzdiskussionen und politik- bzw. verwaltungswissenschaftlicher Forschungsarbeiten. Das Leitbild wurde dabei vor allem im Rahmen der Arbeitsmarktpolitik thematisiert und in deutschen Diskussionen schnell mit den so genannten Hartz-Reformen in Verbindung gebracht. Aber das Leitbild Aktivierender Staat hatte einen sehr viel weiterreichenden Einfluss, als vielleicht auf den ersten Blick sichtbar wurde. Nicht nur bei den Arbeitsmarktreformen, sondern auch in anderen sozialpolitischen Bereichen wie Gesundheit und Familie war und ist das Aktivierungsprinzip Grundsatz vieler Reformen. Die Analyse dieser Arbeit verdeutlicht, wie die Aktivierungsbestrebungen zweier Regierungen in politisches Handeln umgesetzt wurden und welche Konvergenzen und Divergenzen dabei auftraten. Auf diesen Erkenntnissen aufbauend, werden im ersten Teil des Fazits theoretische Schlussfolgerungen im Hinblick auf das Idealmodell und mögliche Lücken, Defizite oder zusätzlichen Forschungsbedarf gezogen. Der mittlere Teil des Fazits besteht aus der Schlussbetrachtung der Grundprinzipien des Wohlfahrtsstaatsansatzes von Esping-Andersen im 
Zusammenhang mit den Faktoren politisch-administrative Kultur und Handlungsakteure. Anhand der erzielten Analyseresultate und ergänzender Literatur in diesem Forschungsbereich werden Lücken bzw. Erweiterungsvorschläge des Idealmodells und der Theorien von EspingAndersen diskutiert, um die Umsetzung des Leitbildes Aktivierender Staat im familienpolitischen Bereich zu beurteilen. Im letzten Teil des Fazits kommt es dann zur abschließenden Bearbeitung der Frage, inwiefern der Aktivierende Staat richtungsweisend für ein europäisches Sozialmodell ist, und wie die mögliche Weiterentwicklung des Leitbildes aussehen könnte.

\subsection{Weiterentwicklung des Idealmodells Aktivierender Familienpolitik}

Zur Basis des Idealmodells Aktivierender Familienpolitik gehören die familienpolitischen Ansätze Esping-Andersens, die familienwissenschaftlichen Prinzipien Kaufmanns und die Definitionen, die Bandemer im Bezug auf das Leitbild Aktivierender Staat entwickelte. Festgelegt wurde in diesem Modell, dass nicht nur die Zielsetzungen und Instrumente des Modells, sondern auch die Art der politischen Umsetzung und die Verteilung von Verantwortung für die Aktivierung entscheidend sind. „Denn es geht sowohl darum, den ,Dritten Sektor' zwischen Markt, Staat und Individuen als auch den einzelnen aktiven Bürger zu stärken, der in vielfältiger Hinsicht seine eigenen Angelegenheiten selbst regelt, aber auch an gesellschaftlichen Problemlösungen mitwirkt und auf diese Weise die Rolle eines ,Koproduzenten’ übernimmt“ (Olk 2000: 114).

\subsubsection{Inhaltliche Bewertung der Zielsetzungen}

Zum Idealmodell Aktivierender Familienpolitik gehören bevölkerungspolitische und emanzipatorische Zielsetzungen sowie die auf das Kindeswohl gerichteten politisch-administrativen Ziele. Bei den bevölkerungspolitischen Zielsetzungen geht es um das Ziel einer nachhaltigen Bevölkerungsentwicklung. Eine Alterung der Gesellschaft kann zwar einerseits durch den Umbau der sozialen Sicherungssysteme (Rentenversicherung, Krankenversicherung usw.) aufgefangen werden, andererseits erfordert demographische Nachhaltigkeit auf 
Zeit und Dauer eine ausreichende Anzahl von Geburten. Die zweite Zielsetzung Aktivierender Familienpolitik richtet sich auf die Gleichstellung von Frauen und Männern in Beruf und Familie. Durch die wirtschaftliche Selbstständigkeit beider Elternteile bzw. von Doppelverdienerhaushalten verkleinert sich das Armutsrisiko von Familien. Außerdem steigt die Anzahl der Beschäftigten, wodurch das System der sozialen Sicherheit entlastet wird. Die dritte Zielsetzung richtet sich auf das Wohlbefinden von Kindern und Jugendlichen. Vor allem in den ersten Lebensjahren kann nicht nur möglichen Entwicklungsschwierigkeiten von Kindern sondern auch Armutsrisiken im späteren Leben entgegen gewirkt werden. Wie sich aus den Prinzipien des Aktivierenden Staates ergibt, ist neben der inhaltlichen Definition aller Zielsetzungen entscheidend, in welcher Form die Verantwortungsteilung bzw. Aktivierung stattfindet.

\subsubsection{Bevölkerungspolitische Zielsetzungen}

Demographische Entwicklungen sind von vielen Faktoren abhängig, und es gibt derzeit keinen wissenschaftlichen Konsens bezüglich der Möglichkeiten zur Beeinflussung dieser Entwicklungen. Schon relativ einfach erscheinende demographische Prognosen hängen von vielen schwer vorhersehbaren Faktoren ab. Deshalb wird in der Demographie oftmals mit Szenarien gearbeitet. Dennoch - oder vielleicht gerade deshalb - ist es wichtig, den zahlenmäßigen Aufbau der Gesellschaft, also eine der wichtigsten Randbedingungen politischen Handelns auch als politisches Ziel in Betracht $\mathrm{zu}$ ziehen (vergl. Wetenschappelijke Raad voor het Regeringsbeleid 2006: 120f). Falls es zu wenig junge Menschen bzw. zu viele ältere Menschen gibt, kann es aus aktivierender bzw. wohlfahrtsstaatlicher Sicht wichtig sein, diesem demographischen Ungleichgewicht entgegen zu steuern. Es wurde daher zu Recht als Ziel in das Idealmodell Aktivierender Familienpolitik aufgenommen.

Unter die demographische Definition dieser Arbeit fallen nur die ,natürlichen Bevölkerungsbewegungen'41. Diese Eingrenzung wurde aus praktischen 
Gründen und aufgrund der Tatsache vorgenommen, dass Esping-Andersen in den hier verwendeten Werken vor allem über natürliche Bevölkerungsentwicklungen geschrieben hat. Allerdings sind neben der natürlichen Bevölkerungsentwicklung auch die Migrationsbewegungen bzw. die Anzahl der Menschen mit Migrationshintergrund und die Art ihres Hintergrundes äußerst relevant. Die Aktivierung von Eltern und Familien mit nicht deutschem oder niederländischem Hintergrund kann durchaus andere Anforderungen an den Staat stellen, ist aber für die Zukunft des Wohlfahrtsstaates sehr interessant und müsste daher im Rahmen des Idealmodells Aktivierender Familienpolitik weiter erforscht werden.

\subsubsection{Emanzipatorische Zielsetzungen}

Die Emanzipation von Frauen ist die zweite Zielsetzung des Idealmodells Aktivierender Familienpolitik. Emanzipationspolitik steht aus Sicht EspingAndersens gemeinsam mit anderen sozialpolitischen Bereichen in direktem Zusammenhang mit der Familienpolitik. Wirtschaftliche Selbstständigkeit wird nicht nur als persönliches Ziel für Frauen, sondern auch als ein wichtiges Ziel zur Armutsbekämpfung in Familien gesehen. Außerdem kann die Steigerung der weiblichen Arbeitsmarktteilnahme zu wirtschaftlichem Wachstum führen. Eine gute Vereinbarkeit von Familie und Beruf kann also sowohl individuell als auch gesamtgesellschaftlich positiv bewertet werden. „Hinter der Aktivierungspolitik verbirgt sich eine doppelte Strategie: Zunahme der Beherrschbarkeit der versorgungsstaatlichen Regelungen und die Förderung der Arbeitsmarktteilnahme als Weg zur Emanzipation“ (Wetenschappelijke Raad voor het Regeringsbeleid 2006: 160). Wichtig zum Gelingen ebendieser Vereinbarkeit ist die Rolle der Väter in Pflege und Erziehung. Je besser die Ausbildung der Frauen und je höher ihre Chancen am Arbeitsmarkt und die Notwendigkeit eines weiteren Familieneinkommens, desto wichtiger ist die Unterstützung der Mütter durch die Väter. Esping-Andersen schreibt in diesem Zusammenhang auch von der „Verhandlungsmacht der Frauen“ (vergl. EspingAndersen 2009: 46) im Hinblick auf die Aufgabenverteilung der Eltern in Familie und Beruf.

Im Idealmodell Aktivierender Familienpolitik wird Emanzipation - ähnlich wie bei Esping-Andersen - als Verteilung von Aufgaben in Familie und Beruf 
zwischen den Partnern und einer eventuellen dritten Person/Institution (außerhäusliche Kinderbetreuung usw.) definiert. Inhaltlich entspricht diese Definition der Wirkungsfunktion des Idealmodells. Die hier verwandte Definition deckt nicht das gesamte Spektrum der Emanzipationspolitik ab, da diese weit mehr beinhaltet als für diese Arbeit relevant war. Ein Großteil der Gleichstellungsthemen wie Gewalt gegen Frauen, Emanzipation gleichgeschlechtlicher Lebensgemeinschaften usw. ist aus emanzipatorischer Sicht von großer Bedeutung. Für den wohlfahrtsstaatlichen Ansatz des Idealmodells Aktivierender Familienpolitik sind diese Themenbereiche allerdings weniger wichtig. Vielleicht wäre eine andere Terminologie der Zielsetzung für die Verwendung im Idealmodell daher passender und könnte man besser den Begriff ,Aufgabenverteilung in Familie und Beruf verwenden. Ein Nachteil wäre allerdings, dass hierdurch die Aufgabenverteilung zwischen beiden Partnern ungenügend verdeutlicht wird, denn Aktivierung ist in diesem Bereich sowohl im Sinne der Arbeitsmarktteilnahme von Frauen als auch im Sinne einer besseren Teilnahme von Vätern an der Betreuung ihrer Kinder wichtig. Diese Ergänzung müsste der Definition hinzugefügt werden.

\subsubsection{Auf das Kindeswohl gerichtete Zielsetzungen}

Die dritte Zielsetzung des Idealmodells richtet sich auf das Kindeswohl. EspingAndersen betont in seinen Arbeiten die besondere Bedeutung der allerersten Lebensphase eines Menschen. Hier werden entscheidende Weichen gestellt für den späteren schulischen Erfolg bzw. der erfolgreichen Teilnahme am Arbeitsmarkt. Die Entwicklung von sehr kleinen Kindern bedarf daher einer besonderen Aufmerksamkeit und entsprechender Sozialinvestitionen. Wenn Gesellschaften erfolgreich in Kinder investieren, können die Armutsrisiken abund die gesellschaftliche Chancengleichheit zunehmen. Hierdurch wird auch die Anzahl derer, die aktiv Verantwortung in Arbeit und Gesellschaft übernehmen können, steigen.

Die untersuchten Länder investieren zunehmend in die frühkindliche Entwicklung von Kindern bzw. ermöglichten Eltern mehr Familienurlaube. Allerdings wurde auch deutlich, dass es - im Zusammenhang mit der Aktivierung in anderen Bereichen - notwendig ist, um auch für ältere Kinder ein gutes Betreuungsangebot anzubieten. Für Jugendliche ist es wichtig, einen 
erfolgreichen Einstieg auf dem Arbeitsmarkt realisieren zu können. Aus diesem Grund investierten die Länder vor allem in wirtschaftlich schwierigen Momenten zunehmend in die Aktivierung von Jugendlichen. Ob diese Zielsetzung eher Bestandteil der Arbeitsmarkt- als der Familienpolitik ist, kann unterschiedlich bewertet werden. Aus wohlfahrtsstaatlicher Sicht kann dabei auch an die demographische Zielsetzung gedacht werden. Für eine zunehmende Zahl von Jugendlichen bzw. jungen Erwachsenen ist die unsichere Lage am Arbeitsmarkt ein wichtiger Grund, um Elternschaft aufzuschieben, bzw. abzustellen. Daher kann man in diesem Zusammenhang argumentieren, dass der Begriff ,Auf das Kindeswohl gerichtete Zielsetzungen' nur begrenzt das beinhaltet, was zur Aktivierung von Kindern und Jugendlichen nötig ist.

Feststeht, dass sowohl in Deutschland als auch in den Niederlanden die qualitative und quantitative Situation der Kindertagesbetreuung einer weiteren Verbesserung bedarf. Diese Zielsetzung wird dann auch in beinahe allen hier untersuchten Dokumenten angeführt bzw. durch entsprechende Instrumente gefördert. Daher ist bzw. war die im Modell verwendete Definition des Ziels in dieser Form richtig. Weiterhin müssen auch Kinder und Jugendliche an den entscheidenden Momenten ihres Lebenslaufs unterstützt werden. Für das Idealmodell wäre daher der Begriff ,Chancengleichheit von Kindern und Jugendlichen' adäquater als der ursprünglich verwendete Begriff. Oder man sollte, so wie im Programm des niederländischen Programmministers für Jugend und Familie von ,Alle(n) Chancen für alle Kinder' sprechen. Diese Formulierung könnte man noch erweitern in: ,Alle Chancen für alle Kinder und Jugendliche‘.

\subsubsection{Inhaltliche Bewertung der Instrumente}

Das Idealmodell Aktivierender Familienpolitik hat vier Instrumentenkategorien. Erstens sind die ökonomischen Instrumente entscheidend. Die Erziehung von Kindern ist gesellschaftlich von großer Bedeutung und daher erhalten Eltern finanzielle Unterstützungen vom Staat. Zur zweiten Kategorie (ökologische Instrumente) gehören finanzierbare Formen der Kinderbetreuung und die Anwesenheit familienfreundlichen Wohnraums. Die dritte Gruppe der Instrumente bilden Maßnahmen, die zur Förderung bzw. Wiederherstellung der Kompetenzen von Familienangehörigen dienen. Dazu gehören die 
Förderung von Kindern und Jugendlichen ebenso wie die Förderung von Frauen und Eltern. Zur vierten Kategorie - der Gruppe der Zeit-Instrumente - gehören beispielsweise die Rechte auf Familienurlaube, die Möglichkeit zur Teilzeitarbeit und die flexible Einrichtung der Arbeitszeit.

Für die in dieser Arbeit untersuchten Zielsetzungen war die Einbindung des Begriffs Verantwortungsteilung ein wichtiges Prinzip. Bei den Maßnahmen kann dieser Ausgangspunkt noch einen Schritt weiter entwickelt, bzw. konkretisiert werden und spricht Bandemer (Bandemer 2005: 31f) von Koproduktion. Bei der Beurteilung der Instrumentengruppen wird daher auch beurteilt wer ein zur Koproduktion geeigneter Partner wäre.

\subsubsection{1 Ökonomische Instrumente}

Familien unterhalten unterschiedliche Formen finanzieller Unterstützung. Dabei kann man zwischen direkter finanzieller Unterstützung und finanziellen Maßnahmen zur Förderung von ökologischen bzw. Zeit-Instrumenten unterscheiden. In dieser Arbeit sind Finanzierungsmaßnahmen, die an ökologische bzw. Zeit- Instrumente gebunden sind in den Kategorien Ökologie bzw. Zeit und nicht in der Kategorie der ökonomischen Instrumente untergebracht. Zur Gruppe der ökonomischen Instrumente gehören hier vor allem finanzielle Leistungen wie beispielsweise das Kindergeld oder Maßnahmen, die eine aktive Teilnahme am Arbeitsmarkt fördern (in vielen Fällen steuerliche Maßnahmen).

In der Analyse wurde deutlich, dass die Niederlande die traditionell stark geförderte ,Versorgerehe' schon während der violetten Koalitionen beenden wollten. Ökonomische Instrumente wurden in den Niederlanden seitdem in zunehmendem Maße einkommensabhängig gestaltet. Selbst beim Kindgebundenen Budget ist nicht nur die Anzahl der Kinder, sondern auch die Höhe des Haushaltseinkommens für die Höhe des Förderbetrages entscheidend. Die Trendwende vom Schutz der Versorgerehe zur Aktivierung beider Partner kann im Vergleich zur deutschen Situation als verhältnismäßig konsequent bezeichnet werden. Auch in Deutschland wird der Doppelverdienerhaushalt zunehmend gefördert durch den entsprechenden Ausbau der ökologischen und Zeit-Instrumente. Parallel wurden aber auch die Familienarbeit bzw. das freiwillige Engagement unterstützt. Opielka schreibt zu 
der Bewertung der bezahlten und unbezahlten Arbeit: „Während die Diskussion zur Frage, ob die Familienarbeit als gesellschaftliche und damit auch sozialpolitisch anzuerkennende Arbeit gelten soll, recht weit gediehen ist (...) erscheint die sozial- und arbeitsmarktpolitische Reflexion des freiwilligen Engagements im internationalen Vergleich ambivalent (Anheier/Toepler 2002). So wird im liberalen und konservativen Wohlfahrtsregime eher der semi-private sowie der bürgerschaftlich-republikanische Aspekt gemeinschaftlichen Engagements betont (Deutscher Bundestag 2002), in sozialdemokratischen und garantistischen Regime-Diskursen (bspw. In internationalen Organisationen; Anheier u.a. 2003) hingegen darüber nachgedacht, ob das zunächst nicht bezahlte Engagement entweder doch an den Arbeitsmarkt angekoppelt werden kann (Stecker 2002) oder durch ein Grundeinkommen quasi pauschal ermöglicht werden soll (Offe 2000; Rifkin 2004)“ (Opielka, 2004: 69). Während sich die Niederlande eher dem sozialdemokratischen Modell angenähert haben, wurden in Deutschland sowohl der konservative als auch der sozialdemokratische Weg eingeschlagen. Aus Sicht des Idealmodells Aktivierender Familienpolitik sind Instrumente die zur Aktivierung einer bestimmten Bevölkerungsgruppe beitragen förderungswürdig. Eine einkommensabhängige Gestaltung ist für aktivierende, ökonomische Instrumente also eine entscheidende Voraussetzung.

An der Koproduktion sind im Bereich der ökonomischen Instrumente einerseits die Eltern beteiligt (z.B. durch eine aktive und flexible Teilnahme der Partner am Arbeitsmarkt) und andererseits die Arbeitgeber, die ihre Arbeitnehmer durch entsprechende Zusatzleistungen zur Arbeitsmarktteilnahme bewegen können.

\subsubsection{2 Ökologische Instrumente}

Innerhalb der Kategorie der ökologischen Instrumente wurden sowohl die Infrastruktur zur Kinderbetreuung als auch die Wohn- und Freizeitmöglichkeiten von Familien behandelt. Um sowohl auf dem Arbeitsmarkt als auch gesellschaftlich aktiv sein zu können, ist es für Eltern wichtig, Zugang $\mathrm{zu}$ qualitativ hochwertigen und dem Alter der Kinder angemessenen Betreuungsmöglichkeiten und Freizeitangeboten zu haben.

Für ein flächendeckendes Betreuungsnetz und Freizeitangebot, das den unterschiedlichen Ansprüchen der Eltern gerecht wird, formen die regionalen 
Unterschiede einen wichtigen Ausgangspunkt. Ein Flächenland wie Niedersachsen hat andere Rahmenbedingungen als beispielsweise der Stadtstaat Berlin. Die Verwaltungskompetenzen sind entsprechend auf verschiedene Verwaltungsebenen verteilt, und Koproduktion ist zur Zielerreichung in dieser Instrumentenkategorie von großer Bedeutung. Hier können Kommunen, Kirchen, Arbeitgeber und andere Parteien eine wichtige Rolle spielen. Auch bei der Bereitstellung geeigneten Wohnraums spielt die Bundes- bzw. Reichsebene eine eher begrenzte Rolle und ist vor allem die Zusammenarbeit mit den Landkreisen, Gemeinden, Wohnungsbaugesellschaften usw. wichtig.

Aus der Analyse wurde deutlich, dass es in beiden Ländern - mehr oder weniger konsequent - zum Ausbau der ökologischen Instrumente gekommen ist. In diesem Zusammenhang wurden unterschiedliche Aktivierungsbeispiele erklärt. Im Prinzip kann der Staat sich in diesem Bereich vor allem auf seine Gewährleistungsverantwortung berufen. In Deutschland ist dieser Ansatz nicht nur bei der Gründung der ,Lokalen Bündnisse für die Familie‘ bzw. dem Aufbau der Mehrgenerationenhäuser, sondern auch beim Ausbau der Kinderbetreuung geltend geworden. Zur Realisierung war man dabei in großem Maße auf gesellschaftliche Kräfte angewiesen. In den Niederlanden gibt es mit dem Ausbau der Breiten Schulen ein ähnliches Projekt, das allerdings etwas weniger Aktivierung erzeugte.

In der Kategorie der ökologischen Instrumente scheinen die Möglichkeiten zur Aktivierung beinahe unbegrenzt. Das Prinzip der Koproduktion kommt in diesem Bereich sehr gut zur Geltung und verspricht auch für die Zukunft noch viel Aktivierungspotential. Das gilt auch für den Bereich des Betreuungsausbaus einerseits und für die Produktion familiengerechten Wohnraums und einer entsprechenden Umgebung mit einer Infrastruktur für größere und kleinere Kinder.

\subsubsection{Förderung oder Wiederherstellung von Kompetenzen der Familienangehörigen}

Um eine aktive Rolle in Familie, Gesellschaft und Arbeit spielen zu können, muss man über entsprechende Grundlagen bzw. Fähigkeiten verfügen. Im Rahmen der Zielsetzungen wurde bereits erläutert, dass die Kleinkindphase für die spätere Entwicklung von Kindern von großer Bedeutung ist. Esping- 
Andersen ruft in seiner Arbeit zu einer verstärkten Investition für Säuglinge und Kleinkinder auf.

In der vorliegenden Arbeit wurde allerdings deutlich, dass zur Aktivierung neben der Stimulierung in der frühkindlichen Phase (I) vor allem der Übergang von der Schule zum Arbeitsmarkt (II) und die Förderung von Müttern, die durch die Erziehung ihrer Kinder die Anbindung an den Arbeitsmarkt verloren haben (III) wichtig sind. Bei den drei beschriebenen Situationen handelt es sich um Übergangsphasen im menschlichen Leben. Im Allgemeinen sollten Kompetenzen natürlich zu jedem Zeitpunkt im Leben gefördert werden. In den Übergangsphasen spielen Unterstützung und Stimulierung allerdings eine besonders große Rolle.

Aus der Analyse geht hervor, dass in beiden Ländern viel in die Kompetenzen der unterschiedlichen Familienmitglieder investiert wurde. Man konzentrierte sich dabei allerdings vor allem auf die Kompetenzen Jugendlicher und weniger auf die Kompetenzen von Frauen. Das gilt vor allem für Deutschland ${ }^{42}$. Lütz schreibt hierzu „Nach wie vor setzen Maßnahmen der (...) Arbeitsmarktpolitik auf Qualifizierung und Weiterbildung der Arbeitnehmer, sind jedoch nicht auf breite Reintegration in den Arbeitsmarkt, etwa durch Subventionierung des Niedriglohnsektors gerichtet (...) Darin unterscheidet sich Deutschland von anderen Wohlfahrtsstaaten des konservativ-kontinentalen Typs wie den Niederlanden, die den Ausbau der Teilzeitbeschäftigung, die gezielte Förderung von Arbeitsplätzen für niedrig qualifizierte Arbeitnehmer und die Rückführung passiver Transferleistungen zugunsten bezahlter Arbeit (welfare for work) in den Mittelpunkt ihrer Arbeitsmarktpolitik der zweiten Hälfte der 9oer Jahre gerückt und damit eine vergleichsweise größere Abkehr vom bisherigen Wohlfahrtsstaatsmodell vollzogen haben (vergl. Hemerijk 2003)“ (Lütz 2004: 30).

42 Eventuell hat diese Schlussfolgerung allerdings mit der Wahl der untersuchten Dokumente zu tun und wurde in anderen Politikfeldern mehr in die Rolle von Frauen investiert. Da hier allerdings vor allem Koalitionsvereinbarungen und Regierungserklärungen analysiert wurden, also Dokumente bei denen alle politischen Themen zum Tragen kommen, kann diese Aussage als relativ valide angesehen werden. 
Auffallend ist die zunehmende Investitionsbereitschaft beider Länder in die frühkindliche Entwicklung. Um Segregation vorzubeugen ist es zukünftig wichtig, diesen Bereich noch weiter auszubauen. Eine interessante Abweichung vom allgemein zunehmenden Trend der Aktivierung formt das niederländische Jugendhilfesystem. Initiativen wie z.B. die ,Operatie JONG' haben zu einer steigenden Reglementierung und kurativen Hilfeleistung in diesem Bereich geführt. Dieser Ansatz entspricht eher dem Leitbild des Aktiven Staates sowie es in den sechziger Jahren galt und hat nur wenig mit der hier untersuchten Form der Aktivierung zu tun. Im letzten der für diese Arbeit verwendeten Dokumente allerdings hat sich eine Trendwende abgezeichnet und wurden auch für das niederländische Jugendhilfesystem Aktivierungselemente eingebaut.

Kompetenzen sind im Zusammenhang mit Aktivierung von großer Bedeutung für alle Bevölkerungsgruppen, die noch nicht - oder im Falle einer beruflichen Auszeit - nicht mehr aktiv in Beruf und Gesellschaft teilnehmen können. Genau wie im Bereich der ökologischen Instrumente, bietet auch diese Kategorie großes Potential zur Koproduktion. Abhängig von der Altersstufe der/des zu Aktivierenden können Kirchen, Schulen und Arbeitgeber dabei eine wichtige Rolle spielen. Für den Staat ist es wichtig, um seiner Gewährleistungsverantwortung - vor allem auch während der hier genannten Übergangsphasen - gerecht zu werden.

\subsubsection{Zeit-Instrumente}

Für die Vereinbarkeit von Beruf, Familie und einer aktiven, gesellschaftlichen Teilnahme ist ein passendes Zeit-Arrangement wichtig. Eltern brauchen zu unterschiedlichen Zeitpunkten im Leben ihrer Kinder mehr oder weniger Betreuungszeit. Flexibilität ist daher ein wichtiges Kriterium im Zusammenhang mit dem Faktor Zeit. Die Reduzierung der Arbeitswochenstunde, um mehr Zeit mit den Kindern verbringen zu können bedeutet natürlich nicht, dass diese Wochenstundenzahl für immer auf einem niedrigen Niveau bleiben muss. In einigen Fällen ist die Anpassung der gearbeiteten Wochenstundenzahl gar nicht nötig und kann die Vereinbarkeit von Familie und Beruf schon durch eine Flexibilisierung der Arbeitszeiten ermöglicht werden, wodurch Eltern ihren beruflichen Tagesrythmus auf ihren Familienrythmus abstimmen können. Obwohl die meisten Frauen und Männer 
die Betreuung der Kinder mit der Teilnahme am Arbeitsmarkt kombinieren wollen, kann es natürlich auch zum Verlassen des Arbeitsmarktes aus familiären Gründen kommen. Zu dem Zeitpunkt an dem der Elternteil wieder auf den Arbeitsmarkt zurückkehren möchte, muss vor allem in die Kompetenzen dieser Mutter oder dieses Vaters investiert werden.

Neben der Flexibilität, um Wochenstunden zu verringern, oder aber die Arbeit teilweise daheim bzw. zu traditionell unüblichen Zeitpunkten ausüben zu können, ist die Unterstützung des Instruments Zeit mit finanziellen Mitteln wichtig. Natürlich braucht dies nicht bei allen Arbeitsreduzierungen der Fall zu sein. Um allerdings gewisse Urlaube (Elternzeit usw.) allen Eltern auch finanziell zu ermöglichen, ist für diese Phase die Kopplung von Zeit und Mitteln relevant. Dazu gibt es verschiedene Möglichkeiten wie unterschiedliche Formen der Beurlaubung (Elternzeit, Zeit für Pflege im Falle kranker Familienangehöriger usw.) aber auch Lebenslaufmodelle in denen Menschen selber sparen für Momente im Leben, in denen sie weniger Wochenstunden oder nicht arbeiten wollen oder können. Bezahlte Urlaubsphasen müssen nicht zu kurz aber auch nicht zu lang sein. Zu kurze Urlaubsphasen können zu Stress und Vereinbarkeitsproblemen und einer erheblichen Reduzierung der Wochenarbeitszeit führen.

Aus dem Vergleich der beiden Länder ergibt sich, dass die Kategorie der ZeitInstrumente in den vergangenen Jahren in beiden Ländern erheblich an Bedeutung gewonnen hat. In Deutschland können die Urlaubszeiten im Vergleich zu den Niederlanden als hoch bezeichnet werden, die Möglichkeiten zur Teilzeitarbeit sind dahingegen begrenzter und die Regelungen sind weniger flexibel. An dieser Stelle besteht für Deutschland noch deutlicher Nachholbedarf. Über die Niederlande schrieb der Wissenschaftliche Rat für Regierungspolitik: "Die Niederlande haben in den letzten Jahren viele Veränderungen an ihrem System der sozialen Sicherheit durchgeführt und sind im internationalen Vergleich keine Ausnahme mehr. Allerdings steht fest, dass innerhalb dieses Systems relativ wenig Akzente auf Versicherungen, die sich auf die Lebensphase mit kleinen Kindern richten, liegen (...) Hierbei kann ergänzt werden, dass die im Jahr 2006 eingeführte Lebenslaufregelung - propagiert, um die Vereinbarkeit von Beruf und Familie zu vereinfachen - (bisher) noch nicht eine Form hat, die als Antwort auf diese Problematik gelten könnte. Dabei gilt auch, dass die Lebenslaufregelung in ihrer heutigen Form vor allem für die 
höheren Einkommensschichten attraktiv ist“ (Wetenschappelijke Raad voor het Regeringsbeleid 2006: 156).

Koproduktion ist auch für die Zeit-Instrumente von großer Bedeutung. Neben dem Staat können auch die Arbeitgeber und die Arbeitnehmer Verantwortung übernehmen. Bei der Bezahlung der Urlaubszeiten sollte die Verantwortung vor allem bei der Regierung liegen. Die Flexibilisierung der Arbeitszeiten und die Anpassung der wöchentlichen Stundenzahl ist allerdings eher Aufgabe der Arbeitgeber. Theoretisch wäre auch die Finanzierungsverantwortung der Familienurlaube durch den Arbeitgeber denkbar (vergleichbar mit der niederländischen Aufgabenverteilung im Bereich der Kinderbetreuung). Für kleine und mittelgroße Betriebe würde diese Anpassung aber voraussichtlich zu erheblichen Finanzierungsproblemen führen. In beiden Ländern gibt es inzwischen Instrumente/Wettbewerbe, die die Einspannungen der Arbeitgeber in diesem Bereich verdeutlichen. Dieses Benchmarking-Instrument ist sehr wichtig. Es zeigt Eltern in welchen Betrieben oder Branchen Arbeitnehmer mit Familien gut unterstützt werden. Hilfreiche Tipps und Beispiele bietet das Instrument außerdem den Betrieben, die ihre Familienfreundlichkeit vergrößern wollen.

\subsubsection{Abschließende Bewertung des Idealmodells}

Aus der Analyse und dem Fazit wurde deutlich, wie groß der Einfluss des Leitbildes Aktivierender Staat auf die Familienpolitik des hier untersuchten Zeitpunkts war. Das verwendete Modell bietet nicht nur für den wissenschaftlichen, sondern auch für den politisch-administrativen Bereich einen hilfreichen Rahmen, der einen Überblick über die diversen familienpolitischen Aktivierungspotenziale verschafft. Bei der Formulierung der Zielgruppen wären, wie bereits festgestellt, einige Anpassungen im Modell hilfreich. So müssten die demographischen Zielsetzungen neben der natürlichen Bevölkerungsentwicklung auch die Migrationsbewegungen und die Anzahl der Familien mit Migrationshintergrund umfassen, um insgesamt von einer nachhaltigen demographischen Entwicklung sprechen zu können. Das hier formulierte Ziel der Emanzipation wäre vielleicht besser als Vereinbarkeit von Familie und Beruf für beide Elternteile zu beschreiben und die Wohlfahrt 
von Kindern könnte man eventuell: ,Alle Chancen für alle Kinder und Jugendlichen' nennen.

Bei der Kategorie der Instrumente gibt es insgesamt weniger Anpassungen als bei den Zielen. Für die ökonomischen Instrumente ist das Kriterium ,Einkommensabhängigkeit' wichtig. Viel Raum für Koproduktion mit einer vor allem gewährleistenden Funktion für den Staat bieten die ökologischen Instrumente und die Instrumente zur Förderung der Kompetenzen der Familienangehörigen. Die Zeit-Instrumente gewinnen durch die steigende Beteiligung der Frauen am Arbeitsmarkt und die demographischen Entwicklungen in den kommenden Jahren vermutlich erheblich an Bedeutung und eine Koproduktion mit den Arbeitgebern ist in diesem Zusammenhang wichtig.

Obwohl dieses Modell wohlfahrtsstaatliche Ausgangspunkte hat, ist es durch den Aktivierungsgedanken und die Frage nach der Art der Verantwortungsteilung und den Möglichkeiten zur Koproduktion vor allem auch für die Arbeit auf dem Mikroniveau geeignet. Einige Aspekte, die vor allem zahlenmäßig kleinere Gruppen betreffen, werden in diesem Modell allerdings vernachlässigt. So hatten beispielsweise die Rechte gleichgeschlechtlicher Familien, vor allem in der niederländischen Politik, einen wichtigen Stellenwert in der hier untersuchten Periode. Sie konnten aber nicht direkt mit dem wohlfahrtsstaatlichen Ansatz des Modells in Zusammenhang gebracht werden.

Es ist nicht das Ziel dieser Arbeit, zu beurteilen welches der beiden Länder die erfolgreichste Familienpolitik führte. Generell kann allerdings festgestellt werden, dass die Effizienz staatlichen Handelns durch Aktivierung vergrößert werden kann, da sie zu einem höheren Nutzen gesellschaftlichen Potentials führt. Die Leistungen für Familien nehmen zu, ohne dass der Staat (oder der Steuerzahler) für die komplette Finanzierung dieser Leistungen verantwortlich ist. Wichtig ist dabei, auf eine günstige Zusammenstellung (bzw. einen Mix) der Instrumente und ihrer konkreten bzw. technischen Einrichtung zu achten.

Aber nicht nur die Instrumente, sondern auch die hier unterschiedenen Zielsetzungen werden für beide Länder in den kommenden Jahren eine große Herausforderung darstellen. Für die Niederlande, die bisher den demographischen Zielbereich nicht oder nur sehr dürftig besetzt haben, ist hier ein Umdenken notwendig. Dies gilt bezüglich der Integration von Migranten 
und der verhältnismäßig schnell verändernden natürlichen Bevölkerungsentwicklung einiger Provinzen.

Insgesamt kann festgestellt werden, dass sowohl die Ziele als auch die Instrumente des Aktivierenden Staates übereinstimmen mit den familienpolitischen Ausgangspunkten, und es in beiden Ländern noch erhebliches Aktivierungspotential gibt. Die Weiterentwicklung des Leitbildes ist Thema des letzten Unterkapitels dieser Arbeit.

\subsection{Erfahrungen aktivierender Familienpolitik im Zusammenhang mit dem Wohlfahrtsstaatsansatz von Esping-Andersen}

\subsubsection{Aufgreifen des Wohlfahrtsstaatsansatzes}

Der Wohlfahrtsstaatsansatz ist nicht nur im Zusammenhang mit dem Idealmodell Aktivierender Familienpolitik interessant sondern hat sowohl in der Wissenschaft als auch in der nationalen und internationalen Politik eine richtungweisende Rolle in der Diskussion über die Entwicklung der europäischen Wohlfahrtsstaaten gespielt. Esping-Andersen basiert seine Theorien zum Großteil auf umfangreichen quantitativen Erhebungen und unterscheidet zwischen konservativen, liberalen und sozialdemokratischen Wohlfahrtsregimen. Obwohl keines dieser drei Regime problemlos sei, bezeichnet er das sozialdemokratische Modell als vorbildhaft im Zusammenhang mit den heutigen Transformationen europäischer Wohlfahrtsstaaten. Dieser Typus kommt dem von ihm favorisierten sozialen Investitionsstaat am nächsten.

Wie bereits in den Ausführungen im zweiten Kapitel dieser Arbeit erläutert, argumentiert Esping-Andersen weiterhin, dass sich Staaten aufgrund ihrer geschichtlichen Entwicklung, bzw. ihrer institutionellen Einbettung entsprechend ihres eigenen Pfades bzw. ihrer eigenen Vergangenheit weiterentwickeln. Für den Weg in die wohlfahrtsstaatliche Zukunft ist das politisch-administrative Erbe also mitentscheidend. Esping-Andersens sozialstaatlicher Ansatz brachte ihm viel Anerkennung, wurde aber auch oft 
kritisiert. Im Laufe der Zeit hat der Autor seine allgemein-wohlfahrtstaatlichen Theorien um einen emanzipatorischen bzw. familienpolitischen Schwerpunkt ergänzt, da er Entscheidungen in diesem Bereich maßgeblich im Hinblick auf die breitere wohlfahrtstaatliche Weiterentwicklung hält.

Während Esping-Andersen zum größten Teil quantitativ und auf Makroniveau arbeitet, steht in diesem Dissertationsvorhaben die qualitative Bearbeitung der Politik zweier Länder auf Mikroniveau im Mittelpunkt. Hierdurch kann seine Forschung inhaltlich ergänzt werden. Im Folgenden werden einige grundlegende Themen seiner Arbeit - Pfadabhängigkeit, DeKommodifizierung und De-Familialisierung - aufgegriffen, hinterfragt und weiterentwickelt. Neben dieser Themenbereiche Esping-Andersens wurde aus der Politikanalyse die Relevanz einiger anderer Faktoren deutlich. Daher werden die Themen Esping-Andersens im Folgenden um die Frage nach der Bedeutung der politisch-administrativen Kultur eines Landes und der verantwortlichen Akteure bzw. Politiker im Zusammenhang mit dem verwendeten Leitbild erweitert.

\subsubsection{Zur Weiterentwicklung der Themen Pfadabhängigkeit und De- Kommodifizierung}

Ein wichtiges Kriterium zur Definition der von Esping-Andersen unterschiedenen Regimetypen ist der Grad der De-Kommodifizierung. Gemeint ist hiermit die Unabhängigkeit privater Wohlfahrt vom Funktionieren des Marktes. Am höchsten ist der Grad der De-Kommodifizierung im sozialdemokratischen Regime. Hier fallen dem Staat verhältnismäßig viele private Aufgaben zu, während im konservativen Regime die Familie und im liberalen Regime der Markt eine größere Rolle spielen. Dementsprechend sind auch der Zugang zu den Einkommensersatzmöglichkeiten und die Absicherung sozialer Grundrisiken in den verschiedenen Ländern eingerichtet. EspingAndersen schreibt hierzu „Unsere Studien dokumentieren die fortgeführte Dominanz national institutionalisierter Traditionen. Das zeigt sich in zwei wichtigen Tatsachen. Erstens, während sich die westlichen Nachkriegsstaaten beinahe vergleichbare Ziele setzten, unterschieden sie sich im Grad ihres Ehrgeizes und in der Art ihrer Ausführung. Zweitens passen sich dieselben Wohlfahrtsstaaten heute den neuen Situationen auf sehr unterschiedliche Weise 
an. Ein wichtiger Grund hierfür sind die institutionellen Vermächtnisse, die in den Systemeigenschaften eingebaut sind, und die gefestigten Interessen, die diese kultivieren“ (vergl. Esping-Andersen 1996: 6). De-Kommodifizierung und Pfadabhängigkeit werden in diesem Paragraphen zusammenhängend betrachtet.

Esping-Andersen ordnete die Familienpolitik Deutschlands und der Niederlande dem konservativen Regime zu. Grund für diese Einteilung waren die Ergebnisse quantitativer Sozialsystemvergleiche anhand von Datenanalysen aus den achtziger Jahren. Wie bereits verdeutlicht, hat sich in der Familienpolitik beider Länder inzwischen vieles verändert. Man investierte zum Großteil entsprechend der Instrumente des Idealmodells Aktivierender Familienpolitik, die dem Ideal eines sozialen Investitionsstaates von Esping-Andersen sehr nahe kommen. In den Niederlanden hat sich vor allem die Violette Regierung, die von der Abschaffung der Hausfrauenehe sprach und das dazugehörige Instrumentarium dementsprechend veränderte, gegen den konservativen Pfad gewandt. In der Politikgestaltung zur Arbeitsmarktteilnahme von Frauen hat man sich am sozialdemokratischen System orientiert, während Instrumente aus anderen Bereichen (z.B. Kinderbetreuung) einen durchaus liberalen Charakter haben. Auch Deutschland verfolgte eine doppelte Strategie. Einerseits wurde das Instrumentarium des konservativen Regimes beibehalten bzw. ausgebaut. Andererseits kam es mehr und mehr zum Aufbau eines Instrumentariums (z.B. Elternzeit) das dem sozialdemokratischen Wohlfahrtsstaat entspricht. Kann man zusammenfassend also feststellen dass in Deutschland die DeKommodifizierung zugenommen hat, da sich die Politik in einigen Bereichen (z.B. Elternurlaube und Ausbau der Kinderbetreuung) am sozialdemokratischen Modell orientierte? Und hat in den Niederlanden der Grad der DeKommodifizierung abgenommen, da man die Marktabhängigkeit von Familien durch die Vielzahl der einkommensabhängigen Instrumente erhöhte?

Anhand der Instrumentenanalyse wurde sichtbar, dass beide Länder inzwischen weniger deutlich dem traditionell konservativen familienpolitischen Regime zuzuordnen sind als z.B. noch in den achtziger Jahren. Auch muss man hinzufügen, dass weder Deutschland noch die Niederlande (komplett) dem sozialen Investitionsmodell bzw. dem Idealmodell Aktivierender Familienpolitik entsprechen. Bezüglich der Regimetypologie scheint die deutsche Familienpolitik der vergangenen 15 Jahre sozialdemokratisch-konservativ und 
die niederländische sozialdemokratisch-liberal zu sein. In beiden Ländern hat also die De-Kommodifizierung durch die Orientierung am sozialdemokratischen Regime hinzugewonnen. Allerdings hat es parallel hierzu in der niederländischen Politik Liberalisierungsbestrebungen gegeben. Daher kann man basierend auf dieser Arbeit nicht abschließend beurteilen, ob die DeKommodifizierung in den Niederlanden letztendlich zunahm, abnahm oder gleich blieb. Fest steht allerdings, dass beide Länder heute als hybride Regime bezeichnet werden können. Dementsprechend hat der Wissenschaftliche Rat für die Regierungspolitik im Jahr 2006 die Schlussfolgerung gezogen, dass es sinnvoller erscheint, die Niederlande nicht „so wie es jetzt oft geschieht, zu kritisieren, weil es ein hybrides Land ist (...) sondern als ein Land mit einer eigenen Geschichte und Entwicklung zu sehen“ (Wetenschappelijke Raad voor het Regeringsbeleid 2006: 263).

Gauthier hat eine familienpolitische Typologie entwickelt, die man mit dem Modell Esping-Andersens verbinden kann. Sie unterscheidet vier Familienpolitikmodelle. „Das pro-natale Modell sieht die Unterstützung von Familien konsequent als Aufgabe staatlicher Intervention aus einem bevölkerungspolitischen Motiv heraus. Großzügige Leistungen bei Mutterschaft und eine hohe Anzahl an Kinderbetreuungseinrichtungen dienen der Reduzierung jener Hürden, die der Vereinbarkeit von Beruf und Familie im Wege stehen. Das pro-traditionelle Modell betont den Erhalt der traditionellen ,Versorgerehe' und investiert finanzielle Mittel in diese (...) Das pro-egalitäre Modell verfolgt das Ziel, die Gleichheit zwischen den Geschlechtern stärker zu fördern. Die Regierung übernimmt die Verantwortung, Frauen die Vereinbarkeit von Beruf und Familie zu erleichtern. Väter werden dazu ermutigt, bei der Erziehung ihrer Kinder eine größere Rolle zu übernehmen. Abschließend betont das nicht-intervenierende Modell die begrenzte Verantwortlichkeit des Staates. Die Partizipation der Frauen am Arbeitsmarkt wird zwar nicht gebremst, jedoch bietet der Staat erwerbstätigen Frauen nur beschränkte Unterstützung. Finanzielle Transfers für Familien werden nur im geringen Umfang gewährt, das Angebot staatlicher Kinderbetreuungseinrichtungen ist marginal bzw. ihre Bereitstellung fällt aus der Verantwortung des Staates" (Schmidt 2006: 86f). Diese vier Muster überträgt Gauthier auf die Typologie Esping-Andersens. Sie ordnet das pronatale und pro-traditionelle Modell dem konservativen Regime zu, das egalitäre 
dem sozialdemokratischen und das nicht-intervenierende dem liberalen Regime. Hierauf aufbauend ordnet sie Deutschland und die Niederlande dem protraditionellen Bereich zu. Durch ihre Typologie fügt sie - ähnlich wie das Idealmodell Aktivierender Familienpolitik - den Regimetypen, die sich hauptsächlich aus der Instrumentenanalyse ergeben, bewusst festgesetzte Zielsetzungen hinzu.

Aus der Analyse der familienpolitischen Zielsetzungen beider Länder wurde deutlich, dass auch Gauthiers Kategorisierung nicht mehr ganz treffend ist. Obwohl Deutschland sich sicherlich auch am skandinavischen Modell orientierte, fällt es in ihrer Kategorie (spätestens) seit der ersten Regierung unter Merkel eher in die pro-natale Kategorie. Auch die Niederlande fallen seit der Ankündigung des Endes der Hausfrauenehe und dem konsequenten Ausbau dieses Ziels nicht mehr in die pro-traditionelle Kategorie. Aufgrund der (verhältnismäßig) konsequenten Unterstützung der Gleichstellung kann man sie im Modell Gauthiers dem pro-egalitären System zuordnen. Inwiefern das pro-natale Motiv den konservativen Familienregimes zuzuordnen ist, bleibt in dieser Einteilung fragwürdig. Aufgrund der Ergebnisse dieser Arbeit scheint eine Zuordnung des pro-natalen Modells zwischen dem pro-traditionellen und dem pro-egalitären Modell logischer, da auch im sozialen Investitionsstaat die demographische Nachhaltigkeit eine wichtige Rolle spielt. Die nicht untersuchten Länder sind mit Fragezeichen versehen, da sich auch in diesen Ländern eventuell Verschiebungen vollzogen haben. 
Tabelle 38: Westeuropäische Staaten nach Wohlfahrtsstaats- bzw. Familienregime und Staatsaufbau (Schmidt 2006: 86f, Bearbeitung Willenborg 2009)

\begin{tabular}{|c|c|c|c|c|}
\hline \multirow{2}{*}{$\begin{array}{l}\text { Wohlfahrtsstaats- } \\
\text { bzw. } \\
\text { Familienregime }\end{array}$} & \multicolumn{2}{|l|}{ Konservativ } & \multirow{2}{*}{$\begin{array}{l}\text { Sozialdemokratisch } \\
\text { Pro-egalitär }\end{array}$} & \multirow{2}{*}{$\begin{array}{l}\text { Liberal } \\
\text { Nicht- } \\
\text { intervenierend }\end{array}$} \\
\hline & Pro-traditionell & Pro-natal & & \\
\hline Zentralistisch & $\begin{array}{l}\text { (Niederlande), } \\
\text { Belgien, Irland }\end{array}$ & Frankreich & $\begin{array}{l}\text { Nieder- } \\
\text { Dänemark, } \\
\text { Finnland, } \\
\text { Norwegen, } \\
\text { Schweden }\end{array}$ & $\begin{array}{l}\text { lande } \\
\text { Großbritannien }\end{array}$ \\
\hline Föderativ & $\begin{array}{l}\text { Österreich, } \\
\text { (Deutschland) }\end{array}$ & Deutsch- & land & Schweiz \\
\hline
\end{tabular}

Die Einteilung in Regimetypen war zu Beginn der neunziger Jahre sehr illustrativ. Sie hat dazu beigetragen, den Zusammenhang verschiedener familienpolitischer Maßnahmen zu erklären und verdeutlicht, welche Richtung für familienpolitische Reformen maßgeblich ist. Opielka schreibt in diesem Zusammenhang: „Die Geschichte der Sozialpolitik ist eine Geschichte sozialpolitischer Reformen. Trotz einer beachtlichen Pfadabhängigkeit der vier Typen des Wohlfahrtsregimes bestehen sowohl innerhalb der Regimetypen erhebliche Variationen und sind (...) immer wieder auch Pfadwechsel möglich gewesen“ (Opielka 2004: 247). Aufgrund der Ergebnisse dieser Arbeit kann dieser Feststellung die Frage hinzugefügt werden, ob sich generell nicht immer mehr europäische Staaten zu hybriden Regimes entwickeln. Dementsprechend wäre dann auch eine eventuelle Neudefinition der Regimes nicht sinnvoll. Also bleibt die Frage, ab welchem Moment es noch hilfreich innerhalb der Europäischen Union verschiedene familienpolitische Regimes zu unterscheiden. Diese Frage wird im Unterkapitel zur europäischen Integration aufgegriffen und weiter ausgeführt.

Die Begriffe Pfadabhängigkeit und De-Kommodifizierung standen in den letzten beiden Werken Esping-Andersens thematisch nicht mehr im Zentrum seiner Betrachtungen. Seine umfangreichen quantitativen Erhebungen aus den 
achtziger Jahren hat er nicht fortgesetzt. Dennoch hält er weiterhin am sozialdemokratischen Regime als ideales Leitbild fest. Ganz konkret geht es ihm vor allem um die Aufgabenverteilung von Männern und Frauen in Haushalt und Beruf (bzw. der strukturellen Arbeitsmarktteilnahme von Frauen) und die Chancengleichheit von Kindern. Um diese beiden Ziele herum entwirft er ein familienpolitisches Grundkonzept, denn die Zielerreichung sei von großer Bedeutung für die Zukunft des Wohlfahrtsstaates. Kern seines familienpolitischen Grundkonzepts ist eine homogenisierte Form der DeFamilialisierung.

\subsubsection{Zur Bedeutung des Begriffs De-Familialisierung}

Esping-Andersen betont in seinen Arbeiten, wie wichtig die interdisziplinäre politische Zusammenarbeit für die Stärkung von Familien sei. Besondere Relevanz hätten neben der Rolle der Frauen, die Armutsbekämpfung oder Prävention von Armut, die demographische Nachhaltigkeit einer Gesellschaft und die Entwicklung der Kinder. All diese Faktoren ständen im Zusammenhang mit dem Begriff der so genannten Familialisierung bzw. De-Familialisierung ${ }^{43}$.

Familien, in denen Männer und Frauen am Arbeitsmarkt teilnehmen können, zeichnen sich im Allgemeinen durch ein niedrigeres Armutsrisiko und mehr Gerechtigkeit zwischen den Geschlechtern aus. Falls die Arbeitsmarktteilnahme von Frauen und die Erziehung der Kinder sich gut kombinieren ließen (z.B. durch entsprechende Kinderbetreuungsmöglichkeiten), könne dies positive Auswirkungen auf die Geburtenrate eines Landes haben. Außerdem werde durch passende Kinderbetreuung ab einem gewissen Lebensalter der Segregation von Kindern vorgebeugt und erhöhe sich hiermit die Chancengleichheit.

Der von Esping-Andersen betonte Begriff der De-Familialisierung ist inzwischen nicht mehr nur auf die skandinavischen Länder beschränkt. De-Familialisierung kann als ein Trend bzw. Prozess interpretiert werden, der sich inzwischen in

43 Wie bereits im zweiten Kapitel verdeutlicht, ist hiermit der Zusammenhang zwischen familiärer Aufgabenteilung und Arbeitsmarktteilnahme, oder konkreter, der Umfang familiärer Aufgaben, die durch die Familien selbst geleistet werden, gemeint. 
vielen Ländern durchgesetzt hat. Sigrid Leitner hat vor diesem Hintergrund vorgeschlagen die Typologie von Esping-Andersen um ein Gender-Regime zu erweitern. Sie unterscheidet zwischen vier familialistischen Kategorien. Wichtig ist es hierbei hinzuzufügen, dass sie die Begriffe Familialismus und DeFamilialisierung anders definiert als Esping-Andersen. Daher wird folgende Einteilung nicht wegen der Definition der Begrifflichkeiten, sondern aufgrund der von ihr verwendeten Ausarbeitungen hinzugefügt:

- „optimaler Familialismus“ mit verbreiteter professioneller, öffentlicher Kinderbetreuung und zugleich Transferleistungen für die Kindererziehung in der Familie (Schweden, Dänemark, Frankreich, Belgien und - als Grenzfall zum ,expliziten Familialismus' - Finnland);

- $\quad$ expliziter Familialismus' mit geringem Angebot an öffentlicher Kinderbetreuung, aber Transferleistungen für die familiäre Kindererziehung (Österreich, Deutschland, Italien, Luxemburg, Niederlande);

- $\quad$,impliziter Familialismus' mit geringem Angebot öffentlicher Kinderbetreuung und wenig Barleistungen für die Familienarbeit (Griechenland, Portugal, Spanien);

- ,De-Familialismus' mit verbreiteter öffentlicher Kinderbetreuung, aber keinen Zahlungen für die familiäre Kindererziehung (Irland, Großbritannien)“ (Opielka, 2004: 112f).

Leitner hat durch diese Beschreibung eine geschlechtssensitive Kategorisierung der Familienarbeit entwickelt und wirft die Frage auf, ob geschlechtsneutrale, familienpolitische Rechte unterschiedlich wirken, wenn sie die entsprechenden Ausgangspositionen bzw. Kulturen eines Landes nicht berücksichtigen. Obwohl ihre Kategorisierung nicht mehr den heutigen Gegebenheiten entspricht und sowohl Deutschland als auch die Niederlande nicht mehr dem expliziten Familialismus zugeordnet werden können, weist Leitners Einteilung noch stets auf einen wichtigen Punkt. Für den Erfolg, bzw. die vorgenommene Zielerreichung politisch-administrativer Reformen sind nicht nur die institutionellen Ausgangspositionen eines Landes entscheidend. Die Rolle der Frauen, bzw. die gesellschaftliche Auffassung von der Art der Aufgabenverteilung in Familie und Beruf ist für den vorzunehmenden familienpolitischen Reformkurs einer Regierung und die zu erzielenden Erfolge 
hinsichtlich der Beteiligung der Frauen am Arbeitsmarkt, der demographischen Nachhaltigkeit einer Gesellschaft, der Entwicklung, bzw. Frühförderung von Kindern und der Armutsprävention von großer Bedeutung. Entscheidend ist für Politiker also, den zur Kultur des Landes passenden Mix aus familienpolitischen Mitteln zu finden.

\subsubsection{Systemergänzungen auf Mikroniveau}

Esping-Andersens Theorien zum sozialen Investitionsstaat lieferten einen entscheidenden, inhaltlichen Baustein für das Idealmodell Aktivierender Familienpolitik. Seine Arbeiten sind allerdings (zum Großteil) auf international vergleichenden Makrodaten basiert. Opielka schlussfolgert, dass eine „rein auf quantitativen Daten basierende Analyse von Sozialpolitik (...) kein Bild der institutionellen und kulturellen Zusammenhänge“ vermittelt. „Man versteht daraus nicht, warum die Sozialpolitik in einem Land beispielsweise die familiäre, im anderen die öffentliche Kinderbetreuung favorisiert" (Opielka 2004: 233f). Kremer konkretisierte diese Kritik anhand der sozialen Normen eines Landes hinsichtlich der Rollenverteilung von Männern und Frauen bzw. der Vereinbarkeit von Familie und Beruf. Parallel zum kulturellen Aspekt weist Opielka aber auch auf die institutionellen Zusammenhänge eines Landes. Die Frage nach der Art der Umsetzung des sozialen Investitionsstaates ist daher besonders interessant.

Beide hier untersuchten Länder folgten sowohl inhaltlich als auch hinsichtlich der Verantwortungsteilung dem Leitbild des Aktivierenden Staates. Sie haben hierfür allerdings - aufgrund ihrer institutionellen Hintergründe, ihrer politisch-administrativen Kultur und der entsprechenden politischen Verantwortungsträger - im untersuchten Zeitraum unterschiedliche Wege gewählt. Um diese Wege besser $\mathrm{zu}$ verstehen sind die unterschiedlichen Kulturen bzw. sozialen Normen relevant. Sie können hilfreiche Erklärungen zum Gelingen der familienpolitischen Reformen bieten. Hiermit wird nicht auf das politische Erbe sozialstaatlicher Investitionen, das Esping-Andersen mit dem Begriff der Pfadabhängigkeit beschreibt, hingewiesen sondern auf administrativinstitutionelle Aspekte wie z.B. der Stellenwert eines bestimmten Politikbereichs. Ein anderer entscheidender Aspekt ist die Rolle der Akteure bzw. Politiker. Neben ihrer politischen Couleur sind sie abhängig von den 
allgemeinen politisch-administrativen Strömungen bzw. Leitbildern und dem Grad, zu dem ihre Politik mit diesen Strömungen harmonisiert (oder: zu vereinbaren ist). In den folgenden Unterkapiteln werden diese Punkte anhand der unterschiedlichen Umsetzung des Idealmodells Aktivierender Familienpolitik in den hier untersuchten Ländern ausführlicher behandelt.

\subsubsection{Politisch-administrative Kultur}

Sicherlich ist die Anwesenheit eines Familienministeriums bzw. eines Ministeramts für Familienangelegenheiten keine direkte Garantie für familienpolitischen Erfolg. Allerdings kann sie - bis zu einem gewissen Grad durchaus als ein Indikator des Stellenwertes des Begriffs Familie in der politischen Kultur eines Landes betrachtet werden. Die Bundesrepublik Deutschland richtete schon im Jahre 1953 das Amt eines Familienministeriums mit zugehörigem Beamtenstab ein. Nicht alle für Familien relevanten Themenbereiche unterstanden diesem Ministerium, aber immerhin konnten die Interessen von Familien (auf nationaler Ebene) durch einen Amtsträger vertreten werden. In den Niederlanden war das nicht der Fall. Verschiedene Minister und Staatssekretäre (Minister und Staatssekretäre für soziale Angelegenheiten, Staatssekretäre für Gesundheit, Wohlbefinden und Sport etc.) waren politisch verantwortlich für die familienpolitischen Teilbereiche. In der ersten Periode des hier untersuchten Zeitraums war z.B. die Staatssekretärin für Gesundheit, Wohlbefinden und Sport verantwortlich für eine gebündelte Berichterstattung über die Lage der Familien. Eine koordinierende Funktion hatte sie allerdings nicht. Diese Form der politischen Organisation änderte sich durch die Einführung eines Programmministeriums für Jugend und Familie im Jahre 2007. Seitdem verfügt der Programmminister unter anderem über koordinierende Verantwortlichkeiten im Hinblick auf die Familienpolitik.

Obgleich in dieser Arbeit nicht die Kausalität zwischen der Anwesenheit eines Familienministers und den familienpolitischen Erfolgen untersucht wurde, fiel in der Analyse die Konsequenz in der Weiterentwicklung der Zielsetzungen an deutscher Seite auf. Die deutsche Familienpolitik hat sich im hier untersuchten Zeitraum immer mehr am Leitbild des Aktivierenden Staates orientiert, während in den Niederlanden die familienpolitischen Zielsetzungen etwas unstetiger und weniger konsequent verfolgt wurden. Die Gründe für diesen 
Unterschied wurden an dieser Stelle nicht untersucht. Es liegt allerdings die Vermutung nahe, dass die permanente Anwesenheit eines Stabs für Familienangelegenheiten eine nicht unwichtige Rolle im Zusammenhang mit der Kontinuität bei der Formulierung familienpolitischer Zielsetzungen spielt. Auch im Bereich der Maßnahmen ist in Deutschland eine gewisse Kontinuität zu beobachten, während die niederländischen Maßnahmen vor der Einführung des Programmministeriums weniger zusammenhängend erschienen.

Ein anderer Grund für die unterschiedliche Art der familienpolitischen Reformen bei Verwendung desselben Idealmodells könnte allerdings auch die Kompetenzverteilung der verschiedenen Verwaltungsebenen sein. Während im niederländischen Zentralstaat verhältnismäßig viele Kompetenzen auf nationaler Ebene liegen, ist Deutschland ein stark föderalistisches Land. Vor allem im Bereich der Schulpolitik bzw. der Kinderbetreuung findet man diesen Verwaltungsunterschied deutlich in den hier untersuchten Dokumenten wieder. Die Konsequenz der Reformen im Hinblick auf die Zielerreichung ist hiervon nur wenig beeinträchtigt. Dies gilt allerdings nicht für die Maßnahmen. Die Durchführung der Maßnahmen kann stark abhängig sein von der Frage der Kompetenzverteilung (national, regional, kommunal oder (teil)liberalisiert). Im Reformentwurf sollte daher Raum eingeplant werden für die Interpretationsmöglichkeiten der an der Koproduktion beteiligten Verantwortungsträger.

Ein weiterer politisch-administrativer Kulturfaktor, der sich im Rahmen dieser Arbeit bemerkbar machte, ist die Bedeutung der Dokumente, in denen familienpolitischen Entscheidungen festgelegt werden. In den beiden hier untersuchten Ländern sind vor allem in den Koalitionsverträgen wichtige familienpolitische Ziele und Maßnahmen festgelegt. Diese sind jedoch nicht zusammenhängend an einer Stelle des Vertrags beschrieben, sondern können im Zusammenhang mit verschiedenen Politikbereichen auftauchen (Familie, Emanzipation, Bildung, Arbeitsmarkt etc.). Wichtig ist daher, dass sich die Maßnahmen in den Verhandlungsdiskussionen der neu gewählten Koalition durchsetzen können. Leitbilder können hierbei von sehr großem Nutzen sein.

Einen Einfluss auf die politische Meinungsbildung können aber auch die Familienberichte (bzw. Jugendberichte usw.) haben. In Deutschland ist diese Familienberichterstattung institutionalisiert und kann daher als fester 
Bestandteil der familienpolitischen Entscheidungsfindung betrachtet werden. Im 12. Kinder- und Jugendbericht findet sich z.B. zum Thema demographischer Wandel eine wissenschaftliche und politische Basis, die dann in den folgenden Dokumenten weiter ausgebaut wurde. In den Niederlanden haben die Familienberichte einen anderen Stellenwert. Sie sind nicht institutionalisiert, sondern können von der Regierung angekündigt oder vom Parlament erbeten werden. Sie können also nicht als ein konstanter politisch-administrativer Faktor gesehen werden. Relativ wenig Gewicht für die politische Entscheidungsfindung haben die Regierungserklärungen beider Länder. Sie wurden an dieser Stelle vor allem zur Verdeutlichung der Koalitionsverträge herangezogen. Der Einfluss der hier untersuchten Dokumente ist für die Reformentscheidungen nicht unerheblich. Hierzu besteht weiterer Forschungsbedarf. Interessant wäre es z.B. $\mathrm{zu}$ untersuchen, inwiefern die Institutionalisierung der Familienberichte die politische Entscheidungsfindung beeinflusst.

\subsubsection{Politische Akteure und politisch-administrative Leitbilder}

Neben der politisch-administrativen Einbettung des Themas Familie ist auch die Rolle der Akteure bzw. der verantwortlichen Politiker relevant für die Unterschiede in der Umsetzung Aktivierender Familienpolitik in Deutschland und den Niederlanden. Familienpolitik ist in beiden Ländern Querschnittspolitik. Arbeitsmarktpolitik, Bildungspolitik, Emanzipationspolitik, Wohnungsbaupolitik und einige andere politische Bereiche haben einen entscheidenden Einfluss auf die Familienpolitik. Obwohl es zur Zeit der Verfassung dieser Arbeit in beiden Ländern für die Familienpolitik verantwortliche Familienminister gibt, müssten für eine umfangreiche Analyse der Akteure auch die verantwortlichen Politiker der eben genannten Felder einbezogen werden. Sicherlich wäre das ein interessantes Thema für eine weitere Forschungsarbeit. Im Rahmen dieser Arbeit würde der Ansatz allerdings $\mathrm{zu}$ weit führen und außerdem an der ursprünglichen Fragestellung vorbeigehen.

Da allerdings die Rolle der Akteure für die Entscheidungsfindung sehr interessant ist, soll die Analyse an dieser Stelle beispielhaft, anhand einer gewissen Zahl von Politikern erfolgen, die eine entscheidende Rolle in der Koordinierung der hier analysierten Jugend- und Familienberichte spielten. An 
deutscher Seite sind dies: Dr. Christine Bergmann (SPD), Renate Schmidt (SPD) und Dr. Ursula von der Leyen (CDU) und an der niederländischen Seite: Erica Terpstra (VVD), Clémence Ross-van Dorp (CDA) und André Rouvoet (CU). Im Folgenden werden sie bzw. ihre politischen Handlungen im Zusammenhang mit der Politik der Kabinette denen sie angehörten analysiert.

Wie bereits festgestellt haben sich die hier untersuchten Koalitionen beider Länder im Großen und Ganzen am Leitbild des Aktivierenden Staates orientiert. An deutscher Seite kam die Umsetzung dieses Leitbildes in die Familienpolitik während der ersten Rot-Grünen Koalition eher in den Zielsetzungen als in den Instrumenten zum Tragen. Maßnahmen, die Bundesministerin Bergmann während dieser Zeit durchführte (z.B. die Erhöhung des Kindergeldes und den Umbau des Erziehungsurlaubs zur Elternzeit, die durch beide Eltern gleichzeitig aufgenommen werden konnte) sind eher klassisch sozialdemokratisch als aktivierend $\mathrm{zu}$ nennen. Eine deutliche Veränderung war während der Regierungsperiode von Bundesministerin Schmidt spürbar. Vor allem die von ihr initiierten Maßnahmen, die in dieser Arbeit als ökologische Instrumente bezeichnet wurden (Gründung der ,Allianz für die Familie‘ und der ,Lokalen Bündnisse für Familie`) sind sehr interessante aktivierende Beispiele. Aber auch der Ausbau der ökonomischen Maßnahmen, z.B. durch die Einführung des Kinderzuschlags, kann deutlich als aktivierend im Sinne des hier untersuchten Modells bezeichnet werden (vergl. http://www.bmfsfj.de/bmfsfj/generator/BMFSFJ/Ministerium/geschichte.html aufgerufen am 04.12.2009). Bundesministerin von der Leyen führte die Umsetzung der von ihrer Vorgängerin eingeleiteten Maßnahmen zur Zeit der Großen Koalition unter Merkel nicht nur fort, sondern baute diese mit der Einführung von Elterngeld, Elternzeit und Mehrgenerationenhäusern weiter aus.

Auch in den Niederlanden hat es eine inhaltliche Orientierung am Leitbild des Aktivierenden Staates gegeben. Da es bis zur Einführung des Programmministeriums im Jahr 2007 nur Staatssekretäre mit einer geringen Koordinierungskompetenz gab, ist eine Analyse der niederländischen Akteure verhältnismäßig schwer durchzuführen. Die Politik des ersten Kabinetts unter Kok, in dem Minister Terpstra eine koordinierende Funktion im familienpolitischen Bereich bekleidete, war stark durch das Aktivierungsprinzip geprägt. Das Kabinett brach mit der traditionell sozialdemokratischen Tradition 
und führte in diesem Sinne nicht nur finanzielle Kürzungen im Bereich des Kindergeldes durch, sondern unterstützte die Aktivierung von Müttern und Vätern in Beruf und Familie durch Zeit-Instrumente wie z.B. das Arbeitszeitengesetz und die Vorbereitungen zur Einführung des Rahmenrechts Arbeit und Pflege. Teilzeitarbeit wurde von diesem Kabinett stark gefördert. Das zweite Kabinett unter Kok führte diese Politik im Großen und Ganzen fort, wird aber an dieser Stelle nicht ausführlich analysiert, da es keine für die Fragestellung relevanten Erneuerungen brachte. Auch auf die erste Regierung unter Balkenende wird aufgrund der kurzen Regierungszeit an dieser Stelle nicht näher eingegangen. Während der ersten großen Regierung unter Balkenende (Balkenende II) war Staatssekretär Ross-van Dorp verantwortlich für die Koordinierung der Familienpolitik. Auch ihre Möglichkeiten waren (vergleichbar mit den Staatssekretärinnen während der violetten Regierungen) stark abhängig von der Politik der übrigen Minister und Staatssekretäre. In dieser Zeit wurden sowohl im ökonomischen und ökologischen Bereich als auch im Rahmen der Zeit-Instrumente aktivierende Instrumente gefördert (Lebenslaufvergünstigung, Ausbau Kinderbetreuung, Einführung der breiten Nachbarschaftsschule etc.). Der Bereich, für den Ross-van Dorp selbst verantwortlich war (Förderung von Kompetenzen Jugendlicher/Jugendpolitik), kann interessanterweise nur als sehr begrenzt aktivierend bezeichnet werden. Sowohl ihr Jugendschutzgesetz als auch die geplante Einführung der Zentren für Jugend und Familie entsprachen eher dem Leitbild des Aktiven Staates, mit einer großen öffentlichen Verantwortung und einer dominanten Rolle der professionellen Hilfskräfte in den Jugendeinrichtungen, als dem Leitbild des Aktivierenden Staates. Das vierte Kabinett unter Balkenende folgt in beinahe allen familienpolitischen Bereichen dem Aktivierungsideal. Obgleich die Verantwortungsfrage mit der Einführung eines Programmministers noch immer nicht bis in alle Teilbereiche deutlich geklärt wurde, kann durch Minister Rouvoet eine Zunahme der Koordinierung familienpolitischer Fragen verzeichnet werden. Er verschreibt sich - entsprechend der Vereinbarungen aus dem Koalitionsabkommen - im Hinblick auf die familienpolitischen Maßnahmen (Einführung eines einkommensabhängigen kindgebundenen Budgets, Verdopplung des Erziehungsurlaubs für Väter und Mütter etc.) der aktivierenden Politik. Im Bereich der Kompetenzförderung von Kindern und Jugendlichen kann seine Politik allerdings als zweigleisig bezeichnet werden. Einerseits führte er die aktive Politik der Staatssekretärin Ross-van Dorp fort, 
andererseits betonte er die Selbstheilungskraft von Familien, die wiederum eher dem Leitbild des Aktivierenden Staates entspricht.

Wie zu Beginn des Kapitels bereits festgestellt, ist eine umfangreiche Analyse der Akteure nicht das Ziel dieser Arbeit. Auffallend ist allerdings, dass vier der sechs beschriebenen Minister bzw. Staatssekretäre eine aktivierende Familienpolitik führen bzw. deutliche Ansätze des Aktivierungsprinzips verkörperten. Gründe hierfür kann es natürlich viele geben. Der politische Werdegang eines Politikers ist hierbei durchaus entscheidend, aber es sind auch noch viele andere Faktoren denkbar. Auffallend ist allerdings, dass bei den aktivierenden Familienpolitikern die Frage zwischen der Verkörperung traditionell linker bzw. traditionell rechter Politik weniger wichtig erschien. Ein gutes Beispiel hierfür sind z.B. die Ministerinnen Schmidt und von der Leyen. Mit der demographischen Nachhaltigkeit hatte Ministerin Schmidt (SPD) ein Ziel, dass vor allem auch die CDU Klientel berührte. Auch die ,Allianz für die Familie‘ bzw. die ,Lokalen Bündnisse für Familie‘ sind nicht nur eindeutig aktivierend, sondern auch für die Wähler der beiden großen Volksparteien in Deutschland ansprechend. Bundesministerin von der Leyen hat in ihrer Politik zum Teil fortgeführt, was bereits von Bundesministerin Bergmann initiiert wurde. Mit z.B. der Einführung von Mehrgenerationenhäusern hat sie weitere aktivierende Elemente hinzugefügt. Für das Führen einer aktivierenden Familienpolitik ist es als Politiker also wichtig, entsprechend der theoretischen Grundprinzipien des Aktivierenden Staates, sowohl vom linken als auch vom rechten Flügel der politischen Mitte angenommen zu werden.

In der Wohlfahrtsstaatsanalyse von Esping-Andersen werden aufgrund des Vergleichs staatlicher Investitionshöhen Schlüsse über die Problemlösungsfähigkeit bzw. die Entwicklungsrichtung eines Staates gezogen. Um die gesellschaftlichen Veränderungen bzw. Transitionen in gute Bahnen zu leiten, fordert Esping-Andersen einen sozialen Investitionsstaat mit gleichberechtigen Lebensläufen von Frauen und Männern und der Förderung von Fähigkeiten aller Kinder vor allem in ihren vorschulischen Lebensjahren. Inhaltlich kommt das Idealmodell Aktivierender Familienpolitik seinem Ideal des sozialen Investitionsstaates sehr nahe. Allerdings wird im hier untersuchten Idealmodell eine konsequente Verantwortungsdiskussion verfolgt, während sich Esping-Andersen (vor allem in seinem jüngsten Werk) kritisch gegenüber der Verantwortungsteilung äußert und die Verantwortung für den sozialen 
Investitionsstaat hauptsächlich beim Staat sieht (vergl. Esping-Andersen 2009: 93f).

Obwohl seine inhaltlichen Problemanalyse und die Familienpolitik der beiden hier untersuchten Länder zunehmend konvergent sind, gibt es bei der politischen Formgebung erhebliche Divergenzen. In beiden Ländern ist die Verantwortungsteilung zwischen dem Wohlfahrtsstaat, dem Markt und den Familien/der Gesellschaft und das entsprechende Instrumentarium, um ebendiese zu realisieren, zunehmend Teil der familienpolitischen Praxis geworden. Die beiden Länder haben sich zu hybriden Regimes entwickelt, die weniger dogmatisch als pragmatisch zu verschiedenen Formen von Aktivierung greifen. Esping-Andersens Schlussfolgerung, dass „es der Fall sein muss, dass alternative Regelungen - im Wesentlichen entweder die Familie oder der Markt - suboptimale Lösungen zur Verfügung stellen“ (Esping-Andersen 2009: 174) kann aus dem deutsch-niederländischen Vergleich nicht unterstrichen werden. Im folgenden Unterkapitel werden in diesem Zusammenhang - vor dem breiteren Rahmen der europäischen Integration - Schlussfolgerungen auf die Haltbarkeit des Aktivierungsprinzips gezogen.

\subsection{Der Aktivierender Staat: Ein europäisches Sozialmodell in Entwicklung?}

Es gibt bisher keinen wissenschaftlichen Konsens darüber, ob man inzwischen von einer konvergenzorientierten europäischen Sozial- und Arbeitsmarktpolitik sprechen kann. „Hartmut Kaelble sieht die Länder Europas auf dem Weg zu einem ,europäischen Wohlfahrtsstaat', nachdem sich unterschiedliche Startpositionen mit fortschreitender Industrialisierung abgeschliffen hätten. Demgegenüber kommt Klaus Armingeon zu dem Ergebnis, dass über einen langen Zeitraum die meisten der von ihm untersuchten 34 politisch-sozialen Indikatoren keine wesentlichen Änderungen aufwiesen, so dass die Wohlfahrtsstaaten in Europa ihre Unterschiede beibehalten, erste recht, wenn man bedenkt, dass die Nationalstaaten derzeit unterschiedlich auf die Herausforderungen des Wohlfahrtsstaates reagieren“ (Kevenhörster 2006: 244).

Aufgrund der vorliegenden Vergleichsstudie kann man für den familienpolitischen Bereich Deutschlands und der Niederlande beide 
Sichtweisen unterstreichen. Konvergenz hat es sicherlich im Bereich der Zielsetzungen der beiden Länder gegeben. Nicht zuletzt aufgrund der LissabonStrategie 44 und der Barcelona-Kriterien 45 kommt es im Bereich der aktivierenden Zielsetzungen $\mathrm{zu}$ einer steigenden Zahl von inhaltlichen Parallelen zwischen den europäischen Ländern. Zum Thema Arbeitsmarktteilnahme von Frauen schreibt Esping-Andersen im Jahre 2009, also kurz vor dem Ende der (Lissabon)-Strategielaufzeit „...diese Nachzügler (Südeuropa) holen jetzt sehr schnell auf (Boeri et al. 2005). Tatsächlich ist die weibliche Beschäftigungsrate in Spanien von 30\% im Jahr 1995 auf 53\% im Jahre 2007 gesprungen: ein Anstieg von 77\% innerhalb eines Dezenniums! Das Tempo dieser Veränderungen wird sogar noch sichtbarer, wenn wir uns auf jüngere Frauen konzentrieren deren Teilnahme am Arbeitsmarkt schon bald 70\% betragen wird“ (Esping-Andersen 2009: 23). Das Tempo der einzelnen Mitgliedstaaten mag unterschiedlich sein, und es mag - wie z.B. während der tschechischen Ratspräsidentschaft im Jahre 2009 - einige Politiker geben, die die Strategie bzw. die Kriterien in Frage stellen. Fakt ist allerdings, dass es die familienpolitischen Zielsetzungen der einzelnen Mitgliedstaaten im Großen und Ganzen dem hier verwendeten Idealmodell ähnlich bzw. gleich sind. Aber nicht nur für die Zielsetzungen sondern auch im Bereich der Maßnahmen, ergeben sich zumindest in den hier untersuchten Ländern, einige prinzipielle Übereinstimmungen. Deutschland und die Niederlande verwendeten im untersuchten Zeitraum beide ein aktivierendes Instrumentarium, auch wenn es unterschiedliche Maßnahmen waren, die sie zur Zielerreichung einsetzten.

Wenn es also aufgrund unterschiedlicher Entwicklungen wie z.B. der LissabonStrategie zu einer Konvergenz der familienpolitischen Zielsetzungen in den Ländern kam, schließt sich die Frage an, ob Europa entsprechend einem nationalen Modell Sozialstaat sein kann. Leibfried äußerte sich in der Vergangenheit kritisch gegenüber dieser Frage, denn „das Terrain ist zu komplex, zu stark und zu lange national vorbesetzt, in Deutschland seit mehr als

\footnotetext{
44 Die Lissabon-Strategie ist ein Programm, um die EU-Zone zum Jahr 2010 zum wettbewerbsfähigsten und dynamischsten Wirtschaftsraum der Welt zu machen. Wichtige Themen der Strategie sind Wirtschaftswachstum, Wissensgesellschaft und Nachhaltigkeit im sozialen wie ökologischen Sinn. Die Agenda 2010 ist inhaltlich mit dieser Strategie verbunden. 45 In den Barcelona-Kriterien sind Wachstumsabsprachen im Zusammenhang mit der Lissabon-Strategie festgelegt (z.B. Indikatoren für die Anzahl der Kinderbetreuungsplätze).
} 
einem Jahrhundert. Weder Bismarck noch Beveridge noch „Volksheim“ bieten hilfreiche Modelle für eine sozialpolitische Entwicklung der EU. Es wird etwas drittes und auch anderes auf der eruropäischen Ebene gesucht werden müssen, wenn es dort wirksame, teilfunktionale Äquivalente geben soll, mit denen sich die Schwierigkeiten bewältigen lassen, die die versammelte westeuropäische Wohlfahrtsstaatlichkeit derzeit hat" (Leibfried 1999: 768f). Der Aktivierende Staat ist kein Modell im Sinne des Bismarck- oder Beveridge-Systems. Das politisch-administrative Leitbild Aktivierender Staat stieß bei den Reformen im familienpolitischen Bereich aber nicht nur in den hier untersuchten Ländern auf breite Zustimmung und führte $\mathrm{zu}$ entsprechenden Konsequenzen. Auch die europäische sozialpolitische bzw. (im hier definierten Sinne) familienpolitische Politik ist von diesem Leitbild geprägt. So schreibt die Kommission der Europäischen Gemeinschaften in ihrer Halbzeitbilanz zu den Fortschritten beim Fahrplan für die Gleichstellung von Frauen und Männern (2006-2010) ${ }^{46}$ zum Thema Verantwortungsteilung: „Gleichstellung kann nur erreicht werden, wenn alle Beteiligten Verpflichtungen angehen“. Dieses Prinzip der Verantwortungsteilung verfolgt sie auch in ihren Maßnahmen.

Als Beispiel einer (weichen) aktivierenden Maßnahme der Europäischen Kommission - im Sinne der seit der Lissabon-Strategie vielfältig verwendeten offenen Koordinierungsmethode (OMK) - kann die Förderung der Europäischen Allianz für Familie verstanden werden. Im Rahmen von Veranstaltungen wie z.B. die ,European Demographic Expert Group' tauschen sich Beamte und Wissenschaftler der verschiedenen Mitgliedstaaten über die Wirkungsweise familienpolitischer Maßnahmen (gute Beispiele) und verwandte Themen aus. Sabine von Oppeln schreibt in diesem Zusammenhang, es habe sich ein Modell durchsetzen können, „das in pragmatischer Weise die institutionellen Blockaden des europäischen Mehrebenensystems zu umgehen versucht und zugleich in der Anpassung wohlfahrtsstaatlicher Politik an das dominierende Paradigma (neo)liberalter Politik ein Mindestmaß an sozialer Kohäsion zu garantieren sucht. Der Erfolg dieses Modells, das den Umbau der nationalen Wohlfahrtsstaaten und tendenziell auch eine Nivellierung der Unterschiede 
zwischen den wohlfahrtsstaatlichen Systemen der Mitgliedstaaten zur Folge haben kann, muss sich erst noch erweisen“ (von Oppeln 2007: 16).

Die Evaluierung des Erfolgs wird relativ sein und ist von den selektierten Indikatoren abhängig. Es wird allerdings stets deutlicher, dass neben der bereits festgestellten Konvergenzen im Bereich der Zielsetzungen durch die Zusammenarbeit innerhalb der Europäischen Union auch im Bereich der Instrumente Konvergenzen stimuliert bzw. den Beamten aller Mitgliedstaaten gute Beispiele vorgestellt werden. Fest steht auch, dass sich die Entscheidungsträger der EU-Mitgliedstaaten (aus eigenem Interesse) an internationalen Vorbildern orientieren und sich hierdurch stets mehr hybride familienpolitische Systeme entwickeln. Eine Kategorisierung zwischen liberalen, konservativen und sozialdemokratischen Ländern, so wie sie vor zwanzig Jahren noch sehr illustrativ war, ist heute nicht mehr sehr aufschlussreich. Leibfried und Zürn schreiben in diesem Zusammenhang: „Ein Vergleich der heutigen wohlfahrtsstaatlichen Arrangements mit jenen der siebziger Jahre macht deutlich: Die einst beträchtlichen Unterschiede zwischen den entwickelten Wohlfahrtsstaaten haben sich verringert" (Liebfried/Zürn 2006: 301). Interessanter wäre es also, um die guten Beispiele der gemeinschaftlichen Wohlfahrtsstaatsproduktion, die sich in den verschiedenen Mitgliedstaaten entwickelt haben genauer auf ihre Effektivität und Effizienz zu überprüfen und mögliche Muster der Verantwortungsteilung zu kategorisieren.

\subsection{Zur Weiterentwicklung des Leitbildes Aktivierender Staat}

Entsprechend der Theorie der ,Langen Wellen der Leitbildentwicklung' entwickelt sich ein politisch-administratives Leitbild nach 15-20 Jahren weiter. In den Niederlanden hat es seit 1994 vielfältige Aktivierungsmaßnahmen gegeben, während in Deutschland spätestens seit Regierungsbeginn der ersten RotGrünen Koalition (1998) der Aktivierende Staat als Leitbild galt. In den nächsten Jahren stände also eine Weiterentwicklung des aktuellen Leitbildes an.

Wie zu Beginn dieser Arbeit beschrieben, handelt es sich bei der Weiterentwicklung nicht um das komplette Verwerfen des bestehenden Leitbildes, sondern um einen Lernprozess bei dem auf die Schwächen bzw. Lücken des vorherigen Modells reagiert wird. Einerseits kann es Kritik an der 
Umsetzung bzw. der Interpretierung des Leitbildes geben. Andererseits können sich aber auch aufgrund interner oder externer gesellschaftlicher Veränderungen neue Herausforderungen ergeben denen die grundlegenden Ideen des bereits bestehenden Leitbildes nicht mehr genügen.

Die erstgenannte Form der Kritik begleitet den Aktivierenden Staat spätestens seit den Hartz-Reformen. Das Leitbild wurde, im Zusammenhang mit diesen Reformen, regelmäßig als aussichtslose Gegenreaktion auf leere Haushaltskassen bezeichnet. Evers schreibt, das von dem Leitbild Aktivierender Staat „vor allem das Begriffspaar des Förderns und Forderns übrig geblieben (ist) und nicht $\mathrm{zu}$ Unrecht meinen viele, dass es dabei vor allem um die Neubestimmung des Maßes an Zumutbarkeit beim Umgang mit Langzeitarbeitslosen geht“ (Evers 2004: 1).

In den Niederlanden wurden die (aktivierenden) Arbeitsmarktreformen von weniger Kritik begleitet als die deutschen Reformen in diesem Bereich. Der Begriff Poldermodell, der wie bereits erläutert zur Familie des Leitbildes Aktivierender Staat gerechnet werden kann, verlor jedoch zum Ende der zweiten Regierung unter Kok an Beliebtheit. Es kam zu einer politisch-administrativen Neuorientierung und einer Phase der politischen Instabilität. Inzwischen wird der Begriff Poldermodell wieder neutraler bewertet, und wie bereits festgestellt, können die hier untersuchten niederländischen Maßnahmen (bis auf wenige Ausnahmen) als aktivierend bezeichnet werden. Allerdings sieht sich auch die heutige niederländische Regierung mit der Kritik konfrontiert, sie wolle (in den verschiedenen sozialen Bereichen) zu viel Einfluss auf den privaten Bereich der Bürger ausüben und ihre Politik habe einen zu normativen Anstrich.

Neben den genannten negativen Kritiken sind aber auch sehr viele positive inhaltliche Reaktionen im Zusammenhang mit dem Aktivierenden Staat zu verzeichnen, denn durch die breite Form der Bürgerbeteiligung und der neuen Aktivierung bestehender Ressourcen kann es nicht nur zu Einsparungen und Effizienzsteigerungen kommen, sondern Leistungsempfänger werden aus ethischer Sicht durch die Verantwortungsteilung zum Kooperations- bzw. Verhandlungspartner aufgewertet. Infolge der Verbesserung des gesellschaftlichen Dialogs kommt der demokratische Gedanke des Rechtstaates deutlich zur Geltung und verfügt der Staat über die Möglichkeit, die Inhalte seiner Reformprozesse besser zu verdeutlichen. 
Evaluiert man die Verwendung des Leitbildes in den beiden untersuchten Ländern aus familienpolitischer Sicht, überraschen vielleicht der große Einfluss und die breite Anwendung des Prinzips der Verantwortungsteilung. Es gibt im Bereich der ökologischen Instrumente und auch der Instrumente zur Förderung der Kompetenzen aktivierende Beispiele wie z.B. die Mehrgenerationenhäuser, die Lokalen Bündnisse für Familie, die breiten Nachbarschaftsschulen und die Entwicklung des Familienlastenausgleichs bzw. der ökonomischen Instrumente in den Niederlanden. Diese Instrumente besitzen zwar einige Schwachstellen (oft geht es um die Art der Einführung bzw. der generellen Führung und werden hierbei technische Fragen gestellt), im Großen und Ganzen kann aber von erheblicher zivilgesellschaftlicher Aktivierung gesprochen werden. Dementsprechend vielfältig ist die Anzahl positiver Formen von Sozialinvestitionen, Öffentlich-Privater Zusammenarbeiten und Liberalisierungen.

Für die Weiterentwicklung des Leitbildes wird sicherlich der Einfluss der Weltwirtschaftskrise relevant sein. Als Reaktion auf diese Krise haben beide Länder erhebliche unvorhergesehene Staatsinvestitionen getätigt, und wird es mittelfristig zu Einsparungen bzw. Umschichtungen kommen müssen. Auch die gesellschaftlichen Herausforderungen im Bereich der demographischen Nachhaltigkeit, der Gleichstellung von Frauen und der Chancengleichheit für Kinder und Jugendliche sind nach wie vor aktuell. Esping-Andersens Forderung nach einem sozialen Investitionsstaat behält ihre Relevanz und viele zivilgesellschaftliche Partner arbeiten inzwischen erfolgreich mit dem Staat zusammen, um die gesellschaftlichen Aufgaben zu lösen. Das Prinzip der Verantwortungsteilung wird weiterhin attraktiv bleiben.

Zusammenfassend kann man feststellen, dass die bisherige Kritik am Aktivierenden Staat die Grundidee des Leitbildes nur periphär berührt. Natürlich ist Aktivierung nicht für alle Menschen eine realistische Option. Das Menschbild von dem der Aktivierende Staat ausgeht, ist für gewisse Bevölkerungsschichten realistischer als für andere. Er bietet aber durch seine vielfältige Anwendungsweise viel Verwendungspotential. Auffallend ist die Kritik, die nicht direkt mit der hier verwendeten Definition des Leitbildes zusammenfällt, sondern eher mit dem Schlagwort des Förderns und Forderns. Diese Terminologie wurde im Zusammenhang mit den Hartz-Reformen in Deutschland viel diskutiert. Den Aspekt des Forderns bewerteten Kritiker der 
Hartz-Reformen als überdurchschnittlich hoch, während die Förderung im Verhältnis zu niedrig sei. Im Jahre 2010 hat das Bundesverfassungsgericht die Hartz-IV-Gesetze als mit dem Grundgesetz für unvereinbar erklärt. Dem Urteil folgend müssen die Regelsätze für Kinder und Erwachsene künftig auf einem neu formulierten Grundrecht auf Gewährleistung eines menschenwürdigen Existensminimus basiert sein. Aber mit diesem Urteilsspruch wird die Debatte über die Hartz-Reformen nicht beendet sein. Es gibt noch viele andere Kritikpunkte wie z.B. den Unterschied zwischen den Hartz-IV Regelsätzen und den Löhnen der nicht arbeitslosen Geringverdiener. Kritikern zufolge ist für die Gruppe der weniger Qualifizierten Arbeit oftmals nicht, lohnend‘.

Kernproblem der Hartz-Reformen ist aber nicht die umstrittene Höhe der finanziellen Regelsätze. Vielmehr ist diese Reform, die als aktivierend gedacht war, in ihrer Umsetzung dem Leitbild des Aktiven Staates nicht unähnlich. Sie geht von einer planbaren Gesellschaft aus, für die der Staat passende Regelsätze für jeden Anspruch habenden formuliert. Die Reformer übersahen die beinahe grotesken praktischen Konsequenzen dieses zentralisierten und $\mathrm{zu}$ weit durchgeführten Verantwortungsverständnisses anstelle von einer viel konsequenteren Verantwortungsteilung zwischen z.B. dem Bund, den Kommunen, den Arbeitgebern und den Arbeitsuchenden auszugehen.

Im Hinblick auf die Weiterentwicklung des Leitbildes Aktivierender Staat wird regelmäßig der Begriff Regulierung verwendet. Dieser Term kann verschiedenartig interpretiert werden. Dass eine Form der Regulierung im Sinne des Aktiven Staates allerdings nicht mehr zeitgemäß erscheint und für den mündigen Bürger unakzeptabel ist, wurde bereits diskutiert. Leibfried und Zürn kommen anhand einer internationalen Wohlfahrtsstaatsanalyse zu dem Schluss, dass es, obwohl sich vereinzelt Dezentralisierungstendenzen bei den Leistungserbringungen nachweisen lassen, kaum Indizien für eine umfassende Devolution im Bereich der sozialen Sicherheit gibt. „Sozialstaatswandel vollzieht sich offenkundig in erster Linie entlang der organisatorischen Achse als Gewichtsverlagerung bzw. stärkere Verzahnung zwischen staatlichen, privaten und marktorientierten Formen von Wohlfahrtsproduktion. Dieser Mix ergibt sich zum einen aus der vertikalen Korridorverengung, kann aber auch als Ergebnis von Lernprozessen interpretiert werden, indem über eine Diversifikation von Steuerungsinstrumenten versucht wird, die genuien Stärken verschiedener Wohlfahrtsproduzenten $\mathrm{zu}$ bündeln bzw. auszuschöpfen“ 
(Leibfried/Zürn 2006: 303). Regulierung im Sinne eines richtungsweisenden Staates, der sich auf die Gewährleistung der Wohlfahrtsstaatsproduktion konzentriert, wäre auch im Hinblick auf die kritisierten ökonomischen Maßnahmen interessant. Um zu einer effizienten Leistungskette aus staatlichen, privaten und familialen Akteuren $\mathrm{zu}$ gelangen, ist es sinnvoll lediglich die Rahmenbedingungen für den Erhalt staatlicher Leistungen zu definieren und die Ausführungsverantwortung sowie die dazugehörige Entscheidungsbefugnis so lokal wie möglich, also in der direkten Nähe des Leistungsempfängers, einzurichten. Diese Form der Weiterentwicklung des Leitbildes wäre vergleichbar mit der Entwicklung vom Aktiven zum Schlanken Staat. Thema ist hier die Produktionsweise der wohlfahrtsstaatlichen Leistungskette und nicht die ethische Diskussion zum Thema ,staatliches Handeln', so wie beim Übergang vom Schlanken zum Aktivierenden Staat. 


\section{Zusammenfassung - deutsch}

In der vorliegenden Arbeit wird der Einfluss, bzw. die Umsetzung des Leitbildes Aktivierender Staat auf die Familienpolitik in Deutschland und den Niederlanden untersucht. Im ersten Kapitel ist die Erörterung des Leitbildes aufgenommen. Einer Beschreibung der Anwendung politisch-administrativer Leitbilder zur Steuerung sozialstaatlicher Entwicklungen folgt die Erläuterung der drei dominierenden Leitbilder Demokratischer Rechtstaat, Aktiver Staat und Schlanker Staat. Da es keine eindeutige und allgemein gültige Definition des Aktivierenden Staates gibt, werden nach einer kurzen Einführung in die Hintergründe die unterschiedlichen Schulen und einflussreichen Theorien zusammengefasst und das Leitbild des Aktivierenden Staates im theoretischen Hintergrund erörtert. Das maßgebliche Prinzip des Aktivierenden Staates ist eine neue Verteilung der Verantwortlichkeiten. Sehr aufschlussreich ist in diesem Zusammenhang die Differenzierung verschiedener Risiken, die Hintergründe der teilhabenden Akteure und eine Gewichtung bzw. Verteilung von Verantwortlichkeiten. Verschiedene betriebswirtschaftliche Prinzipien dienen als Überleitung zu den aktivierenden Instrumenten und dem neuen Aufgabenverständnis. Am Ende des ersten Kapitels wird erklärt, wie sich diese Verantwortlichkeitsteilung in der so genannten Koproduktion des Aktivierenden Staates manifestiert. Eine prinzipielle Umschreibung der neuen Aufgabenwahrnehmung, sowie der Instrumente Privatisierung, ÖffentlichPrivate Zusammenarbeit und Sozialinvestition, rundet das Kapitel ab.

Die Entwicklung des Idealmodells Aktivierender Familienpolitik ist das Thema des zweiten Kapitels. Durch eine allgemeine Abhandlung über familienpolitische Hintergründe werden die Ziele und Instrumente in den familienpolitischen Kontext eingebettet. Ausgangspunkt für die Skizzierung von Zielen und Instrumenten ist das analytische Modell Kaufmanns. Anschließend hieran erfolgt der Aufbau des familienpolitischen Modells Esping-Andersens. In seinem wohlfahrtsstaatlichen Ansatz hat die Familienpolitik eine entscheidende Rolle. Ein zusammenhängendes Modell aktivierender Familienpolitik, welches zum nationalstaatlichen Politikvergleich geeignet wäre, bietet er allerdings nicht. Daher werden im zweiten Unterkapitel, die entscheidenden Kernpunkte seiner Werke ,The Three Worlds of Welfare Capitalism', ,Welfare States in Transition National Adaptions in Global Economies', ,Why We Need a New Welfare State' 
und ,The Incomplete Revolution - Adapting to Women's New Roles' erläutert. Die hieraus folgenden Kernpunkte werden mit dem Modell von Kaufmann zusammengefügt. Zur Erweiterung dieses familienpolitischen Grundmodells um die Prinzipien des Aktivierenden Staates werden die Ergebnisse aus dem ersten Kapitel verwendet. Das hieraus entstandene Idealmodell Aktivierender Familienpolitik bildet, am Ende des zweiten Kapitels, die Basis für die empirisch-analytische Arbeit.

Das dritte und vierte Kapitel haben die Verwendung familienpolitischer Ziele und Instrumente in Deutschland und den Niederlanden zum Thema. Zu Beginn dieser Kapitel ist die verwendete Arbeitsweise näher erläutert. Hierzu sind Hintergrund und Stellenwert der verwendeten Dokumente umschrieben und wird die Erörterungsweise der Ziele und Instrumente erklärt. Aus ihrer textlichen Einbettung wird nicht nur ihr Kontext in den politischen Dokumenten deutlich, sondern ergibt sich auch inwiefern der Staat selbst Verantwortung übernehmen will (und kann) bzw. von geteilten Verantwortlichkeiten ausgeht. Eine schematische Darstellung der Entwicklung dieser Ziele und Instrumente rundet die Beschreibung der untersuchten Dokumente ab. Für die Darstellung der Instrumente gilt, dass sich aus der textlichen Einbettung in den Kontext der politischen Dokumente die Form des Instruments ergibt (z.B. Sozialinvestition, Öffentlich-Private Zusammenarbeit oder Privatisierung).

Im fünften Kapitel folgen, aufbauend auf den Ergebnissen der vorherigen Kapitel, eine allgemeine, vergleichende Analyse der Zielsetzungen und Instrumente aus Sicht des Idealmodells und ein detaillierter Vergleich, bei dem die Konvergenzen und Divergenzen beider Länder herausgearbeitet werden. In den verwendeten Textfragmenten kommen die Ausgangspunkte des Aktivierenden Staates deutlich zur Geltung. Die Instrumentenanalyse beschreibt auf welche Weise die Politiker der beiden Länder die Prinzipien des Idealmodells Aktivierender Familienpolitik verarbeiteten. Außerdem bietet die Zusammensetzung des Instrumentariums gute Beispiele für die politische Praxis. Im Zwischenfazit wird beurteilt, wie die Länder das politische Instrumentarium der Aktivierenden Familienpolitik umgesetzt haben, wo es Ähnlichkeiten und Unterschiede zwischen den Ländern gibt, bzw. welche Ziele und Maßnahmen dem Idealmodell Aktivierender Familienpolitik am Nächsten kommen. 
Die Bewertung der Ergebnisse im Zusammenhang mit dem theoretischen Modell Aktivierender Familienpolitik bzw. den verschiedenen theoretischen Ansätzen von Esping-Andersen erfolgt im abschließenden Fazit dieser Arbeit. An der Stelle wird auch beurteilt, inwiefern das Leitbild für die Familienpolitik realistisch ist, an welchen Stellen es eventuell Lücken und Defizite kennt und welchen Einfluss das aktivierende Leitbild auf die europäische Sozialpolitik haben könnte. 


\section{Zusammenfassung - english}

This publication sets out to investigate how the ideal of an 'activating state' can be translated into German and Dutch family policy. The first chapter focuses on the backgrounds of this particular ideal. The description of the general politicaladministrative ideals that are used for steering developments within the welfare state is preceded by an explanation of three dominating ideals: the democratic state under the rule of law, active government and lean government. As there is no unequivocal and generally accepted definition for the ideal of an activating state, the brief introduction is followed by a summary of the various backgrounds, movements and influential theories that can be discerned, whilst the ideal of an activating state is worked out in greater detail in connection with the aforementioned theoretical backgrounds. The leading principle for the activating state is a new division of responsibilities. Highly illustrative in this regard is the differentiation between the different types of risk, the backgrounds of the parties responsible and the assessment, or rather, division of responsibilities. Various business-economic principles are used to explain the activating instruments and the new division of tasks. At the end of the first chapter, it is explained how this division of responsibilities manifests itself in the so-called co-production of the activating state. The chapter ends with a fundamental explanation of this new fulfilment of duties and the instruments of privatisation, public-private partnership and social investments.

The second chapter deals with developing an ideal model for an 'activating family policy'. A general explanation of the backgrounds of family policy places the various objectives and instruments in the context of current family policy. The point of departure for describing these goals and instruments is Kaufmann's analytical model. This is followed by the structure of the EspingAndersen's family policy model. Family policy plays a decisive role in his approach of the welfare state. However, he does not offer a coherent model for activating family policy that would be suitable for a comparison of international policies. That is why the second subchapter focuses on the essential points from his publications. The Three Worlds of Welfare Capitalism', 'Welfare States in Transition - National Adaptations in Global Economies', 'Why We Need a New Welfare State' and 'The Incomplete Revolution - Adapting to Women's New Roles'. These points, as set out in the above publications, are combined with 
Kaufmann's model. The findings of the first chapter are used to enforce the basic model for family policy with the basic principles of an activating state. The ideal model for activating family policy that is thus created is the foundation of the empirical-analytical work at the end of the second chapter.

Chapter three and four deal with the use of goals and instruments in German and Dutch family policy. Both chapters start with a more detailed explanation of the method that is used. In this context, the background and significance of the documents are explained, as is the method used for the analysis for these goals and instruments. By using selected excerpts, not only does their context within the political documents become clear, but it is also shown to what extent the government itself is willing (and able) to carry responsibility, or whether it proceeds on the basis of shared responsibility. The definition of the examined documents ends with a schematic outline of the development of the goals and instruments. It should be noted with regard to the description of the instruments that the excerpts from the political documents are used to clarify the form of the instrument (e.g. social investments, public-private partnership or privatisation).

Building on the findings from the previous chapters, the fifth chapter offers a general comparative analysis of the goals and instruments from the perspective of the ideal model and an extensive comparison in which the convergences and divergences have been worked out in greater detail. The starting points of an activating state become manifest in the excerpts that are used. The analysis of the various instruments specifies in what manner politicians from both countries have incorporated the ideal of an activating family policy. Furthermore, the composition of the set of instruments used offers good examples for putting the policy into practice. The partial conclusions offer an assessment of how the countries have translated the political set of instruments for activating family policy and describe which parallels and differences exist between both counties, i.e. which goals and instruments resemble the ideal of an activating family policy the most.

The assessment of the results in combination with the theoretical model for activating family policy and the various theoretical starting points of EspingAndersen can be found in the conclusion of this book. In this conclusion, it also assessed to what extent the model used for family policy is realistic, whilst also 
identifying whether any lacunas or shortcomings may occur, and what influence the ideal of an activating state may have on European social policy. 


\section{Zusammenfassung - dutch}

In dit boek wordt onderzocht hoe het ideaalbeeld van een 'activerende overheid' wordt vertaald naar het gezinsbeleid in Duitsland en Nederland. In het eerste hoofdstuk zijn de achtergronden van dit ideaalbeeld toegelicht. Een omschrijving van het gebruik van algemene politiek-administratieve ideaalbeelden ter sturing van welvaartsstaatsontwikkelingen volgt na de uiteenzetting van de drie dominerende ideaalbeelden: de democratische rechtsstaat, de actieve overheid en de slanke overheid. Gezien het feit dat er geen eenduidige en algemeen geldende definitie van het ideaalbeeld van een activerende overheid bestaat, worden na een korte introductie achtergronden, verschillende stromingen en invloedrijke theorieën samengevat en wordt het ideaalbeeld in samenhang met de reeds geschetste theoretische achtergronden toegelicht. Het toonaangevende principe van de activerende overheid is de nieuwe verdeling van verantwoordelijkheden. Bijzonder illustratief is in dit verband de differentiatie tussen de verschillende soorten risico's, de achtergronden van de verantwoordelijke partijen en een verantwoordingsbepaling, oftewel een verdeling van verantwoordelijkheden. Verschillende bedrijfseconomische principes dienen als uitleg voor de activerende instrumenten en de nieuwe taakverdeling tussen de verschillende verantwoordelijke partijen. Aan het einde van het eerste hoofdstuk wordt toegelicht hoe deze verdeling van verantwoordelijkheden zich in de zogenaamde coproductie van de activerende overheid manifesteert. Het hoofdstuk eindigt met een toelichting op de nieuwe taakvervulling en de instrumenten privatisering, publiek-private samenwerking en maatschappelijke investeringen.

De ontwikkeling van het ideaalmodel voor activerend gezinsbeleid is het onderwerp van het tweede hoofdstuk. Door een algemene uiteenzetting van de achtergronden van gezinsbeleid worden de doelen en instrumenten in de context van het hedendaagse gezinsbeleid geplaatst. Vertrekpunt voor het schetsen van doelen en instrumenten is het analytische model van Kaufmann. Aansluitend hierop volgt de opbouw van het gezinsbeleidsmodel van EspingAndersen. In zijn welvaartsstaatsbenadering speelt het gezinsbeleid een doorslaggevende rol. Een samenhangend model van activerend gezinsbeleid dat voor een internationale beleidsvergelijking geschikt zou zijn, biedt hij echter niet. Daarom worden in het tweede subhoofdstuk de belangrijkste kernpunten uit zijn werken, ,The Three Worlds of Welfare Capitalism', ,Welfare States in 
Transition - National Adaptations in Global Economies', ,Why We Need a New Welfare State' en ,The Incomplete Revolution - Adapting to Women's New Roles' toegelicht. De hieruit volgende kernpunten worden met het model van Kaufmann samengevoegd. Om het basismodel voor gezinsbeleid met de uitgangspunten van de activerende overheid te verrijken, worden de resultaten uit het eerste hoofdstuk gebruikt. Het hieruit ontstane ideaalmodel voor activerend gezinsbeleid vormt, aan het einde van het tweede hoofdstuk, de basis voor het empirisch-analytische werk.

Onderwerp van het derde en vierde hoofdstuk is het gebruik van doelen en instrumenten van het Duitse en Nederlandse gezinsbeleid. In het begin van deze hoofdstukken wordt de toegepaste werkwijze nader uitgelegd. In dit verband worden de achtergrond en de betekenis van de gebruikte documenten en de analysemethode van de doelen en instrumenten toegelicht. Aan de hand van geselecteerde tekstfragmenten wordt niet alleen hun context in de politieke documenten duidelijk, maar blijkt ook in hoeverre de overheid zelf verantwoordelijkheid wil (en kan) dragen of van gedeelde verantwoordelijkheden uitgaat. Een schematische schets van de ontwikkeling van de doelen en instrumenten rondt de omschrijving van de onderzochte documenten af. Voor de beschrijving van de instrumenten geldt dat uit het tekstfragment uit de politieke documenten de vorm van het instrument (bijvoorbeeld maatschappelijke investeringen, publiek-private samenwerking of privatisering) duidelijk wordt.

In het vijfde hoofdstuk volgen, voortbouwend op de resultaten van de vorige hoofdstukken, een algemene vergelijkende analyse van de doelen en instrumenten vanuit het perspectief van het ideaalmodel en een gedetailleerde vergelijking waarbij de convergenties en divergenties zijn uitgewerkt. In de toegepaste tekstonderdelen worden de uitgangspunten van de activerende overheid zichtbaar. De instrumentenanalyse verduidelijkt op welke manier de politici van beide landen de principes van het ideaalmodel voor activerend gezinsbeleid hebben verwerkt. Bovendien biedt de samenstelling van het instrumentarium goede voorbeelden voor de beleidspraktijk. In de deelconclusies wordt beoordeeld hoe de landen het politieke instrumentarium voor het activerende gezinsbeleid hebben vertaald, en waar er parallellen en verschillen tussen de landen bestaan oftewel: welke doelen en instrumenten lijken het meeste op het ideaalmodel voor activerend gezinsbeleid. 
De beoordeling van de resultaten in samenhang met het theoretische model voor activerend gezinsbeleid en de verschillende theoretische uitgangspunten van Esping-Andersen volgt in de conclusie van dit proefschrift. In de conclusie wordt ook beoordeeld in hoeverre het toegepaste model voor het gezinsbeleid realistisch is, op welke punten er eventueel lacunes of tekortkomingen bestaan en welke invloed het activerende ideaalbeeld op het sociale beleid van Europa zou kunnen hebben. 


\section{Literaturverzeichnis}

\section{Kapitel 1}

Andres, Gerd (2000): Über die Chancen, die Potenziale eines aktivierenden Sozialstaats in praktische Politik zu verwandeln, in: Mezger, Erika/West Klaus-W. (Hrsg.): Aktivierender Sozialstaat und politisches Handeln, Schüren-Verlag, Marburg, S. 41-49.

Badura, Bernhard (2000): Solidarität im Gesundheitswesen, in: Mezger, Erika/West Klaus-W. (Hrsg.): Aktivierender Sozialstaat und politisches Handeln, Schüren-Verlag, Marburg, S. 125-129.

Bandemer, Stephan von (1999): Der aktivierende Staat: Konturen einer Modernisierungsstrategie von Staat und Gesellschaft, in:

Gewerkschaftliche Monatshefte, Heft 6/99.

Bandemer, Stephan von/Hilbert, Josef (2005): Vom expandierenden zum aktivierenden Staat, in: Blanke, Bernhard et al. (Hg.): Handbuch zur Verwaltungsreform, 3. völlig überarbeitete und erweiterte Auflage, VS Verlag für Sozialwissenschaften, Wiesbaden, S. 26-35.

Blair-Schröder-Papier (1999): Der Weg nach vorn für Europas Sozialdemokraten. Ein Vorschlag von Gerhard Schröder und Tony Blair, London (Ms.).

Blanke, Bernhard/Schridde, Henning (2001): Der Aktivierende Staat als Leitbild für den Wandel der Verwaltungskultur, in: Beitrag für das Schöneberger Forum „Der aktivierende Staat und seine Beschäftigten“, 14/15.11.2001.

Blanke, Bernhard/Schridde, Henning/Plaß, Stefan (2001): Aktivierender Staat aktive Bürgergesellschaft; Eine Analyse für das Bundeskanzleramt, Universität Hannover, Hannover.

Bode, Ingo (2004): Das Ende der Einheit - Die Tranformation von Staatlichkeit im disorganisierten Wohlfahrtskapitalismus, in: Wohlfahrtsstaat Transformationen und Perspektiven, VS Verlag für Sozialwissenschaften, Wiesbaden, S. 67-89. 
Budäus, Dietrich (1998): Von der bürokratischen Steuerung zum New Public Management - Eine Einführung, in: Budäus, Dietrich/Conrad, Peter/Schreyögg, Georg (Hrsg.), New Public Management, Berlin/New York.

Bundesregierung (1998a): Aufbruch und Erneuerung - Deutschlands Weg ins 21. Jahrhundert, Koalitionsvereinbarung zwischen der Sozialdemokratischen Partei Deutschlands und Bündnis 9o/Die Grünen, Bonn.

Bundesregierung (1998b): Regierungserklärung von Bundeskanzler Gerhard Schröder vom 10. November 1998 vor dem Deutschen Bundestag, Bonn.

Bundesregierung (1999): Moderner Staat-Moderne Verwaltung: Das Programm der Bundesregierung. Kabinettsbeschluß vom 1. Dezember 1999, Berlin, Bundesregierung.

Bundesregierung (2002): Erneuerung - Gerechtigkeit - Nachhaltigkeit, Für ein wirtschaftlich starkes, soziales und ökologisches Deutschland. Für eine lebendige Demokratie, Koalitionsvereinbarung 2002 zwischen der Sozialdemokratischen Partei Deutschlands und Bündnis 9o/Die Grünen, Berlin.

Bundesregierung (2003): Regierungserklärung von Bundeskanzler Schröder am 14. März vor dem Deutschen Bundestag, Berlin.

Bundesregierung (2005a): Gemeinsam für Deutschland - mit Mut und Menschlichkeit, Koalitionsvertrag zwischen CDU, CSU und SPD, Berlin.

Bundesregierung (2005b): Regierungserklärung von Bundeskanzlerin Angela Merkel, Berlin.

Butterwege (2006): Krise und Zukunft des Sozialstaates; 3., erweiterte Auflage, VS Verlag für Sozialwissenschaften, Wiesbaden.

Czada, Robert (2004): Die neue deutsche Wohlfahrtswelt - Sozialpolitik und Arbeitsmarkt im Wandel, in Wohlfahrtsstaat - Transformation und Perspektiven, VS Verlag für Sozialwissenschaften, Wiesbaden, S. 127154 . 
Dijstelbloem, Huub/Meurs, Pauline (2007): Leervermogen in een gemengd bestel, in: Van sociale bescherming naar sociale investering; Zoektocht naar een andere verzorgingsstaat. Uitgeverij LEMMA, Den Haag, S. 281- 303.

Dingeldey, Irene (2004): Koordination zwischen Staat, Markt und Familie? Kritik der selektiven Perspektiven in der vergleichenden Wohlfahrts- und Arbeitsmarktforschung, in: Wohlfahrtsstaat - Transformation und Perspektiven, VS Verlag für Sozialwissenschaften, Wiesbaden, S. 107124 .

Engelen, Ewald/Hemerijk, Anton (2007): Proeve van een sociale investeringsagenda, in: Van sociale bescherming naar sociale investering; Zoektocht naar een andere verzorgingsstaat, Uitgeverij LEMMA, Den Haag, S. 305-325.

Engelen, Ewald/Hemerijk, Anton/Trommel, Willem (2007): Van sociale bescherming naar sociale investering; Zoektocht naar een andere verzorgingsstaat, Uitgeverij LEMMA, Den Haag.

Esping-Andersen, Gøsta/Gallie, Duncan/Hemerijk, Anton/Myles, John (2002): Why We Need a New Welfare State, Oxford University Press, Oxford.

Esping-Andersen, Gøsta (1999): Social Foundations of Postindustrial Economies, Oxford University Press, New York.

Esping-Andersen, Gøsta (2007): Gelijkheid in de toekomstige verzorgingsstaat, Drees-lezing 2 Juli 2007, Den Haag.

Evans, Mark/Cerny, Philip G. (2004): "New Labour”, Globalisierung und Sozialpolitik, in: Wohlfahrtsstaat - Transformationen und Perspektiven, VS Verlag für Sozialwissenschaften, Wiesbaden, S. 207230.

Evers, Adalbert (2000): Aktivierender Staat-eine Agenda und ihre möglichen Bedeutungen, in: Mezger, Erika/West Klaus-W. (Hrsg.): Aktivierender Sozialstaat und politisches Handeln, Schüren-Verlag, Marburg, S. 1329.

Evers, Adalbert (2004): Zivilgesellschaft und aktivierender Staat, Vortrag auf dem Kongress "Bürger machen Stadt” in Düsseldorf am 18.06.2004, veranstaltet 
vom Ministerium für Städtebau und Wohnen, Kulturelles und Sport des Landes NRW.

Evers, Adalbert (2007): Aktivierender Staat - Ein Beitrag zur Vitalisierung der Bürgergesellschaft oder nur ein Weg zur Ertüchtigung von Arbeitskraftunternehmen?, www.tup-online.com/media/md2567D.pdf, (aufgerufen am 23.10.2007).

Gilbert, Neil (2005): The "Enabling State?" from public to private responsibility for social protection: Pathways and pitfalls, OECD Social, Employment and Migration Working Papers (26), Paris.

Grundsatzabteilung, DGB-Bundesvorstand (2000): Soziale Gerechtigkeit, Sozialstaat und Innovation, in: Mezger, Erika/West Klaus-W. (Hrsg.): Aktivierender Sozialstaat und politisches Handeln, Schüren-Verlag, Marburg, S. 201-212.

Hemerijk, Anton (2007): Contingente convergentie, De doorontwikkeling van de Europese verzorgingsstaat, in: Van sociale bescherming naar sociale investering; Zoektocht naar een andere verzorgingsstaat. Uitgeverij LEMMA, Den Haag, S.83-125.

Holzapfel, Harmut (2000): Bildung und aktivierender Sozialstaat, in: Mezger, Erika/West Klaus-W. (Hrsg.): Aktivierender Sozialstaat und politisches Handeln, Schüren-Verlag, Marburg, S. 63-79.

Jann, Werner (2005): Governance als Reformstrategie - Vom Wandel und der Bedeutung verwaltungspolitischer Leitbilder, in: Schuppert, Gunnar Folke (Hrsg.): Governance - Forschung. Vergewisserung über Stand und Entwicklungslinien, Nomos, Baden-Baden, S. 21-43.

Jochem, Sven (2004): Wohlfahrtsstaatliche Reformpolitik in Verhandlungsstaaten, in: Wohlfahrtsstaat - Transformationen und Perspektiven, VS Verlag für Sozialwissenschaften, Wiesbaden, S.231-265.

Kaufmann, Franz-Xaver (2003): Sicherheit: Das Leitbild beherrschbarer Komplexität, in: Lessenich Stephan (Hrsg.): Wohlfahrtsstaatliche Grundbegriffe; Historische und aktuelle Diskurse, Campus Verlag, Frankfurt/New York, S. 73-104. 
Kevenhörster, Paul (2006): Politikwissenschaft, Band 2, Ergebnisse und Wirkungen der Politik, VS-Verlag, Wiesbaden.

Knijn, Trudie (2004): Het prijzen van de zorg; Sociaal beleid op het snijvlak van privé en publiek, Rede uitgesproken bij de aanvaarding van het ambt van hoogleraar Algemene Sociale Wetenschappen in het bijzonder Zorg en Welzijn aan de Universiteit Utrecht, Utrecht.

Knijn, Trudie (2007): Tussen kinderdagverblijf en verzorgingstehuis - Gezinsbeleid en levenslopen, in: Van sociale bescherming naar sociale investering; Zoektocht naar een andere verzorgingsstaat. Uitgeverij LEMMA, Den Haag, S. $55-81$.

Kötter, Ute (1997): Das niederländische Wohlfahrtsstaatsmodell - kein Vorbild mehr?, in Sozialer Fortschritt, Jg. 46, H. 1-2, S. 12-16.

Lamping, Wolfgang/Schridde, Henning/Plaß, Stefan/Blanke, Bernhard (2002): Der Aktivierende Staat. Positionen, Begriffe, Strategien. Studie für den Arbeitskreis Bürgergesellschaft und Aktivierender Staat der Friedrich-EbertStiftung, Friedrich-Ebert-Stiftung, Bonn.

Lamping, Wolfgang/Schridde, Henning (2004): Der „Aktivierende Sozialstaat“ordnungs- und steuerungstheoretische Dimensionen, in: Wohlfahrtsstaat Transformationen und Perspektiven, VS Verlag für Sozialwissenschaften, Wiesbaden, S. 39-65.

Lütz, Susanne (2004): Der Wohlfahrtsstaat im Umbruch - Neue Herausforderungen, wissenschaftiche Kontroversen und Umbauprozesse, in: Wohlfahrtsstaat - Transformationen und Perspektiven, VS Verlag für Sozialwissenschaften, Wiesbaden, S. 11-35.

Leggewie, Claus/Evers, Adalbert (1999): Der ermunternde Staat - Vom aktiven Staat zur aktivierenden Politik, in: Gewerkschaftliche Monatshefte. Nr. 6/1999, S. 81-97, S. 332-340.

Mayntz, Renate/Scharpf, Fritz (1973): Planungsorganisation; Die Diskussion um die Reform von Regierung und Verwaltung des Bundes, R. Piper \& Co. Verlag, München, S. 116.

Mezger, Erika/West Klaus-W. (Hrsg.) (2000): Aktivierender Sozialstaat und politisches Handeln, Schüren-Verlag, Marburg. 
Ministerie van Algemene Zaken (1994a): Regeerakkoord van 13 augustus 1994, Den Haag.

Ministerie van Algemene Zaken (1994b): Regeringsverklaring van 31 augustus 1994, Den Haag.

Ministerie van Algemene Zaken (1998a): Regeerakkoord 1998, Den Haag.

Ministerie van Algemene Zaken (1998b): Regeringsverklaring van 25 augustus 1998, Den Haag.

Ministerie van Algemene Zaken (2002a): Werken aan vertrouwen, een kwestie van aanpakken, Strategisch Akkoord voor Kabinet CDA, LPF, VVD, Den Haag.

Ministerie van Algemene Zaken (2002b): Regeringsverklaring kabinet Balkenende, Duidelijkheid en Daadkracht, Den Haag.

Ministerie van Algemene Zaken (2003a): Meedoen, meer werk, minder regels, Hoofdlijnenakkoord voor het kabinet CDA, VVD, D66, Den Haag.

Ministerie van Algemene Zaken (2003b): Regeringsverklaring 11 Juni 2003, Den Haag.

Ministerie van Algemene Zaken (2005): Afspraken 26 maart 2005, Den Haag.

Ministerie van Algemene Zaken (2006): Regeringsverklaring Balkenende-III, Den Haag.

Ministerie van Algemene Zaken (2007a): Coalitieakkoord tussen de Tweede Kamerfracties van CDA, PvdA en ChristenUnie, Den Haag.

Ministerie van Algemene Zaken (2007b): Regeringsverklaring 1 maart 2007, Den Haag.

Ministerie van Volksgezondheid, Welzijn en Sport (1996): Notitie Gezin, de maatschappelijke positie van het gezin, Rijswijk.

Ministerie van Volksgezondheid, Welzijn en Sport (2006): Nota gezinsbeleid, Den Haag.

Ministerie van Volksgezondheid, Welzijn en Sport (2007): Jeugd en Gezin, Programmaministerie, www.minvws.nl, (aufgerufen am 13.08.2007). 
Naschold, Frieder/Bogumil, Jörg (2000): Modernisierung des Staates, New Public Management in deutscher und internationaler Perspektive, 2., vollständig aktualisierte und stark erweiterte Auflage, Leske + Budrich, Opladen.

NiederlandeNet: Das Poldermodell, www.niederlandenet.de, (aufgerufen am 30.07.2007).

NiederlandeNet: Die Niederlande nach 1945: Politik und politische Kultur, www.niederlandenet.de, (aufgerufen am 30.07.2007).

Olk, Thomas (2000): Weder Rund-um-Versorgung noch “pure” Eigenverantwortung - aktivierende Strategien in der Politik für Familien, alte Menschen, Frauen, Kinder und Jugendliche, in: Mezger, Erika/West Klaus-W. (Hrsg.):

Aktivierender Sozialstaat und politisches Handeln, Schüren-Verlag, Marburg, S. 105-124.

Online Verwaltungslexikon (2007a): Aktivierender Staat, http://www.olev.de/a/aktiver_staat.htm, (aufgerufen am 23.10.2007).

Online Verwaltungslexikon (2007b): Managementbegriffe N: Definitionen (Neues Steuerungsmodell), http://www.olev.de/n.htm, (aufgerufen am 23.10.2007).

Osborne, David/Gaebler, Ted (1992): Reinventing Government, How the Entrepeneurial spirit is transforming the Public Sector, PLUME, New York.

Parlement en Politiek (2007): Poldermodel, www.parlement.com, (aufgerufen am 05.07.2007).

Priddat, Birger P. (2003): Umverteilung: Von der Ausgleichssubvention zur Sozialinvestition, in: Lessenich, Stephan (Hrsg.): Wohlfahrtsstaatliche Grundbegriffe; Historische und aktuelle Diskurse, Campus Verlag, Frankfurt/New York, S. 373-394.

Priddat, Birger P. (2004): New governance: Sozialpolitik als human capital investment, in: Lütz, Susanne/Cyada, Roland (Hrsg.): Wohlfahrtsstaat Transformationen und Perspektiven, VS Verlag für Sozialwissenschaften, Wiesbaden, S. 91-106. 
Reichard, Christoph (1996): Umdenken im Rathaus. Neue Steuerungsmodelle in der deutschen Kommunalverwaltung, Edition Sigma, Berlin.

Reichard, Christoph (2002): Verwaltung als öffentliches Management, in: König, Klaus (Hrsg.): Deutsche Verwaltung an der Wende zum 21. Jahrhundert, Nomos Verlagsgesellschaft, Baden-Baden, S. 255-277.

Reichard, Christoph (2006): Öffentliche Dienstleistungen für die Bürger. Wege zu Effizienz, Qualität und günstigen Preisen, Referate eines Symposiums der Gesellschaft für öffentliche Wirtschaft, der Deutschen Sektion des Europäischen Zentralverbandes der öffentlichen Wirtschaft (CEEP), des Verbandes kommunaler Unternehmen, des Verbandes Deutscher Verkehrsunternehmen und des Deutschen Städtetages, (Hrsg.): Gesellschaft für öffentliche Wirtschaft, Berlin, S. 53-79.

Reichard, Christoph/Schuppan, Tino (2000): Wie ernst ist es der Bundesregierung mit dem Thema „Aktivierender Staat"?: Anmerkungen zum Konzept der Bundesregierung zur Staats- und Verwaltungsmodernisierung, in: Mezger, Erika/West, Klaus (Hg.): Aktivierender Sozialstaat und politisches Handeln, Hans-Böckler-Stiftung, Marburg, S. 81-97.

Schmid, Günther (2007): Sociaal risicomanagment door transitionele arbeidsmarkten, in: Van sociale bescherming naar sociale investering; Zoektocht naar een andere verzorgingsstaat. Uitgeverij LEMMA, Den Haag, S. 31-53.

Schmid, Josef (2002): Wohlfahrtsstaaten im Vergleich, 2. Auflage, Leske/Budrich, Opladen, S. 179-200.

Schmidt, Nora (Hrsg.) (2006): Handbuch Kommunale Familienpolitik, Ein Praxishandbuch für mehr Familienfreundlichkeit in Kommunen, Eigenverlag des Deutschen Vereins für öffentliche und private Fürsorge e.V., Berlin.

Schröder, Gerhard (2000): Die zivile Bürgergesellschaft. Anregungen zu einer Neubestimmung der Aufgaben von Staat und Gesellschaft, in: Die Neue Gesellschaft - Frankfurter Hefte, 47. Jg., 2000, H. 4, S. $200-207$. 
Schroeder, Wolfgang/Weinert, Rainer (2004): Arbeitsbeziehungen in Schweden und Deutschland, in: Wohlfahrtsstaat - Transformationen und Perspektiven, VS Verlag für Sozialwissenschaften, Wiesbaden, S. 267295 .

Schröter, Eckhard/Wollmann, Hellmut (2005): New Public Management, in: Blanke, Bernhard/Bandemer von, Stephan/Nullmeier, Frank/Wewer, Göttrik: Handbuch zur Verwaltungsreform, 3. völlig überarbeitete und erweiterte Auflage, VS Verlag für Sozialwissenschaften, Wiesbaden, S. 63-74.

Schubert, Klaus/Bandelow, Nils (2003): Lehrbuch der Politikfeldanalyse, Oldenbourg Wissenschaftsverlag GmbH, München.

Schulze-Böing (2000): Leitbild "aktivierende Stadt" - Konzepte zur aktivierenden

Sozialpolitik und Arbeitsförderung auf kommunaler Ebene in: Mezger,

Erika/West Klaus-W. (Hrsg.): Aktivierender Sozialstaat und politisches Handeln, Schüren-Verlag, Marburg, S. 41-49.

Schuppert, Gunnar Folke (2000): Verwaltungswissenschaft, Verwaltung, Verwaltungsrecht, Verwaltungslehre, Nomos Verlagsgesellschaft, Baden-Baden.

Schuppert, Gunnar Folke (2001): Der moderne Staat als Gewährleistungsstaat, in:

Schröter, Eckhard (Hg.): Empirische Policy- und

Verwaltungsforschung. Lokale, nationale und internationale

Perspektiven, Opladen: Westdeutscher Verlag, S. 399-414.

Schuppert, Gunnar Folke (2005): Der Gewährleistungsstaat; Ein Leitbild auf dem Prüfstand, in: Schriften zur Governance Forschung, Alfred Herrhausen Gesellschaft für internationalen Dialog, Band 2, Nomos Verlagsgesellschaft, Baden-Baden.

Seils, Eric (2004): Sozialstaat und Arbeitsmarkt im Strukturwandel. Eine Analyse des Anpassungsprozesses in der Bundesrepublik, in Wohlfahrtsstaat - Transformation und Perspektiven, VS Verlag für Sozialwissenschaften, Wiesbaden, S. 155-178.

Sociaal Economische Raad/Wetenschapelijke Raad voor het Regeringsbeleid (2007): Toekomst van de Nederlandse verzorgingsstaat, SER/WRR- 
symposium over de toekomst van de Nederlandse verzorgingsstaat op 16 januari 2007.

Sozialberichterstattung Gesellschaftliche Trends Aktuelle Informationen (2002): Allmählicher Umbau bei nach wie vor deutlichen nationalen Unterschieden, Analysen zu jüngsten Wandlungstendenzen in westlichen Wohlfahrtsstaaten, in: Informationsdienst Soziale Indikatoren, Ausgabe 28, Juli 2002, S. 1-6.

Trommel Willem (2007): De stagnatie voorbij? Over de verdiensten van de sociale investeringsgedachte, in: Van sociale bescherming naar sociale investering; Zoektocht naar een andere verzorgingsstaat. Uitgeverij LEMMA, Den Haag, S.247-279.

Visser, Jelle/Hemerijck, Anton (1997): A Dutch Miracle - Job Growth, Welfare Reform and Corporatism in the Netherlands, Amsterdam University Press, Amsterdam.

Walter, Jürgen (2000): Pleite oder Neubeginn einer sozialen Staats- und Bildungs(reform)politik, in: Mezger, Erika/West Klaus-W. (Hrsg.): Aktivierender Sozialstaat und politisches Handeln, Schüren-Verlag, Marburg, S. 151-165.

West, Klaus-W. (2000): Reformen für den Sozialstaat, gesellschaftliche Mehrheiten für Reformen, in: Mezger, Erika/West Klaus-W. (Hrsg.): Aktivierender Sozialstaat und politisches Handeln, Schüren-Verlag, Marburg, S. 173189.

Wetenschappelijke Raad voor het Regeringsbeleid (2006): De verzorgingsstaat herwogen; Over verzorgen, verzekeren, verheffen en verbinden, Amsterdam University Press, Amsterdam.

Wohlfahrt, Norbert (2007a): Der aktivierende Sozialstaat - Ein neues sozialpolitisches Konzept und seine Konsequenzen, in: Transparent, Nr. 61, Oktober 2001, Duisburg, http://www.transparentonline.de (aufgerufen am 19. Mai 2007).

Wohlfahrt, Norbert (2007b): Bürgerschaftliches Engagement, Freie Wohlfahrtspflege und aktivierender Sozialstaat - inszenierter Sozialstaatsumbau oder Stärkung des sozialen Kapitals?, www.efh- 
bochum.de/homepages/wohlfahrt/pdf/engagement.pdf, aufgerufen am 23.10.2007).

\section{Kapitel 2}

Andres, Gerd (2000): Über die Chancen, die Potenziale eines aktivierenden Sozialstaats in praktische Politik zu verwandeln, in: Mezger, Erika/West Klaus-W. (Hrsg.): Aktivierender Sozialstaat und politisches Handeln, Schüren-Verlag, Marburg, S. 41-49.

Badura, Bernhard (2000): Solidarität im Gesundheitswesen, in: Mezger, Erika/West Klaus-W. (Hrsg.): Aktivierender Sozialstaat und politisches Handeln, Schüren-Verlag, Marburg, S. 125-129.

Bahle, Thomas (2000): Familienpolitik in Westeuropa. Ursprünge und Wandel im historischen Vergleich, Frankfurt/M., New York, S. 16-21.

Bandemer, Stephan von (1999): Der aktivierende Staat: Konturen einer Modernisierungsstrategie von Staat und Gesellschaft, in:

Gewerkschaftliche Monatshefte, Heft 6/99.

Bandemer, Stephan von/Hilbert, Josef (2005): Vom expandierenden zum aktivierenden Staat, in: Blanke, Bernhard et al. (Hg.): Handbuch zur Verwaltungsreform, 3. völlig überarbeitete und erweiterte Auflage, VS Verlag für Sozialwissenschaften, Wiesbaden, S. 26-35.

Bertram, Hans (2006): Nachhaltige Familienpolitik im Europäischen Vergleich, in: Peter A. Berger und Heike Kahlert (Hrsg.), Der demographische Wandel. Chancen für die Neuordnung der Geschlechterverhältnisse, Campus, Frankfurt/M., S. 203-236.

Blair-Schröder-Papier (1999): Der Weg nach vorn für Europas Sozialdemokraten. Ein Vorschlag von Gerhard Schröder und Tony Blair, London (Ms.).

Blanke, Bernhard/Schridde, Henning (2001): Der Aktivierende Staat als Leitbild für den Wandel der Verwaltungskultur, in: Beitrag für das Schöneberger Forum „Der aktivierende Staat und seine Beschäftigten“, 14/15.11.2001. 
Blanke, Bernhard/Schridde, Henning/Plaß, Stefan (2001): Aktivierender Staat aktive Bürgergesellschaft; Eine Analyse für das Bundeskanzleramt, Universität Hannover, Hannover.

Blanke, Bernhard/Thörmer, Heinz (1998): Social democratic reform politics and the „activating state“, http://www.aktivierenderstaat.de/literatur/bbbhtsoz.htm (aufgerufen am 23.10.2007).

Bode, Ingo (2004): Das Ende der Einheit - Die Transformation von Staatlichkeit im disorganisierten Wohlfahrtskapitalismus, in: Wohlfahrtsstaat Transformationen und Perspektiven, VS Verlag für Sozialwissenschaften, Wiesbaden, S. 67-89.

Bos, Jasja/Hooghiemstra, Erna (2004): Het gezinsdal: verandering van koopkracht na het krijgen van kinderen, in: Bevolkingstrends, 4e kwartaal 2004, Centraal Bureau voor de Statistiek, Den Haag, 61-67.

Brinkgreve, Christien/Velde, Egbert, te (2006): Wie wil er nu nog moeder worden? Uitgeverij Augustus, Amsterdam/Antwerpen.

Budäus, Dietrich (1998): Von der bürokratischen Steuerung zum New Public Management - Eine Einführung, in: Budäus, Dietrich/Conrad, Peter/Schreyögg, Georg (Hrsg.), New Public Management, Berlin/New York.

Bundesregierung (1998a): Aufbruch und Erneuerung - Deutschlands Weg ins 21. Jahrhundert, Koalitionsvereinbarung zwischen der Sozialdemokratischen Partei Deutschlands und Bündnis 9०/Die Grünen, Bonn.

Bundesregierung (1998b): Regierungserklärung von Bundeskanzler Gerhard Schröder vom 10. November 1998 vor dem Deutschen Bundestag, Bonn.

Bundesregierung (1999): Moderner Staat - Moderne Verwaltung: Das Programm der Bundesregierung. Kabinettsbeschluss vom 1. Dezember 1999, Berlin, Bundesregierung.

Bundesregierung (2002): Erneuerung - Gerechtigkeit - Nachhaltigkeit, Für ein wirtschaftlich starkes, soziales und ökologisches Deutschland. Für eine lebendige Demokratie, Koalitionsvereinbarung 2002 zwischen der 
Sozialdemokratischen Partei Deutschlands und Bündnis 9०/Die Grünen, Berlin.

Bundesregierung (2003): Regierungserklärung von Bundeskanzler Schröder am 14. März vor dem Deutschen Bundestag, Berlin.

Bundesregierung (2005a): Gemeinsam für Deutschland - mit Mut und Menschlichkeit, Koalitionsvertrag zwischen CDU, CSU und SPD, Berlin.

Bundesregierung (2005b): Regierungserklärung von Bundeskanzlerin Angela Merkel, Berlin.

Czada, Robert (2004): Die neue deutsche Wohlfahrtswelt - Sozialpolitik und Arbeitsmarkt im Wandel, in Wohlfahrtsstaat - Transformation und Perspektiven, VS Verlag für Sozialwissenschaften, Wiesbaden, S. 127154 .

Dijstelbloem, Huub/Meurs, Pauline (2007): Leervermogen in een gemengd bestel, in: Van sociale bescherming naar sociale investering; Zoektocht naar een andere verzorgingsstaat. Uitgeverij LEMMA, Den Haag, S. 281303.

Dingeldey, Irene (2004): Koordination zwischen Staat, Markt und Familie? Kritik der selektiven Perspektiven in der vergleichenden Wohlfahrts- und Arbeitsmarktforschung, in: Wohlfahrtsstaat - Transformation und Perspektiven, VS Verlag für Sozialwissenschaften, Wiesbaden, S. 107124 .

Engelen, Ewald/Hemerijk, Anton (2007): Proeve van een sociale investeringsagenda, in: Van sociale bescherming naar sociale investering; Zoektocht naar een andere verzorgingsstaat, Uitgeverij LEMMA, Den Haag, S. 305-325.

Engelen, Ewald/Hemerijk, Anton/Trommel, Willem (2007): Van sociale bescherming naar sociale investering; Zoektocht naar een andere verzorgingsstaat, Uitgeverij LEMMA, Den Haag.

Esch, Karin/Hilbert, Josef/Stöbe-Blossey, Sybille (2001): Der aktivierende Staat Konzept, Potentiale und Entwicklungstrends am Beispiel der Jugendhilfe, in: Heinze, Rolf G./Olk, Thomas (Hrsg.), Bürgerengagement in 
Deutschland: Bestandsaufnahmen und Perspektiven, Leske \& Budrich, Opladen, S. 519-547.

Esping-Andersen, Gøsta (1990): The Three Worlds of Welfare Capitalism, Polity Press, Cambridge.

Esping-Andersen, Gøsta (1996): Welfare States in Transition - National

Adaptations in Global Economies, SAGE Publications, London/Thousand Oaks/New Dehli.

Esping-Andersen, Gøsta/Gallie, Duncan/Hemerijk, Anton/Myles, John (2002): Why We Need a New Welfare State, Oxford University Press, Oxford.

Esping-Andersen, Gøsta (2007): Gelijkheid in de toekomstige verzorgingsstaat, Drees-lezing 2 Juli 2007, Den Haag.

Esping-Andersen, Gøsta (2008a): Investing in children and their Life Chances, http://dcpis.upf.edu/ gosta-esping-andersen/articles.htm (aufgerufen am 17.04.2008).

Esping-Andersen, Gøsta (2008b): Trois Lectures (II); Equal Opportunities in an Increasingly Hostile World, http://dcpis.upf.edu/ gosta-espingandersen/articles.htm (aufgerufen am 17.04.2008).

Esping-Andersen, Gøsta (2009): The Incomplete Revolution - Adapting to Women's New Roles, Polity Press, Cambridge.

Evans, Mark/Cerny, Philip G. (2004): "New Labour", Globalisierung und Sozialpolitik, in: Wohlfahrtsstaat - Transformationen und Perspektiven, VS Verlag für Sozialwissenschaften, Wiesbaden, S. 207230.

Evers, Adalbert (2000): Aktivierender Staat - eine Agenda und ihre möglichen Bedeutungen, in: Mezger, Erika/West Klaus-W. (Hrsg.): Aktivierender Sozialstaat und politisches Handeln, Schüren-Verlag, Marburg, S. 1329.

Evers, Adalbert (2004): Zivilgesellschaft und aktivierender Staat, Vortrag auf dem Kongress "Bürger machen Stadt" in Düsseldorf am 18.06.2004, veranstaltet vom Ministerium für Städtebau und Wohnen, Kulturelles und Sport des Landes NRW. 
Evers, Adalbert (2007): Aktivierender Staat - Ein Beitrag zur Vitalisierung der Bürgergesellschaft oder nur ein Weg zur Ertüchtigung von Arbeitskraftunternehmen?, www.tup-online.com/mediaPM2567D.pdf, (aufgerufen am 23.10.2007).

Gerlach, Irene (2004): Familienpolitik, Lehrbuch, VS Verlag für Sozialwissenschaften, Wiesbaden.

Gerlach, Irene (2010): Familienpolitik - 2., aktualisierte und überarbeitete Auflage, Lehrbuch, VS Verlag für Sozialwissenschaften/GWV Fachverlage $\mathrm{GmbH}$, Wiesbaden.

Gilbert, Neil (2005): The “Enabling State?” from public to private responsibility for social protection: Pathways and pitfalls, OECD Social, Employment and Migration Working Papers (26), Paris.

Grundsatzabteilung, DGB-Bundesvorstand (2000): Soziale Gerechtigkeit, Sozialstaat und Innovation, in: Mezger, Erika/West Klaus-W. (Hrsg.): Aktivierender Sozialstaat und politisches Handeln, Schüren-Verlag, Marburg, S. 201-212.

Hemerijk, Anton (2007): Contingente convergentie, De doorontwikkeling van de Europese verzorgingsstaat, in: Van sociale bescherming naar sociale investering; Zoektocht naar een andere verzorgingsstaat. Uitgeverij LEMMA, Den Haag, S.83-125.

Herlth, Alois/Kaufmann, Franz-Xaver (1982): Zur Einführung: Familiale Probleme und sozialpolitische Intervention, in: Kaufmann, Franz-Xaver (Hrsg.): Staatliche Sozialpolitik und Familie, München/Wien, S. 1-22.

Holzapfel, Hartmut (2000): Bildung und aktivierender Sozialstaat, in: Mezger, Erika/West Klaus-W. (Hrsg.): Aktivierender Sozialstaat und politisches Handeln, Schüren-Verlag, Marburg, S. 63-79.

Jann, Werner (2005): Governance als Reformstrategie - Vom Wandel und der Bedeutung verwaltungspolitischer Leitbilder, in: Schuppert, Gunnar Folke (Hrsg.): Governance - Forschung. Vergewisserung über Stand und Entwicklungslinien, Nomos, Baden-Baden, S. 21-43. 
Jochem, Sven (2004): Wohlfahrtsstaatliche Reformpolitik in Verhandlungsstaaten, in: Wohlfahrtsstaat - Transformationen und Perspektiven, VS Verlag für Sozialwissenschaften, Wiesbaden, S.231-265.

Kaufmann, Franz-Xaver (1982): Staatliche Sozialpolitik und Familie, Oldenbourg, München.

Kaufmann, Franz-Xaver (1993): Familienpolitik in Europa, in: Bundesministerium für Familie und Senioren: 40 Jahre Familienpolitik in der Bundesrepublik Deutschland, Rückblick/Ausblick, Festschrift, Luchterhand, Berlin.

Kaufmann, Franz-Xaver/Kuijsten, Anton/Schulze, Hans-Joachim/Strohmeier, Klaus-Peter (2002): Family Life and Family Policies in Europe, Volume 2, Problems and issues in comparative perspective, Oxford, New York, S. $419-490$.

Kevenhörster, Paul (2006): Politikwissenschaft, Band 2, Ergebnisse und Wirkungen der Politik, VS-Verlag, Wiesbaden.

Knijn, Trudie (2004): Het prijzen van de zorg; Sociaal beleid op het snijvlak van privé en publiek, Rede uitgesproken bij de aanvaarding van het ambt van hoogleraar Algemene Sociale Wetenschappen in het bijzonder Zorg en Welzijn aan de Universiteit Utrecht, Utrecht.

Knijn, Trudie (2007): Tussen kinderdagverblijf en verzorgingstehuis - Gezinsbeleid en levenslopen, in: Van sociale bescherming naar sociale investering; Zoektocht naar een andere verzorgingsstaat. Uitgeverij LEMMA, Den Haag, S. $55-81$.

Kötter, Ute (1997): Das niederländische Wohlfahrtsstaatsmodell - kein Vorbild mehr?, in Sozialer Fortschritt, Jg. 46, H. 1-2, S. 12-16.

Kremer, Monique (2007): How Welfare States Care, Culture, Gender and Parenting in Europe, Amsterdam University Press, Amsterdam.

Lammert, Christian (2005): Still liberal welfare states?; Umverteilung, Armut und die Reform der Wohlfahrtsstaaten in den USA und Kanada im internationalen Vergleich, Zentrum für Nordamerika-Forschung, Frankfurt/Main. 
Lamping, Wolfgang/Schridde, Henning/Plaß, Stefan/Blanke, Bernhard (2002): Der Aktivierende Staat. Positionen, Begriffe, Strategien. Studie für den Arbeitskreis Bürgergesellschaft und Aktivierender Staat der FriedrichEbert-Stiftung, Friedrich-Ebert-Stiftung, Bonn.

Lamping, Wolfgang/Schridde, Henning (2004): Der „Aktivierende Sozialstaat“ordnungs- und steuerungstheoretische Dimensionen, in: Wohlfahrtsstaat Transformationen und Perspektiven, VS Verlag für Sozialwissenschaften, Wiesbaden, S. 39-65.

Lütz, Susanne (2004): Der Wohlfahrtsstaat im Umbruch - Neue Herausforderungen, wissenschaftliche Kontroversen und Umbauprozesse, in: Wohlfahrtsstaat - Transformationen und Perspektiven, VS Verlag für Sozialwissenschaften, Wiesbaden, S. 11-35.

Leggewie, Claus/Evers, Adalbert (1999): Der ermunternde Staat - Vom aktiven Staat zur aktivierenden Politik, in: Gewerkschaftliche Monatshefte. Nr. 6/1999. S. 81-97, S. 332-340.

Michel, Sonya/Mahon, Rianne (2002): Child care policy at the crossroads, gender and welfare state restructuring, Routledge, New York/London.

Mezger, Erika/West Klaus-W. (Hrsg.) (2000): Aktivierender Sozialstaat und politisches Handeln, Schüren-Verlag, Marburg.

Ministerie van Algemene Zaken (1994a): Regeerakkoord van 13 augustus 1994, Den Haag.

Ministerie van Algemene Zaken (1994b): Regeringsverklaring van 31 augustus 1994, Den Haag.

Ministerie van Algemene Zaken (1998a): Regeerakkoord 1998, Den Haag.

Ministerie van Algemene Zaken (1998b): Regeringsverklaring van 25 augustus 1998, Den Haag.

Ministerie van Algemene Zaken (2002a): Werken aan vertrouwen, een kwestie van aanpakken, Strategisch Akkoord voor Kabinet CDA, LPF, VVD, Den Haag.

Ministerie van Algemene Zaken (2002b): Regeringsverklaring kabinet Balkenende, Duidelijkheid en Daadkracht, Den Haag. 
Ministerie van Algemene Zaken (2003a): Meedoen, meer werk, minder regels, Hoofdlijnenakkoord voor het kabinet CDA, VVD, D66, Den Haag.

Ministerie van Algemene Zaken (2003b): Regeringsverklaring 11 Juni 2003, Den Haag.

Ministerie van Algemene Zaken (2005): Afspraken 26 maart 2005, Den Haag.

Ministerie van Algemene Zaken (2006): Regeringsverklaring Balkenende-III, Den Haag.

Ministerie van Algemene Zaken (2007a): Coalitieakkoord tussen de Tweede Kamerfracties van CDA, PvdA en ChristenUnie, Den Haag.

Ministerie van Algemene Zaken (2007b): Regeringsverklaring 1 maart 2007, Den Haag.

Ministerie van Volksgezondheid, Welzijn en Sport (1996): Notitie Gezin, de Maatschappelijke positie van het gezin, Rijswijk.

Ministerie van Volksgezondheid, Welzijn en Sport (2006): Nota gezinsbeleid, Den Haag.

Ministerie van Volksgezondheid, Welzijn en Sport (2007): Jeugd en Gezin, Programmaministerie, www.minvws.nl (aufgerufen am 13.08.2007).

Naschold, Frieder/Bogumil, Jörg (2000): Modernisierung des Staates, New Public Management in deutscher und internationaler Perspektive, 2., vollständig aktualisierte und stark erweiterte Auflage, Leske + Budrich, Opladen.

NiederlandeNet (2007a): Das Poldermodell, www.niederlandenet.de (aufgerufen am 30.07.2007).

NiederlandeNet (2007b): Die Niederlande nach 1945: Politik und politische Kultur, www.niederlandenet.de (aufgerufen am 30.07.2007).

Niejahr Elisabeth (2003): Politik vom Wickeltisch, http://images.zeit.de/text/2003/41/Esping-Andersen (aufgerufen am 17.04.2008).

OECD (2002): Babies and Bosses: Reconciling Work and Family Life, OECD Publications Service, Paris. 
Olk, Thomas (2000): Weder Rund-um-Versorgung noch "pure" Eigenverantwortung - aktivierende Strategien in der Politik für Familien, alte Menschen, Frauen, Kinder und Jugendliche, in: Mezger, Erika/West Klaus-W. (Hrsg.): Aktivierender Sozialstaat und politisches Handeln, Schüren-Verlag, Marburg, S. 105-124.

Online Verwaltungslexikon (2007a): Aktivierender Staat, http://www.olev.de/a/aktiver_staat.htm (aufgerufen am 23.10.2007).

Online Verwaltungslexikon (2007b): Managementbegriffe N: Definitionen (Neues Steuerungsmodell), http://www.olev.de/n.htm (aufgerufen am 23.10.2007).

Opielka, Michael (2002): Die groben Unterschiede, in: Hellmann, KayUwe/Fischer, Karsten/Bluhm, Harald: Das System der Politik. Niklas Luhmanns politische Theorie in der Diskussion, Westdeutscher Verlag, Opladen.

Opielka, Michael (2004): Sozialpolitik; Grundlagen und vergleichende Perspektiven, Rowohlt Verlag GmbH, Reinbeck bei Hamburg.

Osborne, David/Gaebler, Ted (1992): Reinventing Government, How the Entrepeneurial spirit is transforming the Public Sector, PLUME, New York.

Ostner, Ilona (2004): Von der Dekommodifizierung zur De-Familialisierung Esping-Andersens Arbeit an den Welten, http://www.wiso.unikoeln.de/soziologie/altesDesign/o2_stud/2003-04/os/1296_ostner (aufgerufen am 07.05.2008).

Parlement en Politiek (2007): Poldermodel, www.parlement.com (aufgerufen am 05.07.2007).

Pfenning, Astrid/Bahle, Thomas (2000): Families and Family Policies in Europe: Comparative Perspectives, in: Eurodata Newsletter No. 12/13, S. 24 .

Priddat, Birger P. (2003): Umverteilung: Von der Ausgleichssubvention zur Sozialinvestition, in: Lessenich, Stephan (Hrsg.): Wohlfahrtsstaatliche Grundbegriffe; Historische und aktuelle Diskurse, Campus Verlag, Frankfurt/New York, S. 373-394. 
Priddat, Birger P. (2004): New governance: Sozialpolitik als human capital investment, in: Lütz, Susanne/Cyada, Roland (Hrsg.): Wohlfahrtsstaat Transformationen und Perspektiven, VS Verlag für Sozialwissenschaften, Wiesbaden, S. 91-106.

Reichard, Christoph (1996): Umdenken im Rathaus. Neue Steuerungsmodelle in der deutschen Kommunalverwaltung, Edition Sigma, Berlin.

Reichard, Christoph (2002): Verwaltung als öffentliches Management, in: König, Klaus (Hrsg.): Deutsche Verwaltung an der Wende zum 21. Jahrhundert, Nomos Verlagsgesellschaft, Baden-Baden, S. 255-277.

Reichard, Christoph/Schuppan, Tino (2000): Wie ernst ist es der Bundesregierung mit dem Thema „Aktivierender Staat“?: Anmerkungen zum Konzept der Bundesregierung zur Staats- und Verwaltungsmodernisierung, in: Mezger, Erika/West, Klaus (Hg.): Aktivierender Sozialstaat und politisches Handeln, Hans-Böckler-Stiftung, Marburg, S. 81-97.

Schmid, Günther (2007): Sociaal risicomanagment door transitionele arbeidsmarkten, in: Van sociale bescherming naar sociale investering; Zoektocht naar een andere verzorgingsstaat. Uitgeverij LEMMA, Den Haag, S. 31-53.

Schmid, Josef (2002): Wohlfahrtsstaaten im Vergleich, 2. Auflage, Leske/Budrich, Opladen, S. 179-200.

Schmidt, Nora (Hrsg.) (2006): Handbuch Kommunale Familienpolitik, Ein Praxishandbuch für mehr Familienfreundlichkeit in Kommunen, Eigenverlag des Deutschen Vereins für öffentliche und private Fürsorge e.V., Berlin.

Schröder, Gerhard (2000): Die zivile Bürgergesellschaft. Anregungen zu einer Neubestimmung der Aufgaben von Staat und Gesellschaft, in: Die Neue Gesellschaft - Frankfurter Hefte, 47. Jg., 2000, H. 4, S. $200-207$.

Schroeder, Wolfgang/Weinert, Rainer (2004): Arbeitsbeziehungen in Schweden und Deutschland, in: Wohlfahrtsstaat - Transformationen und Perspektiven, VS Verlag für Sozialwissenschaften, Wiesbaden, S. 267295. 
Schröter, Eckhard/Wollmann, Hellmut (2005): New Public Management, in: Blanke, Bernhard/Bandemer von, Stephan/Nullmeier, Frank/Wewer, Göttrik: Handbuch zur Verwaltungsreform, 3. völlig überarbeitete und erweiterte Auflage, VS Verlag für Sozialwissenschaften, Wiesbaden, S. $63-74$.

Schubert, Klaus/Bandelow, Nils (2003): Lehrbuch der Politikfeldanalyse, Oldenbourg Wissenschaftsverlag GmbH, München.

Schulze-Böing (2000): Leitbild "aktivierende Stadt" - Konzepte zur aktivierenden Sozialpolitik und Arbeitsförderung aufkommunaler Ebene in: Mezger, Erika/West Klaus-W. (Hrsg.): Aktivierender Sozialstaat und politisches Handeln, Schüren-Verlag, Marburg, S. 41-49.

Schuppert, Gunnar Folke (2000): Verwaltungswissenschaft, Verwaltung, Verwaltungsrecht, Verwaltungslehre, Nomos Verlagsgesellschaft, Baden-Baden.

Schuppert, Gunnar Folke (2001): Der moderne Staat als Gewährleistungsstaat, in: Schröter, Eckhard (Hg.): Empirische Policy- und Verwaltungsforschung. Lokale, nationale und internationale Perspektiven, Opladen: Westdeutscher Verlag, S. 399-414.

Seils, Eric (2004): Sozialstaat und Arbeitsmarkt im Strukturwandel. Eine Analyse des Anpassungsprozesses in der Bundesrepublik, in Wohlfahrtsstaat Transformation und Perspektiven, VS Verlag für Sozialwissenschaften, Wiesbaden, S. 155-178.

Sociaal Economische Raad/Wetenschapelijke Raad voor het Regeringsbeleid (2007): Toekomst van de Nederlandse verzorgingsstaat, SER/WRRsymposium over de toekomst van de Nederlandse verzorgingsstaat op 16 januari 2007.

Sozialberichterstattung Gesellschaftliche Trends Aktuelle Informationen (2002): Allmählicher Umbau bei nach wie vor deutlichen nationalen Unterschieden, Analysen zu jüngsten Wandlungstendenzen in westlichen Wohlfahrtsstaaten, in: Informationsdienst Soziale Indikatoren, Ausgabe 28, Juli 2002, S. 1-6. 
Trommel Willem (2007): De stagnatie voorbij? Over de verdiensten van de sociale investeringsgedachte, in: Van sociale bescherming naar sociale investering; Zoektocht naar een andere verzorgingsstaat. Uitgeverij LEMMA, Den Haag, S.247-279.

Visser, Jelle/Hemerijck, Anton (1997): A Dutch Miracle - Job Growth, Welfare Reform and Corporatism in the Netherlands, Amsterdam University Press, Amsterdam.

Walter, Jürgen (2000): Pleite oder Neubeginn einer sozialen Staats- und Bildungs(reform)politik, in: Mezger, Erika/West Klaus-W. (Hrsg.): Aktivierender Sozialstaat und politisches Handeln, Schüren-Verlag, Marburg, S. 151-165.

West, Klaus-W. (2000): Reformen für den Sozialstaat, gesellschaftliche Mehrheiten für Reformen, in: Mezger, Erika/West Klaus-W. (Hrsg.): Aktivierender Sozialstaat und politisches Handeln, Schüren-Verlag, Marburg, S. 173189 .

Wetenschappelijke Raad voor het Regeringsbeleid (2006): De verzorgingsstaat herwogen; Over verzorgen, verzekeren, verheffen en verbinden, Amsterdam University Press, Amsterdam.

Wingen, Max (1994): Zur Theorie und Praxis der Familienpolitik, Eigenverlag des deutschen Vereins für öffentliche und private Fürsorge, Frankfurt am Main.

Wohlfahrt, Norbert (2007a): Der aktivierende Sozialstaat - Ein neues sozialpolitisches Konzept und seine Konsequenzen, http://www.transparentonline.de/Nr63_o6.htm (aufgerufen am 19. Mai 2007).

Wohlfahrt, Norbert (2007b): Bürgerschaftliches Engagement, Freie Wohlfahrtspflege und aktivierender Sozialstaat - inszenierter Sozialstaatsumbau oder Stärkung des sozialen Kapitals?, www.efhbochum.de/homepages/wohlfahrt/pdf/engagement.pdf (aufgerufen am 23.10.2007). 


\section{Kapitel 3}

Bundesministerium für Familie und Senioren (1993): 40 Jahre Familienpolitik in der Bundesrepublik Deutschland, Rückblick/Ausblick, Luchterhand Bonn.

Bundesministerium für Familie, Senioren, Frauen und Jugend (1994a): Fünfter Familienbericht. Familien und Familienpolitik im geeinten Deutschland. Zukunft des Humanvermögens, Bonn.

Bundesministerium für Familie, Senioren, Frauen und Jugend (1994b): Neunter Jugendbericht. Bericht über die Situation der Kinder und Jugendlichen und die Entwicklung der Jugendhilfe in den neuen Bundesländern, Bonn.

Bundesministerium für Familie, Senioren, Frauen und Jugend (1998): Zehnter Kinder- und Jugendbericht. Bericht über die Lebenssituation von Kindern und die Leistungen der Kinderhilfen in Deutschland, Bonn.

Bundesministerium für Familie, Senioren, Frauen und Jugend (2000): Sechster Familienbericht. Familien ausländischer Herkunft in Deutschland: Leistungen - Belastungen - Herausforderungen, Berlin.

Bundesministerium für Familie, Senioren, Frauen und Jugend (2002): Elfter Kinder- und Jugendbericht, Bericht über die Lebenssituation junger Menschen und die Leistungen der Kinder- und Jugendhilfe, Berlin.

Bundesministerium für Familie, Senioren, Frauen und Jugend (2005): Zwölfter Kinder- und Jugendbericht, Bericht über die Lebenssituation junger Menschen und die Leistungen der Kinder- und Jugendhilfe, Bildung, Betreuung und Erziehung vor und neben der Schule, Berlin.

Bundesministerium für Familie, Senioren, Frauen und Jugend (2006): Siebter Familienbericht. Familie zwischen Flexibilität und Verlässlichkeit, Perspektiven für eine lebenslaufbezogene Familienpolitik, Berlin.

Bundesministerium für Familie, Senioren, Frauen und Jugend (2009): Bericht über die Lebenssituation junger Menschen und die Leistungen der Kinder- und Jugendhilfe in Deutschland - 13. Kinder- und Jugendbericht - und Stellungnahme der Bundesregierung, Berlin. 
Bundesregierung (1998a): Aufbruch und Erneuerung - Deutschlands Weg ins 21. Jahrhundert, Koalitionsvereinbarung zwischen der Sozialdemokratischen Partei Deutschlands und Bündnis 90/Die Grünen, Bonn.

Bundesregierung (1998b): Regierungserklärung von Bundeskanzler Gerhard Schröder vom 10. November 1998 vor dem Deutschen Bundestag, Bonn.

Bundesregierung (1999): Moderner Staat - Moderne Verwaltung: Das Programm der Bundesregierung. Kabinettsbeschluss vom 1. Dezember 1999, Berlin, Bundesregierung.

Bundesregierung (2002): Erneuerung - Gerechtigkeit - Nachhaltigkeit, Für ein wirtschaftlich starkes, soziales und ökologisches Deutschland. Für eine lebendige Demokratie, Koalitionsvereinbarung 2002 zwischen der Sozialdemokratischen Partei Deutschlands und Bündnis 90/Die Grünen, Berlin.

Bundesregierung (2003): Regierungserklärung von Bundeskanzler Schröder am 14. März vor dem Deutschen Bundestag, Berlin.

Bundesregierung (2005a): Gemeinsam für Deutschland - mit Mut und Menschlichkeit, Koalitionsvertrag zwischen CDU, CSU und SPD, Berlin.

Bundesregierung (2005b): Regierungserklärung von Bundeskanzlerin Angela Merkel, Berlin.

Bundesregierung (2009a): Wachstum. Bildung. Zusammenhalt., Koalitionsvertrag zwischen CDU, CSU und FDP, Berlin.

Bundesregierung (2009b): Regierungserklärung, Berlin.

\section{Kapitel 4}

Jeugd en Gezin (2007): Alle kansen voor alle kinderen - Programma voor Jeugd en Gezin - 2007-2011, Den Haag. 
Jeugd en Gezin (2008): De kracht van het gezin, Nota Gezinsbeleid 2008, Den Haag.

Ministerie van Algemene Zaken (1994a): Regeerakkoord van 13 augustus 1994, Den Haag.

Ministerie van Algemene Zaken (1994b): Regeringsverklaring van 31 augustus 1994, Den Haag.

Ministerie van Algemene Zaken (1998a): Regeerakkoord van 18 juli 1998, Den Haag.

Ministerie van Algemene Zaken (1998b): Regeringsverklaring van 25 augustus 1998, Den Haag.

Ministerie van Algemene Zaken (2002a): Werken aan vertrouwen, een kwestie van aanpakken, Strategisch Akkoord voor Kabinet CDA, LPF, VVD van 3. juli 2002, Den Haag.

Ministerie van Algemene Zaken (2002b): Regeringsverklaring van 26. juli 2002, Duidelijkheid en Daadkracht, Den Haag.

Ministerie van Algemene Zaken (2003a): Meedoen, meer werk, minder regels, Hoofdlijnenakkoord voor het kabinet CDA, VVD, D66 van 16. mei 2003, Den Haag.

Ministerie van Algemene Zaken (2003b): Regeringsverklaring van 11 Juni 2003, Den Haag.

Ministerie van Algemene Zaken (2005): Afspraken 26 maart 2005, Den Haag.

Ministerie van Algemene Zaken (2006): Regeringsverklaring Balkenende-III, Den Haag.

Ministerie van Algemene Zaken (2007a): Coalitieakkoord tussen de Tweede Kamerfracties van CDA, PvdA en ChristenUnie van 7. februari 2007, Den Haag.

Ministerie van Algemene Zaken (2007b): Regeringsverklaring van 1 maart 2007, Den Haag.

Ministerie van Volksgezondheid, Welzijn en Sport (1996): Notitie Gezin, de Maatschappelijke positie van het gezin, Rijswijk. 
Ministerie van Volksgezondheid, Welzijn en Sport (2006): Nota gezinsbeleid, Den Haag.

Ministerie van Volksgezondheid, Welzijn en Sport (2007): Jeugd en Gezin, Programmaministerie, www.minvws.nl (aufgerufen am 13.08.2007).

\section{Kapitel 5}

Bundesregierung (2005a): Gemeinsam für Deutschland - mit Mut und Menschlichkeit, Koalitionsvertrag zwischen CDU, CSU und SPD, Berlin.

Evers, Adalbert (2007): Aktivierender Staat - Ein Beitrag zur Vitalisierung der Bürgergesellschaft oder nur ein Weg zur Ertüchtigung von Arbeitskraftunternehmen?, www.tup-online.com/media/md2567D.pdf, (aufgerufen am 23.10.2007).

Evers, Adalbert (2004): Zivilgesellschaft und aktivierender Staat, Vortrag auf dem Kongress "Bürger machen Stadt" in Düsseldorf am 18.06.2004, veranstaltet vom Ministerium für Städtebau und Wohnen, Kulturelles und Sport des Landes NRW.

Gerlach, Irene (2010): Familienpolitik - 2., aktualisierte und überarbeitete Auflage, Lehrbuch, VS Verlag für Sozialwissenschaften/GWV Fachverlage GmbH, Wiesbaden.

Knijn, Trudie (2007): Tussen kinderdagverblijf en verzorgingstehuis - Gezinsbeleid en levenslopen, in: Van sociale bescherming naar sociale investering; Zoektocht naar een andere verzorgingsstaat. Uitgeverij LEMMA, Den Haag, S. $55-81$.

Kremer, Monique (2007): How Welfare States Care, Culture, Gender and Parenting in Europe, Amsterdam University Press, Amsterdam.

Ministerie van Algemene Zaken (1994a): Regeerakkoord van 13 augustus 1994, Den Haag.

OECD (2002): Babies and Bosses: Reconciling Work and Family Life, OECD Publications Service, Paris. 
Opielka, Michael (2004): Sozialpolitik; Grundlagen und vergleichende Perspektiven, Rowohlt Verlag GmbH, Reinbeck bei Hamburg.

Wetenschappelijke Raad voor het Regeringsbeleid (2006): De verzorgingsstaat herwogen; Over verzorgen, verzekeren, verheffen en verbinden, Amsterdam University Press, Amsterdam.

\section{Kapitel 6}

Bandemer, Stephan, von/Hilbert, Josef (2005): Vom expandierenden zum aktivierenden Staat, in: Blanke, Bernhard et al. (Hg.): Handbuch zur Verwaltungsreform, 3. völlig überarbeitete und erweiterte Auflage, VS Verlag für Sozialwissenschaften, Wiesbaden, S. 26-35.

Bundesministerium für Familie, Senioren, Frauen und Jugend (2009): Geschichte des Ministeriums, http://www.bmfsfj.de/bmfsfj/generator/BMFSFJ/Ministerium/geschic hte.html, aufgerufen am 04.12.2009.

Esping-Andersen, Gøsta (2009): The Incomplete Revolution - Adapting to Women's New Roles, Polity Press, Cambridge.

Evers, Adalbert (2004): Zivilgesellschaft und aktivierender Staat, Vortrag auf dem Kongress "Bürger machen Stadt" in Düsseldorf am 18.06.2004, veranstaltet vom Ministerium für Städtebau und Wohnen, Kulturelles und Sport des Landes NRW.

Leibfried, Stephan (1999): Sozialstaat Europa?, In: Gewerkschaftliche Monatshefte 50, Heft 12, S. 766-770.

Leibfried, Stephan/Zürn, Michael (2006): Transformation des Staates?, Suhrkamp Verlag, Frankfurt am Main.

Jann, Werner (2005): Governance als Reformstrategie - Vom Wandel und der Bedeutung verwaltungspolitischer Leitbilder, in: Schuppert, Gunnar Folke (Hrsg.): Governance - Forschung. Vergewisserung über Stand und Entwicklungslinien, Nomos, Baden-Baden, S. 21-43.

Kevenhörster, Paul (2006): Politikwissenschaft, Band 2, Ergebnisse und Wirkungen der Politik, VS-Verlag, Wiesbaden. 
Knijn, Trudie (2007): Tussen kinderdagverblijf en verzorgingstehuis Gezinsbeleid en levenslopen, in: Van sociale bescherming naar sociale investering; Zoektocht naar een andere verzorgingsstaat. Uitgeverij LEMMA, Den Haag, S.79.

Kommission der Europäischen Gemeinschaften (2008): Mitteilung der Kommission an das Europäische Parlament, den Rat, den Europäischen Wirtschafts- und Sozialausschuss und den Ausschuss der Regionen; Halbzeitbilanz zu den Fortschritten beim Fahrplan für die Gleichstellung von Frauen und Männern (2006-2010), Brüssel.

Lütz, Susanne (2004): Der Wohlfahrtsstaat im Umbruch - Neue

Herausforderungen, wissenschaftliche Kontroversen und Umbauprozesse, in: Wohlfahrtsstaat - Transformationen und Perspektiven, VS Verlag für Sozialwissenschaften, Wiesbaden, S. 11-35.

Olk, Thomas (2000): Weder Rund-um-Versorgung noch "pure" Eigenverantwortung - aktivierende Strategien in der Politik für Familien, alte Menschen, Frauen, Kinder und Jugendliche, in: Mezger, Erika/West Klaus-W. (Hrsg.):

Aktivierender Sozialstaat und politisches Handeln, Schüren-Verlag, Marburg, S. 105-124.

Opielka, Michael (2004): Sozialpolitik; Grundlagen und vergleichende Perspektiven, Rowohlt Verlag GmbH, Reinbeck bei Hamburg, S. 247.

Oppeln, Sabine von (2007): Das Europäische Sozialmodell, Bilanz und Perspektiven, Berliner Arbeitspapier zur Europäischen Integration Nr. 3, Jean Monnet Lehrstuhl für Europäische Integration, Berlin.

Wetenschappelijke Raad voor het Regeringsbeleid (2006): De verzorgingsstaat herwogen; Over verzorgen, verzekeren, verheffen en verbinden, Amsterdam University Press, Amsterdam, S. 120f, S. 156, S. 263. 


\section{Anlagen}

\section{Anlage Kapitel 1}

Politisch-kulturelle Entwicklungen in den Niederlanden seit 1945

Zusammenfassung aus: NiederlandeNet, Die Niederlande nach 1945: Politik und politische Kultur, www.niederlandenet.de, aufgerufen am 30.07.2007.

Während politisch-administrative Leitbilder, die $\mathrm{zu}$ folgende Reformroute beschreiben, gibt es auch politisch-kulturelle Metaphoren, welche rückblickend Politiken und Kulturen charakterisieren und einordnen. Die politischen und politisch-kulturellen Entwicklungen der Niederlande (seit 1945) kann man z.B. in drei Kategorien unterteilen:

- „Phase 1: Wiederaufbau im Konsens 1945-1958“ (NiederlandeNet, Die Niederlande nach 1945: Politik und politische Kultur, www.niederlandenet.de, aufgerufen am 30.07.2007).

Die erste Phase kennzeichnete sich durch Kontinuität einerseits und ersten Brüchen andererseits. Nach „1945 blieb die politische Erneuerung aus, die Versäulung wurde nicht durchbrochen und beinahe alle früheren politischen Parteien kehrten auf die Bühne zurück. Im Gegensatz zu den Vorkriegsjahren erhielt der Staat eine wesentliche Rolle in den wirtschaftlichen und sozialen Verhältnissen“ (NiederlandeNet, Die Niederlande nach 1945: Politik und politische Kultur, www.niederlandenet.de (aufgerufen am 30.07.2007) und war neuerdings die Suche nach dem Konsens zwischen Staat, Arbeitergebern und Arbeitnehmern entscheidend.

- „Phase 2: Wohlstand, Entsäulung und Polarisierung 1958-1977: die langen sechziger Jahre" (NiederlandeNet, Die Niederlande nach 1945: Politik und politische Kultur, www.niederlandenet.de, aufgerufen am 30.07.2007). Sowohl wirtschaftlich als auch kulturell kam es in dieser Periode zu großen Veränderungen. Der Wohlstand, von dem ein sehr großer Teil der Bevölkerung profitieren konnte, nahm in einem relativ schnellen Tempo zu. Gleichzeitig kam es zur Entsäulung, d.h. die vier Säulen in welche sich 
die Gesellschaft zuvor unterteilte (Protestanten, Katholiken, Sozialisten und Liberale) zerfielen durch politische Veränderungen und die

Säkularisierung. Neue politische Strömungen entwickelten sich. Politische Gegensätze wurden langsam größer, die gesellschaftlichen Spannungen nahmen zu. Es kam zu Protesten und Blockaden. „Das herrschende Muster der 5oer Jahre, dass die versäulten Eliten ihren Einfluss auf die eigene Anhängerschaft noch beinahe unangetastet ausüben konnten, (wurde) abgelöst (...) von einer politischen Kultur der Individualisierung, Emanzipation und Demokratisierung“ (NiederlandeNet, Die Niederlande nach 1945: Politik und politische Kultur, www.niederlandenet.de, aufgerufen am 30.07.2007).

- Phase 3: Realismus, ,no nonsense' und erneuter Konsens 1977-2002“ (NiederlandeNet, Die Niederlande nach 1945: Politik und politische Kultur, www.niederlandenet.de, aufgerufen am 30.07.2007). Wie auch in vielen anderen Ländern wurde durch sowohl wirtschaftliche, gesellschaftliche als auch ökologische Krisen politisches Umdenken erforderlich. Reformen kennzeichnete Realismus gepaart mit Sparmaßnahmen, um die staatlichen Ausgaben, bzw. die zu dem Zeitpunkt hohen niederländischen Löhne zu drosseln. „Dieser Prozess verlief weder schnell noch schmerzlos und anfänglich setzte sich die Polarisierung der Goer und 7oer Jahre noch fort. Gleichzeitig wurde mit dem berühmten Abkommen von Wassenaar (1982) zwischen Arbeitgebern und Arbeitnehmern der Weg zu einem erneuten sozialen Konsens (,Poldermodel““) eingeschlagen“ (NiederlandeNet, Die Niederlande nach 1945: Politik und politische Kultur, www.niederlandenet.de, aufgerufen am 30.07.2007). 


\section{Anlage Kapitel 2}

Zusammenfassungen der Instrumente aus dem Modell Esping-Andersens

Instrumente, die die ökonomische Situation der Familienhaushalte betreffen (ökon. I.):

- Zweiverdienermodell,

- $\quad$ Flexible Arbeitszeit,

- $\quad$ Teilzeit,

- Finanzielle Unterstützung Immobilienpreise,

- Lohnzuschüsse bzw. Einkommenszuschüsse,

- Workfare bzw. Sozialinvestition,

- Investitionen in Kindheit,

- $\quad$ Einkommen von Eltern.

Instrumente, die die ökologischen Bedingungen des Familienlebens betreffen (ökol. I.):

- $\quad$ Reguläre, staatliche und bezahlbare Kinderbetreuung,

- $\quad$ Regelungen für Kinderbetreuung im Falle von Krankheit,

- Dienstleistungen zur Pflege älterer Menschen,

- Verstärkung der Arbeitsumgebung arbeitender Mütter,

- Gut ausgebildetes Personal fördert frühkindliche Entwicklung.

Instrumente, welche die Förderung oder Wiederherstellung von Kompetenzen der Familienangehörigen betreffen (I. K.):

- Workfare und Sozialinvestition,

- Lebenslanges Lernen,

- Förderung frühkindlicher Entwicklung durch z.B. Vorlesen,

- Hochwertige Ausbildung ab jungem Alter. 
Instrumente, die Familienangehörigen Flexibilität verschaffen und ihnen Zeit für ihre Familie bieten (Z. I.):

- Flexible Arbeitszeiten,

- Teilzeit,

- Bezahlte Elternzeit,

- $\quad$ Lebenszeitmodelle. 
erschienen in der Reihe Q-Serie der Humboldt-Universität zu Berlin 
In dieser Dissertation wird der Einfluss bzw. die Umsetzung des Leitbildes Aktivierender Staat auf die Familienpolitik in Deutschland und den Niederlanden untersucht. Anhand der Theorien von unter anderem Esping-Andersen, Kaufmann und Bandemer entwickelt der Autor ein Idealmodell Aktivierender Familienpolitik und verwendet es in einer ausführlichen politisch-administrativen Analyse. Hieraus ergeben sich nicht nur eine Fülle von Aktivierungsbeispielen, sondern auch Antworten auf Fragen nach möglichen Konvergenzen zwischen beiden Ländern sowie der Weiterentwicklung des Leitbildes Aktivierender Staat.

ISBN 978-3-86004-266-3 